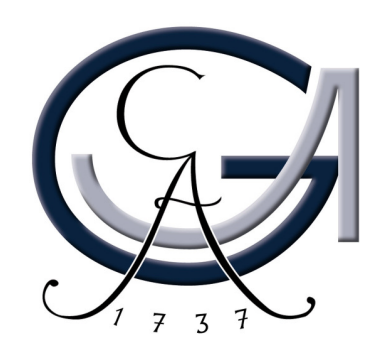

\title{
Single-molecule experiments with mitotic motor proteins
}

\author{
Dissertation \\ zur Erlangung des \\ mathematisch-naturwissenschaftlichen Doktorgrades \\ „Doctor rerum naturalium" \\ der Georg-August-Universität zu Göttingen
}

vorgelegt von

Christina Thiede

aus Athen

Göttingen 2012 
Mitglieder des Betreuungsausschusses:

Prof. Dr. Christoph F. Schmidt (Referent)

Drittes Physikalische Institut

Georg-August Universität, Göttingen

Prof. Dr. Jörg Enderlein (Koreferent)

Drittes Physikalische Institut

Georg-August Universität, Göttingen

Prof. Dr. Helmut Grubmüller

Abteilung für theoretische und computergestützte Biophysik

Max-Planck-Institut für biophysikalische Chemie, Göttingen

Tag der mündlichen Prüfung: 28. September 2012 


\section{Affidavit}

Herewith I affirm that this thesis has been written independently and with no other sources and aids than quoted.

Christina Thiede

Göttingen, 23. October 2012 



\section{Preface}

This cumulative PhD thesis consists of the following accepted publications and submitted manuscript:

\section{Accepted publications:}

Stefan Lakämper, Christina Thiede, Andrè Düselder, Stefanie Reiter, Mikhail J. Korneev, Lukas C. Kapitein, Erwin J. G. Peterman and Christoph F. Schmidt; 2010: The Effect of Monastrol on the Processive Motility of a Dimeric Kinesin-5 Head/Kinesin-1 Stalk Chimera, Journal of Molecular Biology, 399: 1-8.

Adina Gerson-Gurwitz*, Christina Thiede*, Natalia Movshovich, Vladimir Fridman, Maria Podolskaya, Tsafi Danieli, Stefan Lakämper, Dieter R. Klopfenstein, Christoph F. Schmidt and Larisa Gheber; 2011: Directionality of individual kinesin5 Cin 8 motors is modulated by loop 8, ionic strength and microtubule geometry, The EMBO Journal, 30: 4942-4954. *Authors contributed equally.

Christina Thiede, Vladimir Fridman, Adina Gerson-Gurwitz, Larisa Gheber and Christoph F. Schmidt; 2012: Regulation of bi-directional movement of single kinesin5 Cin8 molecules, BioArchitecture, 2: 70-74.

Andrè Düselder*, Christina Thiede*, Christoph F. Schmidt and Stefan Lakämper; 2012: Neck-Linker Length Dependence of Processive Kinesin-5 Motility, Journal of Molecular Biology, 423: 159-168. *Authors contributed equally.

Viviana Valdés, José Ignacio Valenzuela, Matías Jaureguiberry-Bravo, Daniela Salas, Carolina Otero, Christina Thiede, Christoph F. Schmidt and Andrés Couve; 2012: Endoplasmic Reticulum Sorting and Kinesin-1 Command the Targeting of Axonal GABA $_{B}$ Receptors, PLoS ONE, 7: e44168.

\section{Submitted manuscript:}

Christina Thiede*, Stefan Lakämper*, Alok D. Wessel, Stefanie Kramer and Christoph F. Schmidt; 2012: A chimeric Kinesin-1/Kinesin-5 microtubule-sliding motor switches between diffusive and processive motility, submitted to Biophysical Journal. *Authors contributed equally. 



\section{Table of contents}

\begin{tabular}{l}
\hline List of abbreviations \\
Glossary \\
\hline
\end{tabular}

Chapter 2 Directionality of individual kinesin-5 Cin8 motors is modulated by loop 8, ionic strength and microtubule geometry

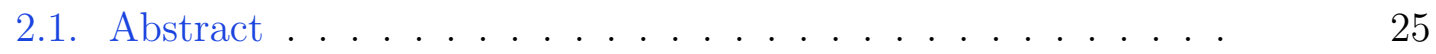

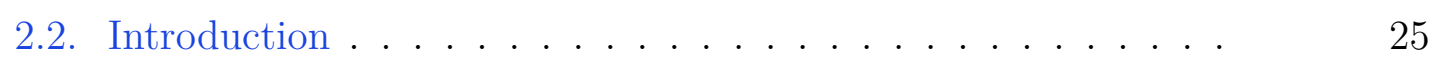

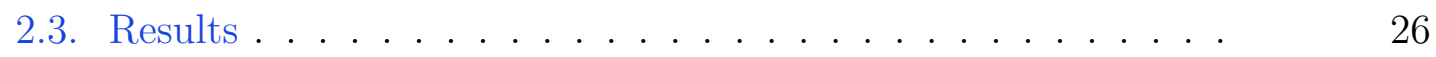

2.4. Discussion . . . . . . . . . . . . . . . . . . . 39

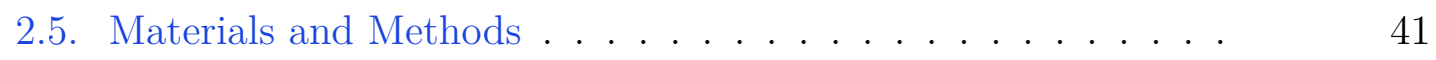

2.6. Acknowledgements .................. . . . . . 42

Chapter 3 Regulation of bi-directional movement of single kinesin-5 Cin8 molecules $\quad 45$

3.1. Abstract ....................... . . . . 45

3.2. Short communication ................. 45

Chapter 4 The effect of monastrol on the processive motility of a dimeric kinesin-5 head/kinesin-1 stalk chimera 51

4.1. Abstract . . . . . . . . . . . . . . . . 51

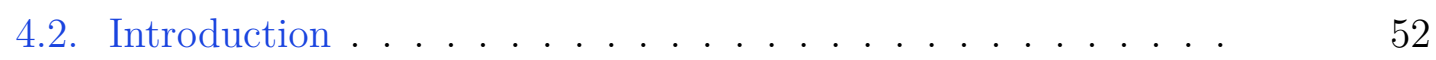

4.3. Results and Discussion . . . . . . . . . . . . . . . 53

4.4. Materials and Methods . . . . . . . . . . . . . . . . . . . 62

4.5. Acknowledgements ................... 64

Chapter 5 Neck-linker length dependence of processive kinesin-5 motility $\quad 65$

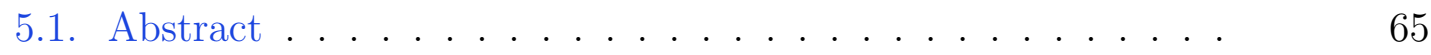

5.2. Introduction . . . . . . . . . . . . . . . 66

5.3. Results and Discussion . . . . . . . . . . . . 69 
5.4. Materials and Methods . . . . . . . . . . . . . . . 75

5.5. Acknowledgements . . . . . . . . . . . . . . . 77

Chapter 6 A chimeric kinesin-1/kinesin-5 microtubule-sliding motor switches between diffusive and processive motility

6.1. Abstract ............................ 79

6.2. Introduction . . . . . . . . . . . . . . . 79

6.3. Results and Discussion . . . . . . . . . . . . . . 81

6.4. Conclusions . . . . . . . . . . . . . . . . 90

6.5. Materials and Methods . . . . . . . . . . . . . . . 91

6.6. Acknowledgements . . . . . . . . . . . . . . . 94

Chapter 7 Endoplasmic reticulum sorting and kinesin-1 command the targeting of axonal $\mathrm{GABA}_{B}$ receptors $\quad 95$

7.1. Abstract . . . . . . . . . . . . . . . 95

7.2. Introduction . . . . . . . . . . . . . . . . 96

7.3. Results . . . . . . . . . . . . . . . . . . . 97

7.4. Discussion . . . . . . . . . . . . . . . . 106

7.5. Materials and Methods . . . . . . . . . . . . . . 109

7.6. Acknowledgements . . . . . . . . . . . . . . . 112

$\begin{array}{lll}\text { Chapter } 8 & \text { Conclusion } & 113\end{array}$

$\begin{array}{lll}\text { Chapter } 9 & \text { Danksagung/Acknowledgements } & 117\end{array}$

\section{Appendices}

Chapter A Supplemetatry data chapter 2: Directionality of individual kinesin-5 Cin8 motors is modulated by loop 8, ionic strength and microtubule geometry I

A.1. Supplementary figures .................... I I

A.2. Supplementary table . . . . . . . . . . . . . VI

A.3. Description of supplementary videos . . . . . . . . . . . . . VII

A.4. Supplementary materials and methods . . . . . . . . . I IX

Chapter B Supplemetatry data chapter 4: The effect of monastrol on the processive motility of a kinesin- 5 head/kinesin-1 stalk chimera XIII

B.1. Supplementary figures . . . . . . . . . . . . . XIII

B.2. Supplementary table ................. X XVI

B.3. Description of supplementary videos . . . . . . . . . XVI 
Chapter C Supplemetatry data chapter 5: Neck-linker length dependence of processive kinesin-5 motility XIX

C.1. Supplementary figures . . . . . . . . . . . . . . .

Chapter D Supplemetatry data chapter 6: A chimeric kinesin-1/kinesin-5 microtubule-sliding motor switches between diffusive and processive motility

D.1. Supplementary figures . . . . . . . . . . . . . . XXIII

D.2. Supplementary table ................ XXVI

D.3. Description of supplementary videos . . . . . . . . XXVI

Chapter E Supplemetatry data chapter 7: Endoplasmic reticulum sorting and kinesin-1 command the targeting of axonal $\mathrm{GABA}_{B}$ receptors XXIX E.1. Supplementary figures . . . . . . . . . . . . . . . XXIX

List of references

\section{Curriculum vitae}





\section{List of abbreviations}

\begin{tabular}{|c|c|}
\hline aa & Amino acid \\
\hline ADP & Adenosine-5'-diphosphate \\
\hline $\mathrm{AM}$ & Assay mix \\
\hline AMP-PNP & 5'-adenylyl- $\beta, \gamma$-imidodiphosphate \\
\hline ATP & Adenosine-5'-triphosphate \\
\hline BME & 2-Mercaptoethanol \\
\hline BRB80 & 80 mM PIPES buffer \\
\hline BSA & Bovine serum albumin \\
\hline BSC-1 cells & Monkey kidney epithelial cells \\
\hline Cdk1 & Cyclin-dependent kinase 1 \\
\hline CEN & Centromeric plasmid \\
\hline COPI & Coat protein complex I \\
\hline COPII & Coat protein complex II \\
\hline $\mathrm{Cy} 3$ & Cyanine 3 \\
\hline DETA & 3-(2-[2-aminoethylamino] ethylamino) propyl-trimethoxysilane \\
\hline $\operatorname{div}$ & Days in vitro \\
\hline DmKHC & Drosophila melanogaster kinesin-1 heavy chain \\
\hline DMSO & Dimethyl sulfoxide \\
\hline dpt & Day post transfection \\
\hline DTT & Dithiothreitol \\
\hline E. coli & Escherichia coli \\
\hline EDTA & Ethylene diamine tetraacetic acid \\
\hline EGTA & Ethylene glycol tetraacetic acid \\
\hline ER & Endoplasmic reticulum \\
\hline ERGIC & ER-to-Golgi intermediate compartment \\
\hline FITC & Fluorescein isothiocyanate \\
\hline GABA & $\gamma$ amino-butyric acid \\
\hline
\end{tabular}




\begin{tabular}{|c|c|}
\hline G1-phase & Gap-1-phase \\
\hline G2-phase & Gap-2-phase \\
\hline GFP & Green fluorescent protein \\
\hline GMP-CPP & Guanosine-5'-[( $\alpha, \beta)$-methyleno] triphosphate \\
\hline GTP & Guanosine-5'-triphosphate \\
\hline $\mathrm{IC}_{50}$ & Half maximal inhibitory concentration \\
\hline iMTs & Interpolar microtubules \\
\hline $\mathrm{kMTs}$ & Kinetochore microtubules \\
\hline NA & Numerical aperture \\
\hline MAP2 & Microtubule-associated protein 2 \\
\hline MB & Motility buffer \\
\hline MD & Mean displacement \\
\hline $\mathrm{MgCl}_{2}$ & Magnesium chloride \\
\hline MMGA & Multi motor gliding assay \\
\hline MSD & Mean square displacement \\
\hline $\mathrm{MT} / \mathrm{MTs}$ & Microtubule/Microtubules \\
\hline NEM & N-ethylmaleimide \\
\hline PEM12 & 12 mM PIPES buffer \\
\hline PIPES & piperazine-N,N'-bis(2-ethanesulfonic acid) \\
\hline S. cerevisiae & Saccharomyces cerevisiae \\
\hline SD & Synthetic defined broth \\
\hline Sf9 & Spodoptera frugiperda \\
\hline SMF & Single-molecule fluorescence \\
\hline SPBs & Spindle-pole bodies \\
\hline TIRF & Total internal reflection fluorescence \\
\hline TMR & Tetramethylrhodamine \\
\hline TPR & Tetratricopeptidrepeat \\
\hline TR & Texas Red \\
\hline Tris & Tris(hydroxymethyl)-aminomethan \\
\hline TRITC & Tetramethyl rhodamine isothiocyanate \\
\hline WT & Wild type \\
\hline YPD & Yeast extract peptone dextrose \\
\hline
\end{tabular}




\section{Glossary}

In this glossary possibly unclear terms and definitions that can - due to copy right restrictions - not be explained in the main text will be shortly explained in order of appearance.

\section{Michaelis-Menten theory}

The Michaelis-Menten theory describes enzyme kinetics. While stepping along the microtubule the motor protein works as enzyme that hydrolyses ATP to ADP. The mean motor velocity $V$ at a given ATP concentration is plotted against the respective ATP concentration $[A T P]$ and fitted with the Michaelis-Menten function:

$$
V=\frac{V_{\max }[A T P]}{K_{M}+[A T P]}
$$

The resulting parameters are the maximal motor velocity $V_{\max }$ and the MichaelisMenten constant $K_{M}$.

$\mathrm{IC}_{50}$

The $\mathrm{IC}_{50}$ gives an account of the half maximal inhibitory concentration of a particular substance (here, monastrol). In other words, this quantitative value indicates how much of the inhibitor is needed to inhibit a given process by half (here, MT gliding velocity). 



\section{Introduction}

In 1985, Vale et al. found a new protein [Vale85], which was

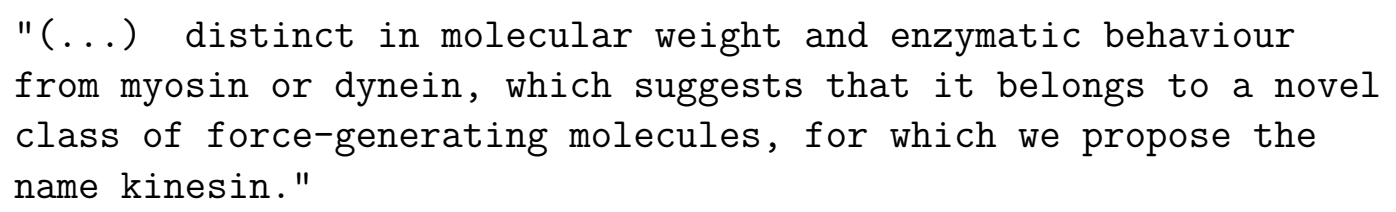

As a possible biological role they hypothesised that

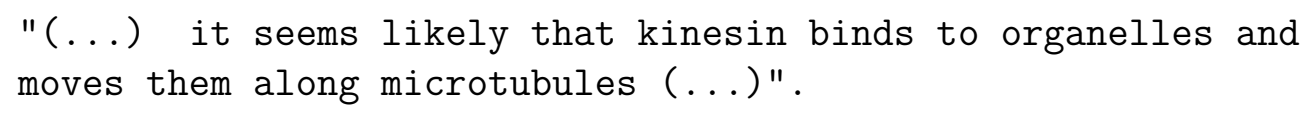

Since then the family of the kinesin motor proteins has grown and stands today at more then 100 members, which are categorised into 14 subfamilies [Lawrence04]. Due to their diverse and crucial roles in the cell, their molecular functions and regulations are still the focus of on-going research. The aim of this thesis is to gain further insight into the regulation of mitotic motor proteins from the kinesin subfamily kinesin-5.

In the subsequent sections, the functions of mitotic motor proteins in cellular processes and kinesin regulation mechanisms will be introduced.

\section{Cell division in eukaryotes $\mid 1.1$}

\section{Stages of mitotic cell division}

Cell division in eukaryotic cells is a highly complex process that divides a mother cell into daughter cells. There are two types of cell division: mitosis and meiosis. In mitosis, two identical sister cells form, leaving each of them capable of dividing again. In meiosis (which will not be described here further), a cell is permanently transformed into a gamete that can fuse with another cell during fertilisation but cannot divide itself again until fertilisation. 
A
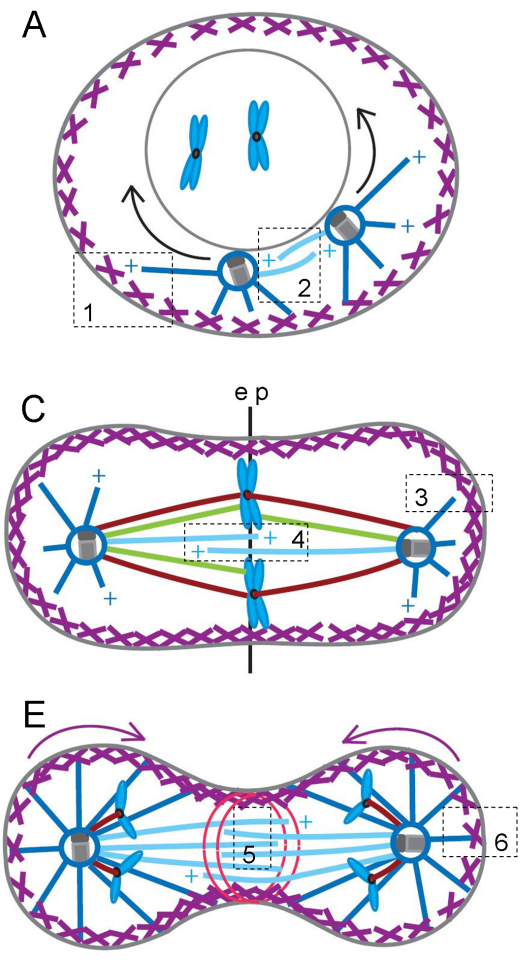

$x \times$ Cell cortex $($ Centrosome

(4) Nucleus

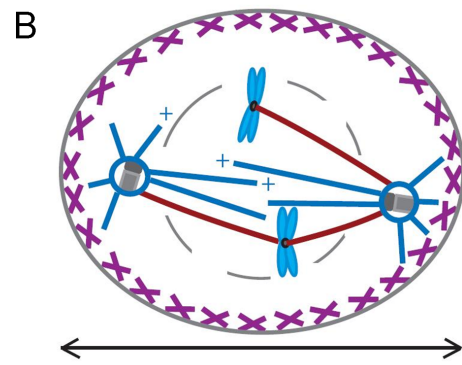

D

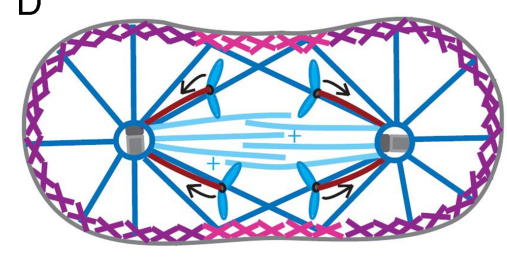

$\mathrm{F}$

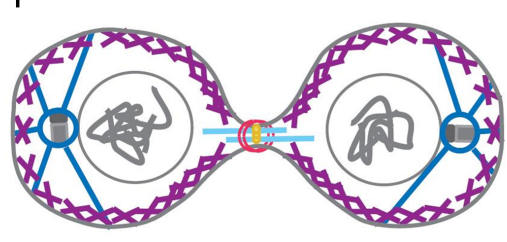

Chromosome $=9=$ Midbody

Kinetochore ( $\because ; \quad$ Contractile ring

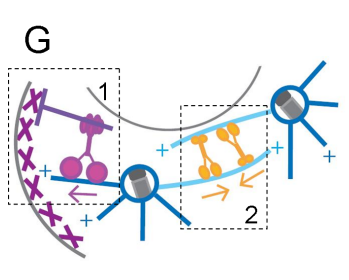

$\mathrm{H}$

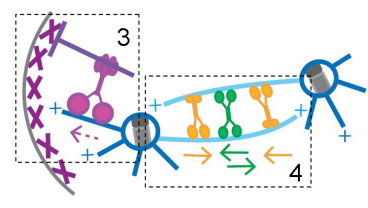

I
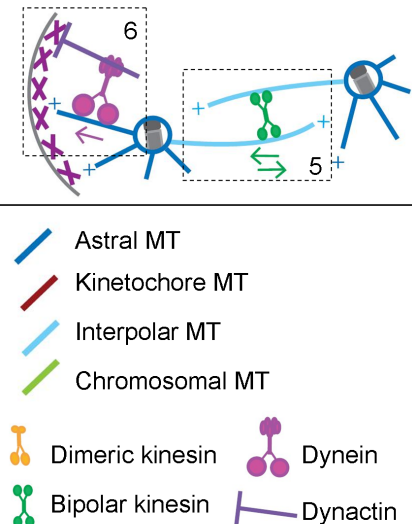

Fig. 1.1: Overview of the processes during mitosis and cytokinesis (based on [Sharp00, Scholey03]).

(A) Prophase, the replicated chromosomes condense and the duplicated centrosomes migrate around the nucleus, initiating the formation of the mitotic spindle.

Prometaphase, the breakdown of the nuclear envelope allows the kinetochore MTs (red) to connect to the chromosomes and to move them towards the equatorial plane (ep). (C) Metaphase, the sister chromatids are aligned in the equatorial plane and via the kinetochore MTs connected to the opposite spindle poles. (D) Anaphase A, in the segregation process the chromatids are moved to opposite poles. Anaphase B, the spindle poles move apart due to reorganisation of the spindle MTs. (E) During late anaphase the chromatids reach the spindle poles and the cleavage furrow containing the contractile ring assembles and begins to contract. (F) Telophase, the sister chromosomes decondense and new nuclear envelopes reassemble around them. Cytokinesis, the cytoplasm of one cell is divided in two by the contractile ring, which causes the furrow to ingress and finally seal, completing the separation of the two daughter cells. (G) - (I) Model of motor proteins in spindle morphogenesis, discussed in section 1.1.3. Not drawn to scale.

The highly complex process of mitotic cell division is driven by the mitotic spindle (sketched in Fig. 1.1, immunofluorescence staining image in Fig. 1.3 A) and the contractile ring (see below). Both are complex systems consisting of numerous cytoskeletal proteins, e.g., microtubules $(\mathrm{MTs})^{1}$, actin filaments and several types of

${ }^{1}$ In the mitotic spindle MTs are organised into four functionally distinct groups:

Astral MTs (dark blue in Fig. 1.1) link the spindle poles to the cell cortex. They also contribute to the separation of the spindle poles and the positioning of the spindle relative to the cell cortex. 
motor proteins which drive directed motion under the consumption of Adenosine-5'triphosphate (ATP) [Forman84]. For proper assembly of the bipolar mitotic spindle, as well as the correct separation of the two spindle poles and by that the distribution of identical copies of the replicated genome to the daughter cells, controlled activity and tight regulation of the multiple complementary and antagonistic motor proteins are required [Sharp00, Mitchison01, Karsenti01, Wittman01, Scholey03]. It is fascinating that in the process of cell division motor proteins that, at single-molecule level, generate forces in the piconewton rage and movements on the nanometer scale [Sharp00, Howard01], work in a system that is able to generate forces up to nanonewton and span distances of tens of micrometers [Rappaport67, Nicklas83, Burton97].

In the past, many studies have investigated the mechanisms by which the microtubule (MT)-based mitotic spindle and the actin-based contractile ring use motor proteins and further cytoskeletal proteins to control mitosis and the division of the cytoplasm of the dividing cell into the daughter cells (cytokinesis) [Sharp00, Howard01, Glotzer01]. The following simplified description of the different stages of mitosis (sketched in Fig. 1.1) only gives a rough overview and does not cover all the proteins and processes involved ${ }^{2}$ in detail (based on [Scholey03]):

During prophase, the replicated chromosomes condense. Outside the nucleus, the duplicated centrosomes migrate around the nuclear envelope and the mitotic spindle starts to assemble between the two centrosomes (Fig. 1.1 A). The centrosome is the primary microtubule-organising centre and consists of a pair of centrioles - short cylindrical arrays of MTs - which act as a helical template for new MTs, growing by subunit addition at their fast-growing (plus) ends [Keating00]. Originating this way from the centrosomes causes the spindle MTs all to be orientated with their plus ends distal to the spindle poles.

Prometaphase starts abruptly with the breakdown of the nuclear envelope. The kinetochore MTs (red in Fig. 1.1) of the mitotic spindle can now attach to the chromosomes via their kinetochores and move them to the equatorial plane (ep in Fig. 1.1). This process is called congression (Fig. 1.1 B).

In metaphase, the pairs of sister chromatids are - via a dynamic process involving the chromosomal MTs (green in Fig. 1.1), further reviewed in [Scholey03] - aligned at the equatorial plane of the spindle, midway between the spindle poles (Fig. 1.1 C). Now the kinetochore MTs connect the sister chromatids to opposite spindle poles. At the beginning of anaphase (anaphase A), the connection between the sister chro-

Kinetochore MTs (red in Fig. 1.1) move the chromosomes relative to the spindle poles.

Interpolar MTs (light blue in Fig. 1.1) exert forces capable of moving the opposite spindle poles relative to one another.

Chromosomal MTs (green in Fig. 1.1) link the centrosomes to the chromosome arms.

2 The spindle assembly described here occurs via the so-called centrosome-directed pathway: originating from two duplicated centrosomes, interpolar and astral MTs assemble, which produce a balance of outward and inward forces that drives separation of the two poles.

An alternative pathway used by some systems is the chromosome-directed pathway: the MTs assemble directed by condensed chromosomes. They are then sorted by MT sliding motor proteins into a bipolar array and crosslinked at their minus ends to form focused poles [Sharp00, Karsenti01, Cytrynbaum03]. 
matids breaks. That allows for the sister chromatids to be moved synchronously toward the spindle pole they face and to form daughter chromosomes. Later in anaphase (anaphase B), the kinetochore MTs shorten and the spindle poles move apart due to reorganisation of the interpolar (light blue in Fig. 1.1) and astral (dark blue in Fig. 1.1) MTs of the mitotic spindle (Fig. 1.1 D). Both processes contribute to chromosome segregation. Also during anaphase, a signal is delivered along the spindle MTs to the cortex that defines the later position (pink in Fig. 1.1) and orientation of the contractile ring, the complex that drives cytokinesis using actin filaments and the motor protein myosin-II [Glotzer01].

In telophase, the two sets of daughter chromosomes reach the spindle poles and decondense (Fig. 1.1 E). Around each set of chromosomes, a new nuclear envelope reassemles, completing the formation of two new nuclei and marking the end of mitosis. The division of the cytoplasm of the cell into the two daughter cells (cytokinesis) begins with the contraction of the contractile ring: Upon contraction of the contractile ring, a barrier develops between the daughter cells and the spindle midzone (the array of interpolar MTs lying between separated chromatids) is constricted into a structure called the midbody (Fig. 1.1 F).

Finally, during cytokinesis, the cytoplasm of one cell is divided in two by the contractile ring which causes the furrow to ingress and finally seal, completing the separation of the two daughter cells.

\section{Cell division in budding yeast Saccharomyces cerevisiae}

The molecular and genetic complexity of eukaryotes makes it desirable to use simple model organisms to study the cellular mechanisms of the eukaryotic cells. One of these simple organisms is budding yeast Saccharomyces cerevisiae (S. cerevisiae) which can reproduce either vegetatively (by simple cell division, described below) or sexually. It is robust, easy to grow and divides almost as rapidly as bacteria. Additional good reasons for using $S$. cerevisiae are (i) that the complete Saccharomyces genomic sequence is available, (ii) the genetic tractability of this organism and (iii) these cells often accomplish cellular processes using mechanisms that are conserved but less complex than homologous functions in higher eukaryotes [Hildebrandt99].

In chapers 2 and 3, this system was used to study mitotic motor proteins in vitro because $S$. cerevisiae represents the only system where all the participating proteins are known and each can be genetically manipulated.

The budding yeast cell division differs in some ways from the above described cell division of higher eukaryotes. First of all $S$. cerevisiae cells divide asymmetrically by budding, producing a daughter cell that is slightly smaller then the mother cell. Also unlike mitosis of higher eukaryotic cells, there is no breakdown of the nuclear envelope during the mitosis of yeast cells (closed mitosis). The nuclear envelope remains as a physical barrier, creating two functionally distinct types of MTs: nuclear and cytoplasmic MTs [Winey95]. The nuclear MTs attach directly to the chromosomes. The highly dynamic cytoplasmic MTs function, equivalent to the astral MTs in higher eucarytotes, in nuclear positioning, e.g. by moving the 


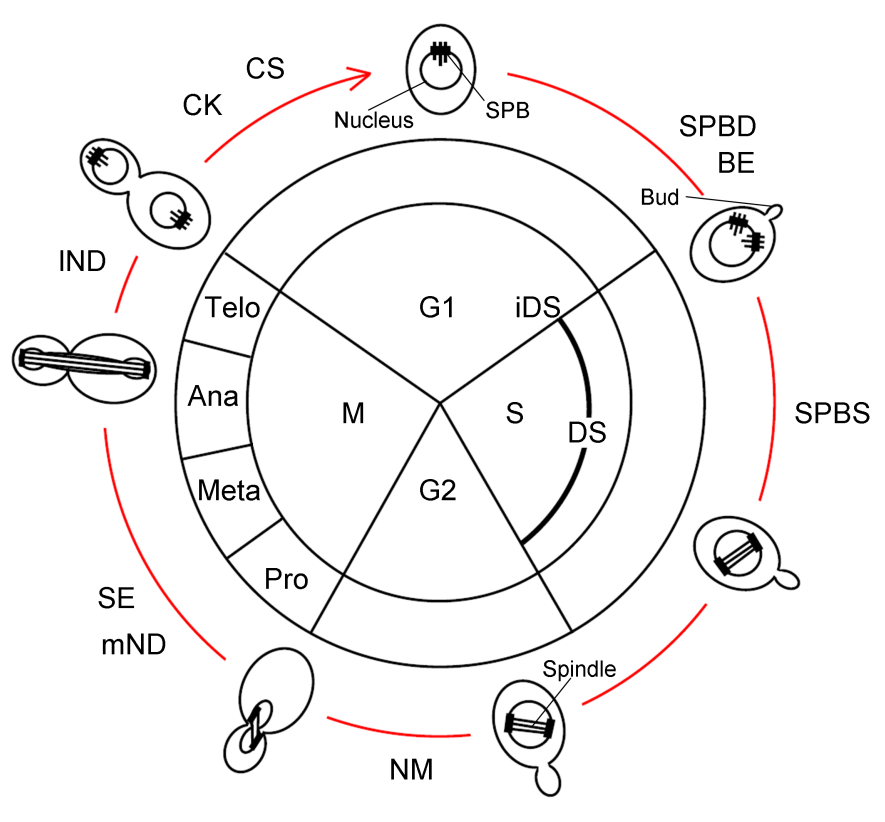

Fig. 1.2: Overview of the processes in the $S$. cerevisiae cell division cycle (based on [Hartwell74]).

SPBD, spindle pole body duplication; BE, bud emergence; iDS, initiation of DNA synthesis; DS, DNA synthesis; SPBS, spindle pole body separation; NM, nuclear migration; $\mathrm{mND}$, medial nuclear division; SE, spindle elongation; IND, late nuclear division; CK, cytokinesis; CS, cell separation. Not drawn to scale.

nucleus to the bud neck during mitosis (M-phase). Similar to the centrosomes in higher eukaryotic cells, the spindle pole body (SPB) - a structure embedded within the nuclear envelope - serves as nucleation site of the two types of MTs [Adams00]. In contrast to higher eukaryotes where MTs and their associated proteins perform other essential roles, such as intracellular transport and signalling, the MTs in yeast perform during vegetative growth only the one single role of mitotic spindle function [Jacobs88].

In the following section, the basic steps of mitosis in budding yeast (referred to simply as yeast ${ }^{3}$ from here on) will be described (based on[Hildebrandt99]).

In the Gap-1 (G1)-phase of the cell cycle, the yeast cell holds no bud and the nucleus contains a single SPB. Three events mark the end of the G1-phase: the SPB duplication in a side-by-side fashion within the nuclear envelope (SPBD in Fig. 1.2), the initiation of DNA synthesis (iDS in Fig. 1.2) and the emergence of a bud (BE in Fig. 1.2).

In S-phase, the SPBs are pushed apart (SPBS in Fig. 1.2) by the assembly of a bipolar microtubule array, forming a short, $\sim 1.5 \mu \mathrm{m}$-long spindle [Hildebrandt99]. In the midzone of this spindle antiparallel MTs from both spindle poles overlap [Winey95]. With the completion of DNA synthesis (DS in Fig. 1.2) the S-phase ends and the cell enters the Gap-2 (G2)-phase, in which the bud grows further.

M-phase begins with the migration of the nucleus (nuclear migration, NM in Fig. 1.2) to the neck of the bud where it undergoes the first stage of nuclear division (medial nuclear division; mND in Fig. 1.2). At the same time the spindle elongates (SE in Fig. 1.2). At some point which is not exactly known yet, the sister chro-

\footnotetext{
${ }^{3}$ Other yeast types are known to divide differently, e.g. the fission yeast Schizosaccharomyces pombe.
} 
matids attach to the spindle. Hereby each chromosome binds to a single MT from each spindle pole via a centromeric DNA/kinetochore complex [O'Toole99]. Unlike higher eukaryotic cells, yeast cells do not appear to feature a congression process (see subsection 1.1.1).

Once all of the chromosomes are bipolarly attached to the fully assembled spindle, the cell enters anaphase, in which the segregation of the chromosomes (anaphase A) and the main spindle elongation (anaphase B) occur. To establish chromosome segregation the spindle becomes positioned parallel to the mother-bud axis. During spindle elongation one spindle pole is pulled into the bud, while the other spindle pole remains in the mother cell.

In anaphase A, the chromosomes separate and migrate along kinetochore MTs toward the opposite spindle poles. During this phase of anaphase the spindle elongation is only small. The main elongation of the spindle and, with that the greatest separation of the chromosomes, occurs in anaphase B.

Anaphase B itself is further subdivided in two stages: first a rapid elongation step occurs, moving at $1-2 \mu \mathrm{m} / \mathrm{min}$ and elongating the spindle to approximately half of it's final length. This step is followed by slower elongation $(0.2 \mu \mathrm{m} / \mathrm{min})$ until the final spindle length of $\sim 10 \mu \mathrm{m}$ is reached [Kahana95, Straight98]. At the end of this elogation process the SPBs and their associated set of chromatids are separated by $\sim 6-7$ times their preanaphase distance [Winey95].

In telophase, the second stage of nuclear division (late nuclear division; IND in Fig. 1.2) is reached and the spindle disassembles, marking the end of mitosis. After cytokinesis (CK in Fig. 1.2) the budding process is completed and mother and daughter cells separate (CS in Fig. 1.2).

\section{Motor proteins in spindle morphogenesis}

The mitotic spindle and the contractile ring segregate chromosomes and divide cells with high fidelity. These "nano-machines" pass through a series of transient steadystate structures, each established by a delicate balance of forces generated by multiple cytoskeletal proteins and in particular by complementary and antagonistic motor proteins. Transitions from one state to the next occur when a change in the activity of a subset of mitotic motor proteins and/or forces generated by growing and shrinking MTs tip the balance [Mitchison01, Sharp00, Wittman01].

In the following description (based on [Sharp00]), a simplified model (focusing only on three types of MT-associated motor proteins) of motor proteins driving spindle assembly, maintenance and elongation will be given to illustrate the idea of balancing forces driving the mitotic spindle from one state of mitosis to the next. The in these processes involved bipolar mitotic motor proteins (forming the kinesin subfamily kinesin-5, further described in 1.2) are the main focus of this thesis and will be discussed in chapters $2,3,4,5$ and 6 .

In the transition from interphase (the long period of the cell cycle between one mitosis and the next) to prophase, the initial assembly of the mitotic spindle in- 
volves a dynamic balance between dynein (purple) on the cortex (inset 1 in Fig. 1.1) and dimeric kinesins (yellow) that move to the plus end of the interpolar MTs in the spindle midzone (inset 2 in Fig. 1.1), pulling the poles apart and together, respectively. The balance between the antagonistic forces of two types of motor proteins can be modified by the extent of the antiparallel MT overlap in the midzone of the spindle. In the beginning of spindle assembly, the force that can be generated by the plus-end directed dimeric kinesins is limited due to a short overlap region of MTs. However, as the poles separate further - due to the outward force of the plus-end directed dynein - and growing MTs continue to interdigitate, the inward force generated by the plus-end directed dimeric kinesin gradually increases until it balances the force generated by dynein (Fig. 1.1 G).

Immediately after the breakdown of the nuclear envelope follows the transition from prometaphase to metaphase. At this point the astral MTs have minimal length and so the outward force generated by dynein is low (inset 3 in Fig. 1.1). In the midzone (inset 4 in Fig. 1.1), a balance between forces generated by bipolar plus-end directed kinesins (green; which were up to this stage in mitosis located within the nucleus), dimeric plus-end directed kinesins and interpolar MT bundles results in the movement of the chromosomes (Fig. 1.1 H). Shortly after that, the forces within the spindle shift again when cortical dynein together with bipolar kinesin activity overwhelms the inward forces generated by the dimeric plus-end directed kinesins and tips the balance of forces in the outward direction. This results in the elongated metaphase spindle.

In anaphase B, sudden loss of the inward forces, caused by the inactivation of the plus-end directed dimeric kinesins, leads to the release of the tension within the metaphase spindle that was generated by antagonistic inward and outward forces (Fig. 1.1 I). This causes a final elongation of the spindle and, just before the disassembly of the mitotic spindle, a final force balanced structure forms at telophase.

\section{The kinesin family and the subfamily kinesin-5}

Next to other motor proteins - like the above mentioned actin-associated myosins and the MT-associated, minus-end directed dyneins - the MT-associated kinesins play an essential role inside the cell. In 1985, conventional kinesin (later termed kinesin-1) was found for the first time in squid axons where it is expressed at very high levels [Vale85]. Apart from cell division, kinesins are involved in a multitude of other cellular processes such as the transport of organelles and vesicles and also in cell signalling. All members of the kinesin family (approximately 150 proteins) share a great similarity in the sequence of their homologous motor domain ( $~ 35-45 \%)$, in which the MT-binding site and the ATP-hydrolyse domain are located [Kashina97]. Kinesins are, apart from the members of the subfamily kinesin- $14^{4}$, processive motor proteins, capable of moving up to several micrometers along a MT without unbinding. In this process, one molecule takes hundreds of

\footnotetext{
${ }^{4}$ Kinesin motor proteins with a C-terminal motor domain are grouped in the subfamily kinesin14. WT kinesin-14 motor proteins, like ncd from Drosophila melanogaster, move in a non-processive fashion by producing isolated minu-end directed power strokes. These motor proteins can only
} 

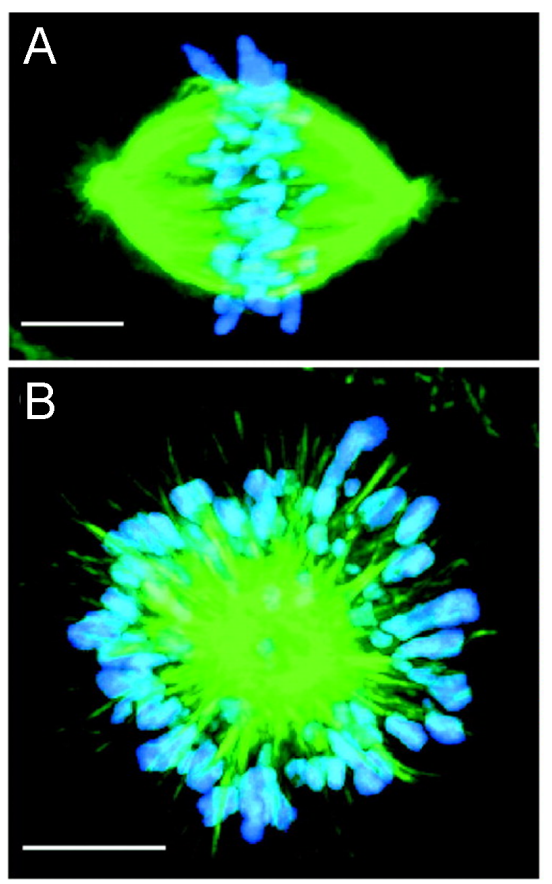

C

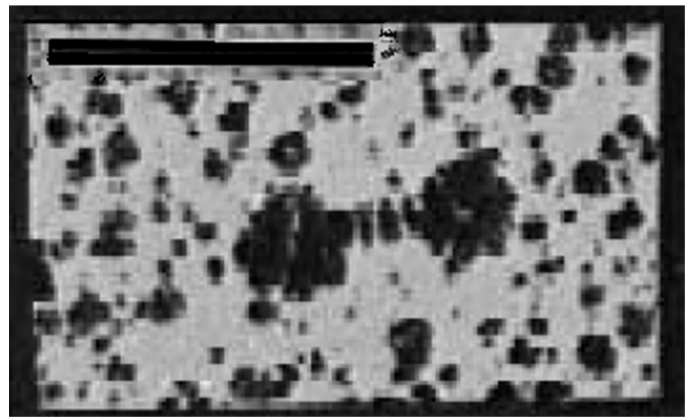

$\mathrm{D}$

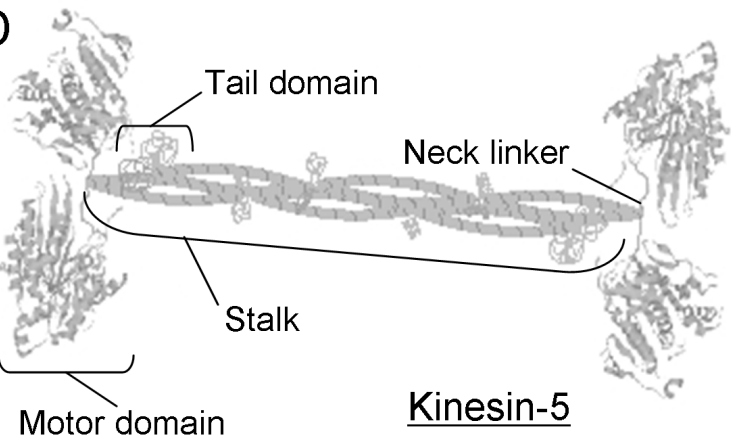

Fig. 1.3: (A and B from [Mayer99]) Inhibition of kinesin-5 (here by monastrol, see section 4.2) causes spindle collapse in mitotic cells. Immunofluorescence staining of BSC1 cells: $\alpha$-tubulin $\widehat{=}$ microtubules (green); chromatin $\widehat{=}$ chromosomes (blue). (A) Cells treated for 4 hours with $0.4 \%$ DMSO as a positive control for a functional mitotic spindle. (B) Cells treated for 4 hours with $68 \mu \mathrm{M}$ monastrol showing the typical monoastral, non-functional phenotype. Scale bars represent $5 \mu \mathrm{m}$. (C from [Kashina97]) Electron micrograph of a rotary shadowed KRP130 molecule, member of the kinesin- 5 family. The kinesin-5 molecule appears elongated, with enlarged globular ends, generally shaped as dumbbells. Scale bar represents $1 \mu \mathrm{m}$. (D based on [Dagenbach04]) Sketch showing the bipolar structure of the homoterameric kinesin- 5 with two pairs of N-terminal motor domains.

$\sim 8$-nm steps, hydrolysing one ATP per step in a complex chemo-mechanical cycle [Hackney95, Hua97, Schnitzer97, Coy99b, Lakämper06]. During processive stepping, the motor proteins move in the so-called hand-over-hand mechanism along the MT which was first proposed by Hancock and Howard [Hancock98]. In the handover-hand model the two motor domains bind, stepwise alternating, along the same protofilament $^{5}$ of the MT [Kural05, Yildiz04].

One of the 14 subfamilies of the kinesin motor proteins is the kinesin-5 subfamily. The founding member of the kinesin-5 family was found as a mutant in a genetic screen for temperature-sensitive, lethal mitotic genes in the fungus aspergillus nidulans [Morris75]. The thereby found mutant blocked the mitosis of the fungal cells and was thus named BimC (blocked in mitosis) [Enos90]. The BimC gene encodes a

produce persistent motion when they act in ensembles [McDonald90, Walker90, Sablin98, Castro00, Block07].

${ }^{5}$ Alternating subunits of $\alpha$ - and $\beta$-tubulin monomers polymerise forming long protofilaments. 13 of these protofilaments associate laterally to form a single hollow and cylindrical MT. 

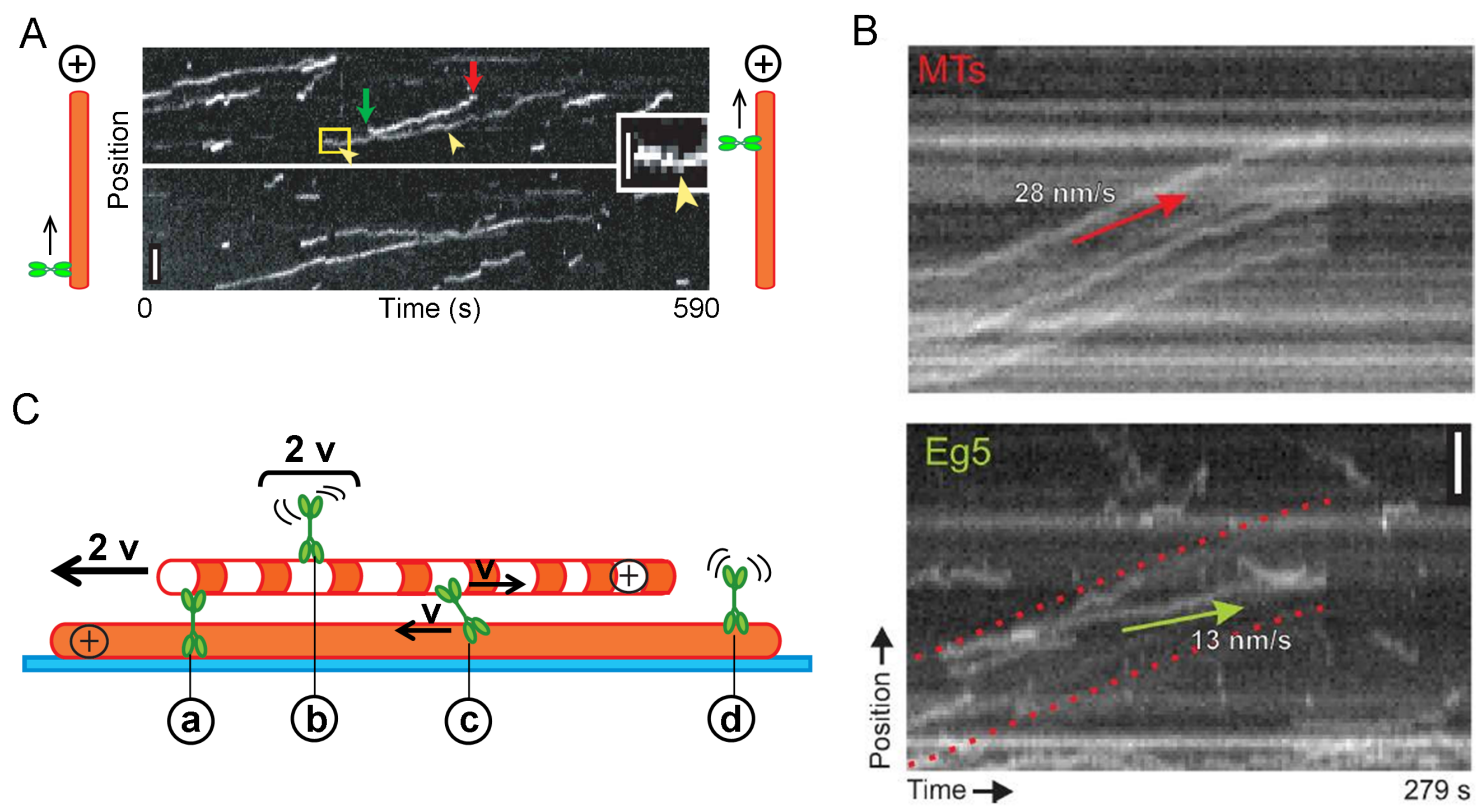

Fig. 1.4: (A based on [Kwok06]) Kymographs (position on MT plotted over time) depicting the motion of single Eg5 molecules labelled with green fluorescent protein (GFP) along MTs in $80 \mathrm{mM}$ Pipes buffer containing $2 \mathrm{mM}$ ATP (direction of Eg5 movement is sketched at the sides). The slope and length of the traces correspond to motor velocity and run length, respectively. Arrows mark the starting point (green) and the ending point (red) of an exemplary single run. Two examples of irregularities in the directional motility (that is, reversal in direction) are marked with yellow arrowheads. Scale bar represents $2 \mu \mathrm{m}$. Inset: 3 times magnification of the framed area; scale bar represents $1 \mu \mathrm{m}$. (B from [Kapitein08]) Top kymograph shows sliding of a MT relative to a surface-attached MT at $\sim 28 \mathrm{~nm} / \mathrm{s}$ in high-ionic-strength buffer ( $80 \mathrm{mM}$ Pipes $+80 \mathrm{mM} \mathrm{KCl})$ containing $2 \mathrm{mM}$ ATP. Below, the corresponding kymograph of GFP-labelled Eg5 shows directional runs $(\sim 13 \mathrm{~nm} / \mathrm{s})$ between two overlapping microtubules (overlap region marked with two red dotted lines) and diffusive motility outside of the overlap region. The slope of the arrows indicates the mean velocity of motors (green) and MTs (red), respectively. Scale bar represents $2 \mu \mathrm{m}$. (C) Sketch illustrating the relative MT sliding assay shown in B: a fluorescently labelled MT is attached to the surface, while another sparsely fluorescently labelled MT binds with an antiparallel orientation and is moved by GFP-labelled Eg5 homotetramers. Four possible types of motor positions and resulting velocities are depicted: a) motor protein bound to surface and sliding MT, b) motor protein attached on top of the sliding MT moving with twice the single-motor velocity $2 \mathrm{v}, \mathrm{c}$ ) motor protein moving in overlap region between two MTs with single-motor velocity v, d) motor protein attached to fixed MT outside of the overlap region.

$132 \mathrm{kDa}$ big and 1184 residues long polypeptide with a $\mathrm{N}$-terminal ${ }^{6}$ motor domain. The motor-domain sequence shows $42 \%$ agreement with the respective domain of kinesin-1 [Enos90].

A similar screen carried out in fission yeast (yeast also belongs to the kingdom of

6 The N-terminus refers to the start of a protein or polypeptide terminated by an amino acid with a free amine group $\left(-\mathrm{NH}_{2}\right)$. The other end of a protein is termed C-terminus. The convention for writing peptide sequences is to put the N-terminus on the left and write the sequence from $\mathrm{N}$ to C-terminus. 
fungi) identified a related kinesin, Cut7 [Hagan90]. Mutations in either BimC or Cut7 blocked SPB separation and thus prevented the successful completion of mitosis in the respective system [Enos90, Hagan90]. Further studies have identified similar mitotic motor proteins in many other systems, e.g. Eg5 in Xenopus leavis, Cin8 and Kip1 in S. cerevisiae, KLP61F in Drosophila melanogaster and hsEg5 in human [Le Guellec91, Hoyt92, Heck93, Blangy95]. This group of related kinesins localises to spindle microtubules as well as structures present at spindle poles and was eventually classified as the kinesin-5 family [Lawrence04].

Unlike kinesin-1, kinesin-5 carries a $\sim 70$ amino acids (aa) long additional domain at the N-terminus, which is highly positively charged and was shown to have a high MT affinity [Stock03]. At the end of their C-terminus, the members of the kinesin-5 family share a 40 aa long part of their sequence, the BimC-Box (see sequence alignment of kinesin-1 and kinesin-5 in chapter 4, Fig. 4.1) [Kashina97]. Also inherent for all kinesin-5 motor proteins is the unique homotetrameric bipolar structure with two pairs of N-terminal motor domains positioned at each end of the tetramer's long axis (Fig. 1.3 C and D) [Cole94, Blangy95, Kashina96, Lawrence04]. This bipolar structure allows kinesin- 5 motor proteins to bind to two MT simultaneously, crosslink them and also slide antiparallel MTs relative to each other (Fig. $1.1 \mathrm{H}$ and I), a behaviour that has been directly observed in vitro [Kapitein05, Wildenberg08, Gerson-Gurwitz11]. In vivo, it was found that kinesin-5 (in this case, Eg5) can be inhibited by the small molecule monastrol (further discussed in chapter 4), resulting in a collapsed monoastral spindle (Fig. 1.4 B) so that cell division is no longer possible [Mayer99, Ferenz10]. Another study in S. cerevisiae showed that previously separated spindle pole bodies collapse in response to kinesin- 5 inhibition [Saunders92].

Compared to kinesin-1, which moves at velocities of $\sim 400-800 \mathrm{~nm} / \mathrm{s}$ [Howard89, Block90, Thorn00], kinesin-5 (that is, Eg5) is a slow motor protein, reaching $\sim 30$ - $50 \mathrm{~nm} / \mathrm{s}$, and has been shown to be modestly processive with a run length of $\sim$ $600 \mathrm{~nm}$ [Sawin92b, Cole94, Kwok06]. In single-molecule fluorescence experiments, where the observation of single fluorescently-labelled motor proteins is possible, it was found that Eg5 moves towards the plus-end of the MT, while its motility is highly complex: single Eg5 motor proteins show a mixture of diffusive and ATPdependent directional motility along a single MT while staying attached for several tens of seconds (Fig. 1.4 A) [Kwok06, Kapitein08]. Interestingly, upon crosslinking of a second MT, diffusive motility is suppressed, and directional motility becomes dominant (Fig. 1.4 B and C) [Kapitein08]. How this molecular switching mechanism is regulated, is to date not fully understood and will be one focus of this thesis.

An even more dramatic switching process was discovered in Cin8 - a kinesin-5 motor protein from S. cerevisiae - by us and an independent study by Roostalu et al. [Gerson-Gurwitz11, Roostalu11]. Under certain conditions (further discussed in chapter 2) Cin8 switched not only from an "on-" to an "off-state" like Eg5, but switched it's direction of motility from minus-end directed to plus-end directed motility. In a continuing study (chapter 3) we were able to confirm that single Cin8 motor proteins can switch direction and we proposed that the main switching 
mechanism is - similar to Eg5 - the binding of a second MT to the bipolar Cin8 molecule (Fig. 3.2; [Thiede12a]). Not only is this the first time that processive minus-end directed kinesin motility was observed, these findings also challenge the 20-year-old dogma that kinesin homologs which carry their catalytic domains at the N-terminus are generally plus-end directed [Dagenbach04].

\section{\begin{tabular}{l|l} 
Mechanisms in kinesin regulation & 1.3
\end{tabular}

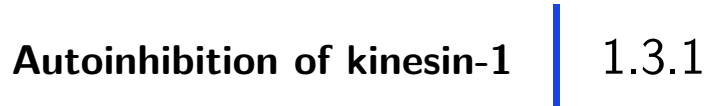

A quite well understood regulation mechanism of kinesins is the autoinhibition of kinesin-1. After a short calculation, it becomes clear why a tight regulation of the motor protein and its ATPase activity is needed in the cell: When the $0.1-1 \mu \mathrm{M}$ kinesin found in tissues [Hollenbeck89] would always be active, regardless of whether bound to cargo or not, and hydrolyse $\sim 100$ ATP molecules per second [Coy99b], the large amount of $10-100 \mu \mathrm{M}$ ATP per second would be wastefully consumed [Hackney92b]. This amount of ATP is comparable to the basic human metabolism rate of $\sim 100 \mathrm{~kJ} \mathrm{~kg}^{-1}$ day $^{-1}$ [Coy99a].

A second problem of kinesin-1 motor proteins moving unregulated without cargo is probably even more serious for the survival of the cell: Since kinesin-1 motor proteins move processively toward the MT plus end at the periphery of the cell, to where they transport cargo generated in the center of the cell, unregulated motor proteins would be stuck at the plus end of the MTs and would not be available for transport anymore.

These reasons make clear that in the cell a mechanism is necessary that inhibits kinesin-1 activity when it is not bound to cargo and only activates kinesin-1 after cargo binding, when it can serve a useful purpose.

Kinesin-1 is a heterotetramer composed of two heavy and two light chains ${ }^{7}$ (Fig. 1.5 A). The light chains (blue in Fig. 1.5 A and B) form - together with Coil-4a,b of the heavy chains - the cargo-binding domain of kinesin-1. The dimerised heavy chains (black in Fig. $1.5 \mathrm{~A}$ and $\mathrm{B}$ ) contain the N-terminal motor domains and a long region with multiple coiled coils followed by a C-terminal tail domain. In the region with multiple coiled coils some parts have a low probability of forming a coiled coil and are probably more flexible regions (e.g., hinge and kink in Fig. 1.5 A) [Hackney92b, Hirokawa89, Woehlke00].

Electron-microscopie studies and hydrodynamic measurements showed that, under physiological ionic conditions, native kinesin-1 is bent with it's tail domain close to its motor domain [Hackney91, Hackney92b]. The light chains of kinesin-1 are thereby not required for either motility or folding into the bent conformation,

\footnotetext{
${ }^{7}$ Unlike kinesin-1 from higher eukaryotes, fungal kinesin-1 does not associate with light chains; although they are otherwise clearly homologous and follow the same chemo-mechanical stepping mechanism.
} 

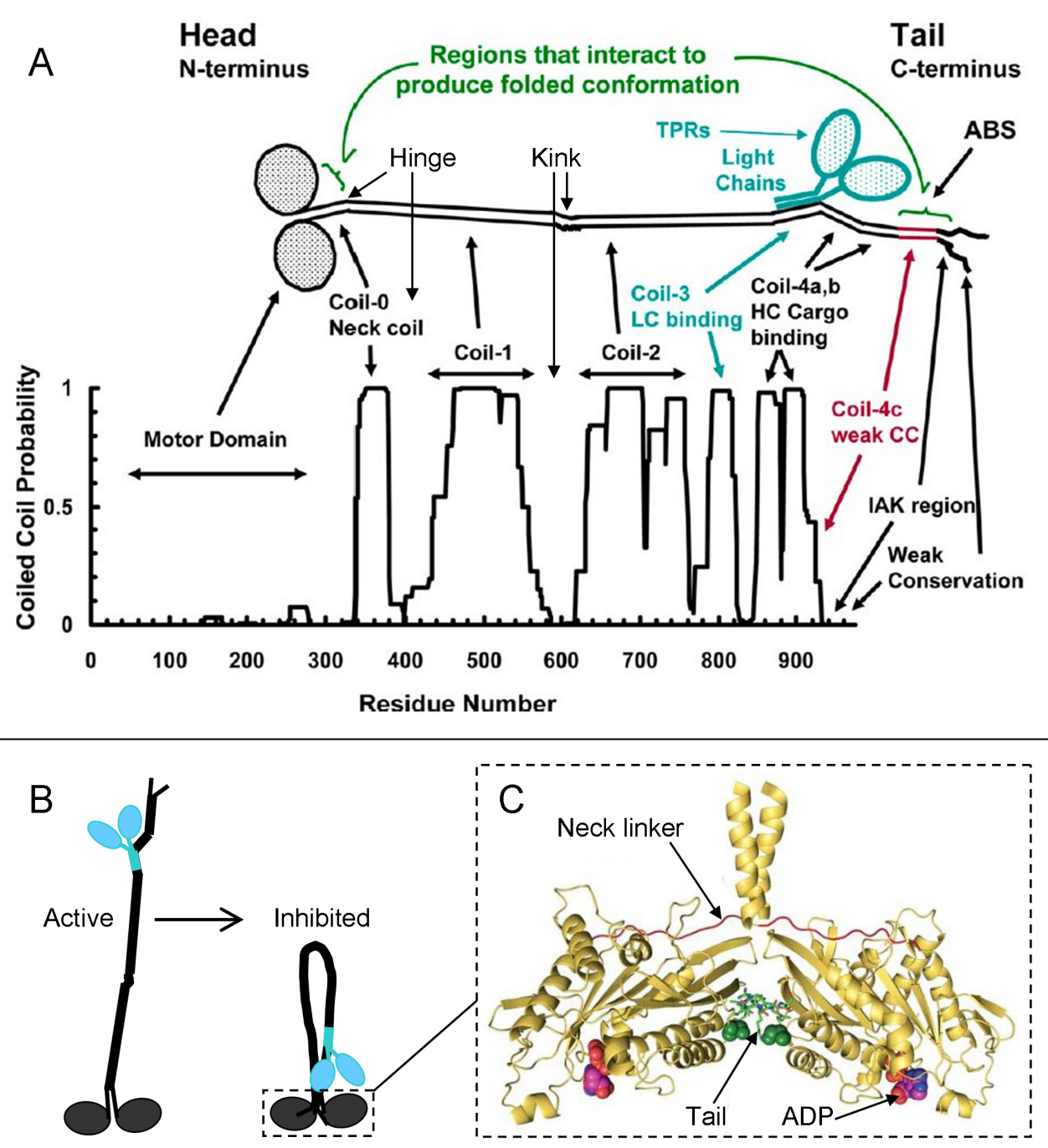

Fig. 1.5: (A based on [Hackney07]) Schematic structure of kinesin-1. The coiled-coil prediction for the heavy chain (HC, black) of Kinesin-1 from Drosophila below leads to the corresponding schematic domain organization on top. The two motor domains are connected to the neck coil by the neck linker and are followed by the long coiled-coil stalk composed of coil-1 and coil-2 [Cuevas92]. At coil-3 of the HCs the coiled-coil region near the N-terminus of the light chains (LCs) binds, anchoring the cargo-binding tetratricopeptidrepeat (TPR) domains of the LCs to the HCs [Diefenbach98]. At Coil-4a,b some specific cargoes can also bind directly to the HC [Seiler00]. Coil-4c, which shows a rather weak coiled-coil prediction, is followed by a region with an excess of positive charge that is critical for both the interaction with the MT as well as for motor domain/neck interaction. This positive region is followed by the highly conserved IAK region that is required for the inhibition of ATPase in the folded conformation of kinesin-1 [Stock99]. The region following the IAK domain at the C-terminus is likely to be unstructured. (B) Tail-inhibition model for the regulation of kinesin- 1 . The motor protein is active in the extended conformation and inhibited in the flexed conformation. (C based on [Kaan11]) Proposed model for "double-lockdown" mechanism of kinesin-1 autoinhibition. The binding of the tail (green, sticks) crosslinks the motor domains (yellow, cartoon) and prevents their free movement, inhibiting neck linker (red) undocking and ADP (red, spheres) release. 
but their presence in the native heterotetramer destabilises the bent confirmation [Hackney92b] and decreases the MT affinity [Verhey98]. These findings led to a first hypothesis of how the motor protein might be regulated: Hackney et al. proposed the tail-inhibition model, where the tail binds to the motor domains and inhibits them, resulting in inactivation of the flexed kinesin-1 molecule (sketched in Fig. 1.5 B) [Hackney92b]. Consistent with this model is the finding that native kinesin-1 has a very low MT-stimulated ATPase rate of $\sim 1$ ATP per second [Saxton88, Hackney91], whereas proteolysed and truncated tail-less kinesins show a high ATPase activity [Kuznetsov89, Huang94].

Coy et al. later directly showed that a 65-amino-acid C-terminal tail domain is an inhibitory regulator of the ATPase and motor activities of kinesin-1's motor domains. They also found that binding of cargo activates kinesin-1 and proposed that the binding of cargo antagonises tail inhibition, acting as an indirect regulation mechanism [Coy99a]. However it remained unclear whether the tail domain directly interacts with the motor domain or whether the tail domain binds outside the motor domain, inhibiting it allosterically.

A further study of Hackney and Stock revealed that both the basal and the MTstimulated ATPase activity of kinesin-1 in the folded confirmation are inhibited by the binding of the tail domains, because tail binding inhibits the rate-limiting step of ADP release [Hackney08]. Still, the molecular mechanism of inhibition of the motor domain by the tail domain remained unclear.

A year later, Hackney et al. could show that binding of only one of the two uncoiled tail domains (containing the highly conserved IAK region and the adjoining positively charged regions, see Fig. $1.5 \mathrm{~A}$ ) to the dimeric motor domains is sufficient to inhibit the activity of both motor domains and that the binding of the second tail domain is much weaker then the binding of the first one (half-side negative cooperativity) [Hackney09]. They hypothesise that the second, weaker bound tail domain might be available to bind to MTs or other interacting proteins.

In a very recent study, Kaan et al. present the crystal structure of the kinesin-1 motor domain dimer in complex with its tail domain. They propose that neither an induced conformational change nor sterical blocking is the cause of motor-domain inhibition. Instead, the tail crosslinks the motor domains at a second position, in addition to the coiled coil (Fig. 1.5 C) [Kaan11]. They term this model for an autoinhibition mechanism the "double-lockdown" mechanism. In the "double-lockdown" state the movement of the motor domains that is needed to undock the neck linker and release ADP is blocked, the kinesin-1 molecule cannot reach the next step in it's chemo-mechanical cycle and is in that way autoinhibited.

After a decade of research, the proposed "double-lockdown" mechanism seems to explain kinesin-1 autoinhibition on the molecular level. In vitro however, many other regulator mechanisms like phosphorylation or other covalent modifications, as well as binding of a number of interaction proteins might be involved in the complex regulation process of kinesin-1 and further studies are required to gain a complete understanding of kinesin-1 regulation in the cell. 


\section{Regulation of kinesin processivity $\quad 1.3 .2$}

To move processively along a MT kinesin uses it's two motor domains alternately to perform successive 8.3-nm displacements. While the leading motor domain (also called head) anchors the dimeric molecule to one tubulin subunit of the MT (see footnote 5), the trailing motor domain moves forward in a diffusive manner and binds to the next tubulin subunit, one step further to the MT plus end. Stepping according to this hand-over-hand mechanism leads to net displacements of $16.6 \mathrm{~nm}$ for the trailing motor domain, whilst the leading motor domain remains stationary. Yildiz et al. could reproduce exactly these displacements [Yildiz04] and therefore rule out other proposed mechanisms for kinesin motility (e.g. the inch worm mechanism [Hua02]).

In the chemo-mechanical cycle each step of a kinesin is tightly bound to the hydrolysis of one ATP molecule. The energy from ATP hydrolysis induces a conformational change ${ }^{8}$ in the leading motor domain which brings the trailing motor domain closer to the MT, increasing the probability that the tethered motor domain finds the next binding side along the MT in it's diffusive search. Each of the two kinesin motor domains are able to bind ATP and hydrolyse it to Adenosine-5'diphosphate (ADP). The specific MT affinity of the motor domains change during stepping depending on what nucleotide (ATP or ADP) is bound to the motor domain [Ma97, Gilbert98, Lakämper06]. Hereby the ATP-kinesin complex and the nucleotide-free state assume strongly bound conformations while the ADP-kinesin complex binds weakly to the MT [Hackney92a].

To generate processive motility for several hundreds of steps, following the handover-hand mechanism, the ATPase cycles of both motor domains have to be kept out of phase in such a way that at each given time at least one of the motor domains is in a strongly MT-binding (rigor state) conformation. During kinesin stepping the leading motor domain remains in the strongly MT-bound, nucleotide-free state as long as the trailing motor domain is in the weak MT-bound ADP state and moves, in a diffusive way, to the next MT-binding site. Once the motor domains have swapped their positions, the now leading motor domain rapidly unbinds its ADP and transits into the rigor state, anchoring the kinesin to the MT for another stepping cycle (alternating site catalysis) [Hackney94].

Although most mechanisms in the chemo-mechanical cycle are accepted in the field, some specific details and proposed sub-steps remain controversial [Schief01].

\footnotetext{
${ }^{8}$ It is widely accepted that binding of ATP to the attached, leading motor domain precedes a conformational change that allows the trailing motor domain to attach to the MT and release it's ADP. However, the details of this conformational change are a matter of debate (reviewed in [Schief01]). The presence of the non-hydrolysable ATP analogue AMP-PNP also accelerates release of the second ADP, showing that hydrolysis is not necessary for ADP release from the second motor domain [Rice99, Vale00]. But it was also shown that hydrolysis usually takes place before attachment of the second head [Ma97, Crevel99].
} 
One of the still debated details of kinesin stepping is the exact way of communication between the two motor domains. To ensure alternating stepping of the two motor domains, one motor domain needs to "sense" the stepping state of the other motor domain at each given time. The mechanical connection between the two motor domains is established through the neck-linker domains (see Fig. 1.5 C) which are also believed to be the communication transmitting elements [Hancock99, Block07]. When both motor domains are bound to the MT intramolecular tension is generated. It was postulated that this tension coordinates the synchronised stepping in two nonexclusive gating mechanisms: (i) Front-head gating, the rearward tension on the leading motor domain (head) prevents ATP binding until the trailing motor domain unbinds from the MT [Rosenfeld03]. This locks the leading motor domain in a strongly MT-bound state and prevents premature detachment of the motor protein. (ii) Rear-head gating, the binding of the leading motor domain to the MT induces strain on the trailing motor domain, accelerating the unbinding of the trailing motor domain [Crevel04b, Schief04]. These models are not mutually exclusive and kinesin might use both mechanisms to coordinate processive stepping.

The processivity (that is here, the run length) of different kinesin motor proteins varies considerably - consistent with their different roles in the cell - between the kinesin subfamilies [Block90, Howard89, Varga06, Rosenfeld09]. Interestingly, the length of the strain transmitting neck linker also varies from 14 to 18 aa between the different kinesin subfamilies [Hariharan09]. Although kinetics experiments showed that isolated motor domains from different kinesin subfamilies also possess different catalytic rates for ATPase and MT detachment [Rosenfeld09, Rosenfeld03], it was proposed that the length of the neck linker plays the crucial role in the kinesin stepping cycle, inducing different run lengths for different kinesin subfamilies. In the chemo-mechanical cycle of kinesin stepping, the neck-linker domain is proposed to have three important roles: (i) Binding of the trailing motor domain to the next free binding site involves diffusion of the unbound motor domain, which is tethered by the entropic spring properties of the neck-linker domain. (ii) Neck-linker docking to the motor domain is thought to be the key conformational change driving kinesin stepping. (iii) When both motor domains are simultaneously bound to the MT, the neck-linker domains transmit the mechanical forces that underlie the coordination of their ATP-hydrolysis cycles.

In a recent paper, Shastry et al. varied the neck-linker length of five chimeric motor-protein constructs, featuring motor domains of five different kinesin subfamilies (that is, kinesin-1, -2, -3, -5 and -7). They found in single-molecule fluorescence assays that, independent of the used motor domain, the run lengths of all chimeras were maximal at the shortest neck-linker length (at which they still saw motility) of 14 aa [Shastry11]. Based on these findings they proposed that the differences in unloaded processivity between different kinesin subfamilies is primarily due to variations in the lengths of their neck-linker domains rather than specific tuning of biochemical rate constants in their ATP hydrolysis cycles. They proposed a simple model, where the shortest possible neck linker leads to the tightest communication between the two motor domains and hence the longest run lengths [Shastry11]. In 
agreement with this model are earlier findings from Yildiz et al., showing that processivity in motor-protein constructs with largely elongated neck-linker lengths can be rescued by appliance of external load [Yildiz08].

In contrast to the study of Shastry et al., we show in chapter 5 that at least the motor domain of Eg5 is optimised for its native neck-linker length of 18 aa. We could also show that neck-linker domains shorter then 14 aa are capable of supporting processive movement [Düselder12]. These findings challenge the simple model proposed by Shastry et al., and suggest instead that different kinesin subfamilies might be optimised for different neck-linker lengths and that other more complex mechanisms, such as neck-linker orientation (as recently proposed by Clany et al. [Clancy11]), might be involved in the control of processive stepping in kinesins.

\section{\begin{tabular}{l|l} 
Structure of this thesis & 1.4
\end{tabular}}

This cumulative thesis covers in five chapters (chapters 2, 3, 4 and 5 consisting of published papers and chapter 6 consisting of a submitted manuscript) new findings on different aspects of the regulation of kinesin-5 motor proteins. Outside the topic of kinesin- 5 motor proteins, chapter 7 consists of an accepted paper about neuronal receptors to which I contributed in vivo measurements in neurons.

In the second chapter the factors that cause directional switching in Cin8, one of the two kinesin-5 from $S$. cerevisiae, were studied. The directionality of kinesin-5 motor proteins was believed to be fixed and uni-directional to the plus-end of the MT [Kashina97, Dagenbach04, Kapitein05, Kwok06]. The findings presented here, and an independent recent report from Roostalu et al. [Roostalu11], showed that surprisingly Cin 8 is able to switch directionality. In this work Cin 8 directionality was examined using single-molecule fluorescence motility experiments and live-cell microscopy.

In-vivo experiments showed that on spindle MTs, Cin8 motor proteins mostly moved slowly towards the spindle midzone where the MTs overlap antiparallel with their plus-ends directed away from the spindle poles. Interestingly, also faster motility towards the spindle poles, where the minus ends of the MTs are located, was occasionally detected.

In vitro, single-molecule fluorescence experiments revealed that individual Cin8 motor proteins could be switched by ionic strength in the buffer solution from fast and processive minus-end to slow plus-end motion on single MTs. Similar to the switching in motility reported for Eg5, Cin8 motility was strongly affected by binding to a second MT: at high ionic strength in the buffer solution, Cin8 motor proteins moved fast and processive on single MTs and switched to rapidly alternated directionalities when bound between antiparallel MTs, while at the same time driving steady plus-end relative sliding.

Unlike many members of the kinesin- 5 family, Cin8 features 99 additional aa in the loop-8 which is involved in MT binding [Kull96, Nitta08, Chee10]. To examine the 
influence of this unique insert on Cin8 motility, the motility of a mutant (Cin8 $\Delta 99$ ) lacking the extra 99 aa was studied. The analysis of single-molecule fluorescence experiments at increasing ionic strength suggested, that the deletion of the insert induced a bias towards minus-end motility and affected the ionic strength-dependent directional switching of Cin8 in vitro. In-vivo experiments showed that the Cin8 $\Delta 99$ mutant cells exhibited reduced midzone-directed motility and efficiency to support spindle elongation. These results indicate the importance of directionality control for the function of Cin8 in the mitotic spindle.

In the third chapter, the capability of single Cin8 molecules of bi-directional switching in low-ionic-strength conditions was confirmed in single-molecule fluorescence in-vitro studies. By intensity analysis of kymographs from GFP-labelled Cin8 motor proteins in low-salt buffer, the possibility that cluster formation of Cin8 is necessary to induce plus-end directed motility, as proposed by Roostalu et al. [Roostalu11], could be ruled out. These findings are further evidence that the switching of directionality of single Cin8 molecules is regulated via cargo binding rather then mechanical coupling between two or more motors.

In the fourth chapter, the effect of the molecule monastrol, which was found to be a kinesin-5 inhibitor [Mayer99], on the Eg5 motor domain was investigated. In this work we constructed a stable dimeric kinesin-1/kinesin-5 chimera (Eg5Kin), which consists of the motor domain and neck linker of Eg5 (Fig. 1.3 D) and the neck coiled coil of Drosophila melanogaster kinesin-1 (DmKHC). Single-molecule fluorescence experiments showed that this chimera, in contrast to Eg5, was highly processive and exhibited no diffusive modes of motility. Adding the Eg5 inhibitor monastrol to the single-molecule assays, resulted in the finding that monastrol reduced the length of processive runs, but surprisingly did not affect the velocity of Eg5Kin. A set of experiments with successively increasing monastrol concentrations then finally indicated that the binding of a single monastrol molecule to an Eg5Kin dimer is not sufficient to stop its processive run, but rather that the simultaneous binding of two monastrol molecules is required to inhibit the dimer, i.e. terminate its run.

In the fifth chapter, the influence of the neck-linker length on the processive motility of twelve dimeric kinesin-1 stalk/kinesin- 5 head chimeras based on Eg5Kin was examined. The processive motility of kinesins relies on tight communication between the two motor domains of a dimeric molecule, such that binding strictly alternates to ensure that always one of the two motor domains is bound to the MT before the other motor domain unbinds to take the next step. The main elements to transmit this communication are believed to be the neck-linker domains connecting stalk and motor domains of the motor protein (Fig. $1.3 \mathrm{D}$ ). One proposed mechanism for the coordination of processive stepping is the transmission of intra-molecular stress through the neck linkers, leading to front-head or rear-head gating [Guydosh06, Shastry10]. In this model of a general mechanism for kinesin processivity, the efficiency of the gating and with that the degree of processivity of a kinesin is believed to be dependent on the length of the 
neck linker. Support for this simple model was presented in several recent studies [Yildiz08, Shastry10, Clancy11, Shastry11].

In the study presented here we analysed the motility of a set of twelve motor-protein constructs - based on the kinesin-1/kinesin-5 chimera Eg5Kin - in which the length of the neck linker was now varied. In these constructs, the neck-linker length ranged from 9 aa over the native 18 aa up to 21 aa of the wild type (WT) Eg5 neck linker. In one additional chimera 3 prolines were added to the native 18 aa of $\operatorname{Eg} 5(18+3 \mathrm{P})$ to gain an artificial neck-linker elongation. In multi-motor gliding assays all of the constructs were positively tested to be active motor proteins. In single-molecule fluorescence as well as in optical-trapping experiments the constructs were further tested on a single-molecule level for velocity, run length and force.

The experiments showed, surprisingly, that neither velocity nor force generation was dependent on the neck-linker length. Another interesting finding was that, contradictory to a recent report from Shastry et al. [Shastry11] in which they claimed that 14 aa is the shortest neck-linker length capable of producing processive motility, the construct with 13 aa in the neck linker was still highly processive. Even the construct with 12 aa still showed processive motility albeit with a shorter run length. For an even shorter neck linker in the construct with 9 aa no processive movement could be detected any more.

The neck-linker lengths close to the native neck-linker length (17 and 18 aa) allowed run lengths twice as long as those of the constructs with 13 to 16 aa. To check if the run length really peaks close to the native neck-linker length of Eg5 constructs with an even longer neck linker (19 to 21 aa) were tested and a decrease in run length with increasing neck-linker length was actually observed.

These findings challenge the simple model in which a short neck linker yields the tightest communication and hence the longest run length, but suggest instead that different kinesins might be optimised for different neck-linker lengths.

In the sixth chapter, the switching of a tetrameric kinesin-1 head/kinesin-5 tail chimera between diffusive and processive motility was studied. In this chimeric motor construct, named DK4mer, the motor domain from Xenopus Eg5 was replaced by the respective parts from Drosophila kinesin- 1 resulting in a fast and processive MT-sliding motor. Since Eg5 was shown to exhibit a complex mixture of diffusive and processive motility modes (Fig. $1.3 \mathrm{~A}$; [Kwok06, Kapitein08]), the aim of this study was to analyse the switching from diffusive to directed motility in more detail.

In single-molecule fluorescence in-vitro assays, DK4mer exhibited fast processive motility on single MTs, interrupted by diffusive pauses. When no ATP was present DK4mer diffused along single MTs, similar to Eg5 [Kwok06]. In MT-sliding assays DK4mer was able to slide antiparallel MTs apart confirming that it is a fully functional tetramer.

Compared to Eg5, DK4mer showed a very clear distinction between diffusive and processive motility modes which made it possible to measure transition rates as a function of ionic-strength in the motility buffer. The findings in this study indicate that the two motility modes of DK4mer reflect two independent and mutually exclusive modes of interaction with MTs which are likely to be relevant for the regulation of native kinesin-5 motors as well. 
Outside the topic of kinesin-5 regulation I participated in a project which investigated the targeting of axonal $\mathrm{GABA}_{B}$ receptors in hippocampal neurons. I contributed in-vivo total-internal-reflection-fluorescence (TIRF) measurements of the motility of fluorescently labelled $\mathrm{GABA}_{B} \mathrm{R} 1$ subunits to this study. For the sake of completeness, the main results of this study will be summarized here and the accepted manuscript is given in chapter 7 .

In neuronal cells the intracellular polarized protein trafficking is critical for synapse formation, synapse maintenance and the regulation of synaptic strength. In all eukaryotic cells the endomembrane trafficking system includes a synthetic route constituted by the endoplasmic reticulum (ER), the ER-Golgi intermediate compartment (ERGIC), the Golgi apparatus and post-Golgi vesicles, and a recyclingdegradative route constituted by endosomes and lysosomes (secretory pathway) [Horton04].

The main inhibitory neurotransmitter in the nervous system is $\gamma$ amino-butyric acid (GABA). Metabotropic GABA type B receptors $\left(\mathrm{GABA}_{B} \mathrm{Rs}\right)$ are neurotransmitter receptors that modulate synaptic transmission by mediating the slow and prolonged responses to GABA in two ways: (i) At presynaptic terminals $\mathrm{GABA}_{B}$ Rs inhibit voltage gated $\mathrm{Ca}^{2+}$ channels thereby inhibiting synaptic vesicle fusion and neurotransmitter release. (ii) At postsynaptic sites they activate inwardly rectifying $\mathrm{K}^{+}$ channels hyperpolarizing the postsynaptic neuron [Bettler04].

$\mathrm{GABA}_{B} \mathrm{Rs}$ are expressed in neurons throughout the brain and spinal cord. They are heteromers composed of two related subunits, $\mathrm{GABA}_{B} \mathrm{R} 1$ and $\mathrm{GABA}_{B} \mathrm{R} 2^{9}$. Only when $\mathrm{GABA}_{B} \mathrm{R} 2$ is associated to $\mathrm{GABA}_{B} \mathrm{R} 1$ a motif, that detains $\mathrm{GABA}_{B} \mathrm{R} 1$ in the ER (ER retention motif), is masked and the assembled $\mathrm{GABA}_{B} \mathrm{R}$ can exit the ER as heteromer destined for the plasma membrane [Couve98, Ramírez09].

From the different isoforms of $\mathrm{GABA}_{B} \mathrm{R} 1$ the most common subunit variants are $\mathrm{GABA}_{B} \mathrm{R} 1 \mathrm{a}$ and $\mathrm{GABA}_{B} \mathrm{R} 1 \mathrm{~b}$. The localisation of $\mathrm{GABA}_{B} \mathrm{Rs}$ in the neuron depends on which $\mathrm{GABA}_{B} \mathrm{R} 1$ variant assembled with $\mathrm{GABA}_{B} \mathrm{R} 2 . \mathrm{GABA}_{B} \mathrm{R}$ heteromers containing $\mathrm{GABA}_{B} \mathrm{R} 1 \mathrm{a}$ are located axonal and somatodendritic whereas those containing $\mathrm{GABA}_{B} \mathrm{R} 1 \mathrm{~b}$ are exclusively located in the somatodendritic domain of the neuron [Vigot06].

The precise targeting machinery for $\mathrm{GABA}_{B} \mathrm{R}$ distribution and the organelles involved in sorting and transport have not been described. Also how the local distribution of secretory components in the neuron influences intracellular trafficking and availability of neurotransmitter receptors is not fully understood.

In the study presented in chapter 7 conventional optical microscopy and live-cell imaging in cultured hippocampal neurons were used. The three main results describe a mechanism for $\mathrm{GABA}_{B} \mathrm{R} 1 \mathrm{a}$ axonal localization based on pre-Golgi sorting and ER transport: (i) The delivery of $\mathrm{GABA}_{B}$ Rs to the plasma membrane is Golgidependent but axonal sorting and targeting of $\mathrm{GABA}_{B} \mathrm{R} 1 \mathrm{a}$ operate in a pre-Golgi compartment. (ii) $\mathrm{GABA}_{B} \mathrm{R} 1 \mathrm{a}$ is targeted and transported within the axonal ER. (iii) The transport of axonal $\mathrm{GABA}_{B} \mathrm{R} 1 \mathrm{a}$ is microtubule-dependent and kinesin-1 determines axonal localization [Valdés12].

\footnotetext{
${ }^{9} \mathrm{~A}$ comprehensive review of $\mathrm{GABA}_{B} \mathrm{R}$ structure, function, localization and pathological implications is given in [Bettler04]
} 
Considering that progression of $\mathrm{GABA}_{B}$ Rs through the secretory pathway is regulated by an ER retention motif this data contributes to understand the role of the axonal ER in sorting and targeting of neurotransmitter receptors. 


\section{Directionality of individual kinesin-5 Cin8 motors is modulated by loop 8, ionic strength and microtubule geometry}

The content of this chapter was published 2011 in the European Molecular Biology Organization (EMBO) Journal [Gerson-Gurwitz11]. I share the first authorship with Adina Gerson-Gurwitz. Co-authors are Natalia Movshovich, Vladimir Fridman, Maria Podolskaya, Tsafi Danieli, Stefan Lakämper, Dieter R. Klopfenstein, Christoph F. Schmidt and Larisa Gheber.

In this work I participated in the purification of proteins. Further I performed all the single-molecule in-vitro experiments at the custom built total internal reflection fluorescence (TIRF) microscope setup and analysed the single-molecule data. The single-molecule relative-sliding experiments were also done and analysed by me. Finally I contributed figures and text to the paper.

\section{\begin{tabular}{l|l} 
Abstract & 2.1
\end{tabular}}

Kinesin-5 motors fulfil essential roles in mitotic spindle morphogenesis and dynamics as slow, processive microtubule (MT) plus-end directed motors. The Saccharomyces cerevisiae kinesin-5 Cin8 was found, surprisingly, to switch directionality. Here, we have examined directionality using single-molecule fluorescence motility assays and live-cell microscopy. On spindles, Cin 8 motors mostly moved slowly $(\sim 25 \mathrm{~nm} / \mathrm{s})$ towards the midzone, but occasionally also faster $(\sim 55 \mathrm{~nm} / \mathrm{s})$ towards the spindle poles. In vitro, individual Cin8 motors could be switched by ionic conditions from rapid ( $\sim 380 \mathrm{~nm} / \mathrm{s})$ and processive minus-end to slow plus-end motion on single MTs. At high ionic strength, Cin8 motors rapidly alternated directionalities between antiparallel MTs, while driving steady plus-end relative sliding. Between parallel MTs, plus-end motion was only occasionally observed. Deletion of the uniquely large insert in loop 8 of Cin 8 induced bias towards minus-end motility and affected the ionic strength-dependent directional switching of Cin8 in vitro. The deletion mutant cells exhibited reduced midzone-directed motility and efficiency to support spindle elongation, indicating the importance of directionality control for the anaphase function of Cin8.

\section{\begin{tabular}{l|l} 
Introduction & 2.2
\end{tabular}}

Members of the kinesin-5 family are homotetrameric motor proteins, which utilize ATP to slide apart antiparallel spindle microtubules (MTs) [Kashina97, Kapitein05]. 
They are conserved among the eukaryotes and fulfil similar functions in spindle dynamics [Enos90, Roof91, Hagan92, Hoyt92, Sawin92a, Heck93, Blangy95, Saunders95, Walczak96, Straight98, Sharp99, Touitou01, Brust-Mascher04, Zhu05]. S. cerevisiae encodes two kinesin-5 homologues, Cin8 and Kip1, which overlap in function, with Cin8 being more important for mitosis progression [Hoyt92, Roof92]. Before the onset of anaphase, Cin8 and Kip1 function in spindle assembly [Hoyt92, Roof92, Chee10] and focus the kinetochore clusters [Tytell06, Gardner08a, Wargacki10]. During spindle elongation in anaphase B, their function is important for (i) maintaining the correct spindle elongation rate [Kahana95, Saunders95, Straight98], (ii) the timely transition from the initial fast phase to the final slow phase of anaphase B [Movshovich08, Gerson-Gurwitz09] and (iii) for stabilizing the spindle midzone [Movshovich08, Fridman09, Gerson-Gurwitz09], which consists of an overlapping array of MTs that emanate from the opposite poles [Winey95]. To guarantee proper mitosis, all components involved, and in particular the motors, have to be tightly regulated. The regulation of kinesin- 5 motors is so far poorly understood.

Several kinesin-5 motors were found to be plus-end directed in vitro [Gheber99], [Kapitein05]. Independent of our findings, however, a recent report has provided evidence that Cin 8 can switch directionality, assumed to be caused by motor-motor coupling [Roostalu11]. Molecular mechanisms as well as physiological relevance have remained unclear.

To track down the mechanisms of directionality control, we have here studied the motor function of the $S$. cerevisiae kinesin-5 Cin8 in parallel in-vivo and in-vitro experiments. We provide evidence that during anaphase spindle elongation, Cin 8 moves in both plus- and minus-end directions on the spindle MTs. We also show that, in vitro, single molecules of Cin8 can switch directionality from fast processive minus-end directed to slow, processive plus-end directed motility. We provide first insights into the mechanism controlling this switch: (i) change of ionic strength, that is, electrostatic interactions within the motor or between motor and MT, activated the switch in vitro; (ii) deletion of the large insert in loop 8 of the Cin 8 motor domain induced bias to minus-end directionality in vitro and reduced Cin8 plus-end directed motility along the anaphase spindles in vivo; (iii) in close to physiological conditions in vitro, individual Cin8 motors switched from fast minus-end motion on single MTs to slow and erratic motion between antiparallel MTs, while they drove plus-end sliding of the linked MTs; (iv) individual motors occasionally moved in the plus-end direction, but mostly maintained fast minus-end motion between parallel MTs.

\section{\begin{tabular}{l|l} 
Results & 2.3
\end{tabular}}

To examine the motile properties of Cin8 in vivo, we imaged live $S$. cerevisiae cells expressing Cin8-3GFP during anaphase B. As spindles elongated, Cin8 was localized both at the spindle midzone and near the poles, likely near the kinetochores as reported before [Tytell06]. In late anaphase, Cin8 detached from the spindle and became diffusive (Fig. 2.1 A; Supplementary Video A.3.1), confirming previous reports [Avunie-Masala11]. Kymograph analysis of fluorescence recordings revealed 


\section{A}

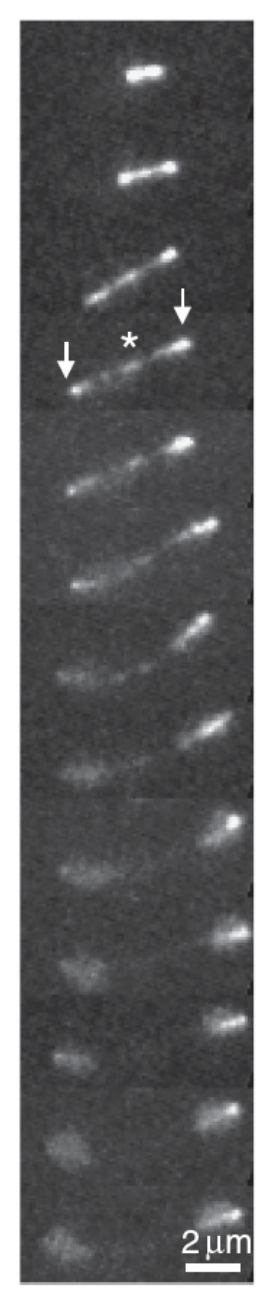

B

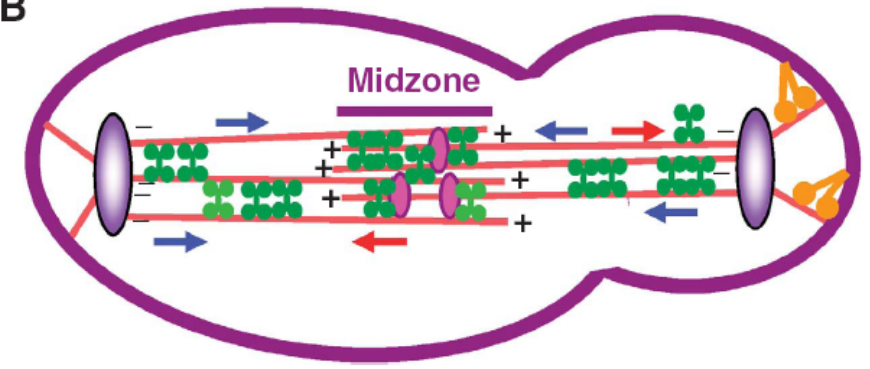
Л $\begin{aligned} & \text { ST } \\ & \text { bodydle-pole }\end{aligned}$
Cin8
C) Kip1
Dyn1
O Ase1
$\rightarrow$ Plus-end directed movements towards the midzone
$\rightarrow$ Minus-end directed movements towards the poles

C

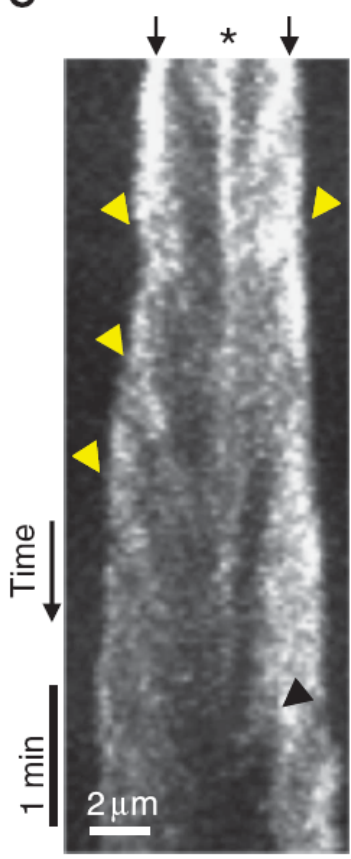

D

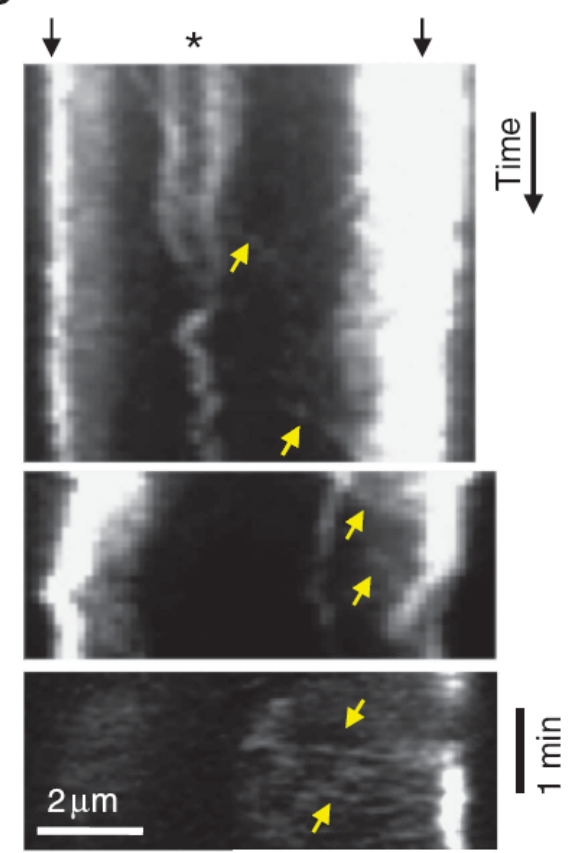

Fig. 2.1: In vivo, Cin8 occasionally switches directionality. (A) 2D-projection time-lapse confocal fluorescence images of Cin8-3GFP localization in WT cells. Time intervals: 1 min. (B) Model of Cin8 plus-end and minus-end directed movement in the anaphase spindle in S. cerevisiae cells. (C, D) Kymographs of Cin8-3GFP localization to the anaphase spindle. (A, C, D) Top arrows: spindle poles; asterisk: spindle midzone; arrowheads: plus-end-directed movements towards the midzone; yellow arrows: minus-end directed movements towards the poles. 
that Cin8-3GFP moved towards the midzone, in the plus-end direction of MTs, with an average velocity of $\sim 25 \mathrm{~nm} / \mathrm{s}$ (Fig. 2.1 C and Fig. 2.7 A; Tab. $2.1 \mathrm{a}$ ).

This slow plus-end directed motility is consistent with the literature on Cin8 and the Xenopus kinesin-5 Eg5 [Gheber99, Kwok04, Kapitein05]. Intriguingly, we also observed occasional movements towards the spindle-pole bodies (SPBs; Fig. $2.1 \mathrm{D}$ and Fig. 2.7 A; Tab. 2.1 b), mainly in long anaphase spindles, when the majority of Cin8 was already detached (Fig. $2.1 \mathrm{D}$ and Fig. $2.7 \mathrm{~A}$ ). The scarcity of SPB-directed movements may be due to their rapidity (Tab. $2.1 \mathrm{~b}$ ) and/or to masking by abundant Cin8-3GFP on the spindle. In intermediate anaphase spindles, shorter than $5 \mu \mathrm{m}$, interpolar MTs (iMTs) can overlap almost for the entire spindle length [Winey95, Gardner08b]. Therefore, in these spindles, the directionality of Cin8 is difficult to determine. In the long anaphase spindles, however, iMTs overlap only in the middle $10-20 \%$ of the spindle length, as determined by electron microscopy [Winey95], direct imaging of GFP-labelled MTs [Avunie-Masala11], or imaging of the midzone-organizing protein Ase1 [Schuyler03, Fridman09]. The fact that Cin8 motility towards the midzone as well as towards the SPBs was observed in spindles longer than $5 \mu \mathrm{m}$ (Fig. 2.1 C, D and Fig. 2.7 A), suggests that these movements occur on parallel arrays of iMTs. Moreover, SPB-directed movements were significantly faster and shorter than midzone-directed movements (Tab.

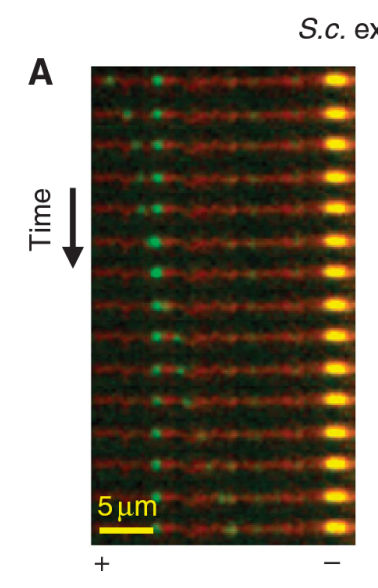

S.c. extracts
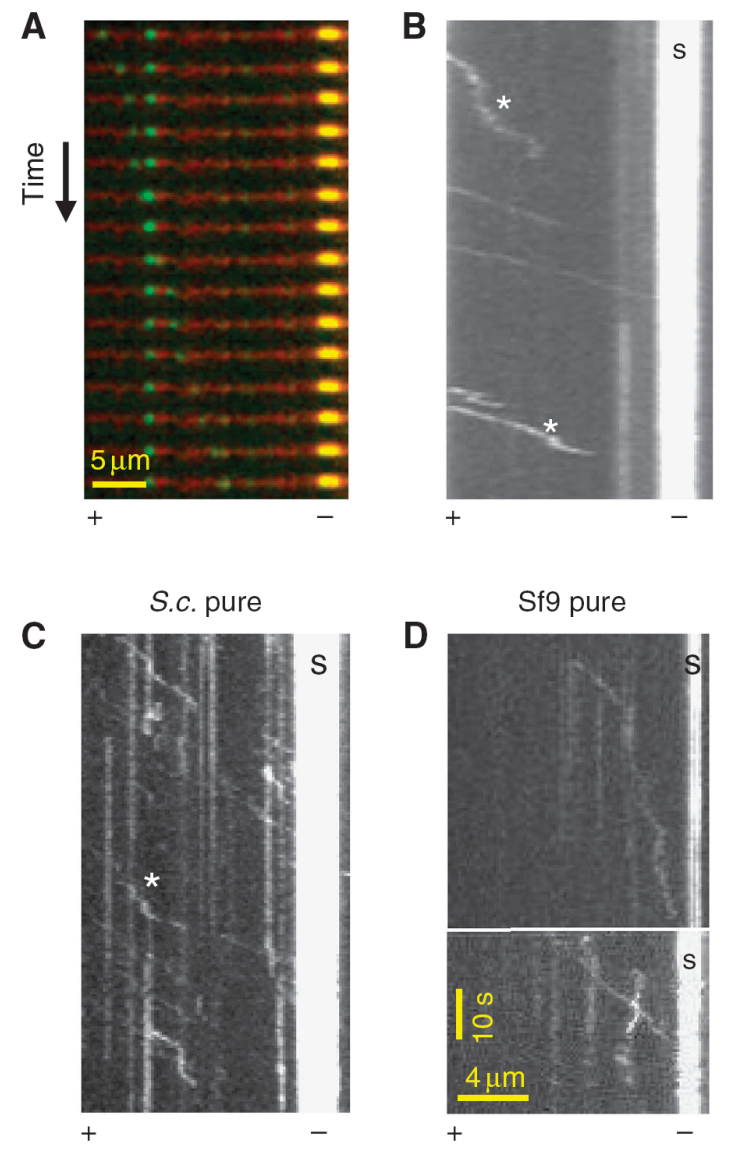

Fig. 2.2: At high salt in vitro, Cin8 is a minus-end directed motor. (A) Timelapse recording showing a single Cin83GFP molecule from whole-cell extract (green) moving directionally along the MT (red) towards the bright seed (yellow). Time intervals: 2 s. (B - D) Kymographs of Cin8 movement on polarity-marked MTs. (B) Cin8-3GFP from whole-cell extract (C) Cin8-GFP purified from yeast cells and (D) Cin8-GFP purified from Sf9 cells. S: bright seed indicating the MT minus end. Scale bars in (D) also apply to (B) and (C). Asterisk indicates diffusive episotes. See also Supplementary Videos A.3.2 and A.3.3. 2.1 ), indicating that movements in the two directions were genuinely different. Thus, taken together, our data indicate that on the anaphase spindles, Cin8 is a bi-directional motor that moves in both plus-end and minus-end directions of the MTs. 


\begin{tabular}{|c|c|c|c|c|}
\hline Cin8 variant & $\mathrm{N}^{a}$ & $\%$ Of spindles ${ }^{b}$ & Velocity $(\mathrm{nm} / \mathrm{s})(\mathrm{n})^{c, d}$ & Run length $(\mathrm{nm})(\mathrm{n})^{c, d}$ \\
\hline$W T \operatorname{Cin} 8$ & 113 & 59 & $26.5 \pm 0.7(120)$ & $1506 \pm 50(120)$ \\
\hline $\operatorname{Cin} 8 \Delta 99$ & 83 & 29 & $15.3 \pm 0.5(123)^{* * * g}$ & $1091 \pm 35(123)^{* * * g}$ \\
\hline Cin8-2A & 102 & 41 & $23.0 \pm 0.8(183)^{* g}$ & $1236 \pm 34(183)^{* * * g}$ \\
\hline
\end{tabular}

(a) Midzone-directed movements of Cin8-3GFP variants in the anaphase spindles.

\begin{tabular}{lrccl} 
Cin8 variant & $\mathrm{N}^{a}$ & \% Of spindles $^{e}$ & Velocity $(\mathrm{nm} / \mathrm{s})(\mathrm{n})^{c}$ & Run length $(\mathrm{nm})(\mathrm{n})^{c}$ \\
\hline \hline$W T$ Cin8 & 113 & 20 & $56.4 \pm 4.2(29)^{* * * f}$ & $845 \pm 43(29)^{* * * f}$ \\
Cin8 $\Delta 99$ & 83 & 18 & $55.8 \pm 2.9(33)^{* * * f}$ & $891 \pm 30(33)^{* f}$ \\
Cin8-2A & 102 & 20 & $63.6 \pm 3.1(32)^{* * * f}$ & $943 \pm 62(33)^{* * f}$ \\
\hline
\end{tabular}

(b) SPB-directed movements of Cin8-3GFP variants in the anaphase spindles.

Tab. 2.1:

${ }^{a} \mathrm{~N}$ - number of intermediate-long $(5-10 \mu \mathrm{m})$ spindles analysed.

$b \%$ Of spindles with at least one midzone-directed movement spanning from SPB to the midzone (see Fig. 2.5).

${ }^{c}$ Values represent average \pm s.e.m., $\mathrm{n}$ - number of movements.

${ }^{d}$ Values determined for movements longer than $0.5 \mu \mathrm{m}$, including movements that do not reach the midzone.

e\% Of spindles with at least one SPB-directed movement, longer than $0.5 \mu \mathrm{m}$.

${ }^{f}$ Compared with the corresponding movements in cells of the same genotype.

${ }^{g}$ Compared with cells expressing $W T$ Cin8.

${ }^{* * *} \mathrm{P}<0.0001 ;{ }^{* *} \mathrm{P}<0.001 ;{ }^{*} \mathrm{P}<0.005$ (Student's t-test).

To explore the unconventional minus-end motility of Cin8 on the spindle, we performed single-molecule fluorescence in-vitro motility assays [Vale96, Kwok06, Kapitein08]. We used whole-cell extracts of $W T$ and cin8 $S$. cerevisiae cells, in which Cin8-3GFP was expressed from its native promoter, either integrated into the genome or carried on a centromeric (CEN) plasmid (Materials and Methods, section 2.5; Supplementary Tab. A.1). We also studied Cin8-GFP purified from Sf9 and yeast cells (Materials and Methods; Supplementary Fig. A.1). Assays were done at saturating ATP (3-5 mM, [Lakämper10]) in a high-salt, close to physiological buffer $(175 \mathrm{mM} \mathrm{NaCl}$, [Gheber99]).

We found, surprisingly, that in these conditions Cin8 moved unidirectionally and processively towards the minus-end of MTs (Fig. 2.2). This is similar to what was recently found in an independent study [Roostalu11]. Runs were often interrupted by diffusive motion (Fig. 2.2 B and C). The average run length between such breaks was $2 \mu \mathrm{m}$ (Fig. 2.3 B). In ADP buffer, motors remained attached to MTs, but only moved diffusively without directional bias. We calculated a diffusion coefficient of $\sim 3 \times 10^{3} \mathrm{~nm}^{2} / \mathrm{s}$ from mean-square-displacement (MSD) analysis of tracks recorded in ADP buffer, comparable to that measured with the closely related Eg5 and other kinesins (Tab. 2.2; [Kwok06, Kapitein08, Bormuth09]). At saturating ATP concentration, we determined an average minus-end velocity of Cin8 molecules of $\sim$ 


\begin{tabular}{|c|c|c|c|}
\hline Genotype & $\mathrm{V}_{K Y M O}{ }^{a}(\mathrm{~nm} / \mathrm{s})$ & $\mathrm{V}_{M D^{b, c}}(\mathrm{~nm} / \mathrm{s})$ & $\mathrm{D}_{A D P} \times 10^{3 c, d}\left(\mathrm{~nm}^{2} / \mathrm{s}\right)$ \\
\hline$W T(\mathrm{pCIN} 8-3 \mathrm{GFP})^{h}$ & $353 \pm 33(116)$ & \multirow{2}{*}{$372 \pm 7^{i}(52)$} & \multirow{2}{*}{$3.7 \pm 0.1^{i}(30)$} \\
\hline CIN8-3GFP ${ }^{k}$ & $365 \pm 27(178)$ & & \\
\hline $\operatorname{cin} 8 \Delta \operatorname{kip} 1 \Delta(\mathrm{pCIN8-3GFP})^{h}$ & $381 \pm 38(100)$ & $345 \pm 8(70)$ & $2.9 \pm 0.1^{* * * l}(24)$ \\
\hline ase1 $(\mathrm{pCIN8-3GFP})^{h}$ & $241 \pm 17^{* * * l}(198)$ & $272 \pm 8^{* * * l}(39)$ & $4.2 \pm 0.2(23)$ \\
\hline
\end{tabular}

\begin{tabular}{|c|c|c|c|}
\hline Genotype & Stall periods ${ }^{e}(\%)$ & $\operatorname{Run~length}^{f}(\mathrm{~nm})$ & Interact. time ${ }^{g}(\mathrm{~s})$ \\
\hline $\begin{array}{l}W T(\mathrm{pCIN} 8-3 \mathrm{GFP})^{h} \\
\text { CIN8-3GFP }^{k}\end{array}$ & $39(1645)^{j}$ & $1700 \pm 200(94)$ & $8.3 \pm 1.0(65)$ \\
\hline $\operatorname{cin} 8 \Delta \operatorname{kip} 1 \Delta(\mathrm{pCIN} 8-3 \mathrm{GFP})^{h}$ & $20(1377)^{j}$ & $2000 \pm 200(132)$ & $9.5 \pm 0.9(115)$ \\
\hline ase1 $(\mathrm{pCIN8}-3 \mathrm{GFP})^{h}$ & $33(1732)^{j}$ & $1200 \pm 100^{* * l}(118)$ & $8.4 \pm 1.0(66)$ \\
\hline
\end{tabular}

Tab. 2.2: In-vitro motile properties of Cin 8 in whole-cell extracts.

Values are average \pm s.e.m. In parentheses, the number of measurements.

${ }^{a} \mathrm{~V}_{K Y M O}$ - average velocity determined from kymograph analysis.

${ }^{b} \mathrm{~V}_{M D}$ - average velocity determined from mean-displacement analysis.

${ }^{c}$ Fit is done to first $15 \mathrm{~s}$.

${ }^{d} \mathrm{D}_{A D P}$ - diffusion coefficient in the presence of $3-5 \mathrm{mM}$ ADP determined from MSD analysis.

${ }^{e}$ Percentage calculated from total motion time.

${ }^{f}$ Run length of directional episodes.

${ }^{g}$ Measurements applied on kymograph traces of complete directional runs, of which both their beginning and end are apparent.

${ }^{h}$ Cin8-3GFP expressed on CEN plasmid.

${ }^{i}$ Measurements carried out on Cin8-3GFP either expressed on CEN plasmid or integrated into the genome.

${ }^{j}$ Total time of measurement (s).

${ }^{k}$ Cin8-3GFP integrated into the genome.

${ }^{l}$ Compared with $W T$.

${ }^{* * *} \mathrm{P}<0.0001 ;{ }^{* *} \mathrm{P}<0.001$ (Student's t-test).

$360 \mathrm{~nm} / \mathrm{s}$ by kymograph and mean-displacement (MD) analyses (Tab. 2.2). This velocity is substantially higher than the plus-end velocity seen in vivo and the velocity reported for other kinesin-5 motors [Valentine06]. In both WT Cin8 batches examined, purified Cin8-GFP and Cin8-3GFP in whole extracts, the distribution of velocities was exceptionally broad in each experiment, ranging from $\sim 17$ to $\sim$ $830 \mathrm{~nm} / \mathrm{s}$ (Supplementary Fig. A.2 A; Fig. 2.4 A(I) and B(I)). We ruled out the possibility that the broad distribution of velocities resulted from motor aggregation by analysing fluorescence intensities of individual spots (Supplementary Fig. A.3).

To make sure that Cin8-3GFP was capable of forming functional tetramers, we examined its MT bundling activity. We found that overexpression of Cin8-3GFP caused extensive bundling of MTs in whole-cell extracts (Supplementary Fig. A.4 
A, left panel) which was not observed in extracts deleted for CIN8 (Supplementary Fig. A.4 A, right panel) or in extracts expressing Cin8-3GFP from a CEN plasmid (not shown). This excludes the possibility that the bundling was caused by other motors or MT-binding proteins in the extracts. Similar MT bundling was previously reported for biotinylated Cin8 (Cin8-BCP; [Gheber99]), which was shown to be a homotetramer [Hildebrandt06]. MT bundling is known to occur only with the full-length tetrameric, but not with truncated dimeric Cin8 (Cin8-871) [Gheber99, Hildebrandt06], suggesting that Cin8-3GFP is a tetramer. Finally, both Cin8-GFP and Cin8-3GFP could maintain the viability of cells deleted for chromosomal copies of CIN8 and KIP1 (Supplementary Fig. A.4 B). Since only tetrameric Cin8 maintains viability of $\operatorname{cin} 8 \Delta \operatorname{kip} 1 \Delta$ cells [Hildebrandt06], our findings indicate that Cin8-3GFP is mostly a functional tetramer.

In summary, our data indicate that the motile properties we observed for Cin8, the broad distribution of velocities, the minus-end directionality and the diffusive motion, did not result from altered oligomeric states or aggregation of the examined Cin8 variants, but rather point to an intrinsic regulation of individual Cin8 motors.

The use of whole-cell yeast extracts from deletion mutants in single-molecule motility assays allowed us to examine the influence of other $S$. cerevisiae cellular factors on Cin 8 motility (Tab. 2.2; Fig. 2.3). We explored the effect, in high-salt buffer, of two major spindle-binding proteins that functionally overlap or interact with Cin8: the kinesin-5 Kip1 [Hoyt92, Roof92] and the MT-binding and midzoneorganizing protein Ase1 [Schuyler03]. Ase1 was shown to physically interact with Cin8 during mitosis and to recruit Cin8 to the spindle midzone [Khmelinskii09].

To examine the influence of Kip1 and Ase1 on Cin8 motor function, we examined

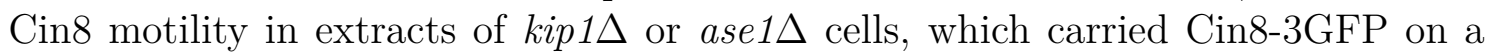
CEN plasmid. We found that deletion of Kip1 or Ase1 did not affect the directionality of Cin8, as it remained minus-end directed in high-salt buffer (Fig. 2.3). In the absence of Kip1, Cin8 was somewhat more processive; its interaction time with MTs was longer, the percentage of runs with stalls was lower, and the diffusion coefficient of Cin8 in the presence of ADP was significantly lower than that of Cin8 in all the other extracts examined (Tab. 2.2; Fig. 2.3 B). Consistently, in kip $1 \Delta$ cells, the detachment of Cin8 from the spindle MTs in late anaphase was reduced compared with $W T$ cells (Fig. $2.3 \mathrm{C}$ and D). In the absence of Ase1, the average run length of Cin8-3GFP directional episodes was significantly decreased, and the average velocity dropped to $\sim 240 \mathrm{~nm} / \mathrm{s}$ (Tab. 2.2; Fig. $2.3 \mathrm{~A}$ and B). In cells, Cin8-3GFP localized abnormally on the anaphase spindle in the absence of Ase1 and was not detectable in the midzone (Fig. 2.3 E). These results suggest that Ase1 regulates Cin8 spindle localization by affecting the motile properties of Cin8.

It remains to understand the circumstances under which the motor switches to plus-end motility, the mode of motion that is believed to drive the poleward relative sliding of antiparallel overlapping MTs in anaphase. Since other kinesins, including the kinesin-5 Eg5, can be turned on and off by varying ionic conditions [Dietrich08, Hancock08, Kapitein08, Hackney09], we tested whether a decrease in ionic strength could affect directionality. We performed single-molecule motility assays with Cin8, either purified or in whole-cell extracts, on polarity-marked MTs at different salt concentrations. We found that with decreasing ionic strength, individ- 
A

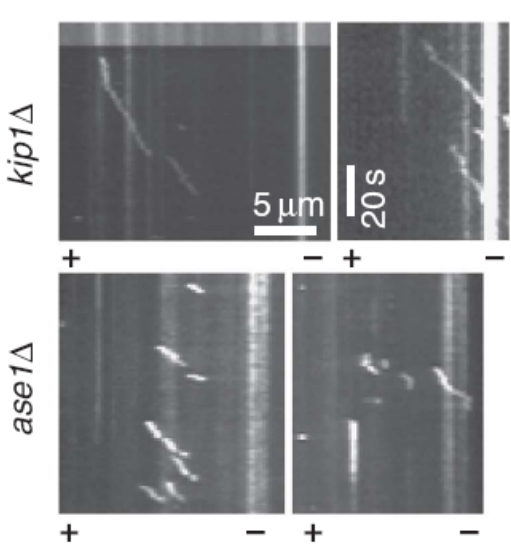

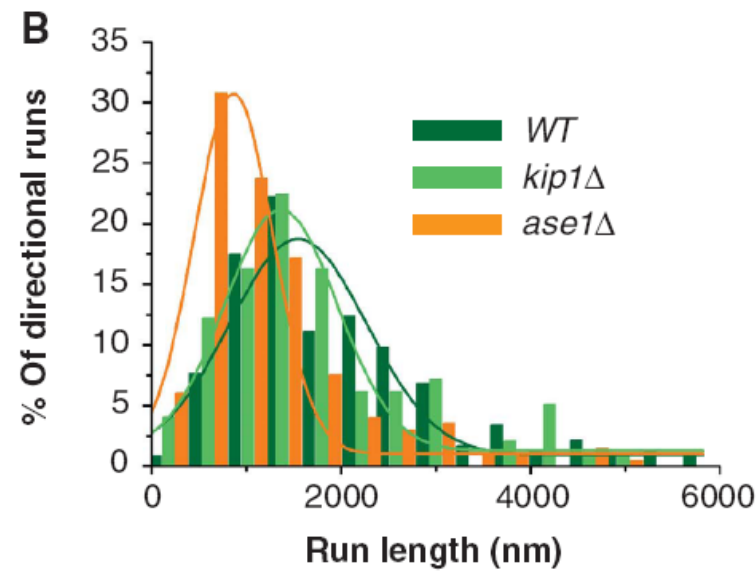

Run length (nm)

\section{Cin8-3GFP}

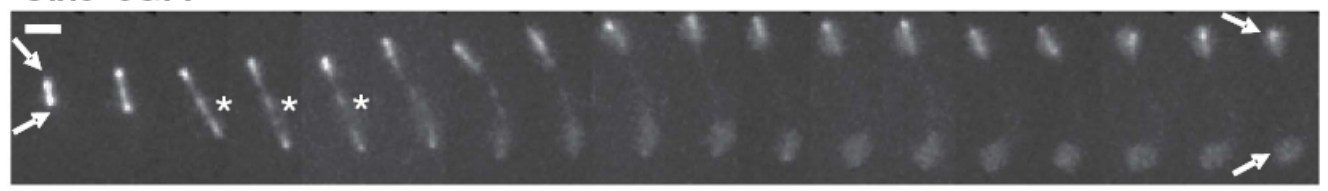

D kip1DCin8-3GFP

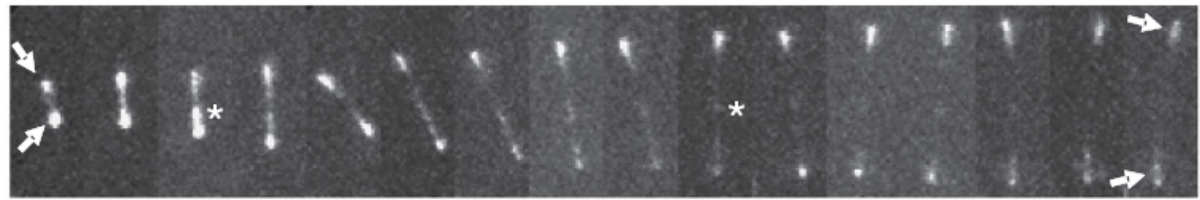

E ase1A Cin8-3GFP

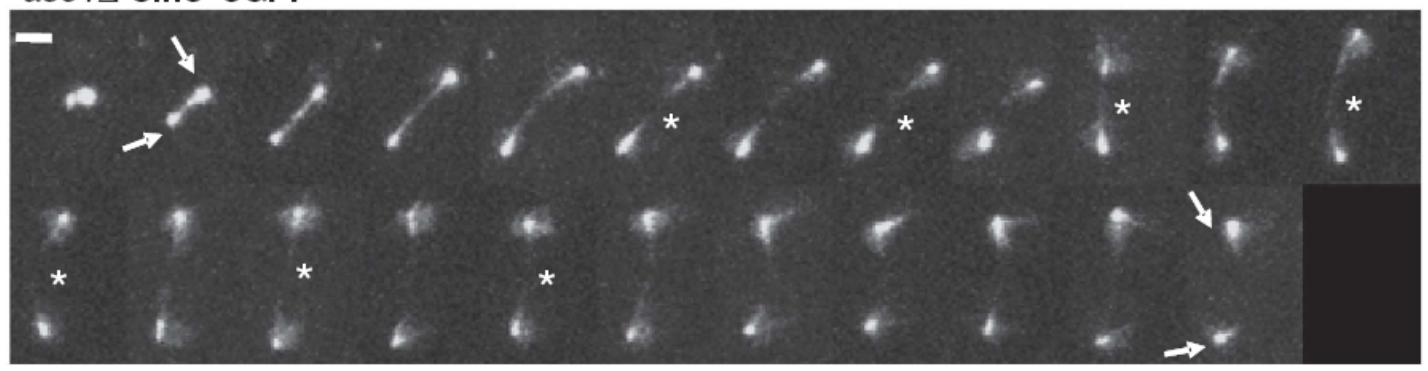

Fig. 2.3: The effect of spindle proteins on Cin8 in-vitro motile properties and in-vivo localization to anaphase spindles. (A, B) Single-molecule fluorescence motility assay was carried out on polarity-marked MTs with kip $1 \Delta$ or ase1 $\Delta$ cell extracts expressing Cin83GFP. Extracts were diluted in high-salt $(175 \mathrm{mM} \mathrm{NaCl})$ buffer. (A) Representative kymographs of in-vitro runs of Cin8-3GFP in the different extracts. (B) Histograms of run lengths of Cin8-3GFP directional episodes in $W T$ (olive), kip1 $\Delta$ (light green) or ase1 $\Delta$ (orange) extracts. Fit: Gaussian distribution. (C - E) Representative 2D-projection timelapse confocal fluorescence images of Cin8-3GFP localization to anaphase spindles in (C) $W T$ (D) kip $1 \Delta$ and (E) ase1 $\Delta$ cells. Asterisk: spindle midzone; arrows: spindle poles; time interval between frames: $1 \mathrm{~min}$; scale bar: $2 \mathrm{~mm}$. Genotypes and Cin8 variants are marked on top. 

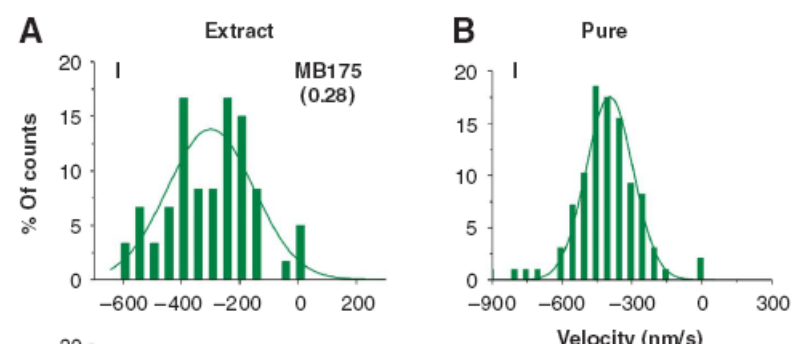

C
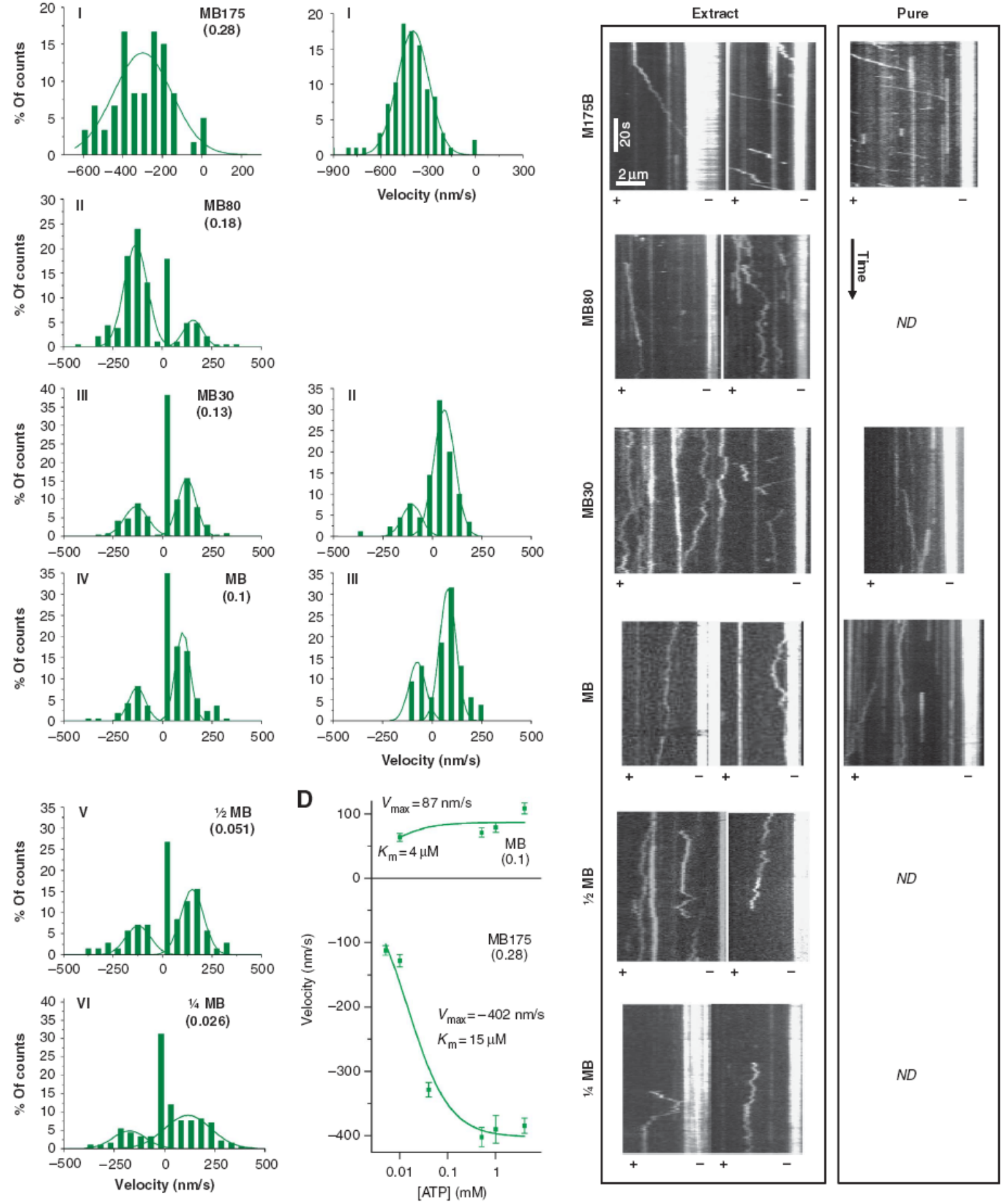

Fig. 2.4: Decrease in ionic strength induces plus-end motility of single molecules of Cin8. (A, B) Histograms of velocities of Cin8 in whole-cell yeast extracts (A) or affinity purified (B) with saturating ATP. Salt and buffer conditions are indicated for each panel; MB: motility buffer; numbers adjacent to "MB" indicate the concentration ( $\mathrm{mM}$ ) of added $\mathrm{NaCl}$. Ionic strength $(\mathrm{M})$ is indicated in parentheses. A control experiment of motility with ADP was carried out using buffer with $30 \mathrm{mM} \mathrm{NaCl}$ (see Supplementary Fig. A.2 C). Velocity histograms were assembled by dividing kymograph traces in $3 \mathrm{~s}$ segments and piecewise linear fitting. Lines: Gaussian distribution fit. (C) Representative kymographs of Cin8 motility along polarity-marked MTs. Expression/purification conditions are indicated on top. Plus- $(+)$ and minus- $(-)$ ends of MTs are indicated. ND: not detected. See also Supplementary Video A.3.4. (D) ATP concentration dependence of plus-end (top) and minus-end directed (bottom) velocity of $\mathrm{Cin} 8$ (average \pm s.e.m.). $\mathrm{NaCl}$ concentration and ionic strengths (parenthesis) are indicated. Michaelis-Menten parameters $\mathrm{V}_{\max }$ and $\mathrm{K}_{m}$ are indicated. 
ual Cin8 motors gradually switched towards plus-end motility (Fig. 2.4 A - C). At ionic strengths $\leq$ 0.13, Cin8 moved bi-directionally, moving $60-80 \%$ of the time towards the plus ends of MTs (Fig. 2.4 A - C). Reduction of ionic strength also significantly decreased the magnitude of the minus-end velocity of Cin8 (Fig. 2.4 A - C) from $\sim 380 \mathrm{~nm} / \mathrm{s}$ in high ionic strength to $\sim 130 \mathrm{~nm} / \mathrm{s}$ in low ionic strength (Supplementary Fig. A.5). The salt dependence of Cin8 motility in whole-cell extracts was similar to that of affinity-purified Cin8 (Fig. 2.4 A and B), although more motors were immobile in the extracts (Fig. $2.4 \mathrm{~A}$ and B), possibly due to the presence of other MT-binding agents. The velocity of movement in both minusand plus-end directions was dependent on ATP concentration (Fig. 2.4 D; Supplementary Fig. A.2 C), proving that bi-directional motion of single Cin8 molecules is not merely driven by thermal forces in the solution. These results demonstrate that a single Cin 8 motor interacting with only one MT can switch directionality. The mechanism for the switch is, therefore, most likely contained in a single motor.

Any switch mechanism is likely to depend, at least in part, on structural elements within the motor's catalytic domains. In comparison with kinesin- 5 homologues of higher eukaryotes, several yeast kinesin- 5 motors carry inserts of considerable length in loop 8 which is involved in MT binding [Kull96, Nitta08, Chee10]. Cin8 and the closely related Candida glabrata kinesin-5 carry the largest inserts, of 99 amino-acid length (99aa) [Chua07]. To probe the role of the large loop 8 in the directionality switch of single Cin8 molecules, we studied a construct in which this segment was replaced with the seven amino acids of loop 8 in the related $S$. cerevisiae kinesin-5 Kip1 (Cin8 $\triangle 99$ ). Yeast cells deleted for the chromosomal copies of CIN8 and KIP1, but expressing Cin8 carrying this deletion were previously shown to be viable, indicating that the mutant Cin8 is, at least partially, functional [Hoyt92]. We found that with decreasing ionic strength, single Cin8 $\Delta 99$ molecules also switched from minusend to plus-end directed motility in whole extracts (Figure $2.5 \mathrm{~A}$ ) and in purified samples (Fig. 2.5 B). However, Cin8 $\triangle 99$-GFP behaved distinctly differently from WT Cin8-GFP (Fig. 2.4 - Fig. 2.6). In whole-cell extracts, at high salt (175 mM $\mathrm{NaCl}$ ), Cin8 $\triangle 99-\mathrm{GFP}$ did not attach to MTs at all. The same was seen for purified $W T$ Cin8, albeit only at $>250 \mathrm{mM} \mathrm{NaCl}$. At $175 \mathrm{mM}$ added $\mathrm{NaCl}$, affinity-purified Cin8 $\Delta 99$ had a slightly lower average velocity than WT Cin8 (Supplementary Fig. A.5). With $30 \mathrm{mM}$ added $\mathrm{NaCl}$, the presence or absence of loop 8 in the WT Cin 8 correlated with clearly opposite behaviours: in whole extracts and purified samples, Cin8 499 remained minus-end directed, while $W T$ Cin8 moved predominantly in the plus-end direction with some bi-directional shuttling (Fig. 2.6 A and B). A systematic comparison between Cin8 and Cin8 $\Delta 99$ motility in different buffers showed that the deletion of the 99aa insert did not eliminate the switch of directionality, but pushed the transition from minus-end to plus-end directionality to lower salt concentrations (Fig. 2.6 C), supporting the notion that the directionality switch of Cin8 involves loop 8.

To examine how the bias to minus-end directionality of the mutant Cin $8 \Delta 99$ affects its function in vivo, we monitored motor localization in cells expressing Cin8 $\Delta 99$. In contrast to $W T$ Cin8-3GFP, Cin8 $499-3 \mathrm{GFP}$ did not detach from the spindles and seemed to be asymmetrically distributed on the anaphase spindles (Supplementary Fig. A.6). The plus-end directed movements of Cin8 $\Delta 99$-3GFP 
A
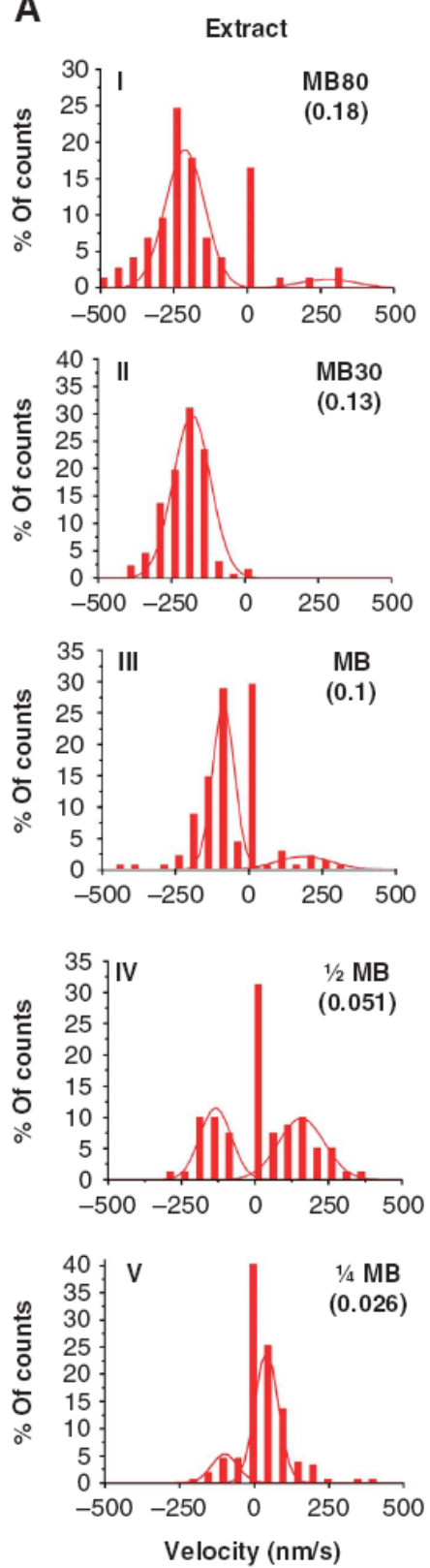

B
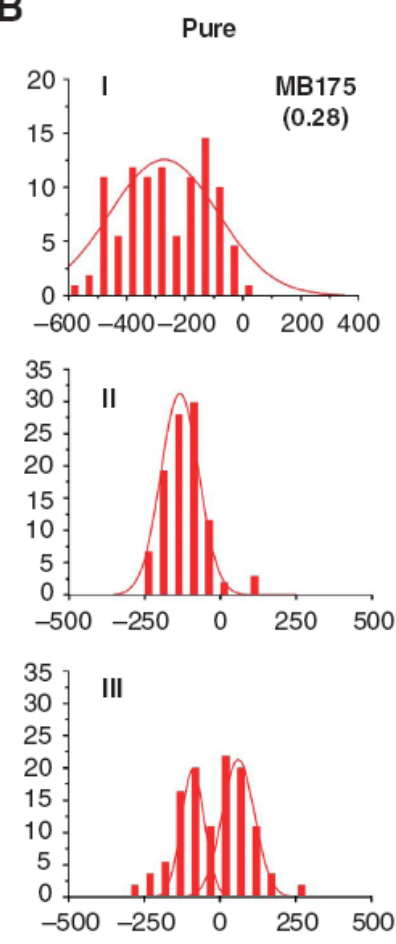

Velocity (nm/s)
D

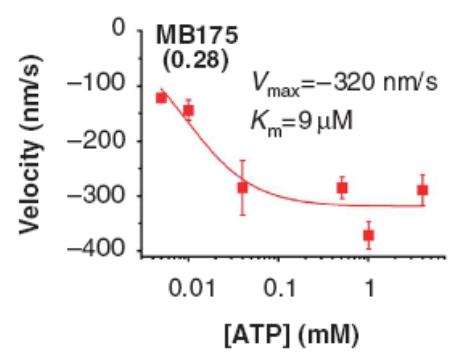

C

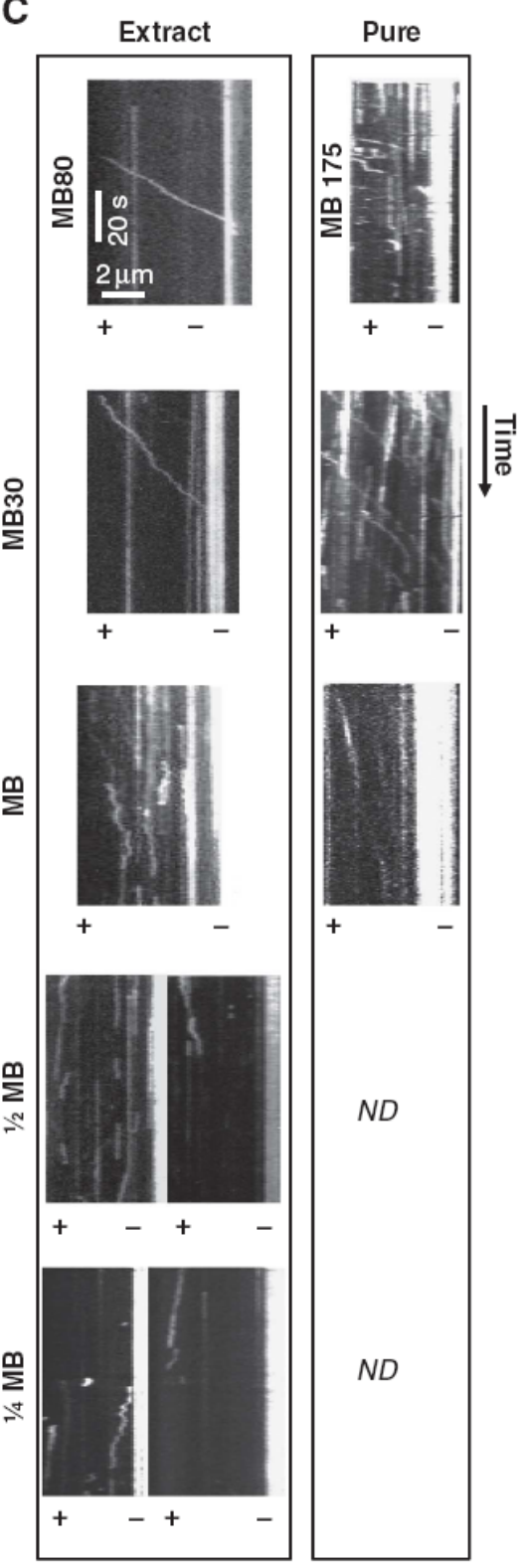

Fig. 2.5: Decrease in ionic strength induces plus-end motility of single molecules of Cin8 $\Delta 99$. (A, B) Histograms of velocities of Cin8 899 in whole-cell yeast extracts (A) or affinity purified (B) with saturating ATP. Salt and buffer conditions are indicated for each panel; MB:motility buffer; numbers adjacent to "MB" indicate the concentration (mM) of added $\mathrm{NaCl}$. Ionic strength $(\mathrm{M})$ is indicated in parentheses. Velocity histograms were assembled by dividing kymograph traces in $3 \mathrm{~s}$ segments and piecewise linear fitting. A control experiment of motility with ADP was carried out using buffer with $30 \mathrm{mM} \mathrm{NaCl}$ (see Supplementary Figure A.2 C). Lines: Gaussian distribution fit. (C) Representative kymographs of Cin8 $\Delta 99$ motility along polarity-marked MTs. Expression/purification conditions are indicated on top. Plus- $(+)$ and minus- (-) ends of MTs are indicated. ND: not detected. See also Supplementary Video A.3.5. (D) ATP concentration dependence of minus-end velocity of $\mathrm{Cin} 8 \Delta 99$ (average \pm s.e.m.) at high ionic strength. $\mathrm{NaCl}$ concentration and ionic strength $\mathrm{M}$ (parenthesis) are indicated. Michaelis-Menten parameters $\mathrm{V}_{\text {max }}$ and $\mathrm{K}_{m}$ are indicated. 

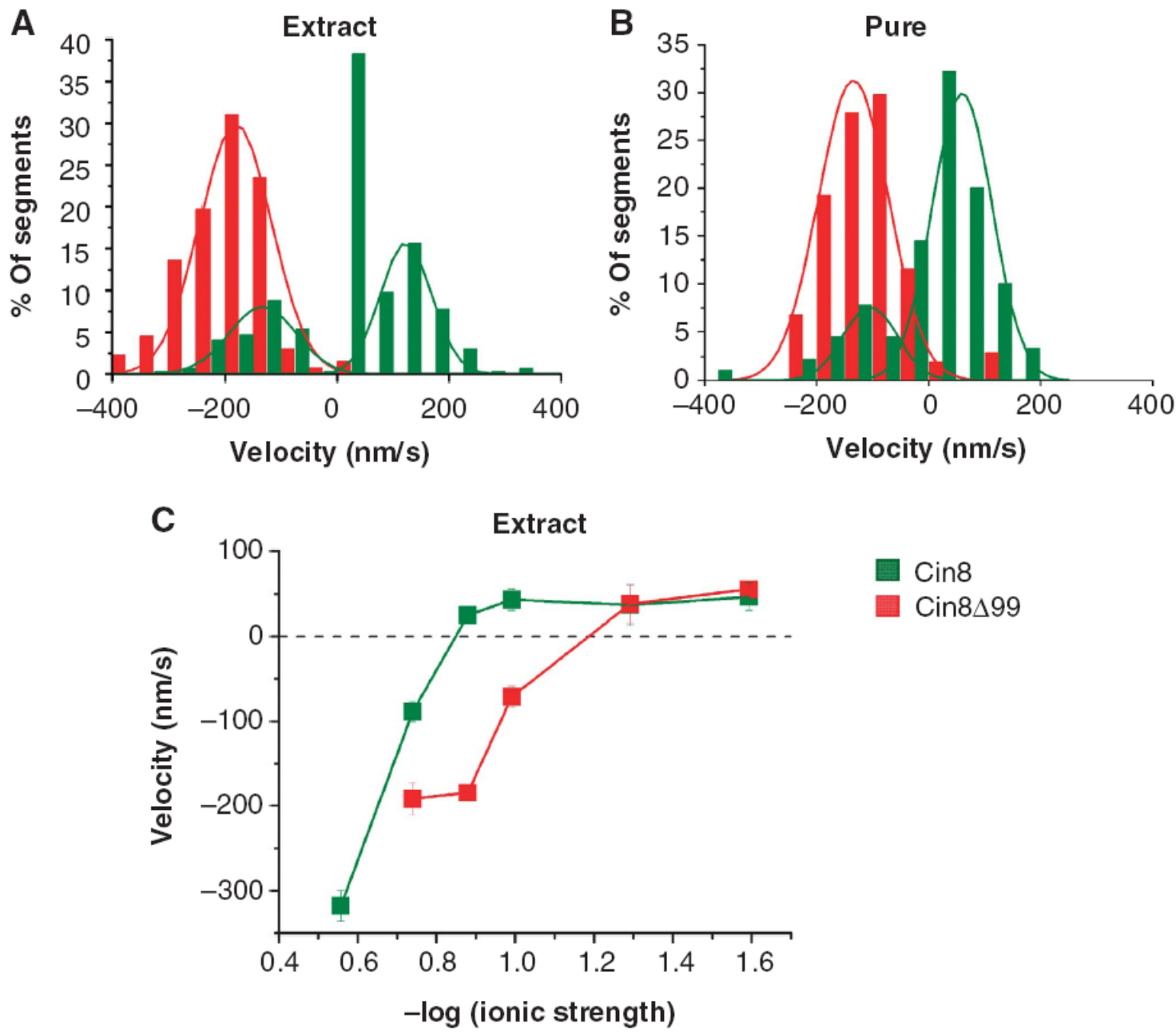

Fig. 2.6: Deletion of the 99aa insert of Cin 8 induces bias towards minus-end motility. (A, B) Histograms of velocities of Cin8 (green) and Cin8 $\Delta 99$ (red) in whole-cell yeast extracts (A) or affinity purified (B) in buffer containing $30 \mathrm{mM} \mathrm{NaCl}$, with saturating ATP. Velocity histograms were assembled by dividing kymograph traces in $3 \mathrm{~s}$ segments and piecewise linear fitting. Lines: Gaussian distribution fit. See also Supplementary Videos A.3.4 and A.3.5. (C) Ionic strength dependence of mean velocity of Cin8 (green) and Cin8 $\Delta 99$ (red) in whole-cell extracts in saturating ATP conditions. Values represent mean \pm s.e.m. of 50 - 200 velocity values.

towards the midzone were fewer, significantly slower than that of Cin8-3GFP and spanned shorter distances (Fig. 2.7; Tab. 2.1). Nonetheless, minus-end directed motility events were also observed (Fig. 2.7 B). Finally, the rate of the initial fast phase of anaphase $B$ in Cin8 $\Delta 99$ cells was $0.61 \pm 0.06 \mu \mathrm{m} / \mathrm{min}$ (average \pm s.e.m., $\mathrm{n}=9)$ which is significantly slower than in $W T$ cells $(0.85 \pm 0.03 \mu \mathrm{m} / \mathrm{min}$, average \pm s.e.m., $\mathrm{n}=8, \mathrm{P}<0.005)$ and is similar to what was observed in cin $8 \Delta$ cells [Straight98]. This result indicates that Cin $8 \Delta 99$ is unable to provide sufficient plusend directed force for spindle elongation. These in-vivo data are consistent with our in-vitro results of diminished plus-end motility of Cin8 899 (Fig. 2.5) and point to a regulatory role of the 99aa insert in loop 8 of Cin 8 in promoting its plus-end motility in cells. 


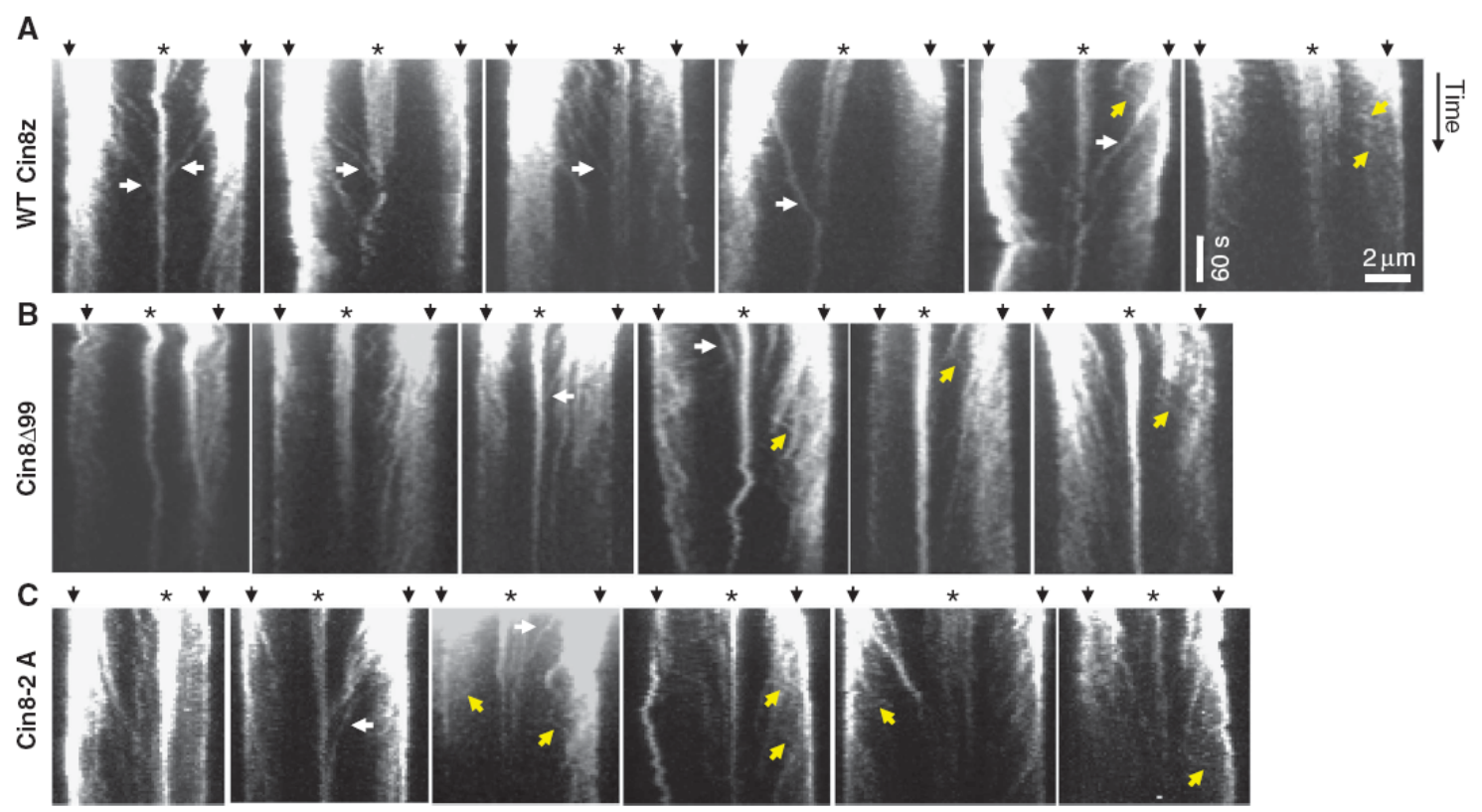

Fig. 2.7: Movements of Cin8-3GFP (A), Cin8 $499-3 G F P(B)$ and Cin8-2A-3GFP (C) in anaphase spindles. Kymographs of movements of Cin8-3GFP variants (indicated on the left) in intermediate to long anaphase spindles. White arrows: midzone-directed movements that span from the SPBs to the midzone; yellow arrows: SPB-directed movements; white arrowheads: movements towards the SPB during spindle disassembly; top arrows: spindle poles; asterisks: midzone.

It is known that during anaphase, Cin8 localization to the spindle is regulated by phosphorylation of the three Cdk1 sites in its catalytic domain, two of which are located in loop 8 of Cin8 [Avunie-Masala11]. To examine if phosphorylation of these sites affects Cin8 directionality on the spindle, we examined spindle movements of a Cin8-3GFP mutant that carried phosphorylation-deficient mutations to alanine at the two Cdk1 sites located in loop 8: Cin8-S277A T285A (Cin8-2A). Similarly to a phosphorylation-deficient Cin8 that carried mutations to alanine at all three catalytic-domain Cdk1 sites [Avunie-Masala11], Cin8-2A-3GFP remained attached to the anaphase spindles for longer times, compared with cells expressing $W T$ Cin 8 (Supplementary Fig. A.6 B, compare with Fig. 2.1 A and Fig. 2.3 C). This result indicates that the two $\mathrm{Cdk} 1$ sites within loop 8 are involved in the regulation of Cin8 spindle localization. Examination of Cin8-2A-3GFP movements on the spindle revealed that this mutant exhibited both midzone- (plus) and SPB-directed (minus) movements (Fig. 2.7 C), with SPB-directed movements being shorter and faster than midzone-directed movements (Tab. 2.1). The movements of Cin8-2A-3GFP were similar to those of Cin8 $\triangle 99$-3GFP: midzone-directed movements were fewer, and significantly slower and shorter compared with the spindle movements of the WT Cin8-3GFP (Fig. 2.7; Tab. 2.1), indicating that Cin8-2A is impaired in its midzone (plus-end)-directed motility on the spindle. This result suggests that the bias towards plus-end directed motility by the 99aa insert in loop 8, stems, at least in part, from phosphorylation of the $\mathrm{Cdk} 1$ sites located in this insert. 
A

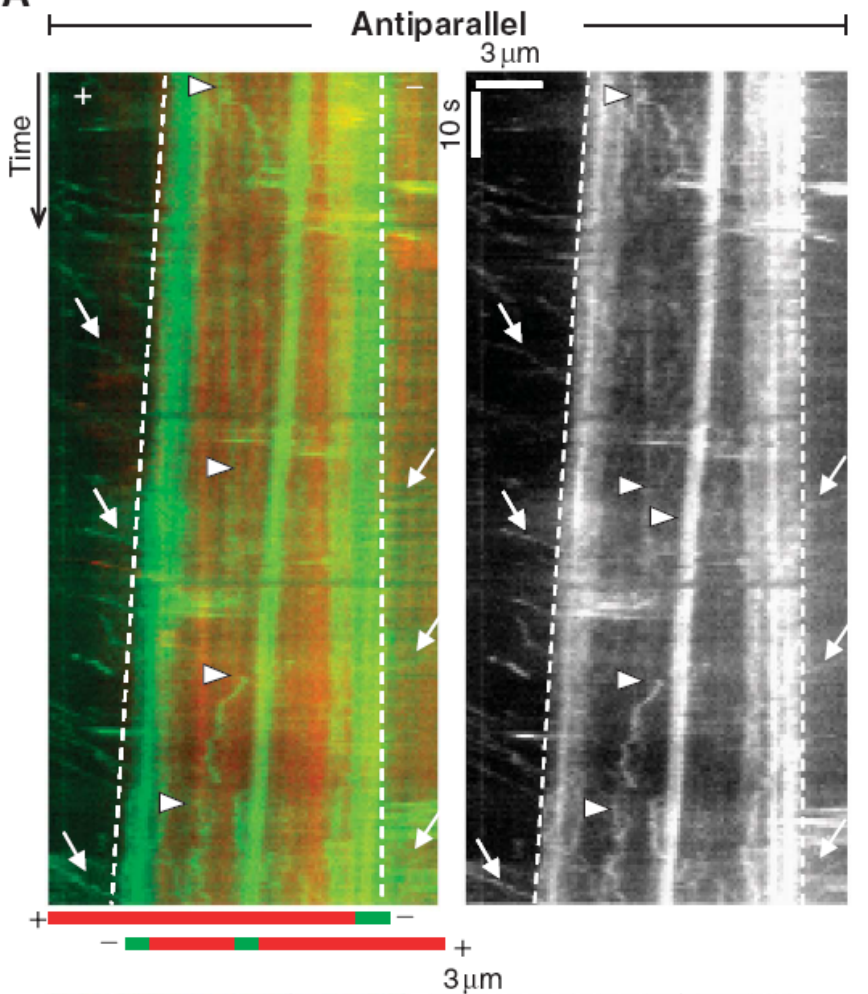

B

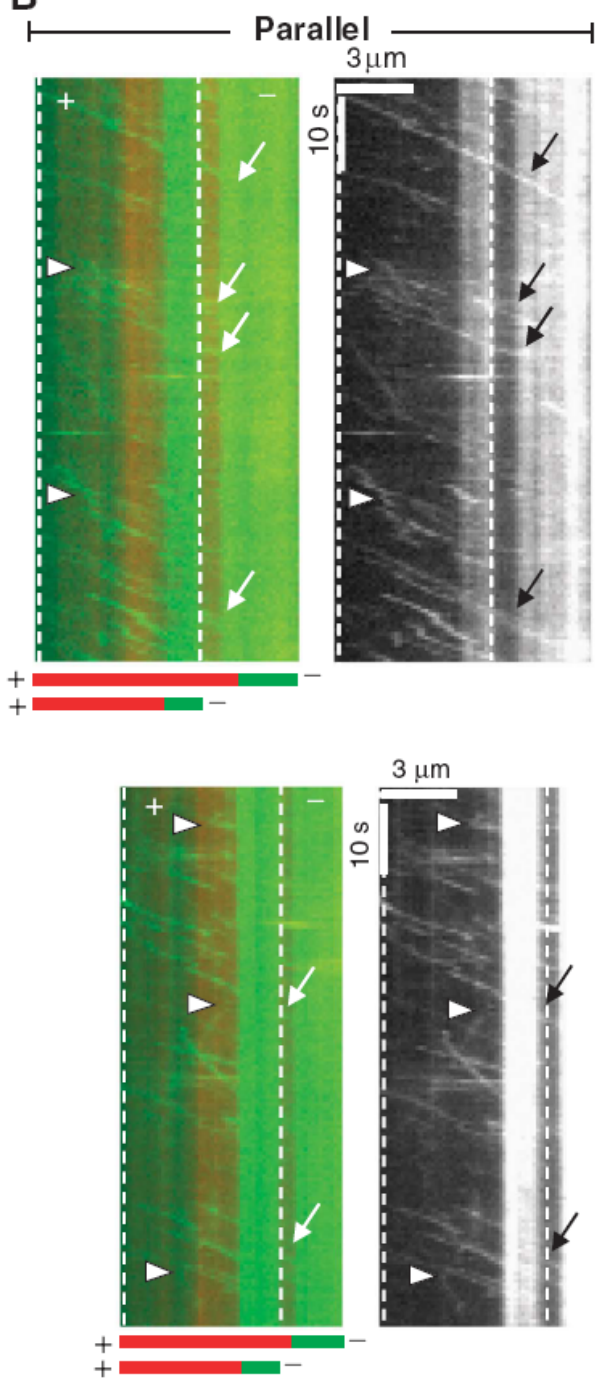

Fig. 2.8: MT orientation changes the motile properties of single Cin 8 molecules. Kymographs of movements of purified Cin8-GFP between antiparallel (A) and parallel (B) MTs in high-salt buffer (MB-175). For each event, a merged kymograph in colour (red: MT; green: Cin8 and minus ends of MTs) is shown on the left and a kymograph of the GFP-channel only is shown on the right. Overlapping region between MTs is marked by dashed lines. Cartoon depicting the orientation of overlapping MTs is shown at the bottom of the colour kymographs. Arrows: minus-end directed motility events; arrowheads: plus-end directed motility events. See also Supplementary Videos A.3.6 - A.3.10.

An important part of the switch mechanism might be related to cargo activation of Eg5. The homologous Xenopus kinesin-5 Eg5 is only turned on to move processively when it crosslinks two MTs, that is, when both of its dimeric catalytic domains are engaged [Kapitein08]. This mechanism was suggested to be related to the cargo activation of dimeric kinesins by straightening of the back-folded stalk and tail [Hackney92a, Stock99, Seiler00].

To see if a similar mechanism might be responsible for switching the directionality of Cin8, we performed single-molecule fluorescence experiments on bundled pairs of polarity-marked MTs. Between antiparallel MTs in high-salt buffer $(175 \mathrm{mM}$ added 
$\mathrm{NaCl}$ ), we saw an immediate switch from fast minus-end directed motion of individual motors to a slow erratic motion without a clear directional bias when motors reached the overlap zone (Fig. 2.8 A). In this geometry, the two crosslinked MTs were typically sliding apart with their minus ends leading with a relative velocity of about $30-60 \mathrm{~nm} / \mathrm{s}$. This reflects force generation by the motors in the plus-end direction. The fact that in the overlap region extended fast motion was no longer observed at all, implies that motors are attracted to the overlap region, likely due to their ability to bind MTs through both ends of the tetramers. In contrast to the antiparallel case, it was evident from kymographs of single motor motility between parallel MTs (Fig. 2.8 B) that most motors kept moving in the minus-end direction at undiminished speed when entering the overlap zone. Occasional short plus-end excursions were observed between parallel MTs (Fig. $2.8 \mathrm{~B}$, arrowheads), which were not observed on single MTs. These events were too rare to reliably evaluate details. We thus conclude that one of the major determinants of Cin8 directionality is binding geometry, with binding between two antiparallel MTs, as it occurs in the spindle midzone, switching the motor from minus-end to plus-end motility in near-physiological salt conditions.

\section{\begin{tabular}{l|l} 
Discussion & 2.4
\end{tabular}}

In the kinesin superfamily, the majority of the members are plus-end directed. Until recently [Roostalu11], minus-end motion was seen only for kinesin-14 family members, which are structurally distinct from all other kinesin subfamilies in that they carry the conserved motor domain at the C-terminus instead of the N-terminus [McDonald90, Walker90, Castro00, Block07]. No full-length kinesin-14 has been found to be processive so far, that is, these motors produce isolated power strokes and can only produce persistent motion when acting in ensembles. A reversal of power stroke directionality has been reported for mutants of the non-processive kinesin-14 ncd from Drosophila melanogaster [Sablin98, Endow00] and could be also generated by swaps of the core and neck domains of ncd and kinesin-1 [Case97, Endow98]. Evidence for active bi-directionality of a given motor construct has been reported for a specific neck-domain mutant of ncd [Endow00] and for cytoplasmic dynein [Dixit08]. Evidence for bi-directional power strokes of individual ncd motors has also been seen in the analysis of single-molecule recordings [Butterfield10].

Here, we show an entirely novel behaviour for a kinesin motor. Individual kinesin5 Cin 8 motors could be switched by varying ionic conditions between processive minus- and plus-directed movements when travelling on single MTs (Fig. 2.4), and they could be switched from processive minus-end motion to plus-end force generation in high-salt conditions by binding and crosslinking two MTs (Fig. 2.8). In vitro, low ionic strength (an unphysiological environment) induced plus-end directed motion of single molecules, while high ionic strength induced minus-end directed motion. Lower ionic strength, in general, reduces electrostatic screening which, in turn, enhances electrostatic interactions between motor subelements or between motor and MT. Thus, the unphysiological change of ionic conditions might mimic the effects of phosphorylation or binding of accessory proteins, which modify electro- 
static interactions under constant physiological conditions. A similar phenomenon was reported for cargo regulation of kinesins. Binding of a cargo vesicle to kinesin-1 or of a second MT to kinesin-5 Eg5, respectively, can activate the motors, but the activation also occurs at low ionic strength. In the case of Cin8, a related mechanism might not just turn the motor on or off, but lead to the switching of directionality when the motor tetramer binds between two antiparallel MTs. An alternative model that was recently proposed [Roostalu11] relies on a collective effect involving physical load on the motors via the binding between MTs. Based on our findings, it appears that more individual mechanisms such as binding of a single motor between two MTs or phosphorylation in the catalytic domain are able to cause or modify directionality switching.

A case in point is the observed regulatory influence of the large 99aa insert in loop 8 of the Cin8 motor domain, deletion of which did not abolish the shift in directionality, but created a strong bias towards minus-end motility (Fig. 2.5 and Fig. 2.6). The mechanism by which phosphorylation in Cin8 catalytic domain regulates its in-vivo function is likely to be a combination of a number of factors such as interaction with the midzone-organizing Ase1 [Khmelinskii09], as was previously suggested [Avunie-Masala11], or with kinetochore proteins. The fact that Cin $8 \Delta 99$ and the phosphorylation-deficient Cin8-2A exhibited reduced motility towards the midzone (Fig. 2.7; Tab. 2.1) suggests that one of the roles of Cin8 phosphorylation in the 99aa insert is to mediate the switch to plus-end directed motility of Cin8 on the spindle.

The observation that switching of directionality at high salt only occurred between antiparallel MTs is consistent with the reported preference of Drosophila kinesin-5 Klp61F for bundling antiparallel MTs [Kapitein08]. A preferred orientation was for that motor conferred by the ATP-independent binding sites in the C-terminal tail of the molecules. An ATP-independent binding mechanism appears to also exist for Cin8 because full-length Cin8 also supports diffusive MT attachment in ADP buffer (Tab. 2.2). Sticky tails with preferred orientation might not prevent parallel crosslinking by the motor, but it was found for Eg5 that all eight binding sites were necessary for motor engagement between MTs [Weinger11]. It is tempting to speculate that this might also be the case for Cin8, but in this case with the further consequence that directionality is reversed to plus-end motion between the antiparallel MTs. As the spindle midzone is the place where antiparallel overlaps occur and as that is the location where the motors need to exert force, such a regulation appears advantageous.

The discovery of the exceptional properties of Cin8 raises the question how these motile properties aid Cin8 in performing its multiple mitotic roles. The ionic strength in S. cerevisiae cells is high, estimated as $\sim 300 \mathrm{mM}$ salt [Olz93, Eunen10]. Under these conditions, Cin8 motors were minus-end directed on single MTs in our in-vitro experiments (Fig. 2.2, Fig. 2.4 and Fig. 2.6). Prior to spindle elongation, Cin8 is known to be important for kinetochore clustering or positioning near the SPBs [Tytell06, Gardner08a, Wargacki10]. The proposed mechanism for this function had been the crosslinking of kinetochore MTs (kMTs) [Tytell06] and the promotion of disassembly of long kMTs [Gardner08a]. Since in S. cerevisiae cells, each kinetochore is attached to a single MT and since on a single MT Cin8 is minus- 
end directed, active motion of Cin8 in the minus-end direction of the kMTs may be an alternative/additional mechanism by which Cin8 contributes to kinetochore positioning.

The slow plus-end directed motility that we observed in vivo in anaphase spindles (Fig. 2.1 C and Fig. 2.7; Tab. 2.1) indicates that Cin8 is switched to plus-end directed motility in the cell, even on single MTs or on parallel bundles. During anaphase spindle elongation, bi-directionality is likely to be important to dynamically partition Cin8 motors between different reservoirs, that is, near the poles where they focus the kinetochore clusters [Tytell06, Gardner08a, Wargacki10] and in the midzone where Cin8 promotes plus-end directed MT sliding (Fig. 2.8; [Roostalu11]) and spindle elongation [Saunders95, Movshovich08]. In fact, we observed that until anaphase spindles reach a length of $\sim 5 \mu \mathrm{m}$, Cin8 is localized throughout the spindle, with no preferential accumulation at the midzone or near the spindle poles, nor obvious detachment (Fig. $2.1 \mathrm{~A}$ and Fig. 2.3 C). A way to maintain this even distribution without detachment might be bi-directional motility of Cin8. Interestingly, higher eukaryotes, which show poleward flux in the spindle MTs, appear not to have kinesin- 5 motors capable of minus-end motility, possibly because it became unnecessary for motor transport to the poles in fluxing spindles.

A factor that appears to be important for directionality is the geometry of binding and allosteric regulation by two bound antiparallel MTs. Intermediate-long $S$. cerevisiae anaphase spindles contain a small number of MTs, two emanating from each pole at the end of anaphase [Winey95]. Motility between antiparallel MTs emanating from opposing poles should move both MTs and keep the motor fixed in the midzone. Therefore, the plus-end motility observed in vivo can only take place on single or between parallel MTs and is likely to utilize a further mode of regulation without which the motors would rapidly converge back to the poles.

In conclusion, Cin8 has turned out to be an exceptional kinesin in that it is truly bi-directional and processive in both directions. This unique feature of Cin 8 appears to play a role in cellular function. First, hints about the molecular mechanism indicate a role of charge interactions and possibly phosphorylation, and most importantly binding geometry between pairs of MTs. It remains to be explored in more detail if Cin8 regulation is a variation of the scheme of the regulation of other kinesins, in particular kinesin-5 motors. In general, our findings demonstrate that in order to fulfil their physiological functions, kinesin motors are much less rigidly programmed than was broadly believed so far and that the extent to which their function is regulated in the cell encompasses much more than simple on-off switches.

\section{\begin{tabular}{l|l} 
Materials and Methods & 2.5
\end{tabular}}

Detailed procedures and $S$. cerevisiae strains used in this study are described in Supplementary materials and methodes (section A.4). In brief, we produced fluorescently labelled Cin8 motors in three ways. We first used whole-cell extracts of S. cerevisiae expressing Cin8 fused with three consecutive C-terminal GFPs (Cin8$3 \mathrm{GFP}$ ) under its own promoter. Cin8-3GFP was either integrated into the yeast genome or expressed from a CEN plasmid. Cells with integrated Cin8-3GFP were 
also used for in-vivo imaging (Supplementary Tab. A.1). Second, we expressed Cin8 fused with a single C-terminal GFP and N-terminal 6HIS tag (6HIS-Cin8-GFP) in Sf9 insect cells, and third, we overexpressed Cin8-GFP-6HIS in S. cerevisiae. For yeast strains and plasmids, see Supplementary Tab. A.1. We purified motor by HIS tag and MT affinity.

Live-cell imaging was done on a spinning-disc confocal microscope (Zeiss Axiovert 200M, UltraView ESR, Perkin-Elmer, UK, [Fridman09]). Z-stacks of $0.2-0.4 \mu \mathrm{m}$ separation were acquired in $1 \mathrm{~min}$ time intervals [Movshovich08]. For the Cin8-3GFP spot-motility analysis, images were acquired every $2 \mathrm{~s}$.

In-vitro motility assays were performed following standard procedures [Howard93, Gheber99, Lakämper10] in motility buffer MB-175 (50 mM Tris/HCl, 30 mM PIPES/$\mathrm{KOH}$, final $\mathrm{pH}$ 7.2, $175 \mathrm{mM} \mathrm{NaCl}, 2 \mathrm{mM}$ EDTA, $1 \mathrm{mM}$ EGTA, $10 \%$ glycerol, 1 $\mathrm{mM}$ phenylmethylsulfonyl fluoride and $1 \mathrm{mM}$ dithiothreitol) as well as versions of this buffer with less $\mathrm{NaCl}$ added. The MTs, polymerized from TMR-labelled porcine tubulin, were polarity marked using Atto-488-labelled seeds marking the minus end of the MTs. Single-molecule fluorescence data were collected on two microscopes, one at Ben-Gurion University (BGU) and one at Göttingen University (GAUG). BGU: Zeiss Axiovert 200M, HBO 100 Mercury Illuminator, cooled CCD (SensiCam, PCO), frame time $0.8 \mathrm{~s}$. Data were processed using ImageJ and MetaMorph (MDS Analytical Technologies) software. GAUG: custom-built total-internal- reflection fluorescence (TIRF) microscope, using a $473 \mathrm{~nm}$ Laser (Viasho, USA) for excitation, a $\times 100$ objective (Nikon, SFluor, NA 1.49, Oil) and a CCD camera (Cascade 512B, Roper Scientific, USA), frame rate $0.5 \mathrm{~s}$. Software was custom written in Labview. Velocity histograms were assembled by drawing lines through consecutive 3 s segments of kymograph traces.

For relative sliding assays, polarity-marked MTs were polymerized as before whereas a solution of shorter MTs was polymerized by incubation at $37{ }^{\circ} \mathrm{C}$ for only 6 min. First, the long polarity-marked MTs were allowed to bind for 3 min to the DETA-coated surface of the assay chamber. The motility buffer (MB-175) was the same as used for the single-molecule assays but with double ATP and $\mathrm{MgCl}_{2}$ concentration. To this buffer, three times the single-motor concentration and $1 \mathrm{ml}$ of short polarity-marked MTs were added, and the mix was washed into the assay chamber. The custom-built TIRF setup described before was expanded such that the emission of the TMR-labelled MTs and the GFP-labelled motor proteins could be detected simultaneously. The TMR and the GFP channel were aligned with ImageJ.

\section{\begin{tabular}{l|l} 
Acknowledgements & 2.6
\end{tabular}}

This work was supported in part by the Lower Saxony Grant no. 11-76251-99-26/08 (ZN2440) awarded to LG, SL and CFS. LG was supported by the ISF grant no. 1043/09 and the BSF grant no. 2003141 and CFS was supported by the Center for Molecular Physiology of the Brain (CMPB), funded by the Deutsche Forschungsgemeinschaft (DFG). We thank Yael Nissenkorn, BGU, Israel for providing Cin8-2A 
plasmids and to Florian Rehfeldt and Marcel Bremerich, GAUG, Germany for data analysis.

\section{Author Contributions}

AGG, CT, NM, VF, MP, TD, SL and DRK performed the experiments; AGG, CT, CFS and LG analysed the data and wrote the paper; CFS and LG supervised and coordinated the project. All authors read and commented on the draft versions of the manuscript and approved the final version.

\section{Supplementary data}

Supplementary data associated with this article can be found online at the EMBO Journal, doi:10.1038/ emboj.2011.403 and in appendix A. 



\section{Regulation of bi-directional movement of single kinesin-5 Cin8 molecules}

The content of this chapter was published 2012 in BioArchitecture [Thiede12a]. I hold the first authorship of the paper and co-authors are Vladimir Fridman, Adina Gerson-Gurwitz, Larisa Gheber and Christoph F. Schmidt.

In this work I participated in the purification of proteins and performed the experiments. Further I analysed the data and contributed figures and text to the paper.

\section{\begin{tabular}{l|l} 
Abstract & 3.1
\end{tabular}}

Kinesin-5 mechanoenzymes drive mitotic spindle dynamics as slow, processive microtubule (MT)-plus-end directed motors. Surprisingly, the Saccharomyces cerevisiae kinesin-5 Cin8 was recently found to be bi-directional: it can move processively in both directions on MTs. Two hypotheses have been suggested for the mechanism of the directionality switch: (1) single molecules of Cin8 are intrinsically minus-end directed, but mechanical coupling between two or more motors triggers the switch; (2) a single motor can switch direction, and "cargo binding ", i.e., binding between two MTs triggers the switch to plus-end motility. Single-molecule fluorescence data we published recently, and augment here, favor hypothesis (2). In low-ionic-strength conditions, single molecules of Cin8 move in both minus- and plus-end direction. Fluorescence photo bleaching data rule out aggregation of Cin8 while they move in the plus and in the minus direction. The evidence thus points toward cargo regulation of directionality, which is likely to be related to cargo regulation in other kinesins. The molecular mechanisms of this regulation, however, remain to be elucidated.

\section{2}

Members of the kinesin-5 family of motor proteins are conserved among eukaryotes, from yeast to humans. Among the cytoskeletal motors, kinesins, myosins and dyneins, kinesin-5 motors are the only ones that function as bipolar homotetramers, with two pairs of catalytic domains located at opposite ends of the active complex [Kashina96, Gordon99]. This special architecture is thought to enable kinesin-5 motors to crosslink and slide apart antiparallel MTs emanating from the opposite poles of the mitotic spindle [Saunders92]. By this mode of action, 
kinesin-5 motors are believed to fulfill their essential roles in spindle dynamics such as spindle assembly, maintenance of the bipolar spindle structure prior to the onset of anaphase [Saunders92, Blangy95, Mayer99], as well as anaphase B spindle elongation [Saunders95, Sharp99, Avunie-Masala11, Fridman09, Gerson-Gurwitz09, Movshovich08]. Since MTs are organized with their plus ends overlapping in the midzone, kinesin-5 can only push spindle poles apart during spindle assembly and elongation via plus-end directed motility between antiparallel MTs. It has indeed been demonstrated in vitro, that the vertebrate kinesin-5 Eg5 moves simultaneously toward the plus ends of two antiparallel MTs that it crosslinks [Kapitein05, Kapitein08]. This finding was consistent with the 20-year-old dogma that kinesin homologs which carry their catalytic domains at the N-terminus are plus-end directed [Dagenbach04].

The majority of the members of the kinesin superfamily are plus-end directed. Minus-end motion was seen only for the structurally distinct kinesin-14 family members which carry the catalytic domain at their C-terminus [Castro00, McDonald90, Walker90]. Being non-processive, these motors produce isolated power strokes and can only produce persistent motion in ensembles. Surprisingly, the S. cerevisiae kinesin-5 Cin8 was recently found to move processively in the minus-end direction of MTs in single-molecule fluorescence motility assays under close-to-physiological conditions [Roostalu11, Gerson-Gurwitz11]. Cin8 was shown to switch directionality to plus-end directed motility in several experimental circumstances: in multi-motor MT gliding assays [Roostalu11, Gheber99], under low-ionic-strength conditions, and when bound between two antiparallel MTs [Roostalu11, Gerson-Gurwitz11]. Two possible mechanisms for this switch have been suggested: one is that single molecules of Cin8 can move only toward the minus end of MTs and that coupling between two or more motors triggers the plus-end directed switch [Roostalu11]; the second possibility is that the ability to switch directionality is contained within a single motor itself and that interaction between Cin8 and MTs can trigger the switch [Gerson-Gurwitz11]. Several lines of evidence support the second mechanism.

The central evidence for a motor-intrinsic switching mechanism is that individual Cin8 molecules can switch to plus-end directed motility under low-ionic-strength conditions [Gerson-Gurwitz11]. In our in-vitro single-molecule fluorescence experiments [Gerson-Gurwitz11], low total ionic strength below $\sim 0.13 \mathrm{M}$ (an unphysiological environment) induced plus-end-directed and high ionic strength promoted minus-end-directed motion of Cin8. In view of the controversy about the mechanism of the directionality switch, it is crucial to prove that, indeed, individual Cin8 molecules move toward the plus ends of MTs and that observed fluorescent traces do not originate from small clusters of motors acting collectively. To address this point, we followed the photo-bleaching of fluorescent Cin8-GFP tetramers, purified from S. cerevisiae cells, while they moved on polarity-marked MTs under low-ionicstrength conditions. Experiments are described in detail in Gerson-Gurwitz et al. [Gerson-Gurwitz11] and additional data is presented here (Fig. 3.1). 
A
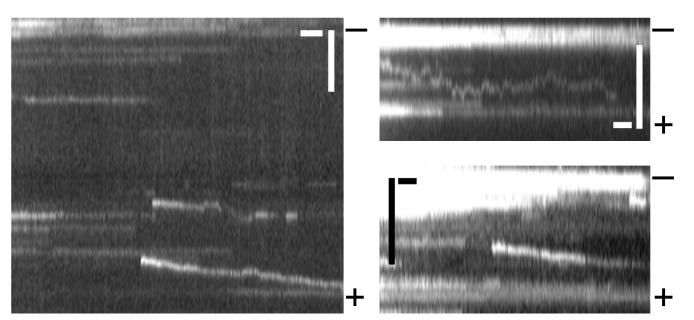

\section{Intensity before \& after final bleaching}

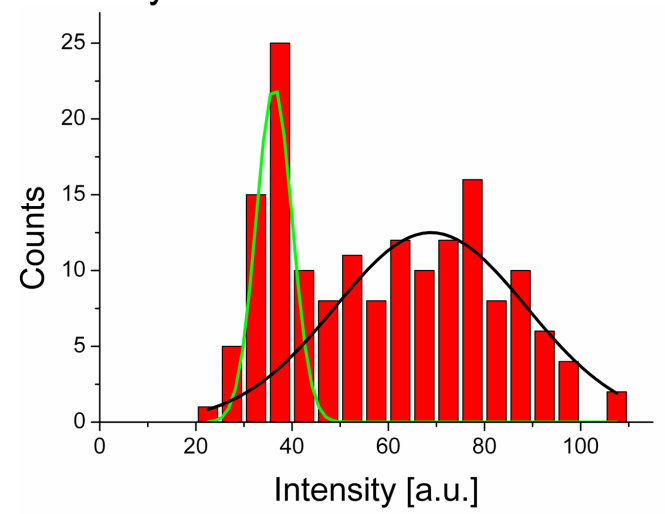

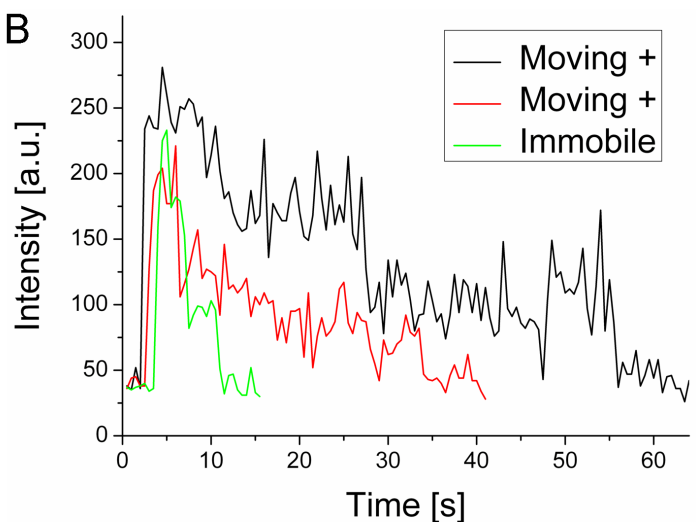

D Initial intensity after landing

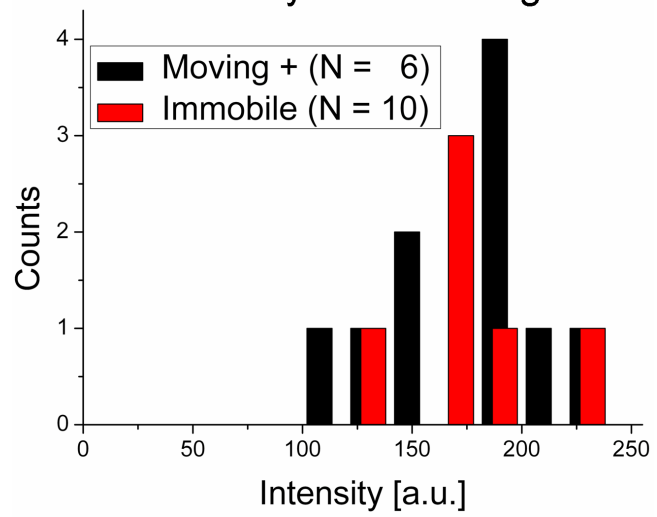

Fig. 3.1: In low-ionic-strength conditions, single Cin 8 motors move toward the plus end of MTs. (A) Kymographs of Cin8 moving away from the brightly labeled seed marking the minus end $(-)$ toward the plus end $(+)$ of the MT. Kymograph in the right bottom panel was captured in MB-30; the other two in MB-0 (exact buffer compositions are given below). Scale bars: horizontal: $10 \mathrm{sec}$; vertical: $3 \mu \mathrm{m}$. (B) Exemplary intensity traces of two motors landing on a MT and moving to the MT plus end (black and red) and of a motor landing on a MT and remaining immobile (green). The traces of the moving motors correspond to the two events shown in the left panel of (A). (C) Histogram of fluorescence intensities before and after final bleaching steps of immobile motors, summed from a 800 $\mathrm{nm} \times 800 \mathrm{~nm}$ square of camera pixels containing the image of the motor (Number of traces /motors: 8). A sum of two Gaussians was fitted to the histogram. The first narrower peak corresponds to the background [compare also to (B)]. The broader second peak represents the intensity of a single GFP. (D) Histogram of initial intensities of Cin8 molecules right after landing, measured in the same arbitrary units as in (C). The intensity of the first 3 frames $(1.5 \mathrm{sec})$ after landing was averaged for both moving and immobile motors. The histogram has a maximum at 4 times the intensity of a single GFP [compare with (C)]. Materials and Methods: Single-molecule fluorescence assays were performed as described in detail in Gerson-Gurwitz et al. [Gerson-Gurwitz11] In short, the custom-built total-internal-reflection-fluorescence (TIRF) microscope consisted of a $473 \mathrm{~nm}$ laser (Viasho, USA) for excitation, a 100× objective (SFluor, NA 1.49, oil, Nikon, USA) and a CCD camera (Cascade 512B, Roper Scientific, USA) for detection. To observe several colors simultaneously, the fluorescence emission signal was split by dichroic mirrors and directed to separate areas on the CCD camera. Fluorescently Cin8-TEV-GFP-6HIS was overexpressed in S. cerevisiae and affinity purified using the his-tag and a Ni-NTA affinity column (Invitrogen, USA). The low-salt buffers for the motility assays were composed as follows: MB-0: $50 \mathrm{mM}$ Tris/HCl, $30 \mathrm{mM}$ PIPES/KOH, pH 7.2, $2 \mathrm{mM}$ EDTA, $1 \mathrm{mM}$ EGTA, $10 \%$ glycerol, $1 \mathrm{mM}$ phenylmethylsulfonyl fluoride and $1 \mathrm{mM}$ dithiothreitol. MB-30: the same as MB-0 but with $30 \mathrm{mM}$ added NaCl. MTs were polymerized from tissue-purified porcine tubulin, essentially as described before [Lakämper10], but with Atto-488 (Atto-Tec GmbH, Siegen, Germany) labeled seeds that also fluoresce in the green and in that way mark the minus end of the MTs [Gerson-Gurwitz11]. Kymographs were generated and analyzed with a custom-written LabView (National Instruments, USA) routine. The fluorescence intensity emitted by single proteins was summed over an area of $5 \times 5$ camera pixels, corresponding to an area of $800 \mathrm{~nm} \times 800 \mathrm{~nm}$ in the specimen plane and was analyzed with ImageJ software (NIH, USA). Histograms and fits were done with OriginPro software (OriginLab Corporation, USA). 
Two buffer conditions were examined: motility buffer (MB) with $30 \mathrm{mM} \mathrm{NaCl}$ added (ionic strength $0.132 \mathrm{M}$ ) and $\mathrm{MB}$ with no added $\mathrm{NaCl}$ (ionic strength $0.102 \mathrm{M}$ ). We have previously shown that under these conditions, Cin8 moves toward the plus end of MTs for $\sim 60 \%$ and $\sim 70 \%$ of the time, respectively [Gerson-Gurwitz11]. To count the number of GFP fluorophores on each moving motor or (possibly) motor aggregate, we measured the intensity of several single spots in a given video recording, both for spots appearing during the recording (i.e., landing from the bulk on the MT) and then moving to the plus end of the MT, and for spots appearing and remaining stationary on the MT during the recording. To obtain a scale for the intensity, i.e., to determine the intensity of a single GFP, intensities before and after the final bleaching step of immobile motors were analyzed (Fig. 3.1 C). The measured intensity distribution was fitted with a sum of two Gaussians, resulting in a value for the background and a value for the intensity of a single GFP. We then measured the distribution of the initial fluorescence intensities right after landing for both moving and immobile motors. Intensities in a fixed area were averaged for the first 3 frames $(1.5 \mathrm{sec})$ right after landing (Fig. 3.1 D), during which time the motors moved much less than the size of the chosen area around the spots. Comparison of initial intensities to monomer intensities confirms that both, the motors moving to the plus end of MTs and the stationary motors were tetramers. Furthermore, some of the bleaching traces of Cin8 molecules that moved toward the plus ends of MTs showed 4 consecutive bleaching steps (Fig. 3.1 B), indicating again that these molecules were tetramers with four GFP molecules. These results demonstrate that, under low-ionic-strength conditions, individual Cin8 molecules can move in the plus-end direction on MTs. While coupling between multiple motors could be an additional mechanism for switching, our results provide support for the existence of the motor-intrinsic switch model.

Since ionic strength generally modulates electrostatic interactions, the unphysiologically low-ionic-strength conditions might mimic the effects of phosphorylation or binding of accessory proteins or of binding geometry to the MTs. A qualitatively similar ionic-strength effect has been reported in the context of cargo regulation of other kinesins [Kapitein08, Seiler00, Hackney09]. Binding of a cargo vesicle to kinesin-1 or of a second MT to kinesin-5 Eg5 activates these motors, but this activation also occurs spontaneously (i.e., without cargo) at low ionic strength in both cases. Tail-head interaction is mediating this regulation in both cases. It is thus tempting to speculate that for Cin8, cargo regulation is also the physiological switch mechanism. In the case of Cin8, a mechanism detecting the binding of a second MT might not just turn the motor on or off, but lead to the observed switching of directionality. Consistent with this hypothesis, we observed plus-end directed antiparallel sliding of MTs by Cin8 when they entered the overlap zone between antiparallel MTs under high-ionic-strength conditions, while motors on single MTs in the same sample were still minus-end directed [Gerson-Gurwitz11]. Similarly, it has been previously demonstrated that, while the vertebrate Eg5 does neither bind to nor move on single MTs under high-ionic-strength conditions, binding between two antiparallel MTs activates MT sliding, driven by plus-end directed Eg5 motility [Kapitein08]. A similar activation effect might also occur in multi-motor MT surface-gliding assays, in which surface-attached Cin8 was also demonstrated to be 
plus-end directed [Gheber99]. In MT sliding assays, Cin8 obviously exerts force in the plus-end direction which is reflected in the relative sliding of the MTs, but individual motors between the coupled MTs move on rather erratic tracks such that clear plus-end-directed periods cannot be detected [Gerson-Gurwitz11]. Cin8 behaves very differently in single-molecule fluorescence experiments between parallel MTs. For the most part, motors continue minus-end motion, apparently not interacting with the second MT [Gerson-Gurwitz11]. The capability to distinguish relative orientation of bound MTs is consistent with the reported preference of Drosophila kinesin-5 Klp61f for bundling antiparallel MTs [Kapitein08]. For this kinesin-5, a preferred orientation was due to the ATP independent binding sites in the C-terminal tails of the molecules. A similar binding mechanism appears to also exist for Cin8 because full-length Cin8 diffusively slides along MTs in ADP buffer [Gerson-Gurwitz11]. For Xenopus laevis kinesin-5 Eg5 it was found that all MT binding sites in the C-terminal tails were necessary for motor engagement between MTs [Weinger11]. It still remains unclear exactly why and how low ionic strength mimics cargo binding. Taking into account the fact that MT attachment of the two pairs of motor domains triggers plus-end directed motility (Fig. $3.2 \mathrm{~A}$ ), a speculative possibility is that under low-ionic-strength conditions, Cin8 can flex in such a way that the two pairs of catalytic domains interact with the same MT (Fig. 3.2 B) and thus trigger plus-end directed motility. Alternatively, low-ionic-strength conditions could modify tail-head interactions as in kinesin-1 to trigger plusend directed motility.

One further piece of evidence in favor of a motor-intrinsic mechanism for directional switching is the regulatory influence of the 99 amino acid insert in loop 8 of the Cin8 motor domain, deletion of which does not abolish the switch of directionality, but shifts the switch to lower ionic strength [Gerson-Gurwitz11]. The mechanism by which phosphorylation in the catalytic domain of Cin8 regulates its in-vivo function is likely to include the modulation of interactions with the midzone-organizing protein Ase1 [Avunie-Masala11, Khmelinskii09], or with kinetochore proteins. The fact that a deletion construct (Cin8D99) and a loop 8 Cdk1 phosphorylation-deficient construct (Cin8-2A) exhibit reduced motility toward the midzone in vivo [Gerson-Gurwitz11], suggests that one of the roles of Cin8 phosphorylation in the 99aa insert is to mediate the switch to plus-end directed motility of Cin8 on the mitotic spindle.

The question remains how its exceptional motile properties aid Cin8 in performing its roles in mitosis. One can speculate on the basis of the localization of Cin8 in the various stages of mitosis. The ionic strength in S. cerevisiae cells is $\sim 300$ $\mathrm{mM}$ salt [Olz93, Eunen10]. At this ionic strength, Cin8 is minus-end directed in vitro [Gerson-Gurwitz11]. Before spindle elongation in anaphase, Cin8 is known to be involved in the positioning of the chromosome kinetochores near the spindle pole bodies [Tytell06, Gardner08a, Wargacki10]. Cin8 could function at that stage by crosslinking of kinetochore MTs (kMTs) [Tytell06] and by aiding the disassembly of long kMTs [Gardner08b]. Since in S. cerevisiae cells each kinetochore is attached to the plus end of a single MT, motion of Cin8 in the minus-end direction of the kMTs might be a part of kinetochore positioning. Cin 8 also shows plus-end directed motility in vivo in anaphase spindles [Gerson-Gurwitz11], even on single MTs or 


\section{A High ionic strength}

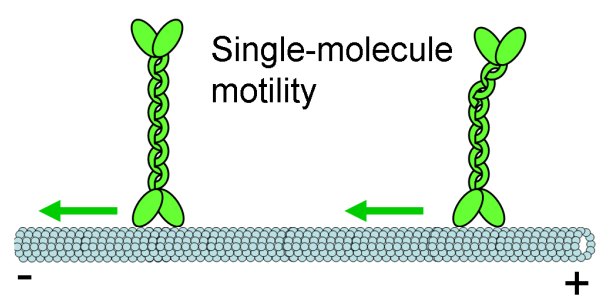

\section{B Low ionic strength}

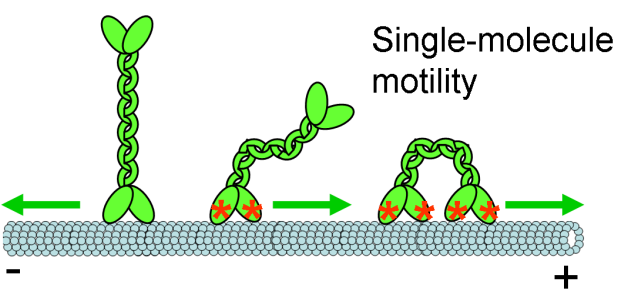

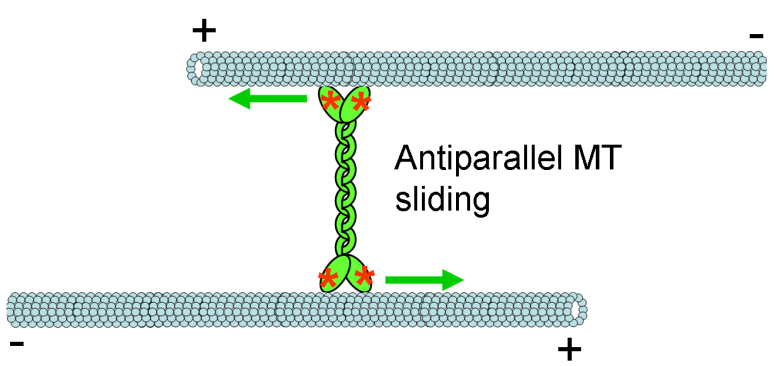

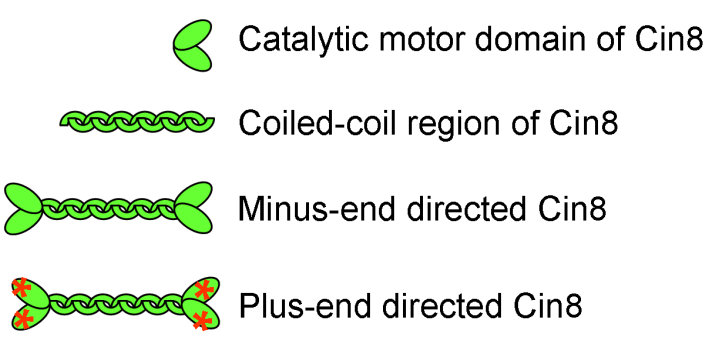

Fig. 3.2: Proposed model for the directionality switch of Cin8. A microtubule is sketched in light blue, with plus and minus end indicated; Cin8 is shown in green; catalytic motor domain and coiled-coil regions are indicated in the legend. Arrows indicate the direction of movement of Cin8 on the MT to which it is attached. (A) On a single MT, in highionic-strength conditions, Cin8 is minus-end directed. Binding between two antiparallel MTs activates Cin8 to move in the plus-end direction of the MTs, causing their antiparallel sliding. (B) Under low-ionic-strength conditions on a single MT, a modified interaction between tails and catalytic domains or flexing of the whole tetramer triggers Cin 8 to move in the plus-end direction of the MT. If the tetramer can flex enough, plus-end directed motility of single molecules of Cin 8 could be triggered by binding of the two heads of Cin 8 to the same MT.

on parallel MT bundles, which implies a further mode of regulation not seen in the in-vitro experiments. Regulated bi-directional motility might be important to distribute Cin8 motors between the different locations where they are known to accumulate, i.e., near the spindle poles and in the midzone.

In summary, Cin8 has rather unexpectedly extended the spectrum of known kinesin capabilities. It is the first known kinesin that is truly bi-directional and processive in both directions. Found in a low eukaryote, this function might have evolved early and might have been lost in higher eukaryotes. The exact molecular mechanism remains to be clarified, but seems likely to be related to cargo switching known for other kinesins. Our results indicate a role of electrostatic interactions and possibly phosphorylation, and, most importantly, binding geometry between pairs of MTs. 


\title{
The effect of monastrol on the processive motility of a dimeric kinesin-5 head/ kinesin-1 stalk chimera
}

The content of this chapter was published 2010 in the Journal of Molecular Biology [Lakämper10]. Co-authors are Stefan Lakämper, Andrè Düselder, Stefanie Reiter, Mikhail J. Korneev, Lukas C. Kapitein, Erwin J. G. Peterman and Christoph F. Schmidt.

In this work I participated in the purification of the truncated mutant of Eg5Kin, the dimeric Eg5Kin-511. Multi-motor gliding assays (MMGA) with Eg5Kin, Eg5Kin511 and monomeric Eg5-369 were done by me. With MMGA I also performed ATPdilution measurements on Eg5Kin and Eg5Kin-511 as well as monastrol-dilution measurements on Eg5Kin-511. Additionally I performed ATPase measurements on Eg5Kin and Eg5Kin-511. I carried out single-molecule fluorescence (SMF) experiments on Eg5Kin and Eg5Kin-511. With SMF I also performed intensity-control measurements on Eg5Kin and Eg5Kin-511 as well as controls on Eg5Kin using a monastrol derivate. Finally I contributed figures and text to the paper.

\begin{abstract}
4.1

Controlled activity of several kinesin motors is required for the proper assembly of the mitotic spindle. Eg5, a homotetrameric bipolar kinesin-5 from Xenopus laevis, can cross-link and slide anti-parallel microtubules apart by a motility mechanism comprising diffusional and directional modes. How this mechanism is regulated, possibly by the tail domains of the opposing motors, is poorly understood. In order to explore the basic unregulated kinesin-5 motor activity, we generated a stably dimeric kinesin-5 construct, Eg5Kin, consisting of the motor domain and neck linker of Eg5 and the neck coiled coil of Drosophila melanogaster kinesin-1 (DmKHC). In single-molecule motility assays, we found this chimera to be highly processive. In addition, we studied the effect of the kinesin-5-specific inhibitor monastrol using single-molecule fluorescence assays. We found that monastrol reduced the length of processive runs, but strikingly did not affect velocity. Quantitative analysis of monastrol dose dependence suggests that two bound monastrol molecules are required to be bound to an Eg5Kin dimer to terminate a run.
\end{abstract}




\section{\begin{tabular}{l|l} 
Introduction & 4.2
\end{tabular}}

The vertebrate kinesin- 5 Eg5 is a homotetrameric motor protein required for proper separation of the poles of the bipolar mitotic spindle during cell division [Enos90, Le Guellec91, Mayer99]. The Drosophila melanogaster homolog (Klp61F) of Eg5 was found to be an extended molecule approximately $80 \mathrm{~nm}$ long, with two motor domains at each end of a central stalk [Kashina96]. It has been demonstrated in invitro microtubule sliding assays that Eg5 can crosslink microtubules and slide antiparallel microtubules apart [Kapitein05], consistent with the structure and in-vivo function of Eg5. Single-molecule fluorescence experiments using green fluorescent protein (GFP)-tagged Eg5 tetramers have shown that Eg5 motility is surprisingly complex: Depending on buffer conditions, single Eg5 motors show a mixture of diffusive and ATP-dependent directional motility modes along single microtubules while staying attached for several tens of seconds [Kwok06, Kapitein08]. When Eg5 crosslinks two microtubules, diffusive motility is suppressed, and directional motility is favored [Kapitein08]. In optical trapping experiments with Eg5 tetramers, processive stepping was observed, but motors detached from the microtubules at a comparatively low force $(\sim 1.6 \mathrm{pN})$ [Korneev07], suggesting a force-sensing mechanism that is not seen in kinesin-1 motors. This sensitivity may, in some way, be caused by interaction with the opposing dimer, because truncated dimeric constructs of human Eg5 (HsEg513His) could withstand higher forces in an optical trap assay. Their processivity was low, however, with only six to eight steps per run [Valentine06], in agreement with single-molecule fluorescence experiments on comparable Xenopus constructs [Kapitein08].

There is evidence that the additional microtubule binding site mediating diffusive interactions of Eg5 is located in parts that are missing in truncations, namely, in the conserved BimC box or the tail [Kashina96, Kashina97]. This conclusion is supported by the ability of tetrameric headless constructs of the Drosophila kinesin-5 homolog Klp61F to cross-link microtubules [Tao06]. Furthermore, the single-molecule and multiple-molecule velocities of the chimeric constructs, as well as the single-molecule velocity of HsEg5-513 measured in trapping experiments $(\sim$ $95 \mathrm{~nm} / \mathrm{s}$ ) [Valentine06], are $\sim 4-8$ times higher than that of the full-length motor [Kapitein05, Kwok06, Korneev07]. Such a discrepancy is actually expected, given the observation that the motility of the full-length motor consists of an alternation of directional and diffusive periods [Kwok06], where the diffusive periods are supported by additional MT binding by the tail and where switching to directional motility appears to be regulated by microtubule binding on the opposite end [Kapitein08]. In earlier single-molecule fluorescence experiments [Kwok06, Kapitein08] the processive motor speed between diffusive periods could not be quantified, since switching between the two states was rapid and could not be resolved. Rapid switching indicates short directional episodes, again consistent with the finding of moderate processivity for the dimeric Eg5 constructs [Valentine06, Crevel97].

A recent study claims, on the basis of microtubule gliding experiments with Eg5 homodimers, monomers, and Eg5-kinesin-1 heterodimers, that kinesin-5 motors move predominantly nonprocessively [Kaseda08]. It has remained unclear whether 
the limited processivity of truncated dimers is (i) an intrinsic property of the motor domains of Eg5, (ii) a consequence of the two coiled-coil domains of the opposing dimer that are lacking, or (iii) a consequence of the absence of the C-terminal tail domains [Kashina97] that, in the tetramer, are in close proximity to the motor domains and might affect processive motility.

We have here constructed a stably dimeric chimeric motor to study the intrinsic capabilities of the Eg5 motor domains and to address the role of coil and tail domains. The chimera consisted of the main force-generating parts of Eg5 - motor domain and neck linker - fused to the neck coiled coil of Drosophila kinesin-1, ensuring stable dimerization. The chimera lacks the opposing pair of motor domains, as well as the tail domains that were found to be responsible for diffusive motion in Klp61F [Wildenberg08]. We hypothesized this chimera to be processive without diffusional episodes. Important questions were would the chimera be more processive than truncated dimeric constructs, how much load could it support and how fast would it move?

A further characteristic feature of wild-type Eg5 is that it can be inhibited specifically by the small molecule monastrol [Mayer99]. In cells, monastrol causes spindle collapse and cell cycle arrest [Mayer99], while in in-vitro microtubule gliding assays, monastrol inhibits microtubule motility. Both effects are due to a so far poorly understood allosteric mechanism, since the binding site of monastrol overlaps neither with the microtubule binding site nor with the nucleotide binding site [Maliga02, Krzysiak06]. A recent cryo-electron microscopy study of the motor domain of Drosophila Klp61F (residues 1 - 368) suggests that the uniquely extended loop 5 plays an important role in the monastrol-induced transition to a diffusive state and in the inhibition of motility [Bodey09, Crevel04a]. In single-molecule fluorescence experiments, the interaction times of single Eg5 homotetramers with microtubules were shown to be shorter in the presence of monastrol. In addition, the motility was less directional and more diffusive [Kwok06] (i.e., average velocity decreased in a dose-dependent manner). An important question that could not be answered in those tetramer assays is: Does the processive velocity of Eg5 decrease in the presence of monastrol, or does the probability to switch to the diffusive state increase? Kinetic experiments [Cochran05] showed that monastrol slows the ADP release rate of monomeric Eg5 truncations both in the absence and in the presence of microtubules. Whether the same effect would occur in the dimer context and whether this would lead to a lower processive velocity or to release from the microtubule remained open. These questions can be directly addressed with our chimera, where opposing tail interactions do not complicate the situation [Korneev07].

\section{Results and Discussion 4.3}

Our first goal was to create a stable dimeric Eg5. A shortened Eg5, containing about half the stalk, is thought to preferentially form dimers; however, unfortunately, these dimers appear to be rather unstable in the absence of the opposing stalk. This may be due to the fact that the full-length motor has evolved a strong preference for tetramer assembly, for which it may help to not get stuck in a stable dimer 
conformation [Kapitein08]. Similar problems with other motors have been successfully addressed by the use of chimeric constructs [Adio09, Tomishige02, Okada99, Kalchishkova08]. We therefore constructed a chimeric motor (Eg5Kin) by replacing the motor domain and the neck linker of Drosophila melanogaster kinesin-1, which is known to form stable dimers (residues 1 - 343 from pPK113, a kind gift from W. O. Hancock), with the corresponding domains of Xenopus laevis Eg5 (residues 1 - 369). We used the full-length chimera and also a truncated version (at residue 421 using the Drosophila numbering) that was tagged with GFP at the C-terminus (Eg5Kin-GFP). All constructs were expressed in Escherichia coli and purified using a C-terminal His tag (Fig. 4.1).

To test the motility of the chimeric motor protein, we performed surface-gliding assays. We found that Eg5Kin motors bound readily to glass surfaces and moved microtubules with a velocity of $95 \pm 10 \mathrm{~nm} / \mathrm{s}(\mathrm{N}=52$; BRB80 buffer; Supplementary Video B.3.1). Truncated Eg5Kin-GFP motors moved microtubules with a comparable velocity of $88 \pm 8 \mathrm{~nm} / \mathrm{s}(\mathrm{N}=45)$. At very low Eg5Kin motor concentrations, we observed pivoting of microtubules around a single point of attachment, while movement relative to this point occurred at a velocity of $92 \pm 15 \mathrm{~nm} / \mathrm{s}(\mathrm{N}=10$; Supplementary Video B.3.2). This behavior is characteristic of processive motility and gives qualitative evidence that Eg5Kin is a processive motor [Howard89].

To further explore the motility and force generation capabilities of Eg5Kin, we performed optical-trapping experiments with single Eg5Kin-GFP motors attached to silica beads with a diameter of $1 \mu \mathrm{m}$. We observed that single motors displaced the beads processively at a somewhat lower average velocity $(56 \pm 10 \mathrm{~nm} / \mathrm{s} ; \mathrm{N}=$ 28; Fig. 4.2 a; Supplementary Video B.3.3) than seen in the surface-gliding assays. As this difference might have been due to the use of a buffer containing only $12 \mathrm{mM}$ Pipes instead of the standard BRB80 buffer, we performed the same experiments in BRB80 with similar results $(63 \pm 2 \mathrm{~nm} / \mathrm{s} ; \mathrm{N}=93)$. We also observed short periods of backward slipping, similar to what has been reported before for truncated Eg 5 dimers [Valentine06]. The motors detached from the microtubules when reaching a force of $4.6 \pm 0.1 \mathrm{pN}(\mathrm{N}=27)$, on average, for $12 \mathrm{mM}$ Pipes (Fig. $4.2 \mathrm{~b}$ ) and a force of $4.4 \pm 0.1 \mathrm{pN}(\mathrm{N}=93)$ for BRB80, substantially higher than the maximal force reported for full-length $\mathrm{Eg} 5$ motors $(\sim 1.6 \mathrm{pN})$ [Korneev07]. A remarkable difference from the behavior of kinesin-1 is that our kinesin- 5 chimera detached mostly without noticeable prior stalling [Korneev07, Visscher99].

In order to determine the number of processive steps that Eg5Kin takes in the absence of an opposing force, we performed single-molecule motility assays with Eg5Kin-GFP using fluorescence microscopy [Kapitein05]. We observed that single fluorescent spots moved unidirectionally at a speed of $96 \pm 0.3 \mathrm{~nm} / \mathrm{s}$ along microtubules ( $\mathrm{N}=82$; BRB80 buffer; Fig. $4.3 \mathrm{a}$ and b; Supplementary Video B.3.4), consistent with the results from optical-trapping and surface-gliding assays. In contrast to full-length Eg5 [Kwok06, Kapitein08], there was no evidence of diffusive periods during motility. Both surface-immobilized and moving spots photobleached in, at most, two consecutive steps (Fig. 4.3 a; Supplementary Fig. B.1), confirming that the observed spots were indeed single dimeric Eg5Kin-GFP motors. Single motors interacted for, on average, $19.1 \pm 3.5 \mathrm{~s}$ with the microtubules (Fig. $4.3 \mathrm{c}$ ), while moving $1.8 \pm 0.3 \mu \mathrm{m}$ on average, corresponding to $\sim 230$ steps of $8 \mathrm{~nm}$. 


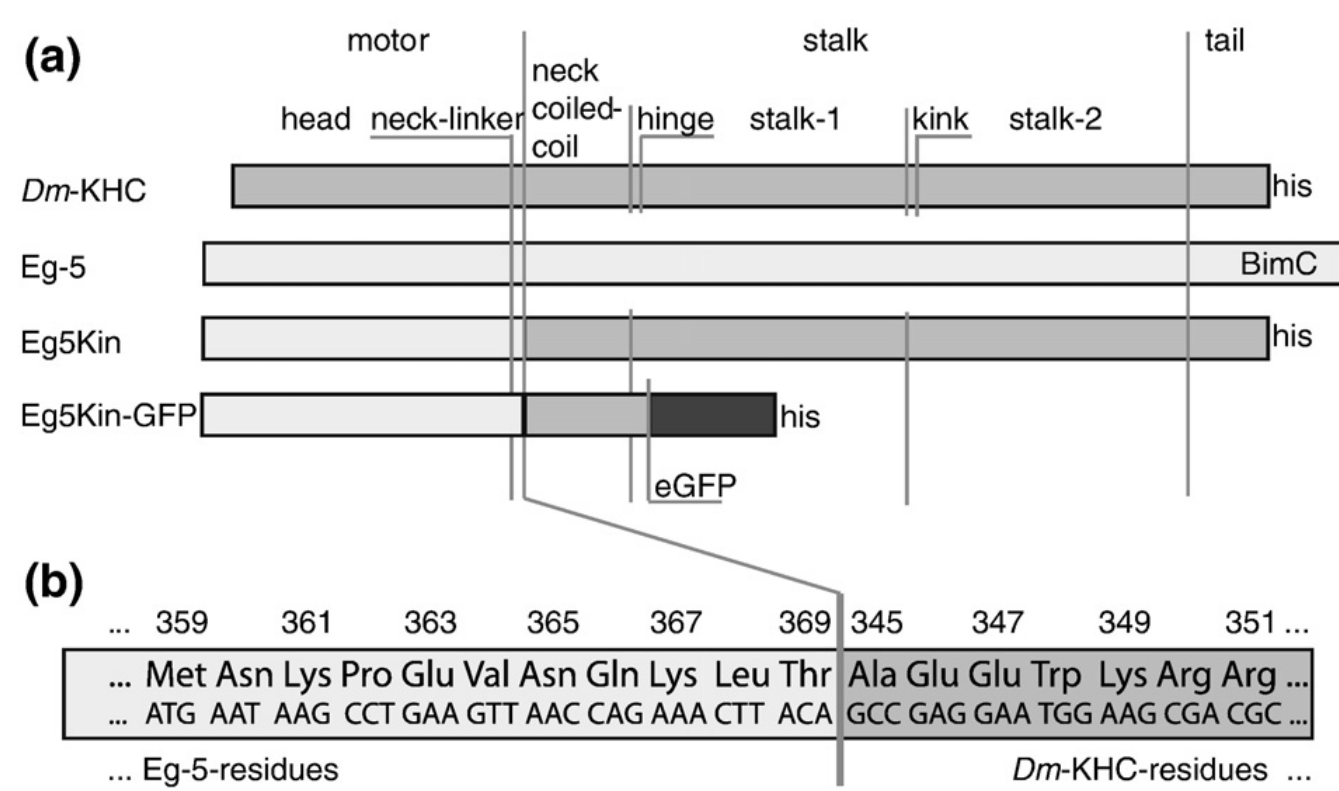

Fig. 4.1: Motor constructs. Constructs were generated by taking advantage of the basic structural organization shared by kinesin-1 (here DmKHC) and kinesin-5 (here Eg5) family members. (a) The motor domain consists of globular head and neck linker in both wild-type motors. Within the extended stalk, there are flexible (hinge and kink) and rigid coiled-coil regions (neck coiled coil; stalks 1 and 2) in kinesin-1, which are not obvious in kinesin-5 motors [Hildebrandt06]. The small globular tail domain of Eg5 contains the BimC box. (b) Details of the junction between the Eg5 motor domain and the DmKHC neck coiled coil: The numbering refers to the amino acid numbering in the respective wild-type motor sequences as indicated in gray. Methods: Eg5Kin was constructed from pPK113 (pET5a-DmKHC-His) [Coy99a] and pBK006 (Eg5-GFP) [Kwok06] using a nested PCR approach to extend the sequence of the Eg5 motor domain (residues 1 - 369) with the DmKHC residues starting at residue 345 towards an existing HindIII restriction site in pPK113. The DmKHC motor domain in pPK113 was subsequently replaced using NdeI and HindIII. The shortened GFP-tagged Eg5Kin-GFP was truncated at residues corresponding to DmKHC421, using PCR primers providing an AscI-XmaI site upstream of a stop codon. The full-length DmKHC in pPK113 was replaced with this truncated DmKHC421 using NdeI and NotI. A GFP-6His cassette flanked by AscI and XmaI was generated in pT7-7 for insertion downstream of the truncated Eg5Kin construct in pPK113. Integrity was confirmed by sequencing. Expression and purification were performed as previously described in Coy et al. [Coy99a] (for a more detailed description of materials and methods, see section 4.4). 
(a)

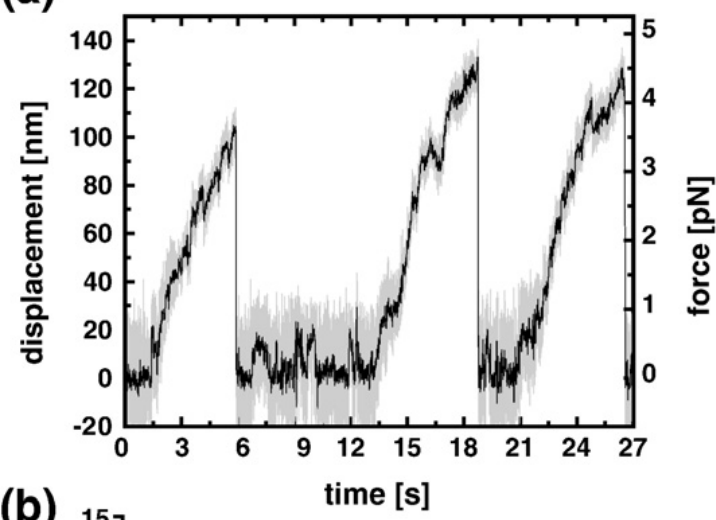

(b)

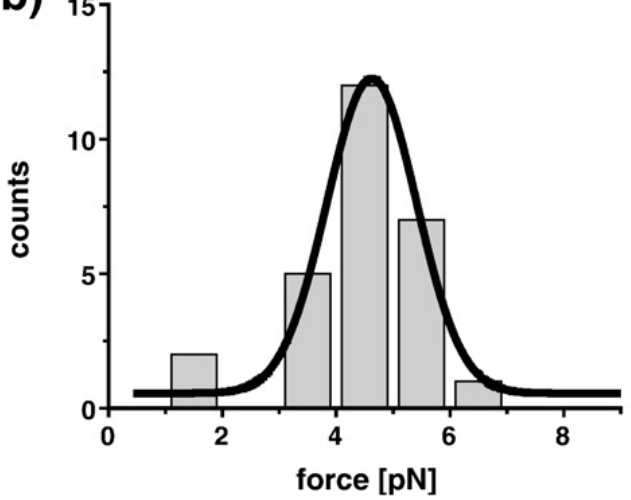

Fig. 4.2: Single-molecule force measurements in an optical trap. (a) Displacement of a $1-\mu \mathrm{m}$ silica bead driven by a single Eg5Kin motor, showing multiple excursions from the center of the trap and detachment without stalling plateaus (average initial velocity of $56 \pm 10$ $\mathrm{nm} / \mathrm{s} ; \quad \mathrm{N}=28$ ). (b) Histogram of the detachment forces of single Eg5Kin motors (average detachment force of $4.6 \pm 0.1 \mathrm{pN} ; \mathrm{N}=$ 27). Motility assays: Experiments were essentially performed as previously described by Korneev et al. [Korneev07] for single-molecule optical trapping assays, by Lakamper and Meyhofer [Lakämper05] for surface-gliding assays, and by Kwok et al. [Kwok06] and Kapitein et al. [Kapitein08] for single-molecule fluorescence assays. Details are presented in the Materials and Methodes (section 4.4).

These results demonstrate that the processive motility of our Eg5Kin chimeras is similar to that reported for truncated dimers in terms of both speed $(\sim 95 \mathrm{~nm} / \mathrm{s})$ and force $(\sim 5 \mathrm{pN})$ [Kapitein08, Valentine06]. The run length of the chimeras was, however, substantially longer (230 steps instead of $6-8$ steps) than that of truncated dimers [Valentine06]. The processive movement of single Eg5Kin dimers proves that the Eg5 motor domains are intrinsically capable of extended processive motility. The increased run length of Eg5Kin, as compared to HsEg5-513, is consistent with two studies recently published by Kalchishkova and Bohm [Kalchishkova08] and Muthukrishnan et al. [Muthukrishnan09] and with a biochemical study by Hackney et al. [Hackney03]. In the first study, in which a human kinesin-5 motor domain, including its neck linker, was fused to a human neuronal kinesin-1 (construct E$\mathrm{K}($ neck $)$ ) [Kalchishkova08], the authors observed an increase in speed as compared to a presumably dimeric truncation of human kinesin-5. Processivity was not assessed. The latter two studies show that extending the neck linker (in Muthukrishnan et al. [Muthukrishnan09] by three homologous aa from kinesin-2) leads to a decrease in the processive motility of kinesin-1. In our construct, we deliberately chose the fusion sites to be homologous to the site in kinesin- 1 that is commonly regarded as the location of the transition from neck linker to neck coiled coil.

One possible structural explanation for the increased processivity of Eg5Kin might be enhanced gating, as compared to kinesin-5 dimers, due to the decreased neck-linker length of Eg5Kin (Eg5Kin and kinesin-1: 14 aa; kinesin-5: 18 aa) [Muthukrishnan09]. The notion that gating is a major factor in determining proces- 


\section{(a)}

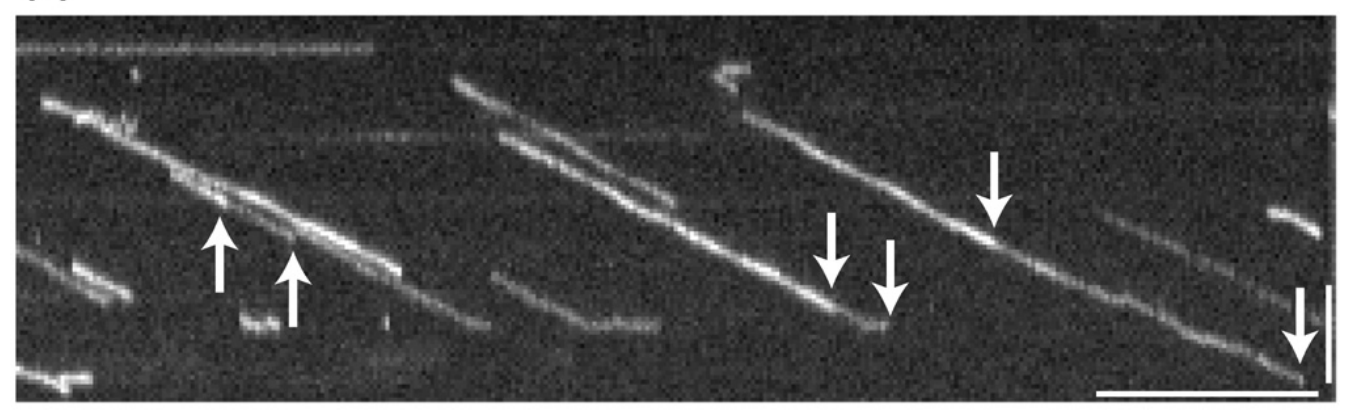

(b)

(c)
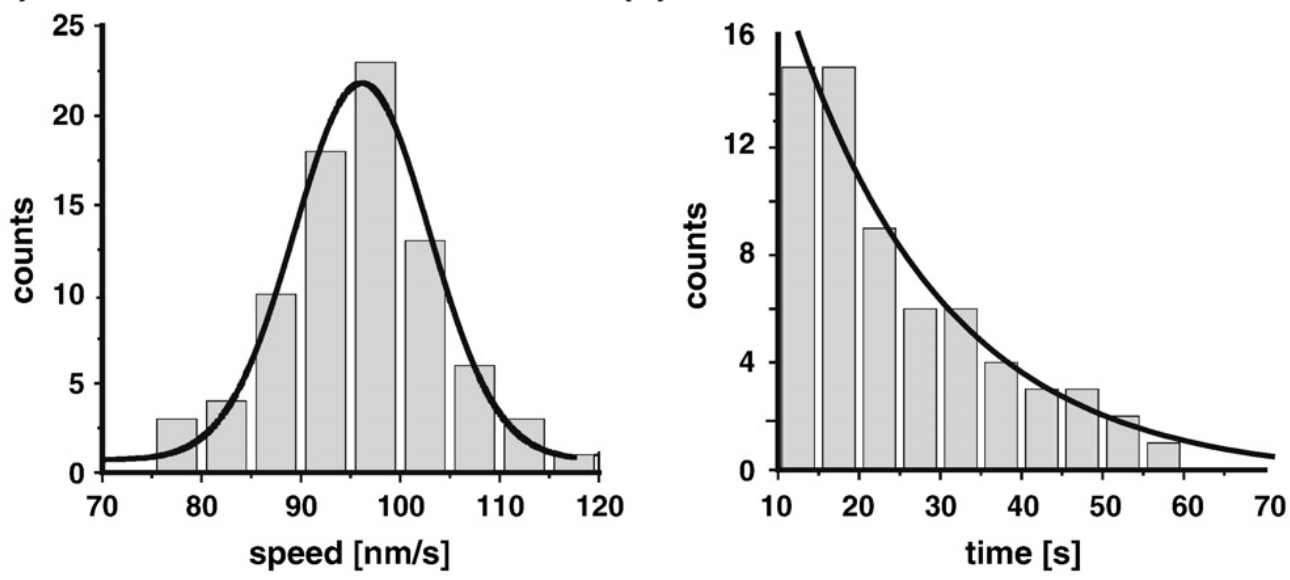

Fig. 4.3: Processivity assays of Eg5Kin-GFP using single-molecule fluorescence. (a) Kymograph of single Eg5Kin-GFP dimers moving on a surface-adsorbed microtubule. Arrows mark incremental bleaching events of moving spots (bleaching data, see Supplementary Fig. B.1). Horizontal scale bar represents $50 \mathrm{~s}$; vertical scale bar represents $3 \mu \mathrm{m}$. (b) Distribution of the speeds of individual Eg5Kin-GFP motors moving unidirectionally along a microtubule. Gaussian fit results in an average speed of $96 \pm 0.3 \mathrm{~nm} / \mathrm{s}(\mathrm{N}=82)$. (c) Histogram of association times exponentially fitted, excluding times below $10 \mathrm{~s}$ : average association time of $19.1 \pm 3.5 \mathrm{~s}$, equivalent to a distance of $1.8 \pm 0.3 \mu \mathrm{m}$ or 230 steps of $8 \mathrm{~nm}$.

sivity is supported by Eg5 dimer trapping experiments in which moderate hindering loads rescue processivity at limiting ATP concentrations [Valentine09]. Our observation of processivity thus suggests that the neck linker of Eg5Kin is of appropriate length to allow effective head-to-head communication.

It has been hypothesized that charge patterns in the neck coiled coil enhance processivity [Romberg98, Thorn00]. We think that this explanation is not likely to apply to the increased processivity we observed here because results with less charged Neurospora kinesin-1 and with microtubules without the negatively charged E-hook removed have ruled out the charge model for extended processivity [Lakämper05, Skiniotis04].

It is intriguing that the motors typically detach before they stall. The relatively high velocity at the point of detachment makes sense, since the detachment forces of $4-5 \mathrm{pN}$ are only about $60 \%$ of typical kinesin- 1 stall forces. This peculiar behavior 
of Eg5, however, demonstrates that there must be a structural feature of the Eg5 head and neck linker that creates force sensitivity. Understanding this mechanism needs further study.

In a further set of experiments, we focused on the inhibition of Eg5Kin motility by monastrol. First, we performed surface-gliding assays at high motor concentrations in the presence of monastrol. Unexpectedly, microtubule motion driven by Eg5Kin motors was not affected by monastrol at concentrations of up to $200 \mu \mathrm{M}$, and velocity remained constant at about $95 \mathrm{~nm} / \mathrm{s}$ (Fig. $4.4 \mathrm{a}$ ). At monastrol concentrations above $250 \mu \mathrm{M}$, motility ceased altogether. This finding is consistent with an all-or-nothing mode of operation of monastrol in the collective mode of motion of a microtubule by many surface-attached motors. Assuming that the motors inhibited by monastrol do impose only a very low friction upon the gliding microtubule, as has been suggested in the literature [Crevel04a], one would - in a situation where many processive motors drive one microtubule - expect full-speed motility to continue until the last of the interacting motors is inhibited.

In order to discover how monastrol affects the processive motility of individual Eg5Kin motors, we performed single-molecule fluorescence assays in the presence of increasing monastrol concentrations. Individual Eg5Kin motors moved unidirectionally, without any diffusive interruptions, even at monastrol concentrations of $100 \mu \mathrm{M}$ (Fig. $4.4 \mathrm{~b}$ ). Just like in the surface-gliding assays, the velocity was not measurably affected by monastrol (Fig. $4.4 \mathrm{a}$ and b). We did, however, observe a monastrol-dependent reduction of the processive run length (Fig. $4.4 \mathrm{~b}$ and c; see also Supplementary Fig. B.2). In other words, monastrol led to an increase in the rate of motor detachment or a decrease in run durations. Since there are two binding sites for monastrol on a motor dimer, one might have expected an intermediate or perturbed motility mode with just one monastrol bound. In fact, we do not see any change in speed or motile properties during processive runs before detachment. There are three possible explanations for this finding: (i) run termination is caused already by the binding of one monastrol to a dimer, or (ii) one monastrol bound has no effect and termination is only triggered by the binding of a second monastrol molecule, or (iii) binding of monastrol is strongly cooperative such that binding of one monastrol is so rapidly followed by binding of a second monastrol and release that intermediate behavior cannot be observed. The functional form of the concentration dependence of the motor detachment rate (inverse association time) can help to distinguish (i) from (ii) and (iii). Our data can be fitted significantly better with a parabolic than with a linear concentration dependence (Fig. 4.4 c). Assuming that the law of mass action applies to the binding of monastrol to the motor heads, this suggests that a processive run terminates only when both binding sites are occupied by monastrol. This rules out possibility (i). Assuming then that two monastrols, one on each motor head, are fatal for motility, it is hard to imagine that binding of one monastrol to one head would not have an effect on dimer motility. This notion is supported by the fact that a monastrol-induced decrease in motor velocity has been observed in other studies of collective motility using monomeric Eg5 constructs and in heterodimeric constructs with one Eg5 motor domain [Kaseda08].

To repeat this control, we generated a set of truncated monomeric Eg5-369(GFP)His and dimeric Eg5-511(GFP)His constructs and performed surface-gliding assays 
(a)
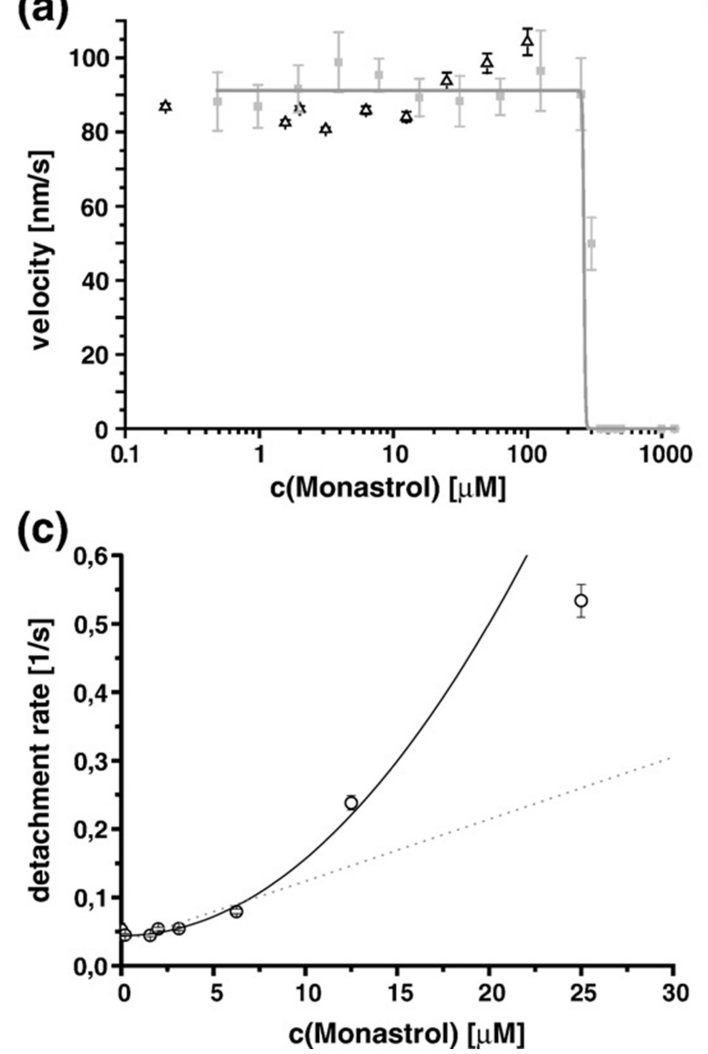

(b)
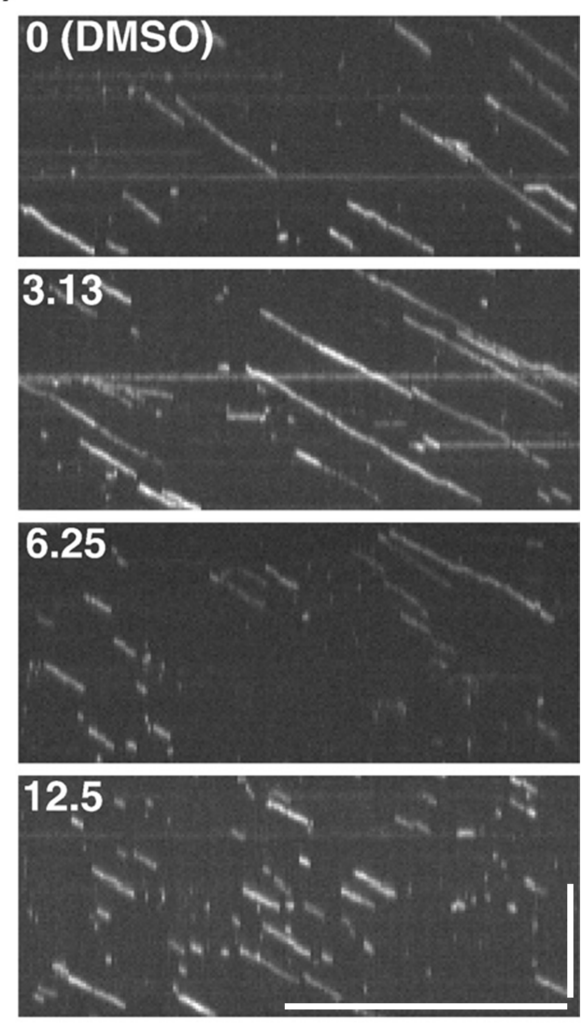

Fig. 4.4: Inhibition of Eg5Kin-GFP motility by monastrol. (a) Velocities observed in Eg5Kin multimotor surface-gliding assays (filled squares, gray line) and in single-molecule fluorescence processivity assays with Eg5Kin-GFP (open triangles, black) as a function of monastrol concentration. Surface-gliding velocities were unaffected up to $200 \mu \mathrm{M}$ monastrol, with an $\mathrm{IC}_{50}$ of $240 \mu \mathrm{M}$. Single-molecule velocities remained unchanged within the observed concentration range. (b) Kymographs of Eg5Kin-GFP in the presence of increasing monastrol concentrations. Kymographs for all other monastrol concentrations are presented in Supplementary Fig. B.2. Motor velocity (i.e., the slope of the lines) did not change with increasing monastrol concentration (see also Fig. 4.3 a), while the lengths of interactions decreased. Horizontal scale bar represents $100 \mathrm{~s}$; vertical scale bar represents $5 \mu \mathrm{m}$. (c) Detachment rates of Eg5Kin-GFP (open circles) as a function of monastrol concentration. Lines: Linear and parabolic fits of the data: $k_{d e t}=k_{0}+\operatorname{const}\left(c_{m o n}\right)^{n}$, where $k_{d e t}$ is the inverse of the measured run duration at a certain monastrol concentration, and $k_{0}$ is the rate at zero monastrol concentration. Gray dotted line: $\mathrm{n}=1$, linear fit with offset. Continuous black line: $\mathrm{n}=2$, parabolic fit with offset; concentration rate for doubling: $\mathrm{C}_{2}=6.3 \pm 0.4 \mu \mathrm{M}$ monastrol. Data deviate from the parabolic fit at high monastrol concentrations because only events visible for two consecutive 0.5-s frames (i.e., lasting longer than $1 \mathrm{~s}$ ) were counted, leading to an apparent saturation at a rate of $\sim$ $1 \mathrm{~s}^{-1}$. Fit results for $\mathrm{n}=2$ were $k_{0}=0.044 \pm 0.002 \mathrm{~s}^{-1}$ and const $=0.001 \pm 0.0001$ $\mu \mathrm{M}^{-1} \mathrm{~s}^{-1}$. 
in the presence of increasing concentrations of monastrol (Supplementary Fig. B.3). For dimeric Eg5 constructs, we observed an overall surface-gliding speed lower than that for Eg5Kin, but a similar inhibitory effect: Above a threshold, microtubule velocity dropped by $\sim 90 \%$. Monomeric Eg5 constructs produced microtubule velocities $(46 \pm 13 \mathrm{~nm} / \mathrm{s})$ similar to those of dimeric constructs $(42 \pm 8 \mathrm{~nm} / \mathrm{s}$; Supplementary Tab. B.1) in surface-gliding assays. The remarkable result was that addition of increasing concentrations of monastrol reduced the average monomer gliding speed only by $\sim 40 \%$, even at micromolar saturating concentrations of monastrol. The inhibition also progressed more gradually than that of dimeric Eg5, without a strong thresholding effect. The monomer data suggest that individual motor domains remain motile even at high monastrol concentration, which could mean that binding of monastrol to isolated monomers is weak (i.e., rapidly reversible). The comparison between the surface gliding experiments of Eg5-369, Eg5-511, and Eg5Kin thus demonstrates that dimerization strongly increases sensitivity to monastrol inhibition, both in terms of strength of inhibition and in terms of a more pronounced threshold behavior. This observation strongly suggests that binding of monastrol to a dimeric Eg5 is cooperative.

It is still difficult, though, to strictly distinguish between scenarios (ii) and (iii) for motor inhibition by monastrol. Since the effect on isolated monomers is weak, binding of one monastrol to a dimer might create an effect that is hidden in experimental uncertainty. Nevertheless, it appears very likely that monastrol can only terminate a processive run when two monastrol molecules are bound to an Eg5Kin dimer.

Bulk kinetic experiments have been mostly performed with Eg5 monomers with and without added microtubules. One cannot expect monastrol binding and unbinding rates to be equal in the case of processive dimers. A comparison is still informative. Luo et al. measured the on-rates of monastrol to ADP and AMP-PNP heads of monomeric Eg5 to be $0.15 \mu \mathrm{M}^{-1} \mathrm{~s}^{-1}$ and $0.97 \mu \mathrm{M}^{-1} \mathrm{~s}^{-1}$, respectively, in the presence of microtubules [Luo04]. Assuming for a moment that one monastrol would terminate a processive run of an Eg5 dimer, we can estimate the corresponding monastrol on-rate from the observed Eg5 run durations. At a monastrol concentration of $12.5 \mu \mathrm{M}$, we observe an average duration of $5 \mathrm{~s}$ corresponding to an on-rate of $\sim 0.2 \mathrm{~s}^{-1}$, which is distinctly lower than $\sim 12.5 \mathrm{~s}^{-1}$ from monomer kinetics even in the slow ADP state. This effect is more pronounced at lower monastrol concentrations. At $1 \mu \mathrm{M}$ monastrol, the average run duration was $20 \mathrm{~s}$, corresponding to an on-rate of $\sim 0.05^{-1}$, compared to $\sim 1 \mathrm{~s}^{-1}$ from monomer kinetics. This comparison supports our conclusions that dimerization of the motor domain affects monastrol binding kinetics, and that it needs two (cooperatively binding) monastrols to terminate a run. Unfortunately, there are no published kinetic data that can be directly compared.

In summary, first, kinetic experiments suggest that monomeric Eg5 constructs bind monastrol on the timescale of our observed dimer run durations. Second, surface gliding of microtubules driven by monomers is measurably slowed by monastrol. Third, velocities do not vary with increasing monastrol in our single-molecule dimer records. In the light of these findings, we are led to conclude that scenario (iii) 
applies and that binding of one monastrol to one head of a dimer is inhibited by a genuinely cooperative mechanism.

Such cooperativity would, at first glance, be easiest to explain if the two heads interacted directly, possibly through a structural element that is affected by monastrol binding, most likely loop 5 on the kinesin back [Bodey09]. It is tempting to speculate that this might occur in a back-to-back conformation of the heads, which could be stabilized by monastrol and thereby block the motor from performing a next step.

The detachment rate-doubling concentration $\mathrm{C}_{2}(6.3 \mu \mathrm{M})$ that we measured for single Eg5Kin-GFP dimers appears, at first glance, to be inconsistent with the monastrol concentration of $\sim 250 \mu \mathrm{M}$ at which surface gliding was inhibited (Fig. 4.4 c). These values can, however (as already discussed above), easily be reconciled if one bears in mind that, in a surface-gliding assay, a microtubule interacts simultaneously with more than one motor. In such a situation, the effect of the reduced interaction time (while speed is constant) of the individual motors would only manifest itself at a monastrol concentration at which interaction periods cease to overlap. Furthermore, the observation that the gliding velocity did not decrease with increasing monastrol up to a concentration where binding and gliding stopped altogether is fully consistent with the finding that individual motor velocity did not change with addition of monastrol. The loss of gliding activity corresponds to the complete inhibition of microtubule binding at high monastrol concentrations.

Our results are, in fact, also consistent with earlier single-molecule fluorescence studies of full-length Eg5-GFP, which showed a reduction of the average velocity of tetrameric Eg5 by monastrol [Kwok06]. The motility of full-length tetrameric Eg5 consists of alternating diffusive and directional episodes, and it was shown that monastrol increases the diffusive character of the motility, also indicating a shortening of the processive episodes by monastrol [Kwok06].

In summary, the kinesin-5 head/kinesin-1 stalk chimera we have generated has proven to be a useful model system for studying, in particular, the regulation of Eg5 motility. Our approach avoids the complexity brought about by the regulatory domains and the additional pair of motor domains in the tetramer, and thereby provides a starting point for the controlled reintroduction of regulation. Eg5Kin is a highly processive motor that moves unidirectionally with a velocity of $95 \mathrm{~nm} / \mathrm{s}$ and an average run length of $\sim 1.9 \mu \mathrm{m}$, and it can sustain forces of $\sim 5 \mathrm{pN}$. These results confirm its basic functional similarity to kinesin-1 motors. The kinesin-5-specific inhibitor monastrol does not affect Eg5Kin velocity, but binding of two monastrols appears to terminate processive runs. Furthermore, we added to the evidence that the domains mediating diffusive interactions observed with Eg5 tetramers reside outside the motor domain. Our findings finally suggest that Eg5, in its native tetrameric form, is highly (down)regulated. Such a regulation makes sense in the context of the mitotic spindle, where various motility processes have to be carefully balanced and adjusted in the different stages of cell division. As a next important step, it now appears possible to study further regulatory mechanisms in the complex mechanical machinery driving mitosis. 


\section{\begin{tabular}{l|l} 
Materials and Methods & 4.4
\end{tabular}}

\section{Cloning and protein purification}

The full-length Eg5Kin chimera was constructed from pPK113 pET5a-FL_DmKHCHis) [Coy99a] and pBK006 (FL-Eg5-GFP) [Kwok06] using a nested-PCR approach in order to extend the sequence of the Eg5 motor domain $(1-369)$ with the DmKHC residues starting at 345 towards an existing HindIII restriction site in pPK113. The DmKHC motor domain in pPK113 was subsequently replaced by sub-cloning of this transition clone using NdeI and HindIII. A shortened GFP-tagged clone was truncated at residues corresponding to DmKHC 421, using PCR primers providing an AscI and XmaI site upstream of a stop-codon and replaced the full-length DmKHC in pPK113 using NdeI and NotI. The truncated and his-tagged wild-type (WT) Eg5 constructs 369 and 511 were generated using primers providing the same flanking restriction sites for insertion into pPK113. A GFP-6His cassette flanked by AscI and XmaI was generated in pT7-7 for insertion downstream of the truncated Eg5Kin construct and the truncated WT constructs in pPK113. Integrity was confirmed by sequencing and expression and purification was performed essentially as previously described in [Coy99a]. In brief, BL21(DE3) cells (Invitrogen, CA, USA) were transformed and grown to a density of $\sim 0.6$ at $37{ }^{\circ} \mathrm{C}$ before induction of expression using $1 \mathrm{mM}$ IPTG for $3 \mathrm{~h}$. Cells were harvested by centrifugation and resuspended in a $20 \mathrm{mM}$ imidazole buffer ( $\mathrm{pH} 7.4)$, supplemented with $1 \mathrm{mM}$ each of DTT, $\mathrm{MgCl}_{2}$, EGTA, BME and $150 \mathrm{mM} \mathrm{NaCl}$ before lysis using ultrasound in the presence of lysozyme and DNAseI. After separation from cell debris by centrifugation, the cytosol was incubated for $1 \mathrm{~h}$ at $4{ }^{\circ} \mathrm{C}$ with Ni-NTA-column material (Qiagen, Germany) and then transferred to a syringe column. After washing the column with $80 \mathrm{mM}$ imidazol and $300 \mathrm{mM} \mathrm{NaCl}$, bound motor was eluted using a $300 \mathrm{mM}$ imidazol buffer containing $10 \mu \mathrm{m}$ ATP and $1 \mathrm{mM}$ DTT. Fractions containing motor were pooled and $3 \times$ dialyzed against $80 \mathrm{mM}$ PIPES/KOH, $1 \mathrm{mM} \mathrm{MgCl}_{2}$, $1 \mathrm{mM}$ EGTA, $1 \mathrm{mM}$ DTT, $10 \mu \mathrm{M}$ ATP. The truncated motors were purified using a MT-affinity purification procedure described in [Lakämper05]. Protein samples were tested for motor activity and stored in aliquots at $-80^{\circ} \mathrm{C}$. Microtubules were purified from pig brain and labeled as described in [Lakämper05].

\section{Surface-gliding assays}

Surface gliding assays were performed at $21{ }^{\circ} \mathrm{C}$ essentially as described previously [Lakämper05]. Full-length Eg5Kin motors were allowed to non-specifically bind to the glass surfaces of assay chambers made from cover-slips and microscope slides assembled with double-stick tape. Subsequently, the chambers were flushed with about three chamber volumes of assay mix in BRB80 buffer (80 mM PIPES/KOH, $1 \mathrm{mM}$ $\mathrm{MgCl}_{2}, 1 \mathrm{mM}$ EGTA) containing $10 \mu \mathrm{m}$ taxol (paclitaxel), $0.022 \mathrm{mg} / \mathrm{ml}$ tetramethylrhodamine (TMR)-labeled microtubules, $2 \mathrm{mM}$ ATP, $4 \mathrm{mM} \mathrm{MgCl}_{2}, 10 \mathrm{mM}$ 
DTT, $0.08 \mathrm{mg} / \mathrm{ml}$ catalase, $0.1 \mathrm{mg} / \mathrm{ml}$ glucose oxidase and $10 \mathrm{mM}$ glucose. Motility was observed in a standard inverted fluorescence microscope (Zeiss Axiovert 100, Germany). In surface-gliding assays in the presence of up to $1250 \mu \mathrm{M}$ monastrol, only moving microtubules were scored to obtain average velocities. A small fraction of microtubules got irreversibly stuck on surface contaminations in the absence of monastrol. Above $\sim 260 \mu \mathrm{M}$ monastrol, no moving microtubules were observed anymore. Images were recorded with a digital CCD camera (CoolSnap ES, Roper Scientific, Germany). The same protocols were used for the truncated motor construct, Eg5Kin-GFP and for non-chimeric Eg5 truncation constructs Eg5-369(GFP)His and Eg5-511(GFP)His. Alternatively, motors were introduced in chambers pre-treated with streptavidin and biotinylated penta-His antibody (Qiagen, Hilden, Germany).

\section{Single-molecule optical-trapping assays}

Assays were performed at $21^{\circ} \mathrm{C}$ using a single-beam optical trap setup built on a custom-designed inverted microscope as described elsewhere [Korneev07]. Infrared laser light (1064 nm, cw, Nd:YVO focused into the flow chamber using an objective (Zeiss Neofluar $100 \times$ oil, $1.3 \mathrm{NA}$, Göttingen, Germany) to form an optical trap. Trap stiffness was varied in the range of $3-6 \times 10^{-5} \mathrm{~N} / \mathrm{m}$. The back-focal plane of the condenser (Zeiss, oil, 1.4 NA) was imaged onto a quadrant photodiode which was operated at a reverse bias of 100 V (YAG-444-4A, Perkin-Elmer, Vaudreuil, Canada) for position detection of the trapped particle. Photo diode signals that correspond to the displacement fluctuations of the trapped particle in the plane normal to the optical axis were processed by custom-built analog electronics [Allersma98] and read into a PC using LabView (National Instruments, Austin, TX, USA). For motility assays, streptavidin-coated silica spheres $(0.5 \mu \mathrm{m}$ diameter, Kisker Biotech, Steinfurt, Germany) were incubated with $0.1 \mathrm{mg} / \mathrm{ml}$ casein in PEM12 buffer (12 mM PIPES/KOH, $2 \mathrm{mM} \mathrm{MgCl}_{2}$, $1 \mathrm{mM}$ EGTA, pH 6.8) prior to incubation with biotinylated antibodies against the GFP tag (Goat Anti-GFP polyclonal antibody, biotin conjugated, Abcam plc, Cambridge, UK) for 30 min. Beads were washed at least three times in PEM12 containing $0.1 \mathrm{mg} / \mathrm{ml}$ casein before dilute motors were added in the presence of $1 \mathrm{mM} \mathrm{ATP}$. Microtubules were added into silanized flow chambers (see below) and allowed to bind to the surface for 5 min before blocking the surface with $0.1 \mathrm{mg} / \mathrm{ml}$ casein in PEM12. An assay mix containing motor-covered beads in PEM12 with added 10 mM dithiothreitol (DTT), $1 \mathrm{mM}$ ATP, $2 \mathrm{mM} \mathrm{MgCl}_{2}, 10 \mathrm{mM}$ glucose, $100 \mu \mathrm{g} / \mathrm{ml}$ glucose oxidase, $80 \mu \mathrm{g} / \mathrm{ml}$ catalase, $0.1 \mathrm{mg} / \mathrm{ml}$ casein was flushed into the pre-treated chamber, and motility was observed as described above. To ensure that beads carried only one functional motor, the motor concentration was reduced such that only about one in ten beads produced motility. 


\section{Single-molecule fluorescence processivity assays}

Tetramethyl-rhodamine labeled microtubules were polymerized in the presence of guanosine-5'-[( $\alpha, \beta)$-methyleno] triphosphate (GMP-CPP) to avoid de-polymerization after dilution. Microtubules were attached to positively charged silanized cover slips. Cover slips were cleaned using $\mathrm{KOH} / \mathrm{EtOH}$ before surface silanization using 3-[2-(2-aminoethylamino)ethylamino] propyl-trimethoxysilane (DETA) (Sigma, Germany) for microtubule immobilization. Microtubules were allowed to bind for 5 min followed by 5 min incubation with $0.1 \mathrm{mg} / \mathrm{ml}$ BSA or casein in BRB80. A 1:20 000 dilution of Eg5Kin-GFP in assay mix was introduced, and fluorescence was observed in a custom-built wide-field fluorescence microscope [Kapitein05]. For assays including monastrol, the assay mix contained at most $5 \%$ DMSO. Digital images were subsequently analyzed for speeds, run lengths, binding rates and association times using kymographs generated with custom-written LabView software. Statistical analysis of data was performed with OriginPro (OriginLab Corporation, Northampton, USA).

\section{\begin{tabular}{l|l} 
Acknowledgements & 4.5
\end{tabular}}

We thank J. van Mameren, M. Noom, and S. van den Wildenberg for software development and help, and W. O. Hancock, and B. Kwok/T. Kapoor for the generous gifts of the plasmids pPK113 and BK006, respectively. This work was partially supported by a research grant from the Human Frontiers Science Program (S.L. and C.F.S.). L.C.K. and E.J.G.P. were supported by a VIDI grant from the Dutch Organization for Scientific Research (NWO) and a travel grant from the Laser Center of the Free University Amsterdam. The work was further supported by the Research Center for Molecular Physiology of the Brain, funded by the Deutsche Forschungsgemeinschaft.

\section{Supplementary data}

Supplementary data associated with this article can be found online at the Journal of Molecular Biology, doi:10.1016/ j.jmb.2010.03.009 and in appendix B. 


\title{
Neck-linker length dependence of processive kinesin-5 motility
}

The content of this chapter was published 2012 in the Journal of Molecular Biology [Düselder12]. I share the first authorship with Andrè Düselder. Co-authors are Stefan Lakämper and Christoph F. Schmidt.

In this work I participated in the purification of proteins. Further I performed the multi-motor surface-gliding experiments, the single-molecule fluorescence experiments, and analysed the respective data. Additionally I contributed figures and text to the paper.

\begin{abstract}
\begin{tabular}{l|l} 
Abstract & 5.1
\end{tabular}
Processive motility of individual molecules is essential for the function of many kinesin motors. Processivity for kinesins relies on communication between the two heads of a dimeric molecule, such that binding strictly alternates. The main communicating elements are believed to be the two neck linkers connecting the motors' stalks and heads. A proposed mechanism for coordination is the transmission of stress through the neck linkers. It is believed that the efficiency of gating depends on the length of the neck linker. Recent studies have presented support for a simple model in which the length of the neck linker directly controls the degree of processivity. Based on a previously published Kinesin-1/Kinesin-5 chimera, Eg5Kin, we have analyzed the motility of 12 motor constructs: we have varied the length of the neck linker in the range between 9 and 21 amino acids using the corresponding native Kinesin-5 sequence (Xenopus laevis Eg5). We found, surprisingly, that neither velocity nor force generation depended on neck-linker length. We also found that constructs with short neck linkers, down to 12 , were still highly processive, while processivity was lost at a length of 9 amino acids. Run lengths were maximal with neck linkers close to the native Kinesin-5 length and decreased beyond that length. This finding generally confirms the coordinating role of the neck linker for kinesin motility but challenges the simplest model postulating a motor-type-independent optimal length. Instead, our results suggest that different kinesins might be optimized for different neck-linker lengths.
\end{abstract}




\section{\begin{tabular}{l|l} 
Introduction & 5.2
\end{tabular}}

Kinesins are motor proteins that use energy from ATP hydrolysis to generate conformational changes. Kinesins drive mechanical processes in cells such as cargotransport along or disassembly of microtubules [Vale85, Brouhard08]. Single homodimeric motor molecules of the Kinesin- 1 family can move processively over micrometer distances along a microtubule [Howard89, Vale96], taking hundreds of $\sim$ 8-nm steps [Svoboda93], and can produce up to $\sim 7 \mathrm{pN}$ force before detachment [Svoboda94, Carter05, Visscher99]. During processive motility, each step is strictly coupled to the hydrolysis of one ATP molecule [Visscher99].

Kinesins from several other subfamilies, such as the Kinesin-3 Kif1A/Unc104 [Klopfenstein02, Tomishige02], the Kinesin-7 CENP-E [Yardimci08], the Kinesin-8 Kip3p [Varga06], or the mitotic Kinesin-5 Eg5 [Kwok06], have been shown to also move processively along microtubules. While structurally adapted to diverse cellular functions, the processive kinesins are likely to employ a similar basic head-head communication mechanism to achieve processive motility [Hirokawa09, Scholey03, Rosenfeld09]. A requirement for processivity of a single kinesin dimer is to keep the kinetic cycles of the two motor domains strictly out of phase, such that at least one motor domain remains strongly attached to the microtubule at any time. Studies on monomeric and dimeric kinesin constructs have led to a basic model of the allosteric mechanisms connecting microtubule binding and the nucleotide state of the motor domain [Schief01, Valentine07]. The head-head communication is believed to rely on tension-mediated gating of chemical reactions, possibly involving both leading and trailing heads [Clancy11]. The mechanically tensed state in the stepping cycle of a dimer is the two-head-bound state. Cryo-electron microscopy images of bound dimers can only be reconciled with dimer X-ray structures if one assumes a stretched conformation of the structural elements linking the two heads [Kozielski97]. The front-head gating model postulates that the leading, nucleotidefree head is unable to bind a new ATP while under backward tension, that is, before the detachment of the rear head. The rear-head gating model assumes that release of phosphate from the rear head, leading to detachment, is accelerated by forward tension [Rosenfeld03]. The tension-transmitting element has been suggested to be the neck linker, a short and mostly unstructured protein segment connecting the head to the stalk coiled coil [Rice99, Hackney05]. When modeled as a simple flexible polymer, the tension in the neck linker at a given end-to-end distance and, therefore, the strength of the head-head communication would scale inversely with contour length, that is, number of amino acids [Kutys10]. Data from three recent studies support such a simple general model of head-head communication [Yildiz08, Clancy11, Shastry11]. For a set of particular Kinesin-1 and Kinesin-2 constructs, it was found that processivity decreases with increasing neck-linker length, while shortening of the native neck linker by one amino acid for that motor abolishes processivity [Kutys10, Hariharan09, Muthukrishnan09, Shastry10]. A continuation of this work showed similar effects for Kinesin-1/Kinesin-2/Kinesin-3 and Kinesin-7 constructs [Shastry11]. In another study [Yildiz08], the insertion of up to 26 prolines in the neck linker of human Kinesin-1 was reported to not only slightly decrease run 
length under low-ionic-strength conditions but also strongly decrease motor speed and stall force. This phenomenon was interpreted to be due to an increasingly loose coupling of ATP hydrolysis to motor stepping. A detailed study of the kinetic pathway of two processive Kinesin-1 constructs with neck linkers extended to 20 and 23 amino acids suggested a different interpretation of new and existing data, based on a model where not only tension alone but also, possibly, neck-linker orientation produces gating [Clancy11].

Interestingly, neck-linker length varies between the different kinesin subfamilies. The highly processive Kinesin-1 motors [Howard89, Vale96, Lakämper03] carry a rather short neck linker of 14 amino acids. Most other kinesin subfamilies carry significantly longer neck linkers (see [Shastry11], Fig. 2 for a sequence alignment) of 16 - 18 amino acids in length. The Xenopus Kinesin-5, Eg5, has one of the longest neck linker with 18 amino acids [Hariharan09]. Nevertheless, wild-type (WT) Kinesin-5 has been shown to move processively [Kwok06]. Dimeric constructs, however, lacking the tetrameric coiled-coil region produced only very short (six to eight steps) processive episodes [Valentine06, Krzysiak06]. This could be due to the neck linker that is 18 amino acids long or to the lack of the opposing dimer and a compromised stalk structure. Full-length tetramers were observed to move over longer distances but detached at relatively small loads and were still less processive than Kinesin-1 [Kwok06]. This behavior, however, appears to be caused by yet another phenomenon, namely, tail-mediated self-inhibition, which leads to a switching off of the motor when bound to only one microtubule [Kapitein08]. Thus, studying intrinsic processivity of Kinesin- 5 has been difficult, both in truncated dimeric constructs and in WT tetramers.

To study the force-generating unit of Kinesin- 5 without interference of tail regulation and without the structurally complex tetrameric coiled coil of Kinesin-5, we have recently constructed a chimeric motor, Eg5Kin, which comprises the motor domain and the 14 N-terminal amino acids of the WT Kinesin-5 neck linker, fused to the coiled coil of Kinesin-1 [Lakämper10]. The initial rationale for using 14 amino acids was to provide a similar mechanical background as in Kinesin- 1 . We had found that Eg5Kin (here referred to as Eg5Kin-NL-14 or, short, NL-14) is capable of long-range processive motility taking on average 240 consecutive $\sim 8$-nm steps and of comparatively high force generation. These findings demonstrated that the Kinesin-5 motor domain is capable of supporting processive motility, provided that it is a stable dimer.

The fact that the neck linker of native Kinesin- 5 has a length of 18 amino acids begs the question if the processivity of the motor dimer increases when the length of the neck linker is shortened from 18 to 14 amino acids. If a shorter-than-native neck linker were to increase processivity, one would have to conclude that the length of the neck linker in Kinesin-5 is optimized for something else than run length and the most efficient head-head communication. A neck linker of the native length of 18 amino acids is known to support processive motility, as shown by the motion of WT Kinesin-5 tetramers between antiparallel microtubules, but the degree of processivity has not been quantified for the WT motor in that scenario. Here, we have generated a set of Eg5Kin chimeras with neck-linker lengths ranging from 9 (NL-09) to the full 18 amino acids of WT Kinesin-5 (NL-18) and beyond to 21 


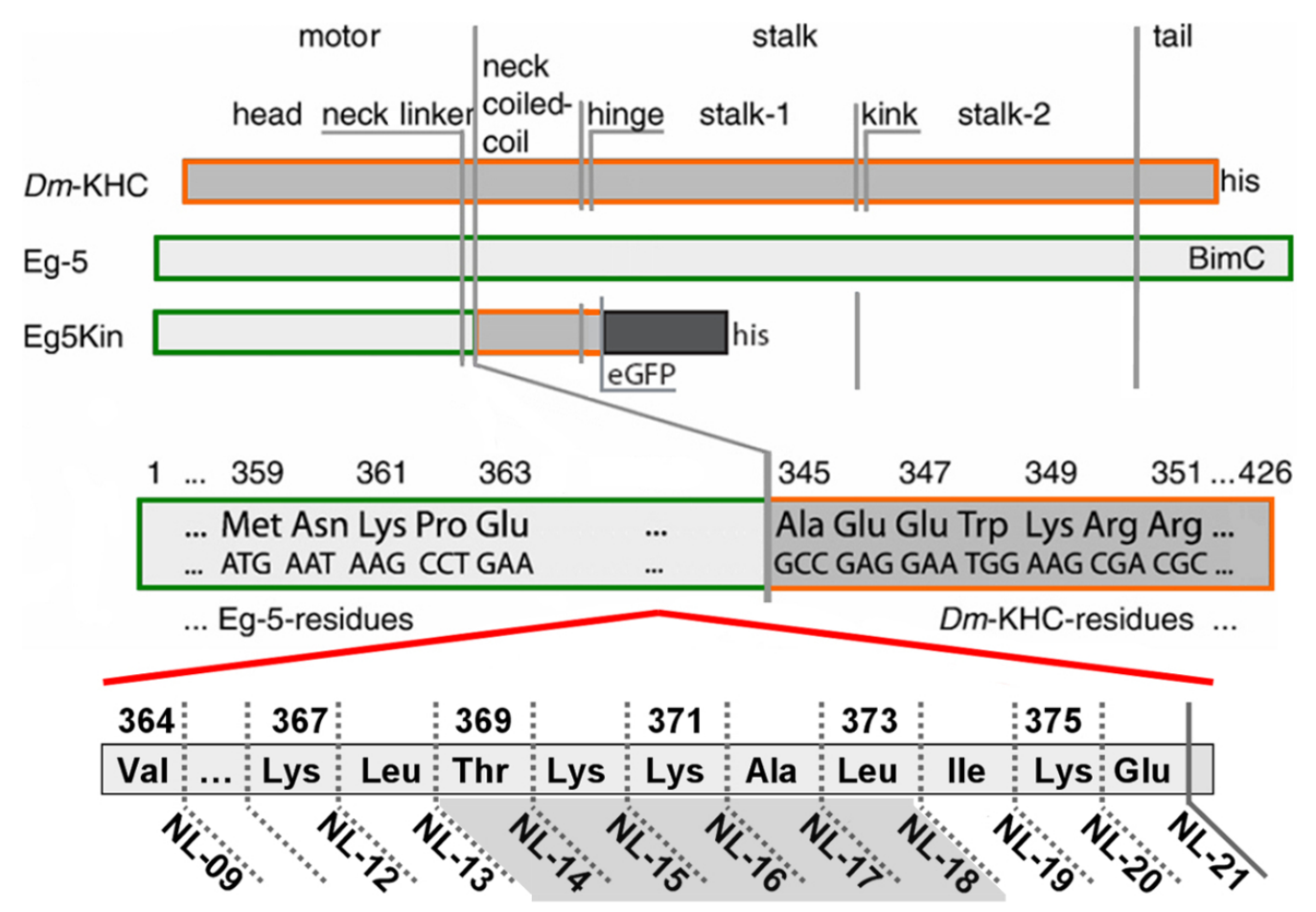

Fig. 5.1: Schematic description of motor constructs. Eg5Kin motor proteins were constructed using the motor domain and part of the neck-linker sequence of Eg5 (light gray; residues 1 - 364 up to 1 - 376 for NL-09 to NL-21, respectively) and the (truncated) stalk of DmKHC (dark gray; DmKHC residues 345 - 426). The numbering refers to the amino acid numbering in the respective WT motor sequences.

amino acids (NL-21) (Fig. 5.1 and Supplementary Fig. C.1). In contrast to other studies, where the length of the neck linker was varied using random or artificial sequences, such as poly-proline inserts and sequences from other distantly related kinesins [Clancy11, Hackney05, Yildiz08, Shastry10], we decided to exclusively use the native Eg5 Kinesin-5 sequence. We shortened the neck linker by successively deleting amino acids, starting with the 18th amino acid in the neck-linker sequence. For the elongation of the neck linker beyond 18 amino acids, we inserted the WT Kinesin-5 sequences using up to three residues from the putative Kinesin-5 neck coiled coil. A coiled-coil prediction for all constructs indicates that these inserted amino acids do not lead to an extension of the DmKHC neck coiled coil toward the N-terminus but that the coiled coil starts for all constructs at the beginning of the DmKHC residues. This prediction provided confidence to interpret our findings assuming steadily increasing neck-linker lengths. To have, as a further control, a motor for which the additional neck-linker amino acids would surely not become incorporated in an extension of the stalk coiled coil, we built one other construct in which we inserted three prolines after the 18th amino acid. This insertion is too short to form a poly-proline structure and is likely to be flexible [Arora02]. With this control, we could also test if new specific interactions between the motor domain and the neck linker, mediated by the nonnative additional proline residues, strongly changed the function of the motor. 


\section{\begin{tabular}{l|l} 
Results and Discussion & 5.3
\end{tabular}}

In a first set of experiments, we used multi-motor surface-gliding assays (MMGAs) to check the activity of the purified motors and to measure the velocities produced by motors acting collectively (Fig. 5.2 a). We found that all constructs were active motor proteins and produced virtually identical surface-gliding velocities of, on average, $\sim 67 \mathrm{~nm} / \mathrm{s}$ (Fig. 5.3 a and Tab. 5.1), the only exception being the shortest construct, NL-09, which showed a clearly diminished velocity $(16 \pm 6 \mathrm{~nm} / \mathrm{s})$. These results differ from the results of Yildiz et al. for human Kinesin-1 [Yildiz08], who saw a strong decrease in velocity with longer neck linkers. This discrepancy may be due to the fact that Kinesin-1 motors have intrinsically a much higher velocity and therefore a different catalytic cycle. Thus, the loss of coupling efficiency found for Kinesin-1 does not appear to occur for Kinesin-5. The magnitude and constancy of velocity are in agreement with the results of Shastry and Hancock with a very similar Kinesin-5 construct [Shastry11].

We then tested the constructs for velocity in optical trapping assays (Fig. 5.2 b) and found, for all the constructs, a similar velocity with an average value of $\sim$ $64 \mathrm{~nm} / \mathrm{s}$ in good agreement with the MMGA results (Fig. 5.3 a and Tab. 5.1). In single-molecule fluorescence assays (Fig. $5.2 \mathrm{c}$ ), we found again very similar velocities for all constructs (Fig. $5.3 \mathrm{a}$ and Tab. 5.1), although the average velocity of $\sim 116$ $\mathrm{nm} / \mathrm{s}$ was higher than the results of MMGAs and optical trapping assays. Although not often explicitly studied, a difference in this direction is not unexpected. Optical trapping assays are not load free, load diminishes velocity [Svoboda93], and we did not attempt here to extrapolate the results to zero load. MMGAs can always suffer from motor attachment artifacts, contamination by defective motors, or strained geometries of motor attachment to the microtubule. Therefore, we do not draw any conclusions from this difference for the present study.

Although NL-09 is still active in MMGAs, it did not show any processive motion, neither in the optical trapping nor in the single-molecule fluorescence assays (Supplementary Fig. C.2). We thus know that NL-09 is an active, albeit non-processive, motor. The lack of processivity most likely results from the neck linker being too short to span the gap between two adjacent binding sites along the microtubule.

These findings are somewhat different from what has been reported for other kinesins and also for a very similar Kinesin-5 construct. In one recent study, Shastry and Hancock investigated the effect of varying neck-linker length on Kinesin-1 and an artificially homodimeric Kinesin-2 construct (Kif3A/A, termed Kin-2) [Shastry10]. For Kinesin-1, the deletion of one amino acid (Kin-1 $\Delta \mathrm{T}$ ) led to a non-processive motor, displaying about $70 \%$ of Kin-1 velocity in surface-gliding assays, whereas the extension of the neck linker by one or several amino acids (Kin-1+L or Kin$1+\mathrm{DAL})$ resulted in a $50 \%$ loss of velocity in single-molecule assays. In contrast, the velocities of their Kin-2 constructs were - similar to our results - not affected by modifications of the neck linker. The only exception was the Kin-2 $\Delta$ DAL, which is not processive and reaches again only about $70 \%$ of the velocity of other Kin-2 constructs in MMGA. In a further study [Shastry11], the same group confirmed earlier results, extended the results to Kinesin-3 and Kinesin-7 motors, and also 

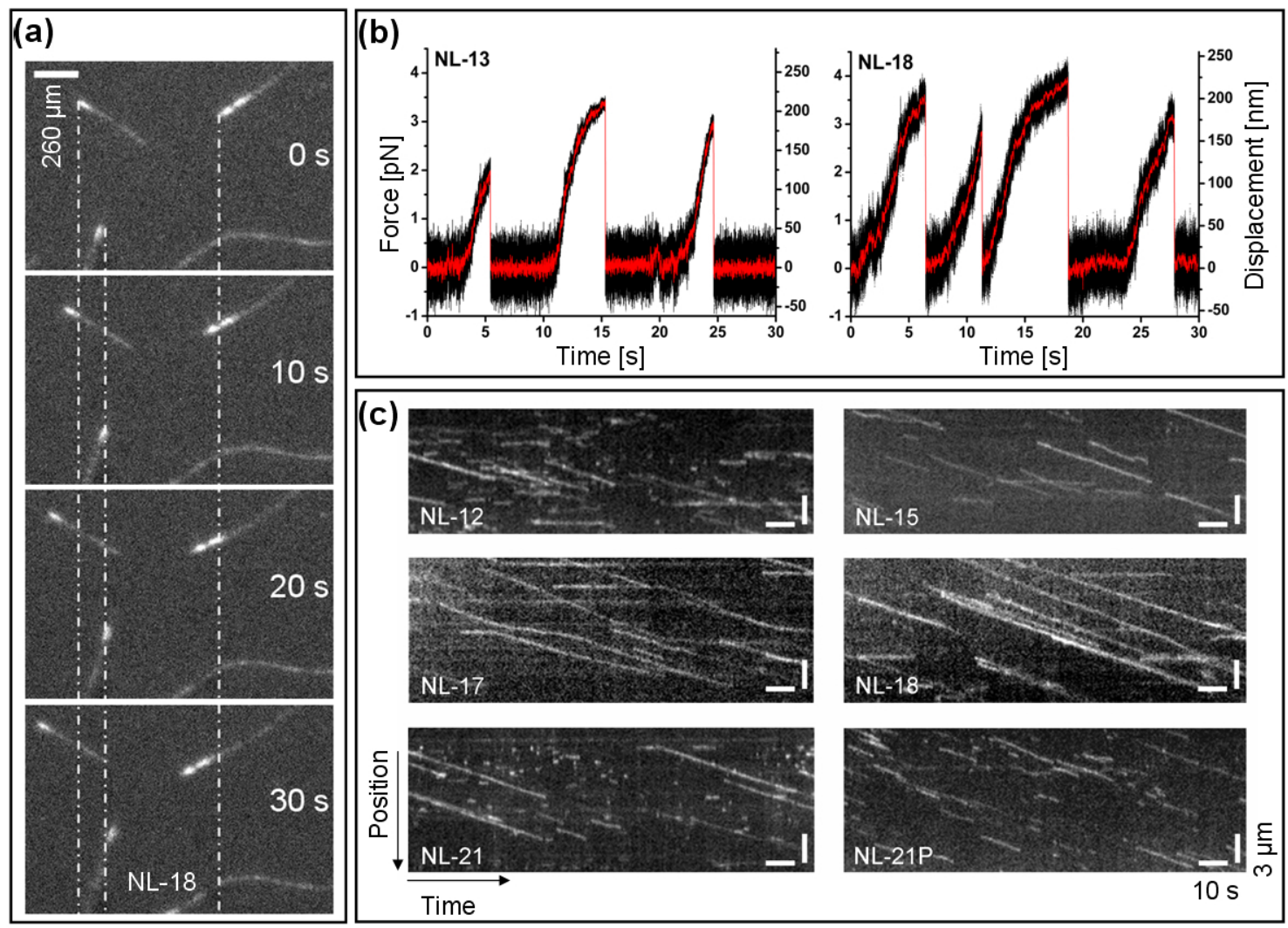

Fig. 5.2: Representative results from single-molecule fluorescence, multi-motor gliding, and optical trapping assays. (a) Time series of a multi-motor gliding assay. The scale bar represents $260 \mu \mathrm{m}$. (b) Bead-displacement time series from the optical trapping assay, showing multiple excursions from the trap centre, ending with detachment without stalling. Initial slope of the excursions is used as an estimate of the unloaded motor velocity (raw signal in black; median-filtered signal in red). (c) Kymographs of single GFP-labelled motor dimers moving on surface-attached microtubules. The number on the lower-left corner indicates the neck-linker length of a given construct. Slope and length of the traces correspond to motor velocity and run length, respectively. Scale bars represent (horizontal) $10 \mathrm{~s}$ and (vertical) $3 \mu \mathrm{m}$.

investigated chimeric Kinesin-5 construct very similar to ours with neck linkers of 14, 15, 17, and 18 amino acids. In contrast to our results, they found diminished processivity for 15 amino acids and no more processivity for 17 and 18 amino acids. Clancy et al. found a decrease in unloaded velocity by about a factor of 2 for a Kinesin-1 construct with a neck linker that is 20 amino acids long, providing evidence that this is due to a decreased motor efficiency leading to back-stepping [Clancy11]. This is an effect that might occur only for extremely long neck-linker lengths, and its possible onset might be masked in our data for the longest constructs NL-20 and NL-21 by statistical errors.

In another study, Yildiz et al. observed a decrease in the single-molecule velocity that was inversely related to the increase in the calculated total length of the neck linker, extended by a poly-proline insert [Yildiz08]. The ATP turnover rate $k_{c a t, A T P}$ was unchanged by the neck-linker extension, and the authors therefore concluded that the coupling between stepping and ATP hydrolysis must have become less 
tight, likely due to an increase in flexibility of the mechanical linkage between the two heads. Velocities of extended kinesins could in part be "rescued" by applying assisting loads. The fact that, in our assays, there was no detectable change or trend in the observed velocities of our constructs suggests that neck-linker length does not influence the rate at which the Kinesin-5 motor domains hydrolyze ATP or the coupling efficiency during their interaction with the microtubule.

Next, we analyzed the maximal forces our motor constructs could develop. In single-bead optical trapping experiments, all our neck-linker constructs displayed stereotypical Kinesin-5 detachment behavior: in contrast to Kinesin-1 motors, detachment occurred at moderate forces of about $3 \mathrm{pN}$, before stall and without much slowing down (Fig. 5.2 b and Supplementary Fig. C.3) [Valentine06, Valentine09, Korneev07]. The individual step size of the different constructs was largely unaffected by the variation of neck-linker length (Supplementary Fig. C.3). All processive neck-linker constructs reached about the same detachment force in the trap of $2.8 \pm 0.1 \mathrm{pN}$, averaged separately for each construct (Fig. $5.3 \mathrm{c}$ and Tab. 5.1). To obtain a detachment force for the non-processive NL-09, we made use of the fact that the motor was still able to bind to a microtubule: motor-coated, optically trapped beads were dragged along a microtubule by moving the microscope stage in a sinusoidal fashion (Fig. $5.3 \mathrm{~d}$ ). Upon binding of the motor, the bead stayed at one position relative to the microtubule and was moved out of the trap by the stage movement. This led to an increase of load on the motor, which eventually resulted in motor unbinding [Uemura02]. Yildiz et al. reported, for their extended Kinesin-1 constructs, a significantly lowered stall force as compared to WT Kinesin-1 [Yildiz08]. Stalling at zero velocity for Kinesin-1 motors and load-dependent detachment for Kinesin-5 motors at finite velocity may reflect somewhat different molecular mechanisms, and effects of neck-linker extension on these processes may not be strictly comparable. Nevertheless, our observations suggest that, for our Kinesin5 constructs, the neck-linker length - and thereby presumably head-head tension - is not controlling force generation and detachment behavior. In contrast to our Kinesin-5 results, keeping in mind the caveat mentioned, Clancy et al. measured stall forces with their extended Kinesin-1 constructs lower than those with WT controls but could show that this was due to increasing backward stepping [Clancy11]. We see no clear evidence of back-stepping with our constructs. This might indicate that the loss of unidirectional motility under hindering loads only occurs at neck linker lengths above 20 or more amino acids, possibly depending on the particular kinesin studied.

Last, we analyzed the run lengths of the constructs as a direct measure of processivity. Using single-molecule fluorescence assays, we observed unidirectional and uninterrupted processive motility with all constructs except NL-09 (Fig. $5.2 \mathrm{c}$ and Supplementary Fig. C.2). Kymograph analysis of the run lengths of all constructs showed that, for the constructs NL-12 up to NL-21, the processive run length decreased continuously toward both short and long neck-linker lengths and exhibited a maximum of about $3 \mu \mathrm{m}$ around NL-17 and NL-18 (Fig. $5.3 \mathrm{~b}$ and Tab. 5.1). There was no significant difference in run lengths between the constructs NL-21 and NL-21P. 
(a)

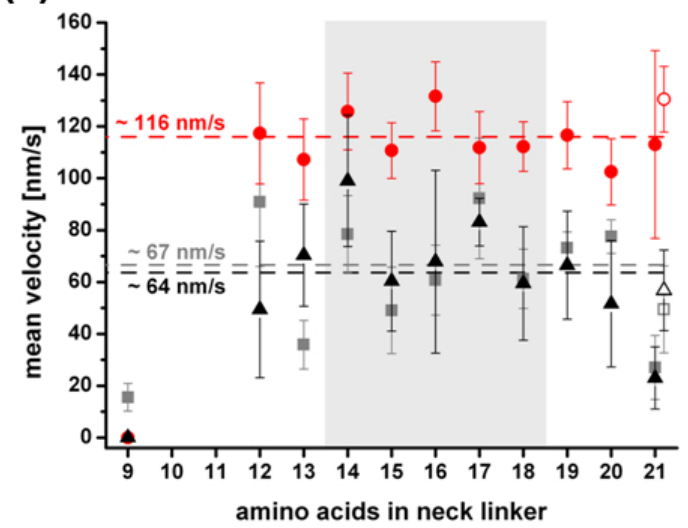

(c)

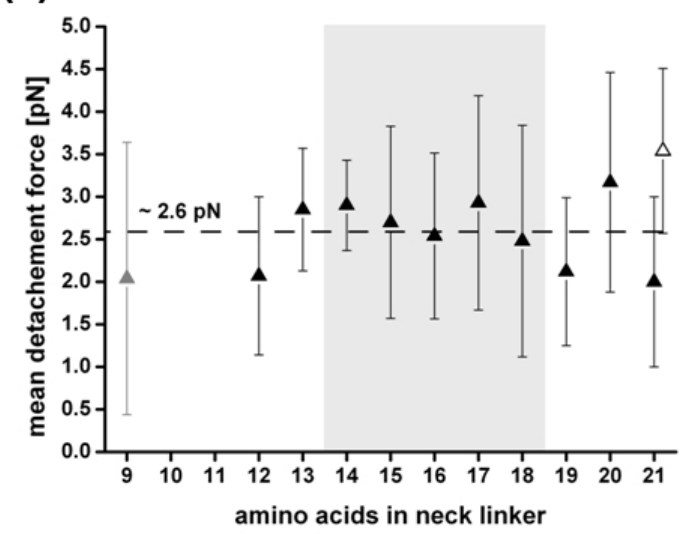

(b)

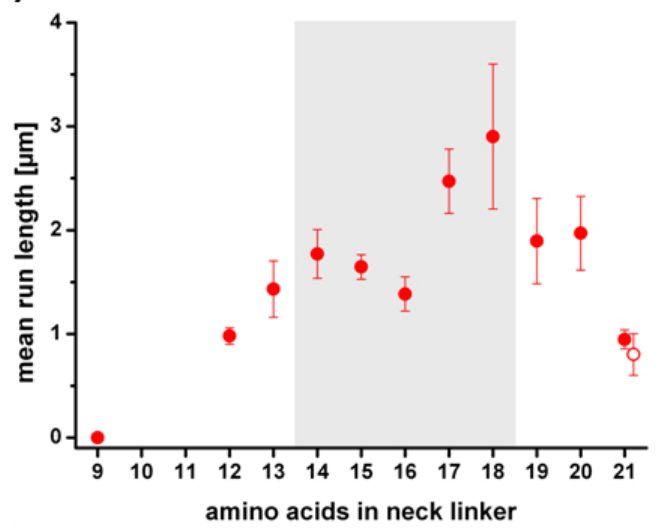

(d)

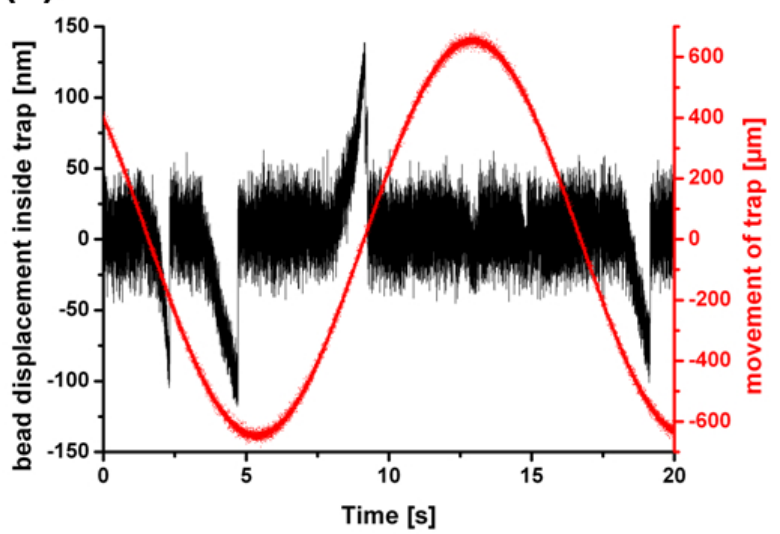

Fig. 5.3: Velocities, run lengths, and detachment forces (also see Tab. 5.1). (a) Mean velocities for all constructs. Triangles: optical trapping assays. Circles: single-molecule fluorescence assays. Squares: multi-motor gliding assays. Broken lines represent the average of values for processive motors and are given to guide the eye. (b) Mean processive run lengths for all constructs in single-molecule fluorescence assays. For NL-09, no processive motility was detected. (c) Detachment forces for all constructs. Since the constructs detached mostly without stalling, the mean force immediately before release is scored. (d) The force given for the non-processive NL-09 construct is the detachment force obtained by dragging a motor-coated bead in a sinusoidal movement (frequency $=0.06 \mathrm{~Hz}$; amplitude $=660 \mathrm{~nm}$ ) along a microtubule. The range between the natural neck-linker lengths of Kinesin-1 and Kinesin-5 (14 - 18 amino acids) is indicated using gray shading. Open symbols refer to NL-21P.

To control against multimerization of the motors, which could strongly affect run lengths, we performed single-molecule fluorescence assays with high enough laser power to cause rapid bleaching. We could confirm two-step bleaching in the intensity signals confirming the dimeric structure of the constructs (Supplementary Fig. C.4). To avoid any possible variations in conditions between the measurements of different constructs, we kept temperature, buffes, microtubule preparations, and illumination conditions as similar as possible between experiments.

The first surprising finding was that NL-12 and NL-13 displayed processive behavior, virtually indistinguishable from that of NL-14 (Fig. 5.3 b). In the study of 
Shastry and Hancock, two sets of constructs showed no processivity when having neck linkers with less than 14 amino acids, presumably because the tethered head was not able to reach the next binding site [Shastry10]. This was thought to be a general mechanism consistent with the fact that 14 amino acids is the length of the shortest neck linker in any known processive kinesin. Our results show that even shorter neck linkers can support processivity. This finding is consistent with and goes beyond the recently reported processivity of Kinesin-2/Kinesin-3 and Kinesin-7 constructs that showed processivity with neck linkers of 13 amino acids [Shastry11]. A construct with the further shortened neck linker, NL-09, was finally lacking processivity (Supplementary Fig. C.2). The second unexpected finding was that upon addition of one or two natural amino acids in constructs NL-15 and NL-16, there was no drop in processivity compared to NL-14 (Fig. $5.3 \mathrm{~b}$ ), as one might have expected based on the findings for Kinesin-1/Kinesin-2/Kinesin-3/Kinesin-5 and Kinesin-7 constructs by Shastry and Hancock [Shastry10, Shastry11]. Our observation suggests that neck-linker length alone might not be sufficient to predict processivity. Our finding that the neck-linker constructs approaching the native Kinesin-5 necklinker length, NL-17 and NL-18, displayed a significantly higher processivity than shorter or longer constructs (Fig. $5.3 \mathrm{~b}$ ) is further evidence for the same point.

Last, we also found processive motility when extending the neck linker beyond the natural 18 amino acids of Eg5. Run length, however, decreased from NL-19 to NL-21 (and NL-21P) below the maximal run length of NL-18 (Fig. $5.3 \mathrm{~b}$ ), as one expects to eventually occur due to diminishing communication between the two heads. An increase in run length of constructs carrying neck linkers longer than 14 amino acids was also seen by Clancy et al. [Clancy11].

Given the new experimental evidence that neck-linker extension can increase processivity, it remains to discuss the consequences for the gating models for Eg5 in particular or possibly for all kinesins. First of all, one has to keep in mind that, independent of head-head communication, interactions between the heads and the microtubule can also affect processivity. Electrostatic interactions, especially under low-ionic-strength conditions, can keep the heads loosely associated with the microtubule, even in the absence of efficient communication. In particular, addition of positive charge in the neck linker was suggested to compensate for a loss of processivity through interactions much similar to the ones discussed for the positively charged neck with the negatively charged E-hook of tubulin [Thorn00, Skiniotis04, Lakämper05]. The native Kinesin-5 neck-linker sequence contains two positively charged lysine residues at positions 15 and 16 of the neck linker. These lysines might compensate for a loss of processivity otherwise observed upon extension of the neck linker. Three points, apart from the relatively high-ionicstrength conditions, argue against this notion. First, the addition of a second lysine should have an additive effect, which we did not observe. Second, only NL-17 and NL-18 showed a marked increase in run length, and it seems unlikely that the charge of the lysine residues needs these further extensions to take effect. Third, loosely bound periods during processive runs should also make the average velocity during events strongly load dependent, which we did not observe. Therefore, we think that neck-linker/microtubule interactions are not causing the variations in run lengths in our experiments. 


\begin{tabular}{lccc|cccc|cc} 
& \multicolumn{5}{c|}{ Trap } & \multicolumn{5}{c|}{ TIRF } & \multicolumn{2}{c}{ MMGA } \\
\cline { 2 - 10 } Construct & $\begin{array}{c}\text { Velocity } \\
(\mathrm{nm} / \mathrm{s})\end{array}$ & $\begin{array}{c}\text { Force } \\
(\mathrm{pN})\end{array}$ & $\mathrm{N}$ & $\begin{array}{c}\text { Velocity } \\
(\mathrm{nm} / \mathrm{s})\end{array}$ & $\begin{array}{c}\text { Run length } \\
(\mu \mathrm{m})\end{array}$ & $\begin{array}{c}\mathrm{N}) \\
\mathrm{C}_{\text {TIRF }} \\
(\mathrm{nM})\end{array}$ & $\begin{array}{c}\text { Velocity } \\
(\mathrm{nm} / \mathrm{s})\end{array}$ \\
\hline \hline NL-09 & $0 \pm 0^{a}$ & $2.0 \pm 1.6$ & 33 & $0 \pm 0^{a}$ & $0 \pm 0^{a}$ & 80 & 178 & $16 \pm 6$ & 41 \\
NL-12 & $70 \pm 20$ & $2.1 \pm 0.9$ & 109 & $117 \pm 20$ & $0.9 \pm 0.1$ & 104 & 157 & $19 \pm 25$ & 37 \\
NL-13 & $99 \pm 25$ & $2.9 \pm 0.7$ & 100 & $107 \pm 16$ & $1.4 \pm 0.3$ & 102 & 105 & $36 \pm 9$ & 29 \\
NL-14 & $60 \pm 19$ & $2.9 \pm 0.5$ & 50 & $125 \pm 15$ & $1.8 \pm 0.2$ & 124 & 131 & $78 \pm 15$ & 28 \\
NL-15 & $68 \pm 35$ & $2.7 \pm 1.1$ & 98 & $111 \pm 11$ & $1.7 \pm 0.1$ & 130 & 52 & $49 \pm 17$ & 20 \\
NL-16 & $83 \pm 9$ & $2.5 \pm 0.9$ & 35 & $132 \pm 13$ & $1.4 \pm 0.2$ & 269 & 189 & $61 \pm 14$ & 17 \\
NL-17 & $60 \pm 22$ & $2.9 \pm 1.3$ & 63 & $112 \pm 14$ & $2.5 \pm 0.3$ & 162 & 53 & $92 \pm 23$ & 23 \\
NL-18 & $59 \pm 22$ & $2.5 \pm 1.4$ & 68 & $112 \pm 10$ & $2.9 \pm 0.7$ & 145 & 316 & $61 \pm 11$ & 20 \\
NL-19 & $66 \pm 21$ & $2.1 \pm 0.9$ & 321 & $117 \pm 13$ & $1.8 \pm 0.4$ & 106 & 158 & $37 \pm 6$ & 34 \\
NL-20 & $52 \pm 24$ & $3.2 \pm 1.3$ & 109 & $103 \pm 13$ & $1.9 \pm 0.3$ & 98 & 132 & $77 \pm 7$ & 30 \\
NL-21 & $23 \pm 12$ & $2.0 \pm 1.0$ & 67 & $113 \pm 36$ & $0.8 \pm 0.2$ & 106 & 317 & $27 \pm 13$ & 32 \\
NL-21P & $57 \pm 16$ & $3.5 \pm 1.0$ & 134 & $131 \pm 13$ & $0.9 \pm 0.1$ & 128 & 95 & $49 \pm 18$ & 39 \\
\hline
\end{tabular}

Tab. 5.1: Results for the three types of measurements for all neck-linker constructs Trap, data from optical trapping assays; TIRF, data from single-molecule fluorescence assay; $\mathrm{C}_{\text {TIRF }}$, protein concentration in single-molecule fluorescence measurement; $\mathrm{N}$, number of beads tracked, single motors tracked, and microtubules tracked, respectively. ${ }^{a}$ No processive motility detected.

Two physically different gating scenarios seem possible. Either (i) neck-linker orientation is the deciding parameter, and inter-head tension is not important for the processivity, or (ii) each kinesin family has a particularly adapted neck-linker length and/or specific interactions between the native neck linker and the rest of the motor that together optimize the transmission of tension and the gating and stepping mechanism. Gating scenario (ii) seemed, on the one hand, to be supported by the finding that replacement and addition of single amino acids in several kinesins alter processivity [Shastry10, Shastry11]. We did not see such strong effects. Furthermore, a comparison between motors with different extension sequences but equal length, NL-21 and NL-21P, indicates that it is unlikely that the particular sequence of the extension played a dominating role in determining processive run lengths.

Gating scenario (i), on the other hand, is supported by Clancy et al., who found an increase of processivity with strongly extended neck linkers for Kinesin-1 constructs [Clancy11]. In that case, the result was interpreted to be caused by a twohead-bound waiting state with a smaller probability of total release. Since this mechanism also entails lower speed, it is likely that this was not exactly the case for our intermediate-length Kinesin-5 constructs, though. In essence, however, headhead communication via neck-linker orientation and not tension is consistent with our results. Interestingly, two studies reporting a structurally unusual neck-linker 
orientation [Turner01] and two-state neck-linker orientation kinetics [Rosenfeld05] further argue for this possibility, particularly for Kinesin-5.

In summary, a simple mechanistic model generally applying to all processive kinesins - equating a shorter neck-linker length directly to more tension and to more efficient gating and thereby to more processivity with one universal optimal number for the neck-linker length - might not exist. In view of the broad variations in function, regulation, and oligomerization properties of the kinesins, it would be indeed surprising if a single parameter, namely, neck-linker length, could determine performance. Coordination between the heads or gating, however, is a necessity for processive kinesins. Some of the existing data, including ours, now point to the interesting possibility that the orientation of the neck linker plays a crucial role, rather than merely length and tension. This opens up the option that different kinesins could achieve optimal head-head communication with different neck-linker lengths. The native neck-linker length and sequence might be optimized, in each case, for both effective communication and processivity, as well as other specific functions such as regulation.

\section{\begin{tabular}{l|l} 
Materials and Methods & 5.4
\end{tabular}

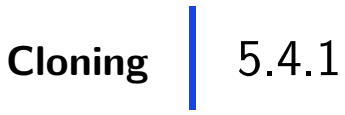

All constructs were based on the previously published pEG5Kin-GFPhis construct [Lakämper10]. Deletions or insertions of codons were performed in a two-step PCR approach. Using a complementary pair of modified primers in the neck-linker region, we created two PCR products: one of the motor domain up to the desired mutation and a second starting with the neck-linker mutation ending with the C-terminal GFP (green fluorescent protein). Both fragments were combined to serve as template for a second round of PCR using the 3'- and 5'-primers flanking the N-terminus and the Cterminus of the full construct. The resulting PCR products were then incorporated into pTOPO using a TOPO Kit (Invitrogen, Carlsbad, CA, USA) for sequencing and amplification before replacing the coding sequence in pEG5Kin-GFPhis by subcloning using the existing restriction sites for NdeI and XmaI as described previously [Lakämper10]. Constructs NL-09, NL-12, NL-19, NL-20, NL-21, and NL-21P were generated by gene synthesis (GeneArt Life Technologies, Regensburg, Germany). Integrity was confirmed by sequencing.

\section{\begin{tabular}{l|l} 
Protein purification & 5.4 .2
\end{tabular}}

Motors were expressed in Escherichia coli and purified essentially as described previously [Lakämper10]. In brief, E. coli BL21(DE3) (Invitrogen) were transformed and grown to a density of $\sim 0.6$ at $37^{\circ} \mathrm{C}$ before induction of expression using $1 \mathrm{mM}$ IPTG for $3 \mathrm{~h}$. Cells were harvested by centrifugation and re-suspended in a $20 \mathrm{mM}$ imidazole buffer, pH 7.4, supplemented with $1 \mathrm{mM}$ each of DTT, $\mathrm{MgCl}_{2}$, ethylene glycol 
bis( $\beta$-aminoethyl ether) N,N'-tetraacetic acid (EGTA), and $\beta$-mercaptoethanol and $150 \mathrm{mM} \mathrm{NaCl}$ before lysis using ultrasound in the presence of lysozyme and DNase I. After separation from cell debris by centrifugation, the cytosol was incubated for 1 $\mathrm{h}$ at $4{ }^{\circ} \mathrm{C}$ with Ni-NTA column material (Qiagen, Hilden, Germany) and then transferred to a syringe column. After washing the column with $80 \mathrm{mM}$ imidazole and 300 $\mathrm{mM} \mathrm{NaCl}$, bound motor was eluted using a $300-\mathrm{mM}$ imidazole buffer containing 10 $\mu \mathrm{m}$ ATP and $1 \mathrm{mM}$ DTT. Fractions containing motor were pooled and $3 \times$ dialyzed

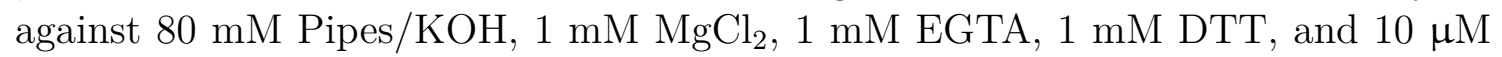
ATP. In addition, motors were also purified using a microtubule-affinity purification procedure described in [Lakämper05]. Protein samples were tested for motor activity and stored in aliquots at $-80^{\circ} \mathrm{C}$. Tubulin was purified from pig brain, and microtubules were polymerized and labeled as described in [Lakämper03, Lakämper05]. All chemicals were obtained from Sigma, Germany, if not specified otherwise.

\section{Multi-motor surface-gliding assays}

MMGAs were performed at $22^{\circ} \mathrm{C}$, essentially as described previously [Lakämper05]. Motor-protein constructs were allowed to nonspecifically bind to the glass surfaces of assay chambers made from coverslips and plasma-cleaned microscope slides assembled with double-stick tape. Subsequently, the chambers were flushed with about three chamber volumes of assay mix in BRB80 buffer $[80 \mathrm{mM}$ Pipes/KOH (pH 6.8), $1 \mathrm{mM} \mathrm{MgCl}_{2}$, and $1 \mathrm{mM}$ EGTA] containing $10 \mu \mathrm{m}$ taxol (paclitaxel), 0.022 $\mathrm{mg} / \mathrm{ml}$ tetramethylrhodamine-labeled microtubules, $2 \mathrm{mM}$ ATP, $4 \mathrm{mM} \mathrm{MgCl} 2,10$ $\mathrm{mM}$ DTT, $0.08 \mathrm{mg} / \mathrm{ml}$ catalase C40, $0.1 \mathrm{mg} / \mathrm{ml}$ glucose oxidase, and $10 \mathrm{mM}$ glucose. Motility was observed in a standard inverted fluorescence microscope (Zeiss Axiovert 200, Göttingen, Germany). Images were recorded with a digital chargecoupled device camera (CoolSnap ES; Roper Scientific Germany).

\section{Silanized assay chambers and single-molecule fluorescence assays}

Coverslips were cleaned using a plasma cleaner (Harrick Plasma, Ithaca, NY, USA) before silanization with a positively charged silane, 3-[2-(2-aminoethylamino)-ethylamino]propyl-trimethoxysilane (Sigma), for microtubule immobilization. Assay chambers were made from 3-[2-(2-aminoethylamino)-ethylamino]propyl-trimethoxysilane coverslips and microscope slides assembled with double-stick tape. Tetramethylrhodamine-labeled microtubules diluted in BRB80 buffer containing $10 \mu \mathrm{m}$ taxol (paclitaxel) were allowed to bind for $5 \mathrm{~min}$ to the silanized glass surfaces of the assay chamber. This was followed by $5 \mathrm{~min}$ of incubation with $0.1 \mathrm{mg} / \mathrm{ml}$ casein in BRB80. The chamber then was flushed with $7-10 \mu$ of assay buffer (BRB80 containing $2 \mathrm{mM}$ ATP and $4 \mathrm{mM} \mathrm{MgCl}_{2}$ and an oxygen scavenging system based on $10 \mathrm{mM}$ DTT, $0.08 \mathrm{mg} / \mathrm{ml}$ catalase C40, $0.1 \mathrm{mg} / \mathrm{ml}$ glucose oxidase, and $10 \mathrm{mM}$ glucose) containing GFP-tagged motor proteins at appropriate single-molecule concentrations (Tab. 5.1). Fluorescence was observed in a custom-built total internal reflection fluorescence (TIRF) microscope described previously [Gerson-Gurwitz11]. 
Digital video recordings (two frames per second) were analyzed for motor speeds and run lengths using kymographs generated with custom-written LabView software. Statistical analysis of the data was performed with OriginPro (OriginLab Corporation, Northampton, MA, USA). All measurements were performed at 22 ${ }^{\circ} \mathrm{C}$.

\section{Optical trapping assays $\quad 5.4 .5$}

We diluted 1- $\mu$ m-diameter plain silica beads (Kisker, Steinfurt, Germany) in BRB80 to a concentration of $0.5 \mathrm{mg} / \mathrm{ml}$ and incubated them for 30 min with $50 \mu \mathrm{g}$ proteinG (Sigma). After centrifugation and re-suspension, the beads were incubated with $1 \mu \mathrm{g}$ penta-his antibody (Sigma) and $0.02 \mathrm{mg}$ casein. After $30 \mathrm{~min}$, the beads were centrifuged and re-suspended to the desired concentration. Diluted his-tagged motor protein was then added to the beads and allowed to bind for $15 \mathrm{~min}$. The motor dilution was chosen so that only about half of the beads showed activity in the assay. This makes it most likely that only one motor on the beads is able to bind to a microtubule at any time [Svoboda93, Skiniotis04]. The motor-coated beads were added to the assay buffer, which was then filled into an assay chamber. Beads were trapped using a focused 4-W 1064-nm NdYVO laser (Coherent Germany) in a custom-built single-beam optical trap described elsewhere [Lakämper05]. After positioning a bead above a microtubule, the interaction between the motor and the microtubule could be monitored. The motion of the bead was measured by back-focal-plane interferometry [Turner01, Lakämper05]. Data were acquired with custom-written LabView software. Further analysis and statistical calculations were performed with MatLab (The Mathworks) and OriginPro (OriginLab Corporation). All measurements were performed at $22{ }^{\circ} \mathrm{C}$.

\section{Acknowledgements $\mid 5.5$}

This work was supported by Lower Saxony Grant No. 11-76251-99-26/08 (ZN2440) and by the Deutsche Forschungsgemeinschaft through the Research Center for Molecular Physiology of the Brain and the Collaborative Research Center SFB 803. We thank Stefanie Kramer and Angelina Schuder for technical support and Kerstin von Roden, Charlotte Willms, and Dieter R. Klopfenstein for help with the protein expression and purification. Kymograph analysis was performed using a LabView program written by Joost van Mameren.

\section{Author Contributions}

SL conceived the project, cloned, and purified some of the motors. SL and CFS designed and supervised experiments, and SL, CFS, CT, and AD wrote the manuscript. CT performed single-molecule fluorescence assays and surface-gliding assays, and AD performed optical trapping experiments. AD and CT analyzed data and created figures. 


\section{Supplementary data}

Supplementary data to this article can be found online at the Journal of Molecular Biology, doi:10.1016/j.jmb.2012.06.043 and in appendix C. 


\title{
A chimeric kinesin-1/kinesin-5 microtubule-sliding motor switches between diffusive and processive motility
}

The content of this chapter was submitted 2012 to the Biophysical Journal. I share the first authorship with Stefan Lakämper. Co-authors are Alok D. Wessel, Stefanie Kramer and Christoph F. Schmidt.

In this work I performed the multi-motor surface-gliding assays and the singlemolecule fluorescence assays. Further I analysed the respective data, contributed the figures and worked on the text of the manuscript.

\begin{abstract}
\begin{tabular}{l|l} 
Abstract & 6.1
\end{tabular}
Homotetrameric bipolar Kinesin-5 motors, such as Eg5 from Xenopus laevis or Cin8 from Saccharomyces cerevisiae are essential for the assembly of the mitotic spindle and for chromosome separation. These kinesins are able to bind between two microtubules (MTs) simultaneously and slide them apart, towards the spindle poles in mitosis. The localization and activity of Kinesin- 5 motors in the spindle must be tightly controlled for the proper function of cell division. In in-vitro assays, single Eg5 molecules move in a mix of diffusive and directed modes on single MTs and switch to a directed mode between MTs. How allosteric communication between opposing motor domains functions remains unclear, but the highly conserved Kinesin-5 tail domains might be involved.

Here we present a single-molecule fluorescence in-vitro study of a tetrameric Kinesin1 head/Kinesin-5 tail chimera, DK4mer. This motor exhibits fast processive motility on single MTs, interrupted by diffusive pauses. Similar to Eg5, DK4mer diffuses along MTs in the absence of ATP. It is also able to slide antiparallel MTs apart. The clear distinction between diffusive and processive periods made it possible to measure transition rates as a function of buffer ionic strength. This together with controls using tail-less dimers indicates that the modes of motility reflect two independent and mutually exclusive modes of interaction with MTs which are likely to be relevant for the regulation of native Kinesin-5 motors as well.
\end{abstract}

\section{\begin{tabular}{l|l} 
& \\
Introduction & 6.2
\end{tabular}}

Microtubules are the functionally most versatile elements of the cytoskeleton. Their complex assembly and disassembly dynamics are an integral part of cellular machineries such as the mitotic spindle [Hoyt96, Scholey03]. MTs in functional struc- 
tures such as cilia, flagella or the mitotic spindle are organized by a large variety of MT-binding proteins [Rosenbaum02, Snow04]. An important class of MTbinding proteins are motor proteins of the kinesin and dynein families [Sharp00]. Some of these motors drive cargo transport along the MTs, for example along axons, others serve to dynamically organize MT superstructures. Motor proteins are typically allosterically regulated on several levels to only consume ATP and produce force and motion when needed. First of all, ATPase activity is strongly suppressed when kinesins are not bound to MTs [Hackney88]. In addition, several kinesins have been found to be cargo activated [Coy99a, Friedman99, Seiler00]. In dimeric Kinesin-1 motors, backfolding of the tail and interaction with the neck region was shown to suppress ATPase activity [Verhey98, Coy99a, Hackney00]. In the homotetrameric Kinesin-5 family member Eg5 from Xenopus laevis, a modified version of cargo activation was found. Kinesin-5 motors are generally plus-end directed, they bind between two MTs and can slide MTs relatively to each other [Kapitein05]. In mitosis they act in the midzone of the spindle to exert poleward force [Sawin92a, Miyamoto04]. In-vitro experiments demonstrated that Eg5 motors are only activated when bound between two MTs [Kapitein08] and that the activation in physiological buffer conditions requires the presence and interplay of a total of eight MT-binding sites, including the four head-MT interactions and in addition the non-ATP-dependent interactions between the four C-terminal tails of the tetramer and the MTs [Weinger11]. The Saccharomyces cerevisiae Kinesin-5 Cin8 adds a further feature to the regulation theme: It was shown to reverse directionality from minus-end directed on single MTs to plus-end directed when bound between two MTs [Gerson-Gurwitz11]. The plus-end motility of Eg5 on single MTs at low-salt conditions differs distinctly from Kinesin-1 motility. It consists of short runs that occasionally feature back-and-forth diffusion [Kapitein08]. It was shown in single-molecule fluorescence assays that ATP-dependent processive motility alternates with diffusive motility not requiring ATP hydrolysis [Kwok06]. The diffusive mode of Eg5 is believed to be maintained by the second binding site in the Eg5 tail domain [Wildenberg08, Weinger11, Sharp99]. The complex motility and regulation of Kinesin-5 motor proteins with their multiple MT-binding sites and their internal interactions remain, at this point, far from understood.

One approach to gain further insight into the function of Kinesin-5 motors is to create truncated constructs, lacking critical parts or forming only dimers [Valentine06, Weinger11, Gerson-Gurwitz11]. A different approach is to generate chimeras by fusing parts from well-studied motors of other families to parts of Kinesin-5 motors. Here we have characterized a model motor in which we replaced the motor domain and the neck linker of Eg5 with the homologous parts of Drosophila melanogaster Kinesin-1, naming the resulting tetrameric chimera DK4 mer. The rationale was to create a fast tetrameric MT-sliding motor without the Kinesin-5 tail-head regulation, but with the additional tail binding sites and the overall bipolar Kinesin-5 structure. In a series of in-vitro assays we studied the chimera's motility at different ionic strengths and ATP concentrations as well as its capability to slide MTs relative to each other. The model motor DK4mer showed fast and highly processive motility as expected from the Kinesin-1 heads, and robust MT 
crosslinking and sliding as expected from the tetrameric dumbbell geometry. The intriguing result was that DK4mer performed extraordinarily long runs and switched in a mutually inhibitory manner between two modes of interaction with the MT, a processive and a diffusive one. This further illuminates the regulatory mechanisms in tetrameric kinesins.

\section{\begin{tabular}{l|l} 
Results and Discussion & 6.3
\end{tabular}}

The tetrameric Kinesin-1/Kinesin-5 chimera (DK4mer) we cloned was intended to combine fast robust motility with the capability to crosslink and slide MTs. Motor domain and neck linker of the fast and processive DmKHC should guarantee the former, the stalk and tail of Eg5 the latter (Fig. 6.1 C). The tail domains of Eg5 contain additional MT-binding sites. The fast motility of the Kinesin-1 heads in the model construct should make it possible to clearly distinguish processive motile periods from tail-mediated diffusive binding periods.

\section{DK4mer in surface-gliding and single-molecule fluorescence assays}

In order to characterize the DK4mer motors, we performed a set of in-vitro motility assays. In standard multi-motor surface-gliding assays we first confirmed that DK4mer is a competent MT motor. In assay mix (AM) based on BRB80 buffer, a gliding velocity of $\sim 550 \mathrm{~nm} / \mathrm{s}$ was observed, both with and without a C-terminal GFP tag (Supplementary Fig. D.1 A and B). This velocity, at saturating ATP concentration, was $\sim 40 \%$ lower than that produced by Kinesin-1 DmKHC (Supplementary Fig. D.1 A and B), but 10- to 20-fold higher than that produced by Eg5 in similar conditions [Kapitein05]. To control if the ATP affinity of DK4mer differs from that of DmKHC, although both motor proteins share the same motor domain, we performed surface-gliding assays with DK4mer and Kinesin-1 under exactly the same assay conditions at varying ATP concentrations (Supplementary Fig. D.1 C). For both motors, the ATP dependence of the gliding velocity was well fit by a Michaelis-Menten equation, resulting in reaction constants of $\mathrm{K}_{M}=0.056 \mathrm{mM}$ for DK4mer-GFP and $\mathrm{K}_{M}=0.061 \mathrm{mM}$ for DmKHC. The results suggest that the affinity of the Kinesin-1 motor domain for ATP is unchanged in the context of the DK4mer-GFP chimera (For simplicity termed DK4mer in the following). As both DK4mer and DmKHC feature the same motor domain with unchanged ATP affinity the discrepancy in velocity might be caused by the differing structures outside of the motor domain, e.g. the neck linker or the Eg5 tail domain present in DK4mer. The difference in velocities might also be caused by the fact that, in multi-motor surface-gliding assays, the observed MT-gliding velocity is produced by an ensemble of motors that might include damaged molecules and that comprises motors that are bound to the surface in different geometries.

In multi-motor surface-gliding assays, non-processive motor proteins also produce motility. It is therefore not simple to test for processivity of individual motors in this assay. In order to determine if DK4mer chimeras are processive, we next per- 

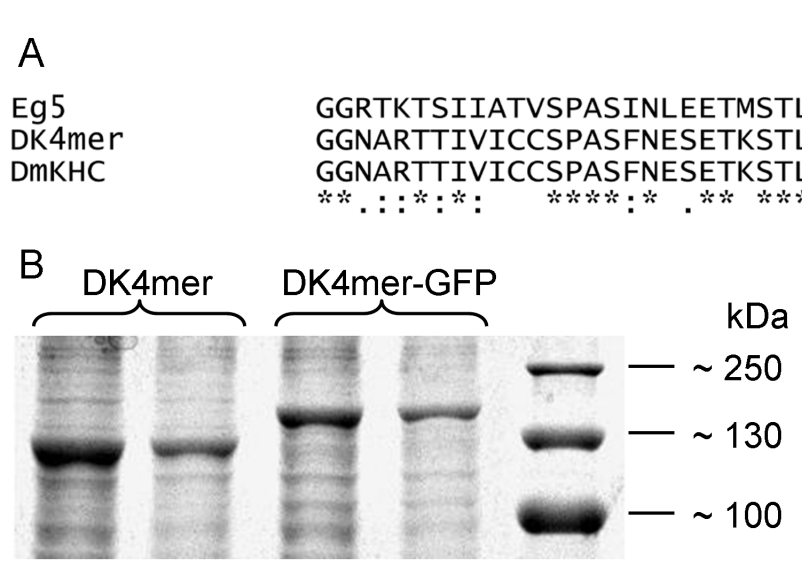

355

Eg5/DK4mer neck-linker |

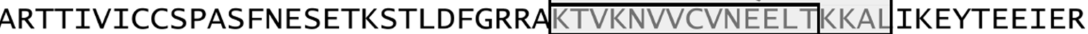
GGNARTTIVICCSPASFNESETKSTLDFGRRA KTVKNVVCVNEELTAEEWKRRYEKEKEK

DK4mer

$\mathrm{B}$
330

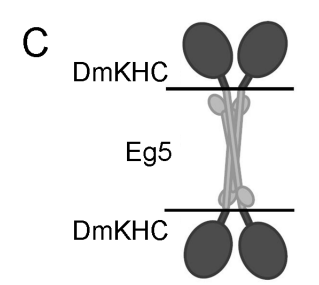

Fig. 6.1: Construction and purification of the tetrameric chimera DK4mer: (A) Details of the junction between the Xenopus laevis Eg5 neck coiled-coil and the Drosophila melanogaster DmKHC motor domain: The numbering refers to the amino acid numbering in the respective $W T$ motor sequences as indicated by grey boxes. (B) SDS-PAGE gel showing DK4mer with $\sim 130 \mathrm{kDa}$ and DK4mer-GFP with $\sim 157 \mathrm{kDa}$. (C) Sketch of the structure of the bipolar homotetrameric chimera DK4mer. (D) Sketch of the structure of a truncated version of DK4mer, the dimeric chimera DK511, lacking the Eg5 tail domains.

formed single-molecule fluorescence assays with DK4mer which was C-terminally tagged with GFP [Lakämper10]. We observed single fluorescent spots interacting with and moving along surface-adsorbed MTs for tens of seconds towards the plusend of the MTs (Fig. 6.2 A and Supplementary Fig. D.2), indicating processive motility of DK4mer. Micrometer-long unidirectional movements were occasionally interrupted by diffusive episodes without a directional bias (Fig. 6.2 A and Fig. 6.3 A). During directed motility phases, single DK4mer molecules moved along a MT at an average velocity of $\sim 500 \mathrm{~nm} / \mathrm{s}$ (Fig. 6.2 B, filled columns), in close agreement with the results from the surface-gliding assays. In order to control against the possibility that we were observing aggregates of multiple motor proteins, we recorded the intensity time course of individual fluorescent spots and subsequently analyzed kymograph traces. This analysis revealed bleaching of moving spots in at most four consecutive steps. This finding strongly suggests that we observed mostly single DK4mer tetramers (Supplementary Fig. D.3), and that these indeed moved processively.

In AM based on BRB80 buffer, single DK4mers traveled processively over micrometer-long distances and tended to pause in a diffusive state before resuming processive travel (Fig. 6.2 A and Fig. 6.3 A). The transitions between runs and pauses were quite distinct. Overall run lengths were remarkably long, in BRB80 buffer on average $\sim 9 \mu \mathrm{m}$ (Fig. 6.4 D and Supplementary Tab. D.1). This behavior is in stark contrast to what was earlier observed with full-length Eg5 [Kwok06], where directed and diffusive motility was difficult to discern, and the average run length was $\sim 600 \mathrm{~nm}$ in conditions equivalent to the $80 \mathrm{mM}$ PIPES buffer we used here. For dimeric Kinesin-1 motors, run lengths typically range between 1 and 3 $\mu \mathrm{m}$ [Howard89, Block90, Thorn00, Shastry11] (also see D421 data in Fig. 6.4 B and 
A

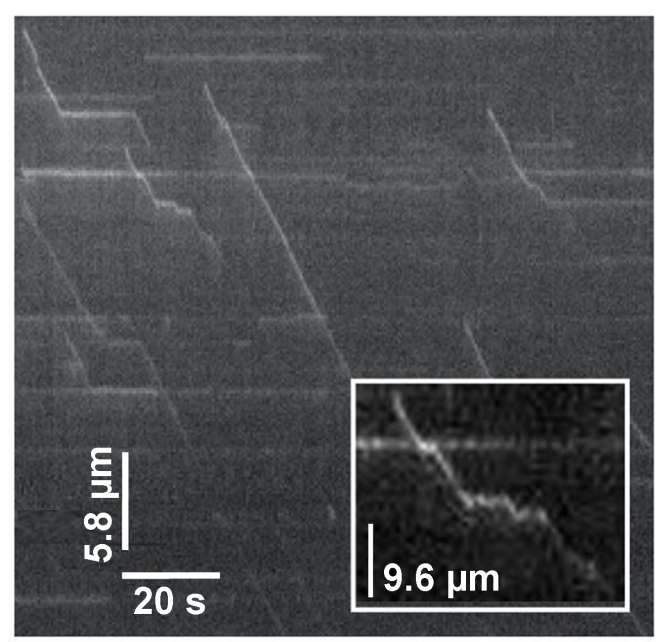

B

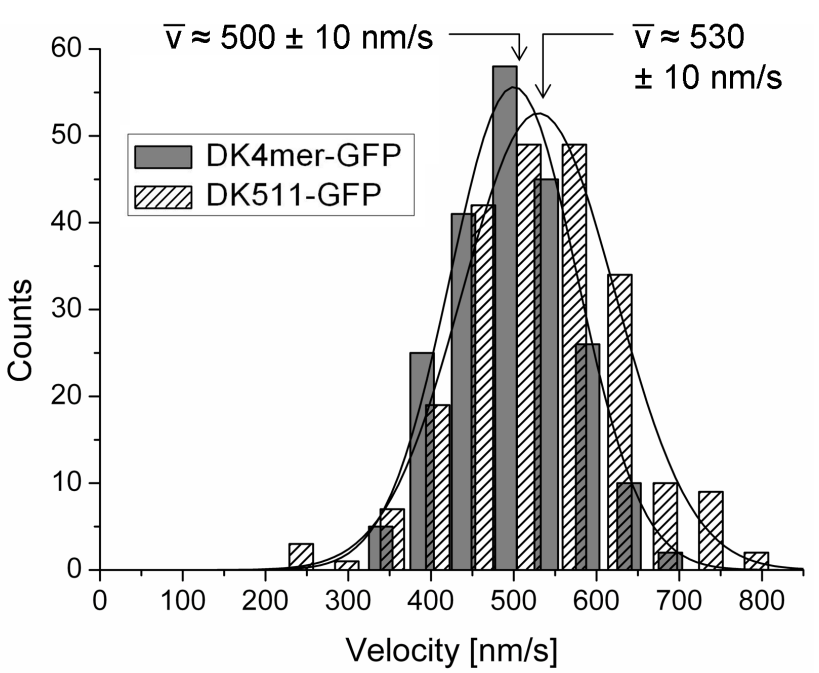

Fig. 6.2: Single-molecule fluorescence assay with $2 \mathrm{mM}$ ATP in BRB 80 buffer: (A) Kymograph of DK4mer motility showing short diffusive and extended processive periods. (B) Velocity distribution of the tetrameric DK4mer-GFP and the dimeric DK511-GFP fitted with Gaussians. Average velocity: $500 \pm 10 \mathrm{~nm} / \mathrm{s}(\mathrm{N}=212)$ for DK4mer and 530 $\pm 10 \mathrm{~nm} / \mathrm{s}(\mathrm{N}=226)$ for DK511. To measure velocities, only straight runs or straight segments of runs were evaluated.

Supplementary Tab. D.1). An earlier study of a dimeric Kinesin-1 tail/Kinesin-5 head chimera (Eg5Kin), lacking the Kinesin-5 tail with its MT binding site, showed a run length of $1.9 \mu \mathrm{m}$ [Lakämper10]. Consistent with that result, we observed a run length of $\sim 1 \mu \mathrm{m}$ for an Eg5 dimer, truncated at aa 511 (data not shown). Valentine et al. [Valentine06] report a run length of only $\sim 68 \mathrm{~nm}$ for a truncated version of human Eg5 (Eg5-513-5His) in an optical trap assay in high-salt buffer. The evidence thus is that bare motor domains of Eg5, at least in intermediate ionic strengths, are capable of producing run lengths similar to Kinesin- 1 motors. The short run lengths of full-length Eg5 motors are known to be caused by tail-mediated cargo regulation which, at physiological ionic strength, completely inhibits the motors unless they are bound between two MTs [Kapitein08]. Lower ionic strength can override the inhibition, and BRB 80 buffer is close to the margin of this effect. The long run length of DK4mer observed here indicates that the Kinesin-1 motor domains are not susceptible to this type of regulation by the Eg 5 tails.

An analysis of the pauses during processive runs showed that the average pause duration remained largely unchanged with increasing ionic strength of the buffer solution (Fig. 6.4 A). We found an average pause time of $3.0 \pm 0.3 \mathrm{~s}$ in low ionic strength (P30 buffer) and $3.9 \pm 0.6 \mathrm{~s}$ in high ionic strength (BRB80 buffer +40 $\mathrm{mM} \mathrm{KCl}$ ), corresponding to a pause exit rate of $0.33 \mathrm{~s}^{-1}$ and $0.26 \mathrm{~s}^{-1}$ respectively. This low exit rate cannot be due to a diffusional search since the motor is kept near the track by tail binding, but rather is evidence for an additional barrier between the tail-bound state and the processive state. In other words, although the tail-MT interaction keeps the motor on the track, tail binding and processive motor activ- 
ity appear to be mutually exclusive. Weinger et al. [Weinger11] showed that the two binding modes show cooperative aspects for full-length Eg5 in MT-MT sliding assays. Taken together, these findings indicate that the tail-head and tail-MT interactions are at the core of the intriguing cargo regulation of Eg5.

When we replaced ATP in AM (based on BRB80) with ADP, purely diffusive interactions were observed (Fig. $6.3 \mathrm{~B}$ ), much like reported for Eg5 before under similar conditions (Kwok et al:: AM based on BRB80 with $0.5 \%$ DMSO added, Kapitein et al.: AM based on BRB70) [Kwok06, Kapitein08]. We determined effective one-dimensional diffusion constants by mean-squared displacement (MSD) analysis. The 1D-diffusion constant of DK4mer in AM (based on BRB80) with 2 mM ADP was $1.2 \times 10^{4} \mathrm{~nm}^{2} \mathrm{~s}^{-1}$ (Fig. $6.5 \mathrm{~B}$ ), about 10 -fold higher than the one reported for $\operatorname{Eg} 5\left(0.7-1.1 \times 10^{3} \mathrm{~nm}^{2} \mathrm{~s}^{-1}\right)$ [Kwok06, Kapitein08]. Diffusion constants measured with other diffusive kinesins under similar buffer conditions lie in the same range as the one of DK4mer, for example: Kinesin-13 MCAK: $38 \times 10^{4}$ $\mathrm{nm}^{2} \mathrm{~s}^{-1}$ [Helenius06], Kinesin-8 kip3p: $0.43 \times 10^{4} \mathrm{~nm}^{2} \mathrm{~s}^{-1}$ [Bormuth09]. At increased ionic strength, the diffusion coefficient of DK4mer increased about 4-fold, reflecting a weaker interaction between motor and MT (Fig. 6.5 B).

\section{The truncated dimer, DK511}

To control that the tail domains are indeed responsible for the diffusive attachment, we generated a shortened dimeric construct on the basis of DK4mer, truncated at Eg5 residue 511 and thereby lacking the Eg5 tail domains (DK511, 6.1 D). We chose the truncation site such that monomers should form dimers, but not tetramers due to the short stalks. It is reasonable to assume that the most C-terminal parts of the stalk before the tail, normally embedded in a tetrameric coiled-coil, do otherwise not influence the motility characteristics of the dimeric end. Single-molecule fluorescence assays confirmed that DK511 was in fact a dimeric motor protein, as we observed uninterrupted and unidirectional processive motility of single motors at an average velocity of $\sim 530 \mathrm{~nm} / \mathrm{s}$ (Fig. 6.2 B and Supplementary Tab. D.1) and with an average run length of $\sim 2.8 \mu \mathrm{m}$ (Supplementary Tab. D.1). Thus the truncation led to a functional and processive dimer with an average velocity similar to DK4mer. These results underline two points: (i) The Eg5 tail has no major influence on the velocity produced by the Kinesin-1 motor domains. In other words, the observed $40 \%$ reduction in velocity of DK4mer compared to Kinesin-1 is not caused by friction that could be introduced by the Eg5 tail domains. The reduced velocity might be caused by a mismatch in the neck linker region. Several studies have shown that a variation in the neck coiled-coil and the neck linker can have influence on the communication between the motor domains and can affect velocity [Shastry10, Yildiz08, Düselder12]. (ii) The pauses observed in DK4mer motility, but not in DK511 motility, are likely to be introduced by the Eg5 tail. Likewise, the extraordinarily long run length of DK4mer is likely to be caused by the additional Eg5 tail-MT interactions. 

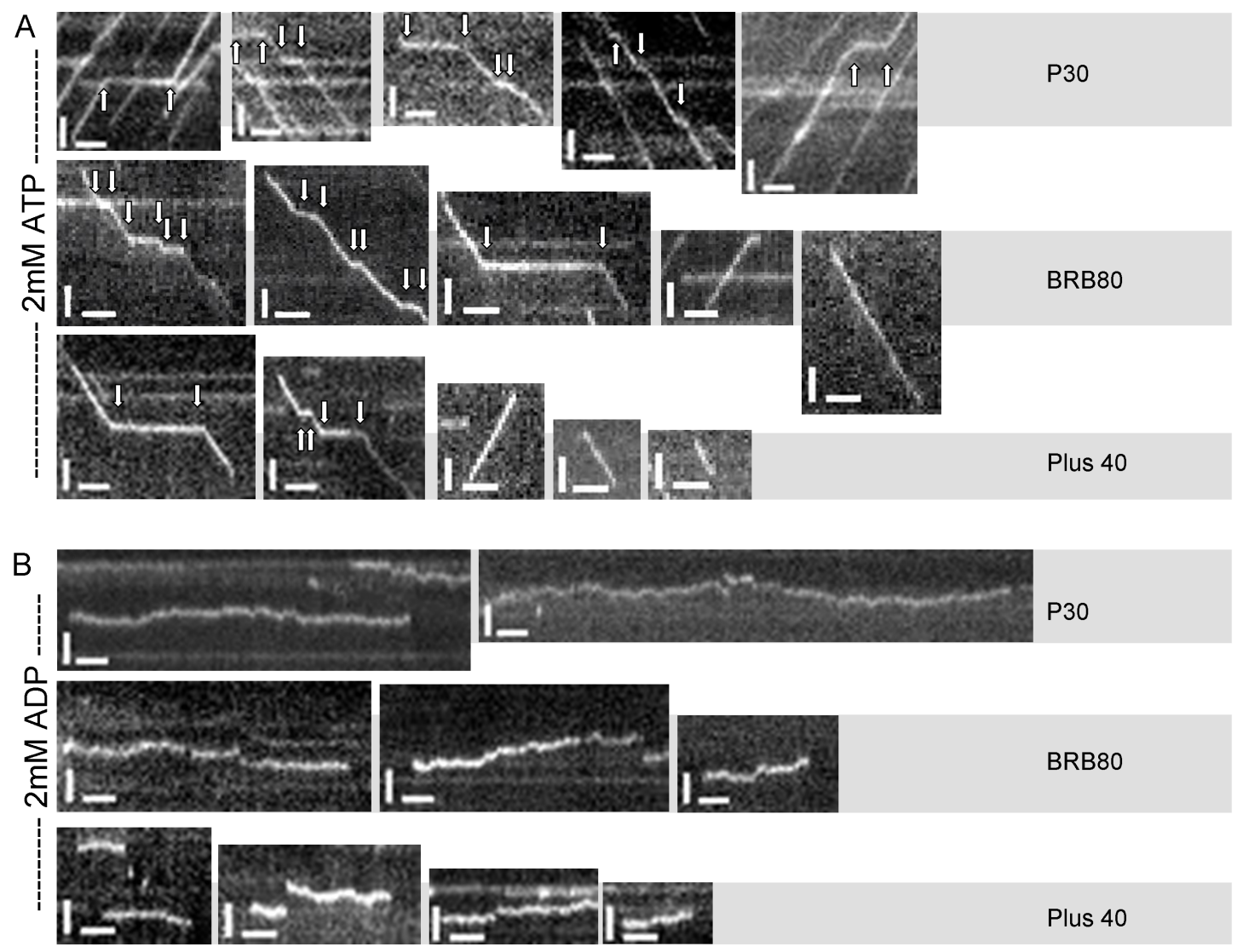

Fig. 6.3: Switching between diffusive and processive motility modes: (A) Kymographs of DK4mer motility at $2 \mathrm{mM}$ ATP in P30 buffer, BRB80 buffer, and BRB80 buffer with an additional $40 \mathrm{mM} \mathrm{KCl}$. Selected tracks approximately represent the frequency of runs and pauses under the respective buffer conditions. Arrows mark the switches between motility modes. (B) Kymographs of motility at $2 \mathrm{mM}$ ADP in P30 buffer, BRB80 buffer, and BRB80 buffer with additional $40 \mathrm{mM} \mathrm{KCl}$. Horizontal scale bars: 5 s, vertical scale bars: $1600 \mathrm{~nm}$.

\section{Motility of single DK4mer molecules at different ionic strengths}

It is known that ionic strength can have a strong influence on the motility of motor proteins by modifying the motor-MT interaction [Kapitein05, Kwok06, Kapitein08, Gerson-Gurwitz11]. To further explore the influence of the tail-MT interaction, we analyzed the effect of increasing ionic strength on the motility of the DK4mer motor construct. DK4mer is better suited for such experiments than native Eg5 because, with DK4mer, the transitions between diffusive pauses and directed motility on single MTs are very clearly detectable. An increase of ionic strength and Debye screening is predicted to reduce interaction strength since the tail domain likely interacts via weak electrostatic interactions with the C-terminus of tubulins [Vale89, Thorn00, Wang00, Cahu09].

Consistent with this expectation, in our experiments, increasing the ionic strength led to reduced overall run lengths, from $9.6 \pm 2.4 \mu \mathrm{m}$ in P30 buffer to $1.7 \pm 0.8$ $\mu \mathrm{m}$ in BRB80 buffer plus $60 \mathrm{mM} \mathrm{KCl} \mathrm{(Fig.} \mathrm{6.3} \mathrm{A,} \mathrm{Fig.} \mathrm{6.4} \mathrm{D} \mathrm{and} \mathrm{Supplementary}$ Tab. D.1). The decrease in run length, however, was caused mainly by shorter 
pauses with an increased likelihood for detachment, while the processive segments of runs appeared less affected (Fig. 6.3 A, Fig. 6.4 A and B, Supplementary Tab. D.1). From $20 \mathrm{mM}$ added $\mathrm{KCl}$ on, motility was purely processive with a run length of $2.6 \pm 0.7 \mu \mathrm{m}$ comparable to that of $\mathrm{D} 421,2.9 \pm 0.8 \mu \mathrm{m}$, (a truncated dimeric Kinesin-1 construct) and DK511, $2.3 \pm 0.9 \mu \mathrm{m}$ (Fig. $6.4 \mathrm{~B}$ and Supplementary Tab. D.1). We thus found that an increase in ionic strength of the buffer mostly reduced tail-MT interactions. This result is consistent with the report by Weinger et al. for Eg5 [Weinger11]. Note that the run length (Fig. 6.4 B) shows a sharp rather than gradual transition from mixed motility at low ionic strength to purely processive motility at high ionic strength. This result further supports the idea that tail-MT and motor-domain-MT interactions are mutually exclusive. Furthermore, increasing the ionic strength from P30 buffer to BRB80 buffer plus $40 \mathrm{mM} \mathrm{KCl}$ led to $\mathrm{a} \sim 4$-fold reduction in the duration of interactions in the presence of ADP (Fig. $6.3 \mathrm{~B})$.

In ADP buffer, MSD analysis showed that DK4mer interactions with single MTs were purely diffusive at all ionic strengths (Fig. 6.5 A). Increasing the ionic strength led to a 4- to 5 -fold increase in the diffusion constant, from $0.5 \pm 0.04 \times 10^{4}$ $\mathrm{nm}^{2} \mathrm{~s}^{-1}$ to $2.2 \pm 0.7 \times 10^{4} \mathrm{~nm}^{2} \mathrm{~s}^{-1}$, consistent with what has been reported for $\mathrm{Eg} 5$ [Kapitein08]. This indicates that the ATP-independent interaction is electrostatic and thus is weakened by increasing ionic strength (Fig. 6.5 B). Interestingly however, the diffusion constant of DK4mer during pauses between processive runs at $2 \mathrm{mM}$ ATP was significantly lower $\left(0.4 \pm 0.2 \times 10^{4} \mathrm{~nm}^{2} \mathrm{~s}^{-1}\right)$ then during ADP-diffusive interactions $\left(1.2 \pm 0.04 \times 10^{4} \mathrm{~nm}^{2} \mathrm{~s}^{-1}\right.$ at otherwise identical buffer conditions (Fig. $6.5 \mathrm{~B}$, circle). These results suggest that the two diffusive modes of DK4mer, the one in the presence of ATP and the one in the presence of ADP, are determined by somewhat different interactions with the MT.

Increasing the ionic strength had very little effect on the average velocity of directed motility. This supports the notion that once the motor protein is moving, the Kinesin-1 motor domains work independently of and undisturbed by the Eg5 tail and are not very sensitive to changes of ionic strength (Fig. 6.4 C).

With increasing ionic strength, slow velocities appeared in the velocity distribution of DK4mer (Fig. 6.4 C). This slow fraction can be explained by the limited resolution of the experiments. With increasing ionic strength the pauses in the processive runs become shorter, and, eventually, the shortest pauses in high salt buffer will not be detected as pauses anymore, but will be hidden in apparently processive runs leading to a distorted overall velocity distribution.

\section{The truncated dimer DK511 at different ionic strengths}

To confirm the result that only the tail domain-MT interaction is influenced by ionic strength, we also measured the motility of the DK511 in low ionic strength (P30 buffer). We found purely processive motility with an average run length of $3.4 \pm 0.4 \mu \mathrm{m}$, only about $30 \%$ of the run length of DK4mer $(9.6 \pm 2.4 \mu \mathrm{m})$ under the same buffer conditions. Interestingly the DK511 run length was very similar to the run length of the truncated dimeric Kinesin-1 construct D421 of $2.7 \pm 0.4$ $\mu \mathrm{m}$ (Fig. $6.4 \mathrm{~B}$ and Supplementary Tab. D.1). This result confirms that the long 

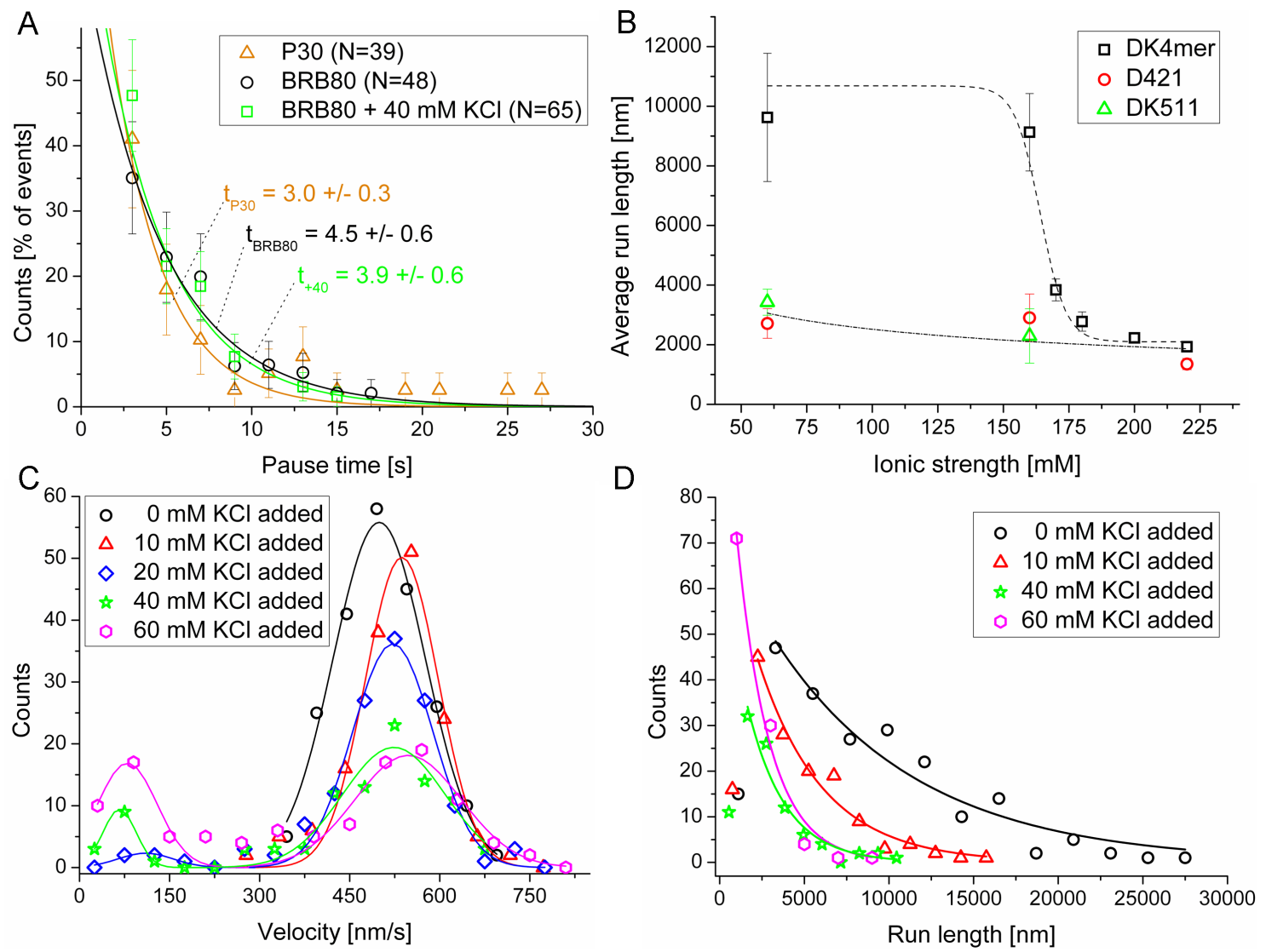

Fig. 6.4: Single-molecule fluorescence assays at $2 \mathrm{mM}$ ATP and different salt concentrations: (A) Distribution of pause durations during runs of single DK4mer molecules in AM based on three different buffers. Distributions are fitted with single-exponentials, using the number of events as weights. The average duration of pauses is given for each buffer condition. Pauses shorter than $2 \mathrm{~s}$ were not scored. (B) Average run length plotted against ionic strength of the buffer solution: DK4mer (squares) and, for comparison, truncated DmKHC Kinesin-1, D421 (circles), and truncated DK4mer, DK511 (triangles). Dashed trend lines serve to guide the eye. (C) Velocity distributions at different concentrations of $\mathrm{KCl}$ added to BRB80 buffer. Distributions were fitted with sums of two Gaussians. Permanently stuck motor proteins $(\mathrm{v}=0 \mathrm{~nm} / \mathrm{s})$ were not taken into account. (D) Run-length distributions at different concentrations of added $\mathrm{KCl}$, fitted with single exponentials (lines). Run lengths $<1 \mu \mathrm{m}$ were not included into the fit because their frequency is likely underestimated due to limited resolution. For runs with pauses, the run lengths of the processive segments were added up. Average velocities and run lengths are summarized in Supplementary Tab. D.1. 
A

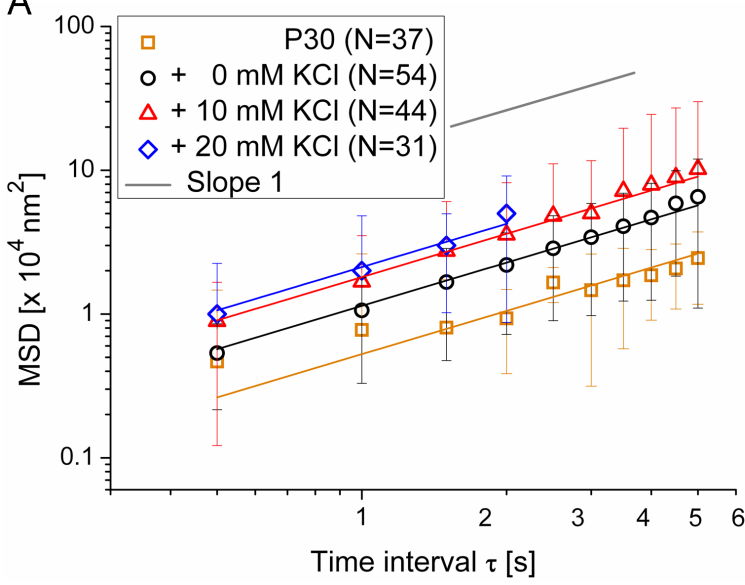

B

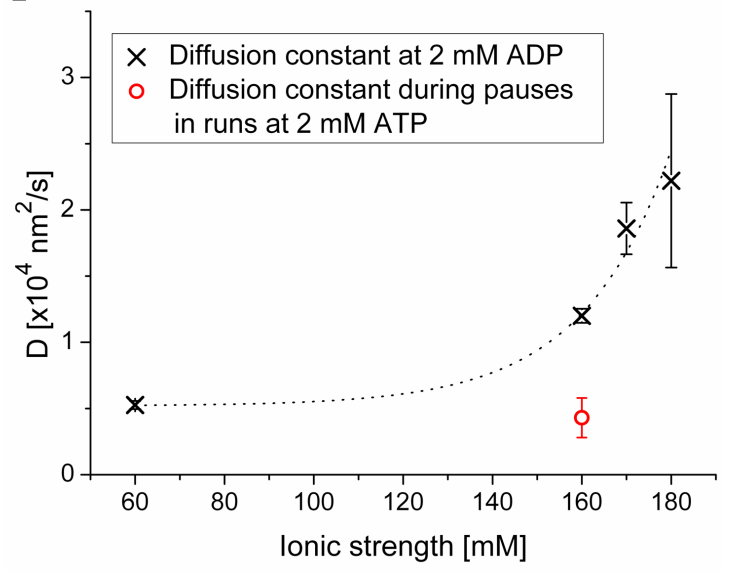

Fig. 6.5: Single-molecule fluorescence assays at $2 \mathrm{mM} \mathrm{ADP}$ at different salt concentrations: (A) Mean-squared displacements (MSD) of single DK4mer motors on MTs at 2 mM ADP in AM based on P30 buffer and BRB80 buffer with different amounts of added $\mathrm{KCl}$, fitted linearly in the log-log plot (lines). The dwell time decreased with increasing salt. For $20 \mathrm{mM}$ added $\mathrm{KCl}$, only dwell times up to $2 \mathrm{~s}$ were measured with sufficient statistics. N: number of analyzed motor traces. Power-law slope of 1 is indicated by grey line. (B) 1D-diffusion constants of DK4mer motors on MTs at $2 \mathrm{mM}$ ADP (crosses, $\mathrm{N}$ for each salt condition equals values given in (A) as a function of buffer ionic strength, dotted line to guide the eye. For comparison: diffusion constant of DK4mer (circle, $\mathrm{N}=$ 11) during pauses between processive runs (marked with arrows in Fig. 6.4 B) at $2 \mathrm{mM}$ ATP in BRB80 buffer. N: number of analyzed motor traces.

run lengths found for DK4mer are caused by the Eg5 tail domains. In AM based on BRB80 buffer, run lengths for both D421 and DK511 were only slightly shorter than in low-ionic-strength buffer. This supports the notion that the function of the Kinesin-1 motor domain is only slightly influenced by a change in the ionic strength of the buffer solution.

\section{Relative sliding of MTs driven by DK4mer at varying ionic strength}

Single-molecule fluorescence assays reported earlier, showed that individual Xenopus laevis Eg5 motors are able to crosslink MTs and drive relative sliding at twice the velocity (2v) of an individual motor on a single MT [Kapitein08]. Twice the single motor velocity (2v-model, Fig. 6.6 B) can only be reached in a sliding assay when relative sliding is driven by the continuous and simultaneous action of both pairs of motor domains, with each pair of motor domains driving motility at single-motor velocity $\mathrm{v}$.

To probe DK4mer for this Kinesin- 5 feature, we performed relative sliding assays as described before [Gerson-Gurwitz11] and could in fact confirm that DK4mer is able to crosslink and slide MTs (Fig. 6.6 A) at a higher than single-motor velocity (Fig. 6.6 C). This result confirms that DK4mer is a fully functional tetrameric motor.

In order to analyze the effect of ionic strength on the motility of DK4mer between crosslinked MTs, we performed relative sliding assays at high enough motor 


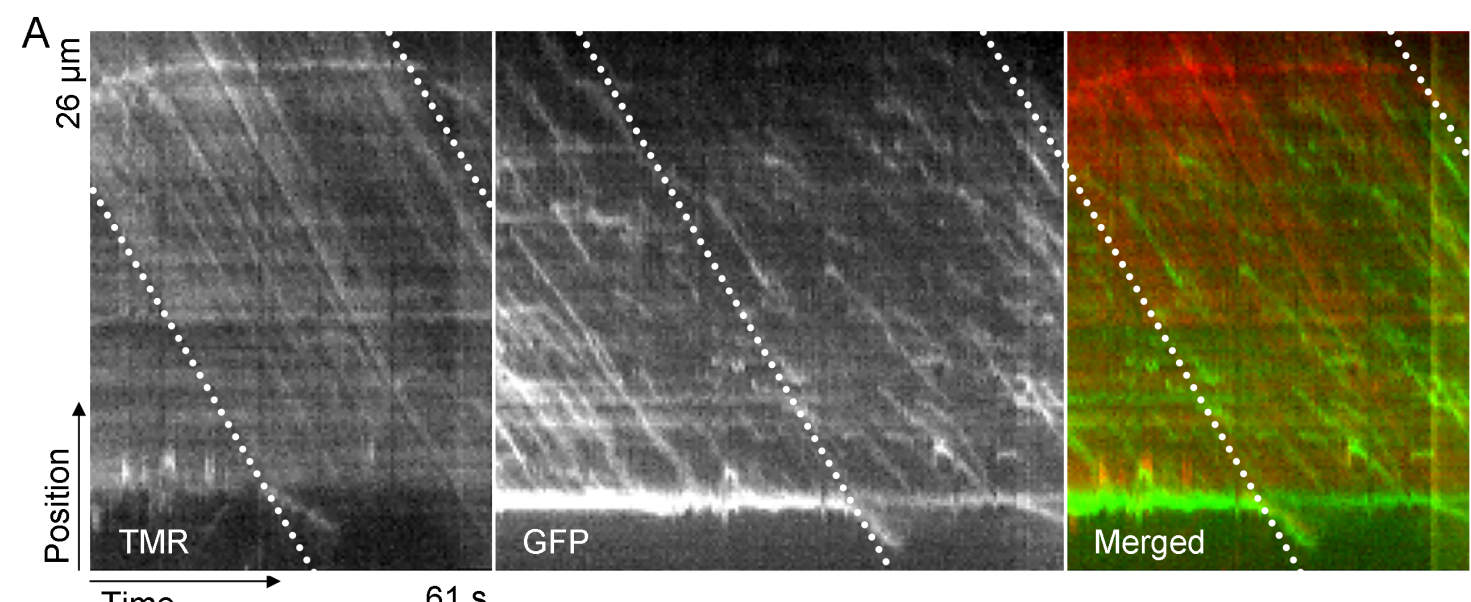

B

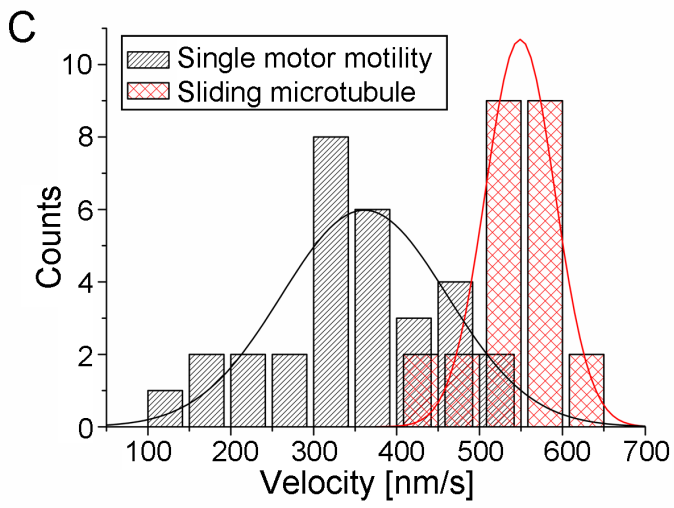

Fig. 6.6: DK4mer motility while sliding a mobile MT over a surface-attached MT: (A) Kymographs of dual-color single-molecule fluorescence assays recording the motility of single DK4mer motors while sliding TMR-labeled MTs in P30 buffer: mobile and stationary MT in TMR-channel (ends of mobile MT are marked with dotted line), DK4mer in GFPchannel (overlap area between MTs between dotted lines), and merged kymograph of MTs (red) and DK4mer (green). (B) Cartoon of four possible types of motor protein localization and resulting velocities: a) motor protein moving on sliding MT with single-motor velocity, leading to velocity $2 \mathrm{v}$ against the surface, b) motor protein moving in overlap region on two MTs with velocity $\mathrm{v}, \mathrm{c}$ ) motor protein moving on fixed MT with velocity $\mathrm{v}$ and d) motor protein bound to surface and to MT, not moving. (C) Histogram of motor (black) and MT (red) velocities in the MT-overlap region.

concentrations to ensure that motility was driven by multiple motors at different ionic strengths. We found that relative sliding occurred over a wide range of salt concentrations (Supplementary Fig. D.5), even at high ionic strengths (BRB80 buffer $+40 \mathrm{mM} \mathrm{KCl}, \mathrm{I}=200 \mathrm{mM}$ ) at which switching to tail-mediated binding on single MTs, i.e. diffusive intervals, were not seen anymore (Fig. 6.4 B). This result suggests that, at high ionic strength, motor domain driven interactions alone could maintain crosslinking and simultaneously drove relative sliding even with diminished tail binding.

The velocity of relative sliding at low ionic strength remained below the expected value of twice the single motor velocity $(2 \mathrm{v})$, but increasing the ionic strength shifted 
the distribution towards 2v (Fig. 6.6 B and C, Supplementary Fig. D.5). Relative sliding speed was $\sim 700 \mathrm{~nm} / \mathrm{s}$ in P30 buffer, but $\sim 1100 \mathrm{~nm} / \mathrm{s}$ in P30 buffer plus $70 \mathrm{mM}$ added $\mathrm{KCl}$. The explanation for the reduced sliding velocity at low ionic strength is probably that sliding MTs also interact with surface-adsorbed motors which would slow them down. In P30 buffer with $80 \mathrm{mM} \mathrm{KCl}$ added (I = $140 \mathrm{mM})$ and higher ionic strengths, we could not observe any relative sliding. In singlemolecule assays we could still see processive runs on single MTs in BRB80 buffer $+60 \mathrm{mM} \mathrm{KCl}(\mathrm{I}=220 \mathrm{mM})$. One explanation for this discrepancy might be Debye screening: the probability of a motor binding to a single MT in high ionic strength is lower than in low ionic strength. One motor binding to two MTs simultaneously in high ionic strength has an even lower probability, which might make it very difficult to observe relative sliding at higher ionic strengths.

\section{\begin{tabular}{l|l} 
Conclusions & 6.4
\end{tabular}}

Much attention has recently been focused on the regulation of motor proteins in complex cellular contexts. Kinesin-5, as essential actuator of the mitotic spindle, is a prime example of a highly regulated mechanoenzyme. We have here used a Kinesin-1 head/Kinesin-5 tail chimera to provide a well-behaved model system to investigate details of the multiple binding modes of tetrameric kinesins to MTs which are believed to be involved in turning the motor "on" and "off". Several earlier approaches have used mutated and chimeric constructs, mainly in the experimental context of the mitotic spindle, observing modifications of its elongation dynamics [Cahu09, Kwok04]. A chimera similar to ours was independently described by Cahu et al., and was used in in-vivo experiments in Xenopus egg extracts. In those experiments it was shown that the chimeric motors mislocalized in mitotic spindles and consequently caused spindle collapse into tightly bundled MT arrays [Cahu09].

Our tetrameric chimera exhibited fast, processive motility and was able to crosslink and slide MTs. This was to be expected from the properties of the parent kinesins that provided the respective components. Additionally, our results clearly show that diffusive and processive episodes alternated during interactions and were mutually exclusive, processive interaction being driven by the motor heads and diffusive attachment caused by the tails, likely by electrostatic interactions. This finding makes it tempting to speculate that tail binding might cause a conformation of the heads with respect to the MT that makes motility impossible and, vice versa, that head binding brings the tail binding sites out of contact with the MT. The latter result is somewhat different from what was found for native Eg5, the heads of which are likely to be particularly sensitive to regulation by the tails. In Eg5 the tail domain is required for proper and efficient orientation and function of Eg5, when it crosslinks and orients MTs in vivo and in vitro [Weinger11]. Further studies of specifically altered or chimeric constructs are necessary and will yield further insight in the structural changes involved in the regulation of the transition from "off-" to "on-state" of Kinesin-5 motors. 


\section{\begin{tabular}{l|l} 
Materials and Methods & 6.5
\end{tabular}}

\section{Cloning and protein purification $\mid 6.5 .1$}

The construction of the tetrameric chimera DK4mer and a further dimeric derivative was based on a detailed analysis of the transition between motor domain and neck coiled-coil in Eg5 and the known limits of the motor domain and neck linker in the Drosophila Kinesin-1 (DmKHC). In DK4mer the first 345 amino acids (aa) of Drosophila Kinesin-1 were fused to Xenopus Kinesin-5 (Eg5) at aa 370. Additional constructs contained either a 6-his tag (DK4mer-his) or a green fluorescent protein (GFP)-6his cassette as described in detail previously (22). The exact sequence we chose for the transition is schematically shown in Fig. 6.1 A. The dimeric derivative DK511 was truncated at aa 511 (Fig. 6.1 D). Donor plasmids were kindly provided by W. O. Hancock (DmKHC) and T. M. Kapoor (FL-Eg5-GFP, BK006). We used a nested PCR approach similar to that described before [Lakämper10] to extend the motor domain and neck linker of DmKHC (DK) with sequences providing a direct and uninterrupted transition using selected restriction sites to a correspondingly amplified neck/stalk/tail-fragment of Eg5 (EK). DK was amplified with a fwd primer, DK1fwd, providing an NdeI site (flanked by additional SalI and XmaI-sequences) and two rev primers, DKrev1 and DKrev2, providing transition sequences of the Eg5 neck up to an AflI site which was generated using a silent mutation in the natural sequence (further flanking regions provide more restrictions sites such as NotI for sub-cloning of fragments). Similarly the EK fragment was generated using a fwdprimer, EKfwd1, and two rev-primers, EKrev1 and EKrev2, providing the same restriction sites at the $\mathrm{N}$-terminus and a cassette containing a sequence containing an AscI and XmaI site followed by a 6his box and a stop codon followed by the cutting sides NotI, Sal and XhoI. The AscI/XmaI site allowed us to insert a previously used GFP-6his casette from pT7-7-GFP-his. The resulting PCR-fragments were initially "parked" in a pTOPO-XL vector (Invitrogen, USA), before sub-cloning into a pFastBac vector (Invitrogen, USA) for expression in Sf9-cells. Truncation constructs (DK511-GFPhis as a truncated version of DK4mer and D421-GFPhis as a truncated version of DmKHC) were generated using a simple PCR approach and subsequent sub-cloning in a pET-21b(+) vector (Novagen, Germany). Expression and purification was performed as described in [Lakämper10]. Chemicals were purchased from Sigma, USA, if not otherwise stated.

\section{\begin{tabular}{l|l} 
Multi-motor surface-gliding assays & 6.5 .2
\end{tabular}}

Multi-motor surface-gliding assays were performed at $22{ }^{\circ} \mathrm{C}$ as described previously [Lakämper10]. The motor proteins were allowed to non-specifically bind to the glass surfaces of assay chambers made from cover slips and microscope slides, assembled with double-stick tape. Subsequently, the chambers were flushed with about three chamber volumes of assay mix (AM) based on BRB80 buffer (80 mM PIPES/KOH, 
pH 6.8, $1 \mathrm{mM} \mathrm{MgCl}_{2}, 1 \mathrm{mM}$ EGTA) containing $10 \mu \mathrm{m}$ taxol (paclitaxel), $2 \mathrm{mM}$ ATP, $4 \mathrm{mM} \mathrm{MgCl}_{2}, 10 \mathrm{mM}$ DTT, $0.08 \mathrm{mg} / \mathrm{ml}$ catalase C40, $0.1 \mathrm{mg} / \mathrm{ml}$ glucose oxidase and $10 \mathrm{mM}$ glucose. For multi-motor surface-gliding assays, $0.022 \mathrm{mg} / \mathrm{ml}$ tetramethylrhodamine (TMR)-labeled MTs, polymerized as described previously [Lakämper10], were added to AM. Motility was observed in a standard inverted fluorescence microscope (Zeiss Axiovert 200, Germany) using a Zeiss EC Plan-Neofluar 100× 1.3 NA oil immersion objective (Zeiss, Germany). Images were recorded with a digital CCD camera (CoolSnap ES, Roper Scientific, Germany) at a frame rate of 2 frames/s. Videos were analyzed with ImageJ (National Institute of Health, USA) to obtain MT velocities.

\section{Single-molecule fluorescence assays $\quad 6.5 .3$}

Cover slips were cleaned using a plasma cleaner (PDC-002, Harrick Plasma, USA) before surface silanization with 3-[2-(2-Aminoethylamino)ethylamino]propyltrimethoxysilane (DETA) (Sigma, Germany) for MT immobilization as described before [Lakämper10]. TMR-labeled MTs were allowed to bind to the surface during an incubation time of $5 \mathrm{~min}$., followed by $5 \mathrm{~min}$. incubation with $0.1 \mathrm{mg} / \mathrm{ml}$ casein in BRB80. Finally DK4mer-GFPhis (DK4mer) diluted in AM to single-molecule concentration $(\sim 150 \mathrm{nM})$ was introduced, and fluorescence was observed in a custombuilt total-internal-reflection-fluorescence (TIRF) microscope.

The TIRF setup was built similarly to the setup described previously by van Dijk et al. [Dijk07] with the following modifications: we used two lasers (473 $\mathrm{nm}$ and 532 $\mathrm{nm}$, both Viasho, USA) to excite the fluorophores (GFP and TMR). The lasers were expanded and coupled via a multi-wave-length beam splitter (z474/488/532/635rpc, Chroma, USA) off-axis into an oilimmersion objective (Nikon, SFluor $100 \times, 1.49$ ) to obtain TIRF illumination. The emitted fluorescent light was split in the GFP and TMR signals using the dichroic mirror (525/50, Chroma, USA), then passed trough bandpass filters (530/50 for GFP and 605/70 for TMR, both Chroma, USA) and finally directed via mirrors to separate areas of the detector area of a frame-transfer EMCCD camera (Cascade 512B, Roper Scientific, USA). In a further modification (not used for the experiments in this work) a commercial image splitter (Optosplit III, Cairn Research, UK) was integrated into the setup (Supplementary Fig. D.4) providing the possibility to detect an additional, third fluorophore. For analysis, the separated signals of GFP and TMR were superimposed using the OptoSplit ImageJ plugin provided by Cairn Research, UK.

For measurements at different salt concentrations, $\mathrm{KCl}$ was added to BRB80 buffer. Measurements with lower than $80 \mathrm{mM}$ salt concentration were done in AM as described above, but based on P30 buffer (30 mM PIPES/KOH, pH 6.8, $1 \mathrm{mM}$ $\mathrm{MgCl}_{2}, 1 \mathrm{mM}$ EGTA). To calculate the total ionic strength of the different buffers, one has to take into account that PIPES buffer is a diprotonic acid $\left(\mathrm{pKa}_{1}<3\right.$, $\mathrm{pKa}_{2}=6.8$ ). To adjust, e.g., $80 \mathrm{mM}$ of PIPES free acid to $\mathrm{pH} 6.8$, the addition of $120 \mathrm{mM} \mathrm{KOH}$ is required. At $\mathrm{pH} 6.8$, additional to the $\mathrm{K}^{+}$and $\mathrm{Cl}^{-}$ions, an equimolar amount of each acid group is in solution, and the $\mathrm{OH}^{-}$ions are buffered 
by water. Ionic strength can be calculated as: $\mathrm{I}=\frac{1}{2} \sum \mathrm{c}_{i} \mathrm{z}_{i}^{2}$, with $\mathrm{c}_{i}$ concentration and $\mathrm{z}_{i}$ valence of ion type i. BRB 80 plus $10 \mathrm{mM} \mathrm{KCl}$, for example, has an ionic strength $\mathrm{I}=1 / 2(40 \times 1+40 \times 4+(120+10) \times 1+10 \times 1) \mathrm{mM}=170 \mathrm{mM}$. All single-molecule-fluorescence measurements were performed at $22{ }^{\circ} \mathrm{C}$. The CCD camera was controlled with WinSpec32 software (Princeton Instruments, USA). Digital images were recorded at a frame rate of 2 frames/s and were subsequently analyzed for velocities and run lengths using kymographs generated with a customwritten LabView (National Instruments, USA) routine. To estimate average velocities, only the straight (judged by eye) runs or straight segments of runs were taken into account. Runs lasting for less then 2 seconds were not scored. Statistical analysis of data was performed with OriginPro (OriginLab Corporation, USA). MSD analysis was done with a custom-written MATLAB (The MathWorks, USA) routine.

\section{\begin{tabular}{l|l} 
Relative sliding of polarity-marked MTs & 6.5 .4
\end{tabular}}

Polarity-marked MTs were assembled in two polymerization steps: First, highly concentrated TMR-labeled tubulin $(3.33 \mathrm{mg} / \mathrm{ml})$ was polymerized in the presence of 0.4 mM guanosine-5'-[( $\alpha, \beta)$-methyleno] triphosphate (GMP-CPP, Jena Bioscience, Germany) to avoid depolymerization after dilution. In the second step, these short MTs (seeds) were diluted and extended by further polymerization in a solution of 0.54 $\mathrm{mg} / \mathrm{ml}$ TMR-labeled tubulin and $0.72 \mathrm{mM}$ GTP in P30 buffer. Additionally to a batch of long polarity-marked MTs, a batch of short polarity-marked MTs was polymerized by increasing the seed concentration and shortening the incubation time. Finally the MTs were stabilized in P30 buffer containing $10 \mu \mathrm{M}$ taxol (paclitaxel, Sigma, USA). DETA-silanized assay chambers were prepared as described above. Long MTs were allowed to bind for $\sim 4$ min., followed by $\sim 6$ min. incubation with $0.5 \mathrm{mg} / \mathrm{ml}$ casein in P30 buffer. Finally, $10 \mu \mathrm{l}$ of AM based on P30 buffer containing 4 - 5 times the single-molecule concentration (1:150 dilution) of DK4mer and short MTs were added into the flow chamber.

For the measurements at different salt concentrations, AMs based on P12 buffer

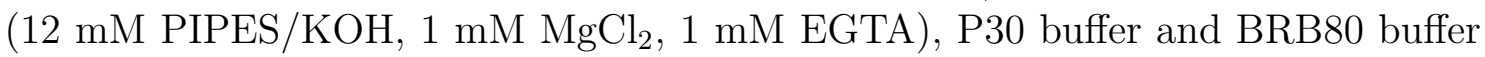
were used. Fluorescence was observed with a wide-field fluorescence microscope (Zeiss Axiovert 200, Germany) equipped with a Zeiss EC Plan-Neofluar 100x 1.3 NA oil immersion objective. Images were recorded with a CCD camera (Coolsnap ES, Roper Scientific, Germany). All measurements were performed at $22{ }^{\circ} \mathrm{C}$ and videos were recorded at a frame rate of 2 frames/s. Digital images were analyzed using kymographs generated with custom-written LabView (National Instruments, USA) routines for MT velocity and binding geometry.

\section{Single-molecule imaging of motors during MT sliding $\quad 6.5 .5$}

Single motor molecules were imaged during relative sliding of polarity-marked MTs in the custom-built TIRF setup. The assay was performed similarly to the (multi- 
motor) relative sliding assay of polarity-marked MTs, but with a 1:280 dilution of the motor protein DK4mer. The measurements were performed at $22{ }^{\circ} \mathrm{C}$, and videos were recorded at a frame rate of 2 frames/s. The TMR- and the GFP-signals, recorded on separate areas of the CCD detector, were aligned using the OptoSplit ImageJ plugin provided by Cairn Research, UK and analyzed for motor protein and MT velocity as described above.

\section{\begin{tabular}{l|l} 
Acknowledgements & 6.6
\end{tabular}}

This work was supported in part by the Lower Saxony Grant no. 11-76251-9926/08 (ZN2440) and in part by the Center for Molecular Physiology of the Brain (CMPB), funded by the Deutsche Forschungsgemeinschaft (DFG). We thank Marcel Bremerich for programming the MSD analysis routine.

\section{Supplementary data}

Supplementary data associated with this manuscript can be found in appendix D. 


\title{
Endoplasmic reticulum sorting and kinesin-1 command the targeting of axonal $\mathrm{GABA}_{B}$ receptors
}

Outside the topic of kinesin-5 regulation I participated in a project which investigated the targeting of axonal $\mathrm{GABA}_{B}$ receptors in hippocampal neurons. The content of this chapter was published 2012 in the Journal PLoS ONE [Valdés12]. Co-authors are Viviana Valdés, José Ignacio Valenzuela, Daniela A. Salas, Matías Jaureguiberry-Bravo, Carolina Otero, Christoph F. Schmidt and Andrés Couve.

In this work I performed in-vivo total-internal-reflection-fluorescence (TIRF) measurements of the motility of fluorescently labelled $\mathrm{GABA}_{B} \mathrm{R} 1$ subunits and analysed the respective single-molecule data. Additionally I contributed figures and text to the paper.

\begin{abstract} 7.1

In neuronal cells the intracellular trafficking machinery controls the availability of neurotransmitter receptors at the plasma membrane, which is a critical determinant of synaptic strength. Metabotropic $\gamma$ amino-butyric acid (GABA) type B receptors $\left(\mathrm{GABA}_{B} \mathrm{Rs}\right)$ are neurotransmitter receptors that modulate synaptic transmission by mediating the slow and prolonged responses to GABA. $\mathrm{GABA}_{B}$ Rs are obligatory heteromers constituted by two subunits, $\mathrm{GABA}_{B} \mathrm{R} 1$ and $\mathrm{GABA}_{B} \mathrm{R} 2 . \mathrm{GABA}_{B} \mathrm{R} 1 \mathrm{a}$ and $\mathrm{GABA}_{B} \mathrm{R} 1 \mathrm{~b}$ are the most abundant subunit variants. $\mathrm{GABA}_{B} \mathrm{R} 1 \mathrm{~b}$ is located in the somatodendritic domain whereas $\mathrm{GABA}_{B} \mathrm{R} 1 \mathrm{a}$ is additionally targeted to the axon. Sushi domains located at the N-terminus of $\mathrm{GABA}_{B} \mathrm{R} 1 \mathrm{a}$ constitute the only difference between both variants and are necessary and sufficient for axonal targeting. The precise targeting machinery and the organelles involved in sorting and transport have not been described. Here we demonstrate that $\mathrm{GABA}_{B}$ Rs require the Golgi apparatus for plasma membrane delivery but that axonal sorting and targeting of $\mathrm{GABA}_{B} \mathrm{R} 1 \mathrm{a}$ operate in a pre-Golgi compartment. In the axon $\mathrm{GABA}_{B} \mathrm{R} 1 \mathrm{a}$ subunits are enriched in the endoplasmic reticulum (ER), and their dynamic behavior and colocalization with other secretory organelles like the ER-to-Golgi intermediate compartment (ERGIC) suggest that they employ a local secretory route. The transport of axonal $\mathrm{GABA}_{B} \mathrm{R} 1 \mathrm{a}$ is microtubule-dependent and kinesin-1, a molecular motor of the kinesin family, determines axonal localization. Considering that progression of $\mathrm{GABA}_{B}$ Rs through the secretory pathway is regulated by an ER retention motif our data contribute to understand the role of the axonal ER in non-canonical sorting and targeting of neurotransmitter receptors.
\end{abstract}




\section{\begin{tabular}{l|l} 
& \\
Introduction & 7.2
\end{tabular}}

Polarized protein trafficking in the neuron is critical for synapse formation, synapse maintenance and the regulation of synaptic strength. In all eukaryotic cells the endomembrane trafficking system includes a forward biosynthetic route constituted by the endoplasmic reticulum (ER), the ER-Golgi intermediate compartment (ERGIC), the Golgi apparatus and post-Golgi vesicles, and a recycling-degradative route constituted by endosomes and lysosomes. The unique architecture and size of neurons does not necessarily imply that the structure/function relationship of these organelles and their contribution to the secretory process are different than in other cell types. However, their spatial arrangement and contribution to local processing may be specially adapted to the complexities of the neuronal morphology [Horton04]. How the neuron orchestrates this highly compartmentalized trafficking is poorly understood. In particular, how the local distribution of secretory components in the neuron impinges on intracellular trafficking and availability of neurotransmitter receptors remains for the most part unexplored.

GABA is the main inhibitory neurotransmitter in the nervous system and the metabotropic $\mathrm{GABA}_{B}$ Rs are obligatory heteromers composed of two related subunits, $\mathrm{GABA}_{B} \mathrm{R} 1$ and $\mathrm{GABA}_{B} \mathrm{R} 2$ (for a comprehensive review of $\mathrm{GABA}_{B} \mathrm{R}$ structure, function, localization and pathological implications see [Bettler04]). Both belong to family $\mathrm{C}$ of $\mathrm{G}$ proteincoupled receptors, and contain a large extracellular N-terminal domain, seven membrane-spanning domains and an intracellular C-terminal domain. GABA $_{B}$ Rs are expressed in neurons throughout the brain and spinal cord. They are mainly perisynaptic receptors located in gabaergic and glutamatergic presynaptic terminals and postsynaptic sites. $\mathrm{GABA}_{B} \mathrm{R} 1$ binds agonists with high affinity whereas $\mathrm{GABA}_{B} \mathrm{R} 2$ couples to $\mathrm{G}_{\alpha i}$ establishing a transactivation mechanism between the two subunits. At presynaptic terminals $\mathrm{GABA}_{B} \mathrm{Rs}$ inhibit voltage gated $\mathrm{Ca}^{2+}$ channels thereby inhibiting synaptic vesicle fusion and neurotransmitter release. At postsynaptic sites they activate inwardly rectifying $\mathrm{K}^{+}$channels hyperpolarizing the postsynaptic neuron. In addition, stimulation of $\mathrm{GABA}_{B}$ Rs decreases the levels of cyclic AMP. GABA ${ }_{B}$ Rs have been implicated in epilepsy, anxiety, stress, sleep disorders, nociception, depression, cognition and addictive mechanisms to drug abuse. The relevance of studying $\mathrm{GABA}_{B} \mathrm{R}$ availability is further supported by clinical observations that report the appearance of tolerance to $\mathrm{GABA}_{B} \mathrm{R}$ agonists, an inconvenient side effect to therapy. $\mathrm{GABA}_{B} \mathrm{R}$ subunits are synthesized in the soma and glycosylated in the ER [Couve98, Ramírez09]. The progression of $\mathrm{GABA}_{B}$ Rs through the secretory pathway is regulated by an RXR-type sequence (RSRR) in the C-terminal domain of $\mathrm{GABA}_{B} \mathrm{R} 1$ that functions as an ER retention motif in the absence of $\mathrm{GABA}_{B} \mathrm{R} 2$ [Margeta-Mitrovic00]. The ER retention motif is masked upon association to $\mathrm{GABA}_{B} \mathrm{R} 2$, and assembled $\mathrm{GABA}_{B} \mathrm{Rs}$ exit the ER as heteromers destined for the plasma membrane. Consistent with ER retention acting as a limiting step $\mathrm{GABA}_{B} \mathrm{Rs}$ are abundant within intracellular compartments, especially the ER [Kulik03]. GABA $_{B}$ R1a and GABA $_{B}$ R1b constitute the most abundant isoforms for $\mathrm{GABA}_{B} \mathrm{R} 1$. Heteromers containing $\mathrm{GABA}_{B} \mathrm{R} 1 \mathrm{a}$ are axonal and somatodendritic whereas those containing $\mathrm{GABA}_{B} \mathrm{R} 1 \mathrm{~b}$ are exclusively 
located in the somatodendritic domain [Vigot06]. GABA $_{B} \mathrm{R} 1 \mathrm{a}$ and $\mathrm{GABA}_{B} \mathrm{R} 1 \mathrm{~b}$ mediate their different functions only as a result of their specific axonal or somatodendritic localization [Vigot06]. The sushi domains located at the N-terminus of $\mathrm{GABA}_{B} \mathrm{R} 1 \mathrm{a}$ are necessary and sufficient for axonal targeting even in a $\mathrm{GABA}_{B} \mathrm{R} 2$ knock-out background [Biermann10]. However, the precise targeting machinery and the organelles involved in sorting and transport have not been described. Combining conventional optical microscopy and live-cell imaging using organelle reporters and trafficking blockers in cultured hippocampal neurons we describe a mechanism for $\mathrm{GABA}_{B} \mathrm{R} 1 \mathrm{a}$ axonal localization based on pre-Golgi sorting and ER transport.

\section{Results 7.3}

\section{The delivery of $\mathrm{GABA}_{B}$ Rs to the plasma membrane is Golgi-dependent but axonal targeting is not.}

First we carried out a control experiment to validate the use of overexpression of recombinant $\mathrm{GABA}_{B} \mathrm{R}$ subunits as a strategy to study receptor trafficking. Cultured hippocampal neurons were transfected with $\mathrm{MYC}-\mathrm{GABA}_{B} \mathrm{R} 1 \mathrm{a}$ and the distribution of the subunit at the plasma membrane or in intracellular compartments was evaluated by immunostaining 1 - 5 dpt. MYC-GABA ${ }_{B}$ R1a was retained in intracellular compartments in the cell body and axons up to $5 \mathrm{dpt}$ in the absence of recombinant GABA $_{B}$ R2 expression (Supplementary Fig. E.1). In contrast, GABA ${ }_{B}$ R1a was readily detectable at the cell surface at $2 \mathrm{dpt}$ upon co-transfection with $\mathrm{GABA}_{B} \mathrm{R} 2$ (Supplementary Fig. E.1, right column). These experiments indicate that the trafficking properties of recombinant receptors mimic the situation of the native subunits, and that the trafficking of recombinant receptors is not affected by the endogenous subunits. More importantly, they demonstrate that our experiments using transfection of recombinant $\mathrm{GABA}_{B} \mathrm{R} 1$ subunits exclusively examine their intracellular population.

To determine whether GABA $_{B}$ Rs employ a Golgi-dependent intracellular trafficking route in neurons, primary cultures of hippocampal neurons were transfected with MYC-GABA ${ }_{B}$ R1a and FLAG-GABA ${ }_{B}$ R2 in the absence or presence of ARF1Q71I-HA, a constitutively active ARF1 mutant that prevents export from the Golgi apparatus [Zhang94]. $1 \mathrm{dpt}$ the distribution of $\mathrm{GABA}_{B} \mathrm{Rs}$ at the plasma membrane and in intracellular compartments was evaluated by immunostaining under non-permeabilized or permeabilized conditions. We examined somatic or axonal domains as shown in the schematic neuron (Fig. 7.1 A). As reported previously co-transfection of MYC-GABA ${ }_{B} \mathrm{R} 1 \mathrm{a}$ and FLAG-GABA ${ }_{B} \mathrm{R} 2$ resulted in a robust localization of both subunits at the cell surface (Fig. 7.1 B). In contrast, ARF1Q71I-HA blocked the appearance of both subunits at the plasma membrane and produced accumulation in intracellular compartments (Fig. 7.1 C). These results indicate that the Golgi apparatus is necessary for the delivery of GABA $_{B}$ Rs to the plasma membrane in hippocampal neurons. 
A

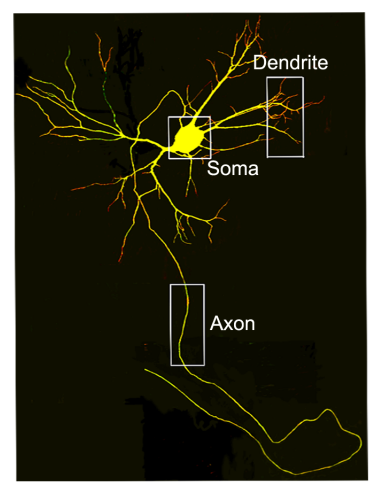

D

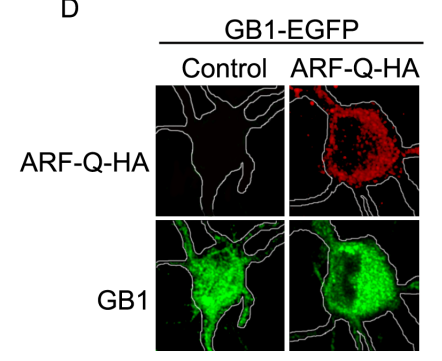

B

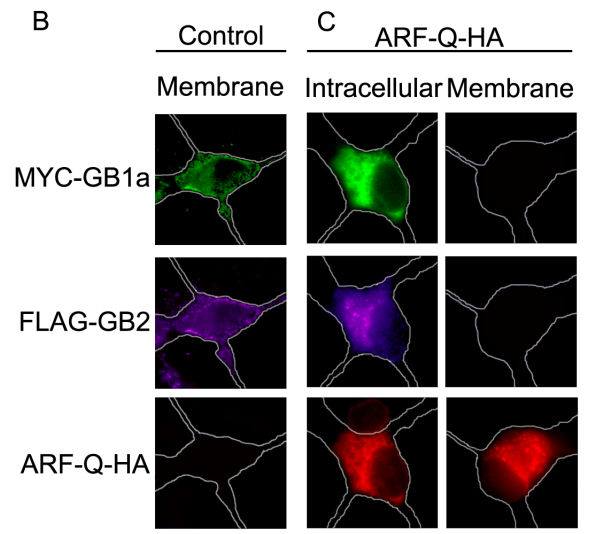

$\mathrm{E}$

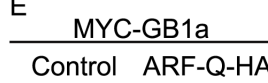

$\mathrm{F}$ MYC-GB1b
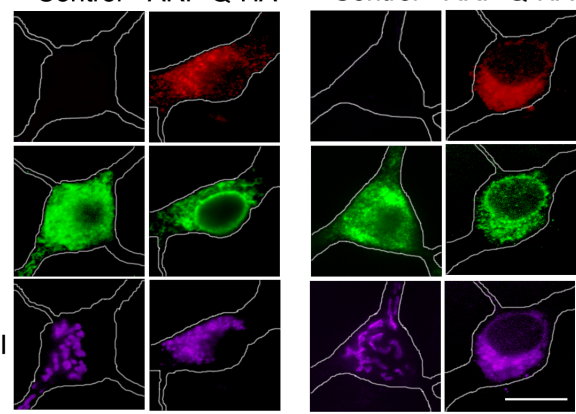

G

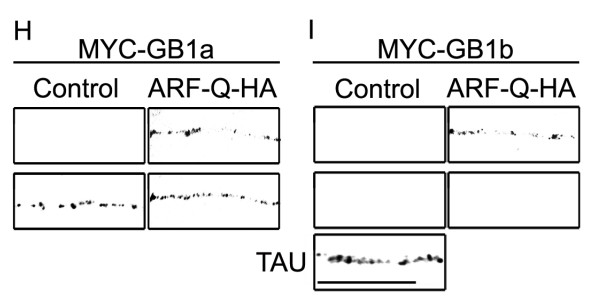

Fig. 7.1: GABA $_{B} \mathrm{R} 1 \mathrm{a}$ is targeted to the axon even after blockade of Golgi export.

(A) Hippocampal neurons were transfected with MYC-GABA ${ }_{B} \mathrm{R} 1 \mathrm{a}$ and RFP, and processed for immunofluorescence under permeabilized conditions. MYC-GABA ${ }_{B}$ R1a was detected with MYC antibodies (green) and RFP was detected without staining (red). Boxes indicate somatic, dendritic and axonal regions used throughout the study. (B) Hippocampal neurons were transfected with MYC-GABA ${ }_{B}$ R1a and FLAG-GABA ${ }_{B}$ R2 and processed for immunofluorescence under nonpermeabilized conditions. MYC-GABA ${ }_{B} \mathrm{R} 1 \mathrm{a}$ was detected with MYC antibodies (MYC-GB1a, green) and FLAG-GABA ${ }_{B}$ R2 was detected with FLAG antibodies (FLAG-GB2, magenta) (representative image of $\mathrm{n}=12$ neurons). (C) Same as above for neurons transfected with MYCGABA $_{B}$ R1a, FLAG-GABA ${ }_{B}$ R2, and ARF1-Q71I-HA (ARF-Q-HA). ARF1-Q71I-HA was detected with HA antibodies (red). Neurons were labeled under permeabilized conditions to visualize intracellular $\mathrm{GABA}_{B} \mathrm{R}$ subunits or non-permeabilized conditions to evaluate their abundance at the plasma membrane (representative image of $\mathrm{n}=28$ neurons). (D) Hippocampal neurons from $\mathrm{GABA}_{B}$ R1-EGFP mice in the absence (control) or presence of ARF1-Q71I-HA (ARF-QHA) and processed for immunofluorescence under permeabilized conditions. ARF1-Q71I-HA was detected with HA antibodies (ARF-Q-HA, red), GABA ${ }_{B}$ R1-EGFP signal was amplified with EGFP antibodies (GB1, green) (representative image of $n=10$ neurons). (E) Hippocampal neurons were transfected with MYC-GABA ${ }_{B}$ R1a in the absence (control) or presence of ARF1-Q71I-HA (ARFQ-HA) and processed for immunofluorescence under permeabilized conditions. ARF-Q-HA was detected with HA antibodies (red), MYC-GABA ${ }_{B}$ R1a was detected with MYC antibodies (green), the Golgi apparatus was detected by expressing pEYFP-Golgi (magenta) (representative image of $n$ $=18$ neurons). (F) Same as above for neurons transfected with MYC-GABA ${ }_{B}$ R1b (representative image of $n=10$ neurons). Scale bar for (B) - (F) represents $20 \mu \mathrm{m}$. (G) - (I) Axons of hippocampal neurons under the experimental conditions of $(\mathrm{D})$ - $(\mathrm{F})$ (representative images of neurons examined above). Tau staining was used to visualize the axon when not labeled by MYC-GABA ${ }_{B}$ R1b. Scale bar for (G) - (I) represents $20 \mu \mathrm{m}$. 
$\mathrm{GABA}_{B} \mathrm{R} 1 \mathrm{a}$ is targeted to the axon in hippocampal neurons [Kulik03, Vigot06]. Thus, we determined whether axonal targeting was also Golgi dependent. First we used cultured hippocampal neurons of transgenic mice that express $\mathrm{GABA}_{B} \mathrm{R} 1$ EGFP under the control of an endogenous promoter [Casanova09]. This experimental model combines the advantages of a nearly physiological scenario and ease of detection. GABA $_{B} \mathrm{R} 1$ localized to the axon in control conditions (Fig. $7.1 \mathrm{D}$ and Fig. 7.1 G, left panel). Importantly, $\mathrm{GABA}_{B} \mathrm{R} 1$ was still targeted to the axon in the presence of ARF1-Q71I-HA (Fig. 7.1 D and Fig. 7.1 G, right panels). According to previous reports the predominant axonal variant of $\mathrm{GABA}_{B} \mathrm{R} 1$ corresponds to $\mathrm{GABA}_{B} \mathrm{R} 1 \mathrm{a}$ [Kulik03, Vigot06]. Therefore, these data are compatible with the idea that $\mathrm{GABA}_{B} \mathrm{R} 1 \mathrm{a}$ is sorted and targeted to the axon at or prior to the Golgi apparatus.

Next we used recombinant receptors to directly compare the axonal targeting of $\mathrm{GABA}_{B} \mathrm{R} 1 \mathrm{a}$ and $\mathrm{GABA}_{B} \mathrm{R} 1 \mathrm{~b}$, and their Golgi dependence. Neurons were transfected with MYC-GABA ${ }_{B}$ R1a or MYC-GABA ${ }_{B}$ R1b in the absence or presence of ARF1-Q71I-HA. Consistent with previous reports, GABA $_{B}$ R1a but not GABA ${ }_{B}$ R1b was predominantly targeted to the axon in hippocampal neurons (Fig. 7.1 E - Fig. $7.1 \mathrm{I}$, control panels). Importantly, recombinant $\mathrm{GABA}_{B} \mathrm{R} 1 \mathrm{a}$ was still targeted to the axon in the presence of ARF1-Q71I-HA (Fig. 7.1 E and Fig. 7.1 H). In contrast, $\mathrm{GABA}_{B} \mathrm{R} 1 \mathrm{~b}$ was absent from the axon under all the conditions examined (Fig. $7.1 \mathrm{~F}$ and Fig. 7.1 I). These findings indicate that axonal targeting is specific to $\mathrm{GABA}_{B} \mathrm{R} 1 \mathrm{a}$. In addition, they demonstrate that the sorting and targeting of $\mathrm{GABA}_{B} \mathrm{R} 1 \mathrm{a}$ to the axon occurs at or prior to the Golgi stage, and therefore suggest a non-conventional modality. They also indicate that axonal targeting of GABA $_{B}$ R1a upon ARF1-Q71I-HA expression is not produced by an overload of the early secretory pathway because the effect was not observed for GABA $_{B} \mathrm{R} 1 \mathrm{~b}$, a conclusion further supported by the results in lower expressing transgenic neurons.

\section{GABA $_{B} \mathbf{R} 1 \mathrm{a}$ is targeted and transported within the axonal ER.}

ER resident proteins and components of the protein folding and export machineries localize to the axon [Willis05, Merianda09, Weclewicz98]. Thus, to determine the intracellular localization of axonal $\mathrm{GABA}_{B} \mathrm{R} 1 \mathrm{a}$ neurons were immunostained with antibodies against the $\mathrm{GABA}_{B} \mathrm{R} 1$ subunit and an antibody against the SEKDEL sequence of the rat ER protein Grp78, including a conserved motif present in luminal ER resident proteins responsible for retrieval from the Golgi apparatus [Munro87]. Endogenous $\mathrm{GABA}_{B} \mathrm{R} 1$ colocalized with the ER in axons (Fig. 7.2 A, arrows). Control labeling without primary antibodies confirmed the specificity of the signal (Fig. 7.2 B and Fig. 7.2 C). In transgenic mouse neurons GABA $_{B}$ R1-EGFP also colocalized with the ER (Fig. 7.2 D, arrows). Importantly, colocalization was still observed upon overexpression of MYC-GABA ${ }_{B}$ R1a and KDEL-RFP, a fluorescent probe widely used for ER visualization that contains the ER targeting sequence of calreticulin and the ER retrieval sequence KDEL (Fig. 7.2 E, arrows). In addition, colocalization was observed with cytochrome b5-EGFP, another fluorescent ER probe (Fig. $7.2 \mathrm{~F}$ ). The colocalization between $\mathrm{GABA}_{B} \mathrm{R} 1 \mathrm{a}$ and ER markers was specific because Piccolo, a marker for dense core vesicles and synapses [Dieck98] 
showed a markedly different axonal localization (Fig. 7.2 G). Staining with MAP2, an exclusive dendritic marker confirmed the co-distribution of $\mathrm{GABA}_{B} \mathrm{R} 1 \mathrm{a}$ and the ER occurs in the axon (Fig. $7.2 \mathrm{H}$ ). These results demonstrate that $\mathrm{GABA}_{B} \mathrm{R} 1 \mathrm{a}$ is enriched in the axonal ER.

To establish whether the ER functions as a transport organelle for $\mathrm{GABA}_{B} \mathrm{R} 1 \mathrm{a}$ we examined the dynamic behavior of fluorescent versions of $\mathrm{GABA}_{B} \mathrm{R} 1 \mathrm{a}$ and the ER in axons of live hippocampal neurons. Discrete GABA $_{B}$ R1a-EGFP and KDEL-RFP puncta were distributed along the axons. While the majority of puncta remained static or showed very limited lateral displacement over the examined period, a subset displayed continuous, long-range mobility (imaged at $0.20-0.25$ frames/s for a total of $\sim 120 \mathrm{~s}$, Fig. $7.3 \mathrm{~A}$ and Fig. 7.3 B). GABA ${ }_{B}$ R1a-EGFP and KDEL-RFP moved bidirectionally, with a moderate retrograde bias, and with modal speeds of $100-200 \mathrm{~nm} / \mathrm{s}$ (Fig. 7.3 E and Fig. 7.3 F). Significantly, some GABA ${ }_{B}$ R1a-EGFP and KDEL-RFP puncta moved in synchrony, with similar speed and retrograde predominance (Fig. $7.3 \mathrm{C}$ and Fig. $7.3 \mathrm{G}$ ). Puncta containing Rab11-GFP, a recycling endosome marker [Jones06], also localized to axons but showed a different dynamic pattern characterized by rapid direction changes and lower overall displacement. We used TIRF microscopy and higher temporal resolution (2 frames/s) to determine the instant velocity of $\mathrm{GABA}_{B} \mathrm{R} 1 \mathrm{a}-\mathrm{EGFP}$ more accurately in hippocampal neurons. Mean anterograde and retrograde instant velocities were comparable $(751.70 \pm 33.20$ and $877.73 \pm 67.59 \mathrm{~nm} / \mathrm{s}$ respectively) (Fig. $7.3 \mathrm{H}$ and Fig. 7.3 I). These values fit conventional kinesin velocities [Cai07] and their slight increase above panels A - G most likely result from excluding stalls in the analysis of our higher temporal resolution imaging.

A proportion of GABA $_{B}$ R1a-EGFP and KDEL-RFP puncta moved independently from each other. This may indicate that a fraction of $\mathrm{GABA}_{B} \mathrm{R} 1 \mathrm{a}-\mathrm{EGFP}$ is transported in a different secretory organelle or that the axonal ER compartment is capable of dynamically segregating cargo. To discriminate between these possibilities we carried out a series of complementary experiments. First we analyzed timelapse microscopy sequences individually. Interestingly, GABA ${ }_{B}$ R1a-EGFP puncta that initially colocalized with the ER sometimes separated from the organelle, remained segregated for a few frames and fused again with a pre-existing ER compartment (Fig. 7.4 A). It is well known that ER cargo recycles between the ER and the ERGIC using export/retrieval motifs such as the RXR-type sequence present in $\mathrm{GABA}_{B} \mathrm{R} 1 \mathrm{a}$ [Bonifacino03]. To determine whether the segregated GABA ${ }_{B} \mathrm{R} 1 \mathrm{a}$ puncta resided temporarily in the ERGIC, we first visualized the axonal distribution of MYC-GABA ${ }_{B}$ R1a and p58-YFP, an established marker of the ERGIC [Ward01], in fixed cells. Sparse ERGIC puncta were observed in axons and a subset of them colocalized with MYC-GABA ${ }_{B}$ R1a (Fig. 7.4 B). Additionally, live-cell imaging was carried out in neurons transfected with $\mathrm{GABA}_{B} \mathrm{R} 1 \mathrm{a}-\mathrm{RFP}$ and p58-YFP. A small fraction of $\mathrm{GABA}_{B} \mathrm{R} 1 \mathrm{a}-\mathrm{RFP}$ displayed synchronous motility with p58-YFP in axons (Fig. $7.4 \mathrm{C}$ ). Taking into account that intra-ER mobility is microtubule dependent but short-range ER to ERGIC transport is not [Ben-Tekaya05], we reasoned that the enrichment of $\mathrm{GABA}_{B} \mathrm{R} 1 \mathrm{a}$ in the ER should increase upon destabilization of 

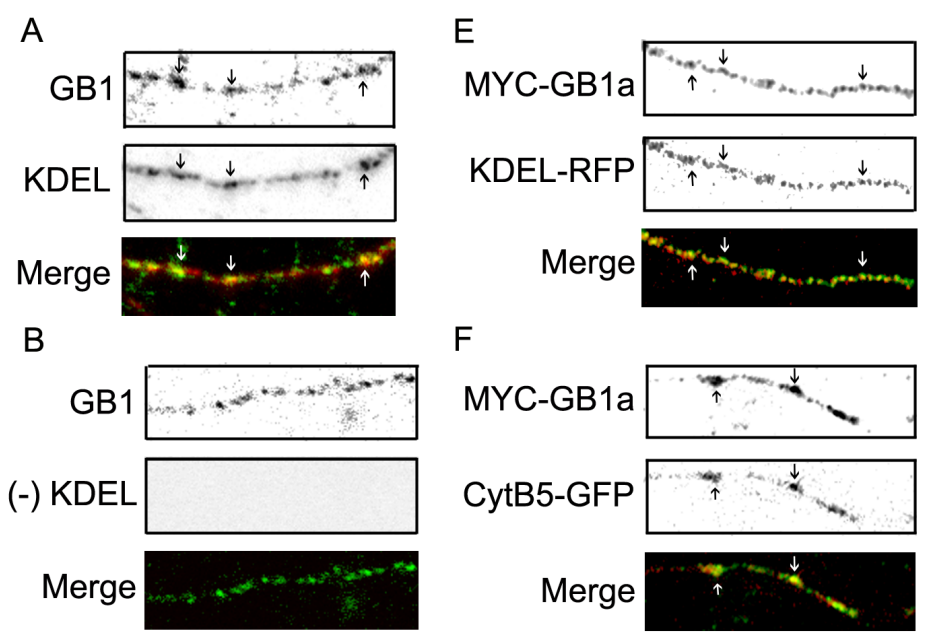

$\mathrm{F}$
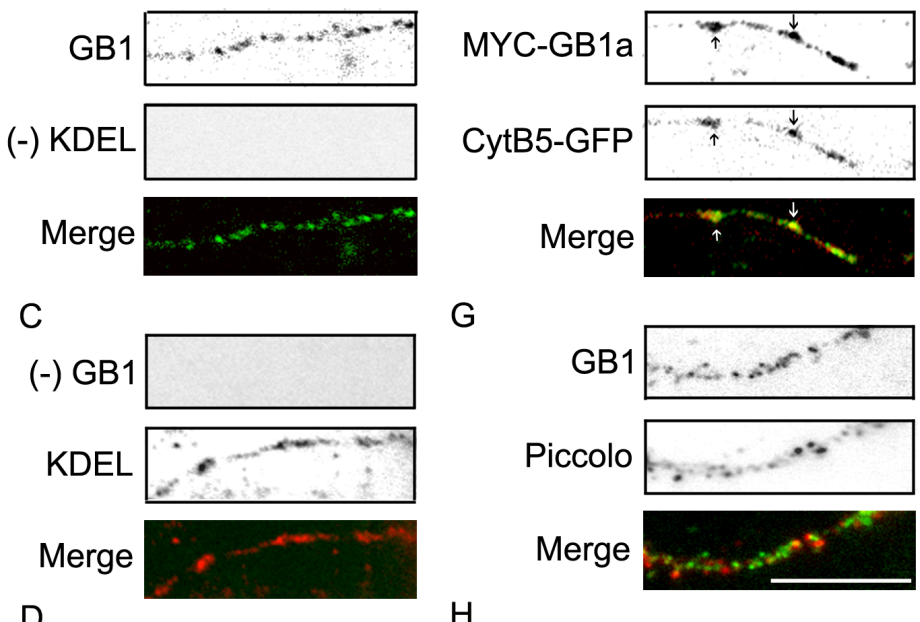

G
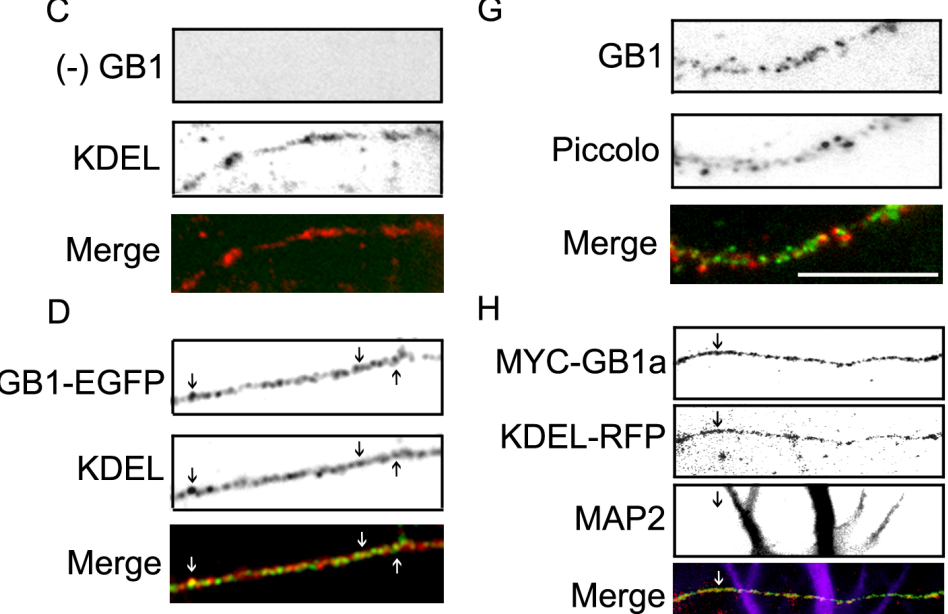

$\mathrm{H}$

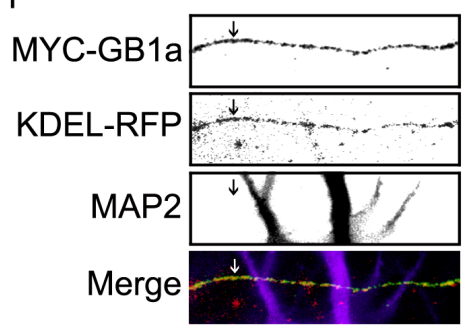

Fig. 7.2: GABA $_{B}$ R1a colocalizes with the ER in axons.

(A) Representative axon of hippocampal neurons processed for immunofluorescence under permeabilized conditions. $\mathrm{GABA}_{B} \mathrm{R} 1$ was detected with $\mathrm{GABA}_{B} \mathrm{R} 1$ antibodies (GB1, green) and the ER was detected with KDEL antibodies (KDEL, red). Merged images are shown on the bottom panel (representative image of $n=17$ neurons). (B) Same as above excluding staining with primary KDEL antibodies. (C) Same as above excluding staining with primary $\mathrm{GABA}_{B} \mathrm{R} 1$ antibodies. (D) Representative axon of hippocampal neurons from $\mathrm{GABA}_{B} \mathrm{R} 1$-EGFP mice processed for immunofluorescence under permeabilized conditions. GABA ${ }_{B}$ R1-EGFP signal was amplified with EGFP antibodies (GB1-EGFP, green) and the ER was detected with KDEL antibodies (KDEL, red). Merged images are shown on the bottom panel (representative image of $n=10$ neurons). (E) Representative axon of hippocampal neurons transfected with MYC-GABA ${ }_{B}$ R1a and KDEL-RFP and processed for immunofluorescence under permeabilized conditions. MYC-GABA ${ }_{B}$ R1a was detected with MYC antibodies (MYC-GB1a, green), KDEL-RFP was visualized without staining (red). Merged images are shown on the bottom panel (representative image of $\mathrm{n}=20$ neurons). ( $\mathrm{F}$ ) Representative axon of hippocampal neurons transfected with MYC-GABA ${ }_{B} \mathrm{R} 1 \mathrm{a}$ and cytochrome b5-EGFP (CytB5-GFP) and processed for immunofluorescence under permeabilized conditions. MYC-GABA $_{B}$ R1a was detected with MYC antibodies (MYC-GB1a, green), CytB5-GFP was visualized without staining (red). Merged images are shown on the bottom panel (representative image of $\mathrm{n}=20$ neurons). (G) Representative axon of hippocampal neurons processed for immunofluorescence under permeabilized conditions. $\mathrm{GABA}_{B} \mathrm{R} 1 \mathrm{a}$ was detected with $\mathrm{GABA}_{B} \mathrm{R} 1$ antibodies (green) and Piccolo was detected with Piccolo antibodies (red). Merged images are shown on the bottom panel (representative image of $n=15$ neurons). (H) Representative axon of hippocampal neurons transfected with MYC-GABA ${ }_{B}$ R1a and KDEL-RFP and processed for immunofluorescence under permeabilized conditions. MYC-GABA ${ }_{B} \mathrm{R} 1 \mathrm{a}$ was detected with MYC antibodies (MYC-GB1a, green), KDEL-RFP was visualized without staining (red) and MAP2 was detected with anti-MAP2 antibodies (MAP2, magenta). The axon is identified as a MAP2negative projection. Merged images are shown on the bottom panel (representative image of $n=$ 15 neurons). Scale bar for $(\mathrm{A})-(\mathrm{H})$ represents $20 \mu \mathrm{m}$. 
A
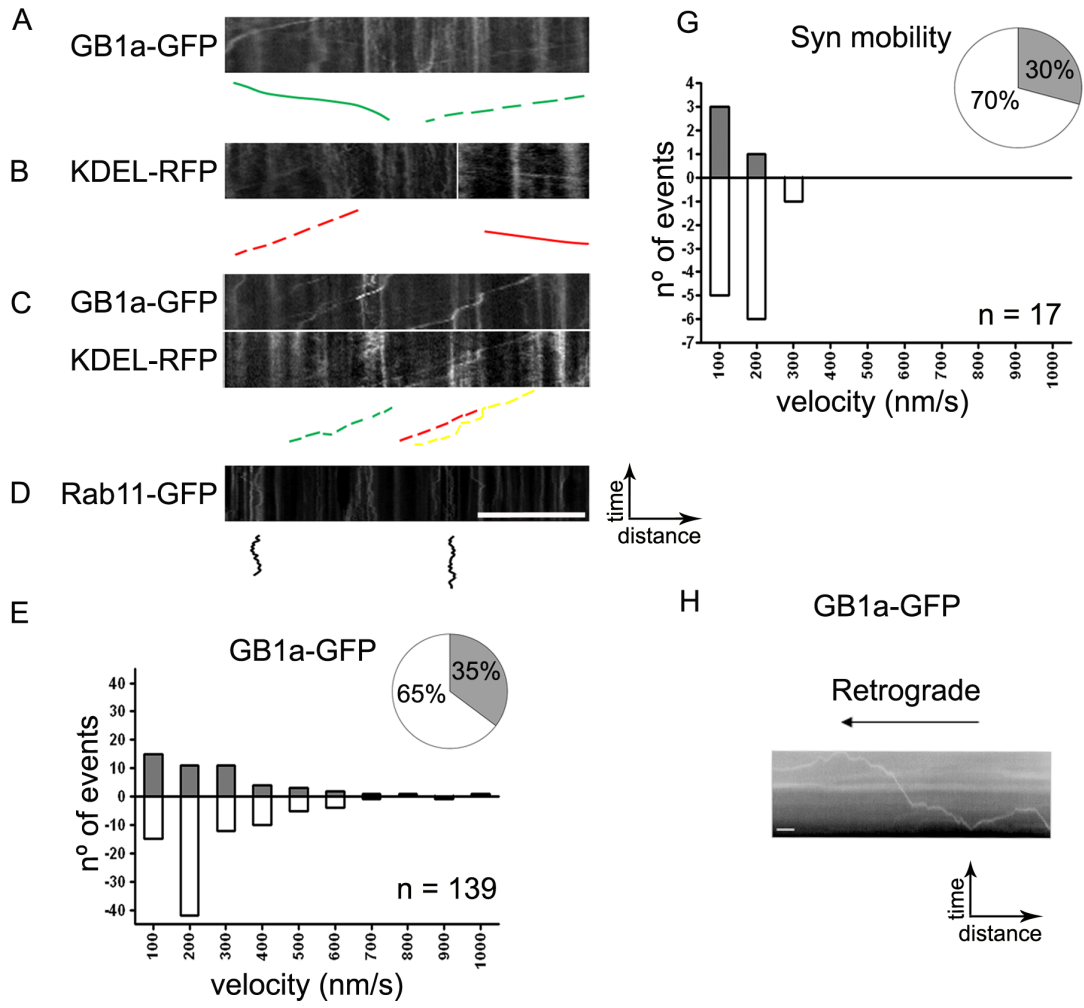

$\mathrm{H}$

GB1a-GFP

$\mathrm{F}$
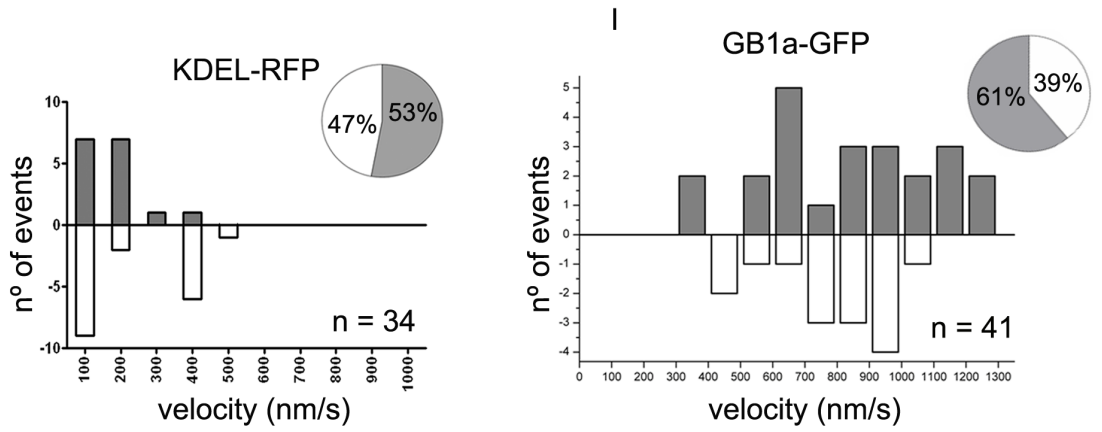

Fig. 7.3: $\mathrm{GABA}_{B} \mathrm{R} 1 \mathrm{a}$ and KDEL are mobile in axons.

(A) Representative kymograph constructed from time series of axons of hippocampal neurons transfected with GABA $_{B}$ R1a-EGFP (GB1a-GFP). Color lines correspond to selected traces within the corresponding kymograph. Solid green line: anterograde mobility; dashed green line: retrograde mobility. (B) Same as above for KDEL-RFP. Solid red line: anterograde mobility; dashed red line: retrograde mobility. (C) Representative kymographs from time series of axons of hippocampal neurons transfected with GABA $_{B}$ R1a-EGFP and KDEL-RFP. Dashed green line: retrograde mobility of $\mathrm{GABA}_{B} \mathrm{R} 1 \mathrm{a}-\mathrm{EGFP}$; dashed red line: retrograde mobility of KDEL-RFP; dashed yellow line: synchronous retrograde mobility of $\mathrm{GABA}_{B} \mathrm{R} 1 \mathrm{a}-\mathrm{EGFP}$ and KDEL-RFP. (D) Representative kymographs constructed from axons of hippocampal neurons transfected with Rab11-GFP. Solid black lines: short-range bidirectional mobility of Rab11-GFP (representative kymograph of $n=$ 14 neurons). Images were acquired at $0.20-0.25$ frames/s for a total of $120 \mathrm{~s}$. Scale bar for (A) (D) represents $20 \mu \mathrm{m}$. (E - G) Average velocity and directionality was quantified from the kymographs for GABA ${ }_{B}$ R1a-EGFP, KDEL-RFP and synchronous mobility of GABA $_{B}$ R1a-EGFP and KDEL-RFP. Bar graphs represent the frequency distribution of velocities, anterograde transport (gray bars), retrograde transport (white bars). Pie charts represent fractions of anterograde (gray) and retrograde transport (white). Average velocities and direction were obtained from 17 - 139 moving puncta from a total of 42 neurons from at least three independent culture preparations. (H) Representative kymograph from time series of axons of hippocampal neurons transfected with GABA $_{B}$ R1a-EGFP imaged by TIRF microscopy at 2 frames/s. Scale bars represent $10 \mathrm{~s}$ and 3 $\mu \mathrm{m}$. (I) Bar graph represents the frequency distribution of velocities: anterograde (gray bars), retrograde (white bars). Pie chart represents fractions of anterograde (gray) and retrograde transport (white). Average velocities and direction were obtained from 41 moving puncta from at least three independent culture preparations. 
microtubules. Consistent with this prediction, nocodazole blocked the mobility of GABA $_{B}$ R1a-EGFP puncta and the subunits accumulated in a KDEL-RFP compartment (Fig. $7.4 \mathrm{D}$, top right panels). As expected, a mutant $\mathrm{GABA}_{B} \mathrm{R} 1 \mathrm{a}$ subunit that is not retained in the ER $\left(\mathrm{GABA}_{B} \mathrm{R} 1 \mathrm{a}-\mathrm{ASA}-\mathrm{EGFP}\right)$ accumulated in a different compartment after nocodazole treatment (Fig. 7.4 D, bottom right panels). These observations suggest that axonal $\mathrm{GABA}_{B} \mathrm{R} 1 \mathrm{a}$ is targeted and transported to the axon within the ER, and possibly engages in a local export/retrieval mechanism between the ER and the ERGIC.

\section{Kinesin-1 contributes to the axonal localization of $\mathrm{GABA}_{B} \mathrm{R} 1 \mathrm{a}$.}

Since kinesin-1, an axonal biased molecular motor [Nakata03, Jacobson06], colocalizes with $\mathrm{GABA}_{B} \mathrm{R} 1$ in neurons, and associates to the subunit in fractionation and coimmunoprecipitation assays [Vidal07] we evaluated its contribution to axonal $\mathrm{GABA}_{B} \mathrm{R} 1 \mathrm{a}$ targeting. To examine the axonal localization of MYC-GABA ${ }_{B} \mathrm{R} 1 \mathrm{a}$ we determined an axon-to-dendrite ratio (A:D ratio) [Biermann10]. To study the role of kinesin-1 we used a dominant negative comprising the cargo-binding domain of Kif5C that interferes with endogenous kinesin-cargo interactions fused to RFP (Kif5C-RFP-DN) [Falley09]. The axonal targeting of MYC-GABA ${ }_{B}$ R1a was markedly reduced in the presence of Kif5C-RFP-DN (Fig. 7.5 A and Supplementary

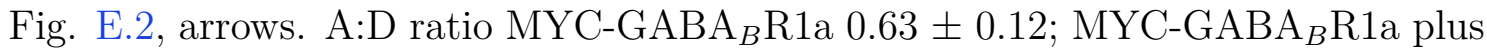
Kif5C-RFP-DN $0.06 \pm 0.02 ; \mathrm{p}<0.01)$. On the contrary, the axonal distribution of the ER marker ER-YFP and GABA $_{B}$ R2-EGFP were not affected by Kif5C-RFPDN (Fig. 7.5 B. A:D ratio ER-YFP $0.64 \pm 0.47$; ER-YFP plus Kif5C-RFP-DN 0.63 $\pm 0.32 ; \mathrm{p}=0.97 ; \mathrm{GABA}_{B} \mathrm{R} 2$-EGFP $0.79 \pm 0.46 ; \mathrm{GABA}_{B} \mathrm{R} 2$-EGFP plus Kif5CRFP-DN $0.69 \pm 0.68 ; \mathrm{p}=0.80)$.

Kinesin-1 may control the axonal localization of MYC-GABA ${ }_{B}$ R1a via an adaptor mechanism through the cytosolic C-terminal domain of $\mathrm{GABA}_{B} \mathrm{R} 1 \mathrm{a}$. To directly test this we first determined whether axonal targeting required the cytosolic C-terminal domain of $\mathrm{GABA}_{B} \mathrm{R} 1 \mathrm{a}$ using two C-terminal subunit mutants, MYCGABA $_{B}$ R1a-AA-ASA and MYC-GABA ${ }_{B}$ R1a- $\Delta$ C [Restituito05]. GABA ${ }_{B}$ R1a, GA$\mathrm{BA}_{B} \mathrm{R} 1 \mathrm{a}-\mathrm{AA}-\mathrm{ASA}$ and $\mathrm{GABA}_{B} \mathrm{R} 1 \mathrm{a}-\Delta \mathrm{C}$ were all abundant in the axon indicating that targeting is independent of the C-terminal domain (Fig. $7.5 \mathrm{C}$, arrows). Using a quantitative colocalization analysis based on Manders coefficients [Ramírez10] all $\mathrm{GABA}_{B} \mathrm{R} 1 \mathrm{a}$ constructs colocalized partially with the ER and no statistically significant difference was observed between $\mathrm{GABA}_{B} \mathrm{R} 1 \mathrm{a}$ and the two mutants (MYCGABA $_{B}$ R1a, $\mathrm{n}=9$ neurons; MYC-GABA ${ }_{B}$ R1a-AA-ASA $\mathrm{p}=0.11, \mathrm{n}=5$ neurons; MYC-GABA ${ }_{B} \mathrm{R} 1 \mathrm{a}-\Delta \mathrm{C} \mathrm{p}=0.30, \mathrm{n}=5$ neurons). These results suggest that the C-terminal domain is not a major determinant of axonal ER distribution and targeting of $\mathrm{GABA}_{B} \mathrm{R} 1 \mathrm{a}$. Finally, we analyzed the kinesin-1 dependence on the axonal targeting of $\mathrm{GABA}_{B} \mathrm{R} 1 \mathrm{a}$ lacking the C-terminal domain. Axonal localization of MYC-GABA ${ }_{B}$ R1a- $\Delta \mathrm{C}$ was still severely impaired by the dominant negative construct (Fig. $7.5 \mathrm{D}$, arrows. A:D ratio MYC-GABA ${ }_{B}$ R1a- $\Delta \mathrm{C} 0.42 \pm 0.06$; MYCGABA $_{B}$ R1a- $\Delta$ C plus Kif5C-RFP-DN $\left.0.05 \pm 0.01 ; \mathrm{p}<0.01\right)$. Combined these experiments demonstrate that kinesin-1 is necessary for the ER axonal targeting and 
A

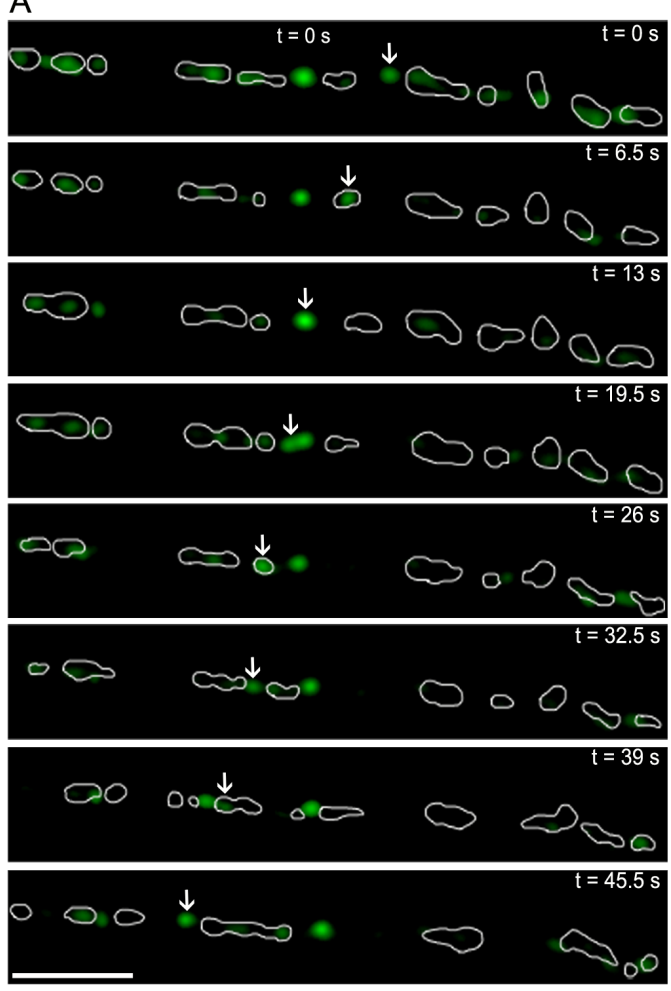

B

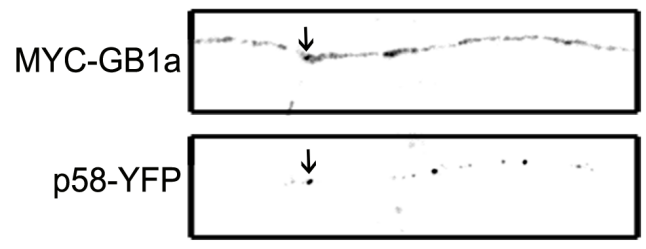

C

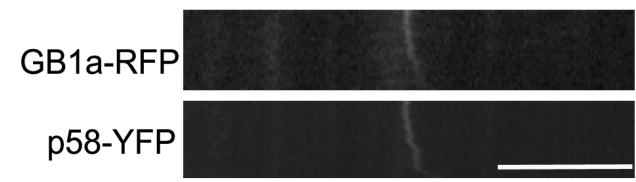

$\mathrm{D}$

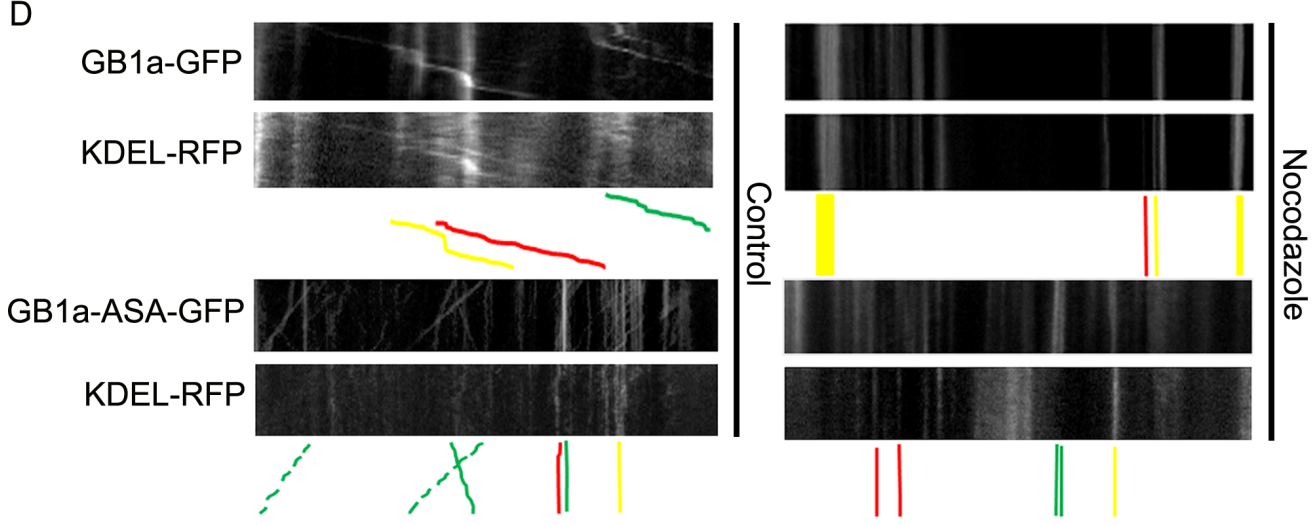

Fig. 7.4: $\mathrm{GABA}_{B} \mathrm{R} 1 \mathrm{a}$ cycles between KDEL compartments in axons.

(A) Representative axon of hippocampal neurons transfected with GABA $_{B}$ R1a-EGFP and KDELRFP, and visualized live. The area originally occupied by the ER (KDEL-RFP) in each time frame was outlined in white. The arrows show a GABA $_{B}$ R1a-EGFP puncta that exits and enters different ER compartments within the observation period (45.5 s) (representative time-lapse sequence of $n=10$ neurons). Scale bar represents $20 \mu \mathrm{m}$. (B) Representative axon of hippocampal neurons transfected with MYC-GABA ${ }_{B}$ R1a (MYC-GB1a) and p58-YFP, and processed for immunofluo-

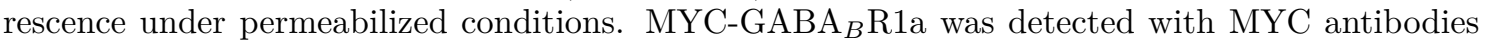
(green); p58-YFP was visualized without staining (red). Merged images are shown on the bottom panel (representative image of $\mathrm{n}=23$ neurons). Scale bar represents $20 \mu \mathrm{m}$. (C) Kymographs were constructed from time series of axons of hippocampal neurons transfected with GABA $_{B}$ R1a-RFP (GB1a-RFP) and p58-YFP. Solid yellow lines: synchronous transport of GABA $_{B}$ R1a-RFP and p58-YFP (representative kymograph of $\mathrm{n}=22$ neurons). Scale bar for $(C)$ - (D) represents $20 \mu \mathrm{m}$. (D) Kymographs were constructed from time series of axons of hippocampal neurons under control conditions or treated with nocodazole after transfection with GABA $_{B}$ R1a-EGFP (GB1a-GFP) or GABA $_{B}$ R1a-ASA-EGFP (GB1a-ASA-GFP) and KDEL-RFP. Top right panels: accumulated $\mathrm{GABA}_{B} \mathrm{R} 1 \mathrm{a}-\mathrm{EGFP}$ and KDEL-RFP in static puncta (solid yellow lines). Bottom right panels: accumulated GABA $_{B}$ R1a-ASA-EGFP and KDEL-RFP in different static puncta (solid green and red lines) (representative kymograph of $\mathrm{n}=21$ neurons). 


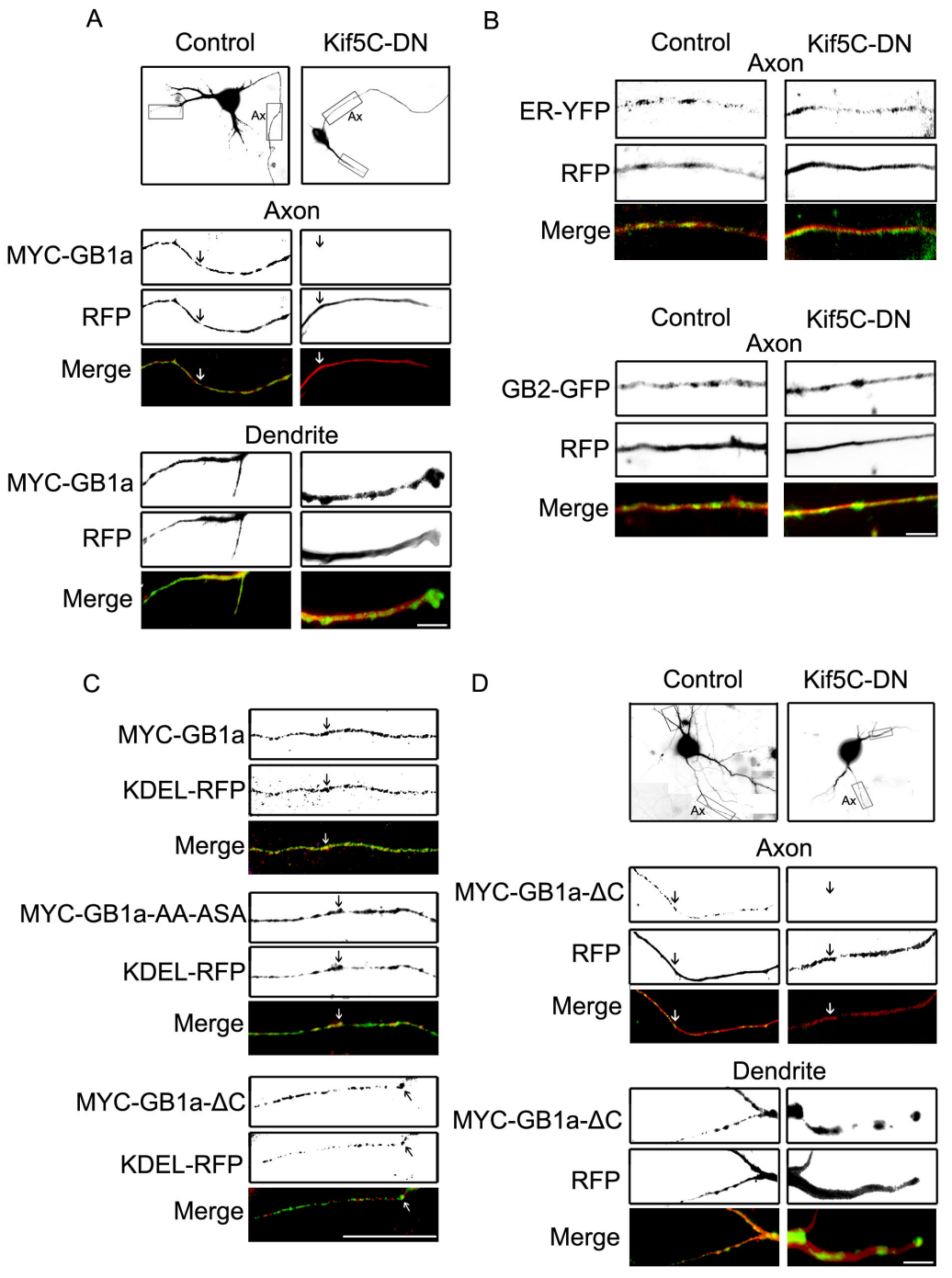

Fig. 7.5: Axonal targeting of $\mathrm{GABA}_{B} \mathrm{R} 1 \mathrm{a}$ is kinesin-1 dependent and C-terminal domain independent.

(A) Hippocampal neurons were transfected with MYC-GABA ${ }_{B}$ R1a and RFP (left, representative image of $\mathrm{n}=12$ neurons) or MYC-GABA ${ }_{B} \mathrm{R} 1 \mathrm{a}$ and Kif5C-RFP-DN, a dominant negative version of kinesin-1 (Kif5C-DN, right, representative image of $\mathrm{n}=13$ neurons). The neuronal volume filled by RFP or Kif5C-RFP-DN is shown. Boxes in top panels correspond to regions of axons $(\mathrm{Ax})$ and dendrites (unlabelled) magnified below. MYC-GABA ${ }_{B} \mathrm{R} 1 \mathrm{a}$ (green) and RFP or Kif5CRFP-DN (red). Merged images are shown on the bottom panel. Scale bar represents $40 \mu \mathrm{m}$. (B) Top panels: representative axon of hippocampal neurons transfected with pEYFP-ER (ER-YFP) and RFP or pEYFP-ER and Kif5C-RFP-DN. Markers were visualized without staining. Merged images are shown on the bottom panel (representative image of $n=15$ neurons). Bottom panels: representative axon of hippocampal neurons transfected with GABA $_{B}$ R2-EGFP (GB2-GFP) and RFP or GABA ${ }_{B}$ R2-EGFP and Kif5C-RFP-DN. Markers were visualized without staining. Merged images are shown on the bottom panel (representative image of $n=15$ neurons). Scale bar for represents $40 \mu \mathrm{m}$. (C) Representative axons of hippocampal neurons transfected with KDEL-RFP and MYC-GABA ${ }_{B}$ R1a, or MYC-GABA ${ }_{B}$ R1a-AA-ASA, or MYC-GABA ${ }_{B}$ R1a- $\Delta$ C, and processed for immunofluorescence under permeabilized conditions. MYC-GABA ${ }_{B} \mathrm{R} 1 \mathrm{a}$ versions were detected with MYC antibodies (MYC-GB1a, MYC-GB1a-AA-ASA, MYC-GB1a- $\Delta$ C); KDEL-RFP was visualized without staining (representative images of $\mathrm{n}=20,9$ and 10 neurons respectively). Merged images are shown on the bottom panels. Scale bar represents $20 \mu \mathrm{m}$. (D) Hippocampal neurons were transfected with MYC-GABA ${ }_{B} \mathrm{R} 1 \mathrm{a}-\Delta \mathrm{C}$ and RFP (left, representative image of $\mathrm{n}=12$ neurons) or MYC-GABA ${ }_{B}$ R1a- $\Delta$ C and Kif5C-RFP-DN (Kif5C-DN, right, representative image of $n$ $=9$ neurons). The neuronal volume filled by RFP or Kif5C-RFP-DN is shown. Boxes in top panels correspond to regions of axons $(\mathrm{Ax})$ and dendrites (unlabelled) magnified below. MYC$\mathrm{GABA}_{B} \mathrm{R} 1 \mathrm{a}-\Delta \mathrm{C}$ (green) and RFP or Kif5C-RFP-DN (red). Merged images are shown on the bottom panel. Scale bar represents $40 \mu \mathrm{m}$. 
localization of $\mathrm{GABA}_{B} \mathrm{R} 1 \mathrm{a}$. In addition, they indicate that the C-terminal domain of $\mathrm{GABA}_{B} \mathrm{R} 1 \mathrm{a}$, which is exposed to the cytosol, is not involved in this transport mechanism, further supporting the role of luminal or ER membrane domains in axonal sorting and targeting.

\section{\begin{tabular}{l|l} 
Discussion & 7.4
\end{tabular}}

We have shown that $\mathrm{GABA}_{B}$ Rs require the Golgi apparatus for plasma membrane delivery. Importantly, to our knowledge we have demonstrated for the first time that the sorting and targeting of an axonal neurotransmitter receptor, namely the $\mathrm{GABA}_{B} \mathrm{R} 1 \mathrm{a}$ subunit, occur in a pre-Golgi compartment. Consistent with these observations, our evidence points to the fact that $\mathrm{GABA}_{B} \mathrm{R} 1 \mathrm{a}$ traffics along the axonal ER and the ERGIC, and is transported by the molecular motor kinesin-1.

\section{Pre-Golgi sorting and targeting of axonal $\mathrm{GABA}_{B} \mathrm{R} 1 \mathrm{a}$}

Our study indicates that axonal sorting of $\mathrm{GABA}_{B} \mathrm{R} 1$ subunits operates in the ER. As reported elsewhere, axonal targeting of $\mathrm{GABA}_{B} \mathrm{R} 1 \mathrm{a}$ is unaltered in $\mathrm{GABA}_{B} \mathrm{R} 2$ knock-out neurons [Biermann10]. Additionally, the sushi domains located at the N-terminus of $\mathrm{GABA}_{B} \mathrm{R} 1 \mathrm{a}$, are sufficient for targeting even when placed in a nonrelated $\mathrm{CD} 8 \alpha$ protein context [Biermann10]. Combined with the results presented here these observations imply that sorting and targeting signals exposed to the ER lumen mediate the axonal localization of $\mathrm{GABA}_{B} \mathrm{R} 1 \mathrm{a}$.

Historically, sorting of plasma membrane proteins to distinct membrane domains has been thought to occur exclusively at the Golgi or the trans-Golgi network, but accumulated evidence now favors the view that decisions are made at almost every step along the secretory pathway including the ER [Rodríguez-Boulan05]. For example, mutations in Sec24p, a component of the coat protein complex II (COPII), selectively disrupt recruitment of cargo for ER to Golgi transport [Miller03]. Likewise, synthetic cell penetrating peptides based on the cytosolic domain of the temperaturesensitive VSVG protein only inhibits the transport of a subset of cargo from the ER to the Golgi apparatus [Soza04].

Two possible targeting mechanisms are conceivable for $\mathrm{GABA}_{B} \mathrm{R} 1 \mathrm{a}$ in axons: (i) a luminal or membrane spanning ER protein enriched in the axonal ER subcompartment may bind the sushi domains and produce the accumulation of $\mathrm{GABA}_{B} \mathrm{R} 1 \mathrm{a}$ but not $\mathrm{GABA}_{B} \mathrm{R} 1 \mathrm{~b}$, in the axon (selective retention); or (ii) a protein or protein complex that spans the ER membrane may function as an adaptor between the $\mathrm{GABA}_{B} \mathrm{R} 1 \mathrm{a}$ subunit and a molecular motor and selectively direct the transport of the subunits to axons (selective transport). Additional axonal scaffolding proteins may anchor the $\mathrm{GABA}_{B} \mathrm{R} 1 \mathrm{a}$ subunit to strengthen axonal localization.

Although we cannot rule out the first alternative our data are consistent with a selective transport mechanism. Axonal targeting of $\mathrm{GABA}_{B} \mathrm{R} 1 \mathrm{a}$ is not altered by its cytosolic C-terminal. Thus, transport may be controlled by a specific $\mathrm{GABA}_{B} \mathrm{R} 1 \mathrm{a}$ N-terminal adaptor or by a general ER adaptor complex. Identification of these molecules in future studies is needed to strengthen this hypothesis. A mechanism 
compatible with (i) has been described for the rotavirus VP7 glycoprotein, which is retained in the ER. VP7 is still transported to the axon after BFA treatment [Weclewicz93]. Similar to GABA $_{B}$ R1a, VP7 uses a Golgi-independent intracellular sorting mechanism to reach the axon. One may envision the participation of chaperones in ER transport and targeting. For example, Hsc70 may provide a mechanism to release kinesin from cargo in specific subcellular domains, thereby producing the delivery of axonally transported cargo [Terada10].

\section{Kinesin-1-dependent ER transport of $\mathrm{GABA}_{B} \mathrm{R} 1 \mathrm{a}$ in axons}

Since $\mathrm{GABA}_{B} \mathrm{R} 1$ is transported along the $\mathrm{ER}$ and has a limited residency period within the organelle under physiological conditions we refer to it as an ER-boarded protein. The precise transport mechanism of ER-boarded $\mathrm{GABA}_{B} \mathrm{R} 1 \mathrm{a}$ in the axon is not clear. A kinesin-1-dependent transport of ER proteins has been described in dendrites [Bannai04]. Likewise, a microtubule-dependent transport of two ER resident proteins in the axon, GFP-SERCA and GFP-IP ${ }_{3} \mathrm{R}$, has been observed in cultured chick dorsal root ganglion neurons [Aihara01]. Their bi-directionality and average velocities $(\sim 0.1 \mu \mathrm{m} / \mathrm{s})$ suggest that the transport is likely non-vesicular, and may represent lateral displacement within the continuous axonal ER membrane or mobility of the organelle itself. Directionality at steady state and average velocities observed for GFP-SERCA and GFP-IP ${ }_{3}$ R range between slow (0.001- 0.03 $\mu \mathrm{m} / \mathrm{s})$ and fast axonal transport $(\sim 1 \mu \mathrm{m} / \mathrm{s})$ [Aihara01, Shah10]. These intermediate velocities are conserved in our study suggesting that similar mechanisms may operate for the transport of ER-boarded $\mathrm{GABA}_{B} \mathrm{R} 1 \mathrm{a}$ in axons. A conserved transport mechanism is also supported by the microtubule dependence of axonal GFP-SERCA, GFP-IP ${ }_{3} \mathrm{R}$ and $\mathrm{GABA}_{B} \mathrm{R} 1 \mathrm{a}$ mobility. Thus, as reported for ER resident proteins at least one component of the mobility of $\mathrm{GABA}_{B} \mathrm{R} 1 \mathrm{a}$ is not mediated by simple diffusion. However, precisely how the microtubule-cytoskeleton mediates the movement of integral membrane proteins within the continuous ER network is still unclear. According to the mechanisms originally put forward by Tsukita and Ishikawa [Tsukita76], Waterman-Storer and Salmon [Waterman-Storer98] and others [Woźniak09] kinesin-1 may control the lateral displacement of the subunit along the ER membrane in a conveyor-like system or mediate the microtubule-dependent ER sliding of the organelle itself, which may contribute to $\mathrm{GABA}_{B} \mathrm{R} 1 \mathrm{a}$ transport. Since components of both mechanisms are dependent on kinesin-1 and stable microtubules, it is difficult to discriminate between them. Because in this study we wanted to eliminate microtubules that serve as tracks to kinesin-1, the concentrations of nocodazole used were $\sim 15$ fold higher than those that affect exclusively the population of dynamic microtubules $(100 \mu \mathrm{M}$ versus $\sim 6 \mu \mathrm{M})$. Thus, additionally, we cannot rule out the contribution of dynamic microtubules to the transport of $\mathrm{GABA}_{B} \mathrm{R} 1 \mathrm{a}$ in the axon [Waterman-Storer98]. The existence of discrete mobile packets for axonal $\mathrm{GABA}_{B} \mathrm{R} 1 \mathrm{a}$ also raises the possibility that these are not continuous with the ER network. Electron microscope studies in central and peripheral axons have revealed that the structure of the ER is predominantly contiguous but that it contains occasional free elements [Tsukita76, Broadwell84]. Additionally, isolated and mobile compartments have been observed with fluorescent reporters [Aihara01]. Puncta-like behavior may also be explained by transitory tubule fis- 
sion/fusion events [Tsukita76] or lateral mobility of protein aggregates. The precise relationship between the mobile structures observed in this and other studies with the continuous ER network requires further studies. Nonetheless, our results support a kinesin-1-mediated axonal transport of ER-boarded $\mathrm{GABA}_{B} \mathrm{R} 1 \mathrm{a}$ that is in agreement with previous reports.

The majority of the axonal $\mathrm{GABA}_{B} \mathrm{R} 1 \mathrm{a}$ subunit was static during the live imaging conditions and intervals examined in this study. Additionally, the mobile fraction was higher for a mutant subunit that escapes the ER (MYC-GABA ${ }_{B}$ R1a-EGFP: 19 $\%$ mobile puncta, $\mathrm{n}=259$; MYC-GABA ${ }_{B}$ R1a-ASA-AA-EGFP $33 \%$ mobile puncta, $\mathrm{n}=247$ ). It will be interesting to understand the significance and properties of the static component of $\mathrm{GABA}_{B} \mathrm{R} 1 \mathrm{a}$ in the ER, and whether ER immobility plays a role in processing or trafficking.

Previous studies have demonstrated that $\mathrm{GABA}_{B} \mathrm{Rs}$ are segregated intracellularly and that blockade of ER exit results in the accumulation of heteromers in the soma and dendrites of hippocampal neurons [Ramírez09]. These results suggest that $\mathrm{GABA}_{B} \mathrm{R}$ subunits are transported into dendrites independently and not as assembled heteromers. They also suggest that newly synthesized GABA $_{B}$ Rs assemble in the ER and exit throughout the somatodendritic compartment prior to insertion at the plasma membrane. This idea is in agreement with transport within the ER and disagrees with a long-haul post-Golgi vesicular transport of $\mathrm{GABA}_{B} \mathrm{Rs}$, suggesting a non-canonical trafficking modality for $\mathrm{GABA}_{B} \mathrm{Rs}$ in dendrites. Thus, long-range ER transport may underlie both dendritic and axonal targeting of $\mathrm{GABA}_{B}$ Rs. Since the local complexity of the ER network influences intracellular trafficking it may be utilized by neurons to control the availability of dendritic and axonal membrane proteins that are spatially restricted [Ramírez11, Cui-Wang12].

\section{Axonal trafficking and ER/ERGIC recycling}

In central and peripheral axons the ER is a continuous three-dimensional network of irregular tubules and cisternae [Tsukita76, Broadwell84]. As mentioned above, several studies have demonstrated the localization of ER resident proteins and components of the protein folding and export machineries in the axon [Merianda09, Weclewicz98]. More importantly, the discovery of local assembly of COPII components in the axon supports the existence of functional ER exit sites required to process newly synthesized proteins that contribute to axonal outgrowth during the early stages of development [Aridor09]. However, direct evidence for local protein trafficking within early biosynthetic organelles in the axon is still lacking. In Drosophila, polarized secretion of the EGFR ligand from photoreceptor neurons includes local processing and secretion in the axon, and both mechanisms are controlled by ER localization [Yogev10].

More functional evidence is needed to conclusively demonstrate the role of a local trafficking pathway for $\mathrm{GABA}_{B} \mathrm{R} 1 \mathrm{a}$ in axons. However, a fraction of $\mathrm{GABA}_{B} \mathrm{R} 1 \mathrm{a}$ colocalizes and moves in synchrony with the ERGIC. This indicates that a local route involving export and retrieval between early biosynthetic organelles may contribute to $\mathrm{GABA}_{B} \mathrm{R} 1 \mathrm{a}$ trafficking in the axon. A coat protein I complex (COPI) dependent retrieval mechanism from the cis-Golgi to the ER has been postulated for 
GABA $_{B}$ R1a [Brock05, Villemure05]. Whether this occurs in the axon and whether the remaining secretory steps operate locally to deliver $\mathrm{GABA}_{B} \mathrm{R} 1 \mathrm{a}$ to the axonal plasma membrane remains unclear. In any case, the presence of the same RXR-type ER retention motif in $\mathrm{GABA}_{B} \mathrm{R} 1 \mathrm{a}$ and $\mathrm{GABA}_{B} \mathrm{R} 1 \mathrm{~b}$ suggests that despite determining a rate-limiting step for ER exit, dwell time in the ER is not a major determinant of spatial range and axonal localization of $\mathrm{GABA}_{B} \mathrm{R} 1 \mathrm{a}$ [Herpers04].

Overall our study demonstrates that ER sorting and local transport are relevant for axonal $\mathrm{GABA}_{B} \mathrm{R}$ trafficking. It is of great interest to determine to what extent the axonal ER is involved in the trafficking of other neurotransmitter receptors and ion channels that travel long distances, especially in long peripheral nerves.

\section{Materials and Methods 7.5

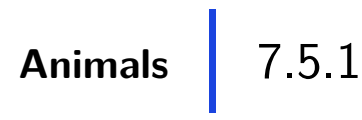

Adult pregnant female Sprague-Dawley rats were purchased from the Central Animal Facility at Universidad Católica de Chile and killed by asphyxia in a $\mathrm{CO}_{2}$ chamber according to the Guide for Care and Use of Laboratory Animals (The National Academy of Sciences, 1996). GABA ${ }_{B}$ R1-EGFP mice were kindly provided by Bernhard Bettler (University of Basel, Switzerland). They correspond to transgenic animals for the $\mathrm{GABA}_{B} \mathrm{R} 1$-EGFP BAC in a homozygous knockout background for $\mathrm{GABA}_{B} \mathrm{R} 1$ as described previously [Schuler01, Casanova09].

\section{Cell lines, neuronal cultures and transfection $\mid 7.5 .2$}

COS-7 cells were maintained and transfected as described previously [Couve98] using a Gene Pulser Xcell (BioRad). Primary hippocampal neurons were cultured from E18 rats or E18 GABA $_{B}$ R1-EGFP transgenic mice according to established procedures [Goslin91] and transfected by $\mathrm{Ca}^{2+}$ phosphate at $14-18$ days in vitro (div) [Jiang06]. All transfected neurons, except for those in Supplementary Fig. E.1, were analyzed 1 day post transfection (dpt). Transfected neurons for Supplementary Fig. E.1 were analyzed between $1-5$ dpt.

\section{Reagents and DNA plasmids $\mid 7.5 .3$}

Nocodazole was purchased from Sigma (St. Louis, MO). MYC-GABA ${ }_{B}$ R1, FLAGGABA $_{B}$ R2, HA-GABA ${ }_{B}$ R2, MYC-GABA ${ }_{B}$ R1-AA-ASA, MYC-GABA ${ }_{B}$ R1- $\Delta$ C in pRK5 have been described previously and contain epitope tags on the extracellular N-terminal domains [Couve98, Calver01, Couve02, Restituito05]. MYC-GABA ${ }_{B}$ R1AA-ASA contains point mutations that replace two leucine residues at a di-leucine motif and two arginine residues at the ER retention motif (RSRR) by alanines. 
MYC-GABA ${ }_{B}$ R1- $\Delta$ C lacks the complete C-terminal domain. Both mutants escape the ER and traffic to the cell surface in the absence of $\mathrm{GABA}_{B} \mathrm{R} 2$. GABA ${ }_{B}$ R1aEGFP, GABA ${ }_{B}$ R2-EGFP and GABA $_{B}$ R1a-monomeric red fluorescent protein have also been described previously and contain the fluorescent proteins attached to the intracellular C-terminal domain [Ramírez09]. pDsRed-C1 (RFP), pEYFP-Golgi, pEYFP-ER and pDsRed2-ER (KDEL-RFP) were obtained from Clontech (Mountain View, CA). Kif5C-RFP-DN was kindly provided by S. Kindler and H. J. Kreienkamp (Institut für Humangenetik, Universitätsklinikum Hamburg-Eppendorf, Hamburg, Germany) and corresponds to amino acids 678 - 955 of KIF5C (NM-001107730) also referred to as DN2 by Falley and collaborators [Falley09]. ARF1-Q71I-HA was kindly provided by O. Jeyifous (University of Chicago, Chicago, IL), Rab11-GFP was kindly provided by F. Bronfman (Pontificia Universidad Católica de Chile), p58YFP was kindly provided by J. Lippincott-Schwartz (National Institutes of Health, Bethesda, MD). Cytochrome b5-EGFP was kindly provided by C. Hetz (Universidad de Chile). All manipulations and fidelity of DNA constructs were verified by sequencing.

\section{Antibodies $\quad 7.5 .4$}

$\mathrm{GABA}_{B} \mathrm{R} 1$ antibodies (which recognize $\mathrm{GABA}_{B} \mathrm{R} 1 \mathrm{a}$ and $\mathrm{GABA}_{B} \mathrm{R} 1 \mathrm{~b}$ ) were raised against the intracellular C-terminal domain in rabbits and affinity purified. GA$\mathrm{BA}_{B} \mathrm{R} 1$ antibodies specifically recognize MYC-GABA ${ }_{B} \mathrm{R} 1 \mathrm{a}$ in transfected COS cells and detect the predicted doublet corresponding to $\mathrm{GABA}_{B} \mathrm{R} 1 \mathrm{a}$ and $\mathrm{GAB}_{B} \mathrm{R} 1 \mathrm{~b}$ in crude brain membranes (Supplementary Fig. E.3). Microtubule-associated protein 2 (MAP2) antibodies were purchased from Chemicon (Temecula, CA). Piccolo antibodies were kindly provided by E. D. Gundelfinger and W. D. Altrock (Leibniz Institute for Neurobiology, Magdeburg Germany). KDEL antibodies (directed against a 6-residue peptide (SEKDEL) of the rat Grp78 protein) and cis-Golgi matrix protein 130 (GM130) were purchased from StressGen (Ann Arbor, MI). MYC antibodies were purchased from Sigma (St. Louis, Missouri). Influenza A Virus epitope (HA) antibodies were purchased from Roche (Indianapolis, IN). Anti-GFP antibodies (ab6556) were purchased from Abcam (Cambridge, UK). Secondary anti-mouse, anti-rabbit, anti-guinea pig or anti-chicken antibodies conjugated to Texas Red (TR), tetramethyl rhodamine isothiocyanate (TRITC), fluorescein isothiocyanate (FITC) or cyanine 3 (Cy3) were purchased from Jackson Immuno Research Laboratories (West Grove, PA).

\section{Immunofluorescence, image capture and processing, and time-lapse microscopy

Immunofluorescence was performed as described previously under non-permeabilized or permeabilized conditions [Ramírez09]. Depending on the type of experiment axons were identified by the presence of a positive marker (Tau), the absence of a negative marker (MAP2), or by morphological criteria that included: longer projection, constant diameter and right angle branching. Image-processing routines were 
developed in our laboratory based on of Interactive Data Language (IDL) (ITT, Boulder, CO), including routines for segmentation [Ramírez09]. Live-cell imaging was performed in a $23{ }^{\circ} \mathrm{C}$ equilibrated microscopy suite. Images were obtained using an Olympus BX61WI upright microscope and an Olympus disk-scanning unit. Consecutive frames were acquired over a period of $\sim 120$ s. Kymographs were constructed from an axonal segment $20 \mu \mathrm{m}$ from the soma using ImageJ from threepixel-wide axonal traces. The axon-to-dendrite (A:D) ratio of $\mathrm{MYC}^{-G A B A}{ }_{B} \mathrm{R} 1 \mathrm{a}$ was determined using ImageJ according to a procedure based on Biermann et al., 2010 and modified [Biermann10]. Briefly, one-pixel-wide lines were traced along the initial $150 \mu \mathrm{m}$ of axons and dendrites in images labeled with soluble RFP or Kif5C-RFP-DN. No measurements were carried out beyond $150 \mu \mathrm{m}$ to prevent artifacts due to axonal length differences between RFP or Kif5C-RFP-DN transfected neurons. Average pixel intensities of $\mathrm{MYC}-\mathrm{GABA}_{B} \mathrm{R} 1 \mathrm{a}$ were determined along the traced lines, background was subtracted and the data was used to determine the A:D ratio. Criteria for cell selection included even distribution of RFP or Kif5C-RFPDN in axons and dendrites, and cells expressing constructs at very high levels were excluded from the analysis. 9 - 12 neurons from at least two independent culture preparations were analyzed for each condition. Images of axons from $\mathrm{GABA}_{B} \mathrm{R} 1-$ EGFP mice were acquired using an Olympus FV-1000 confocal microscope. AntiGFP antibodies were used to amplify the weak axonal signal of GABA $_{B}$ R1-EGFP expressed at physiological levels.

\section{Total Internal Reflection Fluorescence (TIRF) microscopy $\mid 7.5 .6$}

For TIRF microscopy 14 - 18 div hippocampal neurons were transfected with GA$\mathrm{BA}_{B} \mathrm{R} 1 \mathrm{a}-\mathrm{EGFP}$ and motility in axons was analyzed $1 \mathrm{dpt}$. TIRF was carried out on a custom-built TIRF microscope setup: Two lasers (473 nm and $532 \mathrm{~nm}$, both $30 \mathrm{~mW}$, Viasho, USA) were used to excite the fluorophores (GFP and RFP). The lasers were expanded and coupled via a multi-beam splitter (z474/488/532/635rpc, Chroma, USA) off-axis into the oil-immersion objective (Nikon, SFluor $100 \times, 1.49$ ) to obtain TIRF illumination. The emitted fluorescent light was split in the GFP and RFP signals using a dichroic mirror (525/50, Chroma, USA) then passed trough bandpass filters (530/50 for GFP and 605/70 for TMR, both Chroma, USA) and finally directed via mirrors to separate areas on the chip of a frame transfer CCD camera (Cascasde:512B, Roper Scientific, USA). The CCD camera was controlled via WinSpec32 (Princeton Instruments, USA). The penetration depth of TIRF was $\sim 147 \mathrm{~nm}$. Digital images were taken at a frame rate of 2 frames/s and were subsequently analyzed for velocity and direction using kymographs generated with a custom-written LabView (National Instruments, USA) routine. Kymographs were analyzed for velocity and direction by fitting lines to the segments of a trace judged by eye. Stalls were not taken into account. 


\section{\begin{tabular}{l|l} 
Acknowledgements & 7.6
\end{tabular}}

We thank B. Bettler, F. Bronfman, O. Jeyifous, S. Kindler, H. J. Kreienkamp, C. Hetz and J. Lippincott-Schwartz for valuable reagents.

\section{Supplementary data}

Supplementary data associated with this article is given in appendix E and will soon be available online at PLoS ONE where the article is currently in print. 


\section{Conclusion}

The aim of this thesis was to gain further insight into the regulation of motor proteins from the kinesin subfamily kinesin-5. In the following chapter, the main results and new findings concerning the regulation of kinesin- 5 in general, as well as Cin8 and Eg5 in particular, will be shortly summarised and discussed.

\section{New findings on the regulation of Cin8}

Simultaneously coinciding with our publication [Gerson-Gurwitz11], Roostalu et al. observed switching in direction for multiple Cin8 motor proteins in multi-motor gliding assays which was dependent on salt and Cin8 concentration [Roostalu11]. They claimed this switching to be a collective effect of many motor proteins working together under physical load (that is, MTs) [Roostalu11]. In contrast to that study, we reported in chapters 2 and 3 for the first time that the direction of individual kinesin-5 Cin8 motor proteins can be switched by varying ionic buffer conditions between processive minus- and plus-directed movements when travelling on single MTs [Gerson-Gurwitz11, Thiede12a]. An even more dramatic switching process in Cin8 from fast processive minus-end motion to plus-end force generating motion occurred in high-salt conditions upon binding and crosslinking two antiparallel MTs [Gerson-Gurwitz11].

Even though we could show that Cin 8 motility is also influenced by other factors like phosphorylation, binding of other proteins, and manipulation of a Cin8 specific 99 aa-long domain, we proposed that the main switching mechanism of Cin8 directionality is the crosslinking of two antiparallel MTs.

In the cell, under high-ionic-strength conditions [Olz93, Eunen10], the fast processive plus-end directed motion could aid in bringing Cin8 to the SPBs when it is bound to a single MT. It was shown previously that near the SPBs, Cin8 is important for kinetochore clustering or positioning, in which it is proposed to assume the role of crosslinking kinetochore MTs and disassembling long kinetochore MTs [Tytell06, Gardner08a, Wargacki10]. Since in S. cerevisiae cells, each kinetochore is attached to a single MT, active motion of Cin8 in the minus-end direction of the kinetochore MTs may be an alternative or additional mechanism by which Cin8 contributes to kinetochore positioning. The other mode of Cin8 directionality can be advantageous in the midzone of the mitotic spindle: when Cin8 crosslinks two antiparallel MTs, the plus-end force generating motion might be switched "on", promoting plus-end directed MT sliding and spindle elongation.

An additional advantage of bi-directionality in Cin8 is the possibility to dynamically partition Cin 8 motor proteins between different reservoirs, that is, near the spindle poles and in the midzone. In agreement with that notion, we observed in vivo that until anaphase spindles reach a length of $\sim 5 \mathrm{~mm}$, Cin8 is localised throughout the 
spindle, with neither preferential accumulation at the midzone or near the spindle poles, nor obvious detachment [Gerson-Gurwitz11]. This even distribution without detachment from the MTs might be established by bi-directional motility of Cin8.

Even though Cin8 switching introduced by crosslinking of two antiparallel MTs in high-ionic-strength conditions is a strong regulation mechanism in vitro, it is clear that many other mechanisms and interactions inside the cell are involved in Cin 8 regulation in the context of the highly complex and tightly regulated process of mitotic cell division.

\section{New findings on the regulation of Eg5}

In chapter 4, a kinesin-5 head/kinesin-1 stalk chimera (Eg5Kin) was used as a model system to study the regulation of Eg5 motility. In this chimera the complexity of motility seen for WT Eg5 [Kwok06, Kapitein08] is reduced by avoiding the proposed regulatory domains and the additional pair of motor domains of the WT tetramer. In a study from Valentine et al. they used a truncated dimeric human Eg5 (hsEg5) construct and found that dimeric hsEg 5 takes on average only eight processive steps. From this result Valentine et al. concluded that Eg5 is a motor protein with only a modest run length [Valentine06]. Although we used the Eg5 form Xenopus leavis in our study, the motor domain is very similar to that of hsEg5 [Dagenbach04]. We found that Eg5Kin is a highly processive motor protein with a run length of $\sim 1.9$ $\mu \mathrm{m}$ and could thereby show that at least the Eg5 motor domain itself is - fused to a stable dimer - capable of performing highly processive motion [Lakämper10]. Unlike Eg5, Eg5Kin showed no diffusive episodes in its motility, adding to the evidence that the domains mediating this diffusive interaction in Eg5 are located outside of the motor domain.

When taken together, our findings suggest that WT Eg5 is highly (down)regulated in its native tetrameric form, which might be useful in the context of the mitotic spindle, where various motility processes have to be carefully balanced and adjusted in the different stages of cell division.

A second aspect of this study was the mechanism of Eg5 inhibition by the kinesin5 specific inhibitor monastrol [Mayer99]. We could show that monastrol does not affect the velocity of Eg5Kin and that binding of two monastrol molecules to the dimeric motor protein is required to terminate it's processive run [Lakämper10]. This indicates that the processive stepping of Eg5 is only affected when to each Eg5 motor domain a monastrol molecule is bound. The exact molecular mechanism leading to Eg5 inhibition through monastrol is to date not fully understood and is the subject of on-going research [Liu11].

In chapter 5, we used the kinesin-5 head/kinesin-1 stalk chimera Eg5Kin as basis for a study of the controlled reintroduction of one of the motor protein's proposed regulatory elements, that is, the neck-linker length. The variation in neck-linker length for a set of twelve chimeric motor-protein constructs based on Eg5Kin showed that neck-linker length influences neither the velocity nor the force generation of the 
constructs. However, it does influence the run length [Düselder12].

In a recent study, Shastry et al. proposed a simple mechanistic model for the regulation of processivity in kinesins: the shortest neck linker (they proposed a minimal length of $14 \mathrm{aa}$ ) leads to the tightest communication between the motor domains and results in the longest run length [Shastry11]. Our study contradicts this simple model with two findings: First, constructs with 13- and 12-aa-long neck linker domains were still capable of processive motility. Second, we found the longest run length with the motor-protein construct containing an 18-aa-long neck linker, which is the native neck-linker length of WT Eg5 [Düselder12].

After finding that the Eg5 motor domain seems to be optimised for it's native necklinker length of 18 aa and considering that the neck-linker lengths range from 14 to 18 aa in the kinesin family, we proposed that different kinesins might achieve optimal communication with different neck-linker lengths. In the light of the diverse functions, structures and regulation mechanisms found for kinesins, a single and general parameter - like the neck-linker length - for the degree of processivity seems unlikely and a simple general model applying to all processive kinesins might not exist. The native neck-linker length and sequence might, in each case, be optimised for both effective communication and processivity as well as other specific regulatory functions. Nevertheless, processive motility requires coordination between the motor domains and if this is not dominated by tension, then orientation of the neck linker might play a crucial role, as recently proposed by Clancy et al. for motorprotein constructs with largely elongated neck-linker domains [Clancy11].

In chapter 6 , we used a tetrameric kinesin-1 head/kinesin-5 stalk chimera (DK4mer) as a model system to investigate details of the multiple MT-binding modes of tetrameric kinesins which are believed to be involved in the regulation of the motor proteins. This motor-protein construct is the inverse one to Eg5Kin described above. DK4mer exhibited fast, processive motility and was able to crosslink MTs and slide antiparallel MTs apart [Thiede12b]. Additionally, the tetrameric chimera showed an alternation of short diffusive and long processive episodes during MT interaction. Interestingly, a truncated version of DK4mer (DK511), missing the Eg5 tail domains, did not exhibit any diffusive episodes in it's processive motility. Based on these findings, we proposed that the processive MT interaction is driven by the motor domains and the diffusive attachment is caused by the tail domains, likely by electrostatic interactions.

Considering that DK4mer features Eg5 tail domains, we were able to find more evidence that the domains mediating it's diffusive interaction in Eg5 are located outside of the motor domain, and probably in the tail domain. This notion is supported by a recent study from Weinger et al. showing that in Eg5 the tail domain is required for proper and efficient orientation and function of Eg5 when it crosslinks and orients MTs in vivo and in vitro [Weinger11].

\section{New findings on the regulation of kinesin-5 in general}

Until now, it was generally accepted in the field of motor proteins that all N-terminal members of the kinesin family move to the plus-end of the MT [Dagenbach04, Lawrence04]. Minus-end motion was only seen for C-terminal kinesin motor pro- 
teins, now grouped in the subfamily kinesin-14 [McDonald90, Walker90, Castro00, Block07]. WT Kinesin-14 motor proteins, like ncd from Drosophila melanogaster, move in a non-processive fashion by producing isolated power strokes [Sablin98]. These motor proteins can only produce persistent motion when they act in ensembles.

The study of Cin8 showed an entirely novel behaviour for kinesin motor proteins, since this is the first time that an N-terminal kinesin has been shown to move to the minus end of the MT and it is also the first report of processive minus-end directed motility.

Interestingly, higher eukaryotes appear not to have kinesin-5 motor proteins capable of minus-end motility, so this function might have evolved early and might have been lost in higher eukaryotes. The exact molecular mechanism inducing the switch in directionality remains to be clarified, but it seems likely to be related to cargo switching known for other kinesins, like the binding of a cargo vesicle to kinesin-1 or binding of a second MT to kinesin-5 Eg5.

The studies of different chimeric motor-protein constructs - in order to gain further insight into the regulation of Eg5 - have showen that a simple mechanical model for the regulation of processivity of kinesins, as proposed by Shastry et al., might not exist. Consistent with their diverse roles in the cell and the different native neck-linker lengths, each type of kinesin might have a different specifically optimised mechanism to regulate it's processivity.

Altogether, the work presented here has revealed further insights into in the mechanisms of kinesin-5 regulation and demonstrates that in order to fulfil their physiological functions, kinesin motor proteins are much less rigidly programmed than was broadly believed and are even more complex than we thought. After the discovery of kinesin motor proteins almost 20 years ago, the concluding sentence from a paper by Scholey et al. [Scholey03] does still hold:

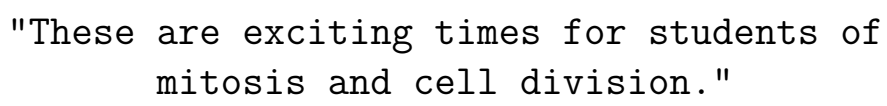




\section{Danksagung/Acknowledgements}

An erster Stelle möchte ich Professor Christoph F. Schmidt dafür danken, dass er mir diese Doktorarbeit mit interessanten Kollaborationen und Projekten ermöglicht hat. Dank geht auch an die weiteren Mitglieder meines Betreuungsausschusses Professor Jörg Enderlein und Professor Helmut Grubmüller dafür, dass sie sich die Zeit genommen haben, den Fortschritt meiner Doktorarbeit zu überprüfen und mit mir zu diskutieren.

The most important collaboration for this thesis was the one with Dr. Leah Gheber and Dr. Andina Guerson-Gurwitz from the Ben-Gurion University of the Negev in Beer-Sheva, Israel (chapters 2 and 3). Thank you very much for the exciting month in Israel and the fruitful collaboration.

In weiteren Kollaborationen habe ich mit Stefan Lakämper (Kapitel 4, 5 und 6) und Carolina Otero (Kapitel 7) gearbeitet. Vielen Dank an euch für die Zusammenarbeit.

Ich möchte mich auch bei unseren TAs Charlotte Willms, Kerstin von Roden und Ulrike Schulz bedanken, ohne deren engagierte Arbeit der „biologische Teil“in unserer Arbeitsgruppe undenkbar wäre und die immer mit Rat und Tat oder einem aufmunterndem Wort zur Stelle waren.

Auch bei Dieter Hille und seinem Team aus der Feinmechanikwerkstatt möchte ich mich für die unkomplizierte Hilfe bedanken. Ebenso wie bei unserem Computerfachmann Thomas Geiling, der bei Computer-Problemen immer sofort zur Stelle war.

Um 12:30 \pm 15 Minuten ist Mensa-Zeit: dieser feste Termin mit den Kollegen hat geholfen, den täglichen Wahnsinn besser zu ertragen. In der guten Atmosphäre der Arbeitsgruppe waren sowohl ernste Diskussionen als auch lockere Gespräch möglich. Besonderer Dank geht dabei an André Düsselder - einem der letzten Mitstreiter in der unbarmherzigen Welt der Motorproteine...

Für die - inzwischen fast Jahrzehntelange - Unterstützung während meiner Zeit an der Universität Göttingen möchte ich mich bei meinen Eltern und meinen beiden Geschwistern bedanken.

Mein größter Dank gilt aber meinem Ansgar, der mich besonders in der „letzten Phase"meiner Doktorarbeit immer wieder bestärkt und aufgebaut hat. 

Appendices 



\section{Supplemetatry data chapter 2: Directionality of individual kinesin-5 Cin8 motors is modulated by loop 8, ionic strength and microtubule geometry}

This is the supplementary data for the chapter 2 titled "Directionality of individual kinesin-5 Cin8 motors is modulated by loop 8, ionic strength and microtubule geometry". It can also be found online at the EMBO Journal, doi:10.1038/ emboj.2011.403.

\section{Supplementary figures}

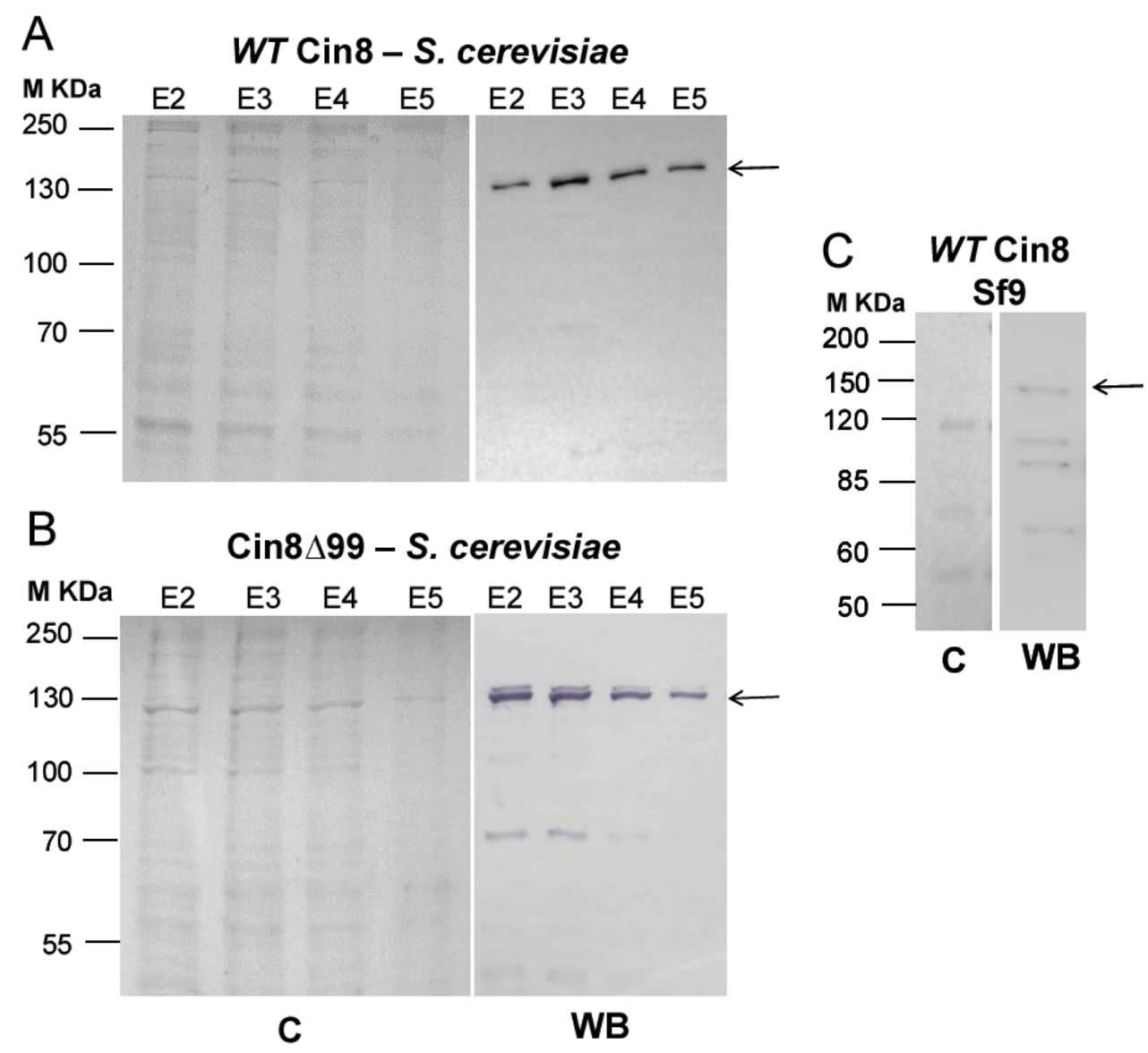

Fig. A.1: Affinity-purification of Cin8 and Cin8 499 . (A - B) S. cerevisiae-expressed Cin8-GFP (A) and Cin8 $\triangle 99$-GFP (B) were partially purified by nickel-affinity column. (C) Sf9-expressed Cin8-GFP was partially purified by MT-affinity column. Arrows indicate the expected size of Cin8. C: Coomassie blot; WB: Western blot; M KDa: molecular weight marker. 

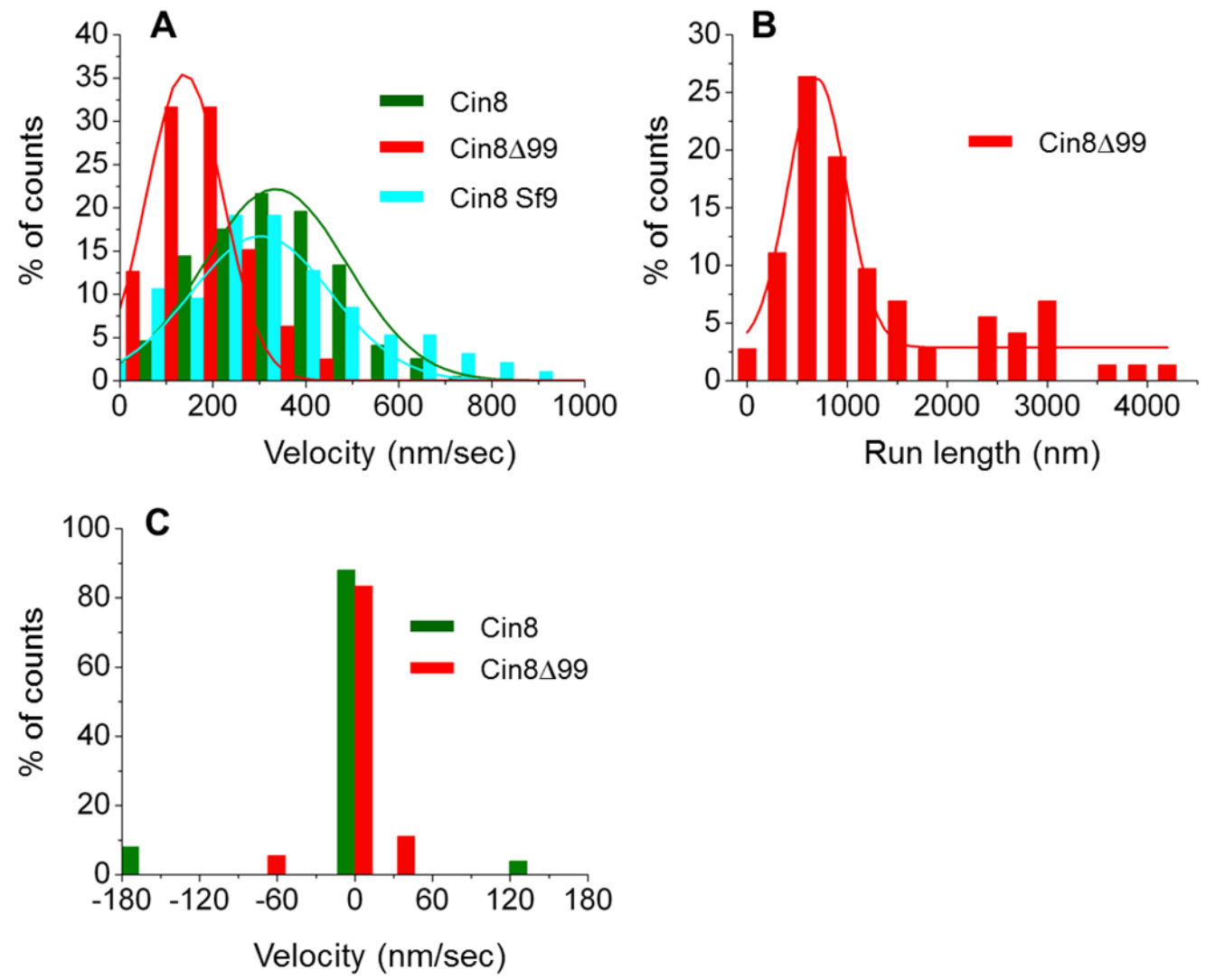

Fig. A.2: Motile properties of Cin8 and Cin8 899 . (A) Histogram of velocities of Cin8 and Cin8 $\Delta 99$ in whole-cell yeast extracts and of MT-affinity purified Cin8 from Sf9 cells. (B) Histogram of run lengths of Cin8 $\Delta 99$ in whole-cell yeast extracts. Measurements were applied on directional episodes in kymograph tracks in different buffer conditions; For Cin8 in whole-cell yeast extract, buffer contained $175 \mathrm{mM} \mathrm{NaCl}$ (MB-175). For Cin8 $\Delta 99$ in whole-cell yeast extract, buffer contained $30 \mathrm{mM} \mathrm{NaCl}$ (MB-30). For MT-affinity purified Cin8 from Sf9 cells, buffer contained $150 \mathrm{mM} \mathrm{NaCl}$ (MB-150). Fit: Gaussian distribution. (C) Control experiments for the distribution of Cin8 and Cin8 899 velocity along polaritymarked MTs in the presence of $3 \mathrm{mM}$ ADP (see Fig. 2.4 and Fig. 2.5, respectively). Analysis was carried out by $3 \mathrm{~s}$ segments along kymographs of runs in buffer with $30 \mathrm{mM}$ NaCl. (A - C) Green: WT Cin8; red: Cin8 $\Delta 99$; cyan: $W T$ Cin8 from Sf9. 
A

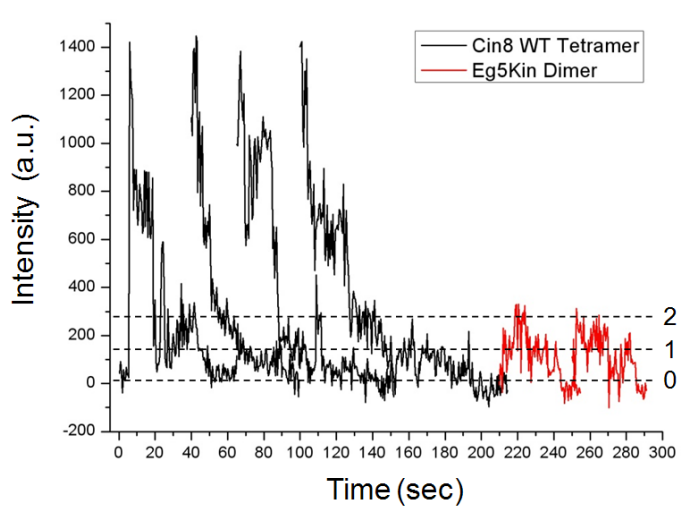

B

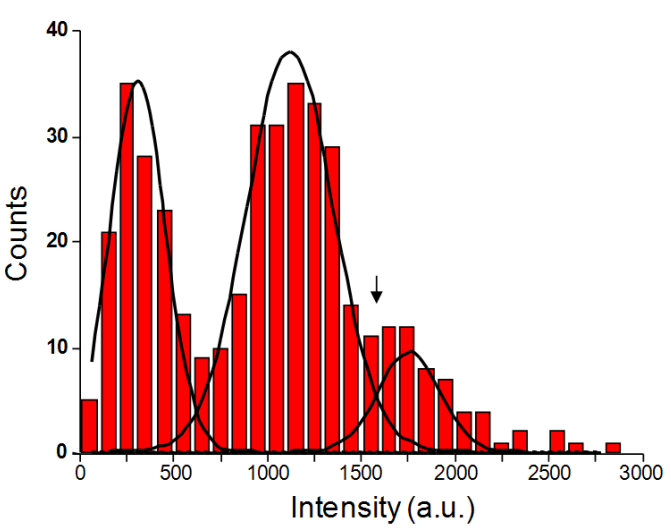

Fig. A.3: Photo-bleaching and fluorescence intensities in the single-molecule motility assay. To rule out the possibility that the broad distribution of velocities of Cin8 is due to aggregation of Cin8-3GFP molecules on the surface, intensities of isolated fluorescent spots and photo-bleaching time courses were monitored. A dimeric motor protein construct, Eg5Kin-GFP [Lakämper10] was used as control. To provide the same optical conditions for both proteins, dimeric Eg5Kin-GFP (with a total of two GFP molecules per dimer) and tetrameric Cin8-3GFP (with a total of twelve GFP molecules per tetramer) were mixed and then bound to the surface of a microscope chamber. Images were taken every $0.5 \mathrm{~s}$. (A) Representative time traces of intensity for Cin8-3GFP (black) and Eg5Kin-GFP (red). For the dimer, two photo-bleaching steps are marked with dashed lines. (B) Histogram of the distribution of fluorescence intensities in a field of mixed Eg5Kin-GFP and Cin8-3GFP molecules. Three peaks are apparent, which correspond to the dimer (left), the tetramer (center) and larger aggregates (right). Bold lines are Gaussian fits; Arrow points at the estimated initial intensity of the tetramers, based on the photo-bleaching steps of the dimer shown in (A). These measurements show that $\sim 16 \%$ of the stationary Cin8-3GFP molecules formed aggregates. This percentage of stationary aggregates is an overestimation of the aggregates for which the velocity was measured, since we did not evaluate spots moving along the MTs that were distinctly brighter than the average. Thus, we rule out the possibility that the broad distribution of velocities of Cin8 is due to aggregation of Cin8 molecules on the surface. 
IV Appendix A. Supplemetatry data chapter 2: Directionality of individual...
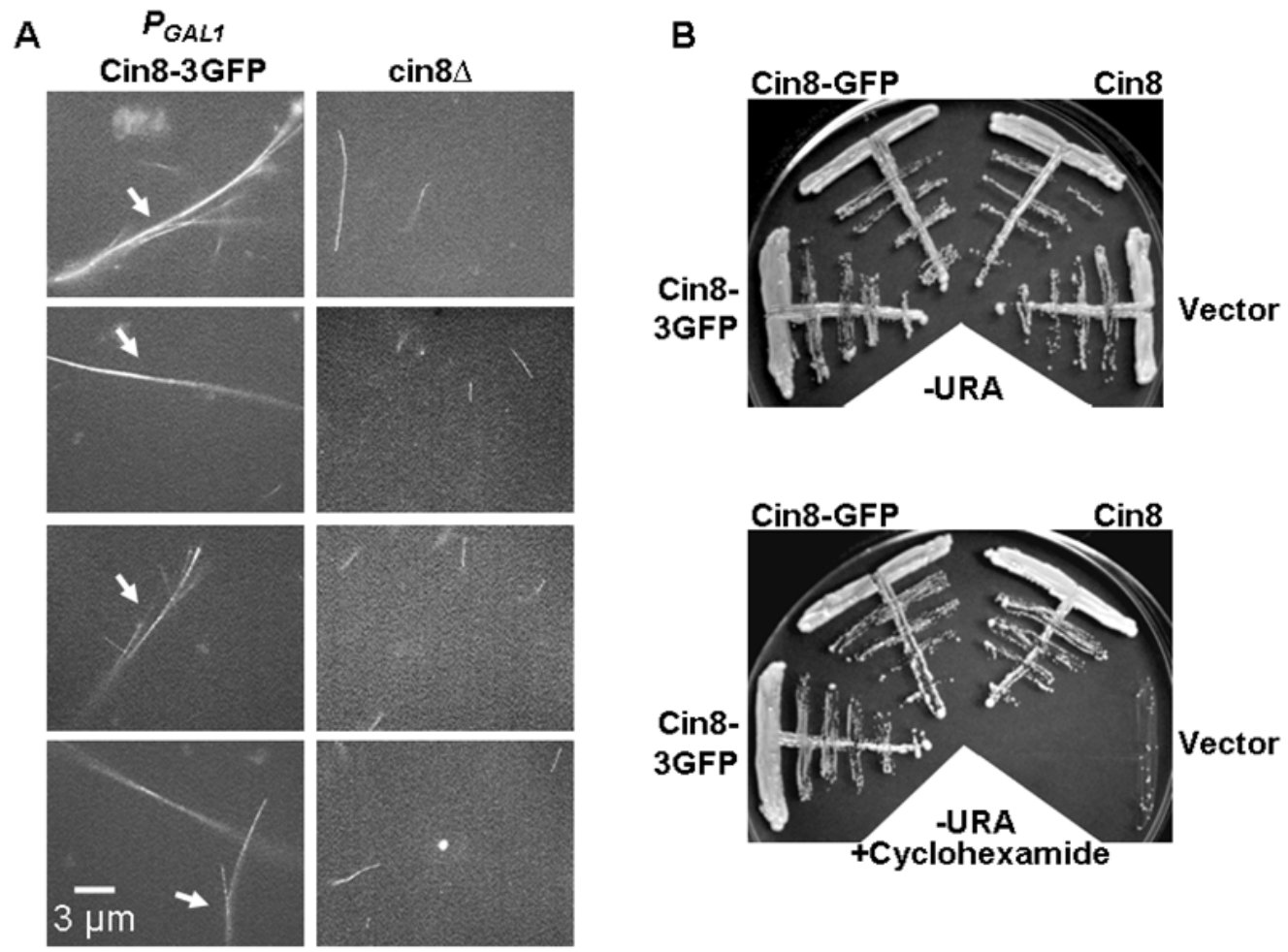

Fig. A.4: Cin8-3GFP bundles MTs; Cin8-GFP and Cin8-3GFP supplement kinesin5 function in S. cerevisiae cells. (A) Whole extracts of cells overexpressing Cin8-3GFP ( $\left.\mathrm{P}_{\text {GAL1 }} \mathrm{Cin} 8-3 \mathrm{GFP}\right)$ or deleted for CIN8 ( $\left.\operatorname{cin} 8 \Delta\right)$, indicated on top, were mixed with taxolstabilized TMR-labelled MTs, in MB buffer supplemented with $100 \mathrm{mM} \mathrm{NaCl}$. Bundles (arrows) were observed with Cin8-3GFP overexpressing extracts but not in $\operatorname{cin} 8 \Delta$ extracts. (B) Viability of cin $8 \Delta$ kip $1 \Delta$ cells was examined in which $W T$ CIN8 shuffle plasmid [Gheber99] was replaced by a centromeric plasmid expressing either Cin8, Cin8-TEV-GFP6HIS (Cin8-GFP), Cin8-3GFP or no motor (vector only), indicated. On plates containing cyclohexamide, shuffle plasmid is removed and only cells expressing functional Cin8 remain viable [Avunie-Masala11, Gheber99]. As can be seen, similarly to cells expressing WT Cin8, cells expressing Cin8-GFP and Cin8-3GFP remained viable on plates containing cyclohexamide. Strain \#: LGY 2038, 2108, 2110, 2562.

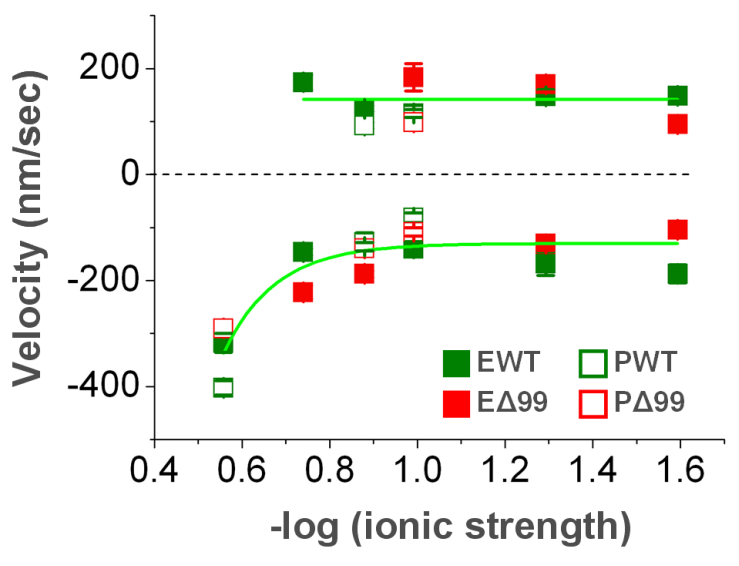

Fig. A.5: Ionic strength dependence of plus- and minus-end directed velocities of $\mathrm{Cin} 8$ (green) and Cin8 499 (red) in whole-cell yeast extracts (EWT, E $\Delta 99$ ) or affinitypurified (PWT, P $\Delta 99$ ). For each sample and buffer condition, the velocities of plus-end and minusend directed segments were averaged separately (Mean \pm s.e.m. of 50 - 200 values). 

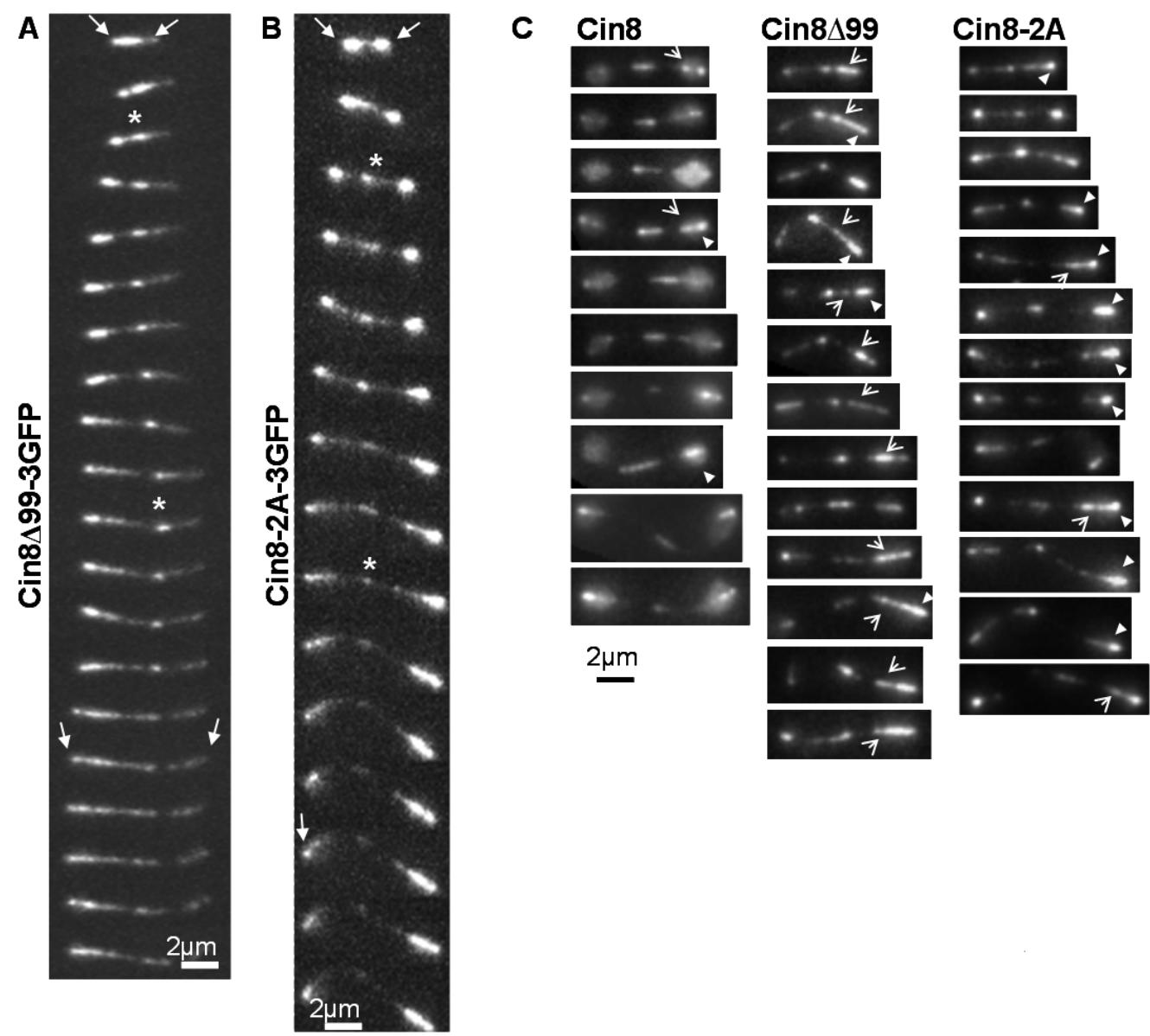

Fig. A.6: Localization of Cin8 $\triangle 99-3 \mathrm{GFP}$ and Cin8-2A-3GFP on anaphase spindles. (A and B) 2D-projection time-lapse confocal fluorescence images. Time intervals: (A) $60 \mathrm{~s} \mathrm{(B)}$ 90 s. Bar: $2 \mu \mathrm{m}$; Arrows: spindle poles; Asterisks: midzone. (C) Additional 2D images of anaphase spindles in cells expressing Cin8, Cin8 499 and Cin8-2A. In (C), enhanced binding of Cin8 to one side of the spindle is indicated by an arrow; enhanced binding of Cin8 to one of the SPBs is indicated by arrowhead. 
VI Appendix A. Supplemetatry data chapter 2: Directionality of individual...

\section{Supplementary table}

\begin{tabular}{|c|c|c|}
\hline Yeast strain & Genotype & Experiment \\
\hline LGY 2038 & $\begin{array}{l}\text { MATa, ura3, leu2, his3, ade2, lys2, cyh2 } \\
\text { cin8::HIS3, kip } 1:: H I S 3 \\
\text { (CIN8 CYH2, LEU2, CEN), (URA3, CEN) }\end{array}$ & $\begin{array}{l}\text { Viability test in } \operatorname{cin} 8 \Delta \\
\text { kip } 1 \Delta \text { cells }\end{array}$ \\
\hline LGY 2055 & $\begin{array}{l}\text { MATa, ura3, his3, ade2, lys2, } \\
\text { cin8::URA3, leu2::CIN8-3GFP-LEU2 }\end{array}$ & $\begin{array}{l}\text { 1) Spindle localization of } \\
W T \text { Cin } 8 \\
\text { 2) Single-molecule assay }\end{array}$ \\
\hline LGY 2108 & $\begin{array}{l}\text { MATa, ura3, leu2, his3, ade2, lys2, cyh2r } \\
\text { cin8::HIS3, kip } 1: \text { HIS3 } \\
\text { (CIN8, URA3, CEN) }\end{array}$ & $\begin{array}{l}\text { Viability test in } \operatorname{cin} 8 \Delta \\
\text { kip } 1 \Delta \text { cells }\end{array}$ \\
\hline LGY 2110 & $\begin{array}{l}\text { MATa, ura3, leu2, his3, ade2, lys2, cyh2r, } \\
\text { cin8::HIS3, kip1::HIS3 } \\
\text { (CIN8-TEV-GFP-6HIS, URA3, CEN) }\end{array}$ & $\begin{array}{l}\text { Viability test in } \operatorname{cin} 8 \Delta \\
\text { kip } 1 \Delta \text { cells }\end{array}$ \\
\hline LGY 2235 & $\begin{array}{l}\text { MATa, ura3, ade2, lys2, cin8::URA3, } \\
\text { leu2::CIN8-3HA-LEU2, his3::TUB1-GFP- } \\
\text { HIS3 }\end{array}$ & Real time anaphase kinetics \\
\hline LGY 2515 & $\begin{array}{l}\text { MAT } \alpha, \text { ura3, leu2, his3, lys2, } G A L^{+} \text {(CIN8- } \\
\text { 3GFP, URA3, CEN) }\end{array}$ & Single-molecule assay \\
\hline LGY 2530 & $\begin{array}{l}\text { MATa, ura3, leu2, his3, ADE }{ }^{+}, \text {lys2, cyh2 } \\
\text { ase1::KanMX (CIN8-3GFP, URA3, CEN) }\end{array}$ & Single-molecule assay \\
\hline LGY 2542 & $\begin{array}{l}\text { MATa, ura3, his3, ade2, lys2, cin8::URA3, } \\
\text { leu2::cin8 } 99-3 G F P-L E U 2\end{array}$ & $\begin{array}{l}\text { 1) Spindle localization of } \\
\text { Cin } 8 \Delta 99 \\
\text { 2) Single-molecule assay }\end{array}$ \\
\hline LGY 2562 & $\begin{array}{l}\text { MATa, ura3, leu2, his3, ade2, lys2, cyh2 }{ }^{r} \text {, } \\
\text { cin8::HIS3, kip1::HIS3 } \\
\text { (CIN8-3GFP, URA3, CEN) }\end{array}$ & $\begin{array}{l}\text { 1) Single molecule assay } \\
\text { 2) Viability test in } \operatorname{cin} 8 \Delta \\
\text { kip } 1 \Delta \text { cells }\end{array}$ \\
\hline LGY 2571 & $\begin{array}{l}\text { MATa, ura3, leu2, his3, ade2, lys2, } \\
\text { cin8::LEU2 (cin8 } 99-3 G F P, \text { URA3, CEN) }\end{array}$ & $\begin{array}{ll}\text { Spindle } & \text { localization of } \\
\text { Cin8 } 899 & \end{array}$ \\
\hline LGY 2719 & $\begin{array}{l}\text { MATa, ura3, his3, ade2, lys2, cin8::URA3, } \\
\text { leu2::cin8-S277A T285A-3GFP-LEU2 }\end{array}$ & $\begin{array}{l}\text { Spindle localization of Cin8- } \\
\text { 2A (Cin8-S277A T285A) }\end{array}$ \\
\hline LGY 2824 & $\begin{array}{l}\text { MATa, ura3, ade2, lys2, cin8::URA3, } \\
\text { leu2::CIN8D99-3HA-LEU2, } \\
\text { his3::TUB1-GFP-HIS3 }\end{array}$ & Real time anaphase kinetics \\
\hline LGY 3079 & $\begin{array}{l}\text { MAT } \alpha, \text { ura3, leu2, his3, ade2, lys2, } G A L^{+}, \\
\left(P_{G A L 1}-C I N 8-T E V-G F P-6 H I S,\right. \\
\text { URA3, CEN) }\end{array}$ & $\begin{array}{l}\text { Single-molecule assay of } \\
\text { affinity-purified } W T \text { Cin } 8\end{array}$ \\
\hline
\end{tabular}




\begin{tabular}{|c|c|c|}
\hline LGY 3082 & 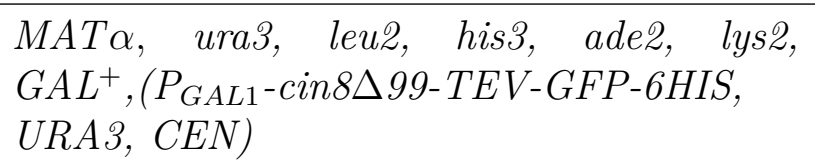 & $\begin{array}{l}\text { Single molecule assay of } \\
\text { affinity-purified Cin } 8 \Delta 99\end{array}$ \\
\hline LGY 3530 & $\begin{array}{l}\text { MATa, ura3, leu2, his3, lys2, cin8::URA3 } \\
\left(P_{G A L 1}-C I N 8-3 G F P, L Y S 2, C E N\right)\end{array}$ & $\begin{array}{l}\text { Overexpression of Cin8- } \\
3 \mathrm{GFP} \text { for MT bundling } \\
\text { assay }\end{array}$ \\
\hline
\end{tabular}

Tab. A.1: List of yeast strains used in this study. For each strain appears a brief description of the genotype and of the experiments in which it was used in this study. In parenthesis are indicated plasmids with their brief description. $\mathrm{P}_{G A L 1}$ : galactose-inducible promoter used for Cin8 overexpression. If not mentioned, Cin8 endogenous promoter was used. CEN: centromeric plasmid.

\section{Description of supplementary videos}

A DVD with supplementary videos is attached to the thesis.

Video A.3.1 is representative video of Cin8-3GFP localization to anaphase spindles. Videos A.3.2 to A.3.5 are representative videos of single-molecule motility assay on polarity-marked MTs, for $W T$ Cin8 and Cin8 499 . At the beginning of each video, the polarity-marked MTs in the field are shown (minus end is bright). Then, filters are changed and set such that the Atto-488-labeled minus-end seeds of the MTs and the GFP-labeled motors are apparent. Images were taken every 0.5 s. Videos A.3.6 to A.3.10 are representative videos of Cin8-GFP movement (green) on polarity-marked MTs in bundles (red) in high salt buffer (MB-175). The Atto488-labeled minus ends of the MTs are visible as bright seeds in the GFP-channel. Images were taken every $0.5 \mathrm{~s}$.

\section{Supplementary Video A.3.1:}

Real time fluorescence recording of Cin8-3GFP localization to an anaphase spindle. Spindle elongates from $\sim 4 \mu \mathrm{m}$ to $\sim 9 \mu \mathrm{m}$. Video corresponds to Fig. 2.1 C. Total time: 8 min. $28 \times$ sped up.

\section{Supplementary Video A.3.2:}

Processive minus-end directed motility of WT Cin8 at high salt buffer. Shown is motility of Cin8-3GFP in S. cerevisiae whole-cell extracts at MB-175 (175 mM $\mathrm{NaCl}$ ). Video corresponds to Fig. 2.2. Total time: 42 s. $3.5 \times$ sped up.

\section{Supplementary Video A.3.3:}

Processive minus-end directed motility of Sf9-expressed Cin8 at high salt buffer. Shown is motility of Cin8-GFP expressed in Sf9 cells and subjected to MT-affinity purification. Motility is assayed at $150 \mathrm{mM} \mathrm{NaCl}$. Video corresponds to Fig. 2.2. Total time: 7 s. $3.5 \times$ sped up. Arrow points on a moving Cin 8 molecule. 


\section{Supplementary Video A.3.4:}

Bi-directional motility of $W T$ Cin 8 at intermediate salt buffer. Shown is motility of Cin8-3GFP in S. cerevisiae whole-cell extracts at MB-30 (30 mM NaCl). Video corresponds to Fig. 2.4 and Fig. 2.6. Total time: 41 s. $3.5 \times$ sped up.

\section{Supplementary Video A.3.5:}

Processive minus-end directed motility of Cin8 $\Delta 99$ at intermediate salt buffer. Shown is motility of Cin8 $\triangle 99-3 \mathrm{GFP}$ in $S$. cerevisiae whole-cell extracts at MB-30 $(30 \mathrm{mM} \mathrm{NaCl})$. Video corresponds to Fig. 2.5 and Fig. 2.6. Total time: 14 s. $3.5 \times$ sped up.

\section{Supplementary Video A.3.6:}

Antiparallel relative sliding of polarity-marked MTs. Shown is the relative sliding of a MT (red) from right to the left over a MT fixed to the surface. The green minus-end of the relative sliding MT is leading. Also in green, single Cin8-GFP molecules are visible both on the single MT as well as in the overlap region. The video corresponds to Fig. 2.8 A, upper panel. Total time: 125 s. $12 \times$ sped up.

\section{Supplementary Video A.3.7:}

Antiparallel relative sliding of polarity-marked MTs. Shown is the landing and relative sliding of a MT (red) from right to the left over a MT fixed to the surface. The green minus end of the relative sliding MT is leading. Also in green single Cin8-GFP molecules are visible both on the single MT as well as in the overlap region. Total time: 52 s. $12 \times$ sped up.

\section{Supplementary Video A.3.8:}

Antiparallel conformation of polarity-marked MTs. Shown is a microtubule (red) crosslinked to a microtubule fixed to the surface in antiparallel conformation. The minus ends of the MTs show up in green. On the single MT and also in the overlap region single Cin8-GFP molecules (green) are visible. The video corresponds to Fig. 2.8 A, lower panel. Total time: 150 s. $12 \times$ sped up.

\section{Supplementary Video A.3.9:}

Parallel conformation of polarity-marked MTs. Shown is a MT (red) crosslinked to a MT fixed to the surface in parallel conformation. The minus-ends of the MTs show up in green. On the single MT and also in the overlap region single Cin8-GFP molecules (green) are visible. The video corresponds to Fig. 2.8 B, upper panel. Total time: 150 s. $12 \times$ sped up.

\section{Supplementary Video A.3.10:}

Parallel conformation of polarity-marked MTs. Shown is a MT (red) crosslinked to a MT fixed to the surface in parallel conformation. The minus ends of the MTs show up in green. On the single MT and also in the overlap region single Cin8-GFP molecules (green) are visible. The video corresponds to Fig. 2.8 B, lower panel. Total time: 150 s. $12 \times$ sped up. 


\section{Supplementary materials and methods}

We produced fluorescently labeled Cin 8 motors in three ways. We first used wholecell extracts of $S$. cerevisiae expressing Cin8 fused with three consecutive C-terminal GFPs (Cin8-3GFP) under its own promoter. Cin8-3GFP was either integrated into the yeast genome or expressed from a centromeric (CEN) plasmid. Cells with integrated Cin8-3GFP were also used for in-vivo imaging (Supplementary Tab. A.1). Second, we overexpressed Cin8 fused with a single C-terminal GFP and N-terminal 6HIS-tag (6HIS-Cin8-GFP) in Sf9 insect cells, and, third, we overexpressed Cin8GFP in $S$. cerevisiae.

\section{Yeast strains, growth conditions and whole-cell extract preparation}

The S. cerevisiae strains (S288C) and yeast expression plasmids are listed in Supplementary Tab. A.1. Rich (YPD) and minimal (SD) media were as described by [Sherman86]. Whole extracts of cells expressing Cin8-3GFP were prepared from log-phase cultures as previously described [Gheber99].

\section{Overexpression and purification of Cin8 from Sf9 cells}

Recombinant baculovirus plasmids (bacmids) expressing 6HIS-Cin8-GFP were constructed according to standard procedures (Bac-to-Bac Expression System, Invitrogen, USA). The CIN8 gene was cloned into the pFastBac ${ }^{T M} \mathrm{HT}$ shuttle vector. DH10Bac cells (Invitrogen, USA) were used to produce a recombinant bacmid. Transfection of Sf9 cells (Invitrogen, USA) with the CIN8 bacmids was carried out with TransIT-insecta reagent (Mirus, USA). The conditioned medium containing the recombinant virus was collected $72 \mathrm{~h}$ post infection and clarified by a $5 \mathrm{~min}$ centrifugation at $1000 \mathrm{~g}$. To produce a potent viral stock, the virus was subjected to four additional rounds of amplification in adherent Sf9 cells, until $70-80 \%$ of the cells expressed Cin8-GFP.

For large-scale overexpression of the Cin8 variant, 2 l of $3 \times 10^{6}$ cells/ml Sf9 cells suspended in serum-free Bioinsect media, supplemented with Pen-Strep-Amphotericine antibiotics (Biological Industries, Israel) were infected with $20 \mathrm{ml}$ of the viral stock. Cells were harvested $48 \mathrm{~h}$ post-infection. About $3 \mathrm{~g}$ of pellet were washed with motility buffer (MB: $50 \mathrm{mM}$ Tris/HCl, $30 \mathrm{mM}$ PIPES/KOH, final $\mathrm{pH}$ 7.2, $175 \mathrm{mM} \mathrm{NaCl}, 2 \mathrm{mM}$ EDTA, $1 \mathrm{mM}$ EGTA, $10 \%$ glycerol, $1 \mathrm{mM}$ phenylmethylsulfonyl fluoride and $1 \mathrm{mM}$ dithiothreitol), supplemented with $175 \mathrm{mM}$ $\mathrm{NaCl}$ (MB-175) [Gheber99]. Cells were lysed by sonication (10 pulses, $10 \mathrm{~s}$ each). Lysate was clarified by 20 min centrifugation at $13000 \mathrm{rpm}$ at $4{ }^{\circ} \mathrm{C}$. Cin 8 was purified from extracts by MT affinity [Vale85]. 


\section{Overexpression and purification of Cin8 from yeast cells $\mid$ A.4.3}

Cells expressing Cin8-TEV-GFP-6HIS under the GAL1 promoter on a CEN plasmid were grown overnight in liquid medium supplemented with $2 \%$ raffinose. For overexpression, cells were pelleted, washed, resuspended in media containing $2 \%$ galactose and grown for additional $7 \mathrm{~h}$. Following overexpression, cells were harvested, pelleted and washed with motility buffer MB-175 [Gheber99]. The washed pellet was ground with a mortar and pestle under liquid nitrogen in $\sim 1 \mathrm{ml}$ of MB175 buffer. Extracts were then clarified by centrifugation at $13000 \mathrm{rpm}$ at $4{ }^{\circ} \mathrm{C}$. Cin8 was purified using a Ni-NTA affinity column (Invitrogen, USA).

\section{\begin{tabular}{l|l} 
Microtubule polymerization & A.4.4
\end{tabular}}

Fluorescent tetramethyl-rhodamine TMR-labeled tubulin and unlabeled tubulin were mixed in a 1:3 ratio and suspended in BRB80 buffer $(80 \mathrm{mM}$ PIPES/KOH, pH 6.8, $1 \mathrm{mM}$ EGTA, $2 \mathrm{mM} \mathrm{MgCl} 2$ ) with, in addition, $1 \mathrm{mM} \mathrm{GTP}$ and $4 \mathrm{mM} \mathrm{MgCl}$. MTs were polymerized at $37^{\circ} \mathrm{C}$ for 20 min. Polymerized MTs were diluted $\sim 1: 150$ into pre-warmed BRB80 containing $10 \mu \mathrm{M}$ paclitaxel (Sigma, USA). To obtain polaritymarked MTs, Atto-488 (Atto-Tec GmbH, Germany) labeled seeds were produced by polymerizing $3.6 \mu \mathrm{M}$ labeled tubulin dimers with $30 \mu \mathrm{M}$ unlabeled tubulin in BRB80 buffer in the presence of $0.42 \mathrm{mM}$ Guanosine-5' $[(\alpha, \beta)$-methyleno]triphosphate (GMPCPP; Jena Bioscience, Germany). The seeds were elongated by adding $1 \mu$ of seed solution to $24 \mu \mathrm{l}$ of a solution containing $4.5 \mu \mathrm{M}$ (TMR)-labeled, $6.4 \mu \mathrm{M}$ unlabeled tubulin, 9.1 $\mathrm{MM}$ N-ethylmaleimide (NEM)-tubulin [Hyman91] $1 \mathrm{mM} \mathrm{GTP,} 1$ $\mathrm{mM} \mathrm{MgCl}_{2}$ and $1 \mathrm{mM}$ dithiothreitol, followed by incubation at $37{ }^{\circ} \mathrm{C}$ for $15 \mathrm{~min}$. NEM-tubulin inhibits minus-end growth, resulting in TMR-labeled MT growth predominantly from the plus-end of the seeds. Polymerized MTs were always kept in buffer containing $10 \mu \mathrm{M}$ paclitaxel.

\section{Buffers for motility assays}

We used motility buffer with several salt concentrations for in-vitro motility assays. The high-salt buffer was MB-175 (see above). Lower-salt buffers were the same as MB-175, except for different concentrations of $\mathrm{NaCl}(0 \mathrm{mM} \mathrm{NaCl}$ in $\mathrm{MB} ; 30 \mathrm{mM}$ $\mathrm{NaCl}$ in $\mathrm{MB}-30 ; 80 \mathrm{mM} \mathrm{NaCl}$ in $\mathrm{MB}-80$ ). $1 / 2 \mathrm{MB}$ and 1/4MB were 2 fold and 4 fold dilutions of MB. For single-molecule motility assays with Sf9-expressed Cin8, we used $150 \mathrm{mM} \mathrm{NaCl}$ in $\mathrm{MB}$.

\section{\begin{tabular}{l|l} 
Single-molecule in-vitro motility assays & A.4.6
\end{tabular}}

The motility assays were performed following standard procedures [Lakämper10, Gheber99, Howard93]. Cover slips were pre-cleaned either by incubation with $0.2 \mathrm{M}$ 
potassium hydroxide or in a plasma cleaner (Harrick Plasma, USA) and then washed three times with purified water. For the single-molecule motility assay, the precleaned coverslips were silanized with positively charged 3-(2-[2-aminoethylamino] ethylamino) propyl-trimethoxysilane (DETA; Sigma-Aldrich, USA). Flow chambers were then assembled by joining slides and coverslips with two strips of double-stick tape as spacers. The flow chambers were first incubated for $5 \mathrm{~min}$ with TMRlabeled MTs, to allow immobilization on the positively charged cover slips. The motor extract containing GFP-tagged Cin8 was diluted 1:25 in motility buffer of the desired salt concentration supplemented with $0.1 \mathrm{mg} / \mathrm{ml}$ glucose-oxidase, 0.8 $\mathrm{mg} / \mathrm{ml}$ catalase, $1 \mathrm{mM}$ DTT, $10 \mathrm{mM}$ glucose (oxygen-scavenging "anti-bleach"). Then $0.2 \mathrm{mg} / \mathrm{ml}$ casein and $3-5 \mathrm{mM} \mathrm{MgATP}$ (for assaying motility) or MgADP (for determining diffusion coefficients) were added to this solution before it was introduced into the flow-chambers.

To confirm that we observed single molecules, we performed fluorescence-intensity measurements. A control solution containing dimeric motor protein Eg5Kin-GFP [Lakämper10] was mixed with extract containing tetrameric Cin8-3GFP in order to provide the exact same assay conditions for both proteins. The proteins were then directly immobilized on DETA surfaces, and images were acquired with time intervals of $0.5 \mathrm{~s}$.

\section{Fluorescence microscopy $\quad$ A.4.7}

Single-molecule motility data was collected on two microscopes, one in Ben Gurion University (BGU), Israel and the other in Georg August University (GAUG), Germany. The BGU setup consists of a motorized inverted microscope (Axiovert 200M; Zeiss, Germany) on a vibration-isolation table (TMC, USA). For fluorescence illumination we used a HBO 100 Mercury Illuminator and for detection a cooled CCD camera (SensiCam, PCO, Germany). Images were acquired with $0.8 \mathrm{~s}$ time intervals and processed using ImageJ and MetaMorph (MDS Analytical Technologies, USA) software. The GAUG setup consists of a custom-built total internal reflection fluorescence (TIRF) microscope using a $473 \mathrm{~nm}$ Laser (Viasho, USA) for fluorescence excitation, and a 100x objective (SFluor, NA 1.49, Oil; Nikon, USA) and a CCD camera (Cascade 512B; Roper Scientific, USA) for detection. In this setup, images were acquired with $0.5 \mathrm{~s}$ time intervals.

\section{Image analysis A.4.8}

Digital images were analyzed by LabView (National Insturemts, USA), ImageJ or MetaMorph software. To track single molecules for the mean-displacement (MD) and mean-square-displacement (MSD) analysis [Kwok06], ImageJ 2D spot-tracking software was used. For velocity and run length analysis, kymographs were generated with MetaMorph or LabView software. To determine run length distributions, directional episodes between diffusive interludes were selected. For a semi-automated velocity analysis, a custom-written LabView procedure divided the kymographs into 
$3 \mathrm{~s}$ segments by setting a marker every 3 seconds. To estimate the velocity in each kymograph segment, lines were fitted by eye to the data segments between the markers using LabView. Gaussian fits to velocity histograms were performed with OriginPro (OriginLab Corporation, USA).

\section{In-vivo assays of anaphase kinetics and Cin8 localization}

A.4.9

Live-cell fluorescence imaging of $S$. cerevisiae cells expressing Cin8-3GFP was performed using a Zeiss Axiovert 200M-based Nipkow spinning-disc confocal microscope (UltraView ESR, Perkin Elmer, UK), equipped with an EMCCD camera (C9100-50, Hamamatsu, Japan) [Fridman09]. For the analysis of anaphase spindle elongation kinetics, Z-stacks of images of $0.2-0.4 \mu \mathrm{m}$ separation between the planes were acquired with 1 min time intervals. The distance between spindle pole bodies was measured in 3D, using MetaMorph software [Movshovich08]. For the Cin8-3GFP spots (speckle) analysis, images were acquired every $2 \mathrm{~s}$. 


\section{Supplemetatry data chapter 4: The effect of monastrol on the processive motility of a kinesin-5 head/kinesin-1 stalk chimera}

This is the supplementary data for the chapter 4 titled "The effect of monastrol on the processive motility of a kinesin- 5 head/kinesin- 1 stalk chimera". It can also be found online at the Journal of Molecular Biology, doi:10.1016/ j.jmb.2010.03.009.

\section{\begin{tabular}{l|l} 
Supplementary figures & B.1
\end{tabular}}
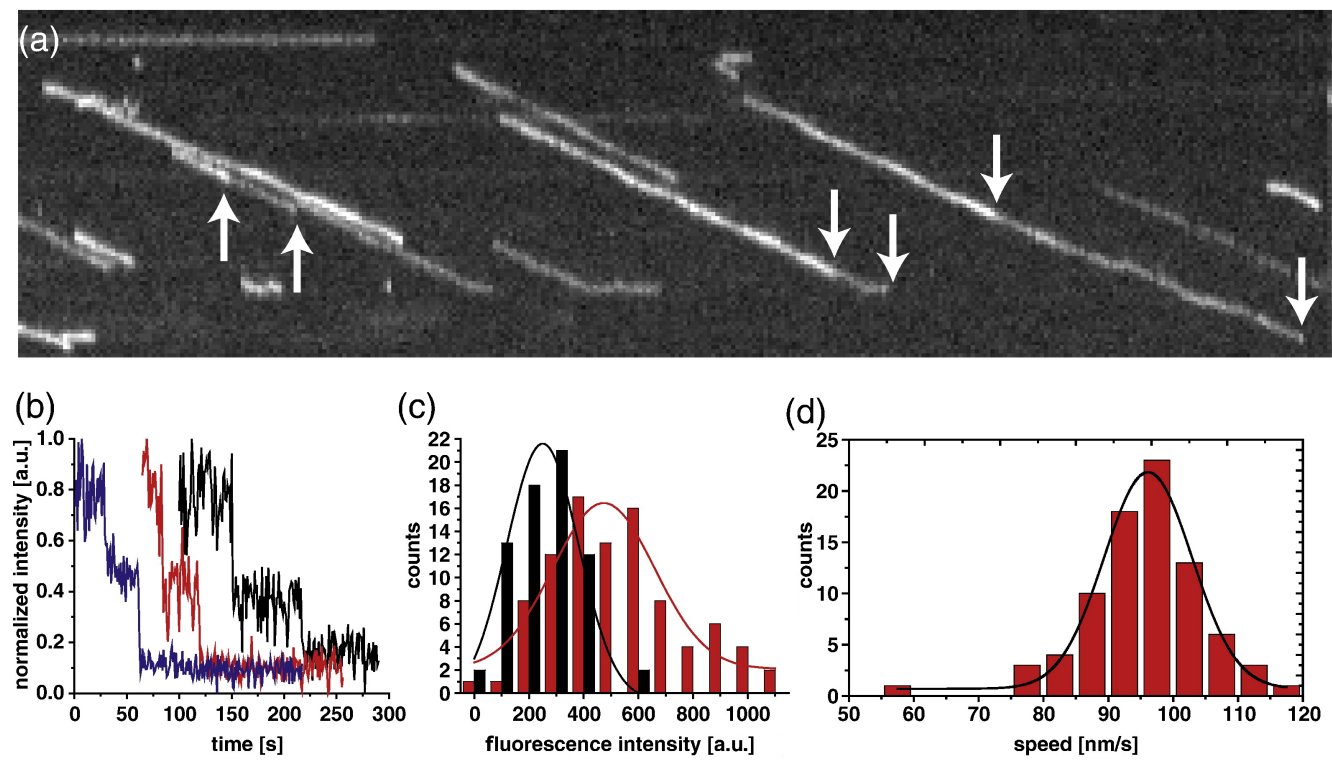

Fig. B.1: Stepwise incremental bleaching of moving and surface-adsorbed GFP-tagged Eg5Kin-451 motors: (a) bleaching events within the kymograph tracks are marked with arrows, (b) fluorescence intensity time traces of single motors adsorbed to surfaces showing clear bleaching steps, (c) histogram of fluorescence intensities of individual motors. Red: initial prebleach intensities of the motors. Black: intensity drops corresponding to clearly identifiable bleaching steps. (d) histogram of single Eg5Kin-451GFP motor speeds. 

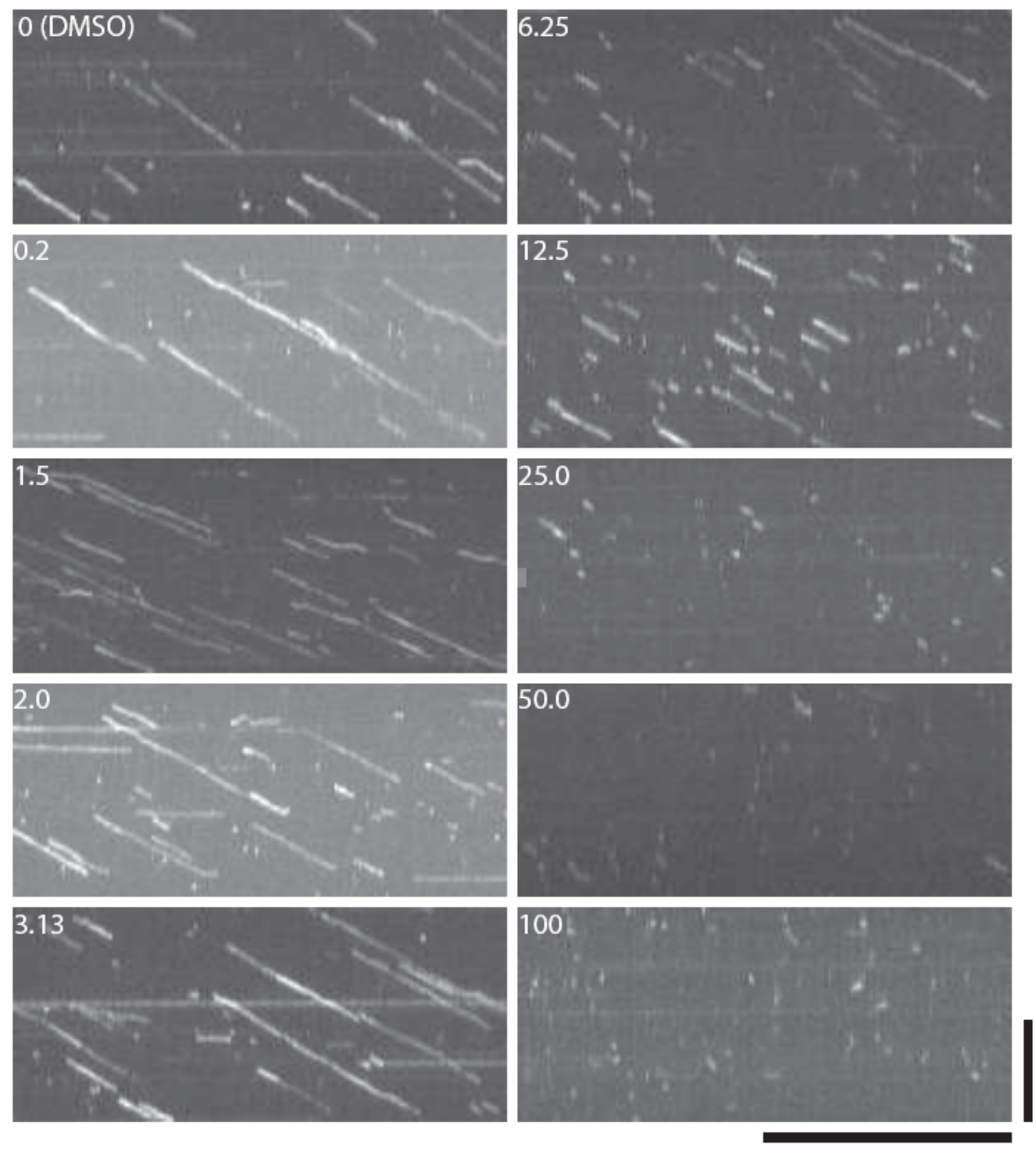

Fig. B.2: Kymographs of single-molecule fluorescence processivity assays with Eg5Kin451GFP motors under control conditions and with increasing monastrol concentrations. Vertical and horizontal scale-bars correspond to $5 \mu \mathrm{m}$ and $100 \mathrm{~s}$, respectively. 
(a)

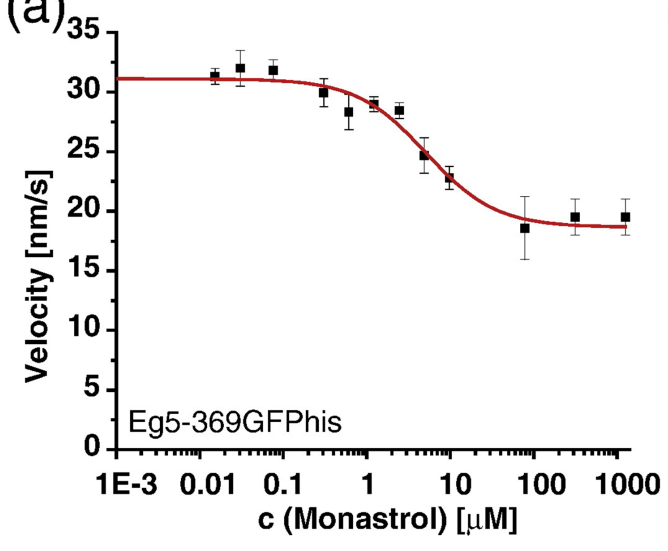

(b)

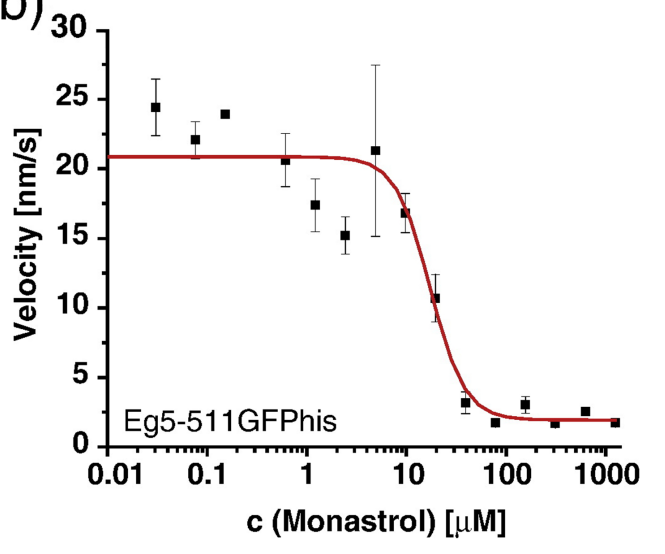

Fig. B.3: Surface-gliding velocities of (a) the monomeric Eg5-369(GFP)His, and (b) the dimeric Eg5-511(GFP)His at increasing monastrol concentrations. In the absence of monastrol, monomeric and dimeric Eg5-constructs produced similar microtubule velocities (see Supplementary Tab. B.1) in surface-gliding assays. Microtubule velocities were averaged from 4 and 3 independent assays, respectively. For the gliding assays with monastrol, we used the MT-affinity purifications that produced the highest fraction of gliding microtubules $(>90 \%)$. 


\section{Supplementary table $\quad$ B.2}

\begin{tabular}{l|l|c|c} 
Construct & Date & Gliding velocity $[\mathrm{nm} / \mathrm{s}]$ & Average velocity $[\mathrm{nm} / \mathrm{s}]$ \\
\hline \hline 369GFPhis & 12.09 .2007 & 43 & \multirow{2}{*}{$46 \pm 13$} \\
\hline 369GFPhis & 04.10 .2007 & 26 & \multirow{2}{*}{$46 \pm$} \\
\hline 369GFPhis & 05.10 .2007 & 81 & \multirow{2}{*}{$42 \pm 8$} \\
\hline 369GFPhis & 24.10 .2007 & 33 & \\
\hline \hline 511GFPhis & 12.09 .2007 & 45 & \\
\hline 511GFPhis & 04.10 .2007 & 35 & \\
\hline 511GFPhis & 05.10 .2007 & 47 &
\end{tabular}

Tab. B.1: : Surface-gliding velocities of the non-chimeric monomeric Eg5-369(GFP)His and the dimeric Eg5-511(GFP) construct in the absence of monastrol. Only those preparations in which at least $75 \%$ of the microtubules were motile were considered. In each individual experiment $\sim 20$ microtubules were evaluated for gliding velocity and averaged. Assay conditions were as described in the text, BRB80 buffer, $2 \mathrm{mM}$ ATP, cover slip coated with protein $\mathrm{G}$ and anti-his antibodies.

\section{\begin{tabular}{l|l} 
Description of supplementary videos & B.3
\end{tabular}}

\section{Supplementary Video B.3.1:}

Multi-motor surface-gliding assay of full-length Eg5Kin under standard conditions (BRB80 buffer; 2 mM ATP; $10 \times$ sped up).

\section{Supplementary Video B.3.2:}

Single-motor surface-gliding assay at low densities of full-length Eg5Kin under standard conditions (BRB80 buffer; $2 \mathrm{mM} \mathrm{ATP}$ ). Nodal point pivoting can be observed. (Field of view: $26 \times 16.5 \mu \mathrm{m} ; 1$ frame/s; $90 \mathrm{~s}$ ).

\section{Supplementary Video B.3.3:}

Silica bead (1 $\mu$ m diameter) sparsely covered with Eg5Kin-451GFP moving in and out of an optical trap (multiple escapes) under standard conditions (BRB80 buffer; $2 \mathrm{mM}$ ATP; trap stiffness $\kappa=0.025 \mathrm{pN} / \mathrm{nm})$.

\section{Supplementary Video B.3.4:}

Single-molecule fluorescence processivity assays of Eg5Kin-451GFP in standard conditions ( $7 \times$ sped up; field of view: $25 \times 4 \mu \mathrm{m} ; 1$ frame/s; $420 \mathrm{~s}$ ). 
Appendix B. Supplemetatry data chapter 4: The effect of monastrol on the ... XVII

\section{Supplementary Video B.3.5:}

Single-molecule fluorescence processivity assay of (non-chimeric) Eg5-511(GFP)His after gel filtration of a standard Ni-NTA-purification. Individual dimers moved along the microtubules at speeds comparable to what was measured in surface-gliding assays $(42 \pm 5 \mathrm{~nm} / \mathrm{s} ; \mathrm{N}=25)$. 



\section{Supplemetatry data chapter 5: \\ Neck-linker length dependence of processive kinesin-5 motility}

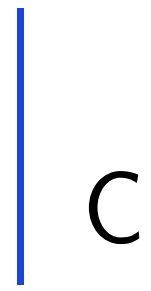

This is the supplementary data for the chapter 5 titled "Neck-linker length dependence of processive kinesin-5 motility". It can also be found online at the Journal of Molecular Biology, doi:10.1016/j.jmb.2012.06.043.

\section{\begin{tabular}{l|l} 
Supplementary figures & C.1
\end{tabular}}

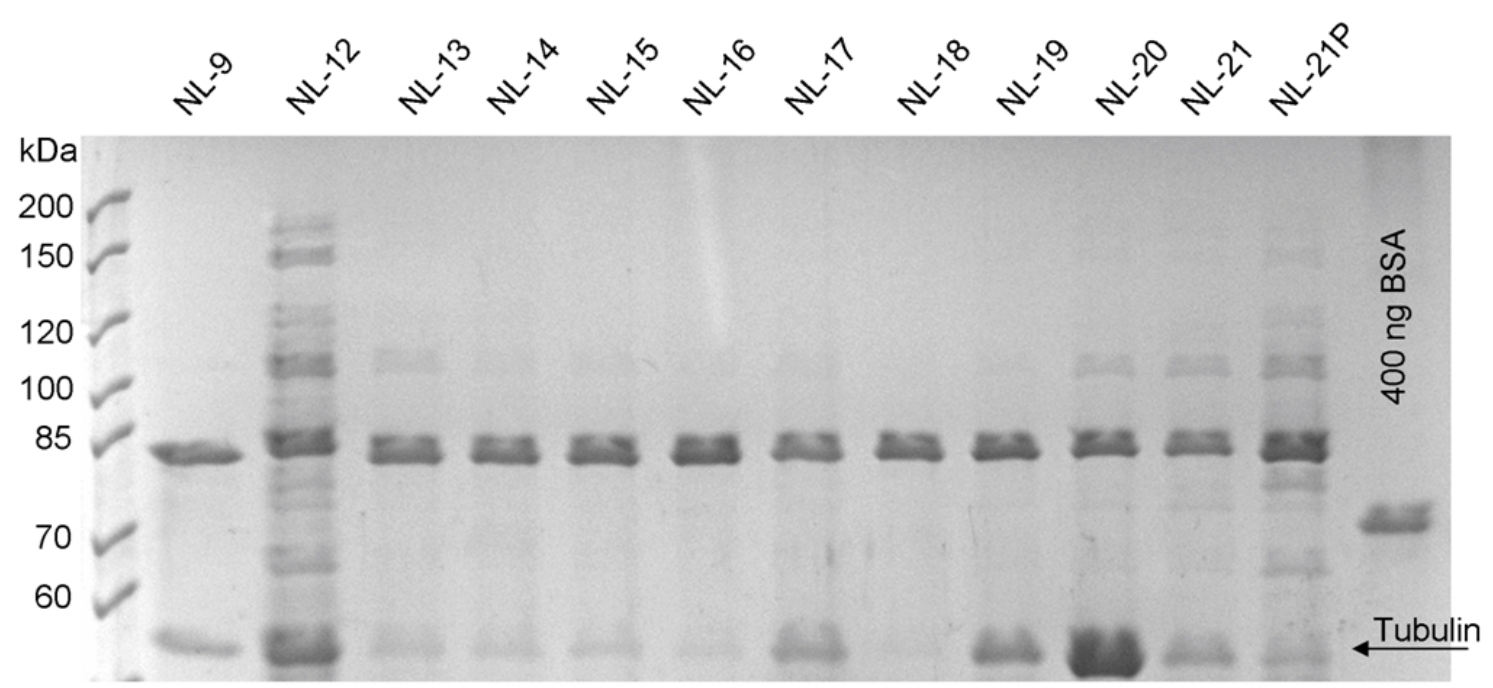

Fig. C.1: Coomassie-stained SDS-PAGE gel (4\% stacking gel and $10 \%$ separation gel) of all neck-linker constructs: Concentrations were chosen to result in an equal amount of protein for each construct ( $400 \mathrm{ng}) .400 \mathrm{ng}$ of BSA is shown for comparison. 

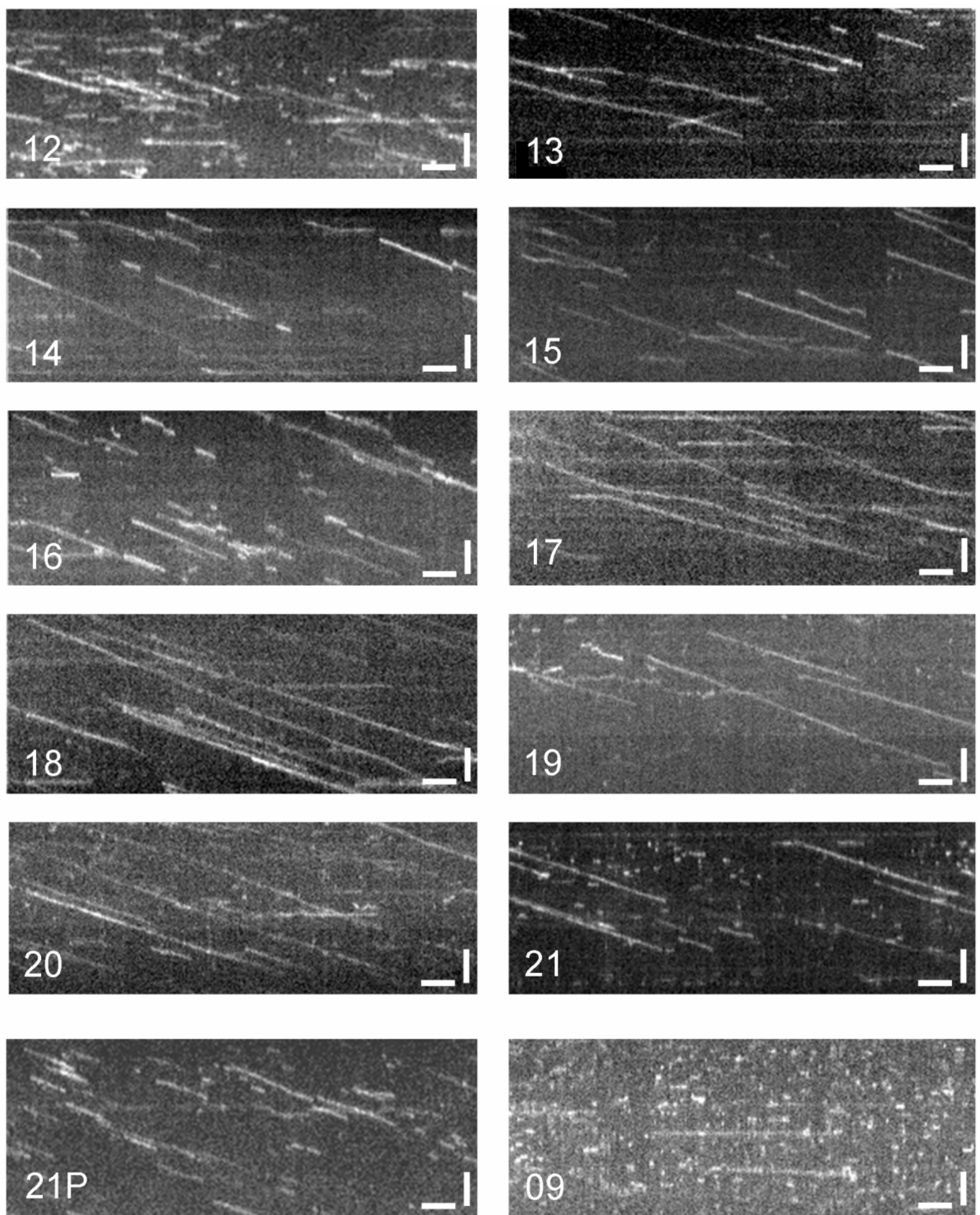

Fig. C.2: Kymographs of motility of all neck-linker constructs: name of construct indicated in lower-left corner. Scale bars: horizontal $10 \mathrm{~s}$, vertical $3 \mu \mathrm{m}$. 
(a)

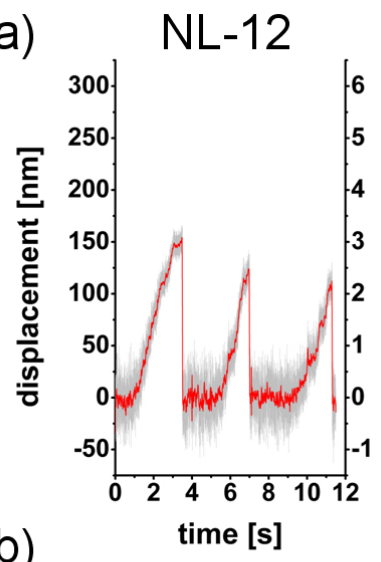

(b)

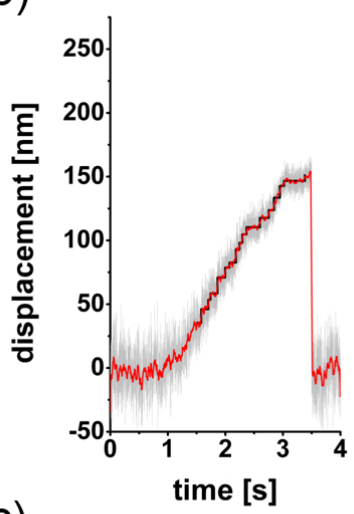

(c)

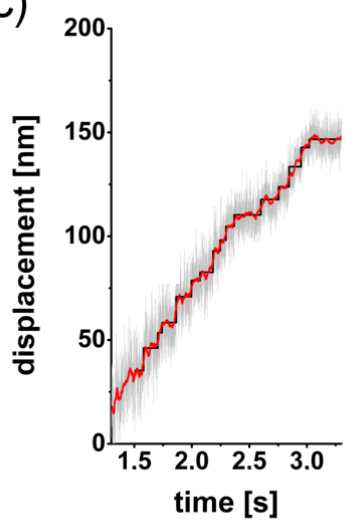

(d)

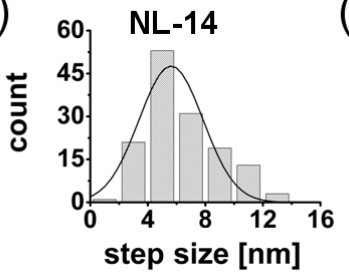

$\mathrm{NL}-14$
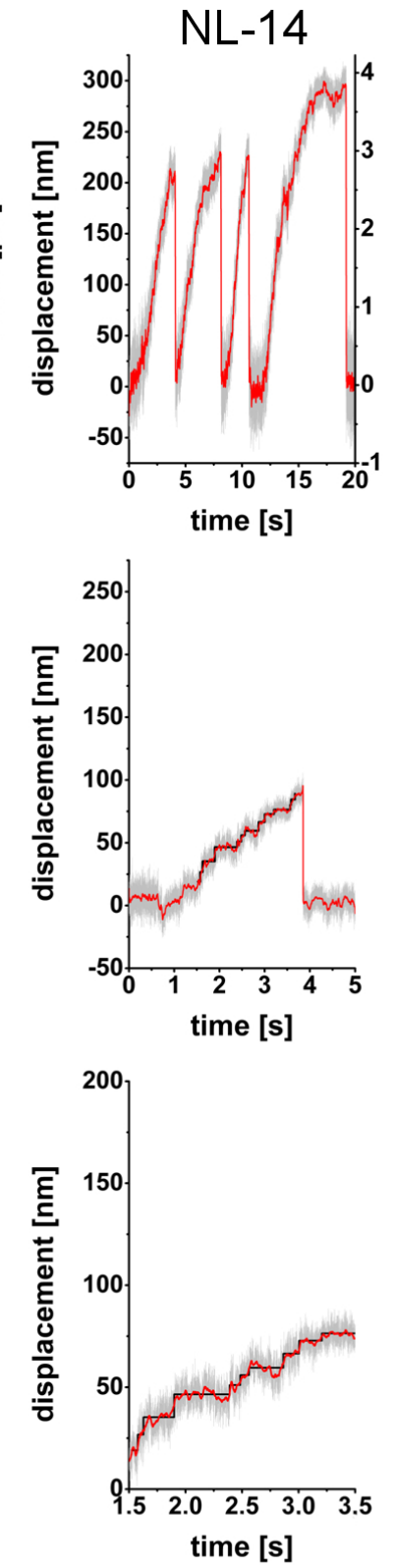

$\mathrm{NL}-21$
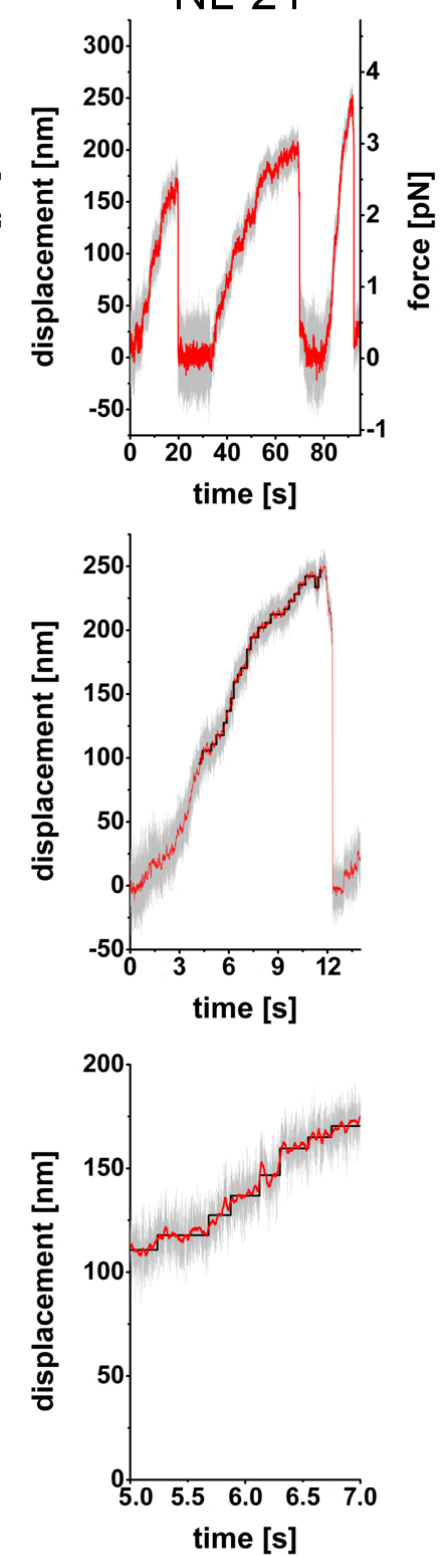

(e)

NL constructs

\begin{tabular}{|c|c|c|c|c|c|c|c|c|c|c|}
\hline 12 & 13 & 14 & 15 & 16 & 17 & 18 & 19 & 20 & 21 & $21 \mathrm{P}$ \\
\hline \hline 6.1 & 6.9 & 5.6 & 8.0 & 8.2 & 9.3 & 6.7 & 7.7 & 5.5 & 7.7 & 6.9 \\
\pm 0.5 & \pm 0.3 & \pm 0.4 & \pm 0.3 & \pm 0.5 & \pm 0.3 & \pm 0.3 & \pm 0.3 & \pm 0.1 & \pm 0.4 & \pm 0.3 \\
\hline 1 & step sizes [nm]
\end{tabular}

Fig. C.3: Results from optical trapping assays - step size analysis: Shown are measurements of three neck-linker constructs NL-12, NL-14 and NL-21. (a) Representative bead-displacement time series. The excursions from the trap centre end prior to any stalling (raw data in gray, $3 \mu$ s median filtered data in red). (b) Single excursions from the trap for the three different constructs. In black is shown the results of a step size analysis performed after Kerssemakers et al. [Kerssemakers09] (raw data in gray, $3 \mu \mathrm{s}$ median filtered data in red). (c) Close up view of the data fitted by the stepfinding algorithm in (b). (d) Exemplary histogram of the step size distribution for NL14. The mean value is $5.6 \pm 0.4 \mathrm{~nm}, \mathrm{~N}=141$. This value, $\Delta_{\text {bead }}$, is attenuated by the finite stiffness of the motor-bead linkage. To estimate the stiffness of this linkage, $\mathrm{k}_{\text {motor }}$, we analysed the variance of the displacement signal from the trapping assays and calculated a linkage stiffness of $0.1 \mathrm{pN} / \mathrm{nm}$. To obtain the motor's step size $\Delta_{\text {motor }}$ we used the correction $\Delta_{\text {motor }}=\Delta_{\text {bead }}$ * $\left(\mathrm{k}_{\text {trap }}+\mathrm{k}_{\text {motor }}\right) / \mathrm{k}_{\text {motor }}$. This results in an average step size of $8.7 \mathrm{~nm}$. (e) Table of the uncorrected results from the stepping analysis of all neck-linker constructs. 
(a)
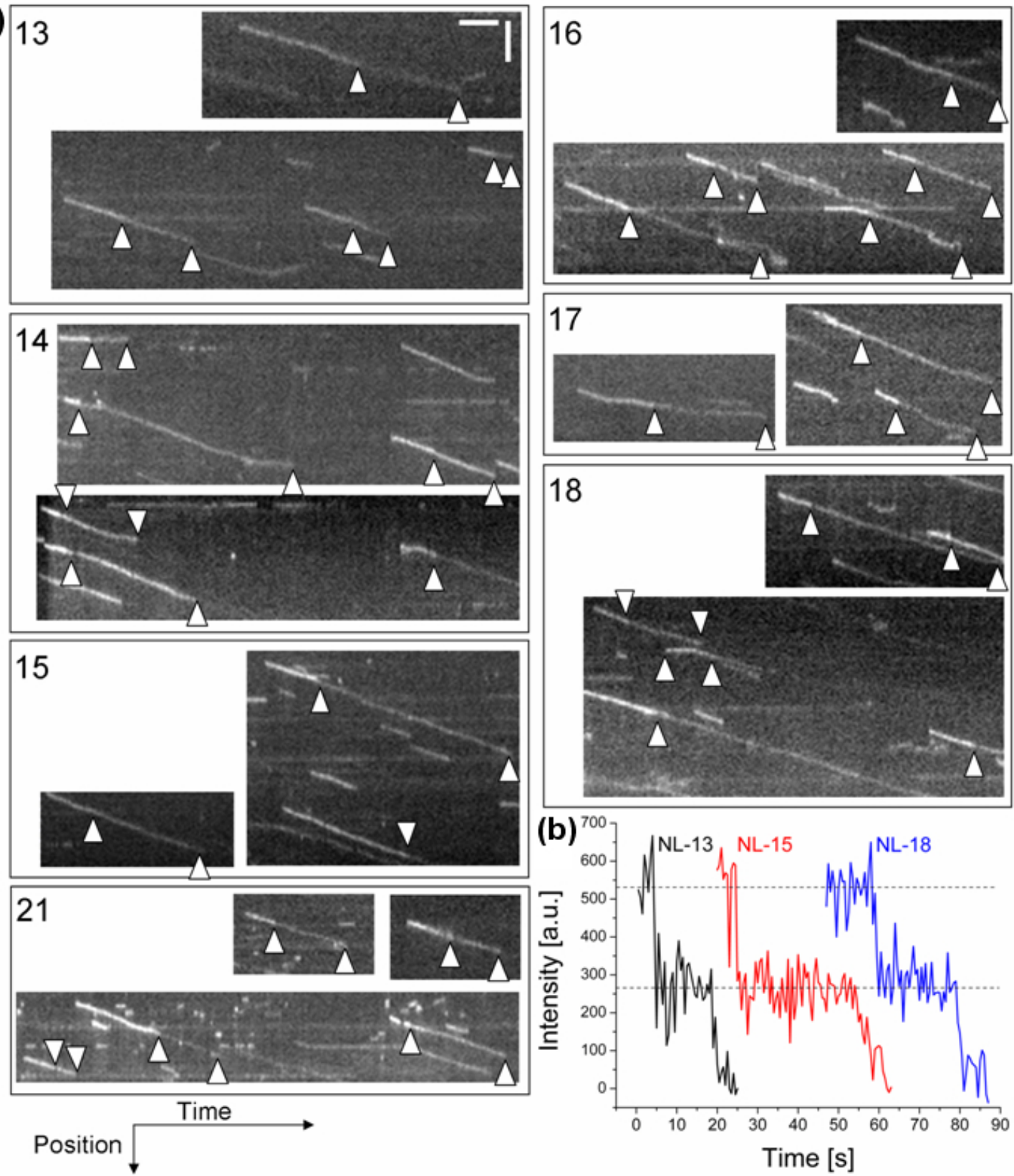

Fig. C.4: Control of motor dimer status by photo-bleaching: (a) Kymographs of single dimers moving on a surface-adsorbed microtubule. Arrowheads mark two-step bleaching of moving motor proteins. Scale bar: horizontal $10 \mathrm{~s}$, vertical $3 \mu \mathrm{m}$. (b) Representative intensity time series of single motors moving on a microtubule. 


\section{Supplemetatry data chapter 6: \\ A chimeric kinesin-1/kinesin-5 \\ microtubule-sliding motor switches between diffusive and processive motility}

This is the supplementary data for the chapter 6 titled "A chimeric kinesin-1/kinesin5 microtubule-sliding motor switches between diffusive and processive motility".

\section{Supplementary figures}

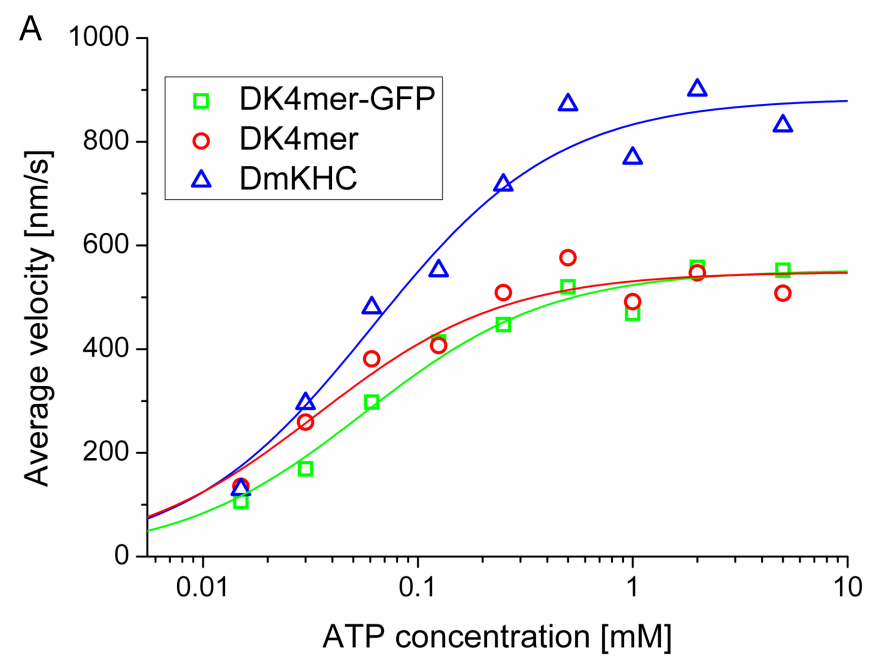

B

\begin{tabular}{l|c} 
Motor protein & Velocity [nm/s] \\
\hline DmKHC & $884 \pm 58(\mathrm{~N}=184)$ \\
\hline DK4mer-GFP & $553 \pm 31(\mathrm{~N}=206)$ \\
\hline DK4mer & $549 \pm 37(\mathrm{~N}=221)$
\end{tabular}

C

\begin{tabular}{l|l} 
Motor protein & $\mathrm{K}_{\mathrm{M}}[\mathrm{mM}]$ \\
\hline DmKHC & $0,061 \pm 0,010$ \\
\hline DK4mer-GFP & $0,056 \pm 0,001$ \\
\hline DK4mer & $0,034 \pm 0,006$
\end{tabular}

Fig. D.1: Multi-motor gliding assay with DK4mer-GFP, DK4mer and DmKHC. (A) Average MT velocity plotted over ATP concentration. The motor constructs DK4mer (circle), DK4mer-GFP (square) and DmKHC (triangle) are fitted with a Michaelis-Menten equation. The resulting maximal velocities and Michaelis-Menten constants $\mathrm{K}_{M}$ are listed in table (B) and (C) respectively.

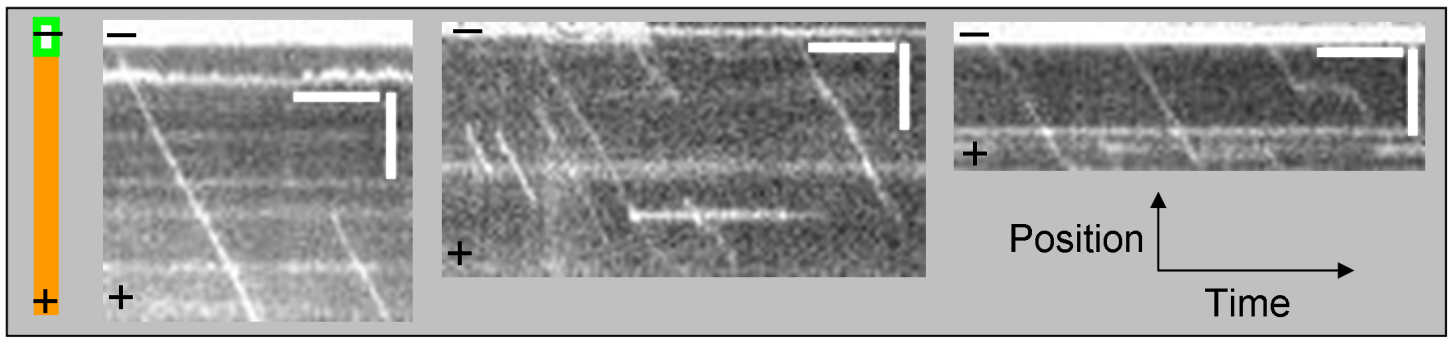

Fig. D.2: Single-molecule fluorescence assay with $2 \mathrm{mM}$ ATP in BRB80 buffer on polarity labeled MTs. Kymographs show single DK4mer motor proteins moving away from the brightly labeled minus-end $(-)$ and towards the plus-end $(+)$. Horizontal scale bars represent $10 \mathrm{~s}$ and vertical scale bars represent $3 \mu \mathrm{m}$. 


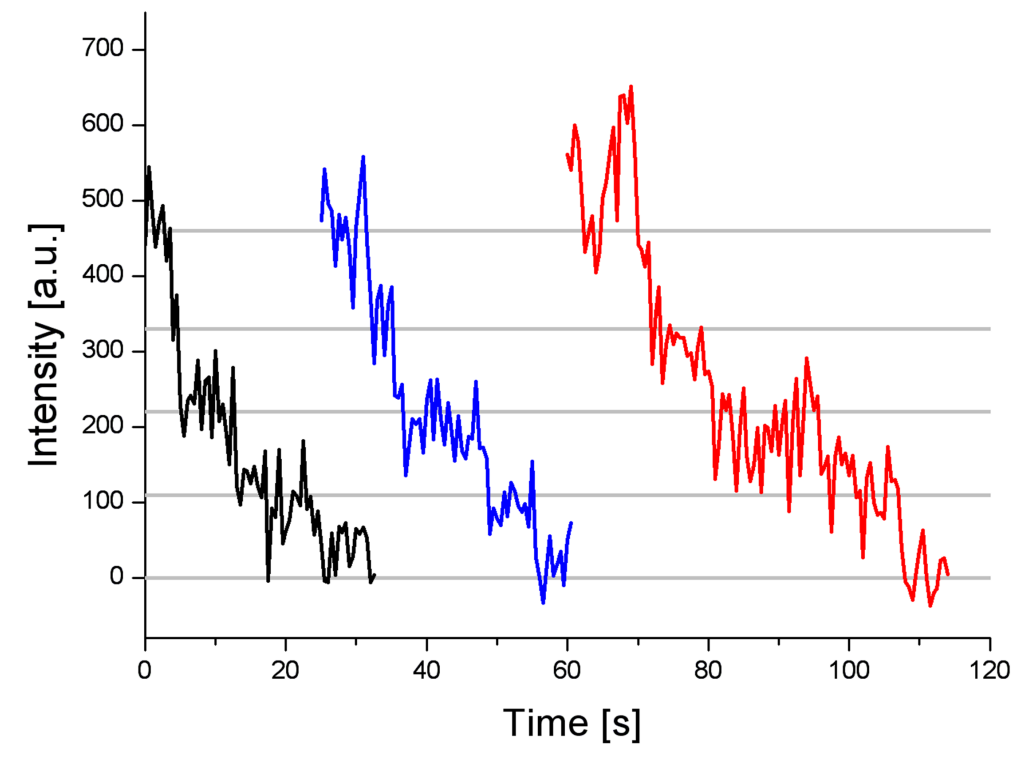

Fig.

D.3:

Intensity measurements of single DK4mer molecule runs. Four bleaching steps are visible confirming that the DK4mer is a tetrameric motor protein with four GFP molecules in total.

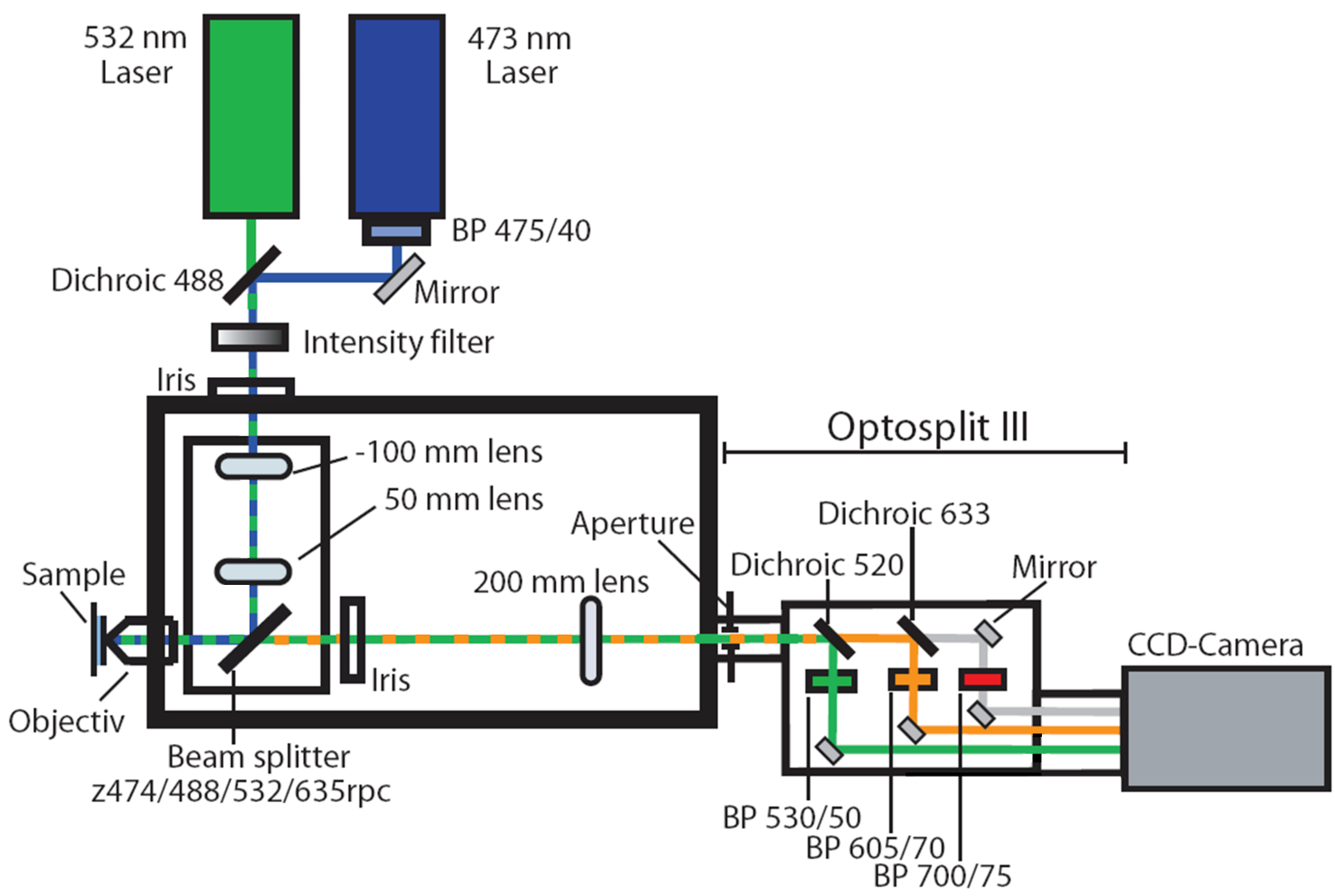

Fig. D.4: Diagram of the custom-build total-internal-reflection-fluorescence (TIRF) microscope used for single-molecule fluorescence experiments. 

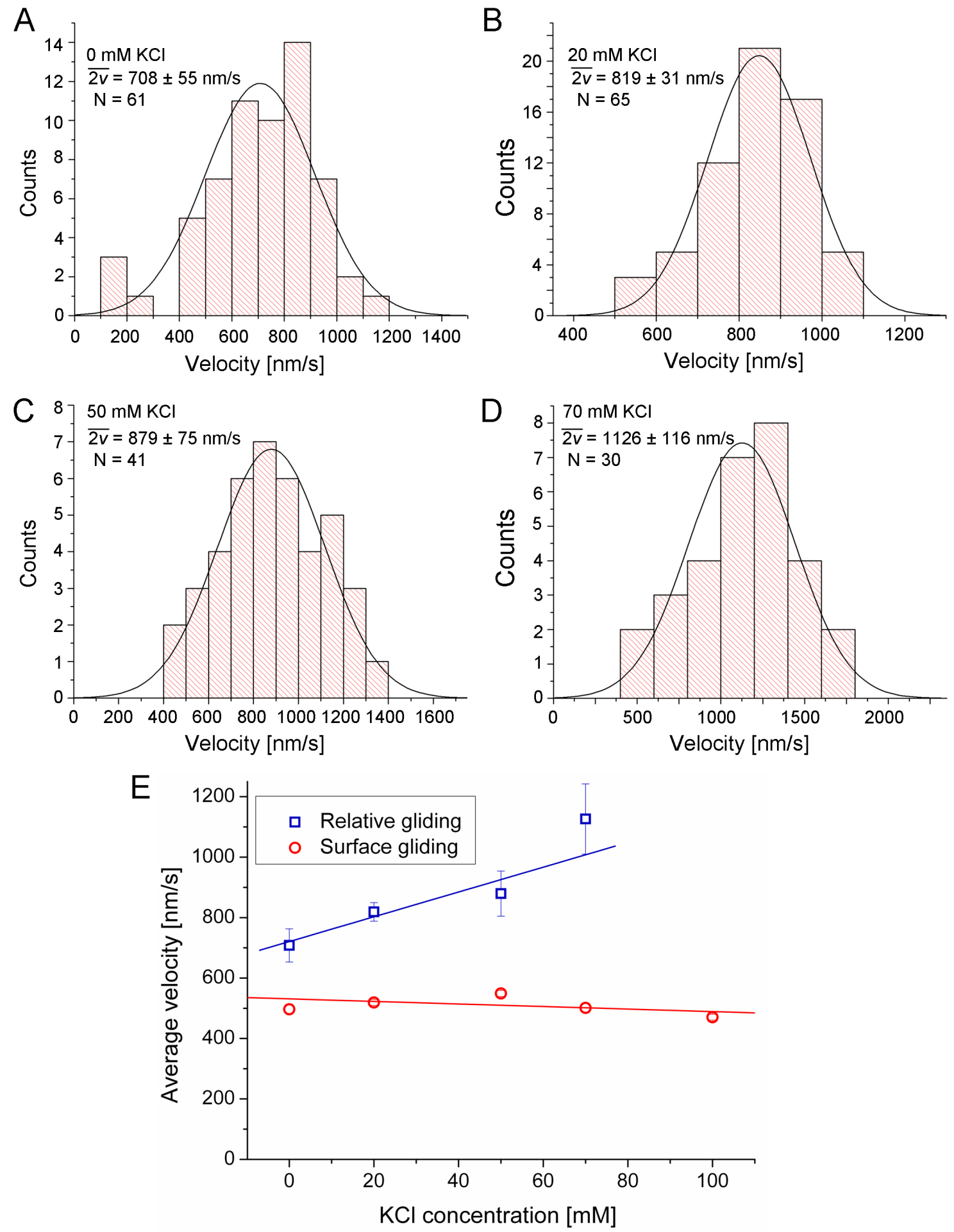

Fig. D.5: Ionic strength dependency of relative MT sliding velocity. (A) - (D) Velocity distribution at increasing $\mathrm{KCl}$ concentration added to P30 buffer ranging from no $\mathrm{KCl}$ added (A) up to $70 \mathrm{mM} \mathrm{KCl}$ added (D). (E) Average relative gliding velocity (squares) of MTs compared to surface gliding velocity (circles) over increasing $\mathrm{KCl}$ concentration. With increasing salt concentration the surface velocity remains constant while the relative gliding velocity increases. At salt concentrations over $60 \mathrm{mM}$ added $\mathrm{KCl}$ the expected sliding velocity of twice the single motor velocity $(\sim 1000 \mathrm{~nm} / \mathrm{s})$ is reached. 
XXVI Appendix D. Supplemetatry data chapter 6: A chimeric kinesin-1/kinesin-5 ...

\section{Supplementary table $\quad$ D.2}

\begin{tabular}{llll} 
Buffer conditions & DK4mer & DK511 & D421 \\
\hline \hline P30 & $492 \pm 4(\mathrm{~N}=202)$ & $517 \pm 5(\mathrm{~N}=106)$ & $679 \pm 7(\mathrm{~N}=166)$ \\
BRB80 & $499 \pm 3(\mathrm{~N}=212)$ & $530 \pm 4(\mathrm{~N}=228)$ & $659 \pm 5(\mathrm{~N}=200)$ \\
BRB80 + 10 mM KCl & $537 \pm 4(\mathrm{~N}=152)$ & & \\
BRB80 + 20 mM KCl & $522 \pm 4(\mathrm{~N}=138)$ & & \\
BRB80 + 40 mM KCl & $524 \pm 9(\mathrm{~N}=101)$ & & \\
BRB80 + 60 mM KCl & $548 \pm 12(\mathrm{~N}=115)$ & & \\
\hline
\end{tabular}

(a) Average velocity $[\mathrm{nm} / \mathrm{s}]$

\begin{tabular}{llll} 
Buffer conditions & DK4mer & DK511 & D421 \\
\hline \hline P30 & $9556 \pm 2367(\mathrm{~N}=202)$ & $3426 \pm 438(\mathrm{~N}=106)$ & $2713 \pm 496(\mathrm{~N}=166)$ \\
BRB80 & $9127 \pm 1259(\mathrm{~N}=212)$ & $2293 \pm 913(\mathrm{~N}=228)$ & $2901 \pm 801(\mathrm{~N}=200)$ \\
BRB80 + 10 mM KCl & $3836 \pm 337(\mathrm{~N}=152)$ & & \\
BRB80 + 20 mM KCl & $2623 \pm 716(\mathrm{~N}=138)$ & & \\
BRB80 + 40 mM KCl & $1981 \pm 308(\mathrm{~N}=101)$ & & $1351 \pm 188(\mathrm{~N}=90)$ \\
BRB80 + 60 mM KCl & $1681 \pm 807(\mathrm{~N}=115)$ & & \\
\hline
\end{tabular}

(b) Average run length $[\mathrm{nm}]$

Tab. D.1: List of the average velocity (a) and the average run length (b) in buffer solutions with different salt concentration for the different motor proteins.

\section{\begin{tabular}{l|l} 
Description of supplementary videos & D.3
\end{tabular}}

\section{Supplementary Video D.3.1:}

Representative movie of a multi-motor gliding assay with $2 \mathrm{mM}$ ATP in the assay buffer. TMR-labeled MTs are moved by surface absorbed DK4mer motor proteins. Images were taken every $1 \mathrm{~s}$ and the total time is $50 \mathrm{~s}$.

\section{Supplementary Video D.3.2:}

Representative movie of a single-molecule fluorescence motility assay. At the beginning a TMR-labeled MT is visible along which after changing the filter the GFPlabeled DK4mer motor proteins are moving. Images were taken every $0.5 \mathrm{~s}$ and the total time is $147 \mathrm{~s}$.

\section{Supplementary Video D.3.3:}

Representative movie of a relative sliding assay with polarity marked MTs (bright 
minus ends). MTs from solution align with a MT fixed to the surface and slide (in this case in antiparallel conformation) along the fixed MT. Images were taken every $0.5 \mathrm{~s}$ and the total time is $31 \mathrm{~s}$.

\section{Supplementary Video D.3.4:}

Representative movie of a dual color single-molecule relative-sliding assay with polarity-marked MTs. TMR-labeled (red) MTs slides over fixed TMR-labeled MTs while single GFP-labeled (green) DK4mer motor proteins move along and between the MTs. Images were taken every $0.5 \mathrm{~s}$ and the total time is $13 \mathrm{~s}$. 



\section{Supplemetatry data chapter 7: Endoplasmic reticulum sorting and kinesin-1 command the targeting of axonal $\mathrm{GABA}_{B}$ receptors}

This is the supplementary data for the chapter 7 titled "Endoplasmic reticulum sorting and kinesin-1 command the targeting of axonal GABA $B$ receptors". The paper is currently in print in PLoS ONE.

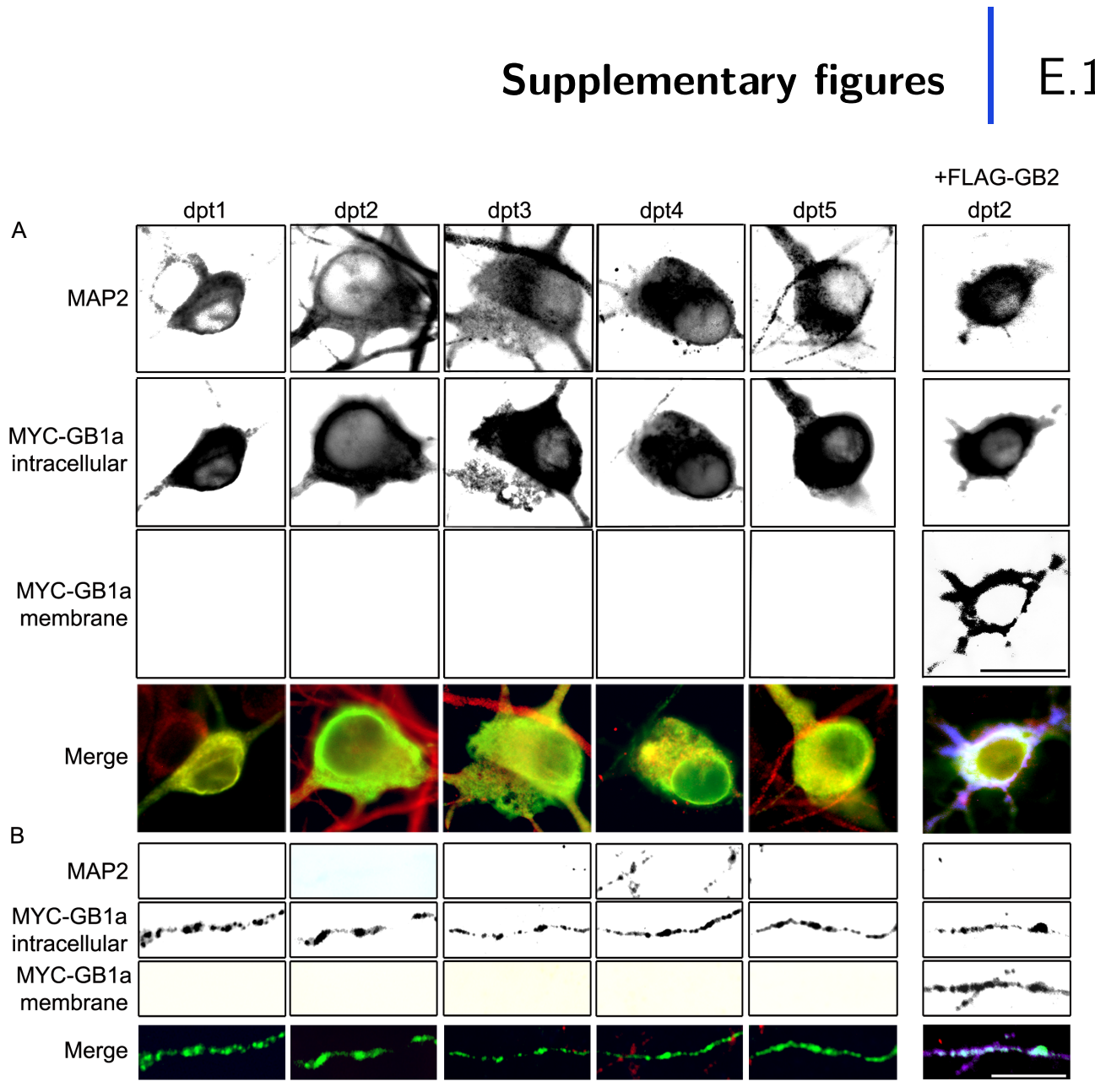

Fig. E.1: Recombinant $\mathrm{GABA}_{B} \mathrm{R} 1 \mathrm{a}$ is retained in intracellular compartments in hippocampal neurons.

(A) Hippocampal neurons were transfected with $\mathrm{MYC}-\mathrm{GABA}_{B} \mathrm{R} 1 \mathrm{a}$ and processed for immunofluorescence under non-permeabilized conditions to detect cell surface epitopes followed by permeabilization to detect intracellular epitopes at the indicated days post transfection (dpt). Intracellular MAP2 (red), intracellular GABA ${ }_{B} \mathrm{R} 1 \mathrm{a}$ (MYC-GB1 intracellular, green), plasma membrane $\mathrm{GABA}_{B} \mathrm{R} 1 \mathrm{a}$ (MYC-GB1 membrane, magenta). Control neurons were transfected with MYC-GABA ${ }_{B}$ R1a and FLAG-GABA ${ }_{B}$ R2 (+FLAG-GB2, right column). Merged images are shown on the bottom panel. (B) Axons of hippocampal neurons under the same experimental conditions. Images are not single focus planes, therefore the intensity represents the signal from the entire cell (representative images of $\mathrm{n}=30$ neurons). Scale bar for (A) and (B) represents $20 \mu \mathrm{m}$. 


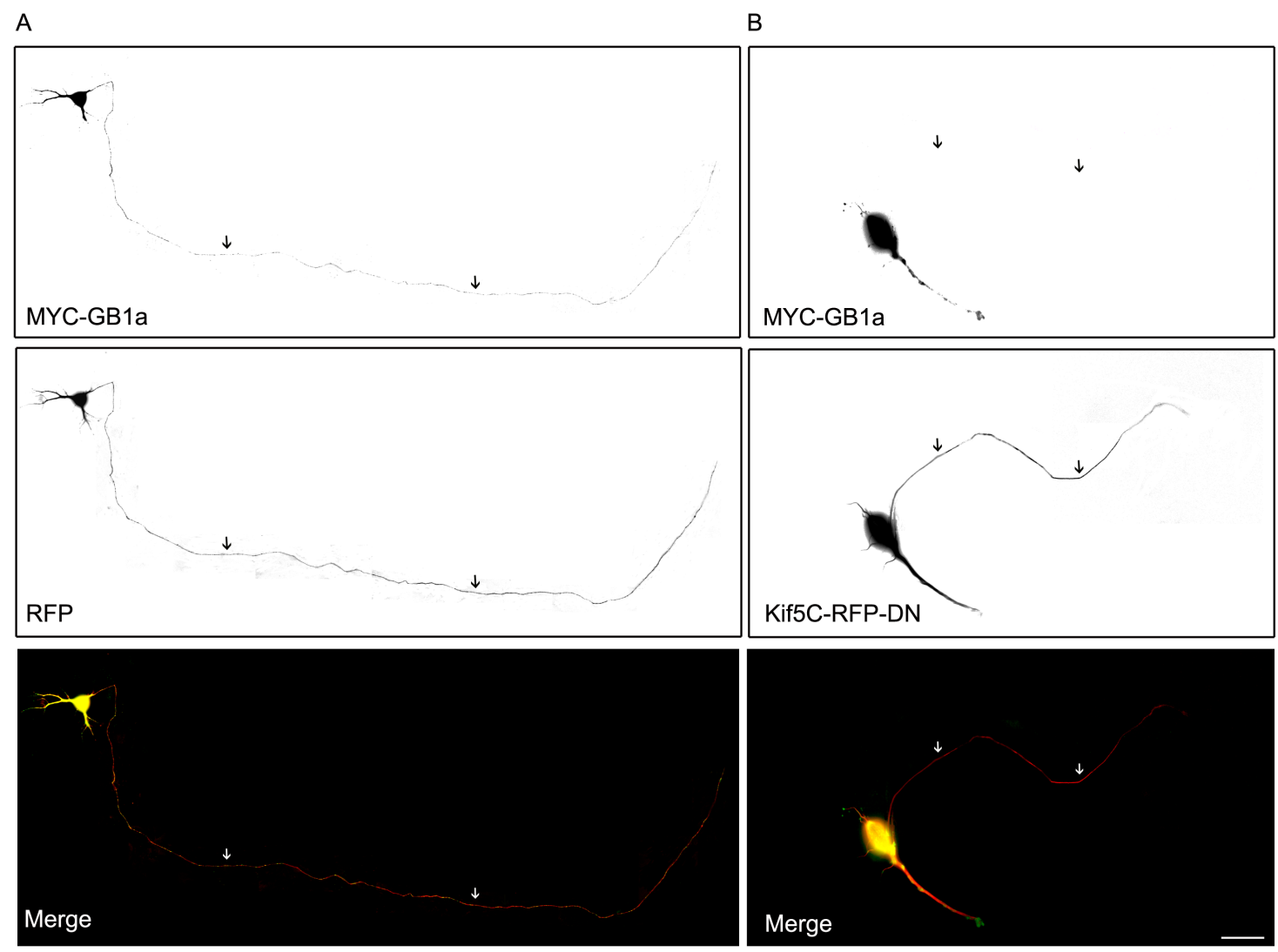

Fig. E.2: Axonal targeting of $\mathrm{GABA}_{B} \mathrm{R} 1 \mathrm{a}$ is kinesin- 1 dependent.

(A) Hippocampal neurons were transfected with MYC-GABA ${ }_{B}$ R1a (MYC-GB1) and RFP. Merged images are shown on the bottom panel. (B) Same as above for MYC-GABA ${ }_{B}$ R1a and Kif5C-RFP-DN. Axonal localization of MYC-GABA ${ }_{B}$ R1a or its absence from the axon is indicated by arrows. Scale bar represents $20 \mu \mathrm{m}$. 

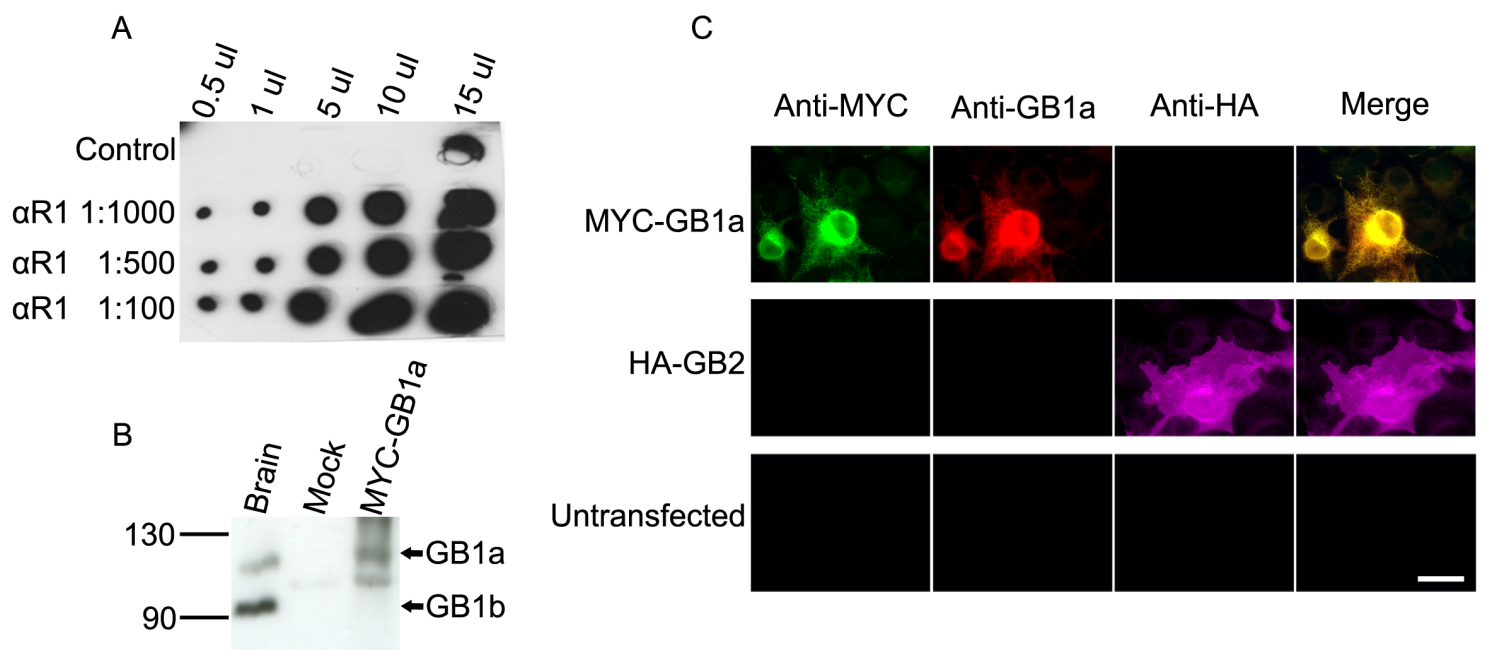

Fig. E.3: Antibodies recognize $\mathrm{GABA}_{B} \mathrm{R} 1$ subunits specifically.

(A) Increasing concentrations of lysates prepared from MYC-GABA ${ }_{B} \mathrm{R} 1 \mathrm{a}$ transfected cells were applied on a nitrocellulose membrane and immunoblotted with control serum (control) or serum from a rabbit immunized with a GST fusion protein containing the Cterminal domain of $\mathrm{GABA}_{B} \mathrm{R} 1(\alpha \mathrm{R} 1)$. (B) Lysates prepared from crude rat brain membranes (Brain), untransfected COS7 cells (Mock), or MYC-GABA ${ }_{B}$ R1a transfected COS7 cells (MYC-GB1a) were immunoblotted with affinity purified $\mathrm{GABA}_{B} \mathrm{R} 1$ antibodies. (C) COS7 were transfected with MYC-GABA ${ }_{B}$ R1a (MYC-GB1a, top), HA-GABA ${ }_{B}$ R2 (HAGB2, middle) or left untransfected (bottom). Cells were fixed and processed for immunofluorescence using MYC antibodies (green), affinity purified $\mathrm{GABA}_{B} \mathrm{R} 1$ antibodies (red) and HA antibodies (magenta). Merged images are shown on the right. Scale bar represents $20 \mu \mathrm{m}$. 



\section{Published papers in pdf format}

Due to copyright restriction the following already printed publications are attached in this section in the original pdf format of the respective journals.

Stefan Lakämper, Christina Thiede, Andrè Düselder, Stefanie Reiter, Mikhail J. Korneev, Lukas C. Kapitein, Erwin J. G. Peterman and Christoph F. Schmidt; 2010: The Effect of Monastrol on the Processive Motility of a Dimeric Kinesin-5 Head/Kinesin-1 Stalk Chimera, Journal of Molecular Biology, 399: 1-8.

Adina Gerson-Gurwitz*, Christina Thiede*, Natalia Movshovich, Vladimir Fridman, Maria Podolskaya, Tsafi Danieli, Stefan Lakämper, Dieter R. Klopfenstein, Christoph F. Schmidt and Larisa Gheber; 2011: Directionality of individual kinesin5 Cin 8 motors is modulated by loop 8, ionic strength and microtubule geometry, The EMBO Journal, 30: 4942-4954. *Authors contributed equally.

Christina Thiede, Vladimir Fridman, Adina Gerson-Gurwitz, Larisa Gheber and Christoph F. Schmidt; 2012: Regulation of bi-directional movement of single kinesin5 Cin8 molecules, BioArchitecture, 2: 70-74.

Andrè Düselder*, Christina Thiede*, Christoph F. Schmidt and Stefan Lakämper; 2012: Neck-Linker Length Dependence of Processive Kinesin-5 Motility, Journal of Molecular Biology, 423: 159-168. *Authors contributed equally.

Viviana Valdés, José Ignacio Valenzuela, Matías Jaureguiberry-Bravo, Daniela Salas, Carolina Otero, Christina Thiede, Christoph F. Schmidt and Andrés Couve; 2012: Endoplasmic Reticulum Sorting and Kinesin-1 Command the Targeting of Axonal GABA $_{B}$ Receptors, PLoS ONE, 7: e44168. 



JMI

\title{
Communication
}

\section{The Effect of Monastrol on the Processive Motility of a Dimeric Kinesin-5 Head/Kinesin-1 Stalk Chimera}

\author{
Stefan Lakämper ${ }^{1,2,3 *}$, Christina Thiede ${ }^{1}$, André Düselder ${ }^{1}$, \\ Stefanie Reiter $^{1,3}$, Mikhail J. Korneev ${ }^{2}$, Lukas C. Kapitein ${ }^{2}$, \\ Erwin J. G. Peterman ${ }^{2}$ and Christoph F. Schmidt ${ }^{1,2,3 *}$
}

\author{
${ }^{1}$ Drittes Physikalisches Institut, \\ Georg-August-Universität \\ Göttingen, Göttingen, Germany \\ ${ }^{2}$ Department of Physics and \\ Astronomy and Laser Center, \\ Free University Amsterdam, \\ Amsterdam, The Netherlands \\ ${ }^{3}$ Deutsche \\ Forschungsgemeinschaft \\ Research Center for Molecular \\ Physiology of the Brain, \\ Göttingen, Germany
}

Received 20 November 2009; received in revised form

26 February 2010; accepted 3 March 2010

Available online

19 March 2010

\section{Edited by J. Karn}

\begin{abstract}
Controlled activity of several kinesin motors is required for the proper assembly of the mitotic spindle. Eg5, a homotetrameric bipolar kinesin-5 from Xenopus laevis, can cross-link and slide anti-parallel microtubules apart by a motility mechanism comprising diffusional and directional modes. How this mechanism is regulated, possibly by the tail domains of the opposing motors, is poorly understood. In order to explore the basic unregulated kinesin-5 motor activity, we generated a stably dimeric kinesin-5 construct, Eg5Kin, consisting of the motor domain and neck linker of Eg5 and the neck coiled coil of Drosophila melanogaster kinesin-1 (DmKHC). In single-molecule motility assays, we found this chimera to be highly processive. In addition, we studied the effect of the kinesin-5-specific inhibitor monastrol using single-molecule fluorescence assays. We found that monastrol reduced the length of processive runs, but strikingly did not affect velocity. Quantitative analysis of monastrol dose dependence suggests that two bound monastrol molecules are required to be bound to an Eg5Kin dimer to terminate a run.
\end{abstract}

(C) 2010 Elsevier Ltd. All rights reserved.

Keywords: kinesin-5; single-molecule fluorescence; processivity; motor cooperativity; monastrol inhibition

\section{Introduction}

The vertebrate kinesin-5 Eg5 is a homotetrameric motor protein required for proper separation of the poles of the bipolar mitotic spindle during cell division. ${ }^{1-3}$ The Drosophila homolog (Klp61F) of Eg5 was found to be an extended molecule approximately $80 \mathrm{~nm}$ long, with two motor domains at each end of a central stalk. ${ }^{4}$ It has been demonstrated in in vitro microtubule sliding assays that Eg5 can crosslink microtubules and slide anti-parallel microtubules apart, ${ }^{5}$ consistent with the structure and in vivo function of Eg5. Single-molecule fluorescence

${ }^{*}$ Corresponding authors. E-mail addresses:

lakaemper@physik3.gwdg.de; cfs@physik3.gwdg.de.

Present address: L. C. Kapitein, Erasmus University

Medical Center, Rotterdam, The Netherlands.

Abbreviation used: GFP, green fluorescent protein. experiments using green fluorescent protein (GFP)tagged Eg5 tetramers have shown that Eg5 motility is surprisingly complex: Depending on buffer conditions, single Eg5 motors show a mixture of diffusive and ATP-dependent directional motility modes along single microtubules while staying attached for several tens of seconds. ${ }^{6,7}$ When Eg5 cross-links two microtubules, diffusive motility is suppressed, and directional motility is favored. ${ }^{7}$ In optical trapping experiments with Eg5 tetramers, processive stepping was observed, but motors detached from the microtubules at a comparatively low force $(\sim 1.6 \mathrm{pN}),{ }^{8}$ suggesting a force-sensing mechanism that is not seen in kinesin- 1 motors. This sensitivity may, in some way, be caused by interaction with the opposing dimer because truncated dimeric constructs of human Eg5 (HsEg513His) could withstand higher forces in an optical trap assay. Their processivity was low, however, with only six to eight steps per run, ${ }^{9}$ in 
agreement with single-molecule fluorescence experiments on comparable Xenopus constructs. ${ }^{7}$

There is evidence that the additional microtubule binding site mediating diffusive interactions of Eg5 is located in parts that are missing in truncations, namely, in the conserved BimC box or the tail. ${ }^{4,10}$ This conclusion is supported by the ability of tetrameric headless constructs of the Drosophila kinesin-5 homolog Klp61F to cross-link microtubules. ${ }^{11}$ Furthermore, the single-molecule and multiple-molecule velocities of the chimeric constructs, as well as the single-molecule velocity of hsEg5-513 measured in trapping experiments $(\sim 95 \mathrm{~nm} / \mathrm{s}),{ }^{9}$ are $\sim 4-8$ times higher than that of the full-length motor. $5,6,8$ Such a discrepancy is actually expected, given the observation that the motility of the full-length motor consists of an alternation of directional and diffusive periods, ${ }^{6}$ where the diffusive periods are supported by additional MT binding by the tail and where switching to directional motility appears to be regulated by microtubule binding on the opposite end. ${ }^{7}$ In earlier single-molecule fluorescence experiments, ${ }^{6,7}$ the processive motor speed between diffusive periods could not be quantified, since switching between the two states was rapid and could not be resolved. Rapid switching indicates short directional episodes, again consistent with the finding of moderate processivity for the dimeric Eg5 constructs. ${ }^{9,12}$

A recent study claims, on the basis of microtubule gliding experiments with Eg5 homodimers, monomers, and Eg5-kinesin-1 heterodimers, that kinesin5 motors move predominantly nonprocessively. ${ }^{13}$ It has remained unclear whether the limited processivity of truncated dimers is (i) an intrinsic property of the motor domains of Eg5, (ii) a consequence of the two coiled-coil domains of the opposing dimer that are lacking, or (iii) a consequence of the absence of the C-terminal tail domains ${ }^{10}$ that, in the tetramer, are in close proximity to the motor domains and might affect processive motility.

We have here constructed a stably dimeric chimeric motor to study the intrinsic capabilities of the Eg5 motor domains and to address the role of coil and tail domains. The chimera consisted of the main force-generating parts of Eg5-motor domain and neck linker-fused to the neck coiled coil of Drosophila kinesin-1, ensuring stable dimerization. The chimera lacks the opposing pair of motor domains, as well as the tail domains that were found to be responsible for diffusive motion in Klp61F. ${ }^{14}$ We hypothesized this chimera to be processive without diffusional episodes. Important questions were would the chimera be more processive than truncated dimeric constructs, how much load could it support and how fast would it move?

A further characteristic feature of wild-type Eg5 is that it can be inhibited specifically by the small molecule monastrol. ${ }^{3}$ In cells, monastrol causes spindle collapse and cell cycle arrest ${ }^{3}{ }^{3}$ while in in vitro microtubule gliding assays, monastrol inhibits microtubule motility. Both effects are due to a so far poorly understood allosteric mechanism, since the binding site of monastrol overlaps neither with the microtubule binding site nor with the nucleotide binding site. ${ }^{15,16}$ A recent cryo-electron microscopy study of the motor domain of Drosophila melanogaster Klp61F (residues 1-368) suggests that the uniquely extended loop 5 plays an important role in the monastrol-induced transition to a diffusive state and in the inhibition of motility. ${ }^{17,18}$ In single-molecule fluorescence experiments, the interaction times of single Eg5 homotetramers with microtubules were shown to be shorter in the presence of monastrol. In addition, the motility was less directional and more diffusive $^{6}$ (i.e., average velocity decreased in a dosedependent manner). An important question that could not be answered in those tetramer assays is: Does the processive velocity of Eg5 decrease in the presence of monastrol, or does the probability to switch to the diffusive state increase? Kinetic experiments ${ }^{19}$ showed that monastrol slows the ADP release rate of monomeric Eg5 truncations both in the absence and in the presence of microtubules. Whether the same effect would occur in the dimer context and whether this would lead to a lower processive velocity or to release from the microtubule remained open. These questions can be directly addressed with our chimera, where opposing tail interactions do not complicate the situation. ${ }^{8}$

\section{Results and Discussion}

Our first goal was to create a stably dimeric Eg5. A shortened Eg5, containing about half the stalk, is thought to preferentially form dimers; however, unfortunately, these dimers appear to be rather unstable in the absence of the opposing stalk. This may be due to the fact that the full-length motor has evolved a strong preference for tetramer assembly, for which it may help to not get stuck in a stable dimer conformation. ${ }^{7}$ Similar problems with other motors have been successfully addressed by the use of chimeric constructs. ${ }^{20-23}$ We therefore constructed a chimeric motor (Eg5Kin) by replacing the motor domain and the neck linker of Drosophila melanogaster kinesin-1, which is known to form stable dimers (residues 1-343 from pPK113, a kind gift from W. O. Hancock), with the corresponding domains of Xenopus laevis Eg5 (residues 1-369). We used the full-length chimera and also a truncated version (at residue 421 using the Drosophila numbering) that was tagged with GFP at the C-terminus (Eg5Kin-GFP). All constructs were expressed in Escherichia coli and purified using a C-terminal His tag (Fig. 1).

To test the motility of the chimeric motor protein, we performed surface-gliding assays. We found that Eg5Kin motors bound readily to glass surfaces and moved microtubules with a velocity of $95 \pm 10 \mathrm{~nm} / \mathrm{s}$ $(N=52$; Supplementary Video 1, BRB80 buffer). Truncated Eg5Kin-GFP motors moved microtubules with a comparable velocity of $88 \pm 8 \mathrm{~nm} / \mathrm{s}(N=45)$. At very low Eg5Kin motor concentrations, we observed pivoting of microtubules around a single 


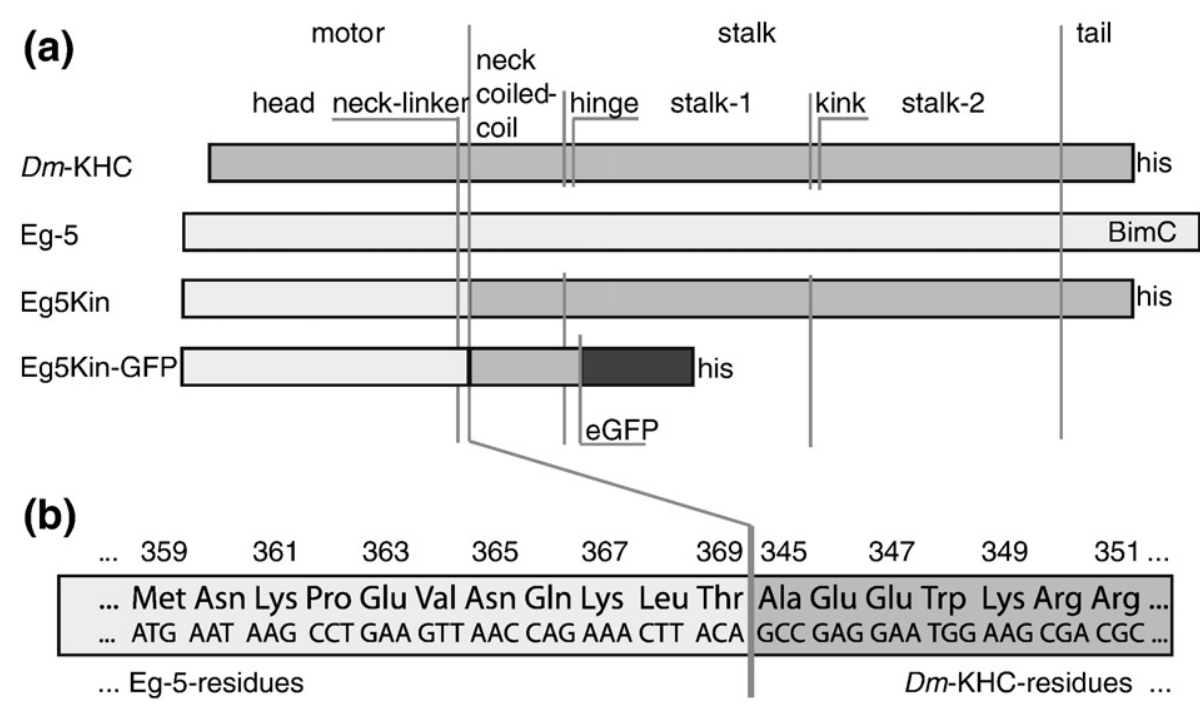

Fig. 1. Motor constructs. Constructs were generated by taking advantage of the basic structural organization shared by kinesin-1 (here DmKHC) and kinesin-5 (here Eg5) family members. (a) The motor domain consists of globular head and neck linker in both wild-type motors. Within the extended stalk, there are flexible (hinge and kink) and rigid coiled-coil regions (neck coiled coil; stalks 1 and 2) in kinesin-1, which are not obvious in kinesin-5 motors. ${ }^{24}$ The small globular tail domain of Eg5 contains the BimC box. (b) Details of the junction between the Eg5 motor domain and the DmKHC neck coiled coil: The numbering refers to the amino acid numbering in the respective wild-type motor sequences as indicated in gray. Methods: Eg5Kin was constructed from pPK113 (pET5a-DmKHC-His) ${ }^{25}$ and pBK006 (Eg5-GFP) ${ }^{6}$ using a nested PCR approach to extend the sequence of the Eg5 motor domain (residues 1-369) with the DmKHC residues starting at residue 345 towards an existing HindIII restriction site in pPK113. The DmKHC motor domain in pPK113 was subsequently replaced using NdeI and HindIII. The shortened GFP-tagged Eg5Kin-GFP was truncated at residues corresponding to DmKHC421, using PCR primers providing an AscI-XmaI site upstream of a stop codon. The full-length DmKHC in pPK113 was replaced with this truncated DmKHC421 using NdeI and NotI. A GFP-6His cassette flanked by AscI and XmaI was generated in pT7-7 for insertion downstream of the truncated Eg5Kin construct in pPK113. Integrity was confirmed by sequencing. Expression and purification were performed as previously described in Coy et al. (for a more detailed description of materials and methods, see Supplementary Information). ${ }^{25}$

point of attachment, while movement relative to this point occurred at a velocity of $92 \pm 15 \mathrm{~nm} / \mathrm{s}(N=10$; Supplementary Video 2). This behavior is characteristic of processive motility and gives qualitative evidence that Eg5Kin is a processive motor. ${ }^{26}$

To further explore the motility and force generation capabilities of Eg5Kin, we performed opticaltrapping experiments with single Eg5Kin-GFP motors attached to silica beads with a diameter of $1 \mu \mathrm{m}$. We observed that single motors displaced the beads processively at a somewhat lower average velocity $(56 \pm 10 \mathrm{~nm} / \mathrm{s} ; N=28)$ (Fig. 2a) than seen in the surface-gliding assays. As this difference might have been due to the use of a buffer containing only $12 \mathrm{mM}$ Pipes instead of the standard BRB80 buffer, we performed the same experiments in BRB80 with similar results $(63 \pm 2 \mathrm{~nm} / \mathrm{s} ; N=93)$. We also observed short periods of backward slipping, similar to what has been reported before for truncated Eg 5 dimers. ${ }^{9}$ The motors detached from the microtubules when reaching a force of $4.6 \pm 0.1 \mathrm{pN}(N=27)$, on average, for $12 \mathrm{mM}$ Pipes (Fig. 2b) and a force of $4.4 \pm 0.1 \mathrm{pN}$ $(N=93)$ for BRB80, substantially higher than the maximal force reported for full-length Eg5 motors $(\sim 1.6 \mathrm{pN}){ }^{8} \quad$ A remarkable difference from the behavior of kinesin- 1 is that our kinesin- 5 chimera detached mostly without noticeable prior stalling. 8,28

In order to determine the number of processive steps that Eg5Kin takes in the absence of an opposing force, we performed single-molecule motility assays with Eg5Kin-GFP using fluorescence microscopy. ${ }^{5}$ We observed that single fluorescent spots moved unidirectionally at a speed of $96 \pm$ $0.3 \mathrm{~nm} / \mathrm{s}$ along microtubules ( $N=82 ; \mathrm{BRB} 80$ buffer) (Fig. 3a and b; see also Supplementary Video 4), consistent with the results from optical-trapping and surface-gliding assays. In contrast to full-length $\mathrm{Eg} 5,6,7$ there was no evidence of diffusive periods during motility. Both surface-immobilized and moving spots photobleached in, at most, two consecutive steps (Fig. 3a; Supplementary Fig. 1), confirming that the observed spots were indeed single dimeric Eg5Kin-GFP motors. Single motors interacted for, on average, $19.1 \pm 3.5 \mathrm{~s}$ with the microtubules (Fig. 3c), while moving $1.8 \pm 0.3 \mu \mathrm{m}$ on average, corresponding to $\sim 230$ steps of $8 \mathrm{~nm}$.

These results demonstrate that the processive motility of our Eg5Kin chimeras is similar to that reported for truncated dimers in terms of both speed $(\sim 95 \mathrm{~nm} / \mathrm{s})$ and force $(\sim 5 \mathrm{pN}){ }^{7,9}$ The run length of the chimeras was, however, substantially longer (230 steps instead of 6-8 steps) than that of truncated dimers. ${ }^{9}$ The processive movement of single Eg5Kin dimers proves that the $\mathrm{Eg} 5$ motor domains are intrinsically capable of extended processive motility. The increased run length of Eg5Kin, as compared to HsEg5-513, is consistent with two studies recently published by Kalchishkova and Bohm $^{23}$ and 


\section{(a)}
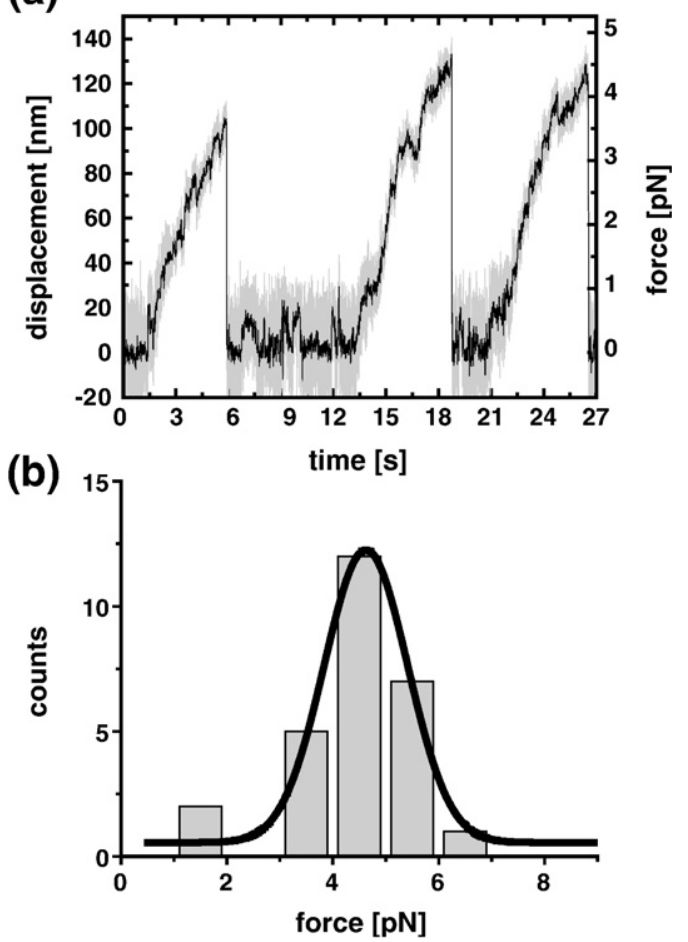

Fig. 2. Single-molecule force measurements in an optical trap. (a) Displacement of a 1- $\mu \mathrm{m}$ silica bead driven by a single Eg5Kin motor, showing multiple excursions from the center of the trap and detachment without stalling plateaus (average initial velocity of $56 \pm 10 \mathrm{~nm} / \mathrm{s}$; $N=28$ ). (b) Histogram of the detachment forces of single Eg5Kin motors (average detachment force of $4.6 \pm 0.1 \mathrm{pN}$; $N=27)$. Motility assays: Experiments were essentially performed as previously described by Korneev et al. ${ }^{8}$ for single-molecule optical trapping assays, by Lakamper and Meyhofer ${ }^{27}$ for surface-gliding assays, and by Kwok et al. ${ }^{6}$ and Kapitein et al. ${ }^{7}$ for single-molecule fluorescence assays. Details are presented in Supplementary Information.

Muthukrishnan et al. ${ }^{29}$ and with a biochemical study by Hackney et al. ${ }^{30}$ In the first study, in which a human kinesin-5 motor domain, including its neck linker, was fused to a human neuronal kinesin-1 (construct E-K(neck)), ${ }^{23}$ the authors observed an increase in speed as compared to a presumably dimeric truncation of human kinesin-5. Processivity was not assessed. The latter two studies show that extending the neck linker (in Muthukrishnan et al. ${ }^{29}$ by three homologous amino acids from kinesin-2) leads to a decrease in the processive motility of kinesin-1. In our construct, we deliberately chose the fusion sites to be homologous to the site in kinesin-1 that is commonly regarded as the location of the transition from neck linker to neck coiled coil.

One possible structural explanation for the increased processivity of Eg5Kin might be enhanced gating, as compared to kinesin-5 dimers, due to the decreased neck-linker length of Eg5Kin (Eg5Kin and kinesin-1: 14 aa; kinesin-5: 18 aa). ${ }^{29}$ The notion that gating is a major factor in determining processivity is supported by Eg5 dimer trapping experiments in which moderate hindering loads rescue processivity at limiting ATP concentrations. ${ }^{31}$ Our observation of processivity thus suggests that the neck linker of Eg5Kin is of appropriate length to allow effective head-to-head communication.

It has been hypothesized that charge patterns in the neck coiled coil enhance processivity. ${ }^{32,33} \mathrm{We}$ think that this explanation is not likely to apply to the increased processivity we observed here because results with less charged Neurospora kinesin-1 and with microtubules without the negatively charged E-hook removed have ruled out the charge model for extended processivity. ${ }^{27,34}$

It is intriguing that the motors typically detach before they stall. The relatively high velocity at the point of detachment makes sense, since the detachment forces of $4-5 \mathrm{pN}$ are only about $60 \%$ of typical kinesin-1 stall forces. This peculiar behavior of Eg5, however, demonstrates that there must be a structural feature of the Eg5 head and neck linker that creates force sensitivity. Understanding this mechanism needs further study.

In a further set of experiments, we focused on the inhibition of Eg5Kin motility by monastrol. First, we performed surface-gliding assays at high motor concentrations in the presence of monastrol. Unexpectedly, microtubule motion driven by Eg5Kin motors was not affected by monastrol at concentrations of up to $200 \mu \mathrm{M}$, and velocity remained constant at about $95 \mathrm{~nm} / \mathrm{s}$ (Fig. 4a). At monastrol concentrations above $250 \mu \mathrm{M}$, motility ceased altogether. This finding is consistent with an all-ornothing mode of operation of monastrol in the collective mode of motion of a microtubule by many surface-attached motors. Assuming that the motors inhibited by monastrol do impose only a very low friction upon the gliding microtubule, as has been suggested in the literature, ${ }^{18}$ one would-in a situation where many processive motors drive one microtubule-expect full-speed motility to continue until the last of the interacting motors is inhibited.

In order to discover how monastrol affects the processive motility of individual Eg5Kin motors, we performed single-molecule fluorescence assays in the presence of increasing monastrol concentrations. Individual Eg5Kin motors moved unidirectionally, without any diffusive interruptions, even at monastrol concentrations of $100 \mu \mathrm{M}$ (Fig. 4b). Just like in the surface-gliding assays, the velocity was not measurably affected by monastrol (Fig. 4a and b). We did, however, observe a monastrol-dependent reduction of the processive run length (Fig. $4 \mathrm{~b}$ and c; see also Supplementary Fig. 2). In other words, monastrol led to an increase in the rate of motor detachment or a decrease in run durations. Since there are two binding sites for monastrol on a motor dimer, one might have expected an intermediate or perturbed motility mode with just one monastrol bound. In fact, we do not see any change in speed or motile properties during processive runs before detachment. There are three possible explanations for this finding: (i) run termination is caused already by the binding of one monastrol to a dimer, or (ii) 
(a)

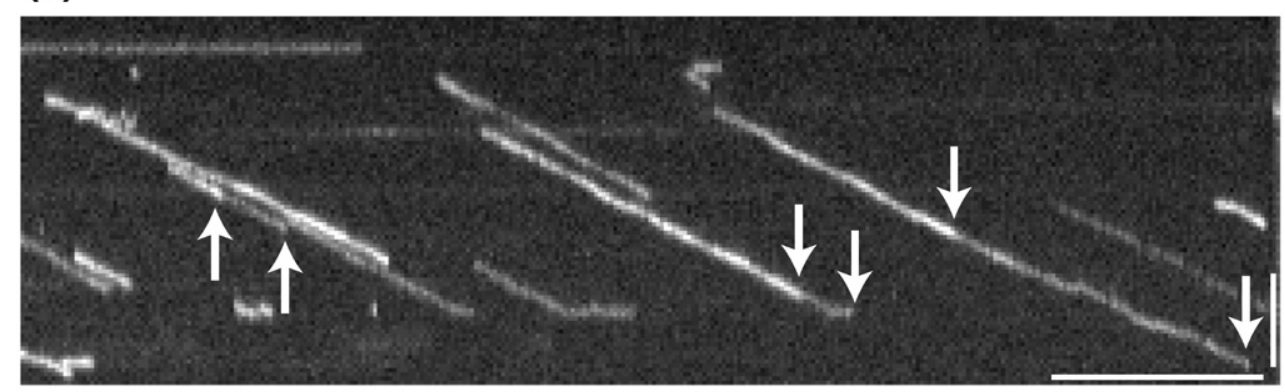

(b)

(c)
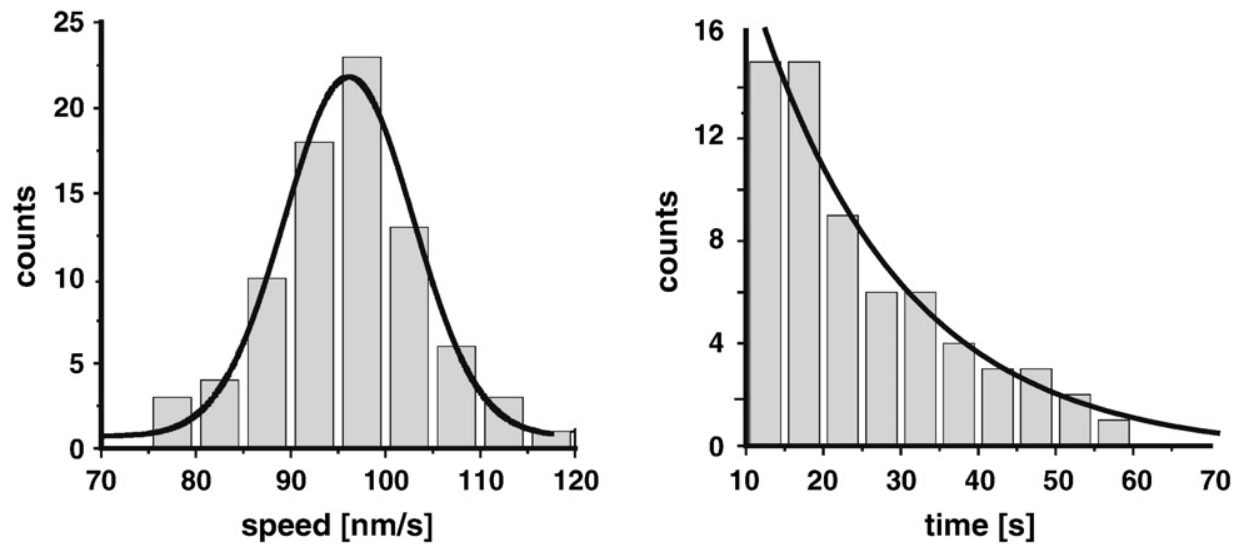

Fig. 3. Processivity assays of Eg5Kin-GFP using single-molecule fluorescence. (a) Kymograph of single Eg5Kin-GFP dimers moving on a surface-adsorbed microtubule. Arrows mark incremental bleaching events of moving spots (bleaching data; see Supplementary Fig. 1). Horizontal scale bar represents 50 s; vertical scale bar represents $3 \mu \mathrm{m}$. (b) Distribution of the speeds of individual Eg5Kin-GFP motors moving unidirectionally along a microtubule. Gaussian fit results in an average speed of $96 \pm 0.3 \mathrm{~nm} / \mathrm{s}(N=82)$. (c) Histogram of association times exponentially fitted, excluding times below $10 \mathrm{~s}$ : average association time of $19.1 \pm 3.5 \mathrm{~s}$, equivalent to a distance of $1.8 \pm 0.3 \mu \mathrm{m}$ or $230 \mathrm{steps}$ of $8 \mathrm{~nm}$.

one monastrol bound has no effect and termination is only triggered by the binding of a second monastrol molecule, or (iii) binding of monastrol is strongly cooperative such that binding of one monastrol is so rapidly followed by binding of a second monastrol and release that intermediate behavior cannot be observed. The functional form of the concentration dependence of the motor detachment rate (inverse association time) can help to distinguish (i) from (ii) and (iii). Our data can be fitted significantly better with a parabolic than with a linear concentration dependence (Fig. 4c). Assuming that the law of mass action applies to the binding of monastrol to the motor heads, this suggests that a processive run terminates only when both binding sites are occupied by monastrol. This rules out possibility (i). Assuming then that two monastrols, one on each motor head, are fatal for motility, it is hard to imagine that binding of one monastrol to one head would not have an effect on dimer motility. This notion is supported by the fact that a monastrol-induced decrease in motor velocity has been observed in other studies of collective motility using monomeric Eg5 constructs and in heterodimeric constructs with one Eg5 motor domain. ${ }^{13}$ To repeat this control, we generated a set of truncated monomeric [Eg5-369(GFP)His] and dimeric [Eg5-511(GFP)His] constructs and performed surface-gliding assays in the presence of increasing concentrations of monastrol (see Supplementary Fig. 3 in Supplementary Material). For dimeric Eg5 constructs, we observed an overall surface-gliding speed lower than that for Eg5Kin, but a similar inhibitory effect: Above a threshold, microtubule velocity dropped by $\sim 90 \%$. Monomeric Eg5 constructs produced microtubule velocities (46 \pm $13 \mathrm{~nm} / \mathrm{s}$ ) similar to those of dimeric constructs $(42 \pm 8 \mathrm{~nm} / \mathrm{s}$; see Supplementary Table 1 in Supplementary Material) in surface-gliding assays. The remarkable result was that addition of increasing concentrations of monastrol reduced the average monomer gliding speed only by $\sim 40 \%$, even at micromolar saturating concentrations of monastrol. The inhibition also progressed more gradually than that of dimeric Eg5, without a strong thresholding effect. The monomer data suggest that individual motor domains remain motile even at high monastrol concentration, which could mean that binding of monastrol to isolated monomers is weak (i.e., rapidly reversible). The comparison between the surface gliding experiments of Eg5-369, Eg5-511, and Eg5Kin thus demonstrates that dimerization 


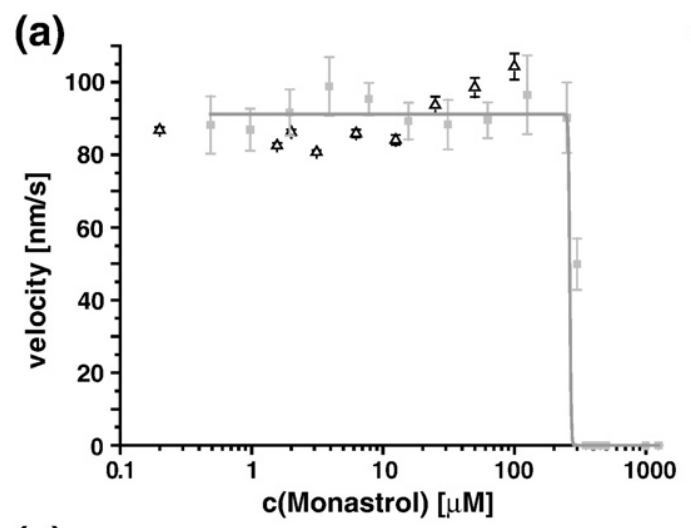

(b)
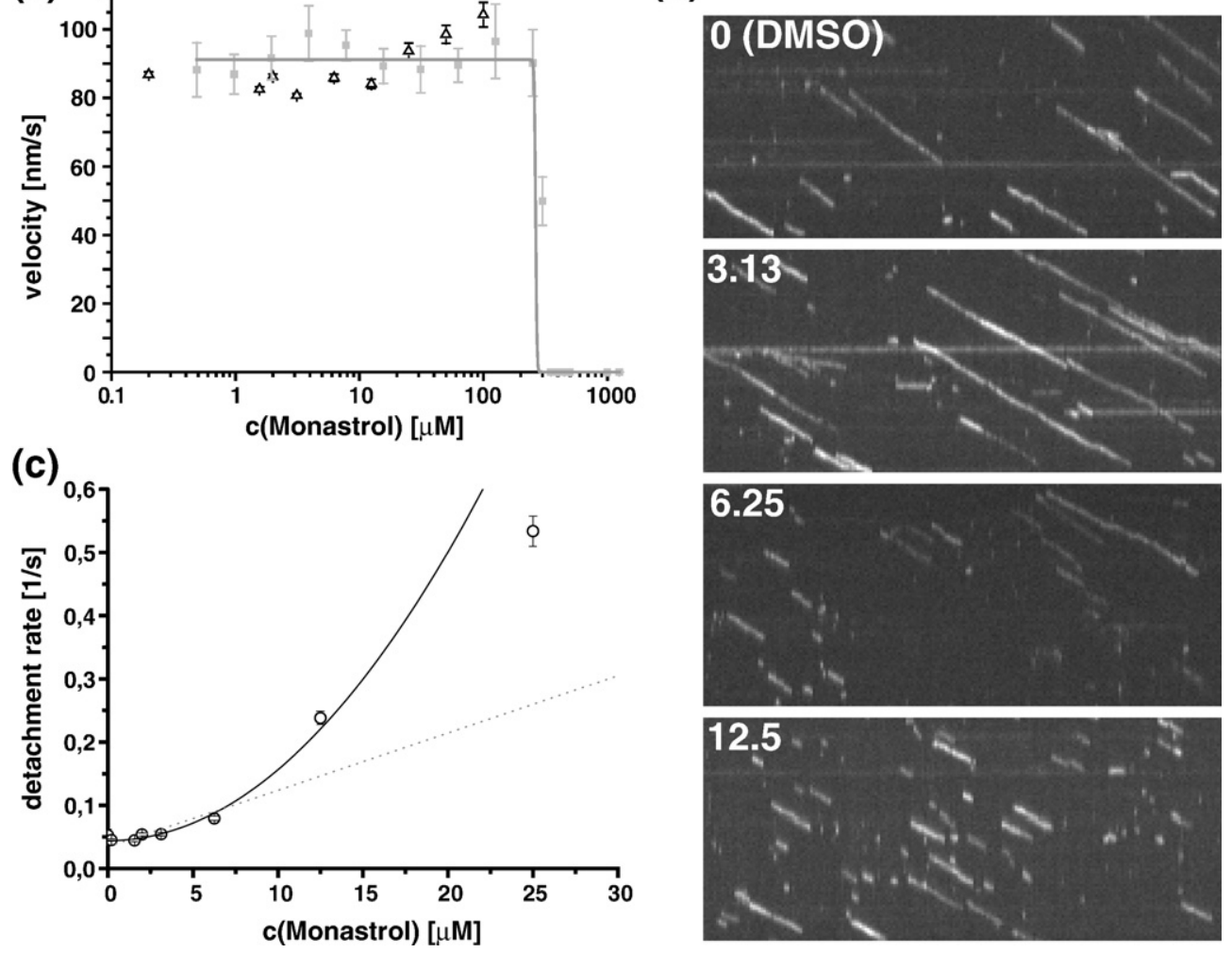

Fig. 4. Inhibition of Eg5Kin-GFP motility by monastrol. (a) Velocities observed in Eg5Kin multimotor surface-gliding assays (filled squares, gray line) and in single-molecule fluorescence processivity assays with Eg5Kin-GFP (open triangles, black line) as a function of monastrol concentration. Surface-gliding velocities were unaffected up to $200 \mu \mathrm{M}$ monastrol, with an $\mathrm{IC}_{50}$ of $240 \mu \mathrm{M}$. Single-molecule velocities remained unchanged within the observed concentration range. (b) Kymographs of Eg5Kin-GFP in the presence of increasing monastrol concentrations. Kymographs for all other monastrol concentrations are presented in Supplementary Fig. 2. Motor velocity (i.e., the slope of the lines) did not change with increasing monastrol concentration (see also Fig. 4a), while the lengths of interactions decreased. Horizontal scale bar represents $100 \mathrm{~s}$; vertical scale bar represents $5 \mu \mathrm{m}$. (c) Detachment rates of Eg5Kin-GFP (open circles) as a function of monastrol concentration. Lines: Linear and parabolic fits of the data: $k_{\text {det }}=k_{0}+\operatorname{const}\left(c_{\text {mon }}\right)^{n}$, where $k_{\text {det }}$ is the inverse of the measured run duration at a certain monastrol concentration, and $k_{0}$ is the rate at zero monastrol concentration. Gray dotted line: $n=1$, linear fit with offset. Continuous black line: $n=2$, parabolic fit with offset; concentration rate for doubling: $C_{2}=6.3 \pm 0.4 \mu \mathrm{M}$ monastrol. Data deviate from the parabolic fit at high monastrol concentrations because only events visible for two consecutive 0.5-s frames (i.e., lasting longer than $1 \mathrm{~s}$ ) were counted, leading to an apparent saturation at a rate of $\sim 1 \mathrm{~s}^{-1}$. Fit results for $n=2$ were $k_{0}=0.044 \pm 0.002 \mathrm{~s}^{-1}$ and const $=0.001 \pm 0.0001 \mu \mathrm{M}^{-1} \mathrm{~s}^{-1}$.

strongly increases sensitivity to monastrol inhibition, both in terms of strength of inhibition and in terms of a more pronounced threshold behavior. This observation strongly suggests that binding of monastrol to a dimeric Eg5 is cooperative.

It is still difficult, though, to strictly distinguish between scenarios (ii) and (iii) for motor inhibition by monastrol. Since the effect on isolated monomers is weak, binding of one monastrol to a dimer might create an effect that is hidden in experimental uncertainty. Nevertheless, it appears very likely that monastrol can only terminate a processive run when two monastrol molecules are bound to an Eg5Kin dimer.

Bulk kinetic experiments have been mostly performed with Eg5 monomers with and without added microtubules. One cannot expect monastrol binding and unbinding rates to be equal in the case of processive dimers. A comparison is still informa- tive. Luo et al. measured the on-rates of monastrol to ADP and AMP-PNP heads of monomeric Eg5 to be $0.15 \mu \mathrm{M}^{-1} \mathrm{~s}^{-1}$ and $0.97 \mu \mathrm{M}^{-1} \mathrm{~s}^{-1}$, respectively, in the presence of microtubules. ${ }^{35}$ Assuming for a moment that one monastrol would terminate a processive run of an $\mathrm{Eg} 5$ dimer, we can estimate the corresponding monastrol on-rate from the observed Eg5 run durations. At a monastrol concentration of $12.5 \mu \mathrm{M}$, we observe an average duration of $\sim 5 \mathrm{~s}$ corresponding to an on-rate of $\sim 0.2 \mathrm{~s}^{-1}$, which is distinctly lower than $\sim 12.5 \mathrm{~s}^{-1}$ from monomer kinetics even in the slow ADP state. This effect is more pronounced at lower monastrol concentrations. At $1 \mu \mathrm{M}$ monastrol, the average run duration was $\sim 20 \mathrm{~s}$, corresponding to an on-rate of $\sim 0.05 \mathrm{~s}^{-1}$, compared to $\sim 1 \mathrm{~s}^{-1}$ from monomer kinetics. This comparison supports our conclusions that dimerization of the motor domain affects monastrol binding kinetics, and that it needs two 
(cooperatively binding) monastrols to terminate a run. Unfortunately, there are no published kinetic data that can be directly compared.

In summary, first, kinetic experiments suggest that monomeric Eg5 constructs bind monastrol on the timescale of our observed dimer run durations. Second, surface gliding of microtubules driven by monomers is measurably slowed by monastrol. Third, velocities do not vary with increasing monastrol in our single-molecule dimer records. In the light of these findings, we are led to conclude that scenario (iii) applies and that binding of one monastrol to one head of a dimer is inhibited by a genuinely cooperative mechanism.

Such cooperativity would, at first glance, be easiest to explain if the two heads interacted directly, possibly through a structural element that is affected by monastrol binding, most likely loop 5 on the kinesin back. ${ }^{17}$ It is tempting to speculate that this might occur in a back-to-back conformation of the heads, which could be stabilized by monastrol and thereby block the motor from performing a next step.

The detachment rate-doubling concentration C2 $(6.3 \mu \mathrm{M})$ that we measured for single Eg5Kin-GFP dimers appears, at first glance, to be inconsistent with the monastrol concentration of $\sim 250 \mu \mathrm{M}$ at which surface gliding was inhibited (Fig. 4c). These values can, however (as already discussed above), easily be reconciled if one bears in mind that, in a surface-gliding assay, a microtubule interacts simultaneously with more than one motor. In such a situation, the effect of the reduced interaction time (while speed is constant) of the individual motors would only manifest itself at a monastrol concentration at which interaction periods cease to overlap. Furthermore, the observation that the gliding velocity did not decrease with increasing monastrol up to a concentration where binding and gliding stopped altogether is fully consistent with the finding that individual motor velocity did not change with addition of monastrol. The loss of gliding activity corresponds to the complete inhibition of microtubule binding at high monastrol concentrations.

Our results are, in fact, also consistent with earlier single-molecule fluorescence studies of full-length Eg5-GFP, which showed a reduction of the average velocity of tetrameric Eg 5 by monastrol. ${ }^{6}$ The motility of full-length tetrameric Eg5 consists of alternating diffusive and directional episodes, and it was shown that monastrol increases the diffusive character of the motility, also indicating a shortening of the processive episodes by monastrol. ${ }^{6}$

In summary, the kinesin-5 head/kinesin- 1 stalk chimera we have generated has proven to be a useful model system for studying, in particular, the regulation of Eg5 motility. Our approach avoids the complexity brought about by the regulatory domains and the additional pair of motor domains in the tetramer, and thereby provides a starting point for the controlled reintroduction of regulation. Eg5Kin is a highly processive motor that moves unidirectionally with a velocity of $95 \mathrm{~nm} / \mathrm{s}$ and an average run length of $\sim 1.9 \mu \mathrm{m}$, and it can sustain forces of $\sim 5 \mathrm{pN}$. These results confirm its basic functional similarity to kinesin-1 motors. The kinesin-5-specific inhibitor monastrol does not affect Eg5Kin velocity, but binding of two monastrols appears to terminate processive runs. Furthermore, we added to the evidence that the domains mediating diffusive interactions observed with Eg5 tetramers reside outside the motor domain. Our findings finally suggest that $\mathrm{Eg} 5$, in its native tetrameric form, is highly (down)regulated. Such a regulation makes sense in the context of the mitotic spindle, where various motility processes have to be carefully balanced and adjusted in the different stages of cell division. As a next important step, it now appears possible to study further regulatory mechanisms in the complex mechanical machinery driving mitosis.

\section{Acknowledgements}

We thank J. V. Mameren, M. Noom, and S. V. D. Wildenberg for software development and help, and W. O. Hancock, and B. Kwok/T. Kapoor for the generous gifts of the plasmids pPK113 and BK006, respectively. This work was partially supported by a research grant from the Human Frontiers Science Program (S.L. and C.F.S.). L.C.K. and E.J.G.P. were supported by a VIDI grant from the Dutch Organization for Scientific Research (NWO) and a travel grant from the Laser Center of the Free University Amsterdam. The work was further supported by the Research Center for Molecular Physiology of the Brain, funded by the Deutsche Forschungsgemeinschaft.

\section{Supplementary Data}

Supplementary data associated with this article can be found, in the online version, at doi:10.1016/ j.jmb.2010.03.009

\section{References}

1. Enos, A. P. \& Morris, N. R. (1990). Mutation of a gene that encodes a kinesin-like protein blocks nuclear division in A. nidulans. Cell, 60, 1019-1027.

2. Le Guellec, R., Paris, J., Couturier, A., Roghi, C. \& Philippe, M. (1991). Cloning by differential screening of a Xenopus cDNA that encodes a kinesin-related protein. Mol. Cell. Biol. 11, 3395-3398.

3. Mayer, T. U., Kapoor, T. M., Haggarty, S. J., King, R. W., Schreiber, S. L. \& Mitchison, T. J. (1999). Small molecule inhibitor of mitotic spindle bipolarity identified in a phenotype-based screen. Science, 286, 971-974.

4. Kashina, A. S., Baskin, R. J., Cole, D. G., Wedaman, K. P., Saxton, W. M. \& Scholey, J. M. (1996). A bipolar kinesin. Nature, 379, 270-272. 
5. Kapitein, L. C., Peterman, E. J., Kwok, B. H., Kim, J. H., Kapoor, T. M. \& Schmidt, C. F. (2005). The bipolar mitotic kinesin Eg5 moves on both microtubules that it crosslinks. Nature, 435, 114-118.

6. Kwok, B. H., Kapitein, L. C., Kim, J. H., Peterman, E. J., Schmidt, C. F. \& Kapoor, T. M. (2006). Allosteric inhibition of kinesin-5 modulates its processive directional motility. Nat. Chem. Biol. 2, 480-485.

7. Kapitein, L. C., Kwok, B. H., Weinger, J. S., Schmidt, C. F., Kapoor, T. M. \& Peterman, E. J. (2008). Microtubule cross-linking triggers the directional motility of kinesin-5. J. Cell Biol. 182, 421-428.

8. Korneev, M. J., Lakamper, S. \& Schmidt, C. F. (2007). Load-dependent release limits the processive stepping of the tetrameric Eg5 motor. Eur. Biophys. J. 36, 675-681.

9. Valentine, M. T., Fordyce, P. M., Krzysiak, T. C., Gilbert, S. P. \& Block, S. M. (2006). Individual dimers of the mitotic kinesin motor Eg5 step processively and support substantial loads in vitro. Nat. Cell Biol. 8, 470-476.

10. Kashina, A. S., Rogers, G. C. \& Scholey, J. M. (1997). The bimC family of kinesins: essential bipolar mitotic motors driving centrosome separation. Biochim. Biophys. Acta, 1357, 257-271.

11. Tao, L., Mogilner, A., Civelekoglu-Scholey, G., Wollman, R., Evans, J., Stahlberg, H. \& Scholey, J. M. (2006). A homotetrameric kinesin-5, KLP61F, bundles microtubules and antagonizes Ncd in motility assays. Curr. Biol. 16, 2293-22302.

12. Crevel, I. M., Lockhart, A. \& Cross, R. A. (1997). Kinetic evidence for low chemical processivity in ncd and Eg5. J. Mol. Biol. 273, 160-170.

13. Kaseda, K., Crevel, I., Hirose, K. \& Cross, R. A. (2008). Single-headed mode of kinesin-5. EMBO Rep. 9, 761-765.

14. van den Wildenberg, S., Tao, L., Kapitein, L. C., Schmidt, C. F.., Scholey, J. M. \& Peterman, E. J. (2008). The homotetrameric kinesin-5, KLP61F, preferentially cross-links microtubules into antiparallel orientations. Curr. Biol. 18, 1860-1864.

15. Maliga, Z., Kapoor, T. M. \& Mitchison, T. J. (2002). Evidence that monastrol is an allosteric inhibitor of the mitotic kinesin Eg5. Chem. Biol. 9, 989-996.

16. Krzysiak, T. C., Wendt, T., Sproul, L. R., Tittmann, P., Gross, H., Gilbert, S. P. \& Hoenger, A. (2006). A structural model for monastrol inhibition of dimeric kinesin Eg5. EMBO J. 25, 2263-2273.

17. Bodey, A. J., Kikkawa, M. \& Moores, C. A. (2009). 9-Angstrom structure of a microtubule-bound mitotic motor. J. Mol. Biol. 388, 218-224.

18. Crevel, I. M., Alonso, M. C. \& Cross, R. A. (2004). Monastrol stabilises an attached low-friction mode of Eg5. Curr. Biol. 14, R411-R412.

19. Cochran, J. C., Gatial, J. E., III, Kapoor, T. M. \& Gilbert, S. P. (2005). Monastrol inhibition of the mitotic kinesin Eg5. J. Biol. Chem. 280, 12658-12667.
20. Adio, S., Jaud, J., Ebbing, B., Rief, M. \& Woehlke, G. (2009). Dissection of kinesin's processivity. PLoS One, e4612, 4 .

21. Tomishige, M., Klopfenstein, D. R. \& Vale, R. D. (2002). Conversion of Unc104/KIF1A kinesin into a processive motor after dimerization. Science, 297, 2263-2267.

22. Okada, Y. \& Hirokawa, N. (1999). A processive singleheaded motor: kinesin superfamily protein KIF1A. Science, 283, 1152-1157.

23. Kalchishkova, N. \& Bohm, K. J. (2008). The role of kinesin neck linker and neck in velocity regulation. J. Mol. Biol. 382, 127-135.

24. Hildebrandt, E. R., Gheber, L., Kingsbury, T. \& Hoyt, M. A. (2006). Homotetrameric form of Cin8p, a Saccharomyces cerevisiae kinesin-5 motor, is essential for its in vivo function. J. Biol. Chem. 281, 26004-26013.

25. Coy, D. L., Hancock, W. O., Wagenbach, M. \& Howard, J. (1999). Kinesin's tail domain is an inhibitory regulator of the motor domain. Nat. Cell Biol. 1, 288-292.

26. Howard, J., Hudspeth, A. J. \& Vale, R. D. (1989). Movement of microtubules by single kinesin molecules. Nature, 342, 154-158.

27. Lakamaper, S. \& Meyhofer, E. (2005). The E-hook of tubulin interacts with kinesin's head to increase processivity and speed. Biophys J. 89, 3223-3234.

28. Visscher, K., Schnitzer, M. J. \& Block, S. M. (1999). Single kinesin molecules studied with a molecular force clamp. Nature, 400, 184-189.

29. Muthukrishnan, G., Zhang, Y., Shastry, S. \& Hancock, W. O. (2009). The processivity of kinesin-2 motors suggests diminished front-head gating. Curr. Biol. 19, 442-447.

30. Hackney, D. D., Stock, M. F., Moore, J. \& Patterson, R. A. (2003). Modulation of kinesin half-site ADP release and kinetic processivity by a spacer between the head groups. Biochemistry, 42, 12011-12018.

31. Valentine, M. T. \& Block, S. M. (2009). Force and premature binding of ADP can regulate the processivity of individual Eg5 dimers. Biophys. J. 97, 1671-1677.

32. Romberg, L., Pierce, D. W. \& Vale, R. D. (1998). Role of the kinesin neck region in processive microtubulebased motility. J. Cell Biol. 140, 1407-1416.

33. Thorn, K. S., Ubersax, J. A. \& Vale, R. D. (2000). Engineering the processive run length of the kinesin motor. J. Cell Biol. 151, 1093-1100.

34. Skiniotis, G., Cochran, J. C., Muller, J., Mandelkow, E., Gilbert, S. P. \& Hoenger, A. (2004). Modulation of kinesin binding by the C-termini of tubulin. $E M B O \mathrm{~J}$. 23, 989-999.

35. Luo, L., Carson, J. D., Dhanak, D., Jackson, J. R., Huang, P. S., Lee, Y. et al. (2004). Mechanism of inhibition of human KSP by monastrol: insights from kinetic analysis and the effect of ionic strength on KSP inhibition. Biochemistry, 43, 15258-15266. 


\section{Directionality of individual kinesin-5 Cin8 motors is modulated by loop 8, ionic strength and microtubule geometry}

\author{
Adina Gerson-Gurwitz ${ }^{1,6}$, \\ Christina Thiede ${ }^{2,6}$, Natalia Movshovich ${ }^{1}$, \\ Vladimir Fridman ${ }^{3}$, Maria Podolskaya ${ }^{3}$, \\ Tsafi Danieli ${ }^{4}$, Stefan Lakämper ${ }^{2,7}$, \\ Dieter R Klopfenstein ${ }^{2}$, Christoph F \\ Schmidt ${ }^{2, *}$ and Larisa Gheber ${ }^{1,3,5, *}$
}

${ }^{1}$ Department of Chemistry, Ben-Gurion University of the Negev, Beer-Sheva, Israel, ${ }^{2}$ Drittes Physikalisches Institut, Georg-AugustUniversität, Göttingen, Germany, ${ }^{3}$ Department of Clinical Biochemistry, Ben-Gurion University of the Negev, Beer-Sheva, Israel, ${ }^{4}$ Protein Expression Facility, Wolfson Centre for Applied Structural Biology, Hebrew University of Jerusalem, Jerusalem, Israel and ${ }^{5}$ Ilse Katz Institute for Nanoscale Science and Technology, Ben-Gurion University of the Negev, Beer-Sheva, Israel

Kinesin-5 motors fulfil essential roles in mitotic spindle morphogenesis and dynamics as slow, processive microtubule (MT) plus-end directed motors. The Saccharomyces cerevisiae kinesin-5 Cin8 was found, surprisingly, to switch directionality. Here, we have examined directionality using single-molecule fluorescence motility assays and live-cell microscopy. On spindles, Cin8 motors mostly moved slowly ( $\sim 25 \mathrm{~nm} / \mathrm{s})$ towards the midzone, but occasionally also faster $(\sim 55 \mathrm{~nm} / \mathrm{s})$ towards the spindle poles. In vitro, individual Cin 8 motors could be switched by ionic conditions from rapid $(380 \mathrm{~nm} / \mathrm{s})$ and processive minusend to slow plus-end motion on single MTs. At high ionic strength, Cin8 motors rapidly alternated directionalities between antiparallel MTs, while driving steady plus-end relative sliding. Between parallel MTs, plus-end motion was only occasionally observed. Deletion of the uniquely large insert in loop 8 of Cin8 induced bias towards minusend motility and affected the ionic strength-dependent directional switching of Cin8 in vitro. The deletion mutant cells exhibited reduced midzone-directed motility and efficiency to support spindle elongation, indicating the importance of directionality control for the anaphase function of $\operatorname{Cin} 8$.

The EMBO Journal advance online publication, 18 November 2011; doi:10.1038/emboj.2011.403

Subject Categories: membranes \& transport; cell \& tissue architecture

\footnotetext{
*Corresponding authors. CF Schmidt, Drittes Physikalisches Institut, Georg-August-Universität, Göttingen, Germany. Tel.: + 49551397740 Fax: + 49551397 720; E-mail: cfs@physik3.gwdg.de or L Gheber, Departments of Clinical Biochemistry and Chemistry, Ben-Gurion University of the Negev, Beer-Sheva, Negev 84105, Israel.

Tel.: + 97286400 633; Fax: +97286281 361;

E-mail: lgheber@bgu.ac.il

${ }^{6}$ These authors contributed equally to this work

${ }^{7}$ Present address: Institute for Mechanical Systems, D-MAVT, ETH

Zurich, Zurich, Switzerland
}

Received: 21 June 2011; accepted: 18 October 2011
Keywords: Cin8; kinesin directionality; kinesin-5; microtubules; mitosis

\section{Introduction}

Members of the kinesin-5 family are homotetrameric motor proteins, which utilize ATP to slide apart antiparallel spindle microtubules (MTs; Kashina et al, 1997; Kapitein et al, 2005). They are conserved among the eukaryotes and fulfil similar functions in spindle dynamics (Enos and Morris, 1990; Roof et al, 1991; Hagan and Yanagida, 1992; Hoyt et al, 1992; Sawin et al, 1992; Heck et al, 1993; Blangy et al, 1995; Saunders et al, 1995; Walczak and Mitchison, 1996; Straight et al, 1998; Sharp et al, 1999; Touitou et al, 2001; BrustMascher et al, 2004; Zhu et al, 2005). S. cerevisiae encodes two kinesin-5 homologues, Cin8 and Kip1, which overlap in function, with Cin8 being more important for mitosis progression (Hoyt et al, 1992; Roof et al, 1992). Before the onset of anaphase, Cin8 and Kip1 function in spindle assembly (Hoyt et al, 1992; Roof et al, 1992; Chee and Haase, 2010) and focus the kinetochore clusters (Tytell and Sorger, 2006; Gardner et al, 2008a; Wargacki et al, 2010). During spindle elongation in anaphase $\mathrm{B}$, their function is important for (i) maintaining the correct spindle elongation rate (Kahana et al, 1995; Saunders et al, 1995; Straight et al, 1998); (ii) the timely transition from the initial fast phase to the final slow phase of anaphase B (Movshovich et al, 2008; Gerson-Gurwitz et al, 2009); (iii) for stabilizing the spindle midzone (Movshovich et al, 2008; Fridman et al, 2009; Gerson-Gurwitz et al, 2009), which consists of an overlapping array of MTs that emanate from the opposite poles (Winey et al, 1995). To guarantee proper mitosis, all components involved, and in particular the motors, have to be tightly regulated. The regulation of kinesin-5 motors is so far poorly understood.

Several kinesin-5 motors were found to be plus-end directed in vitro (Gheber et al, 1999; Kapitein et al, 2005). Independent of our findings, however, a recent report has provided evidence that Cin8 can switch directionality, assumed to be caused by motor-motor coupling (Roostalu et al, 2011). Molecular mechanisms as well as physiological relevance have remained unclear.

To track down the mechanisms of directionality control, we have here studied the motor function of the $S$. cerevisiae kinesin-5 Cin8 in parallel in-vivo and in-vitro experiments. We provide evidence that during anaphase spindle elongation, Cin8 moves in both plus- and minus-end directions on the spindle MTs. We also show that, in vitro, single molecules of Cin8 can switch directionality from fast processive minus-end directed to slow, processive plus-end directed motility. We provide first insights into the mechanism controlling 
this switch: (i) change of ionic strength, that is, electrostatic interactions within the motor or between motor and MT, activated the switch in vitro; (ii) deletion of the large insert in loop 8 of the Cin8 motor domain induced bias to minus-end directionality in vitro and reduced Cin8 plus-end directed motility along the anaphase spindles in vivo; (iii) in close to physiological conditions in vitro, individual Cin8 motors switched from fast minus-end motion on single MTs to slow and erratic motion between antiparallel MTs, while they drove plus-end sliding of the linked MTs; (iv) individual motors occasionally moved in the plus-end direction, but mostly maintained fast minus-end motion between parallel MTs.

\section{Results}

To examine the motile properties of Cin8 in vivo, we imaged live S. cerevisiae cells expressing Cin8-3GFP during anaphase B. As spindles elongated, Cin8 was localized both at the spindle midzone and near the poles, likely near the kinetochores as reported before (Tytell and Sorger, 2006). In late anaphase, Cin8 detached from the spindle and became diffusive
(Figure 1A; Supplementary Movie S1), confirming previous reports (Avunie-Masala et al, 2011). Kymograph analysis of fluorescence recordings revealed that Cin8-3GFP moved towards the midzone, in the plus-end direction of MTs, with an average velocity of $\sim 25 \mathrm{~nm} / \mathrm{s}$ (Figures 1C and 7A; Table I). This slow plus-end directed motility is consistent with the literature on Cin8 and the Xenopus kinesin-5 Eg5 (Gheber et al, 1999; Kwok et al, 2004; Kapitein et al, 2005). Intriguingly, we also observed occasional movements towards the spindle-pole bodies (SPBs; Figures 1D and 7A; Table I), mainly in long anaphase spindles, when the majority of Cin8 was already detached (Figures $1 \mathrm{D}$ and $7 \mathrm{~A}$ ). The scarcity of SPB-directed movements may be due to their rapidity (Table I) and/or to masking by abundant Cin83GFP on the spindle. In intermediate anaphase spindles, shorter than $5 \mu \mathrm{m}$, interpolar MTs (iMTs) can overlap almost for the entire spindle length (Winey et al, 1995; Gardner et al, 2008b). Therefore, in these spindles, the directionality of Cin8 is difficult to determine. In the long anaphase spindles, however, iMTs overlap only in the middle $10-20 \%$ of the spindle length, as determined by Electron Microscopy (Winey et al, 1995), direct imaging of GFP-labelled MTs
A

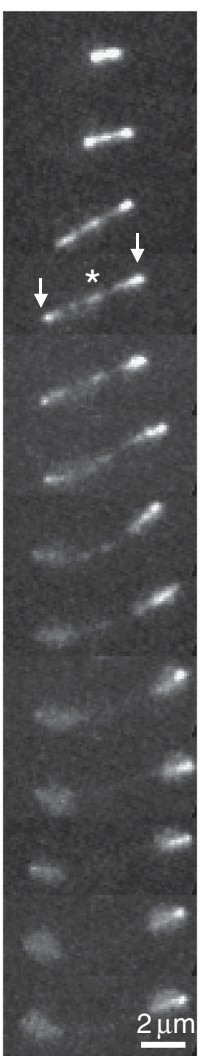

B
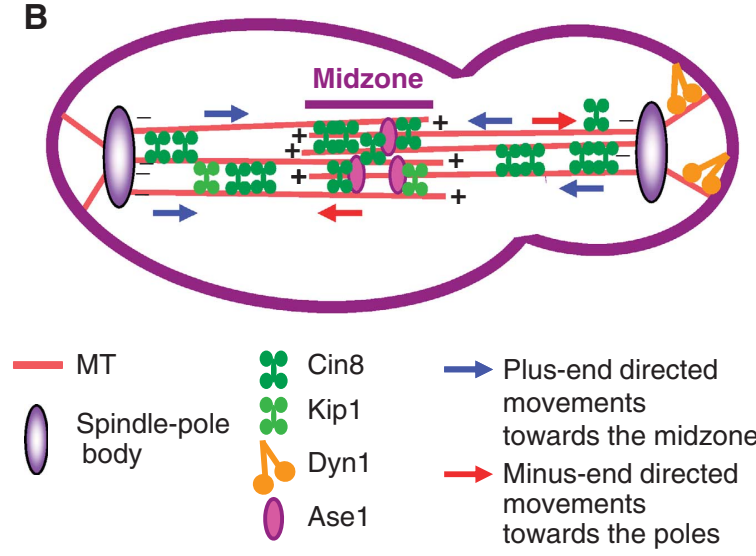

C

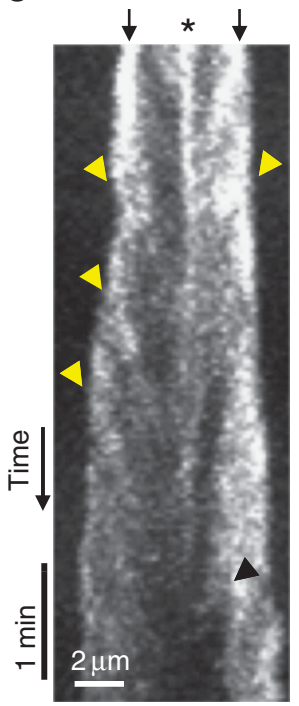

D

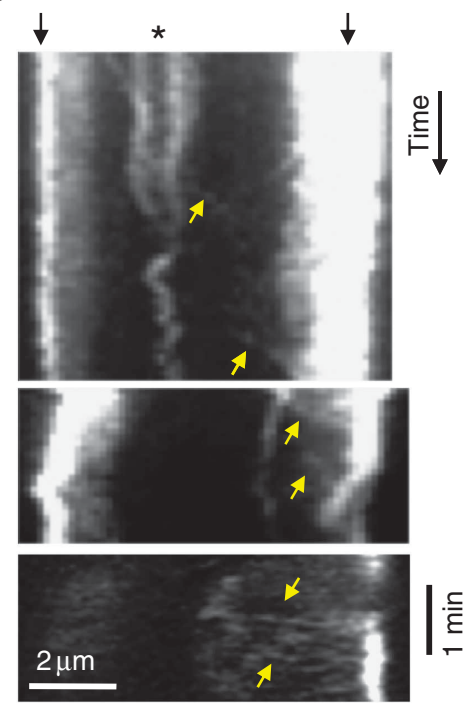

Figure 1 In vivo, Cin8 occasionally switches directionality. (A) 2D-projection time-lapse confocal fluorescence images of Cin8-3GFP localization in WT cells. Time intervals: 1 min. (B) Model of Cin8 plus-end and minus-end directed movement in the anaphase spindle in $S$. cerevisiae cells. (C, D) Kymographs of Cin8-3GFP localization to the anaphase spindle. (A, C, D) Top arrows: spindle poles; asterisk: spindle midzone. Arrowheads: plus-end-directed movements towards the midzone; yellow arrows: minus-end directed movements towards the poles. 
Table I Midzone- and SPB-directed movements of Cin8-3GFP variants in the anaphase spindles

\begin{tabular}{|c|c|c|c|c|c|c|c|}
\hline \multirow[t]{2}{*}{ Cin8 variant } & \multirow[t]{2}{*}{$N^{\mathrm{a}}$} & \multicolumn{3}{|c|}{ Midzone-directed movements } & \multicolumn{3}{|c|}{ SPB-directed movements } \\
\hline & & $\%$ Of spindles ${ }^{\mathrm{b}}$ & $\begin{array}{c}\text { Velocity } \\
(\mathrm{nm} / \mathrm{s})(n)^{\mathrm{c}, \mathrm{d}}\end{array}$ & $\begin{array}{l}\text { Run length } \\
(\mathrm{nm})(n)^{\mathrm{c}, \mathrm{d}}\end{array}$ & $\%$ Of spindles ${ }^{e}$ & $\begin{array}{c}\text { Velocity } \\
(\mathrm{nm} / \mathrm{s})(n)^{\mathrm{c}}\end{array}$ & $\begin{array}{l}\text { Run length } \\
(\mathrm{nm})(n)^{\mathrm{c}}\end{array}$ \\
\hline WT Cin8 & 113 & 59 & $26.5 \pm 0.7(120)$ & $1506 \pm 50(120)$ & 20 & $56.4 \pm 4.2(29) * * * \mathrm{f}$ & $845 \pm 43(29) * * * \mathrm{f}$ \\
\hline Cin8 899 & 83 & 29 & $15.3 \pm 0.5(123) * * * g$ & $1091 \pm 35(123)^{* * * g}$ & 18 & $55.8 \pm 2.9(33) * * * \mathrm{f}$ & $891 \pm 30(33)^{* \mathrm{f}}$ \\
\hline Cin8-2A & 102 & 41 & $23.0 \pm 0.8(183)^{* g}$ & $1236 \pm 34(183)^{* * * g}$ & 20 & $63.6 \pm 3.1(32) * * * f$ & $943 \pm 62(32) * * f$ \\
\hline
\end{tabular}

${ }^{\mathrm{a}} \mathrm{N}$-number of intermediate-long $(5-10 \mu \mathrm{m})$ spindles analysed.

$\mathrm{b}_{\%}$ Of spindles with at least one midzone-directed movement spanning from SPB to the midzone (see Figure 5).

${ }^{\mathrm{c}}$ Values represent average \pm s.e.m., $n$-number of movements.

${ }^{\mathrm{d}}$ Values determined for movements longer than $0.5 \mu \mathrm{m}$, including movements that do not reach the midzone.

$\mathrm{e}_{\%}$ Of spindles with at least one SPB-directed movement, longer than $0.5 \mu \mathrm{m}$.

${ }^{\mathrm{f}}$ Compared with the corresponding movements in cells of the same genotype.

${ }^{g}$ Compared with cells expressing WT Cin8.

${ }^{* * *} P<0.0001 ;{ }^{* *} P<0.001 ;{ }^{*} P<0.005$ (Student's $t$-test).

(Avunie-Masala et al, 2011), or imaging of the midzoneorganizing protein Ase1 (Schuyler et al, 2003; Fridman et al, 2009). The fact that Cin8 motility towards the midzone as well as towards the SPBs was observed in spindles longer than $5 \mu \mathrm{m}$ (Figures 1C, D and 7A), suggests that these movements occur on parallel arrays of iMTs. Moreover, SPBdirected movements were significantly faster and shorter than midzone-directed movements (Table I), indicating that movements in the two directions were genuinely different. Thus, taken together, our data indicate that on the anaphase spindles, Cin8 is a bi-directional motor that moves in both plus-end and minus-end directions of the MTs.

To explore the unconventional minus-end motility of Cin8 on the spindle, we performed single-molecule fluorescence in-vitro motility assays (Vale et al, 1996; Kwok et al, 2006; Kapitein et al, 2008). We used whole-cell extracts of WT and cin8 8 S. cerevisiae cells, in which Cin8-3GFP was expressed from its native promoter, either integrated into the genome or carried on a centromeric (CEN) plasmid (Materials and methods; Supplementary Table S1). We also studied Cin8-GFP purified from Sf9 and yeast cells (Materials and methods; Supplementary Figure S1). Assays were done at saturating ATP (3-5 mM; Lakamper et al, 2010) in a high-salt, close to physiological buffer (175 mM NaCl; Gheber et al, 1999). We found, surprisingly, that in these conditions Cin8 moved unidirectionally and processively towards the minus end of MTs (Figure 2). This is similar to what was recently found in an independent study (Roostalu et al, 2011). Runs were often interrupted by diffusive motion (Figure $2 \mathrm{~B}$ and $\mathrm{C}$ ). The average run length between such breaks was $\sim 2 \mu \mathrm{m}$ (Figure 3B). In ADP buffer, motors remained attached to MTs, but only moved diffusively without directional bias. We calculated a diffusion coefficient of $\sim 3 \times 10^{3} \mathrm{~nm}^{2} / \mathrm{s}$ from mean-square-displacement (MSD) analysis of tracks recorded in ADP buffer, comparable to that measured with the closely related Eg5 and other kinesins (Table II; Kwok et al, 2006; Kapitein et al, 2008; Bormuth et al, 2009). At saturating ATP concentration, we determined an average minus-end velocity of Cin 8 molecules of $\sim 360 \mathrm{~nm} / \mathrm{s}$ by kymograph and mean displacement (MD) analyses (Table II). This velocity is substantially higher than the plus-end velocity seen in vivo and the velocity reported for other kinesin-5 motors (Valentine et al, 2006). In both WT Cin8 batches examined, purified Cin8-GFP and Cin8-3GFP in whole extracts, the distribution of velocities was exceptionally broad in each experiment,
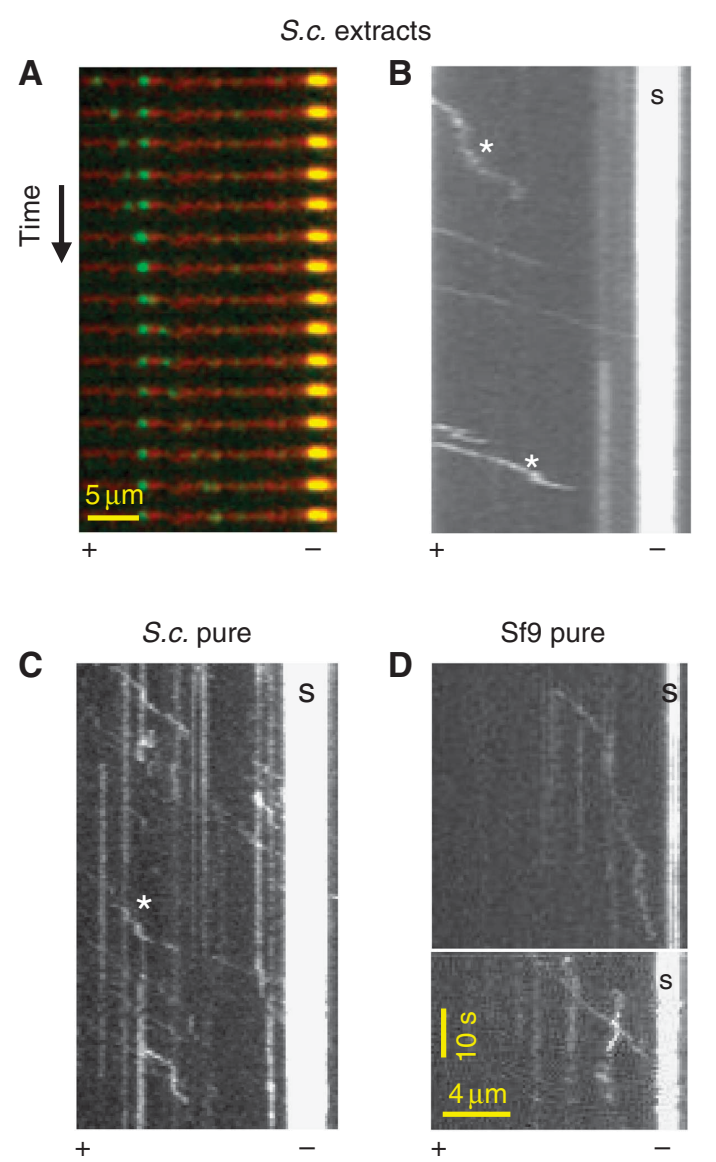

Figure 2 At high salt in vitro, Cin8 is a minus-end directed motor. (A) Time-lapse recording showing a single Cin8-3GFP molecule from whole-cell extract (green) moving directionally along the MT (red) towards the bright seed (yellow). Time intervals: $2 \mathrm{~s}$. (B-D) Kymographs of Cin8 movement on polarity-marked MTs. (B) Cin83GFP from whole-cell extract (C) Cin8-GFP purified from yeast cells and (D) Cin8-GFP purified from Sf9 cells. S: bright seed indicating the MT minus end. Scale bars in (D) also apply to (B) and (C). * indicates diffusive episotes. See also Supplementary Movies S2 and S3.

ranging from $\sim 17$ to $\sim 830 \mathrm{~nm} / \mathrm{s}$ (Supplementary Figure S2A; Figure $4 \mathrm{~A}(\mathrm{I})$ and $\mathrm{B}(\mathrm{I}))$. We ruled out the possibility that the broad distribution of velocities resulted from motor aggregation by analysing fluorescence intensities of individual spots (Supplementary Figure S3). 
A

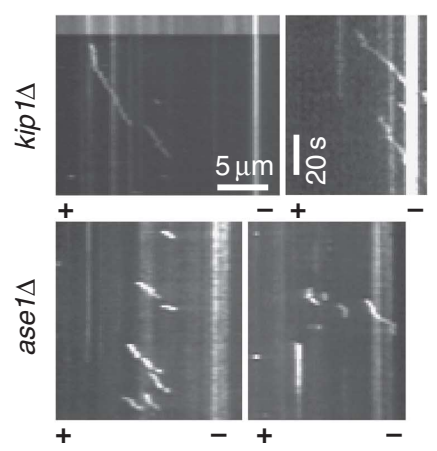

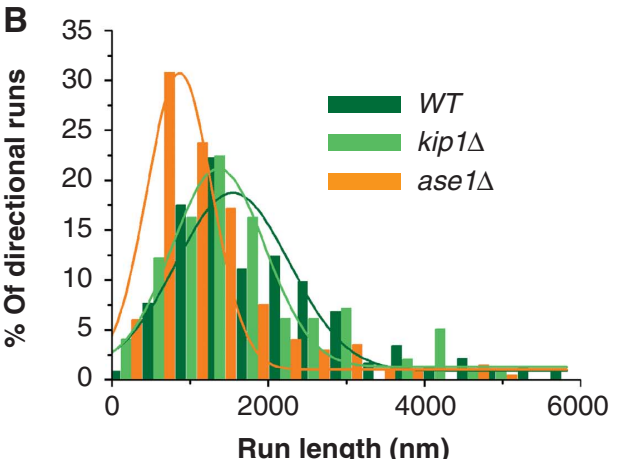

Run length (nm)

C Cin8-3GFP

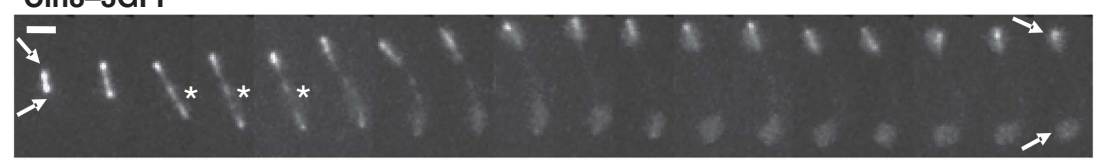

D $k i p 1 \Delta$ Cin8-3GFP

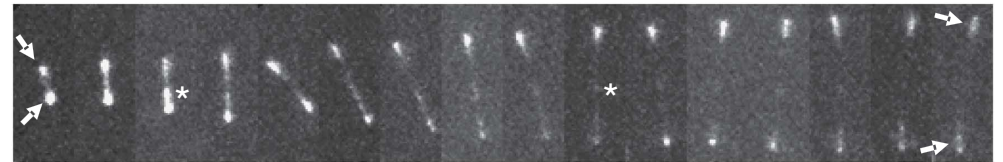

E ase1 $1 \Delta$ Cin8-3GFP

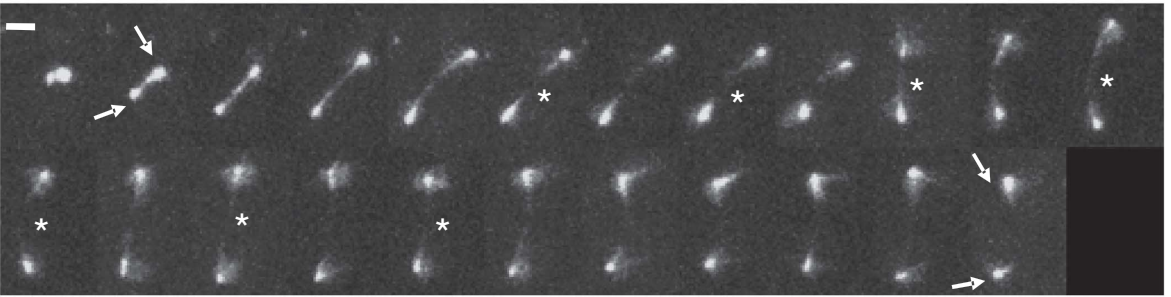

Figure 3 The effect of spindle proteins on Cin8 in-vitro motile properties and in-vivo localization to anaphase spindles. (A, B) Single-molecule fluorescence motility assay was carried out on polarity-marked MTs with kip1 $1 \Delta$ or ase1 $\Delta$ cell extracts expressing Cin8-3GFP. Extracts were diluted in high-salt $(175 \mathrm{mM} \mathrm{NaCl})$ buffer. (A) Representative kymographs of in-vitro runs of Cin8-3GFP in the different extracts. (B) Histograms of run lengths of Cin8-3GFP directional episodes in WT (olive), kip1 $\Delta$ (light green) or ase1 $\Delta$ (orange) extracts. Fit: Gaussian distribution. (C-E) Representative 2D-projection time-lapse confocal fluorescence images of Cin8-3GFP localization to anaphase spindles in (C) WT (D) kip1s and (E) ase1s cells. Asterisk: spindle midzone; Arrows: spindle poles; Time interval between frames: 1 min; Scale bar: $2 \mu \mathrm{m}$. Genotypes and Cin8 variants are marked on top.

Table II In-vitro motile properties of Cin8 in whole-cell extracts

\begin{tabular}{|c|c|c|c|c|c|c|}
\hline Genotype & $\begin{array}{l}V_{\text {KYMO }}{ }^{a} \\
(\mathrm{~nm} / \mathrm{s})\end{array}$ & $\begin{array}{l}V_{\mathrm{MD}}^{\mathrm{b}, \mathrm{c}} \\
(\mathrm{nm} / \mathrm{s})\end{array}$ & $\begin{array}{c}D_{\mathrm{ADP}} \times 10^{3 \mathrm{c}, \mathrm{d}} \\
\left(\mathrm{nm}^{2} / \mathrm{s}\right)\end{array}$ & $\begin{array}{l}\text { Stall periods } \\
\text { (\%) }\end{array}$ & $\begin{array}{l}\text { Run length } \\
\text { (nm) }\end{array}$ & $\begin{array}{l}\text { Interaction } \\
\operatorname{time}^{\mathrm{g}}(\mathrm{s})\end{array}$ \\
\hline $\begin{array}{l}\text { WT }(\mathrm{p} C I N 8-3 G F P)^{\mathrm{h}} \\
\text { CIN8-3GFP }\end{array}$ & $\begin{array}{l}353 \pm 33(116) \\
365 \pm 27(178)\end{array}$ & $372 \pm 7^{i}(52)$ & $3.7 \pm 0.1^{\mathrm{i}}(30)$ & $39(1645)^{j}$ & $1700 \pm 200$ & $8.3 \pm 1.0(65)$ \\
\hline $\begin{array}{l}\text { cins } 8 \text { kip1 } 1 \text { (pCIN8-3GFP })^{\mathrm{h}} \\
\text { ase1 } 1 \text { (pCIN8-3GFP })^{\mathrm{h}}\end{array}$ & $\begin{array}{l}381 \pm 38(100) \\
241 \pm 17^{* * * 1}(198)\end{array}$ & $\begin{array}{l}345 \pm 8(70) \\
272 \pm 8^{* * * 1}(39)\end{array}$ & $\begin{array}{l}2.9 \pm 0.1^{* * * 1}(24) \\
4.2 \pm 0.2(23)\end{array}$ & $\begin{array}{l}20(1377)^{j} \\
33(1732)^{j}\end{array}$ & $\begin{array}{l}2000 \pm 200(132) \\
1200 \pm 100^{* * 1}(118)\end{array}$ & $\begin{array}{l}9.5 \pm 0.9(115) \\
8.4 \pm 1.0(66)\end{array}$ \\
\hline
\end{tabular}

Values are average \pm s.e.m. In parentheses, the number of measurements.

${ }^{a} V_{\mathrm{KYMO}}$ - average velocity determined from kymograph analysis.

${ }^{\mathrm{b}} V_{\mathrm{MD}}$-average velocity determined from Mean Displacement analysis.

${ }^{c}$ Fit is done to first $15 \mathrm{~s}$.

${ }^{\mathrm{d}} \mathrm{D}_{\mathrm{ADP}}$-diffusion coefficient in the presence of 3-5 mM ADP determined from MSD analysis.

ePercentage calculated from total motion time.

${ }^{\mathrm{f}}$ Run length of directional episodes.

${ }^{g}$ Measurements applied on kymograph traces of complete directional runs, of which both their beginning and end are apparent.

${ }^{\mathrm{h}}$ Cin8-3GFP expressed on CEN plasmid.

${ }^{\mathrm{i}}$ Measurements carried out on Cin8-3GFP either expressed on CEN plasmid or integrated into the genome.

${ }^{\mathrm{j}}$ Total time of measurement (s).

${ }^{\mathrm{k}} \mathrm{Cin} 8-3 \mathrm{GFP}$ integrated into the genome.

${ }^{1}$ Compared with WT.

${ }^{* * *} P<0.0001 ;{ }^{* *} P<0.001$ (Student's $t$-test).

$N D$-not detected. 
A
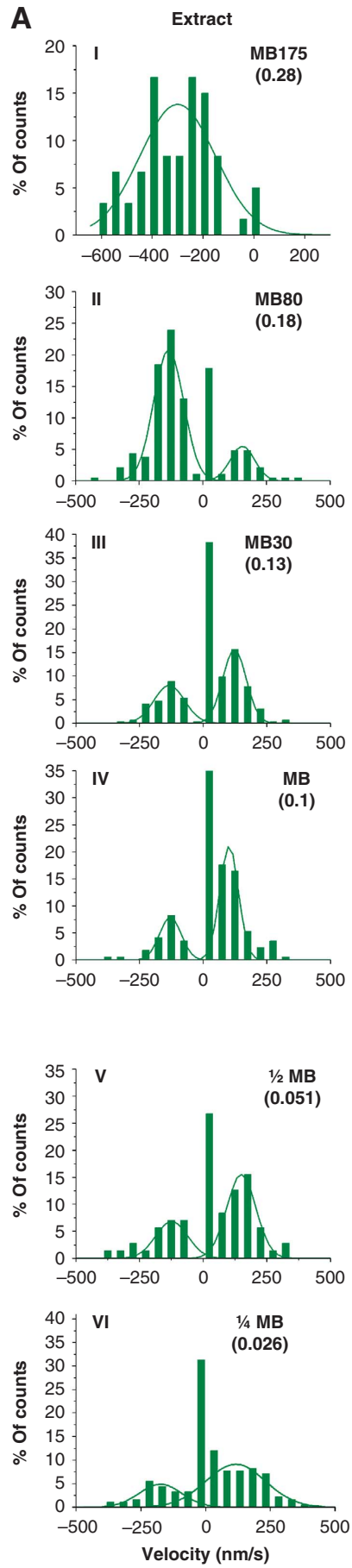
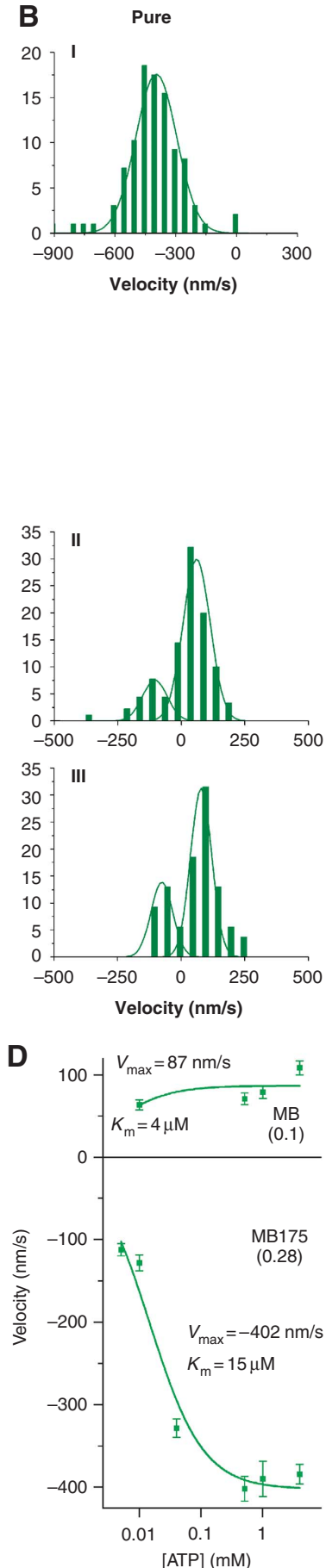

C
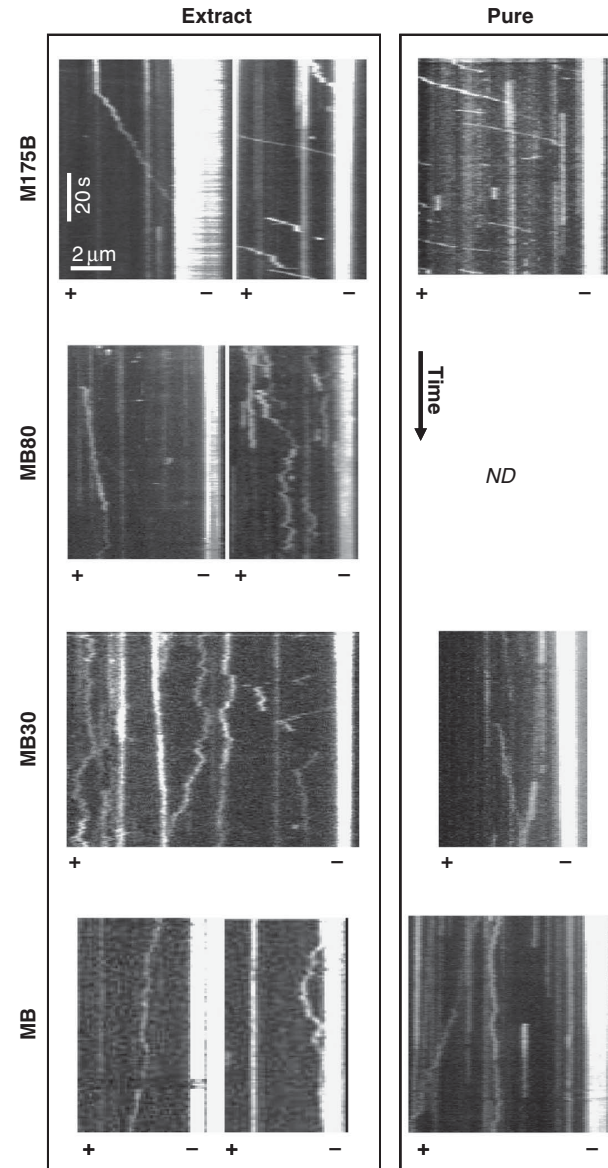

$+$

$\mid \vec{B}$

$N D$

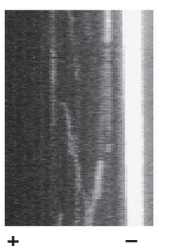

$N D$

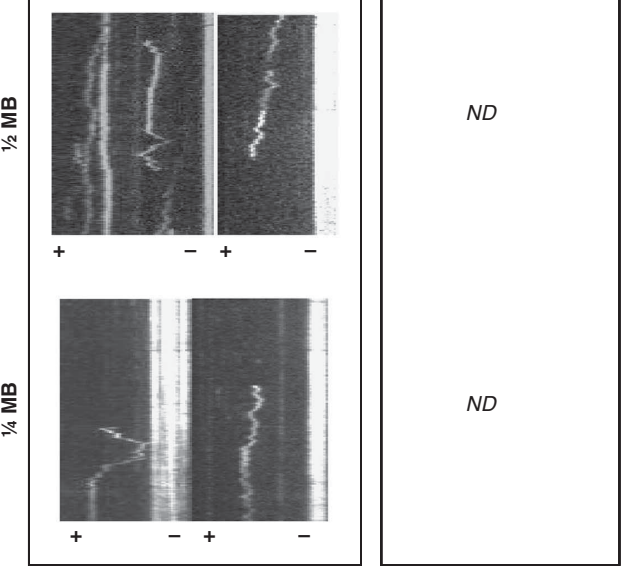

Figure 4 Decrease in ionic strength induces plus-end motility of single molecules of Cin8. (A, B) Histograms of velocities of Cin8 in whole-cell yeast extracts $(\mathbf{A})$ or affinity purified $(\mathbf{B})$ with saturating ATP. Salt and buffer conditions are indicated for each panel: MB-motility buffer; numbers adjacent to 'MB' indicate the concentration (mM) of added $\mathrm{NaCl}$. Ionic strength $(\mathrm{M})$ is indicated in parentheses. A control experiment of motility with ADP was carried out using buffer with $30 \mathrm{mM} \mathrm{NaCl}$ (see Supplementary Figure S2C). Velocity histograms were assembled by dividing kymograph traces in $3 \mathrm{~s}$ segments and piecewise linear fitting. Lines-Gaussian distribution fit. (C) Representative kymographs of Cin8 motility along polarity-marked MTs. Expression/purification conditions are indicated on top. Plus $(+)$ and minus $(-)$ ends of MTs are indicated. See also Supplementary Movie S4. (D) ATP concentration dependence of plus-end (top) and minus-end directed (bottom) velocity of Cin8 (average \pm s.e.m.). $\mathrm{NaCl}$ concentration and ionic strengths (parenthesis) are indicated. Michaelis-Menten parameters $V_{\max }$ and $K_{\mathrm{m}}$ are indicated.

To make sure that Cin8-3GFP was capable of forming functional tetramers, we examined its MT bundling activity. We found that overexpression of Cin8-3GFP caused extensive bundling of MTs in whole-cell extracts (Supplementary Figure S4A, left panel) which was not observed in extracts deleted for CIN8 (Supplementary Figure S4A, right panel) or in extracts expressing Cin8-3GFP from a CEN plasmid (not shown). This excludes the possibility that the bundling was caused by other motors or MT-binding proteins in the extracts. Similar MT bundling was previously reported for biotinylated Cin8 (Cin8-BCP; Gheber et al, 1999), which was shown to be a homotetramer (Hildebrandt 
et al, 2006). MT bundling is known to occur only with the full-length tetrameric, but not with truncated dimeric Cin8 (Cin8-871) (Gheber et al, 1999; Hildebrandt et al, 2006), suggesting that Cin8-3GFP is a tetramer. Finally, both Cin8GFP and Cin8-3GFP could maintain the viability of cells deleted for chromosomal copies of CIN8 and KIP1 (Supplementary Figure S4B). Since only tetrameric Cin8 maintains viability of cins 8 kip $1 \Delta$ cells (Hildebrandt et al, 2006), our findings indicate that Cin8-3GFP is mostly a functional tetramer.

In summary, our data indicate that the motile properties we observed for Cin8, the broad distribution of velocities, the minus-end directionality and the diffusive motion, did not result from altered oligomeric states or aggregation of the examined Cin8 variants, but rather point to an intrinsic regulation of individual Cin8 motors.

The use of whole-cell yeast extracts from deletion mutants in single-molecule motility assays allowed us to examine the influence of other $S$. cerevisiae cellular factors on Cin8 motility (Table II; Figure 3). We explored the effect, in highsalt buffer, of two major spindle-binding proteins that functionally overlap or interact with Cin8: the kinesin-5 Kip1 (Hoyt et al, 1992; Roof et al, 1992) and the MT-binding and midzone-organizing protein Ase1 (Schuyler et al, 2003). Ase1 was shown to physically interact with Cin8 during mitosis and to recruit Cin8 to the spindle midzone (Khmelinskii et al, 2009).

To examine the influence of Kip1 and Ase1 on Cin8 motor function, we examined Cin8 motility in extracts of kip $1 \Delta$ or ase $1 \Delta$ cells, which carried Cin8-3GFP on a CEN plasmid. We found that deletion of Kip1 or Ase1 did not affect the directionality of Cin8, as it remained minus-end directed in high-salt buffer (Figure 3A). In the absence of Kip1, Cin8 was somewhat more processive; its interaction time with MTs was longer, the percentage of runs with stalls was lower, and the diffusion coefficient of Cin8 in the presence of ADP was significantly lower than that of Cin8 in all the other extracts examined (Table II; Figure 3B). Consistently, in kip1 $\Delta$ cells, the detachment of Cin8 from the spindle MTs in late anaphase was reduced compared with $W T$ cells (Figure $3 \mathrm{C}$ and D). In the absence of Ase1, the average run length of Cin8-3GFP directional episodes was significantly decreased, and the average velocity dropped to $\sim 240 \mathrm{~nm} / \mathrm{s}$ (Table II; Figure 3A and B). In cells, Cin8-3GFP localized abnormally on the anaphase spindle in the absence of Ase1 and was not detectable in the midzone (Figure $3 \mathrm{E}$ ). These results suggest that Ase1 regulates Cin8 spindle localization by affecting the motile properties of Cin8.

It remains to understand the circumstances under which the motor switches to plus-end motility, the mode of motion that is believed to drive the poleward relative sliding of antiparallel overlapping MTs in anaphase. Since other kinesins, including the kinesin-5 Eg5, can be turned on and off by varying ionic conditions (Dietrich et al, 2008; Hancock, 2008; Kapitein et al, 2008; Hackney et al, 2009), we tested whether a decrease in ionic strength could affect directionality. We performed single-molecule motility assays with Cin8, either purified or in whole-cell extracts, on polarity-marked MTs at different salt concentrations. We found that with decreasing ionic strength, individual Cin8 motors gradually switched towards plus-end motility (Figure $4 \mathrm{~A}-\mathrm{C}$ ). At ionic strengths $\leqslant 0.13$, Cin 8 moved bi-directionally, moving $60-80 \%$ of the time towards the plus ends of MTs (Figure 4A-C). Reduction of ionic strength also significantly decreased the magnitude of the minus-end velocity of Cin8 (Figure 4A-C) from $\sim 380 \mathrm{~nm} / \mathrm{s}$ in high ionic strength to $\sim 130 \mathrm{~nm} / \mathrm{s}$ in low ionic strength (Supplementary Figure S5). The salt dependence of Cin8 motility in whole-cell extracts was similar to that of affinity-purified Cin8 (Figure 4A and B), although more motors were immobile in the extracts (Figure 4A and B), possibly due to the presence of other MT-binding agents. The velocity of movement in both minus- and plus-end directions was dependent on ATP concentration (Figure 4D; Supplementary Figure S2C), proving that bi-directional motion of single Cin8 molecules is not merely driven by thermal forces in the solution. These results demonstrate that a single Cin8 motor interacting with only one MT can switch directionality. The mechanism for the switch is, therefore, most likely contained in a single motor.

Any switch mechanism is likely to depend, at least in part, on structural elements within the motor's catalytic domains. In comparison with kinesin-5 homologues of higher eukaryotes, several yeast kinesin-5 motors carry inserts of considerable length in loop 8 which is involved in MT binding (Kull et al, 1996; Nitta et al, 2008; Chee and Haase, 2010). Cin 8 and the closely related Candida glabrata kinesin- 5 carry the largest inserts, of 99 amino-acid length (99aa) (Chua et al, 2007). To probe the role of the large loop 8 in the directionality switch of single Cin8 molecules, we studied a construct in which this segment was replaced with the seven amino acids of loop 8 in the related $S$. cerevisiae kinesin-5 Kip1 (Cin8 499 ). Yeast cells deleted for the chromosomal copies of CIN8 and KIP1, but expressing Cin8 carrying this deletion were previously shown to be viable, indicating that the mutant Cin8 is, at least partially, functional (Hoyt et al, 1992). We found that with decreasing ionic strength, single Cin8 499 molecules also switched from minus-end to plus-end directed motility in whole extracts (Figure 5A) and in purified samples (Figure 5B). However, Cin8 $899-G F P$ behaved distinctly differently from WT Cin8-GFP (Figures 4-6). In whole-cell extracts, at high salt (175 mM NaCl), Cin8 $499-G F P$ did not attach to MTs at all. The same was seen for purified WT Cin8, albeit only at $>250 \mathrm{mM} \mathrm{NaCl}$. At $175 \mathrm{mM}$ added $\mathrm{NaCl}$, affinity-purified Cin8 899 had a slightly lower average velocity than WT Cin8 (Supplementary Figure $\mathrm{S} 5$ ). With $30 \mathrm{mM}$ added $\mathrm{NaCl}$, the presence or absence of loop 8 in the WT Cin 8 correlated with clearly opposite behaviours: in whole extracts and purified samples, Cin8 899 remained minus-end directed, while WT Cin8 moved predominantly in the plus-end direction with some bi-directional shuttling (Figure 6A and B). A systematic comparison between Cin8 and Cin $8 \Delta 99$ motility in different buffers showed that the deletion of the 99aa insert did not eliminate the switch of directionality, but pushed the transition from minus-end to plus-end directionality to lower salt concentrations (Figure 6C), supporting the notion that the directionality switch of Cin8 involves loop 8.

To examine how the bias to minus-end directionality of the mutant Cin8 $\Delta 99$ affects its function in vivo, we monitored motor localization in cells expressing Cin8 899 . In contrast to WT Cin8-3GFP, Cin8 $\Delta 99-3 \mathrm{GFP}$ did not detach from the spindles and seemed to be asymmetrically distributed on the anaphase spindles (Supplementary Figure S6). The plus-end directed movements of Cin8 $499-3 \mathrm{GFP}$ towards the 
A
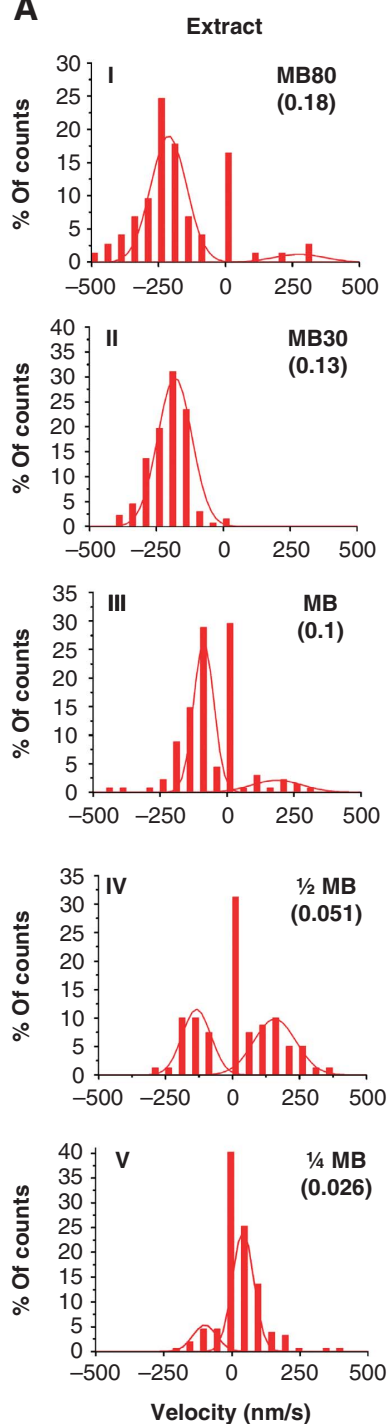

B
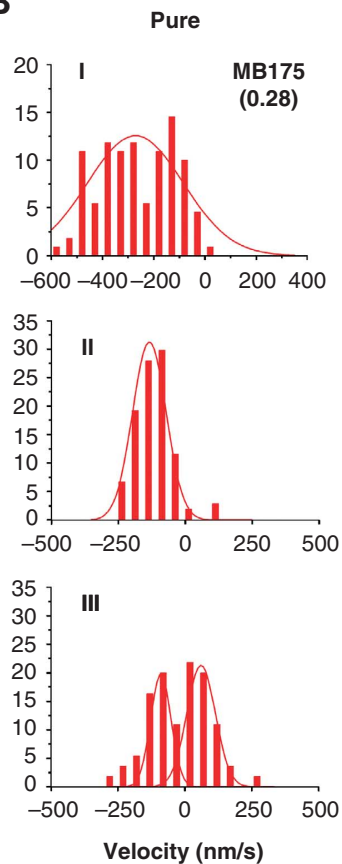

D

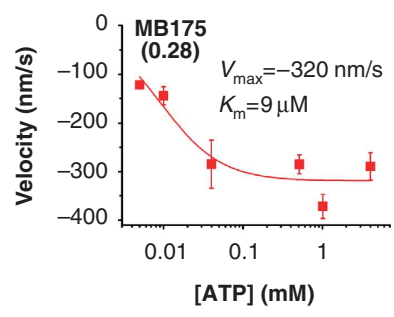

C

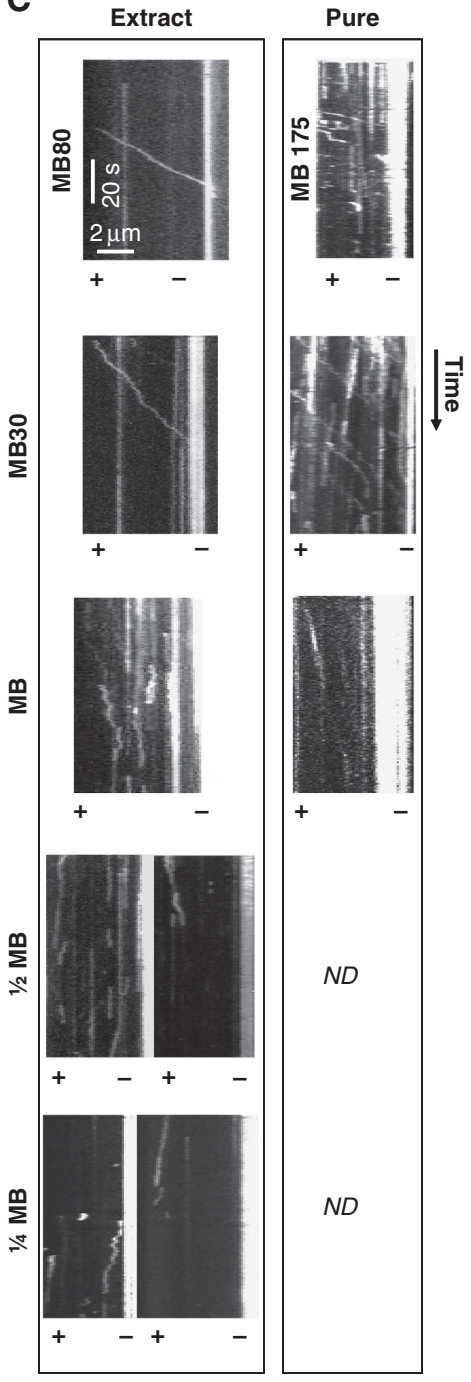

Figure 5 Decrease in ionic strength induces plus-end motility of single molecules of Cin8 899 . (A, B) Histograms of velocities of Cin8 $\Delta 99$ in whole-cell yeast extracts (A) or affinity purified (B) with saturating ATP. Salt and buffer conditions are indicated for each panel: MB-motility buffer; numbers adjacent to 'MB' indicate the concentration (mM) of added $\mathrm{NaCl}$. Ionic strength (M) is indicated in parentheses. Velocity histograms were assembled by dividing kymograph traces in $3 \mathrm{~s}$ segments and piecewise linear fitting. A control experiment of motility with ADP was carried out using buffer with $30 \mathrm{mM} \mathrm{NaCl}$ (see Supplementary Figure S2C). Lines-Gaussian distribution fit. (C) Representative kymographs of Cin8 $\Delta 99$ motility along polarity-marked MTs. Expression/purification conditions are indicated on top. Plus $(+)$ and minus $(-)$ ends of MTs are indicated. See also Supplementary Movie S5. (D) ATP concentration dependence of minus-end velocity of Cin8 499 (average \pm s.e.m.) at high ionic strength. $\mathrm{NaCl}$ concentration and ionic strength, $\mathrm{M}$ (parenthesis) are indicated. Michaelis-Menten parameters $V_{\max }$ and $K_{\mathrm{m}}$ are indicated.

midzone were fewer, significantly slower than that of Cin83GFP and spanned shorter distances (Figure 7; Table I). Nonetheless, minus-end directed motility events were also observed (Figure 7B). Finally, the rate of the initial fast phase of anaphase $B$ in Cin8 899 cells was $0.61 \pm 0.06 \mu \mathrm{m} / \mathrm{min}$ (average \pm s.e.m., $n=9$ ) which is significantly slower than in WT cells $(0.85 \pm 0.03 \mu \mathrm{m} / \mathrm{min}$, average \pm s.e.m., $n=8$, $P<0.005)$ and is similar to what was observed in $\operatorname{cin} 8 \Delta$ cells (Straight et al, 1998). This result indicates that Cin8 899 is unable to provide sufficient plus-end directed force for spindle elongation. These in-vivo data are consistent with our in-vitro results of diminished plus-end motility of Cin8 $\Delta 99$ (Figure 5) and point to a regulatory role of the 99aa insert in loop 8 of Cin8 in promoting its plus-end motility in cells.
It is known that during anaphase, Cin8 localization to the spindle is regulated by phosphorylation of the three Cdk1 sites in its catalytic domain, two of which are located in loop 8 of Cin8 (Avunie-Masala et al, 2011). To examine if phosphorylation of these sites affects Cin8 directionality on the spindle, we examined spindle movements of a Cin8-3GFP mutant that carried phosphorylation-deficient mutations to alanine at the two Cdk1 sites located in loop 8: Cin8-S277A T285A (Cin8-2A). Similarly to a phosphorylation-deficient Cin8 that carried mutations to alanine at all three catalyticdomain Cdk1 sites (Avunie-Masala et al, 2011), Cin8-2A3GFP remained attached to the anaphase spindles for longer times, compared with cells expressing WT Cin8 (Supplementary Figure S6B, compare with Figures 1A and 3C). This result indicates that the two Cdk1 sites within loop 

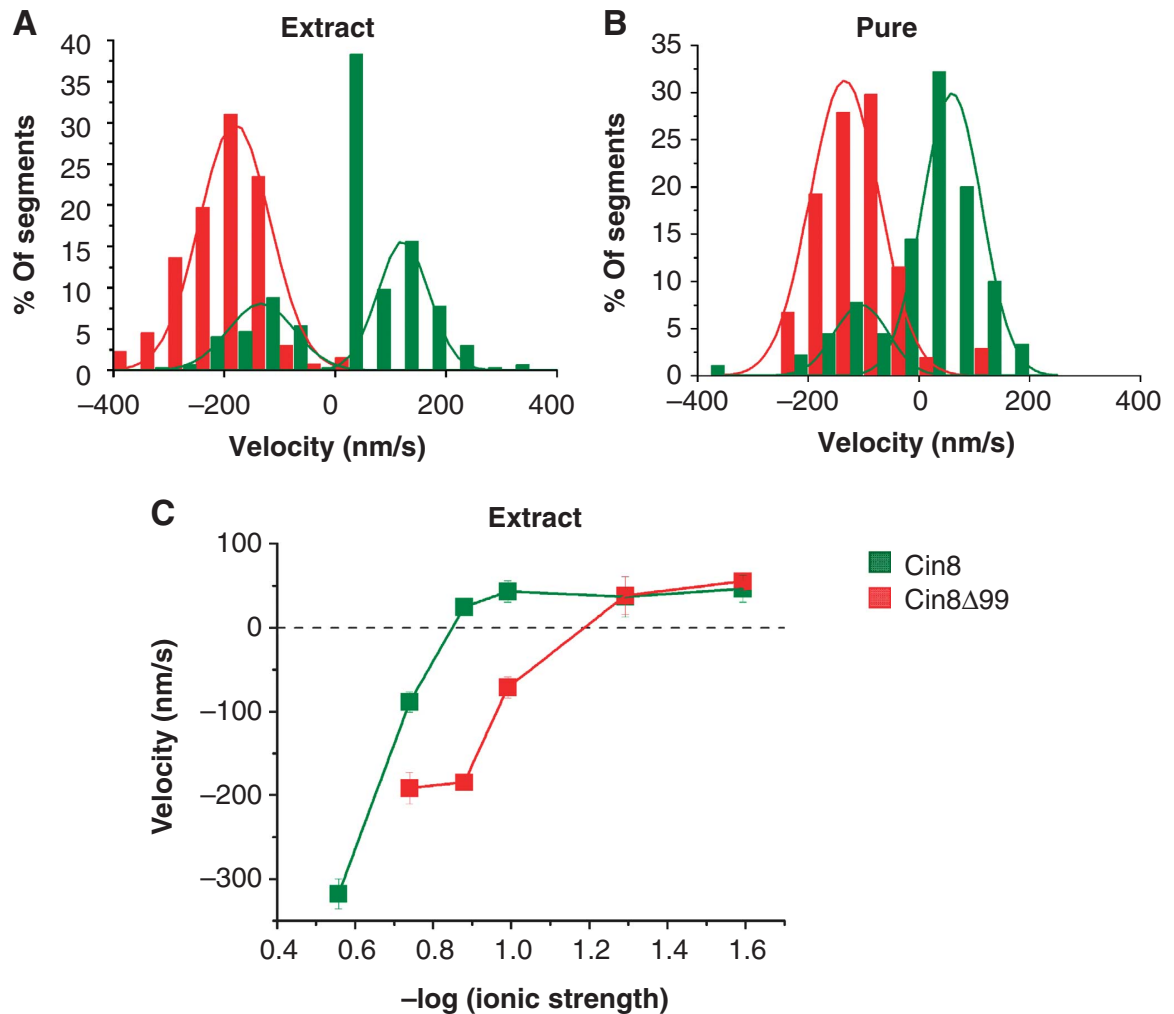

Figure 6 Deletion of the 99aa insert of Cin8 induces bias towards minus-end motility. (A, B) Histograms of velocities of Cin8 (green) and Cin8 899 (red) in whole-cell yeast extracts (A) or affinity purified (B) in buffer containing $30 \mathrm{mM} \mathrm{NaCl}$, with saturating ATP. Velocity histograms were assembled by dividing kymograph traces in $3 \mathrm{~s}$ segments and piecewise linear fitting. Lines-Gaussian distribution fit. See also Supplementary Movies S4 and S5. (C) Ionic strength dependence of mean velocity of Cin8 (green) and Cin8 $\Delta 99$ (red) in whole-cell extracts in saturating ATP conditions. Values represent mean s.e.m. of 50-200 velocity values.

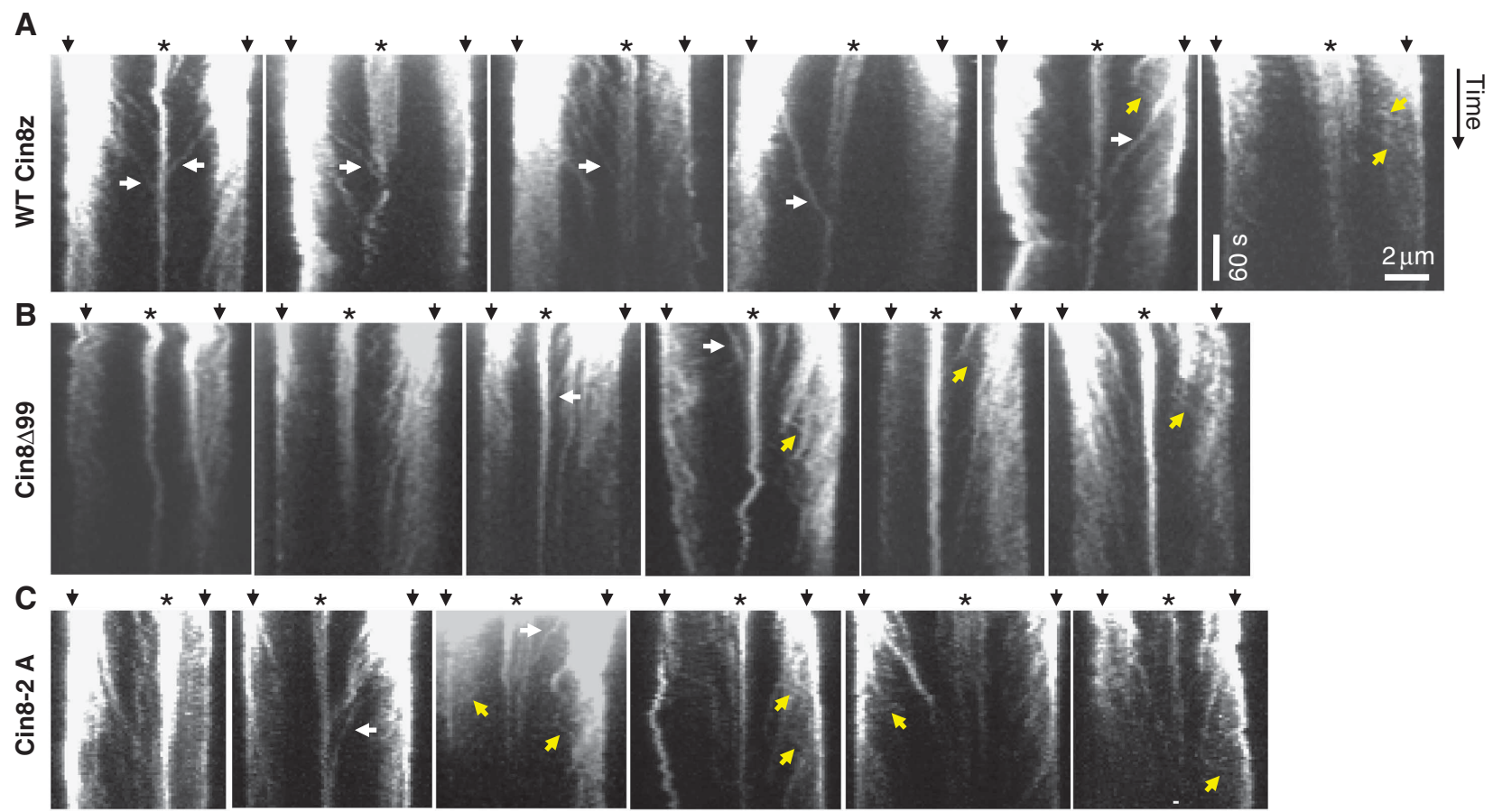

Figure 7 Movements of Cin8-3GFP (A), Cin8 $499-3$ GFP (B) and Cin8-2A-3GFP (C) in anaphase spindles. Kymographs of movements of Cin83GFP variants (indicated on the left) in intermediate to long anaphase spindles. White arrows: midzone-directed movements that span from the SPBs to the midzone; Yellow arrows: SPB-directed movements; White arrowheads: movements towards the SPB during spindle disassembly; Top arrows: spindle poles; Asterisks: midzone. 
8 are involved in the regulation of Cin8 spindle localization. Examination of Cin8-2A-3GFP movements on the spindle revealed that this mutant exhibited both midzone (plus)and SPB-directed (minus) movements (Figure 7C), with SPB-directed movements being shorter and faster than midzone-directed movements (Table I). The movements of Cin82A-3GFP were similar to those of Cin8 $\Delta 99-3 \mathrm{GFP}$ : midzonedirected movements were fewer, and significantly slower and shorter compared with the spindle movements of the WT Cin8-3GFP (Figure 7; Table I), indicating that Cin8-2A is impaired in its midzone (plus-end)-directed motility on the spindle. This result suggests that the bias towards plus-end directed motility by the 99aa insert in loop 8, stems, at least in part, from phosphorylation of the Cdk1 sites located in this insert.

An important part of the switch mechanism might be related to cargo activation of Eg5. The homologous Xenopus kinesin-5 Eg5 is only turned on to move processively when it crosslinks two MTs, that is, when both of its dimeric catalytic domains are engaged (Kapitein et al, 2008). This mechanism was suggested to be related to the cargo activation of dimeric kinesins by straightening of the back-folded stalk and tail (Hackney et al, 1992; Stock et al, 1999; Seiler et al, 2000).

To see if a similar mechanism might be responsible for switching the directionality of Cin8, we performed singlemolecule fluorescence experiments on bundled pairs of polarity-marked MTs. Between antiparallel MTs in high-salt buffer $(175 \mathrm{mM}$ added $\mathrm{NaCl})$, we saw an immediate switch from fast minus-end directed motion of individual motors to a slow erratic motion without a clear directional bias when motors reached the overlap zone (Figure 8A). In this geometry, the two crosslinked MTs were typically sliding apart with their minus ends leading with a relative velocity of about 30$60 \mathrm{~nm} / \mathrm{s}$. This reflects force generation by the motors in the plus-end direction. The fact that in the overlap region, extended fast motion was no longer observed at all implies that motors are attracted to the overlap region, likely due to their ability to bind MTs through both ends of the tetramers. In contrast to the antiparallel case, it was evident from kymographs of single motor motility between parallel MTs (Figure 8B) that most motors kept moving in the minus-end direction at undiminished speed when entering the overlap zone. Occasional short plus-end excursions were observed between parallel MTs (Figure 8B, arrowheads), which were not observed on single MTs. These events were too rare to reliably evaluate details. We thus conclude that one of the major determinants of Cin8 directionality is binding geometry, with binding between two antiparallel MTs, as it occurs in the spindle midzone, switching the motor from minus-end to plus-end motility in near-physiological salt conditions.

\section{Discussion}

In the kinesin superfamily, the majority of the members are plus-end directed. Until recently (Roostalu et al, 2011), minusend motion was seen only for kinesin-14 family members, which are structurally distinct from all other kinesin subfamilies in that they carry the conserved motor domain at the C-terminus instead of the N-terminus (McDonald et al, 1990; Walker et al, 1990; deCastro et al, 2000; Block, 2007). No full-length kinesin-14 has been found to be processive so far, that is, these motors produce isolated power strokes and can only produce persistent motion when acting in ensembles. A reversal of power stroke directionality has been reported for mutants of the non-processive kinesin-14 ncd from Drosophila melanogaster (Sablin et al, 1998; Endow and Higuchi, 2000) and could be also generated by swaps of the core and neck domains of ncd and kinesin-1 (Case et al, 1997; Endow and Waligora, 1998). Evidence for active bi-directionality of a given motor construct has been reported for a specific neck-domain mutant of ncd (Endow and Higuchi, 2000) and for cytoplasmic dynein (Dixit et al, 2008). Evidence for bi-directional power strokes of individual ncd motors has also been seen in the analysis of single-molecule recordings (Butterfield et al, 2010).

Here, we show an entirely novel behaviour for a kinesin motor. Individual kinesin- 5 Cin 8 motors could be switched by varying ionic conditions between processive minus- and plusdirected movements when travelling on single MTs (Figure 4), and they could be switched from processive minus-end motion to plus-end force generation in high-salt conditions by binding and crosslinking two MTs (Figure 8). In vitro, low ionic strength (an unphysiological environment) induced plus-end directed motion of single molecules, while high ionic strength induced minus-end directed motion. Lower ionic strength, in general, reduces electrostatic screening which, in turn, enhances electrostatic interactions between motor subelements or between motor and MT. Thus, the unphysiological change of ionic conditions might mimic the effects of phosphorylation or binding of accessory proteins, which modify electrostatic interactions under constant physiological conditions. A similar phenomenon was reported for cargo regulation of kinesins. Binding of a cargo vesicle to kinesin-1 or of a second MT to kinesin-5 Eg5, respectively, can activate the motors, but the activation also occurs at low ionic strength. In the case of Cin8, a related mechanism might not just turn the motor on or off, but lead to the switching of directionality when the motor tetramer binds between two antiparallel MTs. An alternative model that was recently proposed (Roostalu et al, 2011) relies on a collective effect involving physical load on the motors via the binding between MTs. Based on our findings, it appears that more individual mechanisms such as binding of a single motor between two MTs or phosphorylation in the catalytic domain are able to cause or modify directionality switching.

A case in point is the observed regulatory influence of the large 99aa insert in loop 8 of the Cin8 motor domain, deletion of which did not abolish the shift in directionality, but created a strong bias towards minus-end motility (Figures 5 and 6). The mechanism by which phosphorylation in Cin8 catalytic domain regulates its in-vivo function is likely to be a combination of a number of factors such as interaction with the midzone-organizing Ase1 (Khmelinskii et al, 2009), as was previously suggested (Avunie-Masala et al, 2011), or with kinetohore proteins. The fact that Cin $8 \Delta 99$ and the phosphorylation-deficient Cin8-2A exhibited reduced motility towards the midzone (Figure 7; Table I) suggests that one of the roles of Cin8 phosphorylation in the 99aa insert is to mediate the switch to plus-end directed motility of Cin8 on the spindle.

The observation that switching of directionality at high salt only occurred between antiparallel MTs is consistent with the reported preference of Drosophila kinesin-5 Klp61f for bundling antiparallel MTs (Kapitein et al, 2008). A preferred 
A

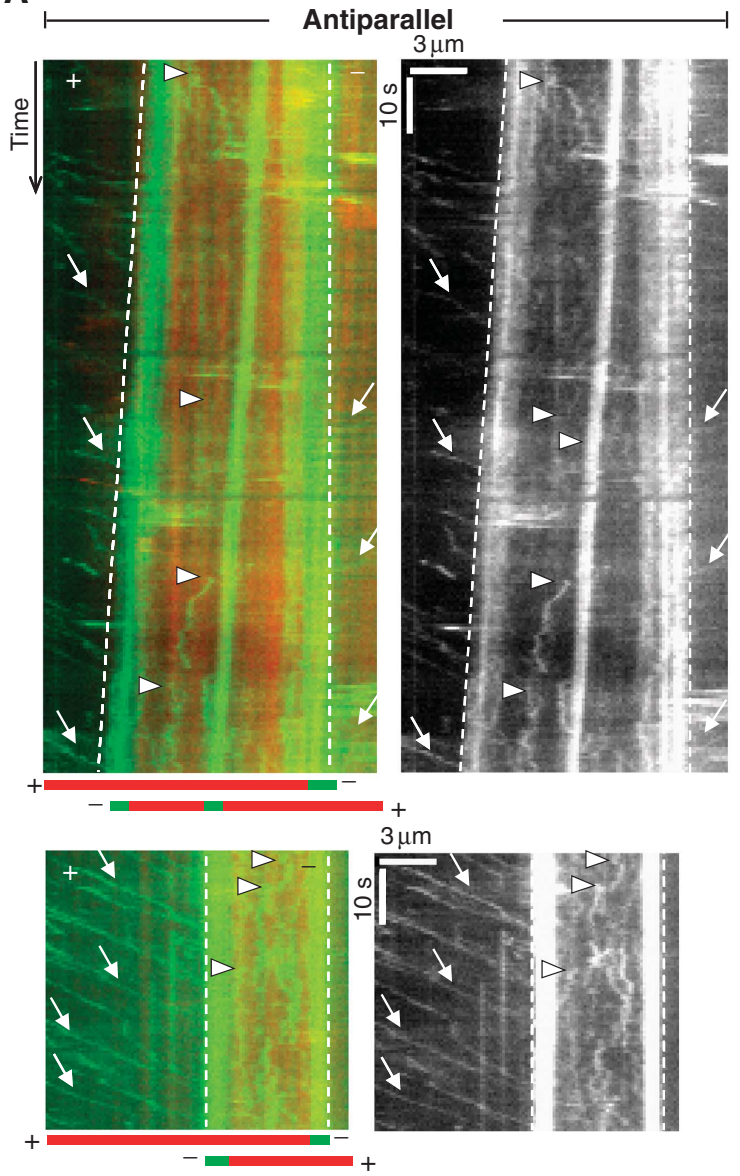

B

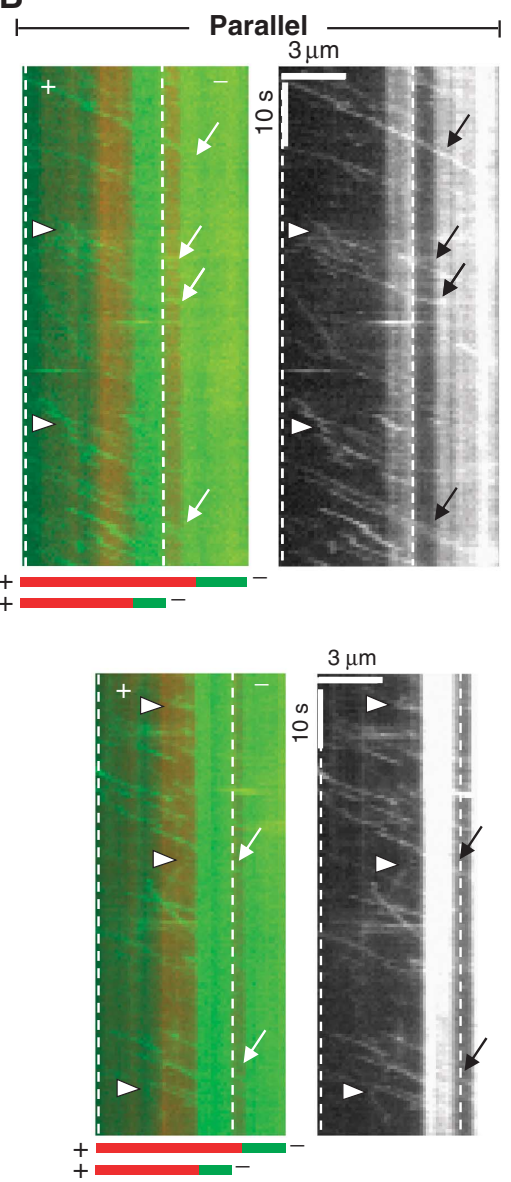

Figure 8 MT orientation changes the motile properties of single Cin8 molecules. Kymographs of movements of purified Cin8-GFP between antiparallel (A) and parallel (B) MTs in high-salt buffer (MB-175). For each event, a merged kymograph in colour (red-MT; green-Cin8 and minus ends of MTs) is shown on the left and a kymograph of the GFP-channel only is shown on the right. Overlapping region between MTs is marked by dashed lines. Cartoon depicting the orientation of overlapping MTs is shown at the bottom of the colour kymographs. Arrows: minus-end directed motility events; arrowheads: plus-end directed motility events. See also Supplementary Movie clips S6-S10.

orientation was for that motor conferred by the ATP-independent binding sites in the C-terminal tail of the molecules. An ATP-independent binding mechanism appears to also exist for Cin8 because full-length Cin8 also supports diffusive MT attachment in ADP buffer (Table II). Sticky tails with preferred orientation might not prevent parallel crosslinking by the motor, but it was found for Eg5 that all eight binding sites were necessary for motor engagement between MTs (Weinger et al, 2011). It is tempting to speculate that this might also be the case for Cin8, but in this case with the further consequence that directionality is reversed to plusend motion between the antiparallel MTs. As the spindle midzone is the place where antiparallel overlaps occur and as that is the location where the motors need to exert force, such a regulation appears advantageous.

The discovery of the exceptional properties of Cin8 raises the question how these motile properties aid Cin8 in performing its multiple mitotic roles. The ionic strength in $S$. cerevisiae cells is high, estimated as $\sim 300 \mathrm{mM}$ salt (Olz et al, 1993; van Eunen et al, 2010). Under these conditions, Cin8 motors were minus-end directed on single MTs in our in-vitro experiments (Figures 2, 4 and 6). Prior to spindle elongation, Cin8 is known to be important for kinetochore clustering or positioning near the SPBs (Tytell and Sorger, 2006; Gardner et al, 2008a; Wargacki et al, 2010). The proposed mechanism for this function had been the crosslinking of kinetochore MTs (kMTs; Tytell and Sorger, 2006) and the promotion of disassembly of long kMTs (Gardner et al, 2008a). Since in $S$. cerevisiae cells, each kinetochore is attached to a single MT and since on a single MT Cin8 is minus-end directed, active motion of Cin8 in the minus-end direction of the kMTs may be an alternative/additional mechanism by which Cin8 contributes to kinetochore positioning.

The slow plus-end directed motility that we observed in vivo in anaphase spindles (Figures 1C and 7; Table I) indicates that Cin8 is switched to plus-end directed motility in the cell, even on single MTs or on parallel bundles. During anaphase spindle elongation, bi-directionality is likely to be important to dynamically partition Cin8 motors between different reservoirs, that is, near the poles where they focus the kinetochore clusters (Tytell and Sorger, 2006; Gardner et al, 2008a; Wargacki et al, 2010) and in the midzone where Cin8 promotes plus-end directed MT sliding (Figure 8; Roostalu et al, 2011) and spindle elongation (Saunders et al, 1995; Movshovich et al, 2008). In fact, we observed that until anaphase spindles reach a length of $\sim 5 \mu \mathrm{m}$, Cin8 is localized throughout the spindle, with no preferential accumulation at the midzone or near the spindle poles, nor obvious 
detachment (Figures 1A and 3C). A way to maintain this even distribution without detachment might be bi-directional motility of Cin8. Interestingly, higher eukaryotes, which show poleward flux in the spindle MTs, appear not to have kinesin5 motors capable of minus-end motility, possibly because it became unnecessary for motor transport to the poles in fluxing spindles.

A factor that appears to be important for directionality is the geometry of binding and allosteric regulation by two bound antiparallel MTs. Intermediate-long $S$. cerevisiae anaphase spindles contain a small number of MTs, two emanating from each pole at the end of anaphase (Winey et al, 1995). Motility between antiparallel MTs emanating from opposing poles should move both MTs and keep the motor fixed in the midzone. Therefore, the plus-end motility observed in vivo can only take place on single or between parallel MTs and is likely to utilize a further mode of regulation without which the motors would rapidly converge back to the poles.

In conclusion, Cin8 has turned out to be an exceptional kinesin in that it is truly bi-directional and processive in both directions. This unique feature of Cin8 appears to play a role in cellular function. First, hints about the molecular mechanism indicate a role of charge interactions and possibly phosphorylation, and most importantly binding geometry between pairs of MTs. It remains to be explored in more detail if Cin8 regulation is a variation of the scheme of the regulation of other kinesins, in particular kinesin-5 motors. In general, our findings demonstrate that in order to fulfil their physiological functions, kinesin motors are much less rigidly programmed than was broadly believed so far and that the extent to which their function is regulated in the cell encompasses much more than simple on-off switches.

\section{Materials and methods}

Detailed procedures and S. cerevisiae strains used in this study are described in Supplementarydata. In brief, we produced fluorescently labelled Cin8 motors in three ways. We first used whole-cell extracts of S. cerevisiae expressing Cin8 fused with three consecutive C-terminal GFPs (Cin8-3GFP) under its own promoter. Cin8-3GFP was either integrated into the yeast genome or expressed from a CEN plasmid. Cells with integrated Cin8-3GFP were also used for in-vivo imaging (Supplementary Table S1). Second, we expressed Cin8 fused with a single C-terminal GFP and $\mathrm{N}$-terminal 6HIS tag (6HIS-Cin8-GFP) in Sf9 insect cells, and third, we overexpressed Cin8-GFP-6HIS in S. cerevisiae. For yeast strains and plasmids, see Supplementary Table S1 (Supplementary data). We purified motor by HIS tag and MT affinity.

Live-cell imaging was done on a spinning-disc confocal microscope (Zeiss Axiovert 200M, UltraView ESR, Perkin-Elmer, UK; Fridman et al, 2009). Z-stacks of $0.2-0.4 \mu \mathrm{m}$ separation were acquired in 1-min time intervals (Movshovich et al, 2008).

\section{References}

Avunie-Masala R, Movshovich N, Nissenkorn Y, Gerson-Gurwitz A, Fridman V, Koivomagi M, Loog M, Hoyt MA, Zaritsky A, Gheber L (2011) Phospho-regulation of kinesin-5 during anaphase spindle elongation. J Cell Sci 124: 873-878

Blangy A, Lane HA, d'Herin P, Harper M, Kress M, Nigg EA (1995) Phosphorylation by $\mathrm{p} 34 \mathrm{cdc} 2$ regulates spindle association of human Eg5, a kinesin-related motor essential for bipolar spindle formation in vivo. Cell 83: 1159-1169

Block SM (2007) Kinesin motor mechanics: binding, stepping, tracking, gating, and limping. Biophys J 92: 2986-2995
For the Cin8-3GFP spot-motility analysis, images were acquired every $2 \mathrm{~s}$.

In-vitro motility assays were performed following standard procedures (Howard et al, 1993; Gheber et al, 1999; Lakamper et al, 2010) in motility buffer MB-175 (50 mM Tris/ $\mathrm{HCl}, 30 \mathrm{mM}$ PIPES/KOH, final pH 7.2, 175 mM NaCl, 2 mM EDTA, 1 mM EGTA, $10 \%$ glycerol, $1 \mathrm{mM}$ phenylmethylsulfonyl fluoride and $1 \mathrm{mM}$ dithiothreitol) as well as versions of this buffer with less $\mathrm{NaCl}$ added. The MTs, polymerized from TMR-labelled porcine tubulin, were polarity marked using Atto-488-labelled seeds marking the minus end of the MTs. Single-molecule fluorescence data were collected on two microscopes, one at Ben-Gurion University (BGU) and one at Göttingen University (GAUG). BGU: Zeiss Axiovert 200M, HBO 100 Mercury Illuminator, cooled CCD (SensiCam, PCO), frame time $0.8 \mathrm{~s}$. Data were processed using ImageJ and MetaMorph (MDS Analytical Technologies) software. GAUG: custom-built totalinternal-reflection fluorescence microscope, using a 473-nm Laser (Viasho, USA) for excitation, and a $\times 100$ objective (Nikon, SFluor, NA 1.49, Oil) and a CCD camera (Cascade 512B, Roper Scientific, USA), frame rate $0.5 \mathrm{~s}$. Software was custom written in Labview. Velocity histograms were assembled by drawing lines through consecutive $3 \mathrm{~s}$ segments of kymograph traces.

For relative sliding assays, polarity-marked MTs were polymerized as before whereas a solution of shorter MTs was polymerized by incubation at $37^{\circ} \mathrm{C}$ for only $6 \mathrm{~min}$. First, the long polarity-marked MTs were allowed to bind for $3 \mathrm{~min}$ to the DETA-coated surface of the assay chamber. The motility buffer (MB-175) was the same as used for the single-molecule assays but with double ATP and $\mathrm{MgCl}_{2}$ concentration. To this buffer, three times the single-motor concentration and $1 \mu \mathrm{l}$ of short polarity-marked MTs were added, and the mix was washed into the assay chamber. The custom-built TIRF set-up described before was expanded such that the emission of the TMR-labelled MTs and the GFP-labelled motor proteins could be detected simultaneously. The TMR and the GFP channel were aligned with ImageJ.

\section{Supplementary data}

Supplementary data are available at The EMBO Journal Online (http://www.embojournal.org).

\section{Acknowledgements}

This work was supported in part by the Lower Saxony Grant no. 1176251-99-26/08 (ZN2440) awarded to LG, SL and CFS. LG was supported by the ISF grant no. 1043/09 and the BSF grant no. 2003141 and CFS was supported by the Center for Molecular Physiology of the Brain (CMPB), funded by the Deutsche Forschungsgemeinschaft (DFG). We thank Yael Nissenkorn, BGU, Israel for providing Cin8-2A plasmids and to Florian Rehfeldt and Marcel Bremerich, GAUG, Germany for data analysis.

Author contributions: AGG, CT, NM, VF, MP, TD, SL and DRK performed the experiments; AGG, CT, CFS and LG analysed the data and wrote the paper; CFS and LG supervised and coordinated the project. All authors read and commented on the draft versions of the manuscript and approved the final version.

\section{Conflict of interest}

The authors declare that they have no conflict of interest.

Bormuth V, Varga V, Howard J, Schaffer E (2009) Protein friction limits diffusive and directed movements of kinesin motors on microtubules. Science 325: 870-873

Brust-Mascher I, Civelekoglu-Scholey G, Kwon M, Mogilner A, Scholey JM (2004) Model for anaphase B: role of three mitotic motors in a switch from poleward flux to spindle elongation. Proc Natl Acad Sci USA 101: 15938-15943

Butterfield AE, Stewart RJ, Schmidt CF, Skliar M (2010) Bidirectional power stroke by ncd kinesin. Biophys J 99: 3905-3915 
Case RB, Pierce DW, Hom-Booher N, Hart CL, Vale RD (1997) The directional preference of kinesin motors is specified by an element outside of the motor catalytic domain. Cell 90: 959-966

Chee MK, Haase SB (2010) B-cyclin/CDKs regulate mitotic spindle assembly by phosphorylating kinesins-5 in budding yeast. PLoS Genet 6: e1000935

Chua PR, Roof DM, Lee Y, Sakowicz R, Clarke D, Pierce D, Stephens T, Hamilton M, Morgan B, Morgans D, Nakai T, Tomasi A, Maxon ME (2007) Effective killing of the human pathogen Candida albicans by a specific inhibitor of non-essential mitotic kinesin Kip1p. Mol Microbiol 65: 347-362

deCastro MJ, Fondecave RM, Clarke LA, Schmidt CF, Stewart RJ (2000) Working strokes by single molecules of the kinesin-related microtubule motor ncd. Nat Cell Biol 2: 724-729

Dietrich KA, Sindelar CV, Brewer PD, Downing KH, Cremo CR, Rice SE (2008) The kinesin-1 motor protein is regulated by a direct interaction of its head and tail. Proc Natl Acad Sci USA 105: 8938-8943

Dixit R, Ross JL, Goldman YE, Holzbaur EL (2008) Differential regulation of dynein and kinesin motor proteins by tau. Science 319: 1086-1089

Endow SA, Higuchi H (2000) A mutant of the motor protein kinesin that moves in both directions on microtubules. Nature 406 913-916

Endow SA, Waligora KW (1998) Determinants of kinesin motor polarity. Science 281: 1200-1202

Enos AP, Morris NR (1990) Mutation of a gene that encodes a kinesin-like protein blocks nuclear division in A. nidulans. Cell 60: 1019-1027

Fridman V, Gerson-Gurwitz A, Movshovich N, Kupiec M, Gheber L (2009) Midzone organization restricts interpolar microtubule plus-end dynamics during spindle elongation. EMBO Rep 10: 387-393

Gardner MK, Bouck DC, Paliulis LV, Meehl JB, O’Toole ET, Haase J, Soubry A, Joglekar AP, Winey M, Salmon ED, Bloom K, Odde DJ (2008a) Chromosome congression by Kinesin-5 motor-mediated disassembly of longer kinetochore microtubules. Cell 135: 894-906

Gardner MK, Haase J, Mythreye K, Molk JN, Anderson M, Joglekar AP, O’Toole ET, Winey M, Salmon ED, Odde DJ, Bloom K (2008b) The microtubule-based motor Kar3 and plus end-binding protein Bim1 provide structural support for the anaphase spindle. $J$ Cell Biol 180: $91-100$

Gerson-Gurwitz A, Movshovich N, Avunie R, Fridman V, Moyal K, Katz B, Hoyt MA, Gheber L (2009) Mid-anaphase arrest in S. cerevisiae cells eliminated for the function of Cin8 and dynein. Cell Mol Life Sci 66: 301-313

Gheber L, Kuo SC, Hoyt MA (1999) Motile properties of the kinesinrelated Cin8p spindle motor extracted from Saccharomyces cerevisiae cells. J Biol Chem 274: 9564-9572

Hackney DD, Baek N, Snyder AC (2009) Half-site inhibition of dimeric kinesin head domains by monomeric tail domains. Biochemistry 48: 3448-3456

Hackney DD, Levitt JD, Suhan J (1992) Kinesin undergoes a 9 S to 6 S conformational transition. J Biol Chem 267: 8696-8701

Hagan I, Yanagida M (1992) Kinesin-related cut7 protein associates with mitotic and meiotic spindles in fission yeast. Nature 356: 74-76

Hancock WO (2008) Intracellular transport: kinesins working together. Curr Biol 18: R715-R717

Heck MM, Pereira A, Pesavento P, Yannoni Y, Spradling AC, Goldstein LS (1993) The kinesin-like protein KLP61F is essential for mitosis in Drosophila. J Cell Biol 123: 665-679

Hildebrandt ER, Gheber L, Kingsbury T, Hoyt MA (2006) Homotetrameric form of Cin8p, a Saccharomyces cerevisiae kinesin-5 motor, is essential for its in vivo function. $J$ Biol Chem 281: 26004-26013

Howard J, Hunt AJ, Baek S (1993) Assay of microtubule movement driven by single kinesin molecules. Methods Cell Biol 39: 137-147

Hoyt MA, He L, Loo KK, Saunders WS (1992) Two Saccharomyces cerevisiae kinesin-related gene products required for mitotic spindle assembly. J Cell Biol 118: 109-120

Kahana JA, Schnapp BJ, Silver PA (1995) Kinetics of spindle pole body separation in budding yeast. Proc Natl Acad Sci USA 92: 9707-9711
Kapitein LC, Kwok BH, Weinger JS, Schmidt CF, Kapoor TM, Peterman EJ (2008) Microtubule cross-linking triggers the directional motility of kinesin-5. J Cell Biol 182: 421-428

Kapitein LC, Peterman EJ, Kwok BH, Kim JH, Kapoor TM, Schmidt CF (2005) The bipolar mitotic kinesin Eg5 moves on both microtubules that it crosslinks. Nature 435: 114-118

Kashina AS, Rogers GC, Scholey JM (1997) The bimC family of kinesins: essential bipolar mitotic motors driving centrosome separation. Biochim Biophys Acta 1357: 257-271

Khmelinskii A, Roostalu J, Roque H, Antony C, Schiebel E (2009) Phosphorylation-dependent protein interactions at the spindle midzone mediate cell cycle regulation of spindle elongation. Dev Cell 17: 244-256

Kull FJ, Sablin EP, Lau R, Fletterick RJ, Vale RD (1996) Crystal structure of the kinesin motor domain reveals a structural similarity to myosin. Nature 380: 550-555

Kwok BH, Kapitein LC, Kim JH, Peterman EJ, Schmidt CF, Kapoor TM (2006) Allosteric inhibition of kinesin-5 modulates its processive directional motility. Nat Chem Biol 2: 480-485

Kwok BH, Yang JG, Kapoor TM (2004) The rate of bipolar spindle assembly depends on the microtubule-gliding velocity of the mitotic kinesin Eg5. Curr Biol 14: 1783-1788

Lakamper S, Thiede C, Duselder A, Reiter S, Korneev MJ, Kapitein LC, Peterman EJ, Schmidt CF (2010) The effect of monastrol on the processive motility of a dimeric kinesin-5 head/kinesin-1 stalk chimera. J Mol Biol 399: 1-8

McDonald HB, Stewart RJ, Goldstein LS (1990) The kinesin-like ncd protein of Drosophila is a minus end-directed microtubule motor. Cell 63: 1159-1165

Movshovich N, Fridman V, Gerson-Gurwitz A, Shumacher I, Gertsberg I, Fich A, Hoyt MA, Katz B, Gheber L (2008) Slk19dependent mid-anaphase pause in kinesin-5-mutated cells. J Cell Sci 121: 2529-2539

Nitta R, Okada Y, Hirokawa N (2008) Structural model for straindependent microtubule activation of Mg-ADP release from kinesin. Nat Struct Mol Biol 15: 1067-1075

Olz R, Larsson K, Adler L, Gustafsson L (1993) Energy flux and osmoregulation of Saccharomyces cerevisiae grown in chemostats under $\mathrm{NaCl}$ stress. J Bacteriol 175: 2205-2213

Roof DM, Meluh PB, Rose MD (1991) Multiple kinesin-related proteins in yeast mitosis. Cold Spring Harb Symp Quant Biol 56: 693-703

Roof DM, Meluh PB, Rose MD (1992) Kinesin-related proteins required for assembly of the mitotic spindle. J Cell Biol 118: 95-108

Roostalu J, Hentrich C, Bieling P, Telley IA, Schiebel E, Surrey T (2011) Directional switching of the Kinesin cin8 through motor coupling. Science 332: 94-99

Sablin EP, Case RB, Dai SC, Hart CL, Ruby A, Vale RD, Fletterick RJ (1998) Direction determination in the minus-end-directed kinesin motor ncd. Nature 395: 813-816

Saunders WS, Koshland D, Eshel D, Gibbons IR, Hoyt MA (1995) Saccharomyces cerevisiae kinesin- and dynein-related proteins required for anaphase chromosome segregation. J Cell Biol 128: 617-624

Sawin KE, LeGuellec K, Philippe M, Mitchison TJ (1992) Mitotic spindle organization by a plus-end-directed microtubule motor. Nature 359: 540-543

Schuyler SC, Liu JY, Pellman D (2003) The molecular function of Ase1p: evidence for a MAP-dependent midzone-specific spindle matrix. Microtubule-associated proteins. J Cell Biol 160: 517-528

Seiler S, Kirchner J, Horn C, Kallipolitou A, Woehlke G, Schliwa M (2000) Cargo binding and regulatory sites in the tail of fungal conventional kinesin. Nat Cell Biol 2: 333-338

Sharp DJ, McDonald KL, Brown HM, Matthies HJ, Walczak C, Vale RD, Mitchison TJ, Scholey JM (1999) The bipolar kinesin, KLP61F, cross-links microtubules within interpolar microtubule bundles of Drosophila embryonic mitotic spindles. J Cell Biol 144: $125-138$

Stock MF, Guerrero J, Cobb B, Eggers CT, Huang TG, Li X, Hackney DD (1999) Formation of the compact confomer of kinesin requires a $\mathrm{COOH}$-terminal heavy chain domain and inhibits microtubule-stimulated ATPase activity. J Biol Chem 274: 14617-14623

Straight AF, Sedat JW, Murray AW (1998) Time-lapse microscopy reveals unique roles for kinesins during anaphase in budding yeast. J Cell Biol 143: 687-694 
Touitou I, Lhomond G, Pruliere G (2001) Boursin, a sea urchin bimC kinesin protein, plays a role in anaphase and cytokinesis. $J$ Cell Sci 114: 481-491

Tytell JD, Sorger PK (2006) Analysis of kinesin motor function at budding yeast kinetochores. JCB 172: 861-874

Vale RD, Funatsu T, Pierce DW, Romberg L, Harada Y, Yanagida T (1996) Direct observation of single kinesin molecules moving along microtubules. Nature 380: 451-453

Valentine MT, Fordyce PM, Block SM (2006) Eg5 steps it up!. Cell Div 1: 31

van Eunen K, Bouwman J, Daran-Lapujade P, Postmus J, Canelas $\mathrm{AB}$, Mensonides FI, Orij R, Tuzun I, van den Brink J, Smits GJ, van Gulik WM, Brul S, Heijnen JJ, de Winde JH, de Mattos MJ, Kettner C, Nielsen J, Westerhoff HV, Bakker BM (2010) Measuring enzyme activities under standardized in vivo-like conditions for systems biology. FEBS J 277: 749-760

Walczak CE, Mitchison TJ (1996) Kinesin-related proteins at mitotic spindle poles: function and regulation. Cell 85: 943-946
Walker RA, Salmon ED, Endow SA (1990) The Drosophila claret segregation protein is a minus-end directed motor molecule. Nature 347: 780-782

Wargacki MM, Tay JC, Muller EG, Asbury CL, Davis TN (2010) Kip3, the yeast kinesin-8, is required for clustering of kinetochores at metaphase. Cell Cycle 9: 2581-2588

Weinger JS, Qiu M, Yang G, Kapoor TM (2011) A nonmotor microtubule binding site in kinesin-5 is required for filament crosslinking and sliding. Curr Biol 21: 154-160

Winey M, Mamay CL, O’Toole ET, Mastronarde DN, Giddings Jr TH, McDonald KL, McIntosh JR (1995) Three-dimensional ultrastructural analysis of the Saccharomyces cerevisiae mitotic spindle. J Cell Biol 129: 1601-1615

Zhu C, Zhao J, Bibikova M, Leverson JD, Bossy-Wetzel E, Fan JB, Abraham RT, Jiang W (2005) Functional analysis of human microtubule-based motor proteins, the kinesins and dyneins, in mitosis/cytokinesis using RNA interference. Mol Biol Cell 16: 3187-3199 



\title{
Regulation of bi-directional movement of single kinesin-5 Cin8 molecules
}

\author{
Christina Thiede, ${ }^{1}$ Vladimir Fridman, ${ }^{2}$ Adina Gerson-Gurwitz, ${ }^{3,+}$ Larisa Gheber ${ }^{2,3,4, *}$ and Christoph F. Schmidtt ${ }^{1, *}$
}

'Drittes Physikalisches Institut; Georg-August-Universität; Göttingen, Germany; ${ }^{2}$ Department of Clinical Biochemistry; Ben-Gurion University of the Negev; Beer-Sheva, Israel; ${ }^{3}$ Department of Chemistry; Ben-Gurion University of the Negev; Beer-Sheva, Israel; ${ }^{4}$ Ise Katz Institute for Nanoscale Science and Technology; Ben-Gurion University of the Negev; Beer-Sheva, Israel

${ }^{+}$Current address: Ludwig Institute for Cancer Research and Department of Cellular and Molecular Medicine; University of California San Diego; La Jolla, CA USA

Keywords: kinesin-5, kinesin directionality, Saccharomyces cerevisiae Cin8, mitosis, microtubules

Kinesin-5 mechanoenzymes drive mitotic spindle dynamics as slow, processive microtubule (MT)-plus-end directed motors. Surprisingly, the Saccharomyces cerevisiae kinesin-5 Cin8 was recently found to be bi-directional: it can move processively in both directions on MTs. Two hypotheses have been suggested for the mechanism of the directionality switch: (1) single molecules of Cin8 are intrinsically minus-end directed, but mechanical coupling between two or more motors triggers the switch; (2) a single motor can switch direction, and "cargo binding" i.e., binding between two MTs triggers the switch to plus-end motility. Single-molecule fluorescence data we published recently, and augment here, favor hypothesis (2). In low-ionic-strength conditions, single molecules of Cin8 move in both minus- and plus-end directions. Fluorescence photo bleaching data rule out aggregation of Cin8 while they move in the plus and in the minus direction. The evidence thus points toward cargo regulation of directionality, which is likely to be related to cargo regulation in other kinesins. The molecular mechanisms of this regulation, however, remain to be elucidated.

Members of the kinesin-5 family of motor proteins are conserved among eukaryotes, from yeast to humans. Among the cytoskeletal motors, kinesins, myosins and dyneins, kinesin-5 motors are the only ones that function as bipolar homotetramers, with two pairs of catalytic domains located at opposite ends of the active complex. ${ }^{1,2}$ This special architecture is thought to enable kinesin5 motors to crosslink and slide apart antiparallel MTs emanating from the opposite poles of the mitotic spindle. ${ }^{3}$ By this mode of action, kinesin-5 motors are believed to fulfill their essential roles in spindle dynamics such as spindle assembly, maintenance of the bipolar spindle structure prior to the onset of anaphase, ${ }^{3-5}$ as well as anaphase B spindle elongation. ${ }^{6-11}$ Since MTs are organized with their plus ends overlapping in the midzone, kinesin-5 can only push spindle poles apart during spindle assembly and elongation via plus-end directed motility between antiparallel MTs. It has indeed been demonstrated in vitro, that the vertebrate kinesin-5 Eg5 moves simultaneously toward the plus ends of two antiparallel MTs that it crosslinks. ${ }^{12,13}$ This finding was consistent with the 20-year-old dogma that kinesin homologs which carry their catalytic domains at the $\mathrm{N}$-terminus are plus-end directed. ${ }^{14}$

The majority of the members of the kinesin superfamily are plus-end directed. Minus-end motion was seen only for the structurally distinct kinesin-14 family members which carry the catalytic domain at their C-terminus. ${ }^{15-17}$ Being non-processive, these motors produce isolated power strokes and can only produce persistent motion in ensembles. Surprisingly, the S. cerevisiae kinesin-5 Cin8 was recently found to move processively in the minus-end direction of MTs in single-molecule fluorescence motility assays under close-to-physiological conditions. ${ }^{18,19} \mathrm{Cin} 8$ was shown to switch directionality to plus-end directed motility in several experimental circumstances: in multi-motor MT gliding assays, ${ }^{18,20}$ under low-ionic-strength conditions, and when bound between two antiparallel MTs. ${ }^{18,19}$ Two possible mechanisms for this switch have been suggested: one is that single molecules of Cin8 can move only toward the minus end of MTs and that coupling between two or more motors triggers the plus-end directed switch; ${ }^{18}$ the second possibility is that the ability to switch directionality is contained within a single motor itself and that interaction between Cin8 and MTs can trigger the switch. ${ }^{19}$ Several lines of evidence support the second mechanism.

The central evidence for a motor-intrinsic switching mechanism is that individual Cin8 molecules can switch to plus-end directed motility under low-ionic-strength conditions. ${ }^{19}$ In our in vitro single-molecule fluorescence experiments, ${ }^{19}$ low total ionic strength below $-0.13 \mathrm{M}$ (an unphysiological environment) induced plus-end-directed and high ionic strength promoted minus-end-directed motion of Cin8. In view of the controversy about the mechanism of the directionality switch, it is crucial to prove that, indeed, individual Cin8 molecules move toward the plus ends of MTs and that observed fluorescent traces do not 
originate from small clusters of motors acting collectively. To address this point, we followed the photo-bleaching of fluorescent Cin8-GFP tetramers, purified from $S$. cerevisiae cells, while they moved on polarity-marked MTs under low-ionic-strength conditions. Experiments are described in detail in Gerson-Gurwitz et al. ${ }^{19}$ and additional data is presented here (Fig. 1). Two buffer conditions were examined: motility buffer (MB) with $30 \mathrm{mM}$ $\mathrm{NaCl}$ added (ionic strength $0.132 \mathrm{M}$ ) and $\mathrm{MB}$ with no added $\mathrm{NaCl}$ (ionic strength $0.102 \mathrm{M}$ ). We have previously shown that under these conditions, Cin8 moves toward the plus end of MTs for $-60 \%$ and $-70 \%$ of the time, respectively. ${ }^{19}$ To count the number of GFP fluorophores on each moving motor or (possibly) motor aggregate, we measured the intensity of several single spots in a given video recording, both for spots appearing during the recording (i.e., landing from the bulk on the MT) and then moving to the plus-end of the MT, and for spots appearing and remaining stationary on the MT during the recording. To obtain a scale for the intensity, i.e., to determine the intensity of a single GFP, intensities before and after the final bleaching step of immobile motors were analyzed (Fig. 1C). The measured intensity distribution was fitted with a sum of two Gaussians, resulting in a value for the background and a value for the intensity of a single GFP. We then measured the distribution of the initial fluorescence intensities right after landing for both moving and immobile motors. Intensities in a fixed area were averaged for the first three frames $(1.5 \mathrm{sec})$ right after landing

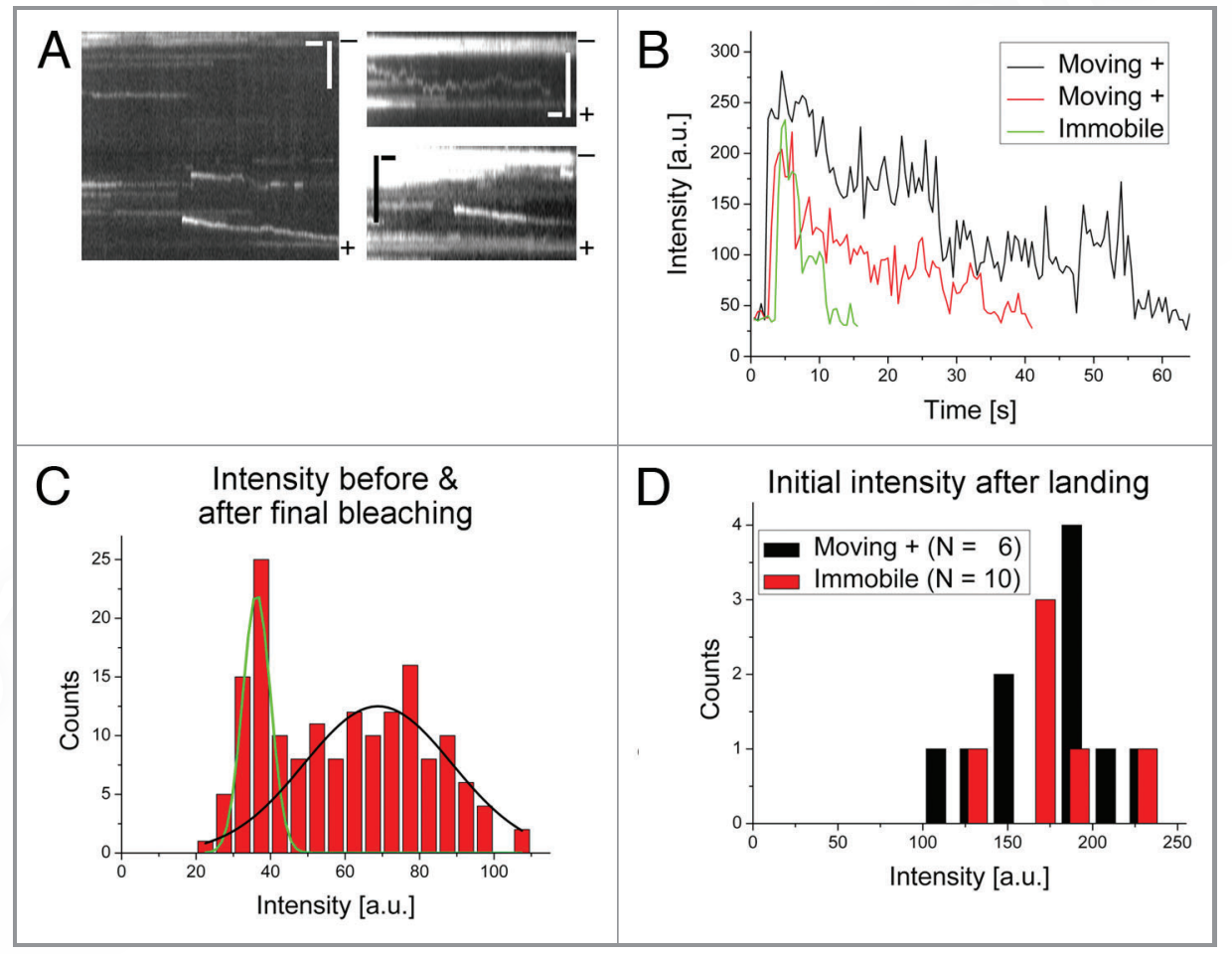

Figure 1. In low-ionic-strength conditions, single Cin8 motors move toward the plus end of MTs. (A) Kymographs of Cin8 moving away from the brightly labeled seed marking the minus end (-) toward the plus end (+) of the MT. Kymograph in the right bottom panel was captured in MB-30; the other two in MB-0 (exact buffer compositions are given below). Scale bars: horizontal: $10 \mathrm{sec}$; vertical: $3 \mu \mathrm{m}$. (B) Exemplary intensity traces of two motors landing on a MT and moving to the MT plus end (black and red) and of a motor landing on a MT and remaining immobile (green). The traces of the moving motors correspond to the two events shown in the left panel of (A). (C) Histogram of fluorescence intensities before and after final bleaching steps of immobile motors, summed from a $800 \mathrm{~nm} \times 800 \mathrm{~nm}$ square of camera pixels containing the image of the motor (number of traces /motors: 8). A sum of two Gaussians was fitted to the histogram. The first narrower peak corresponds to the background [compare also to (B)]. The broader second peak represents the intensity of a single GFP. (D) Histogram of initial intensities of Cin8 molecules right after landing, measured in the same arbitrary units as in (C). The intensity of the first three frames $(1.5 \mathrm{sec})$ after landing was averaged for both moving and immobile motors. The histogram has a maximum at 4 times the intensity of a single GFP [compare with (C)]. Materials and Methods: Single-molecule fluorescence assays were performed as described in detail in Gerson-Gurwitz et al. ${ }^{19}$ In short, the custom-built total-internal-reflection-fluorescence (TIRF) microscope consisted of a $473 \mathrm{~nm}$ laser (Viasho, USA) for excitation, a 100x objective (SFluor, NA 1.49, oil, Nikon, USA), and a CCD camera (Cascade 512B, Roper Scientific, USA) for detection. To observe several colors simultaneously, the fluorescence emission signal was split by dichroic mirrors and directed to separate areas on the CCD camera.

Fluorescently Cin8-TEV-GFP-6HIS was overexpressed in S. cerevisiae and affinity purified using the his-tag and a Ni-NTA affinity column (Invitrogen, USA). The low-salt buffers for the motility assays were composed as follows: MB-0: $50 \mathrm{mM}$ Tris/HCl, $30 \mathrm{mM}$ PIPES/KOH, pH 7.2, 2 mM EDTA, $1 \mathrm{mM} \mathrm{EGTA,} \mathrm{10 \%}$ glycerol, $1 \mathrm{mM}$ phenylmethylsulfonyl fluoride and $1 \mathrm{mM}$ dithiothreitol. MB-30: the same as MB-0 but with $30 \mathrm{mM}$ added NaCl. MTs were polymerized from tissue-purified porcine tubulin, essentially as described before, ${ }^{31}$ but with Atto-488 (Atto-Tec GmbH, Siegen, Germany) labeled seeds that also fluoresce in the green and in that way mark the minus end of the MTs. ${ }^{19}$ Kymographs were generated and analyzed with a custom-written LabView (National Instruments, USA) routine. The fluorescence intensity emitted by single proteins was summed over an area of $5 \times 5$ camera pixels, corresponding to an area of $800 \mathrm{~nm} \times 800 \mathrm{~nm}$ in the specimen plane and was analyzed with ImageJ software (NIH, USA), and histograms and fits were done with OriginPro software (OriginLab Corporation, USA). 
(Fig. 1D), during which time the motors moved much less than the size of the chosen area around the spots. Comparison of initial intensities to monomer intensities confirms that both, the motors moving to the plus end of MTs and the stationary motors were tetramers. Furthermore, some of the bleaching traces of Cin8 molecules that moved toward the plus ends of MTs showed four consecutive bleaching steps (Fig. 1B), indicating again that these molecules were tetramers with four GFP molecules. These results demonstrate that, under low-ionic-strength conditions, individual Cin8 molecules can move in the plus-end direction on MTs. While coupling between multiple motors could be an additional mechanism for switching, our results provide support for the existence of the motor-intrinsic switch model.

Since ionic strength generally modulates electrostatic interactions, the unphysiologically low-ionic-strength conditions might mimic the effects of phosphorylation or binding of accessory proteins or of binding geometry to the MTs. A qualitatively similar ionic-strength effect has been reported in the context of cargo regulation of other kinesins. ${ }^{13,21,22}$ Binding of a cargo vesicle to kinesin- 1 or of a second MT to kinesin- 5 Eg5 activates these motors, but this activation also occurs spontaneously (i.e., without cargo) at low ionic strength in both cases. Tailhead interaction is mediating this regulation in both cases. It is thus tempting to speculate that for Cin8, cargo regulation is also the physiological switch mechanism. In the case of Cin8, a mechanism detecting the binding of a second MT might not just turn the motor on or off, but lead to the observed switching of directionality. Consistent with this hypothesis, we observed plusend directed antiparallel sliding of MTs by Cin8 when they entered the overlap zone between antiparallel MTs under highionic-strength conditions, while motors on single MTs in the same sample were still minus-end directed. ${ }^{19}$ Similarly, it has been previously demonstrated that, while the vertebrate $\mathrm{Eg} 5$ does neither bind to nor move on single MTs under high-ionicstrength conditions, binding between two antiparallel MTs activates MT sliding, driven by plus-end directed $\mathrm{Eg} 5$ motility. ${ }^{13}$ A similar activation effect might also occur in multi-motor MT surface-gliding assays, in which surface-attached Cin8 was also demonstrated to be plus-end directed. ${ }^{20}$ In MT sliding assays, Cin 8 obviously exerts force in the plus-end direction which is reflected in the relative sliding of the MTs, but individual motors between the coupled MTs move on rather erratic tracks such that clear plus-end-directed periods cannot be detected. ${ }^{19}$ Cin8 behaves very differently in single-molecule fluorescence experiments between parallel MTs. For the most part, motors continue minus-end motion, apparently not interacting with the second MT. ${ }^{19}$ The capability to distinguish relative orientation of bound MTs is consistent with the reported preference of Drosophila kinesin-5 Klp61f for bundling antiparallel MTs. ${ }^{13}$ For this kinesin-5, a preferred orientation was due to the ATPindependent binding sites in the C-terminal tails of the molecules. A similar binding mechanism appears to also exist for Cin8 because full-length Cin8 diffusively slides along MTs in ADP buffer. ${ }^{19}$ For Xenopus laevis kinesin-5 Eg5 it was found that all MT binding sites in the C-terminal tails were necessary for motor engagement between MTs. ${ }^{23}$ It still remains unclear exactly why and how low ionic strength mimics cargo binding. Taking into account the fact that MT attachment of the two pairs of motor domains triggers plus-end directed motility (Fig. 2A), a speculative possibility is that under low-ionic-strength conditions, Cin8 can flex in such a way that the two pairs of catalytic domains interact with the same MT (Fig. 2B) and thus trigger plus-end

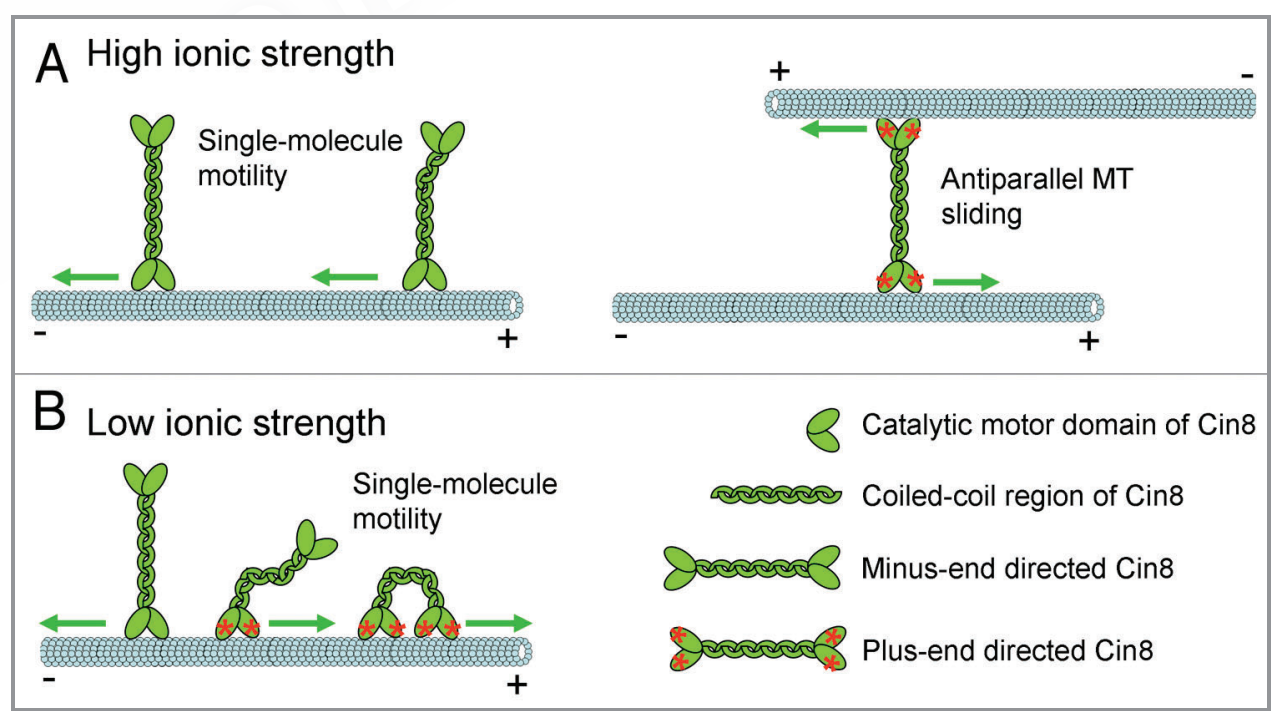

Figure 2. Proposed model for the directionality switch of Cin8. A microtubule is sketched in light blue, with plus and minus end indicated; Cin8 is shown in green; catalytic motor domain and coiled-coil regions are indicated in the legend. Arrows indicate the direction of movement of Cin8 on the MT to which it is attached. (A) On a single MT, in high-ionic-strength conditions, Cin8 is minus-end directed. Binding between two antiparallel MTs activates Cin8 to move in the plus-end directions of the MTs, causing their antiparallel sliding. (B) Under low-ionic-strength conditions on a single MT, a modified interaction between tails and catalytic domains or flexing of the whole tetramer triggers Cin8 to move in the plus-end direction of the MT. If the tetramer can flex enough, plus-end directed motility of single molecules of Cin8 could be triggered by binding of the two heads of Cin8 to the same MT. 
directed motility. Alternatively, low-ionic-strength conditions could modify tail-head interactions as in kinesin-1 to trigger plus-end directed motility.

One further piece of evidence in favor of a motor-intrinsic mechanism for directional switching is the regulatory influence of the 99 amino acid insert in loop 8 of the Cin 8 motor domain, deletion of which does not abolish the switch of directionality, but shifts the switch to lower ionic strength. ${ }^{19}$ The mechanism by which phosphorylation in the catalytic domain of Cin8 regulates its in vivo function is likely to include the modulation of interactions with the midzone-organizing protein Ase1, ${ }^{8,24}$, or with kinetochore proteins. The fact that a deletion construct (Cin8 499$)$ and a loop $8 \mathrm{Cdk} 1$ phosphorylation-deficient construct (Cin8-2A) exhibit reduced motility toward the midzone in vivo, ${ }^{19}$ suggests that one of the roles of Cin8 phosphorylation in the $99 \mathrm{aa}$ insert is to mediate the switch to plus-end directed motility of Cin 8 on the mitotic spindle.

The question remains how its exceptional motile properties aid Cin8 in performing its roles in mitosis. One can speculate on the basis of the localization of $\operatorname{Cin} 8$ in the various stages of mitosis. The ionic strength in $S$. cerevisiae cells is $-300 \mathrm{mM}$ salt. ${ }^{25,26}$ At this ionic strength, Cin8 is minus-end directed in vitro. ${ }^{19}$ Before spindle elongation in anaphase, Cin8 is known to be involved in the positioning of the chromosome kinetochores near the spindle pole bodies. ${ }^{27-29}$ Cin 8 could function at that stage by crosslinking of kinetochore MTs $(\mathrm{kMTs})^{27}$ and by aiding the disassembly of long kMTs. ${ }^{30}$ Since in $S$. cerevisiae cells each kinetochore is attached to the plus end of a single MT, motion of Cin8 in the minus-end direction of the kMTs might be a part of kinetochore positioning. Cin8 also shows plus-end directed motility in vivo in anaphase spindles, ${ }^{19}$ even on single MTs or on parallel MT bundles, which implies a further mode of regulation not seen in the in vitro experiments. Regulated bi-directional motility might be important to distribute Cin 8 motors between the different locations where they are known to accumulate, i.e., near the spindle poles and in the midzone.

In summary, Cin8 has rather unexpectedly extended the spectrum of known kinesin capabilities. It is the first known kinesin that is truly bi-directional and processive in both directions. Found in a low eukaryote, this function might have evolved early and might have been lost in higher eukaryotes. The exact molecular mechanism remains to be clarified, but seems likely to be related to cargo switching known for other kinesins. Our results indicate a role of electrostatic interactions and possibly phosphorylation, and, most importantly, binding geometry between pairs of MTs.

\section{References}

1. Kashina AS, Baskin RJ, Cole DG, Wedaman KP, Saxton WM, Scholey JM. A bipolar kinesin. Nature 1996; 379:270-2; PMID:8538794; http://dx.doi.org/ 10.1038/379270a0

2. Gordon DM, Roof DM. The kinesin-related protein Kip1p of Saccharomyces cerevisiae is bipolar. J Biol Chem 1999; 274:28779-86; PMID:10497250; http:// dx.doi.org/10.1074/jbc.274.40.28779

3. Saunders WS, Hoyt MA. Kinesin-related proteins required for structural integrity of the mitotic spindle. Cell 1992; 70:451-8; PMID:1643659; http://dx.doi. org/10.1016/0092-8674(92)90169-D

4. Blangy A, Lane HA, d'Hérin P, Harper M, Kress M, Nigg EA. Phosphorylation by $\mathrm{p} 34 \mathrm{cdc} 2$ regulates spindle association of human $\mathrm{Eg} 5$, a kinesin-related motor essential for bipolar spindle formation in vivo. Cell 1995; 83:1159-69; PMID:8548803; http://dx.doi.org/ 10.1016/0092-8674(95)90142-6

5. Mayer TU, Kapoor TM, Haggarty SJ, King RW, Schreiber SL, Mitchison TJ. Small molecule inhibitor of mitotic spindle bipolarity identified in a phenotypebased screen. Science 1999; 286:971-4; PMID: 10542155; http://dx.doi.org/10.1126/science.286.5441. 971

6. Saunders WS, Koshland D, Eshel D, Gibbons IR, Hoyt MA. Saccharomyces cerevisiae kinesin- and dyneinrelated proteins required for anaphase chromosome segregation. J Cell Biol 1995; 128:617-24; PMID: 7860634; http://dx.doi.org/10.1083/jcb.128.4.617

7. Sharp DJ, McDonald KL, Brown HM, Matthies HJ, Walczak C, Vale RD, et al. The bipolar kinesin, KLP61F, cross-links microtubules within interpolar microtubule bundles of Drosophila embryonic mitotic spindles. J Cell Biol 1999; 144:125-38; PMID: 9885249; http://dx.doi.org/10.1083/jcb.144.1.125

8. Avunie-Masala R, Movshovich N, Nissenkorn Y, Gerson-Gurwitz A, Fridman V, Kōivomägi $M$, et al. Phospho-regulation of kinesin-5 during anaphase spindle elongation. J Cell Sci 2011; 124:873-8; PMID: 21378308; http://dx.doi.org/10.1242/jcs.077396
9. Fridman V, Gerson-Gurwitz A, Movshovich N, Kupiec M, Gheber L. Midzone organization restricts interpolar microtubule plus-end dynamics during spindle elongation. EMBO Rep 2009; 10:387-93; PMID:19270715; http://dx.doi.org/10.1038/embor. 2009.7

10. Gerson-Gurwitz A, Movshovich N, Avunie R, Fridman V, Moyal K, Katz B, et al. Mid-anaphase arrest in S. cerevisiae cells eliminated for the function of $\mathrm{Cin} 8$ and dynein. Cell Mol Life Sci 2009; 66:301-13; PMID 19099194; http://dx.doi.org/10.1007/s00018-0088479-2

11. Movshovich N, Fridman V, Gerson-Gurwitz A Shumacher I, Gertsberg I, Fich A, et al. Slk19dependent mid-anaphase pause in kinesin-5-mutated cells. J Cell Sci 2008; 121:2529-39; PMID:18628309; http://dx.doi.org/10.1242/jcs.022996

12. Kapitein LC, Peterman EJ, Kwok BH, Kim JH, Kapoor TM, Schmidt CF. The bipolar mitotic kinesin Eg5 moves on both microtubules that it crosslinks. Nature 2005; 435:114-8; PMID:15875026; http://dx.doi.org/ 10.1038 /nature03503

13. Kapitein LC, Kwok BH, Weinger JS, Schmidt CF, Kapoor TM, Peterman EJG. Microtubule cross-linking triggers the directional motility of kinesin-5. J Cell Biol 2008; 182:421-8; PMID:18678707; http://dx.doi.org/ 10.1083/jcb.200801145

14. Dagenbach EM, Endow SA. A new kinesin tree. J Cell Sci 2004; 117:3-7; PMID:14657268; http://dx.doi. org/10.1242/jcs.00875

15. deCastro MJ, Fondecave RM, Clarke LA, Schmidt CF, Stewart RJ. Working strokes by single molecules of the kinesin-related microtubule motor ncd. Nat Cell Biol 2000; 2:724-9; PMID:11025663; http://dx.doi.org/10. $1038 / 35036357$

16. McDonald HB, Stewart RJ, Goldstein LS. The kinesinlike ncd protein of Drosophila is a minus end-directed microtubule motor. Cell 1990; 63:1159-65; PMID: 2261638; http://dx.doi.org/10.1016/0092-8674(90) 90412-8
17. Walker RA, Salmon ED, Endow SA. The Drosophila claret segregation protein is a minus-end directed motor molecule. Nature 1990; 347:780-2; PMID:2146510; http://dx.doi.org/10.1038/347780a0

18. Roostalu J, Hentrich C, Bieling P, Telley IA, Schiebel E, Surrey T. Directional switching of the kinesin Cin8 through motor coupling. Science 2011; 332:94-9; PMID:21350123; http://dx.doi.org/10.1126/science. 1199945

19. Gerson-Gurwitz A, Thiede C, Movshovich N, Fridman V, Podolskaya M, Danieli T, et al. Directionality of individual kinesin-5 Cin 8 motors is modulated by loop 8 , ionic strength and microtubule geometry. EMBO J 2011; 30:4942-54; PMID:22101328; http://dx.doi. org/10.1038/emboj.2011.403

20. Gheber L, Kuo SC, Hoyt MA. Motile properties of the kinesin-related Cin8p spindle motor extracted from Saccharomyces cerevisiae cells. J Biol Chem 1999; 274:9564-72; PMID:10092642; http://dx.doi.org/10. $1074 /$ jbc.274.14.9564

21. Seiler S, Kirchner J, Horn C, Kallipolitou A, Woehlke G, Schliwa M. Cargo binding and regulatory sites in the tail of fungal conventional kinesin. Nat Cell Biol 2000; 2:333-8; PMID:10854323; http://dx.doi.org/10.1038/ 35014022

22. Hackney DD, Baek N, Snyder AC. Half-site inhibition of dimeric kinesin head domains by monomeric tail domains. Biochemistry 2009; 48:3448-56; PMID: 19320433; http://dx.doi.org/10.1021/bi8022575

23. Weinger JS, Qiu M, Yang G, Kapoor TM. A nonmotor microtubule binding site in kinesin-5 is required for filament crosslinking and sliding. Curr Biol 2011; 21:154-60; PMID:21236672; http://dx.doi.org/10. 1016/j.cub.2010.12.038

24. Khmelinskii A, Roostalu J, Roque $\mathrm{H}$, Antony C, Schiebel E. Phosphorylation-dependent protein interactions at the spindle midzone mediate cell cycle regulation of spindle elongation. Dev Cell 2009; 17: 244-56; PMID:19686685; http://dx.doi.org/10.1016/ j.devcel.2009.06.011 
25. Olz R, Larsson K, Adler L, Gustafsson LO. Energy flux and osmoregulation of Saccharomyces cerevisiae grown in chemostats under $\mathrm{NaCl}$ stress. J Bacteriol 1993; 175:2205-13; PMID:8468281

26. van Eunen K, Bouwman J, Daran-Lapujade P, Postmus J, Canelas AB, Mensonides FI, et al. Measuring enzyme activities under standardized in vivo-like conditions for systems biology. FEBS J 2010; 277:749-60; PMID: 20067525; http://dx.doi.org/10.1111/j.1742-4658. 2009.07524.x

27. Tytell JD, Sorger PK. Analysis of kinesin motor function at budding yeast kinetochores. J Cell Biol 2006; 172:861-74; PMID:16533946; http://dx.doi. org/10.1083/jcb.200509101
28. Gardner MK, Bouck DC, Paliulis LV, Meehl JB, O'Toole ET, Haase J, et al. Chromosome congression by Kinesin-5 motor-mediated disassembly of longer kinetochore microtubules. Cell 2008; 135:894-906; PMID:19041752; http://dx.doi.org/10.1016/j.cell. 2008.09.046

29. Wargacki MM, Tay JC, Muller EG, Asbury CL, Davis TN. Kip3, the yeast kinesin-8, is required for clustering of kinetochores at metaphase. Cell Cycle 2010; 9:2581 8; PMID:20603597; http://dx.doi.org/10.4161/cc.9. 13.12076
30. Gardner MK, Haase J, Mythreye K, Molk JN, Anderson M, Joglekar AP, et al. The microtubulebased motor Kar3 and plus end-binding protein Bim1 provide structural support for the anaphase spindle. J Cell Biol 2008; 180:91-100; PMID:18180364; http:// dx.doi.org/10.1083/jcb.200710164

31. Lakämper S, Thiede C, Düselder A, Reiter S, Korneev MJ, Kapitein LC, et al. The effect of monastrol on the processive motility of a dimeric kinesin- 5 head/kinesin- 1 stalk chimera. J Mol Biol 2010; 399:1-8; PMID: 20227420; http://dx.doi.org/10.1016/j.jmb.2010.03.009 



\section{Neck-Linker Length Dependence of Processive Kinesin-5 Motility}

\section{André Düselder $\dagger$, Christina Thiede $\dagger$, Christoph F. Schmidt ${ }^{\star}$ and Stefan Lakämper*}

Drittes Physikalisches Institut, Georg-August-Universität Göttingen, Friedrich-Hund-Platz 1, 37077 Göttingen, Germany

Received 25 April 2012; received in revised form 14 June 2012; accepted 28 June 2012 Available online 9 July 2012

\section{Edited by R. Craig}

\section{Keywords:}

kinesin;

processivity; single-molecule motility; neck linker; gating
Processive motility of individual molecules is essential for the function of many kinesin motors. Processivity for kinesins relies on communication between the two heads of a dimeric molecule, such that binding strictly alternates. The main communicating elements are believed to be the two neck linkers connecting the motors' stalks and heads. A proposed mechanism for coordination is the transmission of stress through the neck linkers. It is believed that the efficiency of gating depends on the length of the neck linker. Recent studies have presented support for a simple model in which the length of the neck linker directly controls the degree of processivity. Based on a previously published Kinesin- 1 /Kinesin- 5 chimera, Eg5Kin, we have analyzed the motility of 12 motor constructs: we have varied the length of the neck linker in the range between 9 and 21 amino acids using the corresponding native Kinesin-5 sequence (Xenopus laevis Eg5). We found, surprisingly, that neither velocity nor force generation depended on neck-linker length. We also found that constructs with short neck linkers, down to 12 amino acids, were still highly processive, while processivity was lost at a length of 9 amino acids. Run lengths were maximal with neck linkers close to the native Kinesin-5 length and decreased beyond that length. This finding generally confirms the coordinating role of the neck linker for kinesin motility but challenges the simplest model postulating a motor-type-independent optimal length. Instead, our results suggest that different kinesins might be optimized for different neck-linker lengths.

(C) 2012 Elsevier Ltd. All rights reserved.
${ }^{*}$ Corresponding authors. E-mail addresses:

cfs@physik3.gwdg.de; lakaemper@imes.mavt.ethz.ch.

$\dagger$ A.D. and C.T. contributed equally to this work.

Present address: S. Lakämper, Institute for Mechanical Systems, Department of Mechanical and Process Engineering, Eidgenössische Technische Hochschule Zürich, Rämistrasse 101, 8092 Zurich, Switzerland.

Abbreviations used: WT, wild-type; MMGA, multi-motor surface-gliding assay; EGTA, ethylene glycol bis( $\beta$-aminoethyl ether) $N, N^{\prime}$-tetraacetic acid; TIRF, total internal reflection fluorescence.

\section{Introduction}

Kinesins are motor proteins that use energy from ATP hydrolysis to generate conformational changes. Kinesins drive mechanical processes in cells such as cargo-transport along or disassembly of microtubules. 1 Single homodimeric motor molecules of the Kinesin-1 family can move processively over micrometer distances along a microtubule, ${ }^{3,4}$ taking hundreds of $\sim 8$-nm steps, ${ }^{5}$ and can produce up to $\sim 7 \mathrm{pN}$ force before detachment. $^{6-8}$ During processive motility, each step is strictly coupled to the hydrolysis of one ATP molecule. $^{8}$ 
Kinesins from several other subfamilies, such as the Kinesin-3 Kif1A/Unc104, ${ }^{9,10}$ the Kinesin-7 $\mathrm{CENP}_{\mathrm{E}}{ }^{11}$ the Kinesin- $8 \mathrm{Kip} 3 \mathrm{p},{ }^{12}$ or the mitotic Kinesin-5 Eg5, ${ }^{13}$ have been shown to also move processively along microtubules. While structurally adapted to diverse cellular functions, the processive kinesins are likely to employ a similar basic headhead communication mechanism to achieve processive motility. ${ }^{14-16}$ A requirement for processivity of a single kinesin dimer is to keep the kinetic cycles of the two motor domains strictly out of phase, such that at least one motor domain remains strongly attached to the microtubule at any time. Studies on monomeric and dimeric kinesin constructs have led to a basic model of the allosteric mechanisms connecting microtubule binding and the nucleotide state of the motor domain. ${ }^{19,18}$ The head-head communication is believed to rely on tensionmediated gating of chemical reactions, possibly involving both leading and trailing heads. ${ }^{19}$ The mechanically tensed state in the stepping cycle of a dimer is the two-head-bound state. Cryo-electron microscopy images of bound dimers can only be reconciled with dimer X-ray structures if one assumes a stretched conformation of the structural elements linking the two heads. ${ }^{20}$ The front-head gating model postulates that the leading, nucleotidefree head is unable to bind a new ATP while under backward tension, that is, before the detachment of the rear head. The rear-head gating model assumes that release of phosphate from the rear head, leading to detachment, is accelerated by forward tension. ${ }^{21}$ The tension-transmitting element has been suggested to be the neck linker, a short and mostly unstructured protein segment connecting the head to the stalk coiled coil. ${ }^{22,23}$ When modeled as a simple flexible polymer, the tension in the neck linker at a given end-to-end distance and, therefore, the strength of the head-head communication would scale inversely with contour length, that is, number of amino acids. ${ }^{24}$ Data from three recent studies support such a simple general model of head-head communication. ${ }^{19,25,26}$ For a set of particular Kinesin-1 and Kinesin-2 constructs, it was found that processivity decreases with increasing neck-linker length, while shortening of the native neck linker by one amino acid for that motor abolishes processivity. ${ }^{24,27-29}$ A continuation of this work showed similar effects for Kinesin-1/ Kinesin-2/Kinesin-3 and Kinesin-7 constructs. ${ }^{25}$ In another study, ${ }^{26}$ the insertion of up to 26 prolines in the neck linker of human Kinesin-1 was reported to not only slightly decrease run length under lowionic-strength conditions but also strongly decrease motor speed and stall force. This phenomenon was interpreted to be due to an increasingly loose coupling of ATP hydrolysis to motor stepping. A detailed study of the kinetic pathway of two processive Kinesin-1 constructs with neck linkers extended to 20 and 23 amino acids suggested a different interpretation of new and existing data, based on a model where not only tension alone but also, possibly, neck-linker orientation produces gating. 19

Interestingly, neck-linker length varies between the different kinesin subfamilies. The highly processive Kinesin-1 motors ${ }^{3,4,30}$ carry a rather short neck linker of 14 amino acids. Most other kinesin subfamilies carry significantly longer neck linkers (see Ref. 25 in Fig. 2 for a sequence alignment) of 1618 amino acids in length. The Xenopus Kinesin-5, Eg5, has one of the longest neck linker with 18 amino acids. ${ }^{27}$ Nevertheless, wild-type (WT) Kinesin-5 has been shown to move processively. ${ }^{13}$ Dimeric constructs, however, lacking the tetrameric coiled-coil region produced only very short (six to eight steps) processive episodes. ${ }^{31,32}$ This could be due to the neck linker that is 18 amino acids long or to the lack of the opposing dimer and a compromised stalk structure. Full-length tetramers were observed to move over longer distances but detached at relatively small loads and were still less processive than Kinesin- $1 .{ }^{13}$ This behavior, however, appears to be caused by yet another phenomenon, namely, tail-mediated self-inhibition, which leads to a switching off of the motor when bound to only one microtubule. ${ }^{33}$ Thus, studying intrinsic processivity of Kinesin- 5 has been difficult, both in truncated dimeric constructs and in WT tetramers.

To study the force-generating unit of Kinesin- 5 without interference of tail regulation and without the structurally complex tetrameric coiled coil of Kinesin-5, we have recently constructed a chimeric motor, Eg5Kin, which comprises the motor domain and the 14 N-terminal amino acids of the WT Kinesin-5 neck linker, fused to the coiled coil of Kinesin-1. ${ }^{34}$ The initial rationale for using 14 amino acids was to provide a similar mechanical background as in Kinesin-1. We had found that Eg5Kin (here referred to as Eg5Kin-NL-14 or, short, NL-14) is capable of long-range processive motility taking on average 240 consecutive $\sim 8-\mathrm{nm}$ steps and of comparatively high force generation. These findings demonstrated that the Kinesin-5 motor domain is capable of supporting processive motility, provided that it is a stable dimer.

The fact that the neck linker of native Kinesin-5 has a length of 18 amino acids begs the question if the processivity of the motor dimer increases when the length of the neck linker is shortened from 18 to 14 amino acids. If a shorter-than-native neck linker were to increase processivity, one would have to conclude that the length of the neck linker in Kinesin-5 is optimized for something else than run length and the most efficient head-head communication. A neck linker of the native length of 18 amino acids is known to support processive motility, as shown by the motion of WT Kinesin- 5 


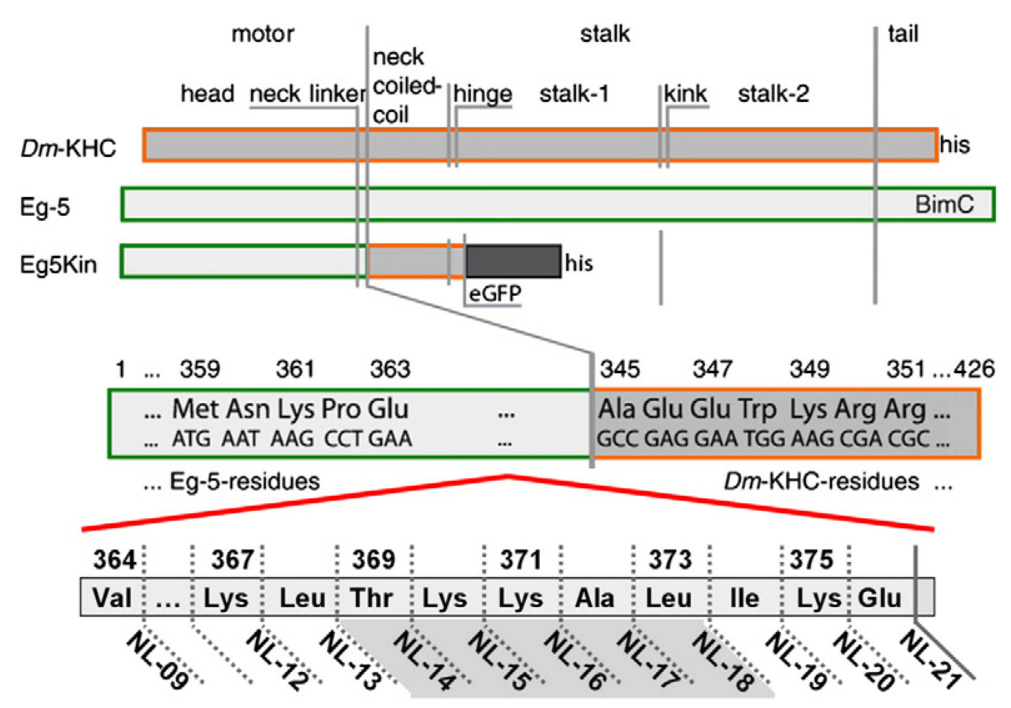

Fig. 1. Schematic description of motor constructs. Eg5Kin motor proteins were constructed using the motor domain and part of the neck-linker sequence of Eg5 (light gray; residues 1-364 up to 1-376 for NL-09 to NL-21, respectively) and the (truncated) stalk of DmKHC (dark gray; DmKHC residues 345426). The numbering refers to the amino acid numbering in the respective WT motor sequences.

tetramers between antiparallel microtubules, but the degree of processivity has not been quantified for the WT motor in that scenario. Here, we have generated a set of Eg5Kin chimeras with necklinker lengths ranging from 9 (NL-09) to the full 18 amino acids of WT Kinesin-5 (NL-18) and beyond to 21 amino acids (NL-21) (Fig. 1 and Supplementary Fig. 1). In contrast to other studies, where the length of the neck linker was varied using random or artificial sequences, such as poly-proline inserts and sequences from other distantly related kinesins, 19,23,26,29 we decided to exclusively use the native Eg5 Kinesin-5 sequence. We shortened the neck linker by successively deleting amino acids, starting with the 18th amino acid in the neck-linker sequence. For the elongation of the neck linker beyond 18 amino acids, we inserted the WT Kinesin-5 sequences using up to three residues from the putative Kinesin-5 neck coiled coil. A coiled-coil prediction for all constructs indicates that these inserted amino acids do not lead to an extension of the DmKHC neck coiled coil toward the $\mathrm{N}$-terminus but that the coiled coil starts for all constructs at the beginning of the DmKHC residues. This prediction provided confidence to interpret our findings assuming steadily increasing neck-linker lengths. To have, as a further control, a motor for which the additional neck-linker amino acids would surely not become incorporated in an extension of the stalk coiled coil, we built one other construct in which we inserted three prolines after the 18th amino acid. This insertion is too short to form a poly-proline structure and is likely to be flexible. ${ }^{35}$ With this control, we could also test if new specific interactions between the motor domain and the neck linker, mediated by the nonnative additional proline residues, strongly changed the function of the motor.

\section{Results and Discussion}

In a first set of experiments, we used multi-motor surface-gliding assays (MMGAs) to check the activity of the purified motors and to measure the velocities produced by motors acting collectively (Fig. 2a). We found that all constructs were active motor proteins and produced virtually identical surface-gliding velocities of, on average, $\sim 67 \mathrm{~nm} / \mathrm{s}$ (Fig. 3a and Table 1), the only exception being the shortest construct, NL-09, which showed a clearly diminished velocity $(16 \pm 6 \mathrm{~nm} / \mathrm{s})$. These results differ from the results of Yildiz et al. for human Kinesin- $1,{ }^{26}$ who saw a strong decrease in velocity with longer neck linkers. This discrepancy may be due to the fact that Kinesin-1 motors have intrinsically a much higher velocity and therefore a different catalytic cycle. Thus, the loss of coupling efficiency found for Kinesin-1 does not appear to occur for Kinesin-5. The magnitude and constancy of velocity are in agreement with the results of Shastry and Hancock with a very similar Kinesin-5 construct. ${ }^{25}$

We then tested the constructs for velocity in optical trapping assays (Fig. 2b) and found, for all the constructs, a similar velocity with an average value of $\sim 64 \mathrm{~nm} / \mathrm{s}$ in good agreement with the MMGA results (Fig. $3 a$ and Table 1). In singlemolecule fluorescence assays (Fig. 2c), we found again very similar velocities for all constructs (Fig. $3 \mathrm{a}$ and Table 1), although the average velocity of $\sim 116 \mathrm{~nm} / \mathrm{s}$ was higher than the results of MMGAs and optical trapping assays. Although not often explicitly studied, a difference in this direction is not unexpected. Optical trapping assays are not load free, load diminishes velocity, ${ }^{5}$ and we did not attempt here to extrapolate the results to zero load. MMGAs can always suffer from motor attachment artifacts, contamination by defective 
(a)

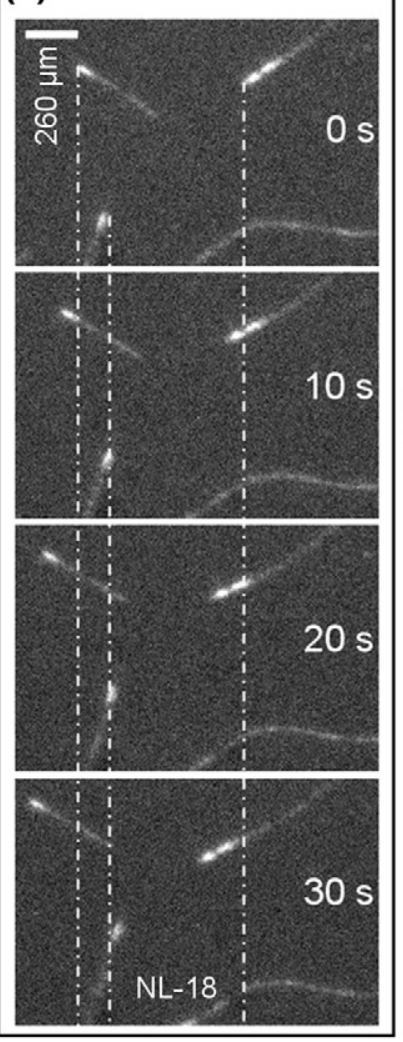

(b)
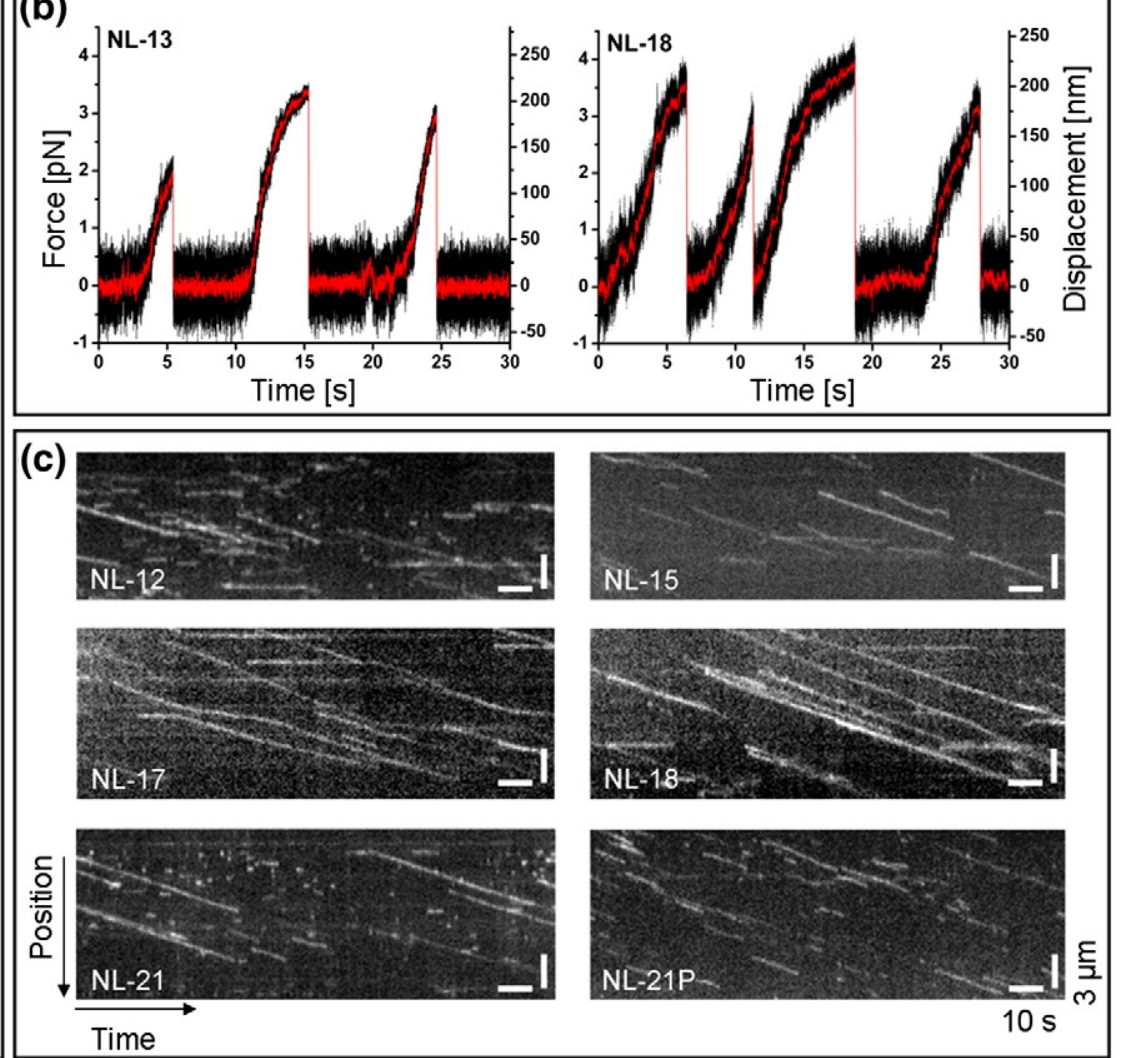

Fig. 2. Representative results from single-molecule fluorescence, multi-motor gliding, and optical trapping assays. (a) Time series of a multi-motor gliding assay. The scale bar represents $260 \mu \mathrm{m}$. (b) Bead-displacement time series from optical trapping assays, showing multiple excursions from the trap centre, ending with detachment without stalling. Initial slope of the excursions is used as an estimate of the unloaded motor velocity (raw signal in black; median-filtered signal in red). (c) Kymographs of single GFP-labelled motor dimers moving on surface-attached microtubules. The number on the lower-left corner indicates the neck-linker length of a given construct. Slope and length of the traces correspond to motor velocity and run length, respectively. Scale bars represent (horizontal) $10 \mathrm{~s}$ and (vertical) $3 \mu \mathrm{m}$.

motors, or strained geometries of motor attachment to the microtubule. Therefore, we do not draw any conclusions from this difference for the present study.

Although NL-09 is still active in MMGAs, it did not show any processive motion, neither in the optical trapping nor in the single-molecule fluorescence assays (Supplementary Fig. 2). We thus know that NL-09 is an active, albeit non-processive, motor. The lack of processivity most likely results from the neck linker being too short to span the gap between two adjacent binding sites along the microtubule.

These findings are somewhat different from what has been reported for other kinesins and also for a very similar Kinesin-5 construct. In one recent study, Shastry and Hancock investigated the effect of varying neck-linker length on Kinesin-1 and an artificially homodimeric Kinesin-2 construct (Kif3A/A, termed Kin-2). ${ }^{29}$ For Kinesin-1, the deletion of one amino acid (Kin- $1 \Delta \mathrm{T}$ ) led to a non-processive motor, displaying about $70 \%$ of
Kin-1 velocity in surface-gliding assays, whereas the extension of the neck linker by one or several amino acids (Kin-1 + L or Kin-1 + DAL) resulted in a $50 \%$ loss of velocity in single-molecule assays. In contrast, the velocities of their Kin-2 constructs were - similar to our results-not affected by modifications of the neck linker. The only exception was the Kin-2 $\Delta \mathrm{DAL}$, which is not processive and reaches again only about $70 \%$ of the velocity of other Kin-2 constructs in MMGA. In a further study, $^{25}$ the same group confirmed earlier results, extended the results to Kinesin-3 and Kinesin-7 motors, and also investigated chimeric Kinesin-5 construct very similar to ours with neck linkers of $14,15,17$, and 18 amino acids. In contrast to our results, they found diminished processivity for 15 amino acids and no more processivity for 17 and 18 amino acids. Clancy et al. found a decrease in unloaded velocity by about a factor of 2 for a Kinesin-1 construct with a neck linker that is 20 amino acids long, providing evidence that this is 
(a)

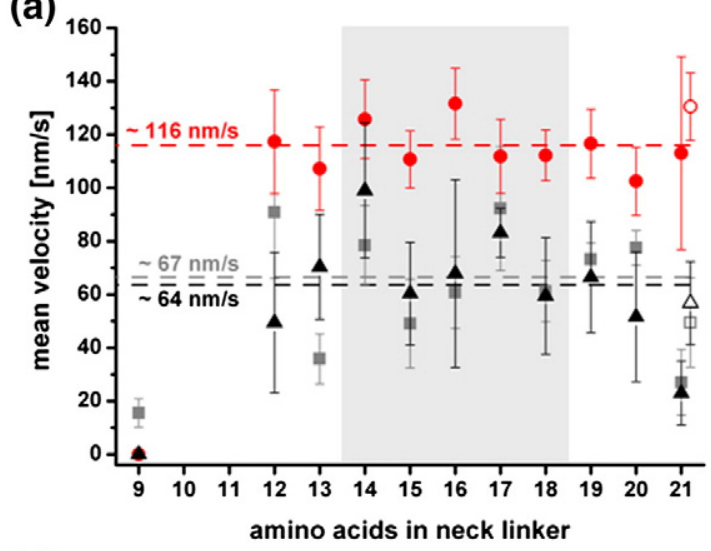

(c)

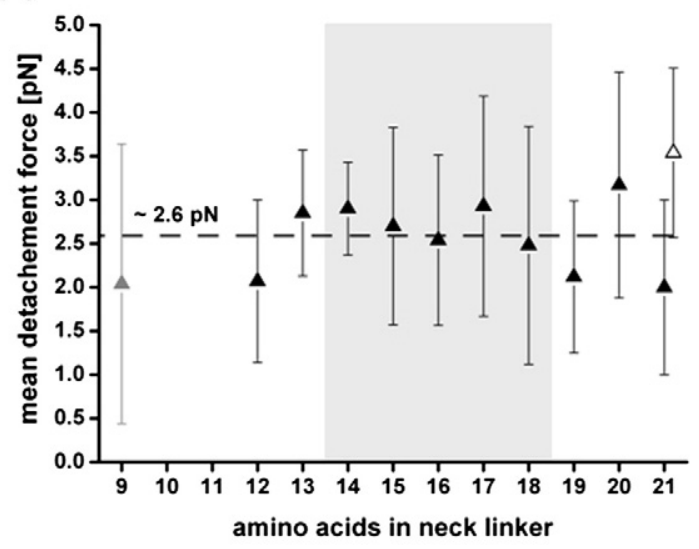

(b)

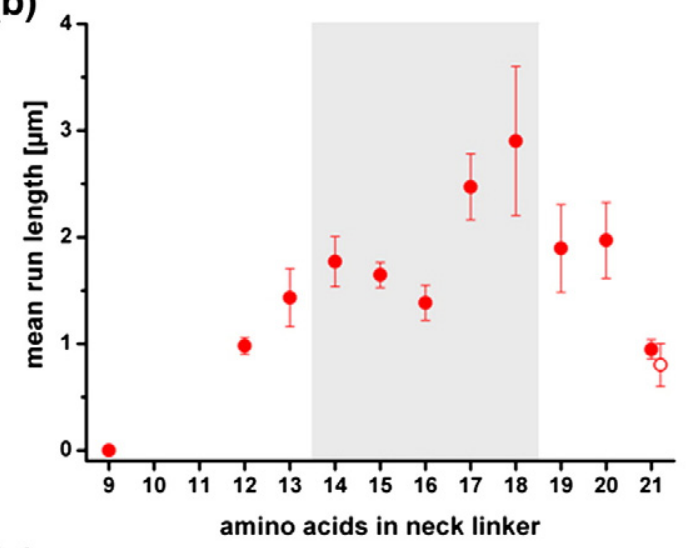

(d)

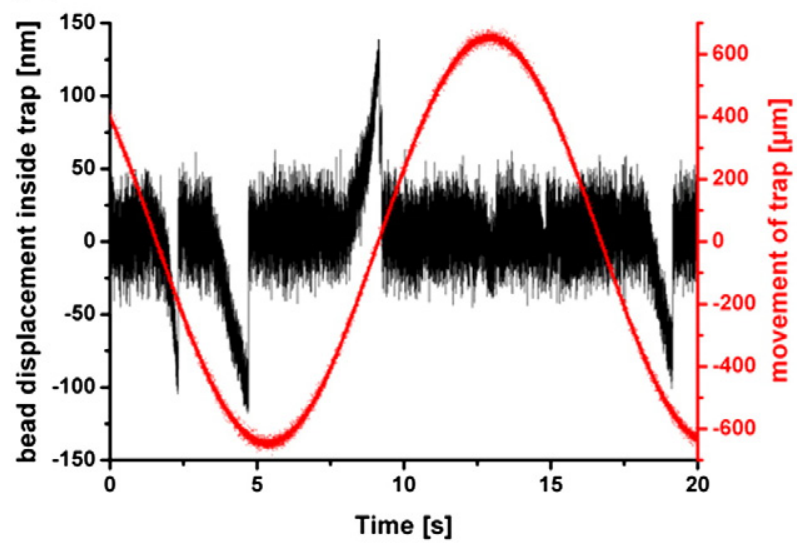

Fig. 3. Velocities, run lengths, and detachment forces (also see Table 1). (a) Mean velocities for all constructs. Triangles: optical trapping assays. Circles: single-molecule fluorescence assays. Squares: multi-motor gliding assays. Broken lines represent the average of values for processive motors and are given to guide the eye. (b) Mean processive run lengths for all constructs in single-molecule fluorescence assays. For NL-09, no processive motility was detected. (c) Detachment forces for all constructs. Since the constructs detached mostly without stalling, the mean force immediately before release is scored. (d) The force given for the non-processive NL-09 construct is the detachment force obtained by dragging a motor-coated bead in a sinusoidal movement (frequency $=0.06 \mathrm{~Hz}$; amplitude $=660 \mathrm{~nm}$ ) along a microtubule. The range between the natural neck-linker lengths of Kinesin-1 and Kinesin-5 (14-18 amino acids) is indicated using gray shading. Open symbols refer to NL-21P.

due to a decreased motor efficiency leading to backstepping. ${ }^{19}$ This is an effect that might occur only for extremely long neck-linker lengths, and its possible onset might be masked in our data for the longest constructs NL-20 and NL-21 by statistical errors.

In another study, Yildiz et al. observed a decrease in the single-molecule velocity that was inversely related to the increase in the calculated total length of the neck linker, extended by a polyproline insert. ${ }^{26}$ The ATP turnover rate $k_{\text {cat,ATP }}$ was unchanged by the neck-linker extension, and the authors therefore concluded that the coupling between stepping and ATP hydrolysis must have become less tight, likely due to an increase in flexibility of the mechanical linkage between the two heads. Velocities of extended kinesins could in part be "rescued" by applying assisting loads. The fact that, in our assays, there was no detectable change or trend in the observed velocities of our constructs suggests that necklinker length does not influence the rate at which the Kinesin-5 motor domains hydrolyze ATP or the coupling efficiency during their interaction with the microtubule.

Next, we analyzed the maximal forces our motor constructs could develop. In single-bead optical trapping experiments, all our neck-linker constructs displayed stereotypical Kinesin-5 detachment behavior: in contrast to Kinesin-1 motors, detachment occurred at moderate forces of about $3 \mathrm{pN}$, before stall and without much slowing down (Fig. $2 \mathrm{~b}$ and Supplementary Fig. 3). ${ }^{31,36,37}$ The individual step size of the different constructs was largely 
Table 1. Results for the three types of measurements for all neck-linker constructs

\begin{tabular}{|c|c|c|c|c|c|c|c|c|c|}
\hline \multirow[b]{2}{*}{ Construct } & \multicolumn{3}{|c|}{ Trap } & \multicolumn{4}{|c|}{ TIRF } & \multicolumn{2}{|l|}{ MMGA } \\
\hline & Velocity $(\mathrm{nm} / \mathrm{s})$ & Force $(\mathrm{pN})$ & $N$ & Velocity (nm/s) & Run length $(\mu \mathrm{m})$ & $N$ & $\mathrm{C}_{\text {TIRF }}(\mathrm{nM})$ & Velocity (nm/s) & $N$ \\
\hline NL-09 & $0 \pm 0^{\mathrm{a}}$ & $2.0 \pm 1.6$ & 33 & $0 \pm 0^{\mathrm{a}}$ & $0 \pm 0^{\mathrm{a}}$ & 80 & 178 & $16 \pm 6$ & 41 \\
\hline NL-12 & $70 \pm 20$ & $2.1 \pm 0.9$ & 109 & $117 \pm 20$ & $0.9 \pm 0.1$ & 104 & 157 & $91 \pm 25$ & 37 \\
\hline NL-13 & $99 \pm 25$ & $2.9 \pm 0.7$ & 100 & $107 \pm 16$ & $1.4 \pm 0.3$ & 102 & 105 & $36 \pm 9$ & 29 \\
\hline NL-14 & $60 \pm 19$ & $2.9 \pm 0.5$ & 50 & $125 \pm 15$ & $1.8 \pm 0.2$ & 124 & 131 & $78 \pm 15$ & 28 \\
\hline NL-15 & $68 \pm 35$ & $2.7 \pm 1.1$ & 98 & $111 \pm 11$ & $1.7 \pm 0.1$ & 130 & 52 & $49 \pm 17$ & 20 \\
\hline NL-16 & $83 \pm 9$ & $2.5 \pm 0.9$ & 35 & $132 \pm 13$ & $1.4 \pm 0.2$ & 269 & 189 & $61 \pm 14$ & 17 \\
\hline NL-17 & $60 \pm 22$ & $2.9 \pm 1.3$ & 63 & $112 \pm 14$ & $2.5 \pm 0.3$ & 162 & 53 & $92 \pm 23$ & 23 \\
\hline NL-18 & $59 \pm 22$ & $2.5 \pm 1.4$ & 68 & $112 \pm 10$ & $2.9 \pm 0.7$ & 145 & 316 & $61 \pm 11$ & 20 \\
\hline NL-19 & $66 \pm 21$ & $2.1 \pm 0.9$ & 321 & $117 \pm 13$ & $1.8 \pm 0.4$ & 106 & 158 & $73 \pm 6$ & 34 \\
\hline NL-20 & $52 \pm 24$ & $3.2 \pm 1.3$ & 109 & $103 \pm 13$ & $1.9 \pm 0.3$ & 98 & 132 & $77 \pm 7$ & 30 \\
\hline NL-21 & $23 \pm 12$ & $2.0 \pm 1.0$ & 67 & $113 \pm 36$ & $0.8 \pm 0.2$ & 106 & 317 & $27 \pm 13$ & 32 \\
\hline NL-21P & $57 \pm 16$ & $3.5 \pm 1.0$ & 134 & $131 \pm 13$ & $0.9 \pm 0.1$ & 128 & 95 & $49 \pm 18$ & 39 \\
\hline
\end{tabular}

Trap, data from optical trapping assays; TIRF, data from single-molecule fluorescence assay; $\mathrm{C}_{\mathrm{TIRF}}$, protein concentration in singlemolecule fluorescence measurement; $N$, number of beads tracked, single motors tracked, and microtubules tracked, respectively.

a No processive motility detected.

unaffected by the variation of neck-linker length (Supplementary Fig. 3). All processive neck-linker constructs reached about the same detachment force in the trap of $2.8 \pm 0.1 \mathrm{pN}$, averaged separately for each construct (Fig. 3c and Table 1). To obtain a detachment force for the non-processive NL-09, we made use of the fact that the motor was still able to bind to a microtubule: motor-coated, optically trapped beads were dragged along a microtubule by moving the microscope stage in a sinusoidal fashion (Fig. 3d). Upon binding of the motor, the bead stayed at one position relative to the microtubule and was moved out of the trap by the stage movement. This led to an increase of load on the motor, which eventually resulted in motor unbinding. ${ }^{38}$ Yildiz et al. reported, for their extended Kinesin-1 constructs, a significantly lowered stall force as compared to WT Kinesin- $1 .^{26}$ Stalling at zero velocity for Kinesin-1 motors and load-dependent detachment for Kinesin-5 motors at finite velocity may reflect somewhat different molecular mechanisms, and effects of neck-linker extension on these processes may not be strictly comparable. Nevertheless, our observations suggest that, for our Kinesin-5 constructs, the neck-linker length-and thereby presumably head-head tension-is not controlling force generation and detachment behavior. In contrast to our Kinesin-5 results, keeping in mind the caveat mentioned, Clancy et al. measured stall forces with their extended Kinesin-1 constructs lower than those with WT controls but could show that this was due to increasing backward stepping. ${ }^{19}$ We see no clear evidence of back-stepping with our constructs. This might indicate that the loss of unidirectional motility under hindering loads only occurs at neck linker lengths above 20 or more amino acids, possibly depending on the particular kinesin studied.
Last, we analyzed the run lengths of the constructs as a direct measure of processivity. Using singlemolecule fluorescence assays, we observed unidirectional and uninterrupted processive motility with all constructs except NL-09 (Fig. 2c and Supplementary Fig. 2). Kymograph analysis of the run lengths of all constructs showed that, for the constructs NL12 up to NL-21, the processive run length decreased continuously toward both short and long necklinker lengths and exhibited a maximum of about $3 \mu \mathrm{m}$ around NL-17 and NL-18 (Fig. $3 \mathrm{~b}$ and Table 1). There was no significant difference in run lengths between the constructs NL-21 and NL-21P.

To control against multimerization of the motors, which could strongly affect run lengths, we performed single-molecule fluorescence assays with high enough laser power to cause rapid bleaching. We could confirm two-step bleaching in the intensity signals confirming the dimeric structure of the constructs (Supplementary Fig. 4). To avoid any possible variations in conditions between the measurements of different constructs, we kept temperature, buffers, microtubule preparations, and illumination conditions as similar as possible between experiments.

The first surprising finding was that NL-12 and NL-13 displayed processive behavior, virtually indistinguishable from that of NL-14 (Fig. 3b). In the study of Shastry and Hancock, two sets of constructs showed no processivity when having neck linkers with less than 14 amino acids, presumably because the tethered head was not able to reach the next binding site. ${ }^{29}$ This was thought to be a general mechanism consistent with the fact that 14 amino acids is the length of the shortest neck linker in any known processive kinesin. Our results show that even shorter neck linkers can support processivity. This finding is consistent with and goes beyond the recently reported processivity of 
Kinesin-2/Kinesin-3 and Kinesin-7 constructs that showed processivity with neck linkers of 13 amino acids. ${ }^{25}$ A construct with the further shortened neck linker, NL-09, was finally lacking processivity (Supplementary Fig. 2). The second unexpected finding was that upon addition of one or two natural amino acids in constructs NL-15 and NL-16, there was no drop in processivity compared to NL14 (Fig. 3b), as one might have expected based on the findings for Kinesin-1/Kinesin-2/Kinesin-3/Kinesin-5 and Kinesin-7 constructs by Shastry and Hancock. ${ }^{25,29}$ Our observation suggests that necklinker length alone might not be sufficient to predict processivity. Our finding that the neck-linker constructs approaching the native Kinesin-5 necklinker length, NL-17 and NL-18, displayed a significantly higher processivity than shorter or longer constructs (Fig. 3b) is further evidence for the same point.

Last, we also found processive motility when extending the neck linker beyond the natural 18 amino acids of Eg5. Run length, however, decreased from NL-19 to NL-21 (and NL-21P) below the maximal run length of NL-18 (Fig. 3b), as one expects to eventually occur due to diminishing communication between the two heads. An increase in run length of constructs carrying neck linkers longer than 14 amino acids was also seen by Clancy et al. ${ }^{19}$

Given the new experimental evidence that necklinker extension can increase processivity, it remains to discuss the consequences for the gating models for Eg5 in particular or possibly for all kinesins. First of all, one has to keep in mind that, independent of inter-head communication, interactions between the heads and the microtubule can also affect processivity. Electrostatic interactions, especially under low-ionic-strength conditions, can keep the heads loosely associated with the microtubule, even in the absence of efficient communication. In particular, addition of positive charge in the neck linker was suggested to compensate for a loss of processivity through interactions much similar to the ones discussed for the positively charged neck with the negatively charged E-hook of tubulin. ${ }^{39-41}$ The native Kinesin- 5 neck-linker sequence contains two positively charged lysine residues at positions 15 and 16 of the neck linker. These lysines might compensate for a loss of processivity otherwise observed upon extension of the neck linker. Three points, apart from the relatively high-ionic-strength conditions, argue against this notion. First, the addition of a second lysine should have an additive effect, which we did not observe. Second, only NL17 and NL-18 showed a marked increase in run length, and it seems unlikely that the charge of the lysine residues needs these further extensions to take effect. Third, loosely bound periods during processive runs should also make the average velocity during events strongly load dependent, which we did not observe. Therefore, we think that neck-linker/microtubule interactions are not causing the variations in run lengths in our experiments.

Two physically different gating scenarios seem possible. Either (i) neck-linker orientation is the deciding parameter, and inter-head tension is not important for the processivity, or (ii) each kinesin family has a particularly adapted neck-linker length and/or specific interactions between the native neck linker and the rest of the motor that together optimize the transmission of tension and the gating and stepping mechanism. Gating scenario (ii) seemed, on the one hand, to be supported by the finding that replacement and addition of single amino acids in several kinesins alter processivity. ${ }^{25,29}$ We did not see such strong effects. Furthermore, a comparison between motors with different extension sequences but equal length, NL-21 and NL-21P, indicates that it is unlikely that the particular sequence of the extension played a dominating role in determining processive run lengths.

Gating scenario (i), on the other hand, is supported by Clancy et al., who found an increase of processivity with strongly extended neck linkers for Kinesin-1 constructs. ${ }^{19}$ In that case, the result was interpreted to be caused by a two-head-bound waiting state with a smaller probability of total release. Since this mechanism also entails lower speed, it is likely that this was not exactly the case for our intermediate-length Kinesin-5 constructs, though. In essence, however, head-head communication via neck-linker orientation and not tension is consistent with our results. Interestingly, two studies reporting a structurally unusual neck-linker orientation $^{42}$ and two-state neck-linker orientation kinetics ${ }^{43}$ further argue for this possibility, particularly for Kinesin-5.

In summary, a simple mechanistic model generally applying to all processive kinesins-equating a shorter neck-linker length directly to more tension and to more efficient gating and thereby to more processivity with one universal optimal number for the neck-linker length-might not exist. In view of the broad variations in function, regulation, and oligomerization properties of the kinesins, it would be indeed surprising if a single parameter, namely, neck-linker length, could determine performance. Coordination between the heads or gating, however, is a necessity for processive kinesins. Some of the existing data, including ours, now point to the interesting possibility that the orientation of the neck linker plays a crucial role, rather than merely length and tension. This opens up the option that different kinesins could achieve optimal head-head communication with different neck-linker lengths. The native neck-linker length and sequence might be optimized, in each case, for both effective communication and processivity, as well as other specific functions such as regulation. 


\section{Materials and Methods}

\section{Cloning}

All constructs were based on the previously published pEG5Kin-GFPhis construct. ${ }^{34}$ Deletions or insertions of codons were performed in a two-step PCR approach. Using a complementary pair of modified primers in the neck-linker region, we created two PCR products: one of the motor domain up to the desired mutation and a second starting with the neck-linker mutation ending with the Cterminal GFP (green fluorescent protein). Both fragments were combined to serve as template for a second round of PCR using the $3^{\prime}$ - and $5^{\prime}$-primers flanking the N-terminus and the C-terminus of the full construct. The resulting PCR products were then incorporated into pTOPO using a TOPO Kit (Invitrogen, Carlsbad, CA, USA) for sequencing and amplification before replacing the coding sequence in pEG5Kin-GFPhis by sub-cloning using the existing restriction sites for $\mathrm{NdeI}$ and $\mathrm{XmaI}$ as described previously. ${ }^{34}$ Constructs NL-09, NL-12, NL-19, NL-20, NL-21, and NL-21P were generated by gene synthesis (GeneArt Life Technologies, Regensburg, Germany). Integrity was confirmed by sequencing.

\section{Protein purification}

Motors were expressed in Escherichia coli and purified essentially as described previously. ${ }^{34}$ In brief, E. coli BL21(DE3) (Invitrogen) were transformed and grown to a density of $\sim 0.6$ at $37^{\circ} \mathrm{C}$ before induction of expression using $1 \mathrm{mM}$ IPTG for $3 \mathrm{~h}$. Cells were harvested by centrifugation and re-suspended in a $20 \mathrm{mM}$ imidazole buffer, pH 7.4, supplemented with $1 \mathrm{mM}$ each of DTT, $\mathrm{MgCl}_{2}$, ethylene glycol bis( $\beta$-aminoethyl ether) $N, N^{\prime}-$ tetraacetic acid (EGTA), and $\beta$-mercaptoethanol and $150 \mathrm{mM} \mathrm{NaCl}$ before lysis using ultrasound in the presence of lysozyme and DNase I. After separation from cell debris by centrifugation, the cytosol was incubated for $1 \mathrm{~h}$ at $4{ }^{\circ} \mathrm{C}$ with Ni-NTA column material (Qiagen, Hilden, Germany) and then transferred to a syringe column. After washing the column with $80 \mathrm{mM}$ imidazole and $300 \mathrm{mM} \mathrm{NaCl}$, bound motor was eluted using a 300-mM imidazole buffer containing $10 \mu \mathrm{m}$ ATP and $1 \mathrm{mM}$ DTT. Fractions containing motor were pooled and $3 \times$ dialyzed against $80 \mathrm{mM}$ Pipes/KOH, $1 \mathrm{mM}$ $\mathrm{MgCl}_{2}, 1 \mathrm{mM}$ EGTA, $1 \mathrm{mM}$ DTT, and $10 \mu \mathrm{M}$ ATP. In addition, motors were also purified using a microtubuleaffinity purification procedure described in Ref. 41. Protein samples were tested for motor activity and stored in aliquots at $-80^{\circ} \mathrm{C}$. Tubulin was purified from pig brain, and microtubules were polymerized and labeled as described in Refs. 30 and 41. All chemicals were obtained from Sigma, Germany, if not specified otherwise.

\section{Multi-motor surface-gliding assays}

MMGAs were performed at $22^{\circ} \mathrm{C}$, essentially as described previously. ${ }^{41}$ Motor-protein constructs were allowed to nonspecifically bind to the glass surfaces of assay chambers made from coverslips and plasma-cleaned microscope slides assembled with double-stick tape.
Subsequently, the chambers were flushed with about three chamber volumes of assay mix in BRB80 buffer [80 mM Pipes/KOH (pH 6.8), $1 \mathrm{mM} \mathrm{MgCl}_{2}$, and $1 \mathrm{mM}$ EGTA] containing $10 \mu \mathrm{m}$ taxol (paclitaxel), $0.022 \mathrm{mg} / \mathrm{ml}$ tetramethylrhodamine-labeled microtubules, $2 \mathrm{mM}$ ATP, $4 \mathrm{mM} \mathrm{MgCl}, 10 \mathrm{mM}$ DTT, $0.08 \mathrm{mg} / \mathrm{ml}$ catalase C40, $0.1 \mathrm{mg} / \mathrm{ml}$ glucose oxidase, and $10 \mathrm{mM}$ glucose. Motility was observed in a standard inverted fluorescence microscope (Zeiss Axiovert 200, Göttingen, Germany). Images were recorded with a digital charge-coupled device camera (CoolSnap ES; Roper Scientific Germany).

\section{Silanized assay chambers and single-molecule fluorescence assays}

Coverslips were cleaned using a plasma cleaner (Harrick Plasma, Ithaca, NY, USA) before silanization with a positively charged silane, 3-[2-(2-aminoethylamino)-ethylamino]propyl-trimethoxysilane (Sigma), for microtubule immobilization. Assay chambers were made from 3-[2-(2-aminoethylamino)-ethylamino]propyl-trimethoxysilane coverslips and microscope slides assembled with double-stick tape. Tetramethylrhodaminelabeled microtubules diluted in BRB80 buffer containing $10 \mu \mathrm{m}$ taxol (paclitaxel) were allowed to bind for $5 \mathrm{~min}$ to the silanized glass surfaces of the assay chamber. This was followed by $5 \mathrm{~min}$ of incubation with $0.1 \mathrm{mg} / \mathrm{ml}$ casein in BRB80. The chamber then was flushed with $7-10 \mu \mathrm{l}$ of assay buffer (BRB80 containing $2 \mathrm{mM}$ ATP and $4 \mathrm{mM}$ $\mathrm{MgCl}_{2}$ and an oxygen scavenging system based on $10 \mathrm{mM}$ DTT, $0.08 \mathrm{mg} / \mathrm{ml}$ catalase $40,0.1 \mathrm{mg} / \mathrm{ml}$ glucose oxidase, and $10 \mathrm{mM}$ glucose) containing GFP-tagged motor proteins at appropriate single-molecule concentrations (Table 1). Fluorescence was observed in a custom-built total internal reflection fluorescence (TIRF) microscope described previously. ${ }^{44}$ Digital video recordings (two frames per second) were analyzed for motor speeds and run lengths using kymographs generated with customwritten LabView software. Statistical analysis of the data was performed with OriginPro (OriginLab Corporation, Northampton, MA, USA). All measurements were performed at $22{ }^{\circ} \mathrm{C}$.

\section{Optical trapping assays}

We diluted 1- $\mu$ m-diameter plain silica beads (Kisker, Steinfurt, Germany) in BRB80 to a concentration of $0.5 \mathrm{mg} / \mathrm{ml}$ and incubated them for $30 \mathrm{~min}$ with $50 \mu \mathrm{g}$ protein-G (Sigma). After centrifugation and re-suspension, the beads were incubated with $1 \mu \mathrm{g}$ penta-his antibody (Sigma) and $0.02 \mathrm{mg}$ casein. After $30 \mathrm{~min}$, the beads were centrifuged and re-suspended to the desired concentration. Diluted his-tagged motor protein was then added to the beads and allowed to bind for $15 \mathrm{~min}$. The motor dilution was chosen so that only about half of the beads showed activity in the assay. This makes it most likely that only one motor on the beads is able to bind to a microtubule at any time. ${ }^{5,40}$ The motor-coated beads were added to the assay buffer, which was then filled into an assay chamber. Beads were trapped using a focused 4-W 1064-nm NdYVO laser (Coherent Germany) in a custom-built single-beam optical trap described elsewhere. ${ }^{41}$ After positioning a bead above a microtubule, the interaction between the motor and the 
microtubule could be monitored. The motion of the bead was measured by back-focal-plane interferometry. ${ }^{41,42}$ Data were acquired with custom-written LabView software. Further analysis and statistical calculations were performed with MatLab (The Mathworks) and OriginPro (OriginLab Corporation). All measurements were performed at $22{ }^{\circ} \mathrm{C}$.

Supplementary data to this article can be found online at http:/ / dx.doi.org/10.1016/j.jmb. 2012.06.043

\section{Acknowledgements}

This work was supported by Lower Saxony Grant No. 11-76251-99-26/08 (ZN2440) and by the Deutsche Forschungsgemeinschaft through the Research Center for Molecular Physiology of the Brain and the Collaborative Research Center SFB 803. We thank Stefanie Kramer and Angelina Schuder for technical support and Kerstin von Roden, Charlotte Willms, and Dieter Klopfenstein for help with the protein expression and purification. Kymograph analysis was performed using a LabView program written by Joost van Mameren.

Author Contributions. S.L. conceived the project, cloned, and purified some of the motors. S.L. and C.F.S. designed and supervised experiments, and S.L., C.F.S., C.T., and A.D. wrote the manuscript. C.T. performed single-molecule fluorescence assays and surface-gliding assays, and A.D. performed optical trapping experiments. A.D. and C.T. analyzed data and created figures.

\section{References}

1. Vale, R. D., Reese, T. S. \& Sheetz, M. P. (1985). Identification of a novel force-generating protein, kinesin, involved in microtubule-based motility. Cell, 42, 39-50.

2. Brouhard, G. J., Stear, J. H., Noetzel, T. L., Al-Bassam, J., Kinoshita, K., Harrison, S. C. et al. (2008). XMAP215 is a processive microtubule polymerase. Cell, 132, 79-88.

3. Howard, J., Hudspeth, A. J. \& Vale, R. D. (1989). Movement of microtubules by single kinesin molecules. Nature, 342, 154-158.

4. Vale, R. D., Funatsu, T., Pierce, D. W., Romberg, L., Harada, Y. \& Yanagida, T. (1996). Direct observation of single kinesin molecules moving along microtubules. Nature, 380, 451-453.

5. Svoboda, K., Schmidt, C. F., Schnapp, B. J. \& Block, S. M. (1993). Direct observation of kinesin stepping by optical trapping interferometry. Nature, 365, 721-727.

6. Svoboda, K. \& Block, S. M. (1994). Force and velocity measured for single kinesin molecules. Cell, 77, 773-784.
7. Carter, N. J. \& Cross, R. A. (2005). Mechanics of the kinesin step. Nature, 435, 308-312.

8. Visscher, K., Schnitzer, M. J. \& Block, S. M. (1999). Single kinesin molecules studied with a molecular force clamp. Nature, 400, 184-189.

9. Klopfenstein, D. R., Tomishige, M., Stuurman, N. \& Vale, R. D. (2002). Role of phosphatidylinositol $(4,5)$ bisphosphate organization in membrane transport by the Unc104 kinesin motor. Cell, 109, 347-358.

10. Tomishige, M., Klopfenstein, D. R. \& Vale, R. D. (2002). Conversion of Unc104/KIF1A kinesin into a processive motor after dimerization. Science, 297, 2263-2267.

11. Yardimci, H., van Duffelen, M., Mao, Y., Rosenfeld, S. S. \& Selvin, P. R. (2008). The mitotic kinesin CENP-E is a processive transport motor. Proc. Natl Acad. Sci. USA, 105, 6016-6021.

12. Varga, V., Helenius, J., Tanaka, K., Hyman, A. A., Tanaka, T. U. \& Howard, J. (2006). Yeast kinesin-8 depolymerizes microtubules in a lengthdependent manner. Nat. Cell Biol. 8, 957-962.

13. Kwok, B. H., Kapitein, L. C., Kim, J. H., Peterman, E. J., Schmidt, C. F. \& Kapoor, T. M. (2006). Allosteric inhibition of kinesin-5 modulates its processive directional motility. Nat. Chem. Biol. 2, 480-485.

14. Hirokawa, N., Noda, Y., Tanaka, Y. \& Niwa, S. (2009). Kinesin superfamily motor proteins and intracellular transport. Nat. Rev., Mol. Cell Biol. 10, 682-696.

15. Scholey, J. M., Brust-Mascher, I. \& Mogilner, A. (2003). Cell division. Nature, 422, 746-752.

16. Rosenfeld, S. S., van Duffelen, M., Behnke-Parks, W. M., Beadle, C., Corrreia, J. \& Xing, J. (2009). The ATPase cycle of the mitotic motor CENP-E. J. Biol. Chem. 284, 32858-32868.

17. Schief, W. R. \& Howard, J. (2001). Conformational changes during kinesin motility. Curr. Opin. Cell Biol. 13, 19-28.

18. Valentine, M. T. \& Gilbert, S. P. (2007). To step or not to step? How biochemistry and mechanics influence processivity in Kinesin and Eg5. Curr. Opin. Cell Biol. 19, 75-81.

19. Clancy, B. E., Behnke-Parks, W. M., Andreasson, J. O., Rosenfeld, S. S. \& Block, S. M. (2011). A universal pathway for kinesin stepping. Nat. Struct. Mol. Biol. 18, 1020-1027.

20. Kozielski, F., Sack, S., Marx, A., Thormahlen, M., Schonbrunn, E., Biou, V. et al. (1997). The crystal structure of dimeric kinesin and implications for microtubule-dependent motility. Cell, 91, 985-994.

21. Rosenfeld, S. S., Fordyce, P. M., Jefferson, G. M., King, P. H. \& Block, S. M. (2003). Stepping and stretching. How kinesin uses internal strain to walk processively. J. Biol. Chem. 278, 18550-18556.

22. Rice, S., Lin, A. W., Safer, D., Hart, C. L., Naber, N., Carragher, B. O. et al. (1999). A structural change in the kinesin motor protein that drives motility. Nature, 402, 778-784.

23. Hackney, D. D. (2005). The tethered motor domain of a kinesin-microtubule complex catalyzes reversible synthesis of bound ATP. Proc. Natl Acad. Sci. USA, 102, 18338-18343.

24. Kutys, M. L., Fricks, J. \& Hancock, W. O. (2010). Monte Carlo analysis of neck linker extension in kinesin molecular motors. PLoS Comput. Biol. 6, e1000980. 
25. Shastry, S. \& Hancock, W. O. (2011). Interhead tension determines processivity across diverse N-terminal kinesins. Proc. Natl Acad. Sci. USA, 108, 16253-16258.

26. Yildiz, A., Tomishige, M., Gennerich, A. \& Vale, R. D. (2008). Intramolecular strain coordinates kinesin stepping behavior along microtubules. Cell, 134, 1030-1041.

27. Hariharan, V. \& Hancock, W. O. (2009). Insights into the mechanical properties of the kinesin neck linker domain from sequence analysis and molecular dynamics simulations. Cell. Mol. Bioeng. 2, 177-189.

28. Muthukrishnan, G., Zhang, Y., Shastry, S. \& Hancock, W. O. (2009). The processivity of kinesin-2 motors suggests diminished front-head gating. Curr. Biol. 19, 442-447.

29. Shastry, S. \& Hancock, W. O. (2010). Neck linker length determines the degree of processivity in kinesin- 1 and kinesin-2 motors. Curr. Biol. 20, 939-943.

30. Lakamper, S., Kallipolitou, A., Woehlke, G., Schliwa, M. \& Meyhofer, E. (2003). Single fungal kinesin motor molecules move processively along microtubules. Biophys. J. 84, 1833-1843.

31. Valentine, M. T., Fordyce, P. M., Krzysiak, T. C., Gilbert, S. P. \& Block, S. M. (2006). Individual dimers of the mitotic kinesin motor Eg5 step processively and support substantial loads in vitro. Nat. Cell Biol. 8, 470-476.

32. Krzysiak, T. C. \& Gilbert, S. P. (2006). Dimeric Eg5 maintains processivity through alternating-site catalysis with rate-limiting ATP hydrolysis. J. Biol. Chem. 281, 39444-39454.

33. Kapitein, L. C., Kwok, B. H., Weinger, J. S., Schmidt, C. F., Kapoor, T. M. \& Peterman, E. J. (2008). Microtubule cross-linking triggers the directional motility of kinesin-5. J. Cell Biol. 182, 421-428.

34. Lakamper, S., Thiede, C., Duselder, A., Reiter, S., Korneev, M. J., Kapitein, L. C. et al. (2010). The effect of monastrol on the processive motility of a dimeric kinesin-5 head/kinesin-1 stalk chimera. J. Mol. Biol. 399, 1-8.

35. Arora, P., Ansari, A., Best, T., Ptashne, M. \& Dervan, P. (2002). Design of artificial transcriptional activators with rigid poly-L-proline linkers. J. Am. Chem. Soc. 124 13067-13071.

36. Valentine, M. T. \& Block, S. M. (2009). Force and premature binding of ADP can regulate the processivity of individual Eg5 dimers. Biophys. J. 97, 1671-1677.

37. Korneev, M. J., Lakamper, S. \& Schmidt, C. F. (2007). Load-dependent release limits the processive stepping of the tetrameric Eg5 motor. Eur. Biophys. J. 36, 675-681.

38. Uemura, S., Kawaguchi, K., Yajima, J., Edamatsu, M., Toyoshima, Y. Y. \& Ishiwata, S. (2002). Kinesinmicrotubule binding depends on both nucleotide state and loading direction. Proc. Natl Acad. Sci. USA, 99, 5977-5981.

39. Thorn, K. S., Ubersax, J. A. \& Vale, R. D. (2000). Engineering the processive run length of the kinesin motor. J. Cell Biol. 151, 1093-1100.

40. Skiniotis, G., Cochran, J. C., Muller, J., Mandelkow, E., Gilbert, S. P. \& Hoenger, A. (2004). Modulation of kinesin binding by the C-termini of tubulin. EMBO J. 23, 989-999.

41. Lakamper, S. \& Meyhofer, E. (2005). The E-hook of tubulin interacts with kinesin's head to increase processivity and speed. Biophys. J. 89, 3223-3234.

42. Turner, J., Anderson, R., Guo, J., Beraud, C., Fletterick, R. \& Sakowicz, R. (2001). Crystal structure of the mitotic spindle kinesin Eg5 reveals a novel conformation of the neck-linker. J. Biol. Chem. 276, 25496-25502.

43. Rosenfeld, S. S., Xing, J., Jefferson, G. M. \& King, P. (2005). Docking and rolling, a model of how the mitotic motor Eg5 works. J. Biol. Chem. 280, 35684-35695.

44. Gerson-Gurwitz, A., Thiede, C., Movshovich, N., Fridman, V., Podolskaya, M., Danieli, T. et al. (2011). Directionality of individual kinesin-5 Cin 8 motors is modulated by loop 8 , ionic strength and microtubule geometry. EMBO J. 30, 4942-4954.

45. Kerssemakers, J., Ionov, L., Queitsch, U., Luna, S., Hess, H. \& Diez, S. (2009). 3D nanometer tracking of motile microtubules on reflective surfaces. Small, 5, 1732-1737. 


\title{
Endoplasmic Reticulum Sorting and Kinesin-1 Command the Targeting of Axonal GABA $A_{B}$ Receptors
}

\author{
Viviana Valdés ${ }^{1,2}$, José Ignacio Valenzuela ${ }^{1,2}$, Daniela A. Salas ${ }^{1,2}$, Matías Jaureguiberry-Bravo ${ }^{1,2,3}$, \\ Carolina Otero ${ }^{4}$, Christina Thiede ${ }^{5}$, Christoph F. Schmidt ${ }^{5}$, Andrés Couve ${ }^{1,2 *}$
}

1 Biomedical Neuroscience Institute (BNI), Faculty of Medicine, Universidad de Chile, Santiago, Chile, 2 Program of Physiology and Biophysics, Institute of Biomedical Sciences (ICBM), Faculty of Medicine, Universidad de Chile, Santiago, Chile, 3 School of Biochemistry, Faculty of Biological Science, Universidad Andrés Bello, Santiago, Chile, 4 Faculty of Medicine, Pontificia Universidad Católica de Chile, Santiago, Chile, $\mathbf{5}$ Georg-August-Universität, Fakultät für Physik, Drittes Physikalisches InstitutBiophysik, Göttingen, Germany

\begin{abstract}
In neuronal cells the intracellular trafficking machinery controls the availability of neurotransmitter receptors at the plasma membrane, which is a critical determinant of synaptic strength. Metabotropic $\gamma$ amino-butyric acid (GABA) type $B$ receptors $\left(G A B A_{B} R s\right)$ are neurotransmitter receptors that modulate synaptic transmission by mediating the slow and prolonged responses to GABA. GABA $A_{B} R$ s are obligatory heteromers constituted by two subunits, $G A B A_{B} R 1$ and $G A B A_{B} R 2$. $G A B A_{B} R 1 a$ and $G A B A_{B} R 1 b$ are the most abundant subunit variants. $G A B A_{B} R 1 b$ is located in the somatodendritic domain whereas $\mathrm{GABA}_{B} \mathrm{R} 1 \mathrm{a}$ is additionally targeted to the axon. Sushi domains located at the N-terminus of $G A B A_{B} R 1 a$ constitute the only difference between both variants and are necessary and sufficient for axonal targeting. The precise targeting machinery and the organelles involved in sorting and transport have not been described. Here we demonstrate that $G A B A_{B} R s$ require the Golgi apparatus for plasma membrane delivery but that axonal sorting and targeting of $G A B A_{B} R 1 a$ operate in a pre-Golgi compartment. In the axon $\mathrm{GABA}_{B} \mathrm{R} 1$ a subunits are enriched in the endoplasmic reticulum (ER), and their dynamic behavior and colocalization with other secretory organelles like the ER-to-Golgi intermediate compartment (ERGIC) suggest that they employ a local secretory route. The transport of axonal $G A B A_{B} R 1$ a is microtubule-dependent and kinesin-1, a molecular motor of the kinesin family, determines axonal localization. Considering that progression of $G A B A_{B} R s$ through the secretory pathway is regulated by an ER retention motif our data contribute to understand the role of the axonal ER in non-canonical sorting and targeting of neurotransmitter receptors.
\end{abstract}

Citation: Valdés V, Valenzuela Jl, Salas DA, Jaureguiberry-Bravo M, Otero C, et al. (2012) Endoplasmic Reticulum Sorting and Kinesin-1 Command the Targeting of Axonal GABA $A_{B}$ Receptors. PLoS ONE 7(8): e44168. doi:10.1371/journal.pone.0044168

Editor: Sergio T. Ferreira, Federal University of Rio de Janeiro, Brazil

Received March 28, 2012; Accepted July 30, 2012; Published August 27, 2012

Copyright: (c) 2012 Valdés et al. This is an open-access article distributed under the terms of the Creative Commons Attribution License, which permits unrestricted use, distribution, and reproduction in any medium, provided the original author and source are credited.

Funding: VV was supported by Iniciativa Científica Milenio (ICM) P09-015-F. JIV was supported by Comisión Nacional de Investigación Científica y Tecnológica (CONICYT). MJB was supported by Fondo Nacional de Desarrollo Científico y Tecnológico (FONDECYT) 1100137 and ICM P09-015-F. CT was supported in part by the Lower Saxony Grant No 11-76251-99-26/08 (ZN2440). CO was supported by Deutsche Forschungsgemeinschaft (DFG)-CONICYT international grant AZ: 444 CHL-113/26/0-1. CFS was supported by the Center for Molecular Physiology of the Brain (CMPB), funded by the DFG. AC was supported by FONDECYT 1100137 and ICM P09-015-F. The funders had no role in study design, data collection and analysis, decision to publish, or preparation of the manuscript.

Competing Interests: The authors have declared that no competing interests exist.

*E-mail: andres@neuro.med.uchile.cl

\section{Introduction}

Polarized protein trafficking in the neuron is critical for synapse formation, synapse maintenance and the regulation of synaptic strength. In all eukaryotic cells the endomembrane trafficking system includes a forward biosynthetic route constituted by the endoplasmic reticulum (ER), the ER-Golgi intermediate compartment (ERGIC), the Golgi apparatus and post-Golgi vesicles, and a recycling-degradative route constituted by endosomes and lysosomes. The unique architecture and size of neurons does not necessarily imply that the structure/function relationship of these organelles and their contribution to the secretory process are different than in other cell types. However, their spatial arrangement and contribution to local processing may be specially adapted to the complexities of the neuronal morphology [1]. How the neuron orchestrates this highly compartmentalized trafficking is poorly understood. In particular, how the local distribution of secretory components in the neuron impinges on intracellular trafficking and availability of neurotransmitter receptors remains for the most part unexplored.

GABA is the main inhibitory neurotransmitter in the nervous system and the metabotropic GABA $A_{B} R$ are obligatory heteromers composed of two related subunits, $\mathrm{GABA}_{\mathrm{B}} \mathrm{R} 1$ and $\mathrm{GABA}_{\mathrm{B}} \mathrm{R} 2$ (for a comprehensive review of $\mathrm{GABA}_{\mathrm{B}} \mathrm{R}$ structure, function, localization and pathological implications see [2]). Both belong to family $\mathrm{C}$ of $\mathrm{G}$ protein-coupled receptors, and contain a large extracellular $\mathrm{N}$-terminal domain, seven membrane-spanning domains and an intracellular $\mathrm{C}$-terminal domain. $\mathrm{GABA}_{\mathrm{B}} \mathrm{Rs}$ are expressed in neurons throughout the brain and spinal cord. They are mainly perisynaptic receptors located in gabaergic and glutamatergic presynaptic terminals and postsynaptic sites. $\mathrm{GABA}_{\mathrm{B}} \mathrm{R} 1$ binds agonists with high affinity whereas $\mathrm{GABA}_{\mathrm{B}} \mathrm{R} 2$ couples to $\mathrm{G}_{\alpha \mathrm{i}}$ establishing a transactivation mechanism between the two subunits. At presynaptic terminals $\mathrm{GABA}_{\mathrm{B}} \mathrm{Rs}$ inhibit voltage gated $\mathrm{Ca}^{2+}$ channels thereby inhibiting synaptic vesicle fusion and neurotransmitter release. At postsynaptic sites they activate inwardly rectifying $\mathrm{K}^{+}$channels hyperpolarizing the postsynaptic 
neuron. In addition, stimulation of $\mathrm{GABA}_{\mathrm{B}} \mathrm{Rs}$ decreases the levels of cyclic AMP. $\mathrm{GABA}_{\mathrm{B}} \mathrm{Rs}$ have been implicated in epilepsy, anxiety, stress, sleep disorders, nociception, depression, cognition and addictive mechanisms to drug abuse. The relevance of studying $\mathrm{GABA}_{\mathrm{B}} \mathrm{R}$ availability is further supported by clinical observations that report the appearance of tolerance to $\mathrm{GABA}_{\mathrm{B}} \mathrm{R}$ agonists, an inconvenient side effect to therapy.

$\mathrm{GABA}_{\mathrm{B}} \mathrm{R}$ subunits are synthesized in the soma and glycosylated in the ER [3], [4]. The progression of $\mathrm{GABA}_{\mathrm{B}} \mathrm{Rs}$ through the secretory pathway is regulated by an RXR-type sequence (RSRR) in the $\mathrm{C}$-terminal domain of $\mathrm{GABA}_{\mathrm{B}} \mathrm{R} 1$ that functions as an ER retention motif in the absence of $\mathrm{GABA}_{\mathrm{B}} \mathrm{R} 2$ [5]. The ER retention motif is masked upon association to $\mathrm{GABA}_{\mathrm{B}} \mathrm{R} 2$, and assembled $\mathrm{GABA}_{\mathrm{B}} \mathrm{Rs}$ exit the ER as heteromers destined for the plasma membrane. Consistent with ER retention acting as a limiting step $\mathrm{GABA}_{\mathrm{B}} \mathrm{Rs}$ are abundant within intracellular compartments, especially the ER [6].

$\mathrm{GABA}_{\mathrm{B}} \mathrm{R} 1 \mathrm{a}$ and $\mathrm{GABA}_{\mathrm{B}} \mathrm{R} 1 \mathrm{~b}$ constitute the most abundant isoforms for $\mathrm{GABA}_{\mathrm{B}} \mathrm{R} 1$. Heteromers containing $\mathrm{GABA}_{\mathrm{B}} \mathrm{R} 1 \mathrm{a}$ are axonal and somatodendritic whereas those containing GA$\mathrm{BA}_{\mathrm{B}} \mathrm{R} 1 \mathrm{~b}$ are exclusively located in the somatodendritic domain [7]. $\mathrm{GABA}_{\mathrm{B}} \mathrm{R} 1 \mathrm{a}$ and $\mathrm{GABA}_{\mathrm{B}} \mathrm{R} 1 \mathrm{~b}$ mediate their different functions only as a result of their specific axonal or somatodendritic localization [7]. The sushi domains located at the Nterminus of $\mathrm{GABA}_{\mathrm{B}} \mathrm{R}$ la are necessary and sufficient for axonal targeting even in a $\mathrm{GABA}_{\mathrm{B}} \mathrm{R} 2$ knock-out background [8]. However, the precise targeting machinery and the organelles involved in sorting and transport have not been described. Combining conventional optical microscopy and live-cell imaging using organelle reporters and trafficking blockers in cultured hippocampal neurons we describe a mechanism for $\mathrm{GABA}_{\mathrm{B}} \mathrm{R}$ la axonal localization based on pre-Golgi sorting and ER transport.

\section{Materials and Methods}

\section{Animals}

Adult pregnant female Sprague-Dawley rats were purchased from the Central Animal Facility at Universidad Católica de Chile and killed by asphyxia in a $\mathrm{CO}_{2}$ chamber according to the Guide for Care and Use of Laboratory Animals (The National Academy of Sciences, 1996). $\mathrm{GABA}_{\mathrm{B}} \mathrm{R}$ 1-EGFP mice were kindly provided by Bernhard Bettler (University of Basel, Switzerland). They correspond to transgenic animals for the $\mathrm{GABA}_{\mathrm{B}} \mathrm{R}$ 1-EGFP BAC in a homozygous knockout background for $\mathrm{GABA}_{\mathrm{B}} \mathrm{R} 1$ as described previously [9], [10].

\section{Cell lines, neuronal cultures and transfection}

COS-7 cells were maintained and transfected as described previously [3] using a Gene Pulser Xcell (BioRad). Primary hippocampal neurons were cultured from E18 rats or E18 $\mathrm{GABA}_{\mathrm{B}} \mathrm{R}$ 1-EGFP transgenic mice according to established procedures [11] and transfected by $\mathrm{Ca}^{2+}$ phosphate at 14-18 days in vitro (div) [12]. All transfected neurons, except for those in Fig. S2, were analyzed 1 day post transfection (dpt). Transfected neurons for Fig. S2 were analyzed between 1-5 dpt.

\section{Reagents and DNA plasmids}

Nocodazole was purchased from Sigma (St. Louis, MO). MYCGABA $_{B}$ R1, FLAG-GABA R2, HA-GABA R2, MYC-GA-

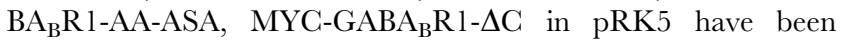
described previously and contain epitope tags on the extracellular N-terminal domains [3], [13-15]. MYG-GABA ${ }_{B}$ R1-AA-ASA contains point mutations that replace two leucine residues at a di-leucine motif and two arginine residues at the ER retention motif (RSRR) by alanines. MYG-GABA ${ }_{B} R 1-\Delta C$ lacks the complete $\mathrm{C}$-terminal domain. Both mutants escape the ER and traffic to the cell surface in the absence of $\mathrm{GABA}_{\mathrm{B}} \mathrm{R} 2$. $\mathrm{GABA}_{\mathrm{B}} \mathrm{R}$ la-EGFP, $\mathrm{GABA}_{\mathrm{B}} \mathrm{R} 2$-EGFP and $\mathrm{GABA}_{\mathrm{B}} \mathrm{R}$ la-monomeric red fluorescent protein have also been described previously and contain the fluorescent proteins attached to the intracellular C-terminal domain [4]. pDsRed-Cl (RFP), pEYFP-Golgi, pEYFP-ER and pDsRed2-ER (KDEL-RFP) were obtained from Clontech (Mountain View, CA). Kif5C-RFP-DN was kindly provided by S. Kindler and H.J. Kreienkamp (Institut für Humangenetik, Universitätsklinikum Hamburg-Eppendorf, Hamburg, Germany) and corresponds to amino acids 678-955 of KIF5C (NM_001107730) also referred to as DN2 by Falley and collaborators [16]. ARF1-Q71I-HA was kindly provided by O. Jeyifous (University of Chicago, Chicago, IL), Rabl1-GFP was kindly provided by F. Bronfman (Pontificia Universidad Católica de Chile), p58-YFP was kindly provided by J. Lippincott-Schwartz (National Institutes of Health, Bethesda, MD). Cytochrome b5EGFP was kindly provided by C. Hetz (Universidad de Chile). All manipulations and fidelity of DNA constructs were verified by sequencing.

\section{Antibodies}

$\mathrm{GABA}_{\mathrm{B}} \mathrm{R} 1$ antibodies (which recognize $\mathrm{GABA}_{\mathrm{B}} \mathrm{R}$ la and $\mathrm{GABA}_{\mathrm{B}} \mathrm{R} 1 \mathrm{~b}$ ) were raised against the intracellular C-terminal domain in rabbits and affinity purified. $\mathrm{GABA}_{\mathrm{B}} \mathrm{R} 1$ antibodies specifically recognize $\mathrm{MYC}_{-} \mathrm{GABA}_{\mathrm{B}} \mathrm{R}$ la in transfected COS cells and detect the predicted doublet corresponding to $\mathrm{GABA}_{\mathrm{B}} \mathrm{R} 1 \mathrm{a}$ and $\mathrm{GABA}_{\mathrm{B}} \mathrm{R} 1 \mathrm{~b}$ in crude brain membranes (Fig. S1). Microtubule-associated protein 2 (MAP2) antibodies were purchased from Chemicon (Temecula, CA). Piccolo antibodies were kindly provided by ED. Gundelfinger and WD. Altrock (Leibniz Institute for Neurobiology, Magdeburg Germany). KDEL antibodies (directed against a 6-residue peptide (SEKDEL) of the rat Grp78 protein) and cis-Golgi matrix protein 130 (GM130) were purchased from StressGen (Ann Arbor, MI). MYG antibodies were purchased from Sigma (St. Louis, Missouri). Influenza A Virus epitope (HA) antibodies were purchased from Roche (Indianapolis, IN). Anti-GFP antibodies (ab6556) were purchased from Abcam (Cambridge, UK). Secondary anti-mouse, antirabbit, anti-guinea pig or anti-chicken antibodies conjugated to Texas Red (TR), tetramethyl rhodamine isothiocyanate (TRITC), fluorescein isothiocyanate (FITC) or cyanine 3 (Cy3) were purchased from Jackson Immuno Research Laboratories (West Grove, PA).

\section{Immunofluorescence, image capture, image processing} and time-lapse microscopy

Immunofluorescence was performed as described previously under non-permeabilized or permeabilized conditions [4]. Depending on the type of experiment axons were identified by the presence of a positive marker (Tau), the absence of a negative marker (MAP2), or by morphological criteria that included: longer projection, constant diameter and right angle branching. Imageprocessing routines were developed in our laboratory based on of Interactive Data Language (IDL) (ITT, Boulder, CO), including routines for segmentation [4]. Live-cell imaging was performed in a $23^{\circ} \mathrm{C}$ equilibrated microscopy suite. Images were obtained using an Olympus BX61WI upright microscope and an Olympus diskscanning unit. Consecutive frames were acquired over a period of $\sim 120$ s. Kymographs were constructed from an axonal segment $20 \mu \mathrm{m}$ from the soma using ImageJ from three-pixel-wide axonal traces. The axon-to-dendrite $(\mathrm{A}: \mathrm{D})$ ratio of MYC-GABA $\mathrm{B}_{\mathrm{B}} \mathrm{R}$ la was determined using Image $\mathrm{J}$ according to a procedure based on 
A

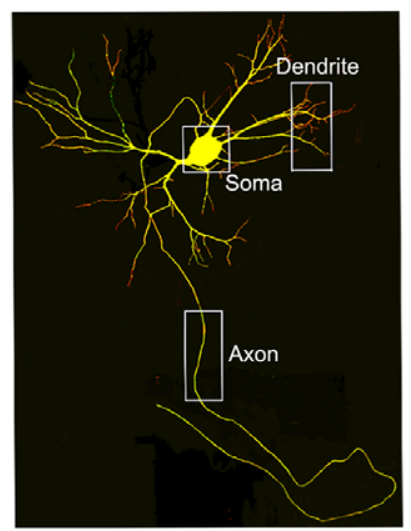

D

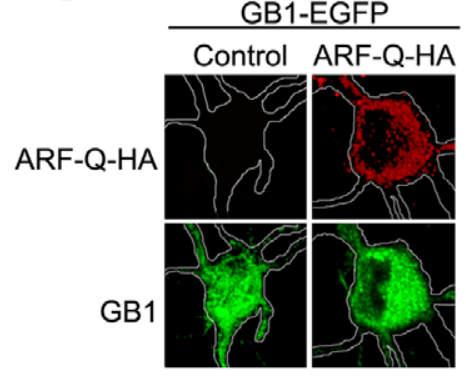

GOLG
B

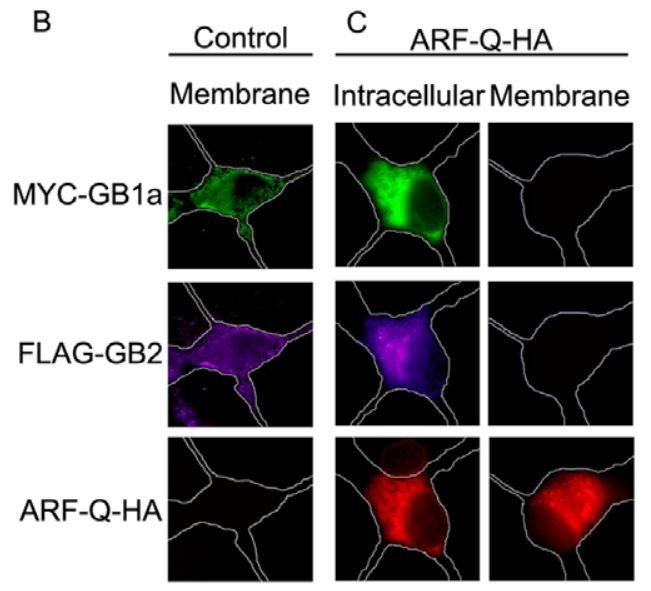

$\mathrm{E}$

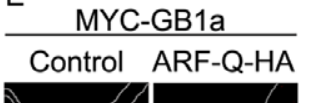

$\mathrm{F}$

F MYC-GB1b
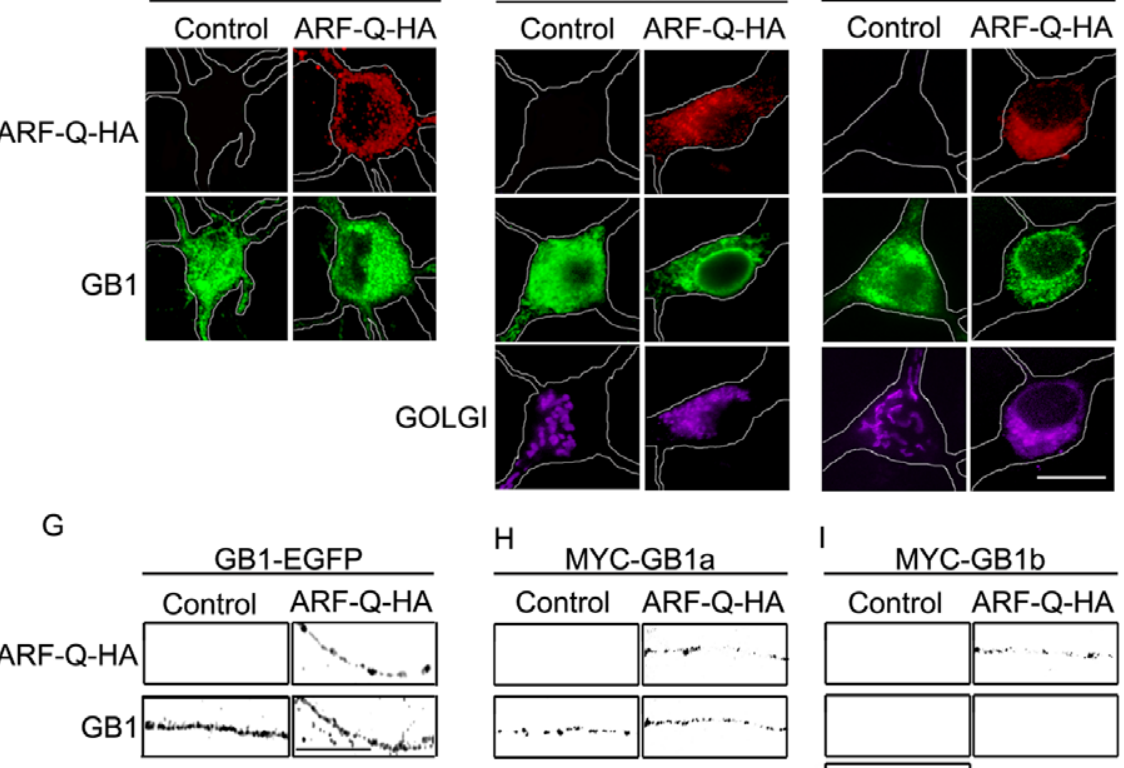
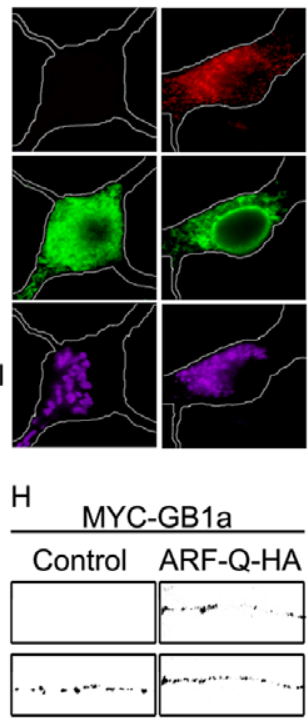

TAU

\section{MYC-GB1b}

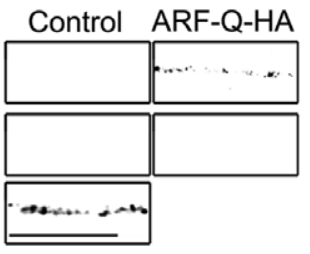

Figure 1. $G A B A_{B} R 1 a$ is targeted to the axon even after blockade of Golgi export. (A) Hippocampal neurons were transfected with MYC$G_{A B A} R 1 a$ and RFP, and processed for immunofluorescence under permeabilized conditions. MYC-GABA $R 1$ a was detected with MYC antibodies (green) and RFP was detected without staining (red). Boxes indicate somatic, dendritic and axonal regions used throughout the study. (B) Hippocampal neurons were transfected with MYC-GABA $B 1$ a and FLAG-GABA ${ }_{B} R 2$ and processed for immunofluorescence under non-permeabilized conditions. MYC-GABA ${ }_{B}$ R1a was detected with MYC antibodies (MYC-GB1a, green) and FLAG-GABA $R 2$ was detected with FLAG antibodies (FLAGGB2, magenta) (representative image of $n=12$ neurons). (C) Same as above for neurons transfected with MYC-GABA ${ }_{B} R 1 a, F_{1}$. Q711-HA (ARF-Q-HA). ARF1-Q711-HA was detected with HA antibodies (red). Neurons were labeled under permeabilized conditions to visualize intracellular $G A B A_{B} R$ subunits or non-permeabilized conditions to evaluate their abundance at the plasma membrane (representative image of $n=28$ neurons). (D) Hippocampal neurons from GABA R1-EGFP mice in the absence (control) or presence of ARF1-Q711-HA (ARF-Q-HA) and processed for immunofluorescence under permeabilized conditions. ARF1-Q711-HA was detected with HA antibodies (ARF-Q-HA, red), GABA $A_{B}$ R1-EGFP signal was amplified with EGFP antibodies (GB1, green) (representative image of $n=10$ neurons). (E) Hippocampal neurons were transfected with MYC$\mathrm{GABA}_{B} \mathrm{R} 1 \mathrm{a}$ in the absence (control) or presence of ARF1-Q711-HA (ARF-Q-HA) and processed for immunofluorescence under permeabilized conditions. ARF-Q-HA was detected with HA antibodies (red), MYC-GABA R1a was detected with MYC antibodies (green), the Golgi apparatus was detected by expressing pEYFP-Golgi (magenta) (representative image of $n=18$ neurons). F) Same as above for neurons transfected with MYC$G_{A B A_{B} R 1 b}$ (representative image of $n=10$ neurons). Scale bar for B-F represents $20 \mu \mathrm{m}$. G-I) Axons of hippocampal neurons under the experimental conditions of D-F (representative images of neurons examined above). Tau staining was used to visualize the axon when not labeled by MYC$\mathrm{GABA}_{\mathrm{B}} \mathrm{R} 1 \mathrm{~b}$. Scale bar for $\mathrm{G}-\mathrm{I}$ represents $20 \mu \mathrm{m}$. doi:10.1371/journal.pone.0044168.g001

Biermann et. al., 2010 and modified [8]. Briefly, one-pixel-wide lines were traced along the initial $150 \mu \mathrm{m}$ of axons and dendrites in images labeled with soluble RFP or Kif5C-RFP-DN. No measurements were carried out beyond $150 \mu \mathrm{m}$ to prevent artifacts due to axonal length differences between RFP or
Kif5C-RFP-DN transfected neurons. Average pixel intensities of MYG-GABA $A_{B}$ Rla were determined along the traced lines, background was subtracted and the data was used to determine the A:D ratio. Criteria for cell selection included even distribution of RFP or Kif5C-RFP-DN in axons and dendrites, and cells 
A

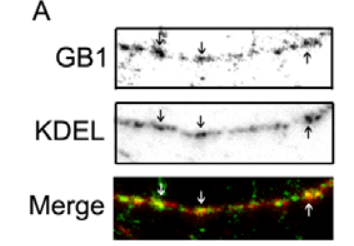

E

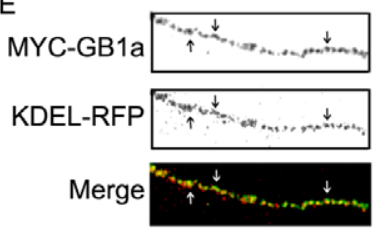

B

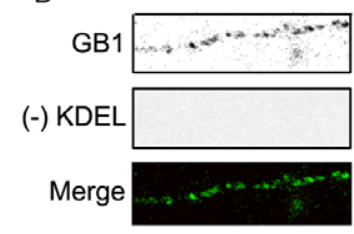

$$
\text { C }
$$

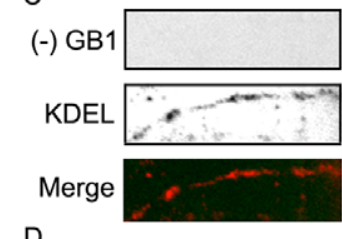

$\mathrm{F}$

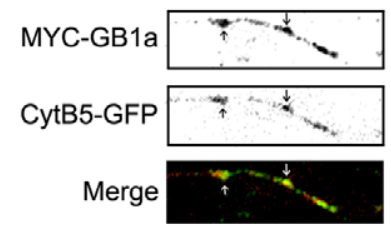

G

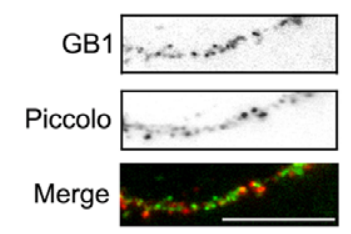

$\mathrm{H}$

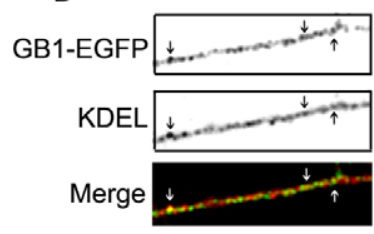

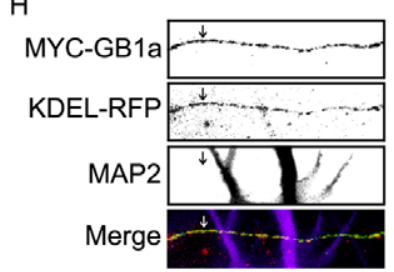

Figure 2. $G A B A_{B} R 1$ a colocalizes with the ER in axons. (A) Representative axon of hippocampal neurons processed for immunofluorescence under permeabilized conditions. $G A B A_{B} R 1$ was detected with $G A B A_{B} R 1$ antibodies (GB1, green) and the ER was detected with KDEL antibodies (KDEL, red). Merged images are shown on the bottom panel (representative image of $n=17$ neurons). (B) Same as above excluding staining with primary KDEL antibodies. (C) Same as above excluding staining with primary $\mathrm{GABA}_{\mathrm{B}} \mathrm{R} 1$ antibodies. (D) Representa-

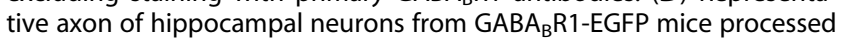
for immunofluorescence under permeabilized conditions. $G A B A_{B} R 1$ EGFP signal was amplified with EGFP antibodies (GB1-EGFP, green) and the ER was detected with KDEL antibodies (KDEL, red). Merged images are shown on the bottom panel (representative image of $n=10$ neurons). (E) Representative axon of hippocampal neurons transfected with MYC-GABA $A_{B}$ R1a and KDEL-RFP and processed for immunofluorescence under permeabilized conditions. MYC-GABA $A_{B} R 1 a$ was detected with MYC antibodies (MYC-GB1a, green), KDEL-RFP was visualized without staining (red). Merged images are shown on the bottom panel (representative image of $n=20$ neurons). (F) Representative axon of hippocampal neurons transfected with MYC-GABA ${ }_{B} R 1 a$ and cytochrome b5-EGFP (CytB5-GFP) and processed for immunofluorescence under permeabilized conditions. MYC-GABA $A_{B}$ R1a was detected with MYC antibodies (MYC-GB1a, green), CytB5-GFP was visualized without staining (red). Merged images are shown on the bottom panel (representative image of $n=20$ neurons). (G) Representative axon of hippocampal neurons processed for immunofluorescence under permeabilized conditions. $G_{A B A} R 1 a$ was detected with $G_{B} A B A_{B} R 1$ antibodies (green) and Piccolo was detected with Piccolo antibodies (red). Merged images are shown on the bottom panel (representative image of $n=15$ neurons). (H) Representative axon of hippocampal neurons transfected with MYC-GABA ${ }_{B} R 1 a$ and KDEL-RFP and processed for immunofluorescence under permeabilized conditions. MYC-GABA${ }_{\mathrm{B}} \mathrm{R} 1 \mathrm{a}$ was detected with MYC antibodies (MYC-GB1a, green), KDEL-RFP was visualized without staining (red) and MAP2 was detected with antiMAP2 antibodies (MAP2, magenta). The axon is identified as a MAP2negative projection. Merged images are shown on the bottom panel (representative image of $\mathrm{n}=15$ neurons). Scale bar for $\mathrm{A}-\mathrm{H}$ represents $20 \mu \mathrm{m}$.

doi:10.1371/journal.pone.0044168.g002 expressing constructs at very high levels were excluded from the analysis. 9-12 neurons from at least two independent culture preparations were analyzed for each condition. Images of axons from $\mathrm{GABA}_{\mathrm{B}} \mathrm{R}$ 1-EGFP mice were acquired using an Olympus FV-1000 confocal microscope. Anti-GFP antibodies were used to amplify the weak axonal signal of $\mathrm{GABA}_{\mathrm{B}} \mathrm{R}$ 1-EGFP expressed at physiological levels.

Total Internal Reflection Fluorescence (TIRF) microscopy

For TIRF microscopy 14-18 div hippocampal neurons were transfected with $\mathrm{GABA}_{\mathrm{B}} \mathrm{R}$ la-EGFP and motility in axons was analyzed $1 \mathrm{dpt}$. TIRF was carried out on a custom-built TIRF microscope setup: Two lasers $(473 \mathrm{~nm}$ and $532 \mathrm{~nm}$, both $30 \mathrm{~mW}$, Viasho, USA) were used to excite the fluorophores (GFP and RFP). The lasers were expanded and coupled via a multi-beam splitter (z474/488/532/635rpc, Chroma, USA) off-axis into the oil-immersion objective (Nikon, SFluor 100x, 1.49) to obtain TIRF illumination. The emitted fluorescent light was split in the GFP and RFP signals using a dichroic mirror (525/50, Chroma, USA) then passed trough bandpass filters (530/50 for GFP and 605/70 for TMR, both Chroma, USA) and finally directed via mirrors to separate areas on the chip of a frame transfer CCD camera (Cascasde:512B, Roper Scientific, USA). The CCD camera was controlled via WinSpec32 (Princeton Instruments, USA). The penetration depth of TIRF was $147 \mathrm{~nm}$. Digital images were taken at a frame rate of 2 frames/s and were subsequently analyzed for velocity and direction using kymographs generated with a customwritten LabView (National Instruments, USA) routine. Kymographs were analyzed for velocity and direction by fitting lines to the segments of a trace judged by eye. Stalls were not taken into account.

\section{Results}

The delivery of $G A B A_{B} R s$ to the plasma membrane is Golgi-dependent but axonal targeting is not

First we carried out a control experiment to validate the use of overexpression of recombinant $\mathrm{GABA}_{\mathrm{B}} \mathrm{R}$ subunits as a strategy to study receptor trafficking. Cultured hippocampal neurons were transfected with MYC-GABA $\mathrm{B}$ la and the distribution of the subunit at the plasma membrane or in intracellular compartments was evaluated by immunostaining $1-5 \mathrm{dpt}$. MYC-GABA $\mathrm{B}$ R la was retained in intracellular compartments in the cell body and axons up to $5 \mathrm{dpt}$ in the absence of recombinant $\mathrm{GABA}_{\mathrm{B}} \mathrm{R} 2$ expression (Fig. S2). In contrast, $\mathrm{GABA}_{\mathrm{B}} \mathrm{R}$ la was readily detectable at the cell surface at 2 dpt upon co-transfection with $\mathrm{GABA}_{\mathrm{B}} \mathrm{R} 2$ (Fig. S2, right column). These experiments indicate that the trafficking properties of recombinant receptors mimic the situation of the native subunits, and that the trafficking of recombinant receptors is not affected by the endogenous subunits. More importantly, they demonstrate that our experiments using transfection of recombinant $\mathrm{GABA}_{\mathrm{B}} \mathrm{R} 1$ subunits exclusively examine their intracellular population.

To determine whether $\mathrm{GABA}_{\mathrm{B}}$ Rs employ a Golgi-dependent intracellular trafficking route in neurons, primary cultures of hippocampal neurons were transfected with MYC-GABA $A_{B} R$ la and $\mathrm{FLAG}_{\mathrm{GABA}} \mathrm{R} 2$ in the absence or presence of ARF1Q71I-HA, a constitutively active ARF1 mutant that prevents export from the Golgi apparatus [17]. $1 \mathrm{dpt}$ the distribution of $\mathrm{GABA}_{\mathrm{B}} \mathrm{Rs}$ at the plasma membrane and in intracellular compartments was evaluated by immunostaining under nonpermeabilized or permeabilized conditions. We examined somatic or axonal domains as shown in the schematic neuron (Fig. 1A). As reported previously co-transfection of MYG- 
A

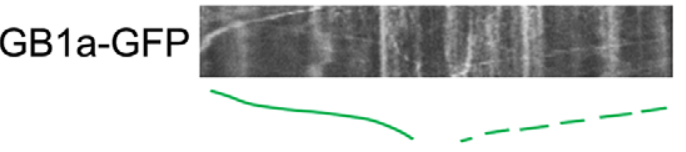

B KDEL-RFP
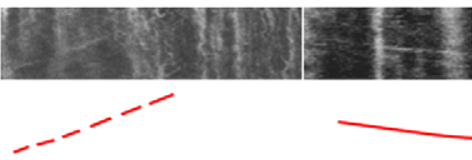

C GB1a-GFP
KDEL-RFP

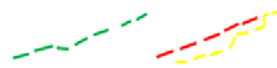

D Rab11-GFP

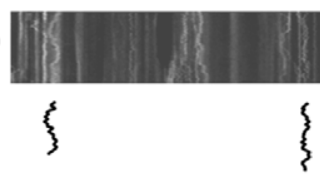

$\mathrm{E}$

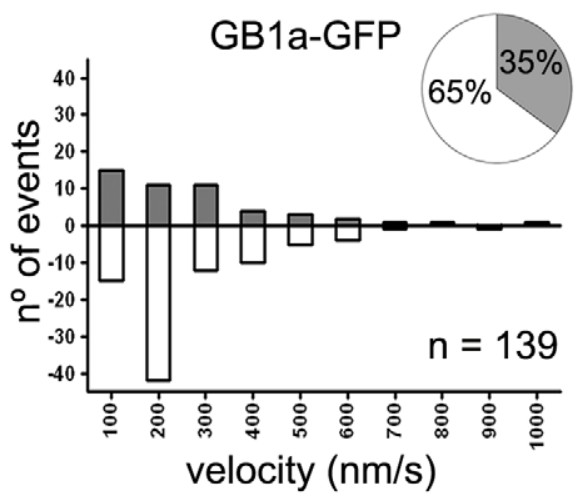

F

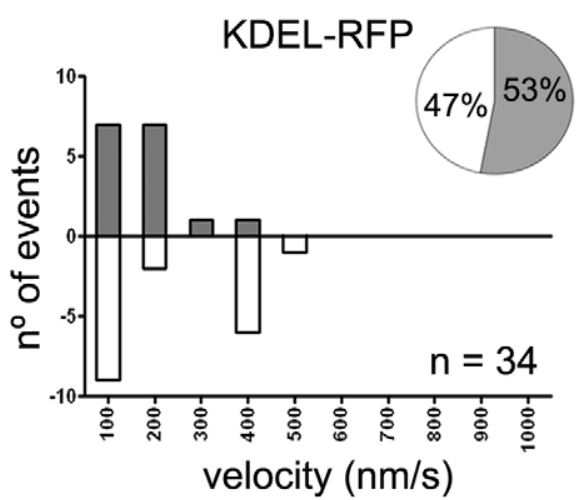

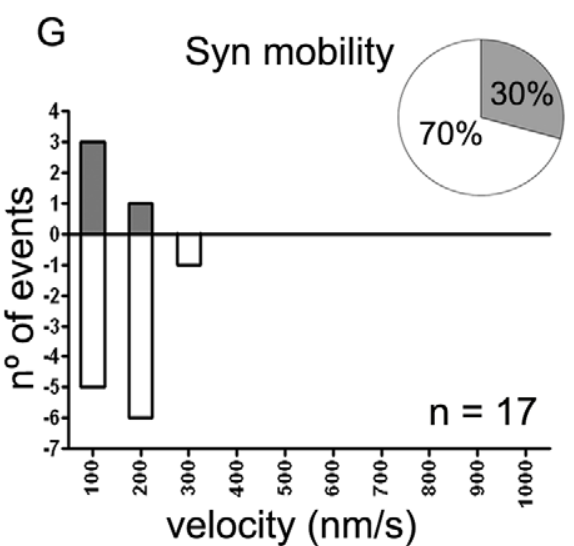

$\underset{\text { distance }}{\stackrel{\oplus}{E}}$

$\mathrm{H}$

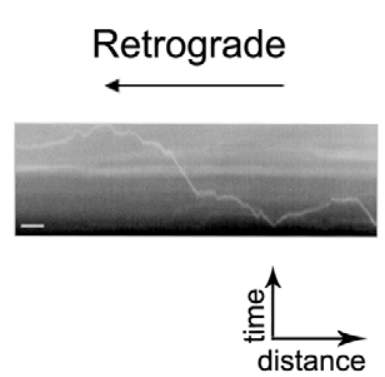

I

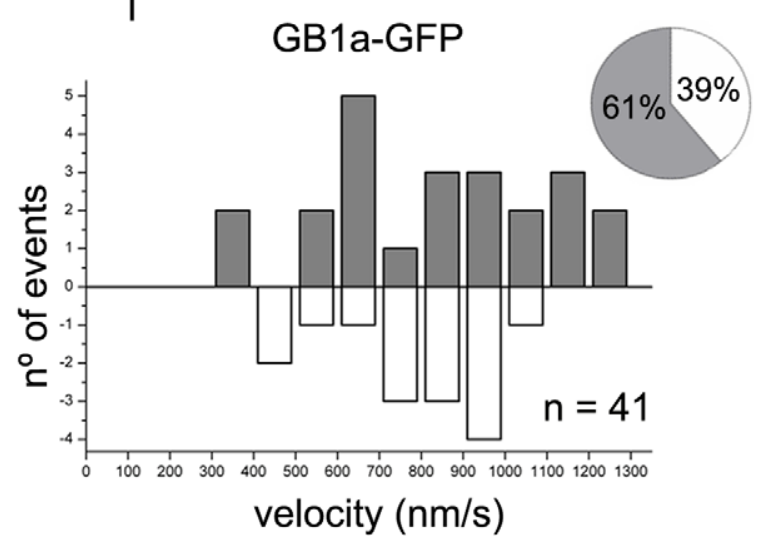

Figure 3. GABA $R$ R1a and KDEL are mobile in axons. (A) Representative kymograph constructed from time series of axons of hippocampal neurons transfected with $G_{A B A}$ R1a-EGFP (GB1a-GFP). Color lines correspond to selected traces within the corresponding kymograph. Solid green line: anterograde mobility; dashed green line: retrograde mobility. (B) Same as above for KDEL-RFP. Solid red line: anterograde mobility; dashed red line: retrograde mobility. (C) Representative kymographs from time series of axons of hippocampal neurons transfected with GABA ${ }_{B} R 1 a-E G F P$ and KDEL-RFP. Dashed green line: retrograde mobility of GABA ${ }_{B} R 1 a-E G F P$; dashed red line: retrograde mobility of KDEL-RFP; dashed yellow line: synchronous retrograde mobility of GABA ${ }_{B} R 1$ a-EGFP and KDEL-RFP. (D) Representative kymographs constructed from axons of hippocampal neurons transfected with Rab11-GFP. Solid black lines: short-range bidirectional mobility of Rab11-GFP (representative kymograph of $n=14$ neurons). Images were acquired at $0.20-0.25$ frames/s for a total of $120 \mathrm{~s}$. Scale bar for A-D represents $20 \mu \mathrm{m}$. (E-G) Average velocity and directionality was quantified from the kymographs for GABA R1a-EGFP, KDEL-RFP and synchronous mobility of GABA $A_{B}$ 1a-EGFP and KDEL-RFP. Bar graphs represent the frequency distribution of velocities, anterograde transport (gray bars), retrograde transport (white bars). Pie charts represent fractions of anterograde (gray) and retrograde transport (white). Average velocities and direction were obtained from 17-139 moving puncta from a total of 42 neurons from at least three independent culture preparations. (H) Representative kymograph from time series of axons of hippocampal neurons transfected with $\mathrm{GABA}_{\mathrm{B}} \mathrm{R} 1 \mathrm{a}-\mathrm{EGFP}$ imaged by TIRF microscopy at 2 frames/s. Scale bars represent $10 \mathrm{~s}$ and $3 \mu \mathrm{m}$. (I) Bar graph represents the frequency distribution of 
velocities: anterograde (gray bars), retrograde (white bars). Pie chart represents fractions of anterograde (gray) and retrograde transport (white). Average velocities and direction were obtained from 41 moving puncta from at least three independent culture preparations.

doi:10.1371/journal.pone.0044168.g003

$\mathrm{GABA}_{\mathrm{B}} \mathrm{R} 1 \mathrm{a}$ and FLAG-GABA $\mathrm{R} 2$ resulted in a robust localization of both subunits at the cell surface (Fig. 1B). In contrast, ARF1-Q71I-HA blocked the appearance of both subunits at the plasma membrane and produced accumulation in intracellular compartments (Fig. 1C). These results indicate that the Golgi apparatus is necessary for the delivery of $\mathrm{GABA}_{\mathrm{B}} \mathrm{Rs}$ to the plasma membrane in hippocampal neurons.

$\mathrm{GABA}_{\mathrm{B}} \mathrm{R}$ la is targeted to the axon in hippocampal neurons [7], [8]. Thus, we determined whether axonal targeting was also Golgi dependent. First we used cultured hippocampal neurons of
A
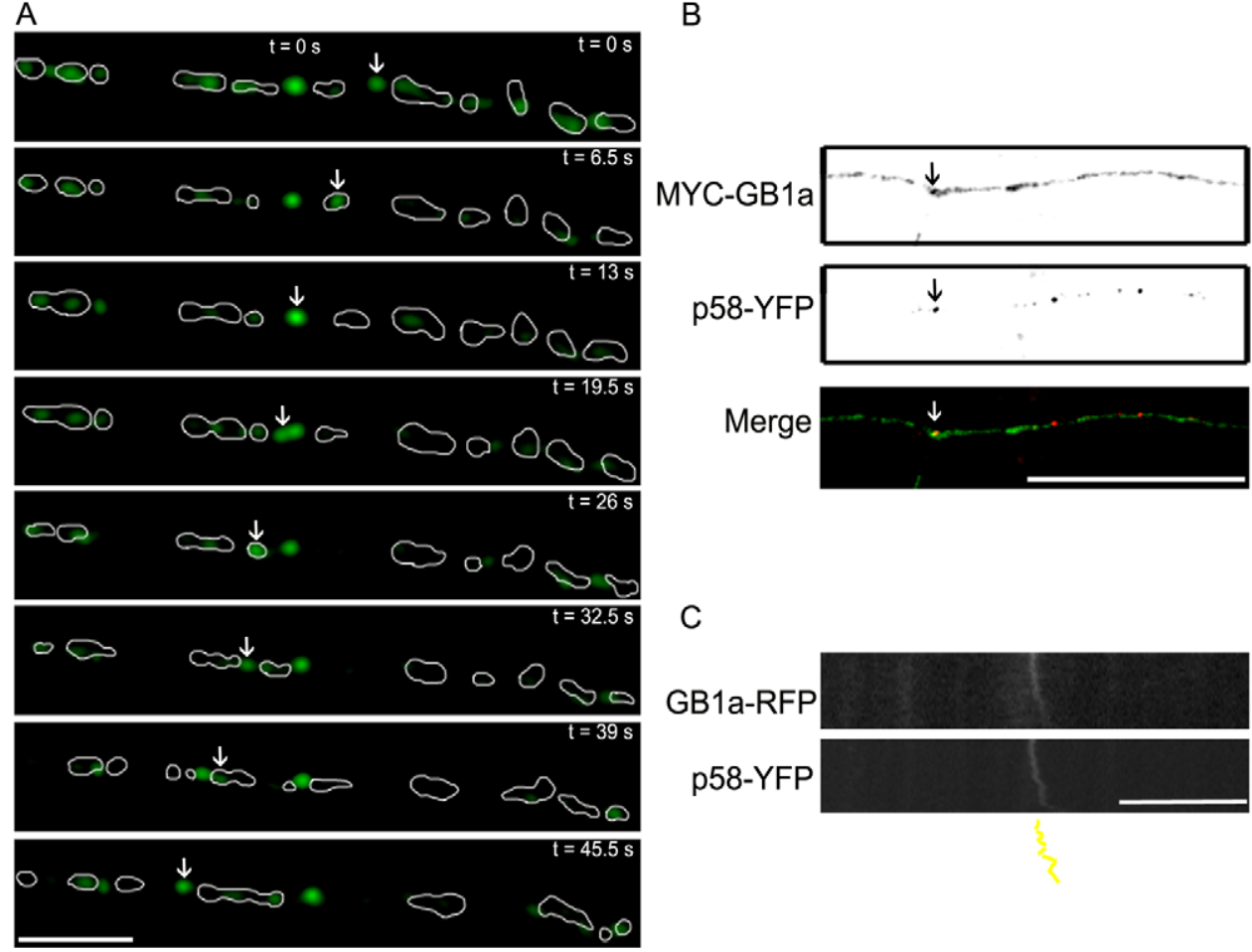

B
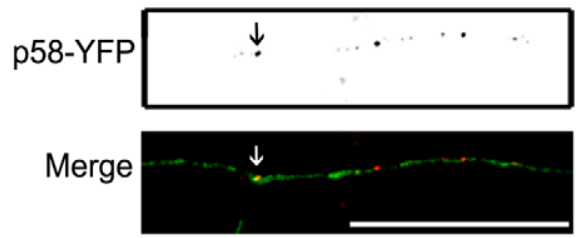

C

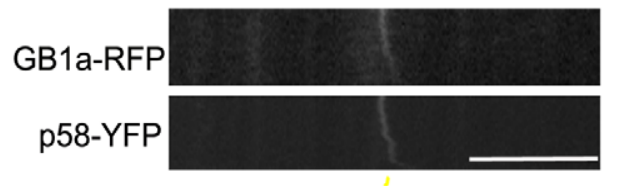

D
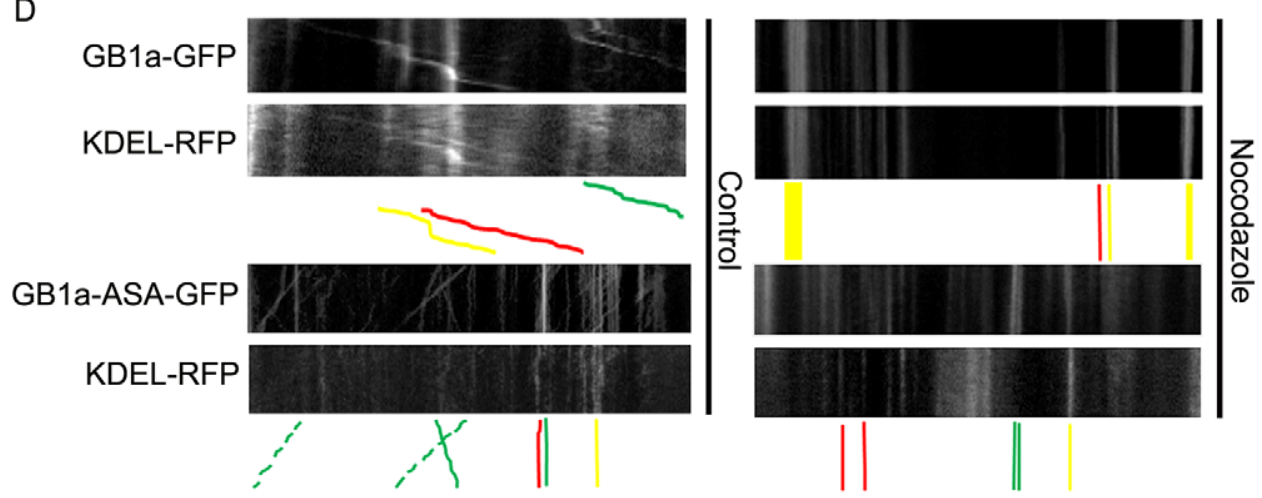

Figure 4. $\mathrm{GABA}_{\mathbf{B}} \mathrm{R} 1 \mathrm{a}$ cycles between KDEL compartments in axons. (A) Representative axon of hippocampal neurons transfected with $G_{A B A} R 1 a-E G F P$ and KDEL-RFP, and visualized live. The area originally occupied by the ER (KDEL-RFP) in each time frame was outlined in white. The arrows show a GABA $A_{B}$ R1a-EGFP puncta that exits and enters different ER compartments within the observation period (45.5 s) (representative timelapse sequence of $n=10$ neurons). Scale bar represents $20 \mu \mathrm{m}$. (B) Representative axon of hippocampal neurons transfected with MYC-GABA $\mathrm{B}_{\mathrm{B}} \mathrm{a}$ (MYC-GB1a) and p58-YFP, and processed for immunofluorescence under permeabilized conditions. MYC-GABA ${ }_{B} R 1$ a was detected with MYC antibodies (green); p58-YFP was visualized without staining (red). Merged images are shown on the bottom panel (representative image of $n=23$ neurons). Scale bar represents $20 \mu \mathrm{m}$. (C) Kymographs were constructed from time series of axons of hippocampal neurons transfected with $\mathrm{GABA}_{B}$ R1a-RFP (GB1a-RFP) and p58-YFP. Solid yellow lines: synchronous transport of GABA ${ }_{B} R 1 a-R F P$ and p58-YFP (representative kymograph of $\mathrm{n}=22$ neurons). Scale bar for C-D represents $20 \mu \mathrm{m}$. (D) Kymographs were constructed from time series of axons of hippocampal neurons under control conditions or treated with nocodazole after transfection with GABA ${ }_{B}$ R1a-EGFP (GB1a-GFP) or GABA ${ }_{B} R 1 a-A S A-E G F P$ (GB1a-ASA-GFP) and KDELRFP. Top right panels: accumulated GABA $A_{B} R 1$ a-EGFP and KDEL-RFP in static puncta (solid yellow lines). Bottom right panels: accumulated GABA ${ }_{B} R 1 a-$ ASA-EGFP and KDEL-RFP in different static puncta (solid green and red lines) (representative kymograph of $n=21$ neurons).

doi:10.1371/journal.pone.0044168.g004 
A
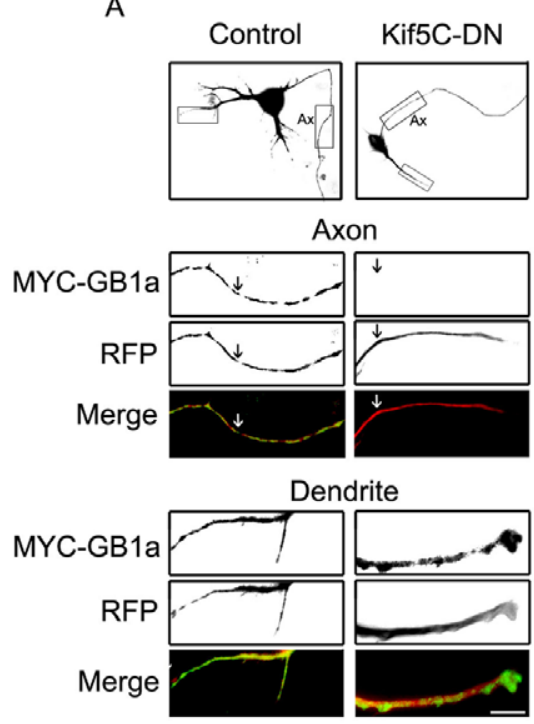

C

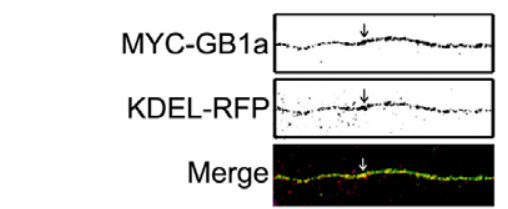

MYC-GB1a-AA-ASA
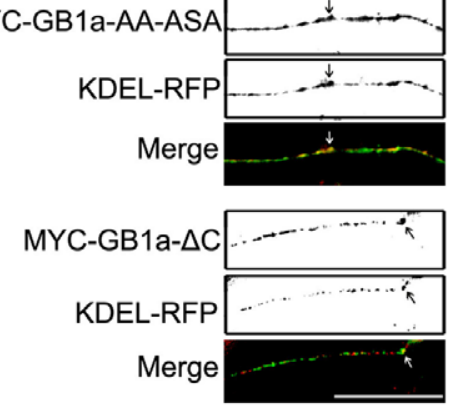

B
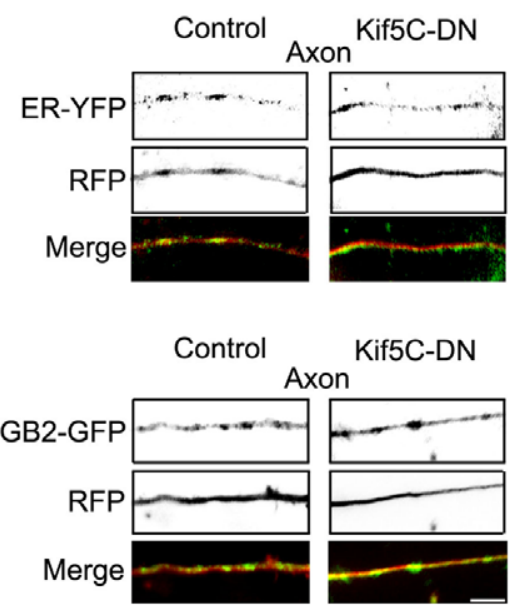

D

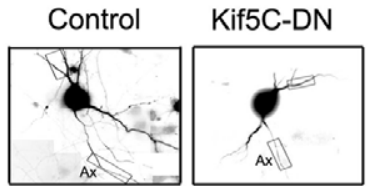

Axon

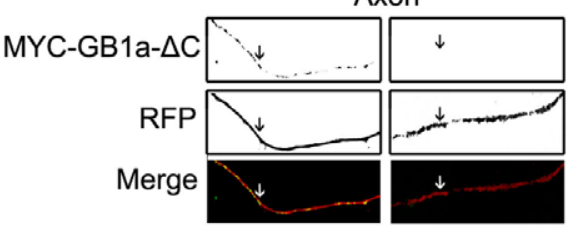

Dendrite

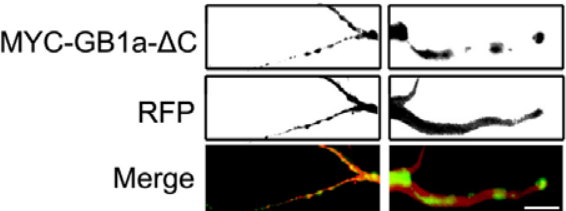

Figure 5. Axonal targeting of $\mathbf{G A B A}_{\mathrm{B}} \mathbf{R} 1 \mathrm{a}$ is kinesin-1 dependent and C-terminal domain independent. (A) Hippocampal neurons were transfected with MYC-GABA $A_{B}$ R1a and RFP (left, representative image of $n=12$ neurons) or MYC-GABA ${ }_{B}$ R1a and Kif5C-RFP-DN, a dominant negative version of kinesin-1 (Kif5C-DN, right, representative image of $n=13$ neurons). The neuronal volume filled by RFP or Kif5C-RFP-DN is shown. Boxes in top panels correspond to regions of axons ( $\mathrm{Ax}$ ) and dendrites (unlabelled) magnified below. MYC-GABA $\mathrm{B}_{\mathrm{B}} \mathrm{R} 1 \mathrm{a}$ (green) and RFP or Kif5C-RFP-DN (red). Merged images are shown on the bottom panel. Scale bar represents $40 \mu \mathrm{m}$. (B) Top panels: representative axon of hippocampal neurons transfected with pEYFP-ER (ER-YFP) and RFP or pEYFP-ER and Kif5C-RFP-DN. Markers were visualized without staining. Merged images are shown on the bottom panel (representative image of $n=15$ neurons). Bottom panels: representative axon of hippocampal neurons transfected with $G A B A_{B} R 2-$ EGFP (GB2-GFP) and RFP or GABA ${ }_{B}$ R2-EGFP and Kif5C-RFP-DN. Markers were visualized without staining. Merged images are shown on the bottom panel (representative image of $n=15$ neurons). Scale bar for represents $40 \mu \mathrm{m}$. (C) Representative axons of hippocampal neurons transfected with KDEL-RFP and MYC-GABA ${ }_{B}$ 1a, or MYC-GABA ${ }_{B}$ 1a-AA-ASA, or MYC-GABA ${ }_{B} R 1 a-\Delta C$, and processed for immunofluorescence under permeabilized conditions. MYC-GABA ${ }_{B}$ R1a versions were detected with MYC antibodies (MYC-GB1a, MYC-GB1a-AA-ASA, MYC-GB1a- $\triangle C$ ); KDEL-RFP was visualized without staining (representative images of $n=20,9$ and 10 neurons respectively). Merged images are shown on the bottom panels. Scale bar represents $20 \mu \mathrm{m}$. (D) Hippocampal neurons were transfected with MYC-GABA $\mathrm{R} 1 \mathrm{a}-\Delta \mathrm{C}$ and RFP (left, representative image of $\mathrm{n}=12 \mathrm{neurons}$ ) or MYC-GABA ${ }_{B}$ R1a- $\triangle C$ and Kif5C-RFP-DN (Kif5C-DN, right, representative image of $n=9$ neurons). The neuronal volume filled by RFP or Kif5C-RFP-DN is shown. Boxes in top panels correspond to regions of axons (Ax) and dendrites (unlabelled) magnified below. MYC-GABA ${ }_{B} R 1 a-\triangle C$ ( $\left.g r e e n\right)$ and RFP or Kif5C-RFP-DN (red). Merged images are shown on the bottom panel. Scale bar represents $40 \mu \mathrm{m}$.

doi:10.1371/journal.pone.0044168.g005

transgenic mice that express $\mathrm{GABA}_{\mathrm{B}} \mathrm{R}$ 1-EGFP under the control of an endogenous promoter [10]. This experimental model combines the advantages of a nearly physiological scenario and ease of detection. $\mathrm{GABA}_{\mathrm{B}} \mathrm{R} 1$ localized to the axon in control conditions (Figs. 1D and $1 \mathrm{G}$, left panel). Importantly, $\mathrm{GABA}_{\mathrm{B}} \mathrm{R} 1$ was still targeted to the axon in the presence of ARF1-Q71I-HA
(Figs. 1D and $1 \mathrm{G}$, right panels). According to previous reports the predominant axonal variant of $\mathrm{GABA}_{\mathrm{B}} \mathrm{R} 1$ corresponds to $\mathrm{GABA}_{\mathrm{B}} \mathrm{R}$ la [7], [8]. Therefore, these data are compatible with the idea that $\mathrm{GABA}_{\mathrm{B}} \mathrm{R}$ la is sorted and targeted to the axon at or prior to the Golgi apparatus. 
Next we used recombinant receptors to directly compare the axonal targeting of $\mathrm{GABA}_{\mathrm{B}} \mathrm{R} 1 \mathrm{a}$ and $\mathrm{GABA}_{\mathrm{B}} \mathrm{R} 1 \mathrm{~b}$, and their Golgi dependence. Neurons were transfected with MYC-GABA $\mathrm{B}_{\mathrm{B}} \mathrm{R}$ la or MYC-GABA ${ }_{B}$ R 1b in the absence or presence of ARF1-Q71I-HA. Consistent with previous reports, $\mathrm{GABA}_{\mathrm{B}} \mathrm{R}$ la but not $\mathrm{GABA}_{\mathrm{B}} \mathrm{R} 1 \mathrm{~b}$ was predominantly targeted to the axon in hippocampal neurons (Figs. 1E-1I, control panels). Importantly, recombinant GABA${ }_{B} R$ la was still targeted to the axon in the presence of ARF1-Q71IHA (Figs. $1 \mathrm{E}$ and $1 \mathrm{H}$ ). In contrast, $\mathrm{GABA}_{\mathrm{B}} \mathrm{R} 1 \mathrm{~b}$ was absent from the axon under all the conditions examined (Figs. $1 \mathrm{~F}$ and $1 \mathrm{I}$ ). These findings indicate that axonal targeting is specific to $\mathrm{GABA}_{\mathrm{B}} \mathrm{R}$ la. In addition, they demonstrate that the sorting and targeting of $\mathrm{GABA}_{\mathrm{B}} \mathrm{R}$ la to the axon occurs at or prior to the Golgi stage, and therefore suggest a non-conventional modality. They also indicate that axonal targeting of $\mathrm{GABA}_{\mathrm{B}} \mathrm{R}$ la upon $\mathrm{ARF} 1-$ Q71I-HA expression is not produced by an overload of the early secretory pathway because the effect was not observed for $\mathrm{GABA}_{\mathrm{B}} \mathrm{R} 1 \mathrm{~b}$, a conclusion further supported by the results in lower expressing transgenic neurons.

\section{$\mathrm{GABA}_{\mathrm{B}} \mathrm{R} 1 \mathrm{a}$ is targeted and transported within the axonal ER}

ER resident proteins and components of the protein folding and export machineries localize to the axon [18-20]. Thus, to determine the intracellular localization of axonal $\mathrm{GABA}_{\mathrm{B}} \mathrm{R} 1 \mathrm{a}$ neurons were immunostained with antibodies against the GA$\mathrm{BA}_{\mathrm{B}} \mathrm{R} 1$ subunit and an antibody against the SEKDEL sequence of the rat ER protein Grp78, including a conserved motif present in luminal ER resident proteins responsible for retrieval from the Golgi apparatus [21]. Endogenous $\mathrm{GABA}_{\mathrm{B}} \mathrm{R} 1$ colocalized with the ER in axons (Fig. 2A, arrows). Control labeling without primary antibodies confirmed the specificity of the signal (Fig. 2B and 2C). In transgenic mouse neurons $\mathrm{GABA}_{\mathrm{B}} \mathrm{R}$ 1-EGFP also colocalized with the ER (Fig. 2D, arrows). Importantly, colocalization was still observed upon overexpression of MYG-GABA $\mathrm{B}$ R la and KDELRFP, a fluorescent probe widely used for ER visualization that contains the ER targeting sequence of calreticulin and the ER retrieval sequence KDEL (Fig. 2E, arrows). In addition, colocalization was observed with cytochrome b5-EGFP, another fluorescent ER probe (Fig. 2F). The colocalization between $\mathrm{GABA}_{\mathrm{B}} \mathrm{R}$ la and ER markers was specific because Piccolo, a marker for dense core vesicles and synapses [22] showed a markedly different axonal localization (Fig. 2G). Staining with MAP2, an exclusive dendritic marker confirmed the co-distribution of $\mathrm{GABA}_{\mathrm{B}} \mathrm{R}$ la and the ER occurs in the axon (Fig. $2 \mathrm{H}$ ). These results demonstrate that $\mathrm{GABA}_{\mathrm{B}} \mathrm{R}$ la is enriched in the axonal ER.

To establish whether the ER functions as a transport organelle for $\mathrm{GABA}_{\mathrm{B}} \mathrm{R}$ la we examined the dynamic behavior of fluorescent versions of $\mathrm{GABA}_{\mathrm{B}} \mathrm{R}$ la and the ER in axons of live hippocampal neurons. Discrete $\mathrm{GABA}_{\mathrm{B}} \mathrm{R}$ la-EGFP and KDEL-RFP puncta were distributed along the axons. While the majority of puncta remained static or showed very limited lateral displacement over the examined period, a subset displayed continuous, long-range mobility (imaged at $0.20-0.25$ frames/s for a total of $\sim 120 \mathrm{~s}$, Figs. $3 \mathrm{~A}$ and $3 \mathrm{~B})$. GABA $\mathrm{B}$ Rla-EGFP and KDEL-RFP moved bidirectionally, with a moderate retrograde bias, and with modal speeds of 100-200 nm/s (Figs. 3E and 3F). Significantly, some $\mathrm{GABA}_{\mathrm{B}} \mathrm{R}$ la-EGFP and KDEL-RFP puncta moved in synchrony, with similar speed and retrograde predominance (Figs. 3C and 3G). Puncta containing Rab1 1-GFP, a recycling endosome marker [23], also localized to axons but showed a different dynamic pattern characterized by rapid direction changes and lower overall displacement. We used TIRF microscopy and higher temporal resolution (2 frames/s) to determine the instant velocity of $\mathrm{GABA}_{\mathrm{B}} \mathrm{R}$ la-EGFP more accurately in hippocampal neurons. Mean anterograde and retrograde instant velocities were comparable $(751.70 \pm 33.20$ and $877.73 \pm 67.59 \mathrm{~nm} / \mathrm{s}$ respectively) (Figs. 3H and 3I). These values fit conventional kinesin velocities [24] and their slight increase above panels A-G most likely result from excluding stalls in the analysis of our higher temporal resolution imaging.

A proportion of $\mathrm{GABA}_{\mathrm{B}} \mathrm{R}$ la-EGFP and KDEL-RFP puncta moved independently from each other. This may indicate that a fraction of $\mathrm{GABA}_{\mathrm{B}} \mathrm{R}$ la-EGFP is transported in a different secretory organelle or that the axonal ER compartment is capable of dynamically segregating cargo. To discriminate between these possibilities we carried out a series of complementary experiments. First we analyzed time-lapse microscopy sequences individually. Interestingly, $\mathrm{GABA}_{\mathrm{B}} \mathrm{R}$ la-EGFP puncta that initially colocalized with the ER sometimes separated from the organelle, remained segregated for a few frames and fused again with a pre-existing ER compartment (Fig. 4A). It is well known that ER cargo recycles between the ER and the ERGIC using export/retrieval motifs such as the RXR-type sequence present in $\mathrm{GABA}_{\mathrm{B}} \mathrm{R}$ la [25]. To determine whether the segregated $\mathrm{GABA}_{\mathrm{B}} \mathrm{R}$ la puncta resided temporarily in the ERGIC, we first visualized the axonal distribution of MYC$\mathrm{GABA}_{\mathrm{B}} \mathrm{R} 1 \mathrm{a}$ and p58-YFP, an established marker of the ERGIC [26], in fixed cells. Sparse ERGIC puncta were observed in axons and a subset of them colocalized with MYG-GABA $\mathrm{B}$ la (Fig. 4B). Additionally, live-cell imaging was carried out in neurons transfected with $\mathrm{GABA}_{\mathrm{B}} \mathrm{R}$ la-RFP and p58-YFP. A small fraction of $\mathrm{GABA}_{\mathrm{B}} \mathrm{R}$ la-RFP displayed synchronous motility with p58-YFP in axons (Fig. 4C). Taking into account that intra-ER mobility is microtubule dependent but short-range ER to ERGIC transport is not [27], we reasoned that the enrichment of $\mathrm{GABA}_{\mathrm{B}} \mathrm{R}$ la in the ER should increase upon destabilization of microtubules. Consistent with this prediction, nocodazole blocked the mobility of $\mathrm{GABA}_{\mathrm{B}} \mathrm{R}$ la-EGFP puncta and the subunits accumulated in a KDEL-RFP compartment (Fig. 4D, top right panels). As expected, a mutant $\mathrm{GABA}_{\mathrm{B}} \mathrm{R}$ la subunit that is not retained in the ER $\left(\mathrm{GABA}_{\mathrm{B}} \mathrm{R}\right.$ la-ASA-EGFP) accumulated in a different compartment after nocodazole treatment (Fig. 4D, bottom right panels). These observations suggest that axonal $\mathrm{GABA}_{\mathrm{B}} \mathrm{R}$ la is targeted and transported to the axon within the $\mathrm{ER}$, and possibly engages in a local export/retrieval mechanism between the ER and the ERGIC.

\section{Kinesin-1 contributes to the axonal localization of $\mathrm{GABA}_{\mathrm{B}} \mathrm{R} 1 \mathrm{a}$}

Since kinesin-1, an axonal biased molecular motor [28], [29], colocalizes with $\mathrm{GABA}_{\mathrm{B}} \mathrm{R} 1$ in neurons, and associates to the subunit in fractionation and coimmunoprecipitation assays [30] we evaluated its contribution to axonal $\mathrm{GABA}_{\mathrm{B}} \mathrm{R}$ la targeting. To examine the axonal localization of $\mathrm{MYC}^{-G_{A B A} \mathrm{R}} \mathrm{la}$ we determined an axon-to-dendrite ratio (A:D ratio) [8]. To study the role of kinesin-1 we used a dominant negative comprising the cargo-binding domain of $\mathrm{Kif5} \mathrm{C}$ that interferes with endogenous kinesin-cargo interactions fused to RFP (Kif5C-RFP-DN) [16]. The axonal targeting of MYC-GABA $\mathrm{B}$ la was markedly reduced in the presence of Kif5C-RFP-DN (Fig. 5A and Fig. S3, arrows. $\mathrm{A}: \mathrm{D}$ ratio $\mathrm{MYC}-\mathrm{GABA}_{\mathrm{B}} \mathrm{R} 1 \mathrm{a} 0.63 \pm 0.12 ; \mathrm{MYC}^{-} \mathrm{GABA}_{\mathrm{B}} \mathrm{R} 1 \mathrm{a}$ plus Kif5C-RFP-DN 0.06 $\pm 0.02 ; \mathrm{p}<0.01)$. On the contrary, the axonal distribution of the ER marker ER-YFP and $\mathrm{GABA}_{\mathrm{B}} \mathrm{R} 2$-EGFP were not affected by Kif5C-RFP-DN (Fig. 5B. A:D ratio ER-YFP 0.64 \pm 0.47 ; ER-YFP plus Kif5C-RFP-DN 0.63 $\pm 0.32 ; p=0.97$; 
GABA $_{B}$ R2-EGFP 0.79 \pm 0.46 ; GABA $_{B}$ R2-EGFP plus Kif5C-RFPDN 0.69 $\pm 0.68 ; \mathrm{p}=0.80$ ).

Kinesin-1 may control the axonal localization of MYC$\mathrm{GABA}_{\mathrm{B}} \mathrm{R}$ la via an adaptor mechanism through the cytosolic C-terminal domain of $\mathrm{GABA}_{\mathrm{B}} \mathrm{R} 1 \mathrm{a}$. To directly test this we first determined whether axonal targeting required the cytosolic Cterminal domain of $\mathrm{GABA}_{\mathrm{B}} \mathrm{R}$ la using two $\mathrm{C}$-terminal subunit mutants, MYC-GABA $A_{B}$ la-AA-ASA and MYC-GABA ${ }_{B} R 1 a-\Delta C$ [15]. $\mathrm{GABA}_{\mathrm{B}} \mathrm{R} 1 \mathrm{a}, \mathrm{GABA}_{\mathrm{B}} \mathrm{R} 1 \mathrm{a}-\mathrm{AA}-\mathrm{ASA}$ and $\mathrm{GABA}_{\mathrm{B}} \mathrm{R} 1 \mathrm{a}-\Delta \mathrm{C}$ were all abundant in the axon indicating that targeting is independent of the C-terminal domain (Fig. 5C, arrows). Using a quantitative colocalization analysis based on Manders coefficients [31] all $\mathrm{GABA}_{\mathrm{B}} \mathrm{R}$ la constructs colocalized partially with the ER and no statistically significant difference was observed between $\mathrm{GABA}_{\mathrm{B}} \mathrm{R}$ la and the two mutants $\left(\mathrm{MYC}-\mathrm{GABA}_{\mathrm{B}} \mathrm{R}\right.$ la, $\mathrm{n}=9$ neurons; MYC-GABA ${ }_{\mathrm{B}} \mathrm{R}$ la-AA-ASA $\mathrm{p}=0.11, \mathrm{n}=5$ neurons; MYG-GABA $A_{B} R 1 a-\Delta C \quad p=0.30, n=5$ neurons). These results suggest that the $\mathrm{C}$-terminal domain is not a major determinant of axonal ER distribution and targeting of $\mathrm{GABA}_{\mathrm{B}} \mathrm{R}$ la.

Finally, we analyzed the kinesin-1 dependence on the axonal targeting of $\mathrm{GABA}_{\mathrm{B}} \mathrm{R}$ la lacking the $\mathrm{C}$-terminal domain. Axonal localization of MYC-GABA $\mathrm{B} l \mathrm{a}-\Delta \mathrm{C}$ was still severely impaired by the dominant negative construct (Fig. $5 \mathrm{D}$, arrows. A:D ratio MYC-GABA ${ }_{B} R 1 a-\Delta C \quad 0.42 \pm 0.06 ; \quad$ MYC-GABA ${ }_{B} R l a-\Delta C$ plus Kif5C-RFP-DN $0.05 \pm 0.01 ; \mathrm{p}<0.01)$. Combined these experiments demonstrate that kinesin- 1 is necessary for the ER axonal targeting and localization of $\mathrm{GABA}_{\mathrm{B}} \mathrm{R}$ la. In addition, they indicate that the $\mathrm{C}$-terminal domain of $\mathrm{GABA}_{\mathrm{B}} \mathrm{R} l \mathrm{a}$, which is exposed to the cytosol, is not involved in this transport mechanism, further supporting the role of luminal or ER membrane domains in axonal sorting and targeting.

\section{Discussion}

We have shown that $\mathrm{GABA}_{\mathrm{B}} \mathrm{R}$ s require the Golgi apparatus for plasma membrane delivery. Importantly, to our knowledge we have demonstrated for the first time that the sorting and targeting of an axonal neurotransmitter receptor, namely the $\mathrm{GABA}_{\mathrm{B}} \mathrm{R} 1 \mathrm{a}$ subunit, occur in a pre-Golgi compartment. Consistent with these observations, our evidence points to the fact that $\mathrm{GABA}_{\mathrm{B}} \mathrm{R}$ la traffics along the axonal ER and the ERGIC, and is transported by the molecular motor kinesin-1.

\section{Pre-Golgi sorting and targeting of axonal $G A B A_{B} R 1 a$}

Our study indicates that axonal sorting of $G_{A B A} R 1$ subunits operates in the ER. As reported elsewhere, axonal targeting of $\mathrm{GABA}_{\mathrm{B}} \mathrm{R} 1 \mathrm{a}$ is unaltered in $\mathrm{GABA}_{\mathrm{B}} \mathrm{R} 2$ knock-out neurons [8]. Additionally, the sushi domains located at the N-terminus of $\mathrm{GABA}_{\mathrm{B}} \mathrm{R}$ la, are sufficient for targeting even when placed in a nonrelated $\mathrm{CD} 8 \alpha$ protein context [8]. Combined with the results presented here these observations imply that sorting and targeting signals exposed to the ER lumen mediate the axonal localization of $\mathrm{GABA}_{\mathrm{B}} \mathrm{R}$ la.

Historically, sorting of plasma membrane proteins to distinct membrane domains has been thought to occur exclusively at the Golgi or the trans-Golgi network, but accumulated evidence now favors the view that decisions are made at almost every step along the secretory pathway including the ER [32]. For example, mutations in Sec24p, a component of the coat protein complex II (COPII), selectively disrupt recruitment of cargo for ER to Golgi transport [33]. Likewise, synthetic cell penetrating peptides based on the cytosolic domain of the temperature-sensitive VSVG protein only inhibits the transport of a subset of cargo from the ER to the Golgi apparatus [34].

Two possible targeting mechanisms are conceivable for $\mathrm{GABA}_{\mathrm{B}} \mathrm{R}$ la in axons: (i) a luminal or membrane spanning ER protein enriched in the axonal ER subcompartment may bind the sushi domains and produce the accumulation of $\mathrm{GABA}_{\mathrm{B}} \mathrm{R}$ la but not $\mathrm{GABA}_{\mathrm{B}} \mathrm{R} 1 \mathrm{~b}$, in the axon (selective retention); or (ii) a protein or protein complex that spans the ER membrane may function as an adaptor between the $\mathrm{GABA}_{\mathrm{B}} \mathrm{R}$ la subunit and a molecular motor and selectively direct the transport of the subunits to axons (selective transport). Additional axonal scaffolding proteins may anchor the $\mathrm{GABA}_{\mathrm{B}} \mathrm{R}$ la subunit to strengthen axonal localization.

Although we cannot rule out the first alternative our data are consistent with a selective transport mechanism. Axonal targeting of $\mathrm{GABA}_{\mathrm{B}} \mathrm{R}$ la is not altered by its cytosolic C-terminal. Thus, transport may be controlled by a specific $\mathrm{GABA}_{\mathrm{B}} \mathrm{R}$ la N-terminal adaptor or by a general ER adaptor complex. Identification of these molecules in future studies is needed to strengthen this hypothesis. A mechanism compatible with (i) has been described for the rotavirus VP7 glycoprotein, which is retained in the ER. VP7 is still transported to the axon after BFA treatment [35]. Similar to $\mathrm{GABA}_{\mathrm{B}} \mathrm{R} 1 \mathrm{a}$, VP7 uses a Golgi-independent intracellular sorting mechanism to reach the axon. One may envision the participation of chaperones in ER transport and targeting. For example, Hsc70 may provide a mechanism to release kinesin from cargo in specific subcellular domains, thereby producing the delivery of axonally transported cargo [36].

\section{Kinesin-1-dependent ER transport of $\mathrm{GABA}_{B} \mathrm{R} 1 \mathrm{a}$ in axons}

Since $\mathrm{GABA}_{\mathrm{B}} \mathrm{R} 1$ is transported along the $\mathrm{ER}$ and has a limited residency period within the organelle under physiological conditions we refer to it as an ER-boarded protein. The precise transport mechanism of ER-boarded $\mathrm{GABA}_{\mathrm{B}} \mathrm{R}$ la in the axon is not clear. A kinesin-1-dependent transport of ER proteins has been described in dendrites [37]. Likewise, a microtubuledependent transport of two ER resident proteins in the axon, GFP-SERCA and GFP-IP ${ }_{3} \mathrm{R}$, has been observed in cultured chick dorsal root ganglion neurons [38]. Their bidirectionality and average velocities $(\sim 0.1 \mu \mathrm{m} / \mathrm{s})$ suggest that the transport is likely non-vesicular, and may represent lateral displacement within the continuous axonal ER membrane or mobility of the organelle itself. Directionality at steady state and average velocities observed for GFP-SERCA and $\mathrm{GFP}_{-} \mathrm{IP}_{3} \mathrm{R}$ range between slow $(0.001-0.03 \mu \mathrm{m} / \mathrm{s})$ and fast axonal transport $(\sim$ $1 \mu \mathrm{m} / \mathrm{s}$ ) [38,39]. These intermediate velocities are conserved in our study suggesting that similar mechanisms may operate for the transport of ER-boarded $\mathrm{GABA}_{\mathrm{B}} \mathrm{R} 1 \mathrm{a}$ in axons. A conserved transport mechanism is also supported by the microtubule dependence of axonal GFP-SERCA, GFP-IP ${ }_{3} \mathrm{R}$ and $\mathrm{GABA}_{\mathrm{B}} \mathrm{R} 1 \mathrm{a}$ mobility. Thus, as reported for ER resident proteins al least one component of the mobility of $\mathrm{GABA}_{\mathrm{B}} \mathrm{R} 1 \mathrm{a}$ is not mediated by simple diffusion. However, precisely how the microtubulecytoskeleton mediates the movement of integral membrane proteins within the continuous ER network is still unclear. According to the mechanisms originally put forward by Tsukita and Ishikawa [40], Waterman-Storer and Salmon [41] and others [42] kinesin-1 may control the lateral displacement of the subunit along the ER membrane in a conveyor-like system or mediate the microtubule-dependent ER sliding of the organelle itself, which may contribute to $\mathrm{GABA}_{\mathrm{B}} \mathrm{R}$ la transport. Since components of both mechanisms are dependent on kinesin-1 and stable microtubules it is difficult to discriminate between them. Because in this study we wanted to eliminate microtubules that 
serve as tracks to kinesin-1, the concentrations of nocodazole used were $\sim 15$ fold higher than those that affect exclusively the population of dynamic microtubules $(100 \mu \mathrm{M}$ versus $\sim 6 \mu \mathrm{M})$. Thus, additionally, we cannot rule out the contribution of dynamic microtubules to the transport of $\mathrm{GABA}_{\mathrm{B}} \mathrm{R} 1 \mathrm{a}$ in the axon [41]. The existence of discrete mobile packets for axonal $\mathrm{GABA}_{\mathrm{B}} \mathrm{R}$ la also raises the possibility that these are not continuous with the ER network. Electron microscope studies in central and peripheral axons have revealed that the structure of the ER is predominantly contiguous but that it contains occasional free elements [40], [43]. Additionally, isolated and mobile compartments have been observed with fluorescent reporters [38]. Puncta-like behavior may also be explained by transitory tubule fission/fusion events [40] or lateral mobility of protein aggregates. The precise relationship between the mobile structures observed in this and other studies with the continuous ER network requires further studies. Nonetheless, our results support a kinesin-1-mediated axonal transport of ER-boarded $\mathrm{GABA}_{\mathrm{B}} \mathrm{R}$ la that is in agreement with previous reports.

The majority of the axonal $\mathrm{GABA}_{\mathrm{B}} \mathrm{R}$ la subunit was static during the live imaging conditions and intervals examined in this study. Additionally, the mobile fraction was higher for a mutant subunit that escapes the ER (MYC-GABA $\mathrm{B}$ Rla-EGFP: $19 \%$ mobile puncta, $\mathrm{n}=259$; MYC-GABA ${ }_{\mathrm{B}} \mathrm{R}$ la-ASA-AA-EGFP $33 \%$ mobile puncta, $n=247$ ). It will be interesting to understand the significance and properties of the static component of $\mathrm{GABA}_{\mathrm{B}} \mathrm{R} 1 \mathrm{a}$ in the ER, and whether ER immobility plays a role in processing or trafficking.

Previous studies have demonstrated that $\mathrm{GABA}_{\mathrm{B}} \mathrm{Rs}$ are segregated intracellularly and that blockade of ER exit results in the accumulation of heteromers in the soma and dendrites of hippocampal neurons [4]. These results suggest that $\mathrm{GABA}_{\mathrm{B}} \mathrm{R}$ subunits are transported into dendrites independently and not as assembled heteromers. They also suggest that newly synthesized $\mathrm{GABA}_{\mathrm{B}} \mathrm{Rs}$ assemble in the ER and exit throughout the somatodendritic compartment prior to insertion at the plasma membrane. This idea is in agreement with transport within the ER and disagrees with a long-haul post-Golgi vesicular transport of $\mathrm{GABA}_{\mathrm{B}} \mathrm{Rs}$, suggesting a non-canonical trafficking modality for $\mathrm{GABA}_{\mathrm{B}} \mathrm{Rs}$ in dendrites. Thus, long-range ER transport may underlie both dendritic and axonal targeting of $\mathrm{GABA}_{\mathrm{B}} \mathrm{R}$ s. Since the local complexity of the ER network influences intracellular trafficking it may be utilized by neurons to control the availability of dendritic and axonal membrane proteins that are spatially restricted [44], [45].

\section{Axonal trafficking and ER/ERGIC recycling}

In central and peripheral axons the ER is a continuous threedimensional network of irregular tubules and cisternae [40], [43]. As mentioned above, several studies have demonstrated the localization of ER resident proteins and components of the protein folding and export machineries in the axon [19-20]. More importantly, the discovery of local assembly of COPII components in the axon supports the existence of functional ER exit sites required to process newly synthesized proteins that contribute to axonal outgrowth during the early stages of development [46]. However, direct evidence for local protein trafficking within early biosynthetic organelles in the axon is still lacking. In Drosophila, polarized secretion of the EGFR ligand from photoreceptor neurons includes local processing and secretion in the axon, and both mechanisms are controlled by ER localization [47].

More functional evidence is needed to conclusively demonstrate the role of a local trafficking pathway for $\mathrm{GABA}_{\mathrm{B}} \mathrm{R} 1 \mathrm{a}$ in axons. However, a fraction of $\mathrm{GABA}_{\mathrm{B}} \mathrm{R}$ la colocalizes and moves in synchrony with the ERGIC. This indicates that a local route involving export and retrieval between early biosynthetic organelles may contribute to $\mathrm{GABA}_{\mathrm{B}} \mathrm{R}$ la trafficking in the axon. A coat protein I complex (COPI) dependent retrieval mechanism from the cis-Golgi to the ER has been postulated for $\mathrm{GABA}_{\mathrm{B}} \mathrm{R}$ la [48], [49]. Whether this occurs in the axon and whether the remaining secretory steps operate locally to deliver $\mathrm{GABA}_{\mathrm{B}} \mathrm{R} 1 \mathrm{a}$ to the axonal plasma membrane remains unclear. In any case, the presence of the same RXR-type ER retention motif in $\mathrm{GABA}_{\mathrm{B}} \mathrm{R}$ la and $\mathrm{GABA}_{\mathrm{B}} \mathrm{R} 1 \mathrm{~b}$ suggests that despite determining a rate-limiting step for ER exit, dwell time in the ER is not a major determinant of spatial range and axonal localization of GABA${ }_{\mathrm{B}} \mathrm{R} 1 \mathrm{a}$ [50].

Overall our study demonstrates that ER sorting and local transport are relevant for axonal $\mathrm{GABA}_{\mathrm{B}} \mathrm{R}$ trafficking. It is of great interest to determine to what extent the axonal ER is involved in the trafficking of other neurotransmitter receptors and ion channels that travel long distances, especially in long peripheral nerves.

\section{Supporting Information}

Figure $S 1$ Antibodies recognize GABA $_{B} R 1$ subunits specifically. (A) Increasing concentrations of lysates prepared from MYC-GABA $A_{B}$ la transfected cells were applied on a nitrocellulose membrane and immunoblotted with control serum (control) or serum from a rabbit immunized with a GST fusion protein containing the $\mathrm{C}$-terminal domain of $\mathrm{GABA}_{\mathrm{B}} \mathrm{R} 1(\alpha \mathrm{R} 1)$. ( $\left.\mathbf{B}\right)$ Lysates prepared from crude rat brain membranes (Brain), untransfected COS7 cells (Mock), or MYG-GABA ${ }_{\mathrm{B}} \mathrm{R}$ la transfected COS7 cells (MYC-GBla) were immunoblotted with affinity purified $\mathrm{GABA}_{\mathrm{B}} \mathrm{R} 1$ antibodies. $(\mathbf{C}) \mathrm{COS} 7$ were transfected with MYG-GABA ${ }_{\mathrm{B}}$ R la (MYG-GB1a, top), HA-GABA ${ }_{\mathrm{B}}$ 2 (HA-GB2, middle) or left untransfected (bottom). Cells were fixed and processed for immunofluorescence using MYC antibodies (green), affinity purified $\mathrm{GABA}_{\mathrm{B}} \mathrm{R} 1$ antibodies (red) and $\mathrm{HA}$ antibodies (magenta). Merged images are shown on the right. Scale bar represents $20 \mu \mathrm{m}$.

\section{(TIF)}

Figure S2 Recombinant GABA $_{B} R 1$ a is retained in intracellular compartments in hippocampal neurons. $(A)$ Hippocampal neurons were transfected with MYG-GABA $\mathrm{B}_{\mathrm{B}} \mathrm{R}$ a and processed for immunofluorescence under non-permeabilized conditions to detect cell surface epitopes followed by permeabilization to detect intracellular epitopes at the indicated days posttransfection (dpt). Intracellular MAP2 (red), intracellular GABA${ }_{B} \mathrm{R} 1 \mathrm{a}$ (MYC-GB1 intracellular, green), plasma membrane GA$\mathrm{BA}_{\mathrm{B}} \mathrm{R}$ la (MYC-GBl membrane, magenta). Control neurons were

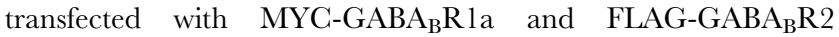
(+FLAG-GB2, right column). Merged images are shown on the bottom panel. (B) Axons of hippocampal neurons under the same experimental conditions. Images are not single focus planes, therefore the intensity represents the signal from the entire cell (representative images of $\mathrm{n}=30$ neurons). Scale bar for $\mathrm{A}-\mathrm{B}$ represents $20 \mu \mathrm{m}$.

(TIF)

Figure S3 Axonal targeting of $\mathrm{GABA}_{\mathrm{B}} \mathrm{R} 1 \mathrm{a}$ is kinesin-1 dependent. (A) Hippocampal neurons were transfected with MYC-GABA ${ }_{\mathrm{B}} \mathrm{R}$ la (MYG-GB1) and RFP. Merged images are shown on the bottom panel. (B) Same as above for MYG$\mathrm{GABA}_{\mathrm{B}} \mathrm{R}$ la and Kif5C-RFP-DN. Axonal localization of MYG$\mathrm{GABA}_{\mathrm{B}} \mathrm{R}$ la or its absence from the axon is indicated by arrows. Scale bar represents $20 \mu \mathrm{m}$. 
(TIF)

\section{Acknowledgments}

We thank B. Bettler, F. Bronfman, O. Jeyifous, S. Kindler, H.J. Kreienkamp, C. Hetz and J. Lippincott-Schwartz for valuable reagents.

\section{References}

1. Horton AC, Ehlers MD (2004) Secretory trafficking in neuronal dendrites. Nat Cell Biol 6: 585-591.

2. Bettler B, Kaupmann K, Mosbacher J, Gassmann M (2004) Molecular structure and physiological functions of GABA(B) receptors. Physiol Rev 84: 835-867.

3. Couve A, Filippov AK, Connolly CN, Bettler B, Brown DA, et al. (1998) Intracellular retention of recombinant GABAB receptors. J Biol Chem 273: 26361-26367.

4. Ramírez OA, Vidal RL, Tello JA, Vargas KJ, Kindler S (2009) Dendritic Assembly of Heteromeric g-Aminobutyric Acid Type B Receptor Subunits in Hippocampal Neurons. J Biol Chem 284: 13077-13085.

5. Margeta-Mitrovic M, Jan YN, Jan LY (2000) A trafficking checkpoint controls GABA(B) receptor heterodimerization. Neuron 27: 97-106.

6. Kulik A, Vida I, Luján R, Haas CA, López-Bendito G (2003) Subcellular localization of metabotropic $\mathrm{GABA}(\mathrm{B})$ receptor subunits $\mathrm{GABA}(\mathrm{B} 1 \mathrm{a} / \mathrm{b})$ and GABA(B2) in the rat hippocampus. J Neurosci 23: 11026-11035.

7. Vigot R, Barbieri S, Bräuner-Osborne H, Turecek R, Shigemoto R (2006) Differential compartmentalization and distinct functions of GABAB receptor variants. Neuron 50: 589-601.

8. Biermann B, Ivankova-Susankova K, Bradaia A, Abdel-Aziz S, Besseyrias V (2010) The Sushi domains of GABAB receptors function as axonal targeting signals. J Neurosci 30: 1385-1394.

9. Schuler V, Lüscher C, Blanchet C, Klix N, Sansig G (2001) Epilepsy, hyperalgesia, impaired memory, and loss of pre- and postsynaptic GABA(B) responses in mice lacking GABA(B(1)). Neuron 31: 47-58.

10. Casanova E, Guetg N, Vigot R, Seddik R, Julio-Pieper M (2009) A mouse model for visualization of GABA(B) receptors. Genesis 47: 595-602.

11. Goslin K, Banker G (1991) Rat hippocampal neurons in low-density cultures. In, Culturing nerve cells, Banker G, Goslin K, eds. 251-281. Cambridge, MA, MIT

12. Jiang M, Chen G (2006) High Ca2+-phosphate transfection efficiency in lowdensity neuronal cultures. Nat Protoc 1: 695-700.

13. Calver AR, Robbins MJ, Cosio G, Rice SQ Babbs AJ (2001) The C-terminal domains of the GABA(B) receptor subunits mediate intracellular trafficking but are not required for receptor signaling. J Neurosci 21: 1203-1210.

14. Couve A, Thomas P, Calver AR, Hirst WD, Pangalos MN, etal. (2002) Cyclic AMP-dependent protein kinase phosphorylation facilitates GABA(B) receptoreffector coupling. Nat Neurosci 5: 415-424

15. Restituito S, Couve A, Bawagan H, Jourdain S, Pangalos MN (2005) Multiple motifs regulate the trafficking of $\mathrm{GABA}(\mathrm{B})$ receptors at distinct checkpoints within the secretory pathway. Mol Cell Neurosci 28: 747-756.

16. Falley K, Schütt J, Iglauer P, Menke K, Maas C, et al. (2009) Shank1 mRNA: dendritic transport by kinesin and translational control by the 5 'untranslated region. Traffic 10: 844-857.

17. Zhang CJ, Rosenwald AG, Willingham MC, Skuntz S, Clark J (1994) Expression of a dominant allele of human ARF1 inhibits membrane traffic in vivo. J Cell Biol 124: 289-300.

18. Willis D, Li KW, Zheng JQ Chang JH, Smit A, et al. (2005) Differential transport and local translation of cytoskeletal, injury-response, and neurodegeneration protein mRNAs in axons. J Neurosci 25: 778-791.

19. Merianda TT, Lin AC, Lam JS, Vuppalanchi D, Willis DE (2009) A functional equivalent of endoplasmic reticulum and Golgi in axons for secretion of locally synthesized proteins. Mol Cell Neurosci 40: 128-142.

20. Weclewicz K, Svensson L, Kristensson K (1998) Targeting of endoplasmic reticulum-associated proteins to axons and dendrites in rotavirus-infected neurons. Brain Res Bull 46: 353-360.

21. Munro S, Pelham HR (1987) A C-terminal signal prevents secretion of luminal ER proteins. Cell 48: 899-907.

22. tom Dieck S, Sanmartí-Vila L, Langnaese K, Richter K, Kindler S (1998) Bassoon, a novel zinc-finger CAG/glutamine-repeat protein selectively localized at the active zone of presynaptic nerve terminals. J Cell Biol 142: 499-509.

23. Jones MC, Caswell PT, Norman JC (2006) Endocytic recycling pathways: emerging regulators of cell migration. Curr Opin Cell Biol 18: 549-557.

24. Cai D, Verhey KJ, Meyhöfer E (2007) Tracking single Kinesin molecules in the cytoplasm of mammalian cells. Biophys J 92: 4137-44.

25. Bonifacino JS, Lippincott-Schwartz J (2003) Coat proteins: shaping membrane transport. Nat Rev Mol Cell Biol 4: 409-414.

26. Ward TH, Polishchuk RS, Caplan S, Hirschberg K, Lippincott-Schwartz J (2001) Maintenance of Golgi structure and function depends on the integrity of ER export. J Cell Biol 155: 557-570.

27. Ben-Tekaya H, Miura K, Pepperkok R, Hauri HP (2005) Live imaging of bidirectional traffic from the ERGIC. J Cell Sci 118: 357-367.

\section{Author Contributions}

Conceived and designed the experiments: VV CFS AC. Performed the experiments: VV JIV MJ-B DS CO CT. Wrote the paper: AC.

28. Nakata T, Hirokawa N (2003) Microtubules provide directional cues for polarized axonal transport through interaction with kinesin motor head. J Cell Biol 162: 1045-1055.

29. Jacobson C, Schnapp B, Banker GA (2006) A change in the selective translocation of the Kinesin-1 motor domain marks the initial specification of the axon. Neuron 49: 797-804.

30. Vidal RL, Ramírez OA, Sandoval L, Koenig-Robert R, Härtel S (2007) Marlin1 and conventional kinesin link GABAB receptors to the cytoskeleton and regulate receptor transport. Mol Cell Neurosci 35: 501-512.

31. Ramírez O, García A, Rojas R, Couve A, Härtel S (2010) Confined displacement algorithm determines true and random colocalization in fluorescence microscopy. J Microsc 239: 173-183.

32. Rodríguez-Boulan E, and Müsch A (2005) Protein sorting in the Golgi complex: shifting paradigms. Biochim Biophys Acta 1744: 455-464.

33. Miller EA, Beilharz TH, Malkus PN, Lee MC, Hamamoto S (2003) Multiple cargo binding sites on the COPII subunit Sec24p ensure capture of diverse membrane proteins into transport vesicles. Cell 114: 497-509.

34. Soza A, Norambuena A, Cancino J, de la Fuente E, Henklein P (2004) Sorting competition with membrane-permeable peptides in intact epithelial cells revealed discrimination of transmembrane proteins not only at the trans-Golgi network but also at pre-Golgi stages. J Biol Chem 279: 17376-17383.

35. Weclewicz K, Kristensson K, Greenberg HB, Svensson L (1993) The endoplasmic reticulum-associated VP7 of rotavirus is targeted to axons and dendrites in polarized neurons. J Neurocytol 22: 616-626.

36. Terada S, Kinjo M, Aihara M, Takei Y, Hirokawa N (2010) Kinesin-1/Hsc70dependent mechanism of slow axonal transport and its relation to fast axonal transport. EMBO J 29: 843-854.

37. Bannai H, Inoue T, Nakayama T, Hattori M, Mikoshiba K (2004) Kinesin dependent, rapid, bi-directional transport of ER sub-compartment in dendrites of hippocampal neurons. J Cell Sci 117: 163-175.

38. Aihara Y, Inoue T, Tashiro T, Okamoto K, Komiya Y, et al. (2001) Movement of endoplasmic reticulum in the living axon is distinct from other membranous vesicles in its rate, form, and sensitivity to microtubule inhibitors. J Neurosci Res 65: 236-246.

39. Shah JV, Cleveland DW (2002) Slow axonal transport: fast motors in the slow lane. Curr Opin Cell Biol 14: 58-62.

40. Tsukita S, Ishikawa H (1976) Three-dimensional distribution of smooth endoplasmic reticulum in myelinated axons. J Electron Microsc 25: 141-149.

41. Waterman-Storer CM, Salmon ED (1998) Endoplasmic reticulum membrane tubules are distributed by microtubules in living cells using three distinct mechanisms. Curr Biol 8: 798-806.

42. Wozniak MJ, Bola B, Brownhill K, Yang YC, Levakova V (2009) Role of kinesin-1 and cytoplasmic dynein in endoplasmic reticulum movement in VERO cells. J Cell Sci 122: 1979-1989.

43. Broadwell RD, Cataldo AM (1984) The neuronal endoplasmic reticulum: its cytochemistry and contribution to the endomembrane system. II. Axons and terminals. J Comp Neurol 230: 231-248.

44. Ramírez OA, and Couve A (2011) The endoplasmic reticulum and protein trafficking in dendrites and axons. Trends Cell Biol 21: 219-227.

45. Cui-Wang T, Hanus C, Cui T, Helton T, Bourne J, Watson D, et al. (2012) Local zones of endoplasmic reticulum complexity confine cargo in neuronal dendrites. Cell 148: 309-321.

46. Aridor M, Fish KN (2009) Selective targeting of ER exit sites supports axon development. Traffic 10: 1669-1684.

47. Yogev S, Schejter ED, Shilo B-Z (2010) Polarized Secretion of Drosophila EGFR Ligand from Photoreceptor Neurons Is Controlled by ER Localization of the Ligand-Processing Machinery. PLoS Biol 8(10): e1000505. doi:10.1371/ journal.pbio. 1000505 .

48. Brock C, Boudier L, Maurel D, Blahos J, Pin JP (2005) Assembly-dependent surface targeting of the heterodimeric GABAB Receptor is controlled by COPI but not 14-3-3. Mol Biol Cell 16: 5572-5578.

49. Villemure JF, Adam L, Bevan NJ, Gearing K, Chénier S (2005) Subcellular distribution of GABA(B) receptor homo- and hetero-dimers. Biochem J 388: 47 55.

50. Herpers B, Rabouille G (2004) mRNA localization and ER-based protein sorting mechanisms dictate the use of transitional endoplasmic reticulum-golgi units involved in gurken transport in Drosophila oocytes. Mol Biol Cell 15: 53065317. 



\section{List of references}





\section{In alphabetical order of labels}

[Adams00]

[Adio09]

[Aihara01]

[Allersma98]

[Aridor09]

[Arora02]

[Avunie-Masala11]

[Bannai04]

[Ben-Tekaya05]

[Bettler04]

[Biermann10]

[Blangy95]
I. R. Adams and J. V. Kilmartin; 2000: Spindle pole body duplication: a model for centrosome duplication?, Trends in Cell Biology, 10: 329335 .

S. Adio, J. Jaud, B. Ebbing, M. Rief and G. Woehlke; 2009: Dissection of kinesin's processivity, PLoS ONE, e4612: 4.

Y. Aihara, T. Inoue, T. Tashiro, K. Okamoto, Y. Komiya and K. Mikoshiba; 2001: Movement of endoplasmic reticulum in the living axon is distinct from other membranous vesicles in its rate, form, and sensitivity to microtubule inhibitors, The Journal of Neuroscience Research, 65: 236-246.

M. W. Allersma, F. Gittes, M. J. de Castro, R. J. Stewart and C. F. Schmidt; 1998: Two-Dimensional Tracking of ncd Motility by Back Focal Plane Interferometry, Biophysical Journal, 74: 1074-1085.

M. Aridor and K. N. Fish; 2009: Selective targeting of ER exit sites supports axon development, Traffic, 10: 1669-1684.

P. S. Arora, A. Z. Ansari, T. P. Best, M. Ptashne and P. B. Dervan; 2002: Design of Artificial Transcriptional Activators with Rigid Poly-1-proline Linkers, Journal of the American Chemical Society, 124: $13067-13071$.

R. Avunie-Masala, N. Movshovich, Y. Nissenkorn, A. Gerson-Gurwitz, V. Fridman, M. Koivomagi, M. Loog, M. A. Hoyt, A. Zaritsky and L. Gheber; 2011: Phospho-regulation of kinesin-5 during anaphase spindle elongation, Journal of Cell Science, 124: 873-878.

H. Bannai, T. Inoue, T. Nakayama, M. Hattori and K. Mikoshiba; 2004: Kinesin dependent, rapid, bi-directional transport of $\mathrm{ER}$ sub-compartment in dendrites of hippocampal neurons, Journal of Cell Science, 117: 163-175.

H. Ben-Tekaya, K. Miura, R. Pepperkok and H. P. Hauri; 2005: Phospho-regulation of kinesin-5 during anaphase spindle elongation, Journal of Cell Science, 118: 357-367.

B. Bettler, K. Kaupmann, J. Mosbacher and M. Gassmann; 2004: Molecular structure and physiological functions of $\mathrm{GABA}_{B}$ receptors, Physiological Reviews, 84: 835-867.

B. Biermann, K. Ivankova-Susankova, A. Bradaia, S. Abdel-Aziz and V. Besseyrias; 2010: The Sushi domains of $\mathrm{GABA}_{B}$ receptors function as axonal targeting signals, The Journal of Neuroscience, 30: $1385-1394$.

A. Blangy, H. A. Lane, P. d'Hérin, M. Harper, M. Kress and E. A. Nigg; 1995: Phosphorylation by p34cdc2 regulates spindle association of human Eg5, a kinesin-related motor essential for bipolar spindle formation in vivo, Cell, 83: 1159-1169. 
[Block90]

[Block07]

[Bodey09]

[Bonifacino03]

[Bormuth09]

[Broadwell84]

[Brock05]

[Brouhard08]

[Brust-Mascher04]

[Burton97]

[Butterfield10]

[Cahu09]

[Cai07]

[Calver01]

[Carter05]
S. M. Block, L. S. B. Goldstein and B. J. Schnapp; 1990: Bead movement by single kinesin molecules studied with optical tweezers, Nature, 348: 348-352.

S. M. Block; 2007: Kinesin motor mechanics: binding, stepping, tracking, gating, and limping, Biophysical Journal, 92: 2986-2995.

A. J. Bodey, M. Kikkawa and C. A. Moores; 2009: 9-Ångstrom structure of a microtubule-bound mitotic motor, Journal of Molecular Biology, 388: $218-224$.

J. S. Bonifacino and J. Lippincott-Schwartz; 2003: Coat proteins: shaping membrane transport, Nature Reviews Molecular Cell Biology, 4: 409-414.

V. Bormuth, V. Varga, J. Howard and E. Schaffer; 2009: Protein friction limits diffusive and directed movements of kinesin motors on microtubules, Science, 325: 870-873.

R. D. Broadwell and A. M. Cataldo; 1984: The neuronal endoplasmic reticulum: its cytochemistry and contribution to the endomembrane system. II. Axons and terminals, The Journal of Comparative Neurology, 230: 231-248.

C. Brock, L. Boudier, D. Maurel, J. Blahos and J. P. Pin; 2005: Assembly-dependent surface targeting of the heterodimeric $\mathrm{GABA}_{B}$ Receptor is controlled by COPI but not 14-3-3, Molecular Biology of the Cell, 16: $5572-5578$.

G. J. Brouhard, J. H. Stear, T. L. Noetzel, J. Al-Bassam, K. Kinoshita, S. C. Harrison, J. Howard and A. A. Hyman; 2008: XMAP215 is a processive microtubule polymerase, Cell, 132: 79-88.

I. Brust-Mascher, G. Civelekoglu-Scholey, M. Kwon, A. Mogilner and J. M. Scholey; 2004: Model for anaphase B: role of three mitotic motors in a switch from poleward flux to spindle elongation, PNAS (USA), 101: 15938-15943.

K. Burton and D. L. Taylor; 1997: Traction forces of cytokinesis measured with optically modified elastic substrata, Nature, 385: $450-454$.

A. E. Butterfield, R. J. Stewart, C. F. Schmidt and M. Skliar; 2010: Bidirectional power stroke by ncd kinesin, Biophysical Journal, 99: 3905-3915.

J. Cahu and T. Surrey; 2009: Motile microtubule crosslinkers require distinct dynamic properties for correct functioning during spindle organization in Xenopus egg extract, Journal of Cell Science, 122: 1295-1300.

D. Cai, K. J. Verhey and E. Meyhöfer; 2007: Tracking single Kinesin molecules in the cytoplasm of mammalian cells, Biophysical Journal, 92: 4137-4144.

A. R. Calver, M. J. Robbins, C. Cosio, S. Q. Rice and A. J. Babbs; 2001: The C-terminal domains of the $\mathrm{GABA}_{B}$ receptor subunits mediate intracellular trafficking but are not required for receptor signaling, The Journal of Neuroscience, 21: 1203-1210.

N. J. Carter and R. A. Cross; 2005: Mechanics of the kinesin step, Nature, 435: 308-312. 
[Casanova09]

[Case97]

[Castro00]

[Chee10]

[Chua07]

[Clancy11]

[Cochran05]

[Cole94]

[Couve98]

[Couve02]

[Coy99a]

[Coy99b]

[Crevel97]

[Crevel99]

[Crevel04a]
E. Casanova, N. Guetg, R. Vigot, R. Seddik and M. Julio-Pieper; 2009: A mouse model for visualization of $\mathrm{GABA}_{B}$ receptors, Genesis, 47: 595-602.

R. B. Case, D. W. Pierce, N. Hom-Booher, C. L. Hart and R. D. Vale; 1997: The directional preference of kinesin motors is specified by an element outside of the motor catalytic domain, Cell, 90: 959-966.

M. J. de Castro, R. M. Fondecave, L. A. Clarke, C. F. Schmidt and R. J. Stewart; 2000: Working strokes by single molecules of the kinesin-related microtubule motor ncd, Nature Cell Biology, 2: 724729 .

M. K. Chee and S. B. Haase; 2010: B-cyclin/CDKs regulate mitotic spindle assembly by phosphorylating kinesins-5 in budding yeast, PLoS Genetics, 6: e1000935.

P. R. Chua, D. M. Roof, Y. Lee, R. Sakowicz, D. Clarke, D. Pierce, T. Stephens, M. Hamilton, B. Morgan, D. Morgans, T. Nakai, A. Tomasi and M. E. Maxon; 2007: Effective killing of the human pathogen Candida albicans by a specific inhibitor of non-essential mitotic kinesin Kip1p, Molecular Microbiology, 65: 347-362.

B. E. Clancy, W. M. Behnke-Parks, J. O. Andreasson, S. S. Rosenfeld and S. M. Block; 2011: A universal pathway for kinesin stepping, Nature Structural and Molecular Biology, 18: 1020-1027.

J. C. Cochran, J. E. Gatial, T. M. Kapoor and S. P. Gilbert; 2005: Monastrol inhibition of the mitotic kinesin Eg5, The Journal of Biological Chemistry, 280: 12658-12667.

D. G. Cole, W. M. Saxton, K. B. Sheehan and J. M. Scholey; 1994: A 'slow', homotetrameric kinesin-related motor protein purified from Drosophila embryos, The Journal of Biological Chemistry, 269: 22913-22916.

A. Couve, A. K. Filippov, C. N. Connolly, B. Bettler, D. A. Brown and S. J. Moss; 1998: Intracellular retention of recombinant GABA $_{B}$ receptors, The Journal of Biological Chemistry, 273: 26361-26367.

A. Couve, P. Thomas, A. R. Calver, W. Hirst, M. N. Pangalos, F. S. Walsh, T. G. Smart and S. J. Moss; 2002: Cyclic AMP-dependent protein kinase phosphorylation facilitates $\mathrm{GABA}_{B}$ receptor-effector coupling, Nature Neuroscience, 5: 415-424.

D. L. Coy, W. O. Hancock, M. Wagenbach and J. Howard; 1999: Kinesin's tail domain is an inhibitory regulator of the motor domain, $\mathrm{Na}$ ture Cell Biology, 1: 288-292.

D. L. Coy, M. Wagenbach and J. Howard; 1999: Kinesin takes one eight-nanometer step for each ATP that it hydrolyzes, The Journal of Biological Chemistry, 276: 3667-3671.

I. M. Crevel, A. Lockhart and R. A. Cross; 1997: Kinetic evidence for low chemical processivity in ncd and Eg5, Journal of Molecular Biology, 273: 160-170.

I. M. Crevel, N. Carter, M. Schliwa and R. A. Cross; 1999: Coupled chemical and mechanical reaction steps in a processive Neurospora kinesin, The EMBO Journal, 18: 5863-5872.

I. M. Crevel, M. C. Alonso and R. A. Cross; 2004: Monastrol stabilises an attached low-friction mode of Eg5, Current Biology, 14: 411-412. 
[Crevel04b]

[Cuevas92]

[Cui-Wang12]

[Cytrynbaum03]

[Dagenbach04]

[Dieck98]

[Diefenbach98]

[Dietrich08]

[Dijk07]

[Dixit08]

[Düselder12]

[Endow98]

[Endow00]

[Enos90]

[Eunen10]
I. M. Crevel, M. Nyitrai, M. C. Alonso, S. Weiss, M. A. Geeves and R. A. Cross; 2004: What kinesin does at roadblocks: the coordination mechanism for molecular walking, The EMBO Journal, 23: 23-32.

M. de Cuevas, T. Tao and L. S. Goldstein; 1992: Evidence that the stalk of Drosophila kinesin heavy chain is an alpha-helical coiled coil, The Journal of Cell Biology, 116: 957-965.

T. Cui-Wang, C. Hanus, T. Cui, T. Helton, J. Bourne, D. Watson, K. M. Harris and M. D. Ehlers; 2012: Local zones of endoplasmic reticulum complexity confine cargo in neuronal dendrites, Cell, 148: 309321 .

E. Cytrynbaum, J. M. Scholey and A. Mogilner; 2003: Force-balance model for early spindle pole separation in Drosophila embryonic mitotic spindles, Biophysical Journal, 84: 757-769.

E. M. Dagenbach and S. A. Endow; 2004: A new kinesin tree, Journal of Cell Science, 117: 3-7.

S. T. Dieck, L. Sanmartí-Vila, K. Langnaese, K. Richter and S. Kindler; 1998: Bassoon, a novel zinc-finger CAG/glutamine-repeat protein selectively localized at the active zone of presynaptic nerve terminals, The Journal of Cell Biology, 142: 142-509.

R. J. Diefenbach, J. P. Mackay, P. J. Armati and A. L. Cunningham; 1998: The C-terminal region of the stalk domain of ubiquitous human kinesin heavy chain contains the binding site for kinesin light chain, Biochemistry, 37: 16663-16670.

K. A. Dietrich, C. V. Sindelar, P. D. Brewer, K. H. Downing, C. R. Cremo and S. E. Rice; 2008: The kinesin-1 motor protein is regulated by a direct interaction of its head and tail, PNAS (USA), 105: 8938-8943.

M. A. van Dijk, L. C. Kapitein, J. van Mameren, C. F. Schmidt and E. J. G. Peterman; 2007: Combining Optical Trapping and Single-Molecule Fluorescence Spectroscopy: Enhanced Photobleaching of Fluorophores, Journal of Physical Chemistry, 108: 6479-6484.

R. Dixit, J. L. Ross, Y. E. Goldman and E. L. Holzbaur; 2008: Differential regulation of dynein and kinesin motor proteins by tau, Science, 319: 1086-1089.

A. Düselder, C. Thiede, C. F. Schmidt and S. Lakämper; 2012: Neck-Linker Length Dependence of Processive Kinesin-5 Motility, Journal of Molecular Biology, 423: 159-168.

S. A. Endow and K. W. Waligora; 1998: Determinants of kinesin motor polarity, Science, 281: 1200-1202.

S. A. Endow and H. Higuchi; 2000: A mutant of the motor protein kinesin that moves in both directions on microtubules, Nature, 406: 913-916.

A. P. Enos and N. R. Morris; 1990: Mutation of a gene that encodes a kinesin-like protein blocks nuclear division in $A$. nidulans, Cell, 60: 1019-1027.

K. van Eunen, J. Bouwman, P. Daran-Lapujade, J. Postmus, A. B. Canelas, F. I. Mensonides, R. Orij, I. Tuzun, J. van den Brink, G. J. Smits, W. M. van Gulik, S. Brul, J. J. Heijnen, J. H. de Winde, 
[Falley09]

[Ferenz10]

[Forman84]

[Fridman09]

[Friedman99]

[Gardner08a]

[Gardner08b]

[Gerson-Gurwitz09]

[Gerson-Gurwitz11]

[Gheber99]

[Gilbert98]

[Glotzer01]

[Gordon99]
M. J. de Mattos, C. Kettner, J. Nielsen, H. V. Westerhoff and B. M. Bakker; 2010: Measuring enzyme activities under standardized in vivo-like conditions for systems biology, FEBS Journal, 277: 749760 .

K. Falley, J. Schütt, P. Iglauer, K. Menke, C. Maas, M. Kneussel, S. Kindler, F. S. Wouters, D. Richter and H. J. Kreienkamp; 2009: Shank1 mRNA: dendritic transport by kinesin and translational control by the 5'untranslated region, Traffic, 10: 844-857.

N. P. Ferenz, A. Gable and P. Wadsworth; 2010: Mitotic functions of kinesin-5, Seminars in Cell and Developmental Biology, 21: 255-259.

D. S. Forman, K. J. Brown, M. W. Promersberger and M. R. Adelman; 1984: Nucleotide specificity for reactivation of organelle movements in permeabilized axons, Cell Motility, 4: 121-128.

V. Fridman, A. Gerson-Gurwitz, N. Movshovich, M. Kupiec and L. Gheber; 2009: Midzone organization restricts interpolar microtubule plus-end dynamics during spindle elongation, EMBO Reports, 10: 387-393.

D. S. Friedman and R. D. Vale; 1999: Single-molecule analysis of kinesin motility reveals regulation by the cargo-binding tail domain, Nature Cell Biology, 1: 293-297.

M. K. Gardner, D. C. Bouck, L. V. Paliulis, J. B. Meehl, E. T. O'Toole, J. Haase, A. Soubry, A. P. Joglekar, M. Winey, E. D. Salmon, K. Bloom and D. J. Odde; 2008: Chromosome congression by Kinesin-5 motor-mediated disassembly of longer kinetochore microtubules, Cell, 135: 894-906.

M. K. Gardner, J. Haase, K. Mythreye, J. N. Molk, M. Anderson, A. P. Joglekar, E. T. O'Toole, M. Winey, E. D. Salmon, D. J. Odde and K. Bloom; 2008: The microtubule-based motor Kar3 and plus end-binding protein Bim1 provide structural support for the anaphase spindle, The Journal of Cell Biology, 180: 91-100.

A. Gerson-Gurwitz, N. Movshovich, R. Avunie, V. Fridman, K. Moyal, B. Katz, M. A. Hoyt and L. Gheber; 2009: Mid-anaphase arrest in $S$. cerevisiae cells eliminated for the function of Cin8 and dynein, Cellular and Molecular Life Science, 66: 301-313.

A. Gerson-Gurwitz, C. Thiede, N. Movshovich, V. Fridman, M. Podolskaya, T. Danieli, S. Lakämper, D. R. Klopfenstein, C. F. Schmidt and L. Gheber; 2011: Directionality of individual kinesin-5 Cin8 motors is modulated by loop 8, ionic strength and microtubule geometry, The EMBO Journal, 30: 4942-4954.

L. Gheber, S. C. Kuo and M. A. Hoyt; 1999: Motile properties of the kinesin-related Cin8p spindle motor extracted from Saccharomyces cerevisiae cells, The Journal of Biological Chemistry, 274: 9564-9572.

S. P. Gilbert, M. L. Moyer and K. A. Johnson; 1998: Alternating Site Mechanism of the Kinesin ATPase, Biochemistry, 37: 792-799.

M. Glotzer; 2001: Animal cell cytokinesis, Annual Review of Cell and Developmental Biology, 17: 351-386.

D. M. Gordon and D. M. Roof; 1999: The kinesin-related protein Kip1p of Saccharomyces cerevisiae is bipolar, The Journal of Biological Chemistry, 274: 28779-28786. 
[Goslin91]

[Guydosh06]

[Hackney88]

[Hackney91]

[Hackney92a]

[Hackney92b]

[Hackney94]

[Hackney95]

[Hackney00]

[Hackney03]

[Hackney05]

[Hackney07]

[Hackney08]

[Hackney09]

[Hagan90]

[Hagan92]

[Hancock98]
K. Goslin and G. Banker; 1991: Rat hippocampal neurons in low-density cultures, Culturing nerve cells from Banker and Goslin, $251-281$.

N. R. Guydosh and S. M. Block; 2006: Backsteps induced by nucleotide analogs suggest the front head of kinesin is gated by strain, PNAS (USA), 103: 8054-8059.

D. D. Hackney; 1988: Kinesin ATPase: rate-limiting ADP release, PNAS (USA), 85: 6314-6318.

D. D. Hackney, J. D. Levitt and D. D. Wagner; 1991: Characterization of $\alpha_{2} \beta_{2}$ and $\alpha_{2}$ forms of kinesin, The Journal of Biological Chemistry, 174: $810-815$.

D. D. Hackney; 1992: Kinesin and Myosin ATPases: Variations on a Theme, Philosophical Transactions of the Royal Society B: Biological Sciences, 336: 13-18.

D. D. Hackney, J. D. Levitt and J. Suhan; 1992: Kinesin Undergoes a 9-S to 6-S Conformational Transition, The Journal of Biological Chemistry, 267: 8696-8701.

D. D. Hackney; 1994: Evidence for alternating head catalysis by kinesin during microtubule-stimulated ATP hydrolysis, PNAS (USA), 91: 6865-6869.

D. D. Hackney; 1995: Highly processive microtubule-stimulated ATP hydrolysis by dimeric kinesin head domains, Nature, 377: 448-450.

D. D. Hackney and M. F. Stock; 2000: Kinesin's IAK tail domain inhibits initial microtubule-stimulated ADP release, Nature Cell Biology, 2: 257-260.

D. D. Hackney, M. F. Stock, J. Moore and R. A. Patterson; 2003: Modulation of kinesin half-site ADP release and kinetic processivity by a spacer between the head groups, Biochemistry, 42: $1211-1218$.

D. D. Hackney; 2005: The tethered motor domain of a kinesin-microtubule complex catalyzes reversible synthesis of bound ATP, PNAS (USA), 102: 18338-18343.

D. D. Hackney; 2007: Jump-starting kinesin, The Journal of Cell Biology, 176: 7-9.

D. D. Hackney and M. F. Stock; 2008: Kinesin tail domains and $\mathrm{Mg}^{2+}$ directly inhibit release of ADP from head domains in the absence of microtubules, Biochemistry, 47: 7770-7778.

D. D. Hackney, N. Baek and A. C. Snyder; 2009: Half-site inhibition of dimeric kinesin head domains by monomeric tail domains, Biochemistry, 48: 3448-3456.

I. Hagan and M. Yanagida; 1990: Novel potential mitotic motor protein encoded by the fission yeast cutrt gene, Nature, 347: $563-566$.

I. Hagan and M. Yanagida; 1992: Kinesin-related cut 7 protein associates with mitotic and meiotic spindles in fission yeast, Nature, 356: 74-76.

W. O. Hancock and J. Howard; 1998: Processivity of the Motor Protein Kinesin Requires Two Heads, The Journal of Cell Biology, 140: 1395-1405. 
[Hancock99]

[Hancock08]

[Hariharan09]

[Hartwell74]

[Heck93]

[Helenius06]

[Herpers04]

[Hildebrandt99]

[Hildebrandt06]

[Hirokawa89]

[Hirokawa09]

[Hollenbeck89]

[Horton04]

[Howard89]

[Howard93]

[Howard01]
W. O. Hancock and J. Howard; 1999: Kinesin's processivity results from mechanical and chemical coordination between the ATP hydrolysis cycles of the two motor domains, PNAS (USA), 96: $13147-13152$.

W. O. Hancock; 2008: Intracellular transport: kinesins working together, Current Biology, 18: 715-717.

V. Hariharan and W. O. Hancock; 2009: Insights into the Mechanical Properties of the Kinesin Neck Linker Domain from Sequence Analysis and Molecular Dynamics Simulations, Cellular and Molecular Bioengineering, 2: 177-189.

L. H. Hartwell; 1974: Saccharomyces cerevisiae Cell Cycle, Bacteriological Reviews, 38: 164-198.

M. M. Heck, A. Pereira, P. Pesavento, Y. Yannoni, A. C. Spradling and L. S. Goldstein; 1993: The kinesin-like protein KLP61F is essential for mitosis in Drosophila, The Journal of Cell Biology, 123: 665-679.

J. Helenius, G. Brouhard, Y. Kalaidzidis, S. Diez and J. Howard; 2006: The depolymerizing kinesin MCAK uses lattice diffusion to rapidly target microtubule ends, Nature, 441: 115-119.

B. Herpers and C. Rabouille; 2004: mRNA localization and ER-based protein sorting mechanisms dictate the use of transitional endoplasmic reticulum-golgi units involved in gurken transport in Drosophila oocytes, Molecular Biology of the Cell, 15: 5306-5317.

E. R. Hildebrandt and M. A. Hoyt; 1999: Mitotic motors in Saccharomyces cerevisiae, Biochimica et Biophysica Acta, 1496: 99116.

E. R. Hildebrandt, L. Gheber, T. Kingsbury and M. A. Hoyt; 2006: Homotetrameric form of Cin8p, a Saccharomyces cerevisiae kinesin-5 motor, is essential for its in vivo function, The Journal of Biological Chemistry, 281: 26004-26013.

N. Hirokawa, K. K. Pfister, H. Yorifuji, M. C. Wagner, S. T. Brady and G. S. Bloom; 1989: Submolecular domains of bovine brain kinesin identified by electron microscopy and monoclonal antibody decoration, Cell, 56: 867-878.

N. Hirokawa, Y. Noda, Y. Tanaka and S. Niwa; 2009: Kinesin superfamily motor proteins and intracellular transport, Nature Reviews Molecular Cell Biology, 10: 682-696.

P. J. Hollenbeck; 1989: The distribution, abundance and subcellular localization of kinesin, The Journal of Cell Biology, 108: 2335-2342.

A. C. Horton and M. D. Ehlers; 2004: Secretory trafficking in neuronal dendrites, Nature Cell Biology, 6: 585-591.

J. Howard, A. J. Hudspeth and R. D. Vale; 1989: Movement of microtubules by single kinesin molecules, Nature, 342: 154-158.

J. Howard, A. J. Hunt and S. Baek; 1993: Assay of microtubule movement driven by single kinesin molecules, Methods of Cell Biology, 39: 137-147.

J. Howard: Mechanics of Motor Proteins and the Cytoskeleton, chapter 14 (Sinauer Associates, 2001). 
[Hoyt92]

[Hoyt96]

[Hua97]

[Hua02]

[Huang94]

[Hyman91]

[Jacobs88]

[Jacobson06]

[Jiang06]

[Jones06]

[Kaan11]

[Kahana95]

[Kalchishkova08]

[Kapitein05]

[Kapitein08]

[Karsenti01]
M. A. Hoyt, L. He, K. K. Loo and W. S. Saunders; 1992: Two Saccharomyces cerevisiae kinesin-related gene products required for mitotic spindle assembly, The Journal of Cell Biology, 118: 109-120.

M. A. Hoyt and J. R. Geiser; 1996: Genetic analysis of the mitotic spindle, Annual Review of Genetics, 30: 7-33.

W. Hua, E. C. Young, M. L. Fleming and J. Gelles; 1997: Coupling of kinesin steps to ATP hydrolysis, Nature, 388: 390-393.

W. Hua, J. Chung and J. Gelles; 2002: Distinguishing inchworm and hand-over-hand processive kinesin movement by neck rotation measurements, Science, 295: 844-848.

T. G. Huang, J. Suhan and D. D. Hackney; 1994: Drosophila kinesin motor domain extending to amino acid position 392 is dimeric when expressed in Escherichia coli, The Journal of Biological Chemistry, 269: 16502-16507.

A. A. Hyman, D. Drexel, D. Kellog, S. Salser, K. Sawin, P. Steffen, L. Wordeman and T. J. Mitchison; 1991: Preparation of modified tubulins, Methods in Enzymology, 196: 478-485.

C. W. Jacobs, A. E. Adams, P. J. Szaniszlo and J. R. Pringle; 1988: Functions of microtubules in the Saccharomyces cerevisiae cell cycle, The Journal of Cell Biology, 107: 1409-1426.

C. Jacobson, B. Schnapp and G. A. Banker; 2006: A change in the selective translocation of the Kinesin-1 motor domain marks the initial specification of the axon, Neuron, 49: 797-804.

M. Jiang and G. Chen; 2006: High $\mathrm{Ca}^{2+}$-phosphate transfection efficiency in low-density neuronal cultures, Nature Protocols, 1: 695-700.

M. C. Jones, P. T. Caswell and J. C. Norman; 2006: Endocytic recycling pathways: emerging regulators of cell migration, Current Opinion in Cell Biology, 18: 549-557.

H. Y. Kaan, D. D. Hackney and F. Kozielski; 2011: The structure of the kinesin-1 motor-tail complex reveals the mechanism of autoinhibition, Science, 333: 883-885.

J. A. Kahana, B. J. Schnapp and P. A. Silver; 1995: Kinetics of spindle pole body separation in budding yeast, PNAS (USA), 92: 9707-9711.

N. Kalchishkova and K. J. Bohm; 2008: The role of kinesin neck linker and neck in velocity regulation, Journal of Molecular Biology, 382: 127-135.

L. C. Kapitein, E. J. G. Peterman, B. H. Kwok, J. H. Kim, T. M. Kapoor and C. F. Schmidt; 2005: The bipolar mitotic kinesin Eg5 moves on both microtubules that it crosslinks, Nature, 435: 114-118.

L. C. Kapitein, B. H. Kwok, J. S. Weinger, C. F. Schmidt, T. M. Kapoor and E. J. G. Peterman; 2008: Microtubule cross-linking triggers the directional motility of kinesin-5, The Journal of Cell Biology, 182: $421-428$.

E. Karsenti and I. Vernos; 2001: The mitotic spindle, a self-made machine, Science, 294: 543-547. 
[Kaseda08]

[Kashina96]

[Kashina97]

[Keating00]

[Kerssemakers09]

[Khmelinskii09]

[Klopfenstein02]

[Korneev07]

[Kozielski97]

[Krzysiak06]

[Kulik03]

[Kull96]

[Kural05]

[Kutys10]

[Kuznetsov89]
K. Kaseda, I. Crevel, K. Hirose and R. A. Cross; 2008: Single-headed mode of kinesin-5, The EMBO Journal, 9: 761-765.

A. S. Kashina, R. J. Baskin, D. G. Cole, K. P. Wedaman, W. M. Saxton and J. M. Scholy; 1996: A bipolar kinesin, Nature, 379: 270-272.

A. S. Kashina, G. C. Rogers and J. M. Scholey; 1997: The bimC family of kinesins: essential bipolar mitotic motors driving centrosome separation, Biochimica et Biophysica Acta, 1357: 257-271.

T. J. Keating and G. G. Borisy; 200: Immunostructural evidence for the template mechanism of microtubule nucleation, Nature Cell Biology, 2: 352-357.

J. Kerssemakers, L. Ionov, U. Queitsch, S. Luna, H. Hess and S. Diez; 2009: 3D nanometer tracking of motile microtubules on reflective surfaces, Small, 5: 1732-1737.

A. Khmelinskii, J. Roostalu, H. Roque, C. Antony and E. Schiebel; 2009: Phosphorylation-dependent protein interactions at the spindle midzone mediate cell cycle regulation of spindle elongation, Developmental Cell, 17: 244-256.

D. R. Klopfenstein, M. Tomishige, N. Stuurman and R. D. Vale; 2002: Role of phosphatidylinositol $(4,5)$ bisphosphate organization in membrane transport by the Unc104 kinesin motor, Cell, 109: $347-358$.

M. J. Korneev, S. Lakämper and C. F. Schmidt; 2007: Load-dependent release limits the processive stepping of the tetrameric Eg5 motor, European Biophysics Journal, 36: 675-681.

F. Kozielski, E. Schönbrunn, S. Sack, J. Müller, S. T. Brady and E. Mandelkow; 1997: Crystallization and Preliminary X-Ray Analysis of the Single-Headed and Double-Headed Motor Protein Kinesin, Journal of Structural Biology, 119: 28-34.

T. C. Krzysiak, T. Wendt, L. R. Sproul, P. Tittmann, H. Gross, S. P. Gilbert and A. Hoenger; 2006: A structural model for monastrol inhibition of dimeric kinesin Eg5, The EMBO Journal, 25: 2263-2273.

A. Kulik, I. Vida, R. Luján, C. A. Haas and G. López-Bendito; 2003: Subcellular localization of metabotropic $\mathrm{GABA}_{B}$ receptor subunits $\mathrm{GABA}_{B 1 a / b}$ and $\mathrm{GABA}_{B 2}$ in the rat hippocampus, The Journal of Neuroscience, 23: 11026-11035.

F. J. Kull, E. P. Sablin, R. Lau, R. J. Fletterick and R. D. Vale; 1996: Crystal structure of the kinesin motor domain reveals a structural similarity to myosin, Nature, 380: 550-555.

C. Kural, H. Balci and P. R. Selvin; 2005: Molecular motors one at a time: FIONA to the rescue, Journal of Physics: Condensed Matter, 17: 3979-3995.

M. L. Kutys, J. Fricks and W. O. Hancock; 2010: Monte Carlo analysis of neck linker extension in kinesin molecular motors, PLoS Computational Biology, 6: e1000980.

S. A. Kuznetsov, Y. A. Vaisberg, S. W. Rothwell, D. B. Murphy and V. I. Gelfand; 1989: Isolation of a 45-kDa fragment from the kinesin heavy chain with enhanced ATPase and microtubule-binding activities, The Journal of Biological Chemistry, 264: 589-595. 
[Kwok04]

[Kwok06]

[Lakämper03]

[Lakämper05]

[Lakämper06]

[Lakämper10]

[Lawrence04]

[Le Guellec91]

[Liu11]

[Luo04]

[Ma97]

[Maliga02]

[Margeta-Mitrovic00]
B. H. Kwok, J. G. Yang and T. M. Kapoor; 2004: The rate of bipolar spindle assembly depends on the microtubule-gliding velocity of the mitotic kinesin Eg5, Current Biology, 14: 1783-1788.

B. H. Kwok, L. C. Kapitein, J. H. Kim, E. J. G. Peterman, C. F. Schmidt and T. M. Kapoor; 2006: Allosteric inhibition of kinesin-5 modulates its processive directional motility, Nature Chemical Biology, 2: 480-485.

S. Lakämper: 2003: Characterization of the processivity of fast fungal kinesin, NKin, from Neurospora crassa, on the level of single molecules, Ph.D. thesis, Universität Hannover.

S. Lakämper and E. Meyhöfer; 2005: The E-Hook of Tubulin Interacts with Kinesin's Head to Increase Processivity and Speed, Biophysical Journal, 89: 3223-3234.

S. Lakämper and E. Meyhöfer; 2006: Back on track - on the role of the microtubule for kinesin motility and cellular function, Journal of Muscle Research and Cell Motility, 27: 161-171.

S. Lakämper, C. Thiede, A. Düselder, S. Reiter, M. J. Korneev, L. C. Kapitein, E. J. G. Peterman and C. F. Schmidt; 2010: The Effect of Monastrol on the Processive Motility of a Dimeric Kinesin-5 Head/Kinesin-1 Stalk Chimera, Journal of Molecular Biology, 399: 1-8.

C. J. Lawrence, R. K. Dawe, K. R. Christie, D. W. Cleveland, S. C. Dawson, S. A. Endow, L. S. B. Goldstein, H. V. Goodson, N. Hirokawa, J. Howard, R. L. Malmberg, J. R. McIntosh, H. Miki, T. J. Mitchison, Y. Okada, A. S. N. Reddy, W. M. Saxton, M. Schliwa, J. M. Scholey, R. D. Vale, C. E. Walczak and L. Wordeman; 2004: A standardized kinesin nomenclature, The Journal of Cell Biology, 167: 19-22.

R. Le Guellec, J. Paris, A. Couturier, C. Roghi and M. Philippe; 1991: Cloning by differential screening of a Xenopus cDNA that encodes a kinesin-related, Molecular and Cellular Biology, 11: 33953398 .

L. Liu, S. Parameswaran, J. Liu, S. Kim and E. J. Wojcik; 2011: Loop 5-directed compounds inhibit chimeric kinesin-5 motors: implications for conserved allosteric mechanisms, The Journal of Biological Chemistry, 286: 6201-6210.

L. Luo, J. D. Carson, D. Dhanak, J. R. Jackson, P. S. Huang, Y. Lee, R. Sakowicz and R. A. Copeland; 2004: Mechanism of inhibition of human KSP by monastrol: insights from kinetic analysis and the effect of ionic strength on KSP inhibition, Biochemistry, 43: $15258-15266$.

Y. Z. Ma and E. W. Taylor; 1997: Interacting head mechanism of microtubule-kinesin ATPase, The Journal of Biological Chemistry, 272 $724-730$.

Z. Maliga, T. M. Kapoor and T. J. Mitchison; 2002: Evidence that monastrol is an allosteric inhibitor of the mitotic kinesin Eg5, Chemistry and Biology, 9: 989-996.

M. Margeta-Mitrovic, Y. N. Jan and L. Y. Jan; 2000: Interacting head mechanism of microtubule-kinesin ATPase, Neuron, 27: 97-106. 
[Mayer99]

[McDonald90]

[Merianda09]

[Miller03]

[Mitchison01]

[Miyamoto04]

[Morris75]

[Movshovich08]

[Munro87]

[Muthukrishnan09]

[Nakata03]

[Nicklas83]

[Nitta08]

[Okada99]

[Olz93]

[O'Toole99]
T. U. Mayer, T. M. Kapoor, S. J. Haggarty, R. W. King, S. L. Schreiber and T. J. Mitchison; 1999: Small molecule inhibition of mitotic spindle bipolarity identified in a phenotype-based screen, Science, 286: 971-974.

H. B. McDonald, R. J. Stewart and L. S. Goldstein; 1990: The kinesin-like ncd protein of Drosophila is a minus end-directed microtubule motor, Cell, 63: 1159-1165.

T. T. Merianda, A. C. Lin, J. S. Lam, D. Vuppalanchi and D. E. Willis; 2009: A functional equivalent of endoplasmic reticulum and Golgi in axons for secretion of locally synthesized proteins, Molecular and Cellular Neuroscience, 40: 128-142.

E. A. Miller, T. H. Beilharz, P. N. Malkus, M. C. Lee and S. Hamamoto; 2003: Multiple cargo binding sites on the COPII subunit Sec24p ensure capture of diverse membrane proteins into transport vesicles, Cell, 114: 497-509.

T. J. Mitchison and E. D. Salmon; 2001: Mitosis: a history of division, Nature Cell Biology, 3: 17-22.

D. T. Miyamoto, Z. E. Perlman, K. S. Burbank, A. C. Groen and T. J. Mitchison; 2004: The kinesin Eg5 drives poleward microtubule flux in Xenopus laevis egg extract spindles, The Journal of Cell Biology, 167: 813-818.

N. R. Morris; 1975: Mitotic mutants of Aspergillus nidulans, Genetics Research, 26: 237-254.

N. Movshovich, V. Fridman, A. Gerson-Gurwitz, I. Shumacher, I. Gertsberg, A. Fich, M. A. Hoyt, B. Katz and L. Gheber; 2008: Slk19-dependent mid-anaphase pause in kinesin- 5-mutated cells, Journal of Cell Science, 121: 2529-2539.

S. Munro and H. R. Pelham; 1987: A C-terminal signal prevents secretion of luminal ER proteins, Cell, 48: 899-907.

G. Muthukrishnan, Y. Zhang, S. Shastry and W. O. Hancock; 2009: The processivity of kinesin-2 motors suggests diminished front-head gating, Current Biology, 19: 442-447.

T. Nakata and N. Hirokawa; 2003: Microtubules provide directional cues for polarized axonal transport through interaction with kinesin motor head, The Journal of Cell Biology, 162: 1045-1055.

R. B. Nicklas; 1983: Measurements of the force produced by the mitotic spindle in anaphase, The Journal of Cell Biology, 97: 542-548.

R. Nitta, Y. Okada and N. Hirokawa; 2008: Structural model for strain-dependent microtubule activation of $\mathrm{Mg}-\mathrm{ADP}$ release from kinesin, Nature Structural and Molecular Biology, 15: 1067-1075.

Y. Okada and N. Hirokawa; 1999: A processive single-headed motor: kinesin superfamily protein KIF1A, Science, 283: 1152-1157.

R. Olz, K. Larsson, L. Adler and L. O. Gustafsson; 1993: Energy flux and osmoregulation of Saccharomyces cerevisiae grown in chemostats under NaCl stress, Journal of Bacteriology, 175: 2205-2213.

E. T. O'Toole, M. Winey and J. R. McIntosh; 1999: High-voltage electron tomography of spindle pole bodies and early mitotic spindles in the yeast saccharomyces cerevisiae, Molecular Biology of the Cell, 10: 2017-2031. 
[Ramírez09]

[Ramírez10]

[Ramírez11]

[Rappaport67]

[Restituito05]

[Rice99]

[Rodríguez-Boulan05] E. Rodríguez-Boulan and A. Müsch; 2005: Protein sorting in the Golgi complex: shifting paradigms, Biochimica et Biophysica Acta, 1744: 455-464.

[Romberg98]

[Roof91]

[Roof92]

[Roostalu11]

[Rosenbaum02]

[Rosenfeld03]

[Rosenfeld05]

[Rosenfeld09]

O. A. Ramírez, R. L. Vidal, J. A. Tello, K. J. Vargas and S. Kindler; 2009: Dendritic Assembly of Heteromeric $\gamma$-Aminobutyric Acid Type B Receptor Subunits in Hippocampal Neurons, The Journal of Biological Chemistry, 284: 13077-13085.

O. A. Ramírez, A. García, R. Rojas, A. Couve and S. Härtel; 2010: Confined displacement algorithm determines true and random colocalization in fluorescence microscopy, Journal of Microscopy, 239: $173-183$

O. A. Ramírez and A. Couve; 2011: The endoplasmic reticulum and protein trafficking in dendrites and axons, Trends in Cell Biology, 21: $219-227$.

R. Rappaport; 1967: Cell division: direct measurement of maximum tension exerted by furrow of echinoderm eggs, Science, 156: $1241-1243$.

S. Restituito, A. Couve, H. Bawagan, S. Jourdain and M. N. Pangalos; 2005: Multiple motifs regulate the trafficking of $\mathrm{GABA}_{B}$ receptors at distinct checkpoints within the secretory pathway, Molecular and Cellular Neuroscience, 28: 747-756.

S. Rice, A. W. Lin, D. Safer, C. L. Hart, N. Naber, B. O. Carragher, S. M. Cain, E. Pechatnikova, E. M. Wilson-Kubalek, M. Whittaker, E. Pate, R. Cooke, E. W. Taylor, R. A. Milligan and R. D. Vale; 1999: A structural change in the kinesin motor protein that drives motility, Nature, 402: 778-784.

L. Romberg, D. W. Pierce and R. D. Vale; 1998: Role of the kinesin neck region in processive microtubule-based motility, The Journal of Cell Biology, 140: 1407-1416.

D. M. Roof, P. B. Meluh and M. D. Rose; 1991: Multiple kinesin-related proteins in yeast mitosis, Cold Spring Harbor Symposia on Quantitative Biology, 56: 693-703.

D. M. Roof, P. B. Meluh and M. D. Rose; 1992: Kinesin-related proteins required for assembly of the mitotic spindle, The Journal of Cell Biology, 118: 95-108.

J. Roostalu, C. Hentrich, P. Bieling, I. A. Telley, E. Schiebel and T. Surrey; 2011: Directional switching of the Kinesin Cin8 through motor coupling, Science, 332: 94-99.

J. L. Rosenbaum and G. B. Witman; 2002: Intraflagellar transport, Nature Reviews Molecular Cell Biology, 3: 813-825.

S. S. Rosenfeld, P. M. Fordyce, G. M. Jefferson, P. H. King and S. M. Block; 2003: Stepping and Stretching. How Kinesin uses internal strain to walk processively, The Journal of Biological Chemistry, 278: 1855018556.

S. S. Rosenfeld, J. Xing, G. M. Jefferson and P. H. King; 2005: Docking and Rolling, a Model of How the Mitotic Motor Eg5 Works, The Journal of Biological Chemistry, 280: 35684-35695.

S. S. Rosenfeld, M. van Duffelen, W. M. Behnke-Parks, C. Beadle, J. Corrreia and J. Xing; 2009: The ATPase cycle of the mitotic motor CENP-E, The Journal of Biological Chemistry, 284: 32858-32868. 
[Sablin98]

[Saunders92]

[Saunders95]

[Sawin92a]

[Sawin92b]

[Saxton88]

[Schief01]

[Schief04]

[Schnitzer97]

[Scholey03]

[Schuler01]

[Schuyler03]

[Seiler00]

[Shah10]

[Sharp99]

[Sharp00]
E. P. Sablin, R. B. Case, S. C. Dai, C. L. Hart, A. Ruby, R. D. Vale and R. J. Fletterick; 1998: Direction determination in the minus-end-directed kinesin motor ncd, Nature, 395: 813-816.

W. S. Saunders and M. A. Hoyt; 1992: Kinesin-related proteins required for structural integrity of the mitotic spindle, Cell, 70: $451-458$.

W. S. Saunders, D. Koshland, D. Eshel, I. R. Gibbons and M. A. Hoyt; 1995: Saccharomyces cerevisiae kinesin- and dynein-related proteins required for anaphase chromosome segregation, The Journal of Cell Biology, 128: 617-624.

K. E. Sawin, K. LeGuellec, M. Philippe and T. J. Mitchison; 1992: Mitotic spindle organization by a plus-end-directed microtubule motor, Nature, 359: 540-543.

K. E. Sawin, T. J. Mitchison and L. G. Wordeman; 1992: Evidence for kinesin-related proteins in the mitotic apparatus using peptide antibodies, Journal of Cell Science, 101: 303-313.

W. M. Saxton, M. E. Porter, S. A. Cohn, J. M. Scholey, E. C. Raff and J. R. McIntosh; 1988: Drosophila kinesin: characterization of microtubule motility and ATPase, PNAS (USA), 85: 1109-1113.

W. R. Schief and J. Howard; 2001: Conformational changes during kinesin motility, Current Opinion in Cell Biology, 13: 19-28.

W. R. Schief, R. H. Clark, A. H. Crevenna and J. Howard; 2004: Inhibition of kinesin motility by ADP and phosphate supports a hand-over-hand mechanism, PNAS (USA), 101: 1183-1188.

M. J. Schnitzer and S. M. Block; 1997: Kinesin hydrolyses one ATP per 8-nm step, Nature, 388: 386-390.

J. M. Scholey, I. Brust-Mascher and A. Mogilner; 2003: Cell division, Nature, 422: 746-752.

V. Schuler, C. Lüscher, C. Blanchet, N. Klix and G. Sansig; 2001: Epilepsy, hyperalgesia, impaired memory, and loss of preand postsynaptic $\mathrm{GABA}_{B}$ responses in mice lacking $\mathrm{GABA}_{B(1)}$, Neuron, 31: 47-58.

S. C. Schuyler, J. Y. Liu and D. Pellman; 2003: The molecular function of Ase1p: evidence for a MAP-dependent midzone-specific spindle matrix, The Journal of Cell Biology, 160: 517-528.

S. Seiler, J. Kirchner, C. Horn, A. Kallipolitou, G. Woehlke and M. Schliwa; 2000: Cargo binding and regulatory sites in the tail of fungal conventional kinesin, Nature Cell Biology, 2: 333-338.

J. V. Shah and D. W. Cleveland; 2010: Slow axonal transport: fast motors in the slow lane, Current Opinion in Cell Biology, 14: 58-62.

D. J. Sharp, K. L. McDonald, H. M. Brown, H. J. Matthies, C. Walczak, R. D. Vale, T. J. Mitchison and J. M. Scholey; 1999: The bipolar kinesin, KLP61F, cross-links microtubules within interpolar microtubule bundles of Drosophila embryonic mitotic spindles, The Journal of Cell Biology, 144: 125-138.

D. J. Sharp, G. C. Rogers and J. M. Scholey; 2000: Microtubule motors in mitosis, Nature, 407: 41-47. 
[Shastry10]

[Shastry11]

[Sherman86]

[Skiniotis04]

[Snow04]

[Soza04]

[Stock99]

[Stock03]

[Straight98]

[Svoboda93]

[Svoboda94]

[Tao06]

[Terada10]

[Thiede12a]
S. Shastry and W. O. Hancock; 2010: Neck Linker Length Determines the Degree of Processivity in Kinesin-1 and Kinesin-2 Motors, Current Biology, 20: 939-943.

S. Shastry and W. O. Hancock; 2011: Interhead tension determines processivity across diverse N-terminal kinesins, PNAS (USA), 108: $16253-16258$.

F. Sherman, J. B. Hicks and G. R. Fink; 1986: Methods in Yeast Genetics: A Laboratory Manual, Cold Spring Harbor Laboratory.

G. Skiniotis, J. C. Cochran, J. Müller, E. Mandelkow, S. P. Gilbert and A. Hoenger; 2004: Modulation of kinesin binding by the C-termini of tubulin, The EMBO Journal, 23: 989-999.

J. J. Snow, G. Ou, A. L. Gunnarson, M. R. Walker, H. M. Zhou, I. Brust-Mascher and J. M. Scholey; 2004: Two anterograde intraflagellar transport motors cooperate to build sensory cilia on C. elegans neurons, Nature Cell Biology, 6: 1109-1113.

A. Soza, A. Norambuena, J. Cancino, E. de la Fuente and P. Henklein; 2004: Sorting competition with membrane-permeable peptides in intact epithelial cells revealed discrimination of transmembrane proteins not only at the trans-Golgi network but also at pre-Golgi stages, The Journal of Biological Chemistry, 279: 1737617383.

M. F. Stock, J. Guerrero, B. Cobb, C. T. Eggers, T. G. Huang, X. Li and D. D. Hackney; 1999: Formation of the compact confomer of kinesin requires a $\mathrm{COOH}$-terminal heavy chain domain and inhibits microtubule-stimulated ATPase activity, The Journal of Biological Chemistry, 274: 14617-14623.

M. F. Stock, J. Chu and D. D. Hackney; 2003: The Kinesin Family Member BimC Contains a Second Microtubule Binding Region Attached to the N-terminus of the Motor Domain, The Journal of Biological Chemistry, 278: 52315-52322.

A. F. Straight, J. W. Sedat and A. W. Murray; 1998: Time-lapse microscopy reveals unique roles for kinesins during anaphase in budding yeast, The Journal of Cell Biology, 143: 687-694.

K. Svoboda, C. F. Schmidt, B. J. Schnapp and S. M. Block; 1993: Direct observation of kinesin stepping by optical trapping interferometry, Nature, 365: 721-727.

K. Svoboda and S. M. Block; 1994: Force and velocity measured for single kinesin molecules, Cell, 77: 773-784.

L. Tao, A. Mogilner, G. Civelekoglu-Scholey, R. Wollman, J. Evans, H. Stahlberg and J. M. Scholey; 2006: A Homotetrameric Kinesin-5, KLP61F, Bundles Microtubules and Antagonizes Ncd in Motility Assays, Current Biology, 16: 2293-2302.

S. Terada, M. Kinjo, M. Aihara, Y. Takei and N. Hirokawa; 2010: Kinesin-1/Hsc70-dependent mechanism of slow axonal transport and its relation to fast axonal transport, The EMBO Journal, 29.

C. Thiede, V. Fridman, A. Gerson-Gurwitz, L. Gheber and C. F. Schmidt; 2012: Regulation of bi-directional movement of single kinesin-5 Cin8 molecules, BioArchitecture, 2: 70-74. 
[Thiede12b]

[Thorn00]

[Tomishige02]

[Touitou01]

[Tsukita76]

[Turner01]

[Tytell06]

[Uemura02]

[Valdés12]

[Vale85]

[Vale89]

[Vale96]

[Vale00]

[Valentine06]

[Valentine07]
C. Thiede, S. Lakämper, A. D. Wessel, S. Kramer and C. F. Schmidt; 2012: A chimeric Kinesin-1/Kinesin-5 microtubule-sliding motor switches between diffusive and processive motility, Submitted to Biophysical Journal.

K. S. Thorn, J. A. Ubersax and R. D. Vale; 2000: Engineering the processive run length of the kinesin motor, The Journal of Cell Biology, 151: 1093-1100.

M. Tomishige, D. R. Klopfenstein and R. D. Vale; 2002: Conversion of Unc104/KIF1A kinesin into a processive motor after dimerization, Science, 297: 2263-2267.

I. Touitou, G. Lhomond and G. Pruliere; 2001: Boursin, a sea urchin bimC kinesin protein, plays a role in anaphase and cytokinesis, Journal of Cell Science, 114: 481-491.

S. Tsukita and H. Ishikawa; 1976: Three-dimensional distribution of smooth endoplasmic reticulum in myelinated axons, Journal of Electron Microscopy, 25: 141-149.

J. Turner, R. Anderson, J. Guo, C. Beraud, R. Fletterick and R. Sakowicz; 2001: Crystal Structure of the Mitotic Spindle Kinesin Eg5 Reveals a Novel Conformation of the Neck-linker, The Journal of Biological Chemistry, 276: 25496-25502.

J. D. Tytell and P. K. Sorger; 2006: Analysis of kinesin motor function at budding yeast kinetochores, The Journal of Cell Biology, 172: $861-874$.

S. Uemura, K. Kawaguchi, J. Yajima, M. Edamatsu, Y. Y. Toyoshima and S. Ishiwata; 2002: Kinesin-microtubule binding depends on both nucleotide state and loading direction, PNAS (USA), 99: 59775981 .

V. Valdés, J. I. Valenzuela, D. A. Salas, M. JaureguiberryBravo, C. Otero, C. Thiede, C. F. Schmidt and A. Couve; 2012: Endoplasmic reticulum sorting and kinesin-1 command the targeting of axonal $\mathrm{GABA}_{B}$ receptors, PLoS ONE, 7: e44168.

R. D. Vale, T. S. Reese and M. P. Sheetz; 1985: Identification of a novel force-generating protein, kinesin, involved in microtubule-based motility, Cell, 42: 39-50.

R. D. Vale, D. R. Soll and I. R. Gibbons; 1989: One-Dimensional Diffusion of Microtubules Bound to Flagellar Dynein, Cell, 59: 915-925.

R. D. Vale, T. Funatsu, D. W. Pierce, L. Romberg, Y. Harada and T. Yanagida; 1996: Direct observation of single kinesin molecules moving along microtubules, Nature, 380: 451-453.

R. D. Vale and R. A. Milligan; 2000: The way things move: looking under the hood of molecular motor proteins, Science, 288: 88-95.

M. T. Valentine, P. M. Fordyce and S. M. Block; 2006: Eg5 steps it up!, Cell Division, 1: 1-8.

M. T. Valentine and S. P. Gilbert; 2007: To step or not to step? How biochemistry and mechanics influence processivity in Kinesin and Eg5, Current Opinion in Cell Biology, 19: 75-81. 
[Valentine09]

[Varga06]

[Verhey98]

[Vidal07]

[Vigot06]

[Villemure05]

[Visscher99]

[Walczak96]

[Walker90]

[Wang00]

[Ward01]

[Wargacki10]

[Waterman-Storer98]

[Weclewicz93]

[Weclewicz98]
M. T. Valentine and S. M. Block; 2009: Force and premature binding of ADP can regulate the processivity of individual Eg5 dimers, Biophysical Journal, 97: 1671-1677.

V. Varga, J. Helenius, K. Tanaka, A. A. Hyman, T. U. Tanaka and J. Howard; 2006: Yeast kinesin-8 depolymerizes microtubules in a length-dependent manner, Nature Cell Biology, 8: 957-962.

K. J. Verhey, D. L. Lizotte, T. Abramson, L. Barenboim, B. J. Schnapp and T. A. Rapoport; 1998: Light Chain-dependent Regulation of Kinesin's Interaction with Microtubules, The Journal of Cell Biology, 143: 1053-1066.

R. L. Vidal, O. A. Ramírez, L. Sandoval, R. Koenig-Robert and S. Härtel; 2007: Marlin-1 and conventional kinesin link $\mathrm{GABA}_{B}$ receptors to the cytoskeleton and regulate receptor transport, Molecular and Cellular Neuroscience, 35: 501-512.

R. Vigot, S. Barbieri, H. Bräuner-Osborne, R. Turecek and R. Shigemoto; 2006: Differential compartmentalization and distinct functions of $\mathrm{GABA}_{B}$ receptor variants, Neuron, 50: 589-601.

J. F. Villemure, L. Adam, N. J. Bevan, K. Gearing and S. Chénier; 2005: Subcellular distribution of $\mathrm{GABA}_{B}$ receptor homo- and hetero-dimers, Biochemical Journal, 388: 47-55.

K. Visscher, M. J. Schnitzer and S. M. Block; 1999: Single kinesin molecules studied with a molecular force clamp, Nature, 400: 184189.

C. E. Walczak and T. J. Mitchison; 1996: Kinesin-related proteins at mitotic spindle poles: function and regulation, Cell, 85: 943946.

R. A. Walker, E. D. Salmon and S. A. Endow; 1990: The Drosophila claret segregation protein is a minus-end directed motor molecule, Nature, 347: 780-782.

Z. Wang and M. P. Sheetz; 2000: The C-Terminus of Tubulin Increases Cytoplasmic Dynein and Kinesin Processivity, Biophysical Journal, 78: $1955-1964$.

T. H. Ward, R. S. Polishchuk, S. Caplan, K. Hirschberg and J. Lippincott-Schwartz; 2001: Maintenance of Golgi structure and function depends on the integrity of ER export, The Journal of Cell Biology, 155: 557-570.

M. M. Wargacki, J. C. Tay, E. G. Muller, C. L. Asbury and T. N. Davis; 2010: Kip3, the yeast kinesin-8, is required for clustering of kinetochores at metaphase, Cell Cycle, 9: 2581-2588.

C. M. Waterman-Storer and E. D. Salmon; 1998: Endoplasmic reticulum membrane tubules are distributed by microtubules in living cells using three distinct mechanisms, Current Biology, 8: 798-806.

K. Weclewicz, K. Kristensson, H. B. Greenberg and L. Svensson; 1993: The endoplasmic reticulum-associated VP7 of rotavirus is targeted to axons and dendrites in polarized neurons, Journal of Neurocytology, 22: 616-626.

K. Weclewicz, L. Svensson and K. Kristensson; 1998: Targeting of endoplasmic reticulum-associated proteins to axons and dendrites in rotavirus-infected neurons, Brain Research Bulletin, 46: $353-360$. 
[Weinger11]

[Wildenberg08]

[Willis05]

[Winey95]

[Wittman01]

[Woehlke00]

[Woźniak09]

[Yardimci08]

[Yildiz04]

[Yildiz08]

[Yogev10]

[Zhang94]

[Zhu05]
J. S. Weinger, M. Qiu, G. Yang and T. M. Kapoor; 2011: A nonmotor microtubule binding site in kinesin-5 is required for filament crosslinking and sliding, Current Biology, 21: 154-160.

S. van den Wildenberg, L. Tao, L. C. Kapitein, C. F. Schmidt, J. M. Scholey and E. J. G. Peterman; 2008: The homotetrameric kinesin-5, KLP61F, preferentially cross-links microtubules into antiparallel orientations, Current Biology, 18: 1860-1864.

D. Willis, K. W. Li, J. Q. Zheng, J. H. Chang, A. Smit, T. Kelly, T. T. Merianda, J. Sylvester, J. van Minnen and J. L. Twiss; 2005: Differential transport and local translation of cytoskeletal, injury-response, and neurodegeneration protein mRNAs in axons, The Journal of Neuroscience, 25: 778-791.

M. Winey, C. L. Mamay, E. T. O'Toole, D. N. Mastronarde, T. H. Giddings, K. L. McDonald and J. R. McIntosh; 1995: Three-dimensional ultrastructural analysis of the Saccharomyces cerevisiae mitotic spindle, The Journal of Cell Biology, 129: 1601-1615.

T. Wittman, A. Hyman and A. Desai; 2001: The spindle, a dynamic assembly of microtubules and motors, Nature Cell Biology, 3: 28-34.

G. Woehlke and M. Schliwa; 2000: Walking on two heads: the many talents of kinesin, Nature Reviews Molecular Cell Biology, 1: 50-58.

M. J. Woźniak, B. Bola, K. Brownhill, Y. C. Yang and V. Levakova; 2009: Role of kinesin-1 and cytoplasmic dynein in endoplasmic reticulum movement in VERO cells, Journal of Cell Science, 122: 1979 1989.

H. Yardimci, M. van Duffelen, Y. Mao, S. S. Rosenfeld and P. R. Selvin; 2008: The mitotic kinesin CENP-E is a processive transport motor, PNAS (USA), 105: 6016-6021.

A. Yildiz, M. Tomishige, R. D. Vale and P. R. Selvin; 2004: Kinesin walks hand-over-hand, Science, 30: 676-678.

A. Yildiz, M. Tomishige, A. Gennerich and R. D. Vale; 2008: Intramolecular Strain Coordinates Kinesin Stepping Behavior along Microtubules, Cell, 134: 1030-1041.

S. Yogev, E. D. Schejter and B. Z. Shilo; 2010: Polarized Secretion of Drosophila EGFR Ligand from Photoreceptor Neurons Is Controlled by ER Localization of the Ligand-Processing Machinery, PLoS Biology, 8: e1000505.

C. J. Zhang, A. G. Rosenwald, M. C. Willingham, S. Skuntz and J. Clark; 1994: Expression of a dominant allele of human ARF1 inhibits membrane traffic in vivo, The Journal of Cell Biology, 124: 289-300.

C. Zhu, J. Zhao, M. Bibikova, J. D. Leverson, E. Bossy-Wetzel, J. B. Fan, R. T. Abraham and W. Jiang; 2005: Functional analysis of human microtubule-based motor proteins, the kinesins and dyneins, in mitosis/cytokinesis using RNA interference, Molecular Biology of the Cell, 16: 3187-3199. 



\section{Curriculum vitae}

Name: Christina Thiede

Nationality: German

Born: 20.11.1982 in Athens, Greece

PhD studies in Physics (since 2009)

Georg-August-Universität Göttingen

Third Institute of Physics - Biophysics

Group of Prof. C. F. Schmidt

$\mathrm{PhD}$ thesis: Single-molecule fluorescence experiments with motor proteins

Acceptance at the GGNB graduate school (2009)

Göttinger Graduate School for Neurosciences, Biophysics, and Molecular Biosciences (GGNB)

PhD program: Physics of Biological and Complex Systems

Diploma in Physics (2003-2008)

Georg-August-Universität Göttingen

Diploma thesis: Funktionsanalyse von Kinesin-Motormolekülen mittels EinzelmolekülFluoreszenz

Third Institute of Physics - Biophysics

Group of Prof. C. F. Schmidt

Work and travel visa (2002-2003)

Australia and New Zealand

Abitur (2002)

Gymnasium Johanneum Lüneburg 